
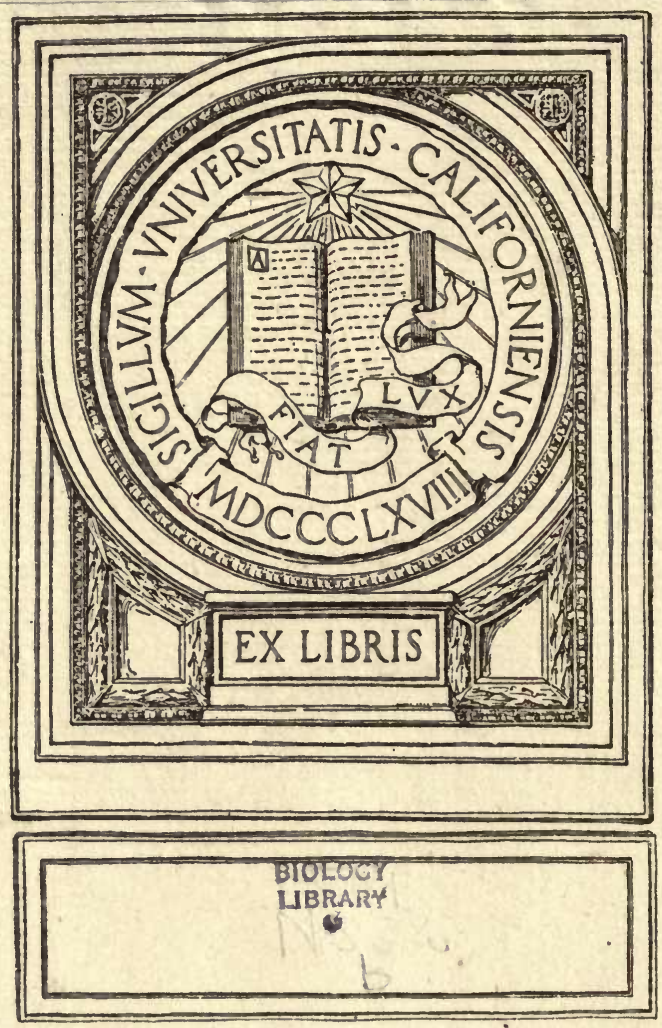


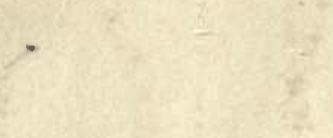




BACTERIOLOGY AND THE PUBLIC HEALTH 


\section{BACTERIOLOGY AND THE PUBLIC HEALTH}

BY GEORGE NEWMAN, M.D., F.R.S.E., D.P.H. II

FORMERLY DEMONSTRATOR OF BACTERIOLOGY IN KING'S COLLEGE, LONDON, ETC. MEDICAL OFFICER OF HEALTH OF THE METROPOLITAN BOROUGH OF FINSIURY JOINT-AUTHOR OF "BACTERIOLOGY OF MILK."

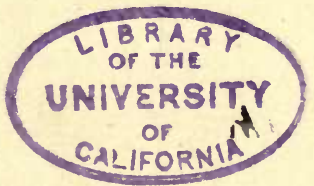

THIRD EDITION

PHILADELPHIA

P. BLAKISTON'S SON ANI CO. 


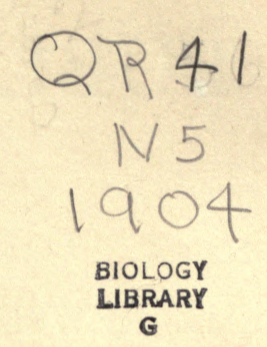




\section{PREFACE}

Though nominally a third edition of Bacteria in Relation to the Economy of Nature, Industrial Processes, and the Public Health, this is, speaking generally, a new book. Several new chapters have been added, and the whole has been enlarged and revised.

The book is an attempt to set forth a simple general statement of our present knowledge of bacteria, especially as they are related to the public health. Theoretical and practical text-books of bacteriology abound, but as a rule they deal largely, and rightly so, with laboratory methods and technique. The general student of hygiene and the medical officer of health require, however, an elementary book in which, whilst ample laboratory facts are recorded, the subject is viewed broadly and particularly as it concerns the practical everyday problems of health and preventive medicine. This book is aimed to meet that requirement.

I am indebted to many friends and colleagues for suggestions and criticisms, and for a number of illustrations. In addition to a number of clichés used in former editions, some of which were kindly lent by the Scientific Press, Limited, from the Atlas of Bacteriology, by Slater \& Spitta, I have to express my obligations to the Controller of His Majesty's Stationery Office, the Secretary of the Royal Commission on Sewage Disposal, and the Chairman of the Main Drainage Committee of the London County Council, for permission to use several blocks illustrating sewage bacteria derived from cultures obtained by my friend, Dr Houston, in the course of his sewage investigations. I am in a similar way much indebted to $\mathrm{Mr}$ 
Foulerton of the Middlesex Hospital, and Dr Harold Spitta of St George's Hospital, for the use of some excellent photographs. My colleague, Mr Harold Swithinbank, has kindly allowed me to use three coloured plates of "acid-fast" cultures from our book on the Bacteriology of Milk, and he has also supplied me with several original plates. To each of these gentlemen I am glad to have the opportunity of expressing my sincere thanks.

G. N.

London, August 1904. 


\section{INTRODUCTION}

THE science. of biology has for its object the study of organic beings, and for its end the knowledge of the laws of their growth, organisation, and function. From the earliest times of man that life has been studied and the observations recorded. Thus there has come slowly to be a considerable accumulation of knowledge concerning the various forms (morphology) and functions (physiology) of organised life. In the midst of this gradual accumulation of facts we begin to see incoherence becoming coherent, chaos becoming cosmos, and apparent chance and accident becoming law.

Bacteriology is a part, a chapter, of general biology, and is concerned with the facts, as at present known, of some of the lowest forms of micro-organic life. Owing to a variety of circumstances, the chief of which is the relation of these micro-organisms to disease, the study of bacteria has assumed a place among the branches of biology of somewhat exceptional importance. The application of biology to daily life and its problems has in recent years been nowhere more marked than in the realm of bacteriology, where the great names of Pasteur, Koch, and Lister, represent the modern epochs of advance. Turn where we will, we shall find the work of the unseen hosts of bacteria daily claiming more and more attention from practical people, and thus biology, even when concerned with the work of microscopic cells, is coming to occupy a new place in the minds of men. Its evolution begins to form part of the general social evolution.

Certainly the recent development of bacteriology forms a remarkable feature in the scientific advance of our time. Not only in the diagnosis and treatment of disease, nor even in the various applications of preventive medicine, but in every increasing degree and sphere micro-organisms are recognised as agents of good or ill no longer to be ignored. They occur in our drinking water, in our milk supply, in the air we breathe. They ripen cream, and flavour butter. They purify sewage, and remove waste organic products from the land. They are the active agents in a dozen industrial fermentations. They 
assist in the fixation of free nitrogen, and they build up assimilable compounds. Their activity assumes innumerable phases and occupies many spheres, probably more frequently proving itself beneficial than injurious, for bacteria are both economic and industrious in the best sense of the terms.

Yet bacteriology has its limitations. It is well to recognise this, for the new science has in some measure suffered in the past from over-zealous and sanguine friends. It cannot achieve everything demanded of it, nor can it furnish a causal agent for every disease to which human flesh is liable. It is a science which even yet is fuller of hope than of proved and established knowledge, for we are at present but upon the threshold of the matter. As in the neighbouring realm of chemistry, it is to be feared that bacteriology has not been without its alchemy. The interpretations and conclusions which have been drawn from time to time respecting bacteriological findings have led to alarmist or optimist views which have not, by later investigations, been fully confirmed. For the science has had devotees who have fondly believed, like the Alchemists, that the twin secret of "transmuting the baser metals into gold," and of indefinitely prolonging human life, was at last to be known. Neither the worst fears of the alarmist nor the sanguine hopes of the optimist have been verified. Science does not progress at such speed or with such kindly accommodation. It holds many things in its hand, but not finally life or death. It has not yet brought to light either "the philosopher's stone" or "the vital essence."

What has already been said affords ample reason for a wider dissemination of the elementary facts of bacteriological science. But there are other reasons of a more practical nature. Municipalities and other bodies are expending public moneys in water analysis, in the examination of milk and the control of its supply, in the inspection of cows and dairies, in the bacterial treatment of sewage, in protecting the oyster trade, in the ventilation of workshops and factories, in disinfection, in the prevention of epidemic diseases, and in other branches of public health administration. Furthermore, our increasing colonial possessions with their tropical diseases, and the growth of preventive medicine generally, make an increasing claim upon public opinion and those engaged in raising the physical condition of the people. The successful accomplishment and solution of these questions depends in measure upon a correct appreciation of the elements of bacteriology.

The present is a transition period in this department of knowledge. A very large body of facts has been collected, and there has been a natural tendency to draw somewhat sweeping deductions which subsequent knowledge has not supported. What is now required is that our experience in the laboratory and outside should be patiently 
and repeatedly checked and tested. If the science of bacteriology is to be built solidly, the two necessities of accumulating accurate facts and making generalisations and deductions must proceed side by side, the former being well established before the latter are accepted. It is the danger of a new science that too much is expected of it. Bacteriology, except in a few well-defined spheres, cannot yet stand alone as reliable basis for legislation. The bacteriologist must be content at present to serve as indicator rather than as dictator. The detection, for instance, of certain bacteria in milk or in oysters is an indication, and not an absolute proposition, of unsatisfactory dairying or oyster culture. Common sense and a broad view of all the ascertainable facts must guide those whose business it is to apply the findings of bacteriology to preventive measures.

In the pages that follow; a large number of statements occur as to the external circumstances and conditions affecting the life of bacteria, and to understand these rightly and hold them in right proportion to each other, it is necessary to bear in mind that many, if not most of them, are of relative importance. They are of value, not as isolated units, but as parts of a whole. It is their co-ordination, relativity, and correlation which must be sought after. Again, the presence of a diphtheria bacillus in the throat of a healthy man appears at first sight to be a fact of absolute and critical importance until the life-history of the bacillus is inquired into and determined, and the relation of the healthy tissues to the performance of its function understood. The bacteriologist and worker in preventive medicine can never afford to neglect the inter-relationship which exists between the seed and the soil. It is not wholly the one or the other with which he has to deal as a practical man. It is the combination and the inter-action between the two. If that principle, and the relativity of our knowledge of bacteria and the rôle which they play are borne in mind, there is little to fear from a transition period.

Whilst there can then be no doubt as to the advantage of a wide dissemination of the ascertained facts concerning bacteria, especially in relation to water, air, milk, and other foods, it must not be forgotten that only patient and skilled observation, and experimental research in well-equipped laboratories, can advance this branch of science or indeed train bacteriologists. The lives of Darwin and Pasteur adequately illustrate this truth. As the world learns its intimate relation to science, and the inter-dependence between its life and scientific truth, States and public authorities may be expected more heartily to support science. 



\title{
CONTENTS
}

\author{
CHAPTER I \\ THE BIOLOGY OF BACTERIA
}

Early work-Place of Bacteria in Nature-Biology of Bacteria; Morphology, Composition, Reproduction, Influence of External Conditions-Light -Modes of Bacterial Action-Seed and Soil-Specificity of Bacteria -Association, Antagonism, Attenuation-Bacterial Diseases of Plants

PAGE

\section{CHAPTER II}

\section{BACTERIA IN WATER}

Quantity of Bacteria in Water-Quality of Water Bacteria: (a) Ordinary Water Bacteria; (b) Sewage Bacteria; B. coli communis; (c) Pathogenic Bacteria in Water-Interpretation of the Findings of Bacteriology-Natural Purification of Water-Artificial Purification of Water -Sand Filtration-Domestic Purification of Water . . .

\section{CHAPTER III}

\section{BACTERIA IN THE AIR}

Methods of Examination of Air-Conditions of Bacterial Contamination of Air : (1) Dust and Air Pollution; (2) Moisture or Dampness of Surfaces : Bacteria in Sewer Air ; (3) the Influence of Gravity ; (4) Air Currents. The Relation of Bacteria to $\mathrm{CO}_{2}$ in the Atmosphere: in Workshops, in Bakehouses, in Railway Tubes, in the House of Commons . 73-91 


\section{CHAPTER IV}

\section{BACTERIA AND FERMENTATION}

Early Work-Kinds of Fermentation: (1) Alcoholic Fermentation, Ascospores, Pure Cultures, Films; (2) Acetous Fermentation; (3) Lactic Acid Fermentation; (4) Butyric Fermentation; (5) Ammoniacal Fermentation-Diseases of Wine and Beer: Turbidity, Ropiness, Bitterness, etc.-Industrial Applications of Bacterial Ferments

\section{CHAPTER V}

\section{BACTERIA IN THE SOIL}

Methods of Examination-Methods of Anaërobic Culture-Place and Function of Micro-organisms in Soil - Denitrification, Nitrification, Nitrogenfixation, Bacterial Symbiosis-Saprophytic and Pathogenic Organisms in Soil-Tetanus-Quarter-Evil-Malignant OEdema-The Relation of Soil to Bacterial Diseases, such as Typhoid Fever . . 116-150

\section{CHAPTER VI}

\section{THE BACTERIOLOGY OF SEWAGE AND THE BACTERIAL} TREATMENT OF SEWAGE

Composition of Sewage-Quantity and Quality of Bacteria in Sewage-Treatment of Sewage : (1) Disposal without Purification ; (2) Chemical Treatment; (3) Bacterial Treatment-Evolution of Bacterial Methods-Septic Tank Method-Contact-Bed Method-Manchester Experiments-Effect of Bacterial Treatment on Pathogenic Organisms

\section{CHAPTER VII}

\section{BACTERIA IN MILK AND MILK PRODUCTS}

General Principles-Sources of Pollution-Number of Bacteria in MilkInfluence of Time and Temperature-Species of Bacteria found in Milk -Fermentations of Milk-Pathogenic Organisms in Milk-Milk-borne Disease: Tuberculosis, Typhoid Fever, Scarlet Fever, Sore-Throat Illnesses, Cholera, Epidemic Diarrhœea-Preventive Measures-Protection of Milk Supply-Control of Milk Supply : Refrigeration, Straining, Sterilisation, Pasteurisation-Specialised Milk-Bacteria in Milk Products-Cream-Ripening-Butter-Making-Cheese-Making-Abnormal Cheese-Ripening-Poisonous Cheese . 


\section{CHAPTER VIII}

\section{BACTERIA IN OTHER FOODS}

1. Shell-fish, Oysters, Cockles, Clams, and their Relation to Disease; Symptoms of Oyster-borne Disease; Channels of Infection; Preventive Methods-2. Meat Poisoning; Tuberculous Meat-3. Ice-cream and Ice-4. Bacterial Infection of Bread-5. Miscellaneous Foods, Watercress, etc.

\section{CHAPTER IX}

\section{BACTERIA AND DISEASE}

Growth of Knowledge of Bacteria as Disease Producers-Channels of Infection-How Bacteria cause Disease-Diphtheria: Conditions of Infection-Scarlet Fever, Typhoid Fever, Epidemic Diarrhœa: Conditions of Infection-Suppuration and Abscess Formation-Anthrax-Pneumonia-Influenza-Actinomycosis-Glanders . . . 280-324

\section{CHAPTER X}

\section{TUBERCULOSIS AS A TYPE OF BACTERIAL DISEASE}

Pathology and Bacteriology of Tuberculosis-The Bacillus of Koch-Animal Tuberculosis, Bovine, Avian, etc. -Bovine and Human Tubercle Bacilli compared-Inter-communicability-Diagnosis of Bovine Tubercle-The Prevention of Tuberculosis-Pseudo-Tuberculosis-Acid-fast Bacteria Allied to the Tubercle Bacillus : in Man, in Animals, in Butter and Milk, in Grass-Differential Diagnosis-Streptothrix Group 325-369

\section{CHAPTER XI}

\section{THE ETIOLOGY OF TROPICAL DISEASES}

Malaria: Forms of Malarial Fever, the Mosquito Theory, Prevention of Malaria-Cholera: Methods of Diagnosis-Plague: Symptoms, Rats and Plague, Bacteriology, Administrative Considerations-LeprosyYellow Fever-Malta Fever-Sleeping Sickness-Beri-beri . 


\section{CHAPTER XII}

\section{THE QUESTION OF IMMUNITY AND ANTITOXINS}

Bacterial Products-Toxins-Question of Immunity-Kinds of ImmunityTheories of Immunity-Applications of Immunity-Vaccination for Small-pox : Effect of Vaccination-Pasteur's Treatment for RabiesInoculations for Cholera, Typhoid, and Plague-Antitoxin Treatment of Diphtheria and its Effects

\section{CHAPTER XIII}

\section{DISINFECTION}

General Principles-Means of Disinfection: by Heat; by ChemicalsPractical Disinfection: Rooms, Walls, Bedding, Clothing, Excreta, Books, Linen, Stables, etc.-Disinfection of Hands-Disinfection after Special Diseases : Phthisis, Small-pox, Scarlet Fever, Diphtheria, Typhoid, Plague

Appendix on Technique . . . . . . . . $453-488$ INDEX . . . . . . . . . . . . 489-497 


\section{LIST OF FIGURES}

FI(s.

PAGE

1. Various Forms of Bacteria $\quad . \quad$.

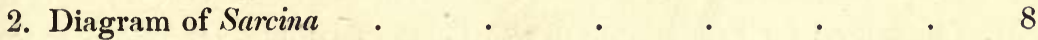

3. Diagrams of Normal and Polymorphic Forms of Tubercle Bacilli . . . . . . . $\quad$. 10

4. Various Forms of Spore Formation and Flagella _ . 13

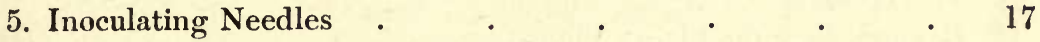

6. Media for Surface and Depth Culture . • . _ 17

7. Method of Producing Hydrogen by Kipp's Apparatus for Cultivation of Anaërobes . . . . 23

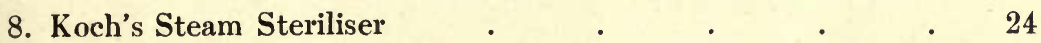

9. Diagrams of B. typhosus and B. coli . . . . . $\quad$. 47

10. Pasteur-Chamberland Filter . . . . . 71

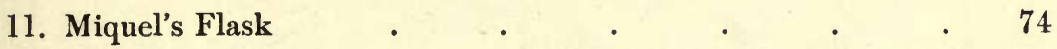

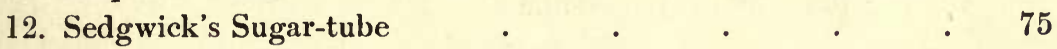

13. Diagram of Ascospore Formation ․ $\quad$ • $\quad$ • $\quad$. 98

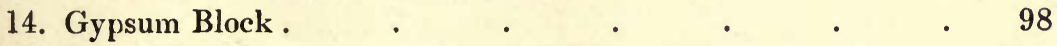

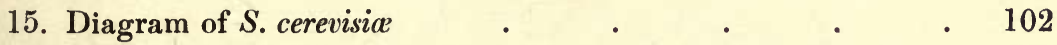

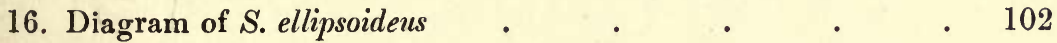

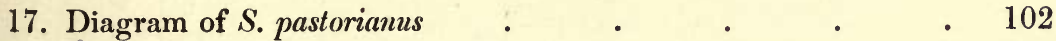

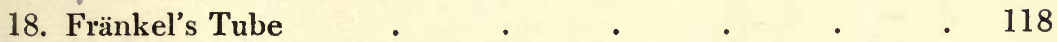

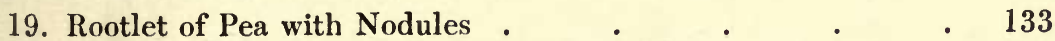

20. Diagram of Bacillus of Symptomatic Anthrax • • • 143

21. A Plan of Septic T'ank and Filter-beds . . . 167

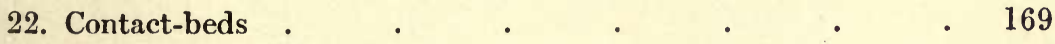

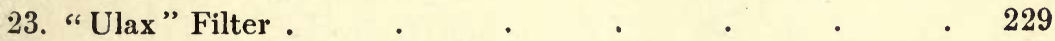

24. Diagram of Bacillus diphtheria . $\quad$. $\quad$. $\quad$ • $\quad 289$

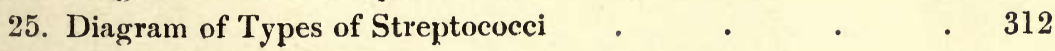
xvii 
FIG.

26. Diagram of Micrococcus tetragonus

27. Diagram of Gonococcus

28. Diagram of Bacillus of Anthrax and Blood Corpuscles.

29. Quartan Malaria Parasite

30. Tertian Malaria Parasite

31. Malignant Malaria Parasite

32. Anopheles maculipennis .

33. Diagram of Culex and Anopheles

34. Human and Mosquito Cycles of the Malaria Parasite

35. Diagram of the Comma Bacillus of Cholera

36. Suspended Spinal Cord

37. Flask used for Preparation of the Toxin of Diphtheria

374

38. Petri Dish

39. A Diagram of Colonies of Bacteria on a Gelatine Plate

40. The Hanging Drop

377

378

41. Drying Stage for Fixing Films

380

42. Types of Liquefaction of Gelatine

385

43. Levelling Apparatus for Koch's Plate

421

44. Moist Chamber for Koch's Plate

426

453

454

455

45. Wolfhügel's Counter

46. Filter-brushing Method

47. Buchner Tube .

48. Another Form of Buchner Tube 


\section{LIST OF PLATES}

[Note.-Photographs marked with an asterisk $\left({ }^{*}\right)$ have been kindly lent by the Scientific Press Company; those marked $\dagger$ are taken by permission from the Report of Royal Commission on Sewage Disposal; and those marked $\ddagger$ from Reports to the London County Council.]

PLATE

1. A Form of Room Temperature Incubator . - To face page 18

2. Hot-air Steriliser and Blood-heat Incubator 24

3. B. coli communis *; Proteus vulgaris ${ }_{\ddagger}$. . . 46

4. B. coli communis; Gas Production in Gelatine $\dagger$. " 50

5. Small Centrifuge; Sedgwick's Sugar-tube . 74

6. Air-Plate Culture from Labourer's Cottage 76

7. Air-Plate Cultures from Bakehouses 86

8. Saccharomyces cerevisiae; Ascospores; Pathogenic Yeast . . . . . "

9. Buchner's Tube ; Kipp's Apparatus for Anaërobic Culture

10. A Vacuum Method of Anaërobic Culture .

11. Nitrous Organism; Nitric Organism; Nitrogenfixing Organisms .. . $^{\circ}$

12. Nitrogen-fixing Bacteria in Nodule on Rootlet of Pea

13. B. tetani $^{*}$; B. mycoides ${ }^{*}$; Streptothrix actinomyces; B. mallei.

14. Sewage Proteus, Organism and Plate Culture $\ddagger$.

15. Sewage Streptococci $\downarrow$ and Streptococcus pyogenes* .

16. B. mesentericus, Organism and Plate Culture $\ddagger$

17. B. anthracis, from Septic Tank Liquor and in

18. B. tuberculosis (old culture); Tubercle Bacilli in 
PLATE

19. B. diphtheria ; B. von Hofinann To face page 288

20. B. typhosus ; B. typhosus (flagella)*; Widal-Grüber Reaction * ${ }^{*}$ B. typhosus in Human Mesenteric Gland . . . . . .

21. B. enteritidis sporogenes $\ddagger$; "Enteritidis change" in Milk Cultures $\dagger$

22. B. anthracis (Stab Culture) $\dagger ; B$. anthracis from Blood *; Fränkel's Pneumococcus . .

23. B. tuberculosis, from Sputum, ${ }^{*}$ Tissues, and Culture

24. Comparative Cultures of Tubercle Bacillus (Bird, Mammal, Butter) 318

25. Cultures of Butter Bacillus of Rabinowitsch and Mœller's Milk Bacillus (Chromo) . . ,

26. Cultures of B. friburgensis, Nos. I. and II. (Chromo)

27. Cultures of Butter Bacilli of Binot and Grassberger (Chromo)

28. Comparative Cultures of Acid-fast Bacteria (Grass

29. Streptothrix luteola (Foulerton); Streptothrix hominis

(Foulerton) . . . . . " 368

30. B. lepra, B. pestis *; Staphylococcus pyogenes aureus 398

31. Apparatus for Filter-brushing Method in Water Examination . 


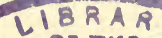

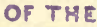 \\ UNIVERSITY \\ of \\ CALIFORNIS \\ BAC'TERIOLOGY AND PUBLIC HEALTH
}

\author{
CHAPTER I
}

\section{THE BIOLOGY OF BACTERIA *}

Early work-Place of Bacteria in Nature-Biology of Bacteria; Morphology, Composition, Reproduction, Influence of External Conditions-LightModes of Bacterial Action-Seed and Soil-Specificity of BacteriaAssociation, Antagonism, Attenuation-Bacterial Diseases of Plants.

THE first scientist who demonstrated the existence of micro-organisms was Antony von Leeuwenhoek. He was born at Delft, in Holland, in 1632, and enthusiastically pursued microscopy with primitive instruments. He corroborated Harvey's discovery of the circulation of the blood, in the web of a frog's foot; he defined the red blood corpuscles of vertebrates, the fibres of the lens of the human eye, the scales of the skin, and the structure of hair. He was neither educated nor trained in science, but in the leisure time of his occupation as a linen-draper he learned the art of grinding lenses, in which he became so proficient that he was able to construct a microscope of greater power than had been previously manufactured. The compound microscope dates from 1590, and when Leeuwenhoek

* We propose throughout to use the term bacterium (pl. bacteria) in its generic meaning, unless especially stated to the contrary. It will also be synonymous with the terms microbe, germ, and micro-organism. The term bacillus will, of course, be restricted to a rod-shaped bacterium. 
was about forty years old, Holland had already given to the world both microscope and telescope. Robert Hooke did for England what Hans Janssen had done for Holland, and established the same conclusion that Leeuwenhoek arrived at independently, viz., that a simple globule of glass mounted between two metal plates which were pierced with a minute aperture to allow rays of light to pass was a contrivance which would magnify more highly than the recognised microscopes of that day. It was with some such instrument as this that the first micro-organisms were observed in a drop of water. It was not until more than a hundred years later that these "animalcula," as they were termed, were thought to be anything more than accidental to any fluid or substance containing them. Plenciz, of Vienna, was one of the first to conceive the idea that decomposition could only take place in the presence of some of these "animalcula." This was in the middle of the eighteenth century. Just about a century later, by a series of important discoveries, it was established beyond dispute that these micro-organisms had an intimate causal relation to fermentation, putrefaction, and disease. Spallanzani, Pasteur, and Tyndall are the three workers who more than others contributed to this discovery. Spallanzani was an Italian who studied at Bologna, and was in 1754 appointed to the Chair of Logic at Reggio. But his inclinations led him into the realm of natural history. Amongst other things, his attention was directed to the doctrine of spontaneous generation, which had been propounded by Needham a few years previously. In 1768 Spallanzani became Professor of Natural History at Pavia, and whilst there he demonstrated that if infusions of vegetable matter were placed in flasks and hermetically sealed, and then brought to the boiling point, no living organisms could thereafter be detected, nor did the vegetable matter decompose. When, however, the flasks were but slightly cracked, the air gained admittance, then invariably both organisms and decomposition appeared. Schwann, the founder of the celltheory, and Schultze, both showed that if the air gaining access to the flask were either calcined or drawn through strong acid the result was the same as if no air entered at all, namely, there were no organisms and there was no decomposition. The result of these investigations was that scientific men began to believe that no form of life arose de novo (abiogenesis), but had its source in previous life (biogenesis). It remained for Pasteur and Tyndall to demonstrate this beyond dispute, and to put to rout the fresh argurnents for spontaneous generation which Pouchet had advanced as late as 1859 . Pasteur collected the floating dust of the air, and found by means of the microscope many organised particles, which he sowed on suitable infusions, and thus obtained rich crops of "animalcula." $\mathrm{He}$ also demonstrated that these organisms existed in varying 
degrees in different atmospheres, few in the pure air of the Mer de Glace, more in the air of the plains, most in the air of towns. He further proved that it was not necessary to insist upon hermetic sealing or cotton filters to keep these living organisms in the air from gaining access to a flask of infusion. If the neck of the flask were drawn out into a long tube and turned downwards, and then a little upwards, even though the end be left open, no contamination gained access. Hence, if the infusion were boiled, no putrefaction would occur. The organisms which fell into the open end of the tube were arrested in the condensation water in the angle of the tube; but even if that were not so, the force of gravity acting upon them prevented them from passing up the long arm of the tube into the neck of the flask. A few years after Pasteur's first work on this subject, Tyndall conceived a precise method of determining the absence or presence of dust particles in the air by passing a beam of sunlight through a glass box before and after its walls had been coated with glycerine. Into the floor of the box were fixed the mouths of flasks containing an infusion. These were boiled, after which they were allowed to cool, and might then be kept for weeks or months without putrefying or revealing the presence of germ life. Here all the conditions of the infusions were natural, except that in the air above them there was no dust.

The sum-total of result arising from these investigations was to the effect that no spontaneous generation was possible, that the atmosphere contained unseen germs of life, that the smallest of organisms responded to the law of gravitation and adhered to moist surfaces, and that micro-organisms were in some way or other the cause of putrefaction.

The final refutation of the hypothesis of spontaneous generation was followed by an awakened interest in the unseen world of microorganic life. Investigations into fermentation and putrefaction followed each other rapidly, and in 1863 Davaine claimed that Pollender's bacillus of anthrax, which was found in the blood and tissues of animals which had died of anthrax, was the cause of that disease. From that time to this, in every department of biology, bacteria have been increasingly found to play an important part. They cause changes in milk, and flavour butter; they decompose animal matter, yet build up the broken-down elements into compounds suitable for use in nature's economy; they assist in the fixation of free nitrogen; they purify sewage; in certain wellestablished cases they are the cause of specific disease, and in many other cases they are the probable cause. No doubt the disposal of spontaneous generation did much to arouse interest in this branch of science. Yet it must not be forgotten that the advance of the 
microscope and bacteriological method and technique have played a large share in this development. The sterilisation of culture fluids by heat, the use of aniline dyes as staining agents, the introduction of solid culture media (such as gelatine and agar), and Koch's "plate" method, have all contributed not a little to the enormous advance of bacteriology.

\section{The Place of Bacteria in Nature}

As we have seen, for a considerable period of time after their first detection these unicellular organisms were considered to be members of the animal kingdom. As late as 1838, when Ehrenberg and Dujardin drew up their classification, bacteria were placed among the Infusorians. This was in part due to the powers of motion which these observers detected in bacteria. It is now, of course, recognised that animals have no monopoly of motion. But what, after all, are the differences between animals and vegetables so low down in the scale of life? Chiefly two: there is a difference in life-history (in structure and development), and there is a difference in pabulum. A plant secures its nourishment from much simpler elements than is the case with animals; for example, it obtains its carbon from the carbonic acid gas in air and water. This it is able to do, as regards the carbon, by means of the green colouring matter known as chlorophyll, by the aid of which, with sunlight, carbonic acid is decomposed in the chlorophyll corpuscles, the oxygen passing back into the atmosphere, the carbon being stored in the plant in the form of starch or other organic compound. The supply of carbon in the chlorophyll-free plants, amongst which are the bacteria, is obtained by breaking up different forms of carbohydrates. Beside albumen and peptone, they use sugar and similar carbohydrates and glycerine as a source of carbon. Many of them also have the capacity of using organic matters of complex constitution by converting such into water, carbonic acid gas, and ammonia. Their hydrogen comes from water, their nitrogen from the soil, chiefly in the form of nitrates. From the soil, too, they obtain other necessary salts. Now all these substances are in elementary conditions, and as such plants can absorb them. Animals, on the other hand, are only able to utilise compound food products which have been, so to speak, prepared for them, for example albuminoids and proteids. They cannot directly feed upon the elementary substances forming the diet of vegetables. This distinction, however, did not at once clear up the difficult matter of the classification of bacteria. It is true, they possess powers of motion, are free from chlorophyll, and even feed occasionally upon products of decomposition-three physiological characters which 
would ally them to the animal kingdom. Yet by their structure and capsule of cellulose and by their life-history and mode of growth they unmistakably proclaim themselves to be of the vegetable kingdom. In 1853 Cohn arrived at a conclusion to this effect, and since that date bacteria have become more and more limited in classification and restricted in definition.

Even yet, however, we are far from a scientific classification of bacteria. Nor is this matter for surprise. The development in this branch of biology has been so rapid that it has been impossible to assimilate the facts collected. The facts themselves by their remarkable variety have not aided classification. Names which a few years ago were applied to individual species, like Bacillus subtilis, or Bacterium termo, or Bacillus coli, are now representative, not of individuals, but of families and species. Again, isolated characteristics of certain microbes such as motility, power of liquefying gelatine, size, colour, and so forth, which at first sight might appear as likely to form a basis for classification, are found to vary not only between similar germs, but in the same germ. Different physical conditions have so powerful an influence upon these microscopic cells that their individual characters are constantly undergoing change. For example, bacteria in old cultures assume a different size, and often a different shape, from younger members of precisely the same species; Bacillus pyocyaneus produces a green to olive colour on gelatine, but a brown colour on potato; the bacillus of Tetanus is virulently pathogenic, and yet may not act thus unless in company with certain other micro-organisms. Hence it will at once appear to the student of bacteriology that, though there is great need for classification amongst the six or seven hundred named "species" of microbes, our present knowledge of their life-history is not yet advanced enough to form more than a provisional arrangement.

We know that bacteria are allied to Hyphomycetes on the one hand and Saccharomycetes on the other, and that they have no differentiation into root, stem, or leaf; we know that they are fungi (having no chlorophyll), in which no sexual reproduction occurs, and that their mode of multiplication is by division. From such facts as these we may build up a classification as follows:- 


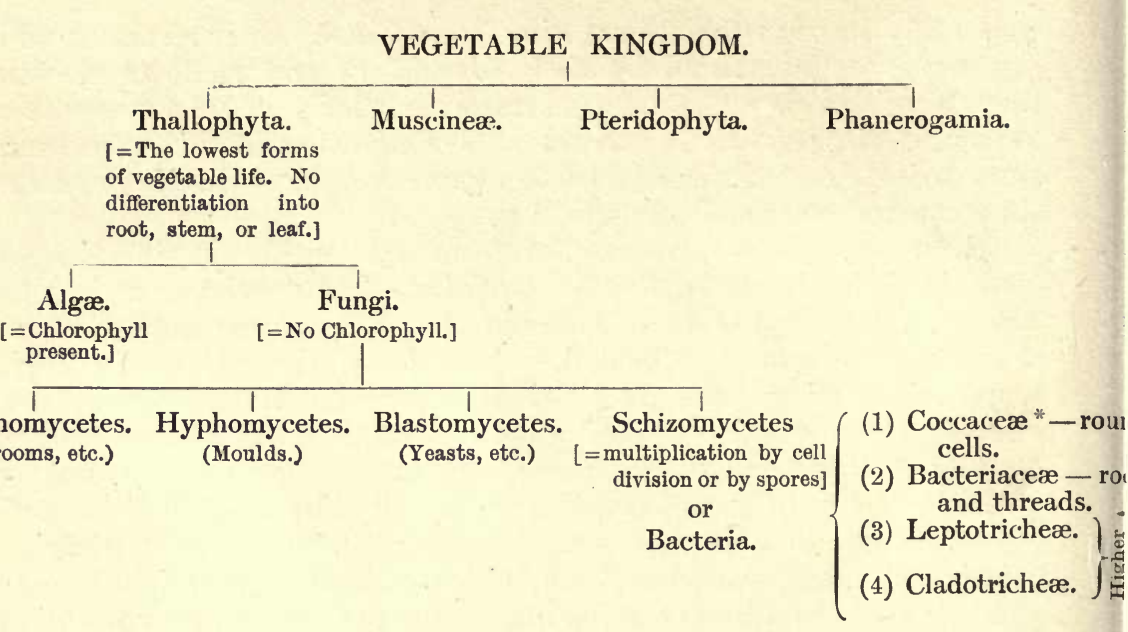

* Migula has suggested that the Schizomycetes should be subdivided into Coccacece, Bacteriacece, Spirillac (spirilla, spirochœeta), Chlamydobacteriacece (Streptothrix, Crenothrix, Cladothrix), and Beggiatoa.

\section{Morphology : Structure and Form}

Having now located micro-organisms in the economy of nature, we may proceed to describe their subdivisions and form. For practical convenience rather than theoretical accuracy, we may accept the simple division of the family of bacteria into three chief forms, viz. :-
Lower Bacteria $\left\{\begin{array}{l}\text { (1) Round cell form-coccus. } \\ \text { (2) Rod form-bacillus. } \\ \text { (3) Thread form-spirillum. }\end{array}\right.$

Higher Bacteria-Leptothrix, Streptothrix, Cladothrix, etc.

A classification dependent as this is upon the form alone is not by any means ideal, for it ignores all the complicated functions of bacteria, but it is, as we have said, practically convenient.

1. The Coccus.-This is the group of round cells. They vary in size as regards species, and as regards the conditions, artificial or natural, under which they have been grown. Some are less than $\frac{1}{25000}$ of an inch in diameter; others are half as large again, if the word large may be used to describe such minute objects. No regular standard can be laid down as reliable with regard to their size. Hence the subdivisions of the cocei are dependent not upon the individual elements so much as upon the relation of those elements to each other. A simple round cell of approximately the size already

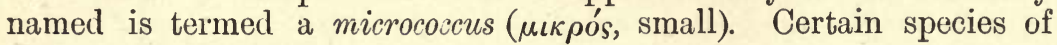


micrococci always or almost always occur in pairs, and such a combination is termed a diplococcus. Some diplococci are united by a thin capsule, which may be made apparent by special methods of staining; in others no limiting or uniting membrane can be seen with the ordinary high powers of the microscope. Again, one frequently finds a species which is exactly described by saying that two micrococci are in contact with each other, and move and act as one individual, but otherwise show no alteration; whilst others are seen which show a flattening of the side of each micrococcus which is in relation to its partner. Perhaps the diplococci in an even greater degree than the micrococci respond to external conditions both as regards size and shape. It must. further be borne in mind that a dividing micrococcus assumes the exact appearance of a diplococcus during the transition stage of the fission. Hence, with the exception of several well-marked species of diplococci, this form is somewhat arbitrary. The third kind of micrococcus is that formed by a number of elements in a twisted chain, named streptococcus ( $\sigma \tau \rho \epsilon \pi \tau o ́ s$, twisted). This form is produced by cells dividing in one axis, and remaining in contact with each other. It occurs in a number of different species,
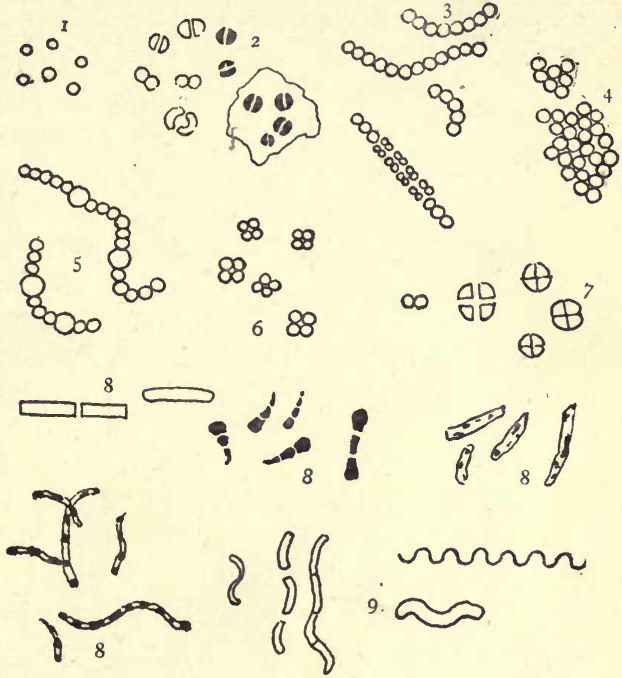

Fig. 1.-Diagrams of Various forms of Bacteria

1. Micrococcus.

2. Diplococcus.

3. Streptococcus.
7. Sarcina.

8 Bacillus.

9. Spirillum.

\section{Staphylococcus.}

5. Leuconostoc, show. ing Arthrospores.

6. Merismopedia. or what are supposed by many authorities to be different species, owing to their different effects. Morphologically all the streptococci are similar, though a somewhat abortive attempt has been made to divide them into two groups, according as to whether they were long chains or short. As a matter of fact, the length of streptococci depends in some cases upon biological properties, in others upon external treatment or the medium of cultivation which has been used. Sometimes they occur as straight chains of only half a dozen elements; at other times they may contain thirty or forty elements, and twist in various ways, even forming rosaries. The elements, too, differ not only in size, but in shape, appearing occasionally as oval 
cells united to each other at their sides. The fourth form is constituted by the micrococci being arranged in masses like grapes, the staphylococcus ( $\sigma \tau \check{\alpha} \phi \breve{u} \lambda i_{s}$, a bunch of grapes). The elements are often smaller than in the streptococcus, and the name itself describes the arrangement. There is no matrix and no capsule. This is the commonest organism found in abscesses, etc. The sarcina is best

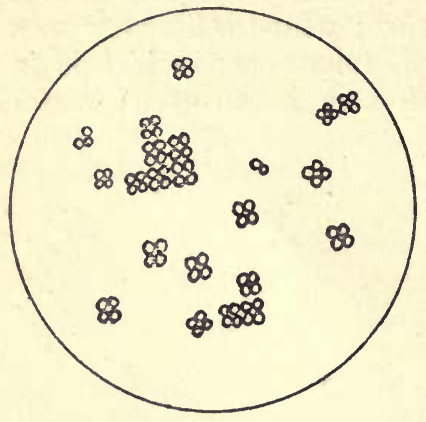

Fig. 2.-Diagram of Sarcina. classified amongst the cocci, for it is composed of them, in packets of four or multiples of four, produced by division vertically in two planes. If the division occurs in one plane, we have as a result small squares of round cells known as merismopedia. In both these conditions it frequently happens that the contiguous sides of the elements of packets become faceted or straightened against each other. It may happen, too, particularly in the sarcino, that segmentation is not complete, and that the elements are larger than in any other class of cocci. They stain very readily. Nearly all the cocci are non-motile, though Brownian movement (see p. 11) may readily be observed.

2. The Bacillus.-This group consists of rods, having parallel sides and being longer than they are broad. They differ in every other respect according to species, but these two characteristics remain to distinguish them. Many of them are motile, others not. The ends or poles of a bacillus may be pointed, round, or almost exactly square and blocked. They all, or nearly all, possess a capsule. Individuals of the same species may differ greatly, according to whether they have been naturally or artificially grown, and pleomorphic forms are abundant.

3. The Spirillum.-This wavy-thread group is divisible into a number of different forms, to which authorities have given special names. It is sufficient, however, to state that the two common forms are the non-septate spiral thread (e.g. the Spirillum Obermeier of relapsing fever), which takes no other form but a lengthened spirillum; and the spirillum which breaks up into elements or units, each of which appears comma-shaped (e.g. the cholera bacillus). The degree of curvature in the spirilla, of course, varies. They are the least important of the lower bacteria.

The Higher Bacteria group includes more highly organised members of the Schizomycetes. They possess filaments, which may be branched, and almost always have septa and a sheath. Perhaps the most marked difference from the lower bacteria is in their 
reproduction. In the higher bacteria we may have what is in fact a flower-terminal fructification by conidia. In this group of vegetables we have the Beggiatoa, Leptothrix, Cladothrix, and, at the top, the Streptothrix. It has been demonstrated that Streptothrix actinomycotica and Streptothrix madurce are the organismal cause, respectively, of Actinomycosis and Madura-foot, two diseases which had hitherto been obscure.

Polymorphism (or Pleomorphism).- This term is used to designate an inconstancy of form or a tendency towards biological variation. Vibrios may become spirilla, the ray fungus passes through a coccoid and bacillary stage, and the diphtheria bacillus may either be long, short, straight, or clubbed. This diversity of form appears to belong to many species, and is transmitted from generation to generation; or the various forms may occur in succession, and represent different stages in the life-history. In B. diphtherioe, B. pestis, and B.tuberculosis and other forms, polymorphism undoubtedly occurs. It is particularly marked in very old cultures of the last named. The ordinary well-known bacillus may grow out into threads with bulbous endings, granular filaments, "drumsticks," and diplococcal forms. It is now known that amongst the causes of polymorphism are certain adverse conditions of medium or other physical influences (moisture, temperature, age, etc.), and thus some bacteria, especially bacilli or vibrios, become altered in shape, losing their ordinary form. On transferring such aberrant and abnormal forms to fresh medium or favourable conditions, they are generally able to assume their original morphology. Indeed the aberrant form is in all probability only a stage in their life-history. Involution forms usually imply degeneration.

\section{Biology of Bacteria}

Composition.-From what we have seen of the pabulum of micro-organisms, we should conclude that in some form or other they contain the elements nitrogen, carbon, and hydrogen. All three substances are combined in the mycoprotein or protoplasm of which the body of the microbe consists. This is generally homogeneous, proteid material, and there is no sign of a nucleus. It possesses a marked affinity for aniline dyes, and by this means organisms are stained for the microscope. Besides the variable quantity of nitrogen present, mycoprotein may also contain various mineral salts. The uniformity of the cell-protoplasm may be materially affected by disintegration and segmentation due to degenerative changes. Vacuoles, which it is necessary to differentiate from spores, also may appear from a like cause. Vacuolation may also occur as a result of a process of osmosis in salt solutions, the protoplasm of the bacillus becoming contracted and disintegrated (plasmolysis). 
Two other signs of degeneration are the appearance of granules in the body of the cell-protoplasm known as metachromatic granules, owing to their different staining propensities, and the polar bodics which are seen in some species of bacteria. Surrounding the mass of mycoprotein, we find in most organisms a capsule or membrane composed, in part at least, of cellulose. This sheath plays a protective part in several ways. During the adult stage of life it protects the mycoprotein, and holds it together. At the time of reproduction or degeneration it not infrequently swells up, and forms a viscous hilum or matrix, inside which are formed the new sheaths of the younger generation. It may be rigid, and so maintain the normal shape of the species, or, on the other hand, flexible, and so adapted to rapid movement of the individual.

Here, then, we have the major parts in the constitution of a bacillus-its body, mycoprotein; its capsule, cellulose. But, further
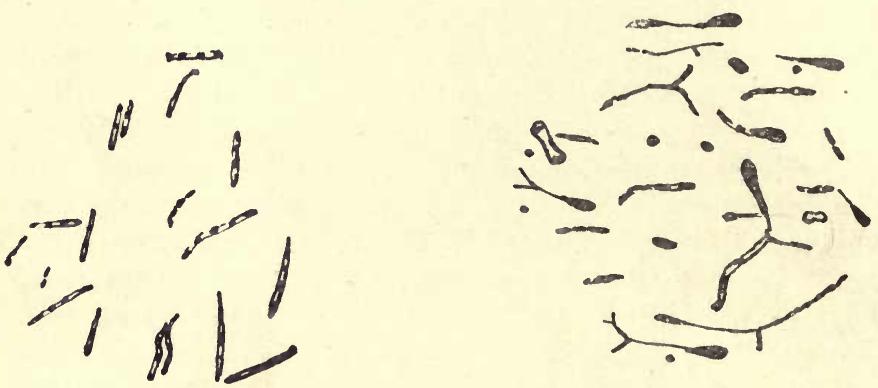

FIG. 3.-Diagrams of Normal and Polymorphic Forms of Tubercle Bacilli.

than this, there are a number of additional distinctive characteristics as regards the contents inside the capsule which call for mention. Sulphur occurs in the Beggiatoa which thrive in sulphur springs. Starch is commoner still. Iron as oxide or other combination is found in several species. Many contain pigments, though these are generally the "innocent" bacteria, in contradistinction to the disease-producing. A pigment has been found which is designated bacterio-purpurin. According to Zopf, the colouring agents of bacteria are the same as, or closely allied to, the colouring matters occurring widely in nature. Migula holds that most of the bacterial pigments are non-nitrogenous bodies. There are a very large number of chromogenic bacteria, some of which produce exceedingly brilliant colours. Among some of the commoner forms possessing this character are Bacillus et micrococcus violaceus, $B$. et $M$. aurantiacus (orange); B. et $M$. luteus; $M$. roseus (pink); many of the Sarcino; $B$. aureus; $B$. fluorescens liquefaciens et 
non-liquefaciens (green); B. pyocyaneus (green); B. prodigiosus (blood-red).

Motility.-When a drop of water containing bacteria is placed upon a slide, a clean cover-glass superimposed, and the specimen examined under an oil immersion lens, various rapid movements will generally be observed in the micro-organisms. These are of four chief kinds: (1) A dancing, stationary motion known as Brownian movement. This is molecular, and depends in some degree upon heat and the medium of the moving particles. It is non-progressive, and is well seen in gamboge particles. (2) An undulatory, serpentine movement, with apparently little advance being made. (3) A rotatory movement, which in some water bacilli is very marked, and consists of spinning round, sometimes with considerable velocity, and maintained for some seconds or even minutes. (4) A progressive, darting movement, by which the bacillus passes over some considerable distance.

The conditions affecting the motility of bacteria are but partly understood. Heating the slide or medium accelerates all movement. A fresh supply of oxygen, or indeed the addition of some nutrient substance, like broth, will have the same effect. There are also the somewhat mysterious powers by which cells possess inherent attraction or repulsion for other cells, known as positive and negative chemiotaxis. These powers have been observed in bacteria by Pfeiffer and Ali-Cohen.

The essential condition in the motile bacilli is the presence of flagella.* These cilia, or hairy processes, project from the sides or from the ends of the rod, and are freely motile and elastic. Sometimes only one or two terminal flagella are present; in other cases, like the bacillus of typhoid fever, five to twenty may occur all round the body of the bacillus, varying in length and size, sometimes being of greater length even than the bacillus itself. It is not yet established as to whether these cilia are prolongations of capsule only, or whether they contain something of the body protoplasm. Migula holds the former view, and states that the position of flagella is constant enough for diagnostic purposes. They are but rarely recognisable except by means of special staining methods. Micrococcus agilis (Ali-Cohen) is one of the rare cases of a coccus which has flagella and powers of active motion.

Modes of Reproduction.-Budding, division, and spore formation are the three chief ways in which Schizomycetes and Saccharomycetes (yeasts) reproduce their kind. Budding occurs in many kinds of yeast-cells, and generally takes place when the nutriment and

A Alagellum is a hair-like process arising from the poles or sides of the bacillus. It must not be confused with a filament, which is a thread-like growth of the bacillus itself. 
environment are favourable. The capsule of a large, or "mother" cell, shows a slight protrusion outwards, which is gradually enlarged into a "daughter" yeast, and later on becomes constricted at the neck. Eventually it separates as an individual. The protoplasm of the spores of yeasts differs, as Hansen has pointed out, according to the conditions of culture.

Division, or fission, is the commonest method of reproduction. It occurs transversely. A small indentation occurs in the capsule, which appears to make its way slowly through the whole body of the bacillus or micrococcus until the two parts are separate, and each contained in its own capsule. It has been pointed out already that in the incomplete division of micrococci we observe a stage precisely similar to a diplococcus. So also in the division of bacilli an appearance occurs described as a diplobacillus.

Simple fission requires but a short period of time to be complete. Hence multiplication is very rapid, for within half an hour a new adult individual can be produced. It has been estimated that at this rate one bacillus will in twenty-four hours produce millions of similar individuals; or, expressed otherwise, Cohn calculated that in three days, under favourable circumstances, the rate of increase would be such as to form a mass of living organisms weighing many tons, and numboring billions of individuals. Favourable conditions do not occur, fortunately, to allow of such increase, which, it is evident, can only be roughly estimated. But the above facts illustrate the enormous fertility of micro-organic life. When we remember that in some species it requires 10,000 or 15,000 fully-grown bacilli placed end to end to stretch the length of an inch, we see also how exceedingly minute are the individuals composing these unseen hosts.

Spore formation may result in the production of germinating cells inside the capsule of the bacillus, endospores, or as modified individuals, arthrospores. The body of a bacillus, in which sporulation is about to occur, loses its homogeneous character and becomes granular, owing to the appearance of globules in the protoplasm. In the course of three or four hours the globule enlarges to fill the diameter of the rod, and assumes a more concentrated condition than the parent cell. At its maturity, and before its rupture of the bacillary capsule, a spore is observed to be bright and shining, oval and regular in shape, with concentrated contents, and frequently causing a local expansion of the bacillus. In a number of rods lying endwise, these local swellings produce a beaded or varicose appearance, even simulating a streptococcus. In the meantime the rod itself has become slightly broader and pale. Eventually it breaks down by segmentation or by swelling up into a gelatinous mass. The spore now escapes and commences its individual existence. Under favourable circumstances it will germinate. The tough capsule gives way at one point, 
generally at one of the poles, and the spore sprouts like a seed. In the space of about one hour's time the oval refractile cell has become a new bacillus. One spore produces by germination one bacillus. Spores never multiply by fission, nor reproduce themselves.

Hueppe has stated that there are certain organisms (like Leuconostoc, and some streptococci) which reproduce by the method of arthrospores. Defined shortly, this is simply an enlargement of one or more cell elements in the chain which thus takes on the function of maternity. On either side of the large coccus may be seen the smaller ones, which it is supposed have contributed of their protoplasm to form a mother cell. An arthrospore is said to be larger, more refractile, and more resistant than an ordinary endospore. Many bacteriologists of repute have declined hitherto definitely to accept arthrospore formation as a proved fact.

Spore formation in bacteria is not to be considered as a method of multiplication. The general rule is undoubtedly that one bacillus produces one spore, and one spore germinates into one bacillus. It is a reproduction, not a multiplication. Indeed, the
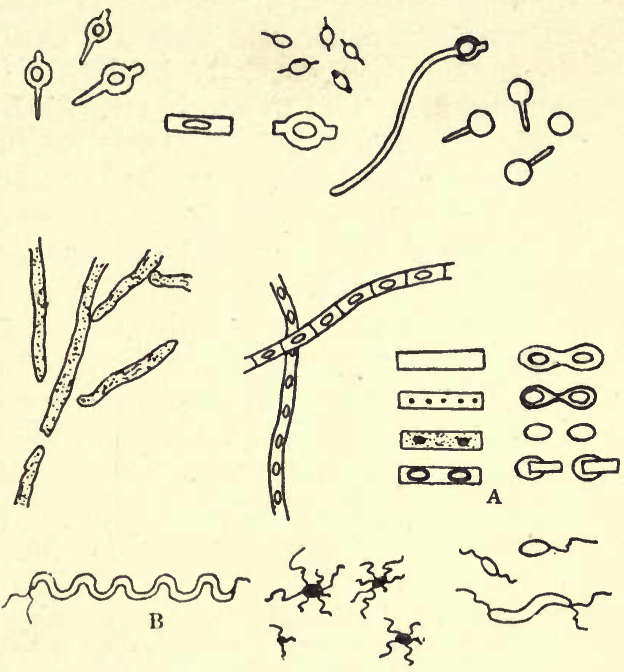

Fig. 4.-Diagrams of Various Forms of Spore Formation and Flagella.

A. Stages in formation of spore and its after development. B. Spirillum with terminal flagella.

whole process is of the nature of a resting stage, and is due (a) to the arrival of the adult bacillus at its biological zenith, or (b) to the conditions in which it finds itself being unfavourable to further vegetative growth, and so it endeavours to perpetuate its species. Most authorities are probably of the latter opinion, though there is not a little evidence for the former. Exactly what conditions are favourable to sporulation is not known. Nutriment has probably an intimate effect upon it. The temperature must not be below $16^{\circ} \mathrm{C}$., nor much above $40^{\circ} \mathrm{C}$. Oxygen, as we have seen, is favourable, if not necessary, to many species, which will in cultivation in broth rise to the surface and lodge in the pellicle to form their seeds. Moisture, too, is considered a necessity.

Koch found that spore formation in $B$. anthracis occurred in six 
hours. The spores may be situated in the middle of the bacillus (as in B. anthracis, B. acidi butyrici, etc.), towards one end (Bacillus of Malignant (Edema), or actually terminal (B. tetani). Those spores produced inside the capsule of the bacillus are termed endospores. Hueppe has described the spores of certain streptococci as arthrospores. The spores of yeast are termed ascospores. The spores of all bacillary species possess, however, certain characters in common. They are as follow. The spore is generally oval, though more spherical in the Hyphomycetes: it is bright and glistening in aspect; it is often greater in diameter than the bacillus giving rise to it; its capsule is thicker and stronger than the capsule of the parent bacillus; and it is generally held that the contained protoplasm is more concentrated, so to speak, than that of the bacillus. These two last characters are of chief importance to us, for it is owing to them that spores possess such marked power of resistance. Cohn has suggested that the capsule of a spore is in reality a double envelope, an inner one of fatty and an outer one of gelatinous nature, and it is owing to this that its resistance to heat and dessication is due. The protoplasm of the spore contains, of course, the essential constituents of the mother cell. It is the method by which "the continuity of germ plasm" is secured in these lowly forms of life. Under favourable circumstances this spore-protoplasm will germinate into a new bacillus.

It should be understood that whilst holding the view that spores are a resting stage during adverse conditions,* we fully recognise that certain favouring external conditions are essential

* Yeast can be effectually starved by cultivating on a small block of plasterof-Paris kept moist under a bell jar; under these circumstances the yeast is supplied with nothing but water. In a few days the protoplasm of yeast cells thus circumstanced becomes filled with vacuoles and fat cells. The protoplasm has been undergoing destructive metabolism, and, there being nothing to supply new material, has diminished in quantity and at the same time been partly converted into fat. Both in plants and animals fatty degeneration is a more or less constant phenomenon of starvation, and to this bacteria are no exception. After a time the protoplasm collects towards the centre of the cell, and divides simultaneously into four masses arranged like a pyramid of four billiard balls, three at the base and one above. These are the ascospores, and sooner or later they are liberated by the rupture of the mother-cell wall. Certain of the Streptothrix family also "sporulate" when they find themselves, like yeast upon gypsum, surrounded by an unfavourable environment. Again, in old cultures, it will be found that when the food supply has been exhausted the bacteria have either sporulated or have died. For these reasons sporulation may be looked upon not as a method of multiplication but one of reproduction, of carrying on the species under adverse conditions. With regard to the rapid formation of spores under apparently favourable circumstances (B. filamentosus, B. anthracis, etc.), it must be borne in mind that the medium may not be by any means so favourable as appears to be the case (Flügge). It is clear that the food supply immediately around many of the bacteria in a culture must soon be exhausted. Besides, there is the toxic influence early at work, often as an inimical agency acting unfavourably towards the bacillus producing it. So that the appearance of spores in such a culture may still be due to conditions which are actually unfavourable. 
to spore formation. Of these, there are at least three of which bacteriologists have knowledge, namely, moisture, oxygen, and a certain temperature. Fluid media forms an excellent nidus for sporulation so long as some oxygen can gain access to the sporulating germs. But many organisms will not sporulate if lying deep in such a medium. In moulds and yeasts oxygen is essential, and for some spore-bearing bacilli a supply of oxygen is a sine qua non (the exceptions are strict anaërobes like $B$. tetani, $B$. butyricus, etc.) of sporulation. Prazmowski has pointed out that it is characteristic of these forms that they are non-motile during sporulation. B. tetani, $B$. butyricus, and other strict anaërobes continue to remain motile during sporing. Temperature exerts a marked influence on the process.* In the case of $B$. subtilis, an organism frequently present in milk, spore formation did not occur below $6^{\circ} \mathrm{C}$; ; at $18^{\circ} \mathrm{C}$. it required two days; at $22^{\circ} \mathrm{C}$. one day; and at $30^{\circ} \mathrm{C}$. only twelve hours. $\dagger^{\circ}$.

When free in the field of the microscope, spores must be distinguished from fat cells, micrococci, starch cells, some kinds of ova, yeast cells, and other like objects. Spores are detected frequently by their resistance to ordinary stains and the necessity of colouring them by special staining methods. When, however, a spore has taken on the desired colour, it retains it with tenacity. In addition to their shape, size, thickened capsule, and staining characteristics, spores also resist desiccation and heat in a much higher degree than bacilli not bearing spores. It has been suggested that bacteria should be classified according to their method of spore formation.

\section{The Influence of External Conditions on the Growth of Bacteria}

In the earliest days of the study of micro-organisms it was observed that they mostly congregate where there is suitable food for their nourishment. The reason why fluids such as milk, and dead animal matter such as a carcase, and living tissues such as a man's body, contain many microbes, is because each of these three media is favourable to their growth. Milk affords almost an ideal food and environment for microbes. Its temperature and constitution frequently meet their requirements. Dead animal matter, too, yields a rich diet for certain species (saprophytes). In the living tissues bacteria obtain not only nutriment, but a favourable

* Koch has shown in the case of $B$. anthracis that at least $16^{\circ} \mathrm{C}$. is necessary for spore formation, and at this temperature limited formation of spores did not occur until after seven days. At $21^{\circ} \mathrm{C}$. spores had formed after seventy-two hours, at $25^{\circ} \mathrm{C}$. after thirty-five to forty hours, and between $30^{\circ} \mathrm{C}$. and $40^{\circ} \mathrm{C}$. in about twenty-four hours; ; the best and strongest cultivations were obtained from $20^{\circ}$ to $25^{\circ} \mathrm{C}$.

$\dagger$ Flïgge.-Micro-organisms. Translation by W. Watson Cheyne, 1890, p. 539. 
temperature and moisture. Outside the human body it has been the endeavour of bacteriologists to provide media as similar to the above as possible, and containing many of the same elements of food, in order that the life-history may be carried on outside the body and under observation. By means of cover-glass preparations for the microscope we are able to study the form, size, motility, flagella, spore formation, and peculiarities of staining, all of which characters aid us in determining to what species the organism under examination belongs. By means of artificial nutrient media we may further learn the characters of the organism in "pure culture,"* its favourable temperature, its power or otherwise of liquefaction, of curdling of milk, or of gas or acid production; its behaviour towards oxygen; its power of producing indol, pigment, and other bodies; as well as its thermal death-point and resistance to light and disinfectants. It is well known that under artificial cultivation an organism may be greatly modified in its morphology and physiology, and yet its conformity to type remains much more marked than any divergence which may occur.

Nutritive Medium. The basis of many of these artificial media is broth. This is made from good lean beef, free from fat and gristle, which is finely minced up and extracted in sterilised water (one pound of lean beef to every 1000 c.c. of water). It is then filtered and sterilised. To provide peptone beef-broth, ten grammes of peptone and five grammes of common salt are added to every litre of acid beef-broth. It is rendered slightly alkaline by the addition of sodium carbonate or sodium hydrate, and is filtered and sterilised. In glycerine-broth 6 to 8 per cent. of glycerine has been added after filtration, in glucose-broth 1 or 2 per cent. of grape-sugar. This latter is used for anaërobic organisms. The use of broth as a culture medium is of great value. It is undoubtedly the best fluid medium, and in it may not only be kept pure cultures of bacteria which it is desired to retain for a length of time, but in it also emulsions and mixtures may be placed preparatory to further examination. Gelatine consists of broth solidified by the addition of 100 grams of best French gelatine to the litre. Its advantage is twofold : it is transparent, and it allows manifestation of the power of liquefaction. When we speak of a liquefying organism we mean a germ having the power of producing a peptonising ferment which can at the temperature of the room break down solid gelatine into a liquid. Grape-sugar gelatine is made like grape-sugar broth. Agar was introduced as a medium which would not like gelatine melt at $25^{\circ} \mathrm{C}$., but remain solid at blood-heat $\left(37.5^{\circ}\right.$ C. ; $98.5^{\circ} \mathrm{F}$.). It is a seaweed generally obtained in dried strips from the Japanese market. Ten to fifteen grammes are added to every litre of peptone-broth. Glycerine and grape-sugar may be added as elsewhere. Blood agar is ordinary agar with fresh sterile blood smeared over its surface. Blood serum is drawn from a jar of coagulated horse-blood, in which the serum has risen to the top. This is collected in sterilised tubes and coagulated in a special apparatus (the serum inspissator). Potato is prepared by scraping ordinary potatoes, washing in corrosive sublimate, and sterilising. It may then be cut into various shapes convenient for cultivation. Upon any of these forms of solid media the characteristic

* A " pure culture" is a growth, in an artificial medium outside the body, of one species of micro-organism only.

+ The facts here given are obviously only general indications. The accurate preparation of medium is of vital importance in Bacteriology, and for its accomplishment text-books should be consulted (Eyre's Bacteriological Technique, 125-174). 
growth of the organism can be observed. Of the nutrient elements required, nitrogen is obtained from albumens and proteids, carbon from milk-sugar, cane-sugar, or the splitting up of proteids; salts (particularly phosphates and salts of potassium) are readily obtainable from those incorporated in the media; and the water which is required is obtainable from the moisture of the media.

There are two common formis of test-tube culture, viz., on the surface and in the depth of the medium. In the former the medium is sloped, and the inoculating needle is drawn along its surface; in the latter the needle is thrust vertically downwards into the depth of the solid

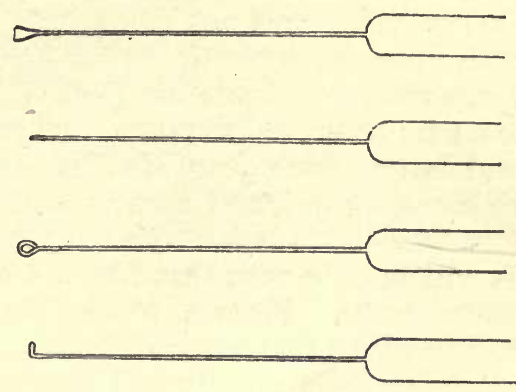

Fia. 5.-Inoculating Needles.

Platinum wire fused into glass handles. medium. Plate cultures and anaërobic cultures will be described at a later stage.

Temperature.-When the medium has been inoculated the culture is placed at a temperature which will be favourable. For

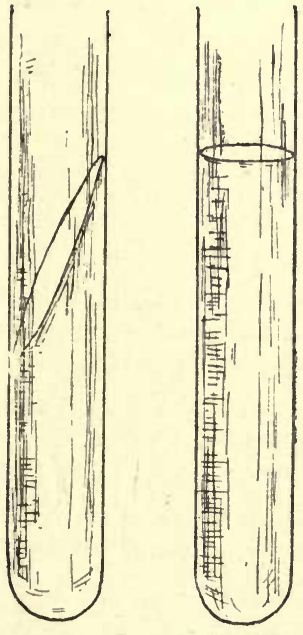

FiG. 6.-Media for Surface and Depth Culture. every species of bacteria there is a favourable temperature, termed the optimum temperature. This is usually the temperature of the natural habitat of the organism. Two standards of temperature are in use in bacteriological laboratories. The one, room temperature, varies from $18^{\circ}-22^{\circ} \mathrm{C}$.; the other is blood-heat, and varies from $35^{\circ}-38^{\circ} \mathrm{C}$. (Plates 1 and 2). It is true some species will grow below $18^{\circ} \mathrm{C}$, and others above $38^{\circ} \mathrm{C}$. The pathogenic (disease-producing) bacteria thrive best as a rule at $37^{\circ} \mathrm{C}$., and the nonpathogenic at the ordinary temperature of the room. The different degrees of temperature are obtained by means of incubators. For the low temperatures gelatine is chosen as a medium, for the higher temperatures agar. Most bacteria grow well at room temperature (about $60^{\circ} \mathrm{F}$.), but they will grow more luxuriantly and speedily at blood-heat.

Whilst these are the ordinary limits of temperature affecting bacteria, they do not by any means include the extremes of heat and cold which micro-organisms can withstand. The average thermal death-point is about $55^{\circ} \mathrm{C}$, but certain species, termed thermophilic, 
isolated from the intestine, horse manure, etc., grow at $60^{\circ}-70^{\circ} \mathrm{C}$. On the other hand, investigations have shown that bacteria can withstand exceedingly low temperatures. Koch showed that the cholera vibrio was not killed by a temperature of $-32^{\circ} \mathrm{C}$. In 1900 , Swithinbank exposed cultures of the tubercle bacillus to the temperature of liquid air $\left(-193^{\circ} \mathrm{C}\right.$.) for continuous periods varying from six hours to forty-two days, without their vitality being affected; and in the same year MacFadyen and Rowland found that Proteus vulgaris, B. coli, and several other species were not killed after an exposure of ten hours to a temperature of liquid hydrogen $\left(-252^{\circ} \mathrm{C}\right)$. It will thus be seen that bacteria can withstand great alternations of temperature. From a public health point of view, it is important to remember that organisms can exist in freezing mixtures and ice, retaining their vitality and virulence. For example, B. coli and the typhoid bacillus can exist from the low temperatures above mentioned to $80^{\circ} \mathrm{C}$., although the usual thermal death-point for these species is between $50^{\circ}-60^{\circ} \mathrm{C}$.*

Moisture has been shown to have a favourable effect upon the growth of microbes. Drying will of itself kill many species (e.g. the spirillum of cholera), and other things being equal, the more moist a medium is, the better will be the growth upon it. Thus it is that the growth in broth is always more luxuriant than that on solid media. Yet the growth of Bacillus subtilis and some other species are an exception to this rule, for they prefer a dry medium. Desiccation as a rule diminishes virulence and lessens growth. But some species can withstand long-continued drying without injury.

Light acts as an inhibitory, or even germicidal, agent. This fact was first established by Downes and Blunt in a memoir to the Royal Society in 1877. They found by exposing cultures to different degrees of sunlight that the growth of the culture was partially or entirely prevented, being most damaged by the direct rays of the sun, although diffuse daylight acted prejudicially. Further, these same investigators proved that the rays of the spectrum which acted most inimically upon bacteria were the blue and violet rays, next to the blue being the red and orange-red rays. The action of light, they explain, is due to the gradual oxidation which is induced by the sun's rays in the presence of oxygen. Duclaux, who worked at this question at a later date, concluded that the degree of resistance to the bactericidal influence of light, which some bacteria possess, might be due to difference in species, difference in culture media, and difference in the degrees of intensity of light. Tyndall tested the growth of organisms in flasks exposed to air and light on the Alps,

* For the latest researches on this point, see Proc. Roy. Soc., 1900 and 1901 ; and the 'Thirty-fourth Annual Report of the State Board of Health, Massachusetts, 1903, pp. 269-281. Dewar commenced experiments of this character in 1892 . 


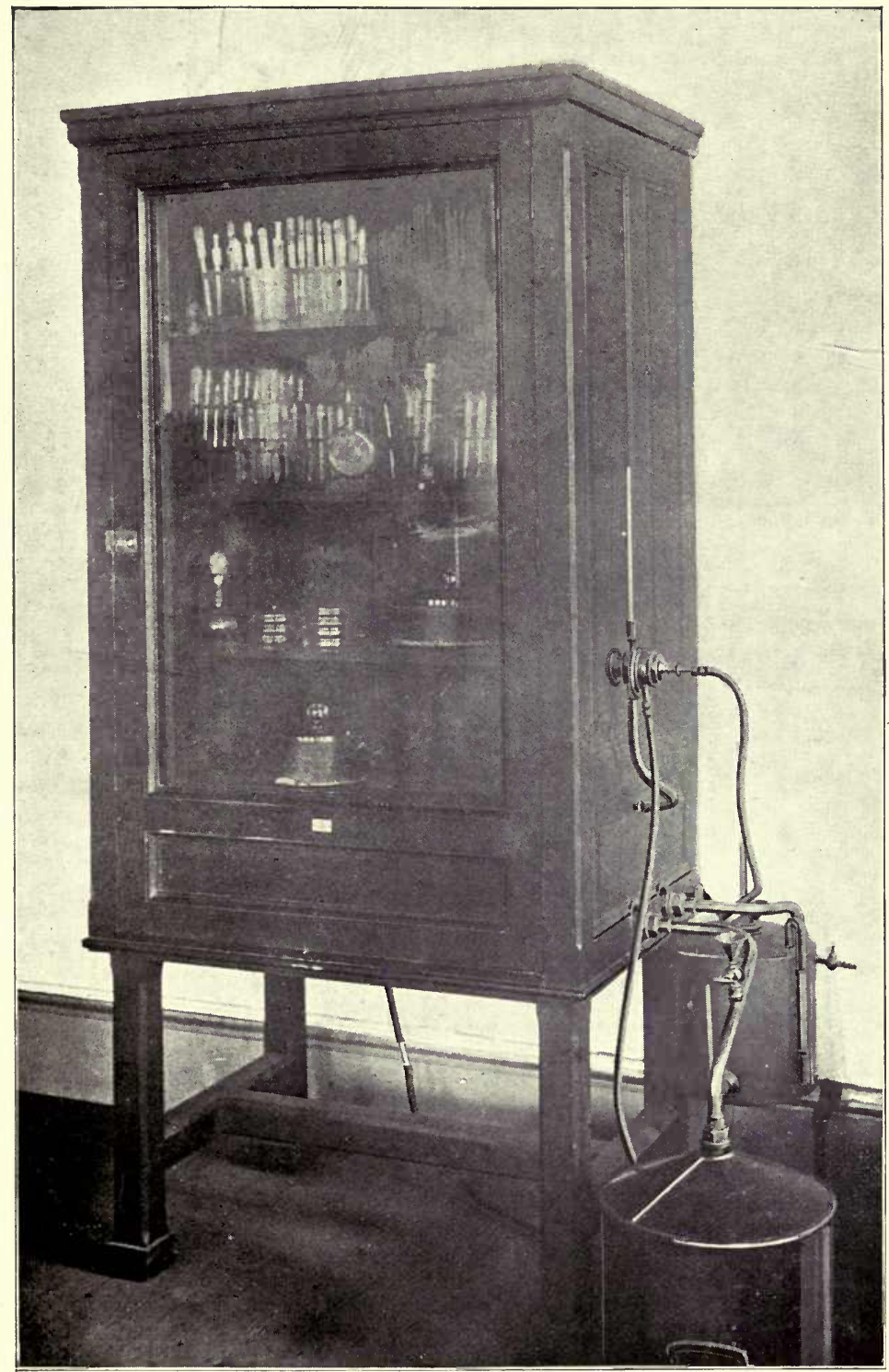

A form of Pasteur's Large Incubator for Cultivation at Room Thmperature. 


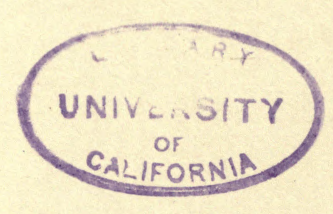


and found that sunlight inhibited the growth temporarily. A large number of experimenters on the Continent and in England have worked at this fascinating subject since 1877, and though many of their results appear contradictory, we may be satisfied in adopting the following conclusions respecting the matter:-

(1) Sunlight has a deleterious effect upon bacteria, and to a less extent on their spores.

(2) This inimical effect can be produced by light irrespectively of rise in temperature.

(3) The ultra-violet rays are the most bactericidal, and the infra-red the least so, which indicates that the phenomenon is due to chemical action.

(4) The presence of oxygen and moisture greatly increase this action, the process being largely an oxidation.

(5) Sunlight also acts prejudicially upon the culture medium, and thereby exerts an injurious action on the culture.

(6) The time occupied in the bactericidal action depends upon the intensity of the light and the inherent vitality of the organism.

(7) With regard to the action of light upon pathogenic organisms, some results have recently been obtained with Bacillus typhosus. Janowski maintains that direct sunlight exerts a distinctly depressing effect on typhoid bacilli. At present more cannot be said than that sunlight and fresh air are two of the most powerful agents we possess with which to combat pathogenic germs.

A very simple method of demonstrating the influence of light is to grow a pure culture in a favourable medium, either in a testtube or upon a glass plate, and then cover the whole with black paper or cloth. A little window may then be cut in the protective covering, and the whole exposed to the light. Where it reaches in direct rays, it will be found that little or no growth has occurred; where, on the other hand, the culture has been in the dark, abundant growth occurs. In diffuse light the growth is merely somewhat inhibited.

A number of experiments in this direction were made at Lawrence, Massachusetts, ${ }^{*}$ with cultures of typhoid and B. coli.

In two experiments, each with typhoid bacillus and $B$. coli, water dilutions were made from fresh cultures of the germs, 1 c.c. of this water being placed in Petri dishes in the sun for definite periods. After exposure, the water in the plates was mixed with agar, and all plates were incubated twenty-four hours at $38^{\circ}$, after which the number of colonies was counted. In one experiment the water dilution of typhoid was mixed with melted agar, and plates made as p. 275 .

Thirty-fourth Ann. Rep. State Bd. of Health of Massachusetts, 1903, 
usual. After the agar had set, these plates were then exposed to the sunlight. In one experiment with $B$. coli, the water culture was not exposed to the sunlight in plates, but the exposure was made in a clear, white glass bottle of the Blake pattern, holding 100 c.c., samples being taken from this at the proper intervals, and plated as usual.

In all cases control cultures were made under exactly the same conditions as were the cultures exposed, these, however, being protected from the sunlight by a heavy, opaque cloth, or some similar material. The temperature of these cultures was, of course, considerably lower than was the temperature in the sun. The numbers of bacteria in the controls showed the usual variation to be expected under the circumstances, usually a slight reduction in numbers being noted during two or three hours' standing, although in one instance the numbers increased quite materially. The data of these control cultures are not shown in the accompanying tables.

The brightness of the sun also varied considerably, and attempts were made to measure the amount of light by photographic means, but these measurements were unsatisfactory, and the data are not included here.

With typhoid, from 95 to 99 per cent. of all the germs were destroyed by ten to fifteen minutes' exposure to direct sunlight. A few germs may resist the sunlight for a somewhat longer time; usually, however, all the germs were destroyed by three or more hours' exposure to bright sunlight. The results of the experiments with typhoid are shown in the following tables:-

\section{Table showing Elimination of Typhoid Germs in Water on Exposure to Sunlight.}

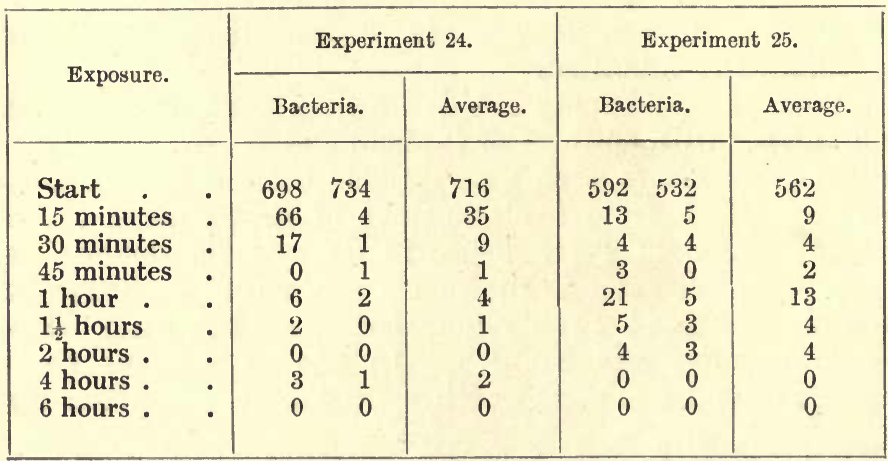


Table shoving Elimination of Typhoid Germs in Agar Plates on Exposure to Sunlight.

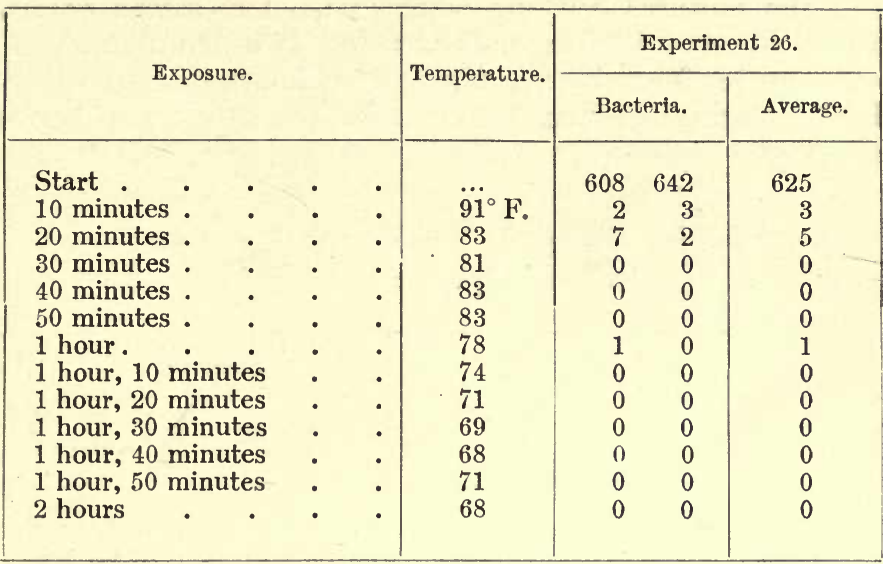

With $B$. coli the results have been somewhat more variable, probably due to more changeable conditions. In one experiment, something over 80 per cent. of the germs were destroyed by fifteen minutes' exposure, all being destroyed after four hours. The results of this experiment were undoubtedly influenced greatly by clouds in the sky, so that at times the sunlight was not very bright, after about two and one-half hours the sun being entirely overcast. In one experiment about 96 per cent. of the germs were eliminated at the end of fifteen minutes, and after thirty minutes all of the germs were destroyed. In these two experiments the water cultures were exposed in plates, the results being shown in the following table:-

\section{TABle showing Elimination of B. coli in Water Cullures on Exposure to Sunlight.}

\begin{tabular}{|c|c|c|c|c|c|c|c|c|}
\hline \multirow{2}{*}{ Exposure. } & \multicolumn{4}{|c|}{ Experiment 78.} & \multicolumn{4}{|c|}{ Experiment 79.} \\
\hline & $\begin{array}{c}\text { Tem- } \\
\text { perature. }\end{array}$ & $\begin{array}{l}\text { Bac } \\
\text { per }\end{array}$ & $\begin{array}{l}\text { teria } \\
\text { c.c. }\end{array}$ & Average. & $\begin{array}{c}\text { Tem. } \\
\text { perature. }\end{array}$ & & $\begin{array}{l}\text { teria } \\
\text { c.c. }\end{array}$ & Average. \\
\hline Start. & 78 & 70,000 & 72,800 & 71,400 & & 445,40 & 824,300 & 634,850 \\
\hline 15 minutes & 78 & 6,564 & 16,166 & 12,365 & 106 & 19,10 & 31,800 & 25,400 \\
\hline 30 minutes & 80 & 4,473 & 2,663 & 3,568 & 107 & & 0 & 0 \\
\hline 45 minutes & 80 & 2,130 & 0 & 1,065 & 110 & & 0 & 0 \\
\hline 1 hour . & 80 & 590 & 0 & 295 & 100 & & 0 & 0 \\
\hline $1 \frac{1}{2}$ hours & 82 & 2,130 & 93 & 1,111 & 108 & & 0 & 0 \\
\hline 2 hours & 62 & 10 & 70 & 40 & 105 & & 0 & 0 \\
\hline 3 hours & $\ldots$ & $\ldots$ & $\ldots$ & $\ldots$ & 100 & & 0 & 0 \\
\hline 4 hours & 61 & 0 & 0 & 0 & 78 & & 0 & 0 \\
\hline
\end{tabular}


In one experiment the water was exposed in bottles. In this case about 98 per cent. of the germs were destroyed after fifteen minutes, the cultures varying somewhat. The germs persisted in the water in considerable numbers for two hours and in small numbers up to four hours, after five hours the sample being completely sterilised. The results of this experiment are shown as follows:-

Table showing Change in Numbers of $\mathrm{B}$. coli in Water in Bulk on Exposure to Sunlight.

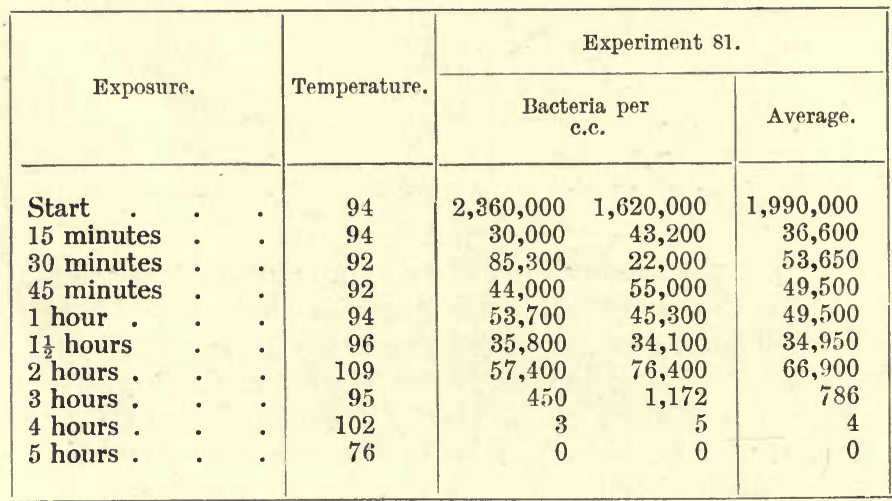

It has been found that the electric light has but little action upon bacteria, though that which it has is similar to sunlight. Recent experiments with the Röntgen rays have not given bactericidal results.

In 1890 Koch stated that tubercle bacilli were killed after an exposure to direct sunlight of from a few minutes to several hours. The intluence of diffuse light would obviously be much less. Professor Marshall Ward has experimented with the resistant spores of Bacillus anthracis by growing these on agar plates and exposing to sunlight. From two to six hours' exposure had a germicidal effect.*

It should be remembered that several species of sea-water bacteria themselves possess the property of phosphorescence. Pflüger was the first to point out that it was such organisms which provided the phosphorescence upon decomposing wood or decaying fish. To what this light is due, whether capsule, or protoplasm, or chemical product, is not yet known. The only facts at present established are to the effect that certain kinds of media and pabulum favour or deter phosphorescence.

* See Trans. Jenner Inst. (second series), 1899, p. 81. 
Aërobiosis.-Pasteur was the first to lay emphasis upon the effect which free air had upon micro-organisms. He classified them according to whether they grew in air, aërobic, or whether they flourished most without it, ancërobic. Some have the faculty of growing with or without the presence of oxygen, and are designated as facultative aërobes or anaërobes. As regards the cultivation of anaërobic germs, it is only necessary to say that hydrogen, nitrogen, or carbonic acid gas may be used in place of oxygen, or they may

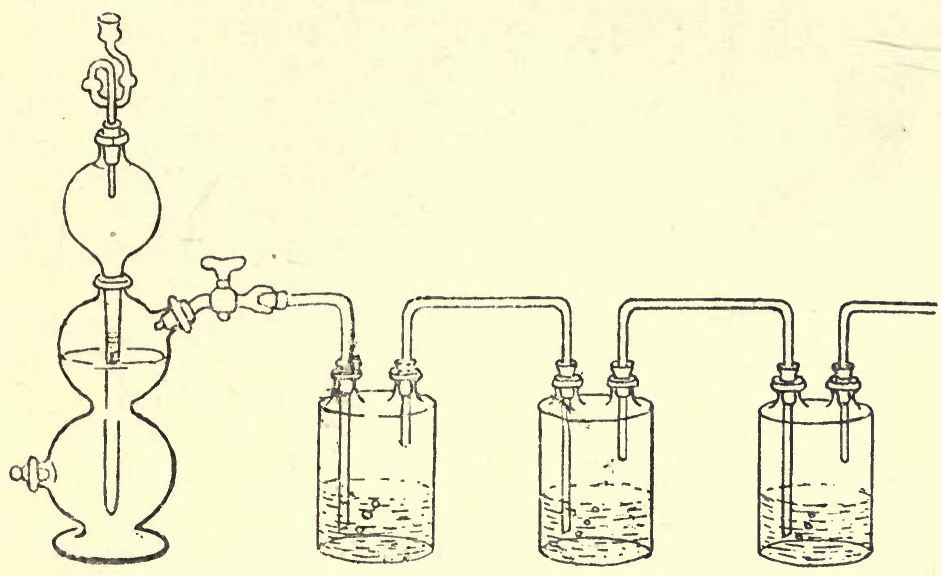

Fic. 7.-Method of producing Hydrogen by Kipp's Apparatus for Cultivation of Anaërobes (see p. 117.

be grown in a medium containing some substance which will absorb the oxygen (see p. 117).

Means of Sterilisation.-As this term occurs frequently even in books of an elementary nature, and as it is expressive of an idea which must always be present to the mind of the bacteriologist, it may be desirable to make allusion to it here.

Chemical substances, perfect filtration, and heat are the three means at our command in order to secure germ-free conditions of apparatus or medium. The first two, though theoretically admissible, are practically seldom used, the former of the two because the addition of chemical substances annuls or modifies the operation, the latter of the two on account of the great practical difficulties in securing efficiency. Hence in the investigations involved in bacteriological research heat is the common sterilising agent. A sustained temperature of $70^{\circ} \mathrm{C} .\left(158^{\circ} \mathrm{F}\right.$.) will kill all bacilli; even $58^{\circ}$ C. will kill most kinds. Boiling at $100^{\circ}$ C. $\left(212^{\circ} \mathrm{F}\right.$.) for five minutes will kill anthrax spores, and for thirty to sixty minutes will kill all bacilli and their spores. This difference in the thermal 
death-point between bacilli and their spores enables the operator to obtain what are called "pure cultures" of a desired bacillus from its spores which may be present. For example, if a culture contains

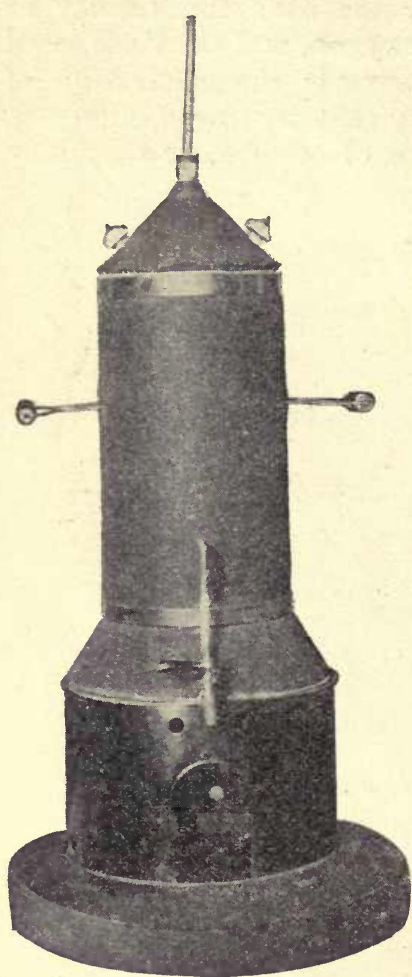

FIG. 8.-Koch's Steam Steriliser. spores of anthrax and is contaminated with micrococci, heating to $70^{\circ} \mathrm{C}$. $\left(158^{\circ} \mathrm{F}\right.$.) will kill all the micrococci, but will not affect the spores of anthrax, which can then grow into a pure culture of anthrax bacilli. Fractional or discontinuous sterilisation depends on the principle of heating to the sterilising point for bacilli (say $70^{\circ} \mathrm{C}$.) on one day, which will kill the bacilli, but leave the spores uninjured. But by the following day the spores will have germinated into bacilli, and a second heating to $70^{\circ} \mathrm{C}$. will kill them before they in their turn have had time to sporulate. Thus the whole will be sterilised, though at a temperature below boiling.

Successful sterilisation, therefore, depends upon killing both bacteria and their spores, and nothing short of that can be considered as sterilisation. The following methods are those generally used in the laboratory. For dry heat (which is never so injurious to organisms as moist heat*): (a) the Bunsen burner, in the flame of which platinum needles, etc., are sterilised; (b) hot-air chamber, in which flasks and test-tubes are heated to a temperature of $150^{\circ}-170^{\circ} \mathrm{C}$. for an hour or more. For moist heat: (c) boiling, for knives and

* It will be observed that there is a marked difference between the effects of dry heat and moist heat. Moist heat is able to kill organisms much more readily than dry, owing to its penetrating effect on the capsule of the bacillus. Dry heat at $140^{\circ} \mathrm{C}$. $\left(284^{\circ} \mathrm{F}\right.$.), maintained for three hours, is necessary to kill the resistant spores of Bacillus anthracis and B. subtilis, but moist heat at forty degrees less will have the same effect. It is from data such as these that in laboratories and in disinfecting apparatus moist heat is invariably preferred to dry heat. For with the latter such high temperatures would be required that the articles being disinfected would be damaged. Koch states the following figures for general guidance: Dry heat at a temperature of $120^{\circ} \mathrm{C}$. $\left(248^{\circ} \mathrm{F}\right.$.) will destroy spores of mould fungi, micrococci, and bacilli in the absence of their spores; for the spores of bacilli $140^{\circ} \mathrm{C}$. $\left(284^{\circ} \mathrm{F}\right.$.), maintained for three hours, is necessary; moist heat at $100^{\circ} \mathrm{C}$. $\left(212^{\circ} \mathrm{F}\right.$.) for fifteen minutes will kill bacilli and their spores. 
PI.ATE 2.

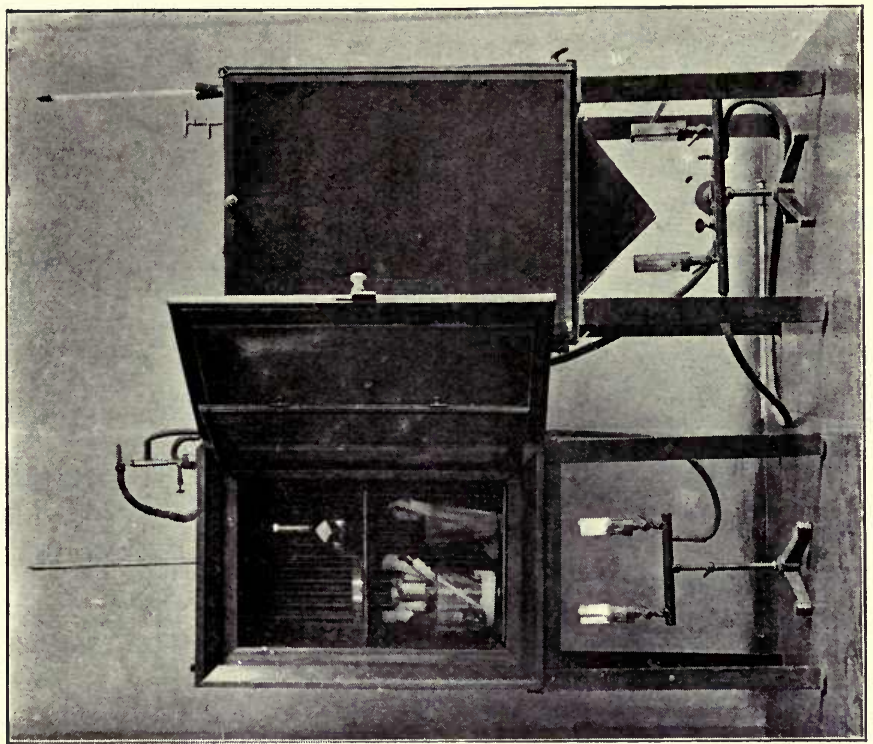

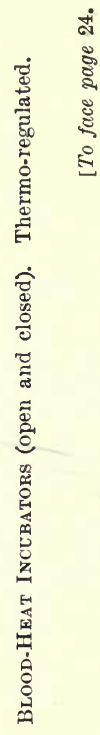

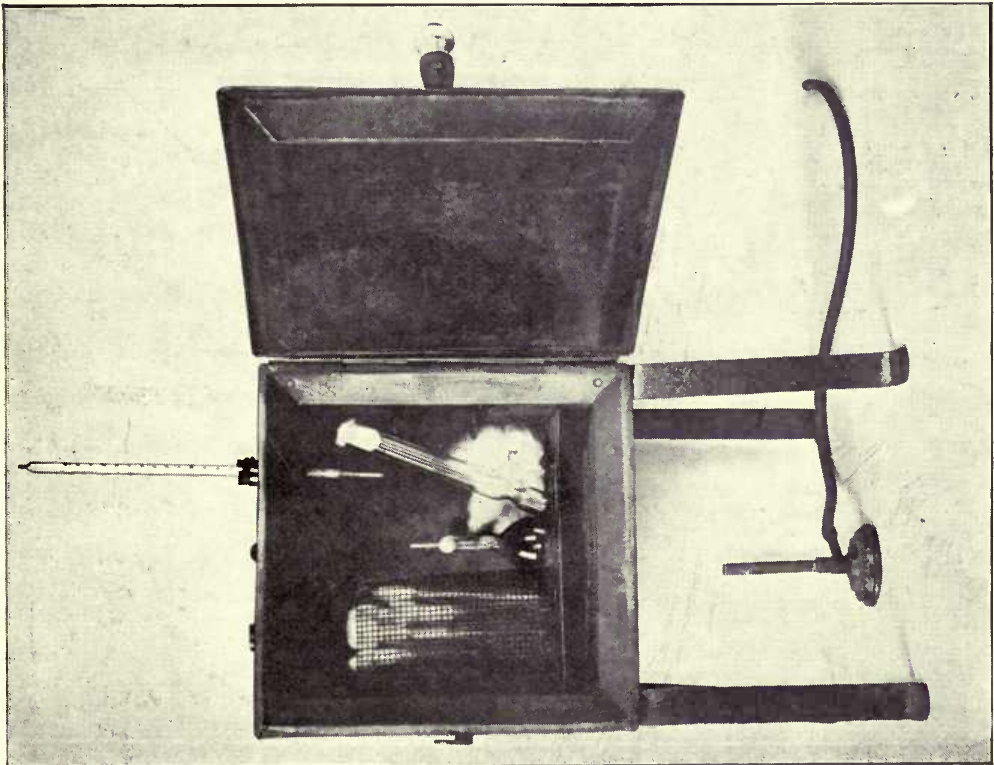

先 

instruments; (d) Koch's steam steriliser, by means of which a crate is slung in a metal cylinder, at the bottom of which water is boiled; (e) the autoclave, which is the most rapid and effective of all the methods. This is in reality a Koch steriliser, but with apparatus for obtaining high pressure. The last two $(d, e)$ are used for sterilising the nutrient media upon which bacteria are cultivated outside the body. Blood serum would, however, coagulate at a temperature over $60^{\circ} \mathrm{C}$. $\left(124^{\circ} \mathrm{F}\right.$.), and hence a special steriliser has been designed to carry out fractional sterilisation daily for a week at about $55^{\circ} \mathrm{C} .-58^{\circ} \mathrm{C}$.

\section{Modes of Bacterial Action}

In considering the specific action of micro-organisms, it is desirable, in the first place, to remember the two great functional divisions of saprophyte and parasite. A saprophyte is an organism that obtains its nutrition from dead organic matter. Its services, of whatever nature, lie outside the tissues of living animals. Its life is spent apart from a "host." A parasite, on the other hand, lives always at the expense of some other organism which is its host, in which it lives or upon which it lives. There is a third or intermediate group, known as "facultative," owing to their ability to act as parasites or saprophytes, as the exigencies of their life may demand.

The saprophytic organisms are, generally speaking, those which contribute most to the benefit of man, and the parasitic the reverse, though this statement is only approximately true. In their relation to the processes of fermentation, decomposition, nitrification, etc., we shall see how great and invaluable is the work which saprophytic microbes perform. Their result depends, in nearly all cases, upon the organic chemical constitution of the substances upon which they are exerting their action, as well as upon the varieties of bacteria themselves. Nor must it be understood that the action of saprophytes is wholly that of breaking down and decomposition. As a matter of fact, some of their work is, as we shall see, of a constructive nature; but, of whichever kind it is, the result depends upon the organism and its environment. This, too, may be said of the pathogenic species, all of which are in a greater or less degree parasitic. It is well known how various are the constitutions of man, how the bodies of some persons are more resistant than those of others, and how the invading microbe will meet with a different reception according to the constitution and idiosyncrasy of the body which it attacks. Indeed, even after invasion the infectivity of the special disease, whatever it happens to be, will be materially modified by the tissues. When we come to turn to the micro-organisms which are pathogenic parasites 
we shall further have to keep clear in our minds that their action is complex, and not simple. In the first place, we have an infection of the body due to the bacteria themselves. It may be a general and widespread infection, as in anthrax, where the bacilli pass, in the blood or lymph current, to each and every part of the body; or it may be a comparatively local one, as in diphtheria, where the invader remains localised at the site of entrance. But, be that as it may, the micro-organisms themselves, by their own bodily presence, set up changes and perform functions which may have far-reaching effects, It is obvious that the wider the distribution the wider is the area of tissue change, and vice verst. Yet there is something of far greater importance than the mere presence of bacteria in human or animal tissues, for the secondary action of disease-producing germsand possibly it is present in other bacteria-is due to their poisonous products, or toxines, as they have been termed. These may be of the nature of ferments, and they become diffused throughout the body, whether the bacteria themselves occur locally or generally. They may bring about very slight and even imperceptible changes during the course of the disease, or they may kill the patient in a few hours. Latterly bacteriologists have come to understand that it is not so much the presence of organisms which is injurious to man and other animals as it is their products, which cause mischief; and the amount of toxic product bears no known proportion to the degree of invasion by the bacteria. The various and widely differing modes of action in bacteria are therefore dependent upon these three elements (1) the tissues or medium, (2) the bacteria or agents, and (3) the products of the bacteria or toxins; and in all organismal processes these three elements act and react upon each other.

Seed and Soil.-It is of essential importance to the right understanding of the rôle which bacteria play in the production of disease to give full place to the part taken by the soil on which they are implanted. Few ideas in bacteriology are more erroneous, or likely to lead to graver misconception, than to suppose that bacteria produce the same effect under all conditions, and that the human tissues play a small part. One might equally well expect seed to behave in the same way in all kinds of soil. We know that as a fact, seeds only flourish under certain conditions, and that the soil is only second in importance to the seed-life itself. It is somewhat the same in the production of disease. The early school of preventive medicine declared for the health of the individual and laid the emphasis upon predisposition; the modern school have declared for the infecting agent, and have laid emphasis upon the bacillus. The truth is to be found in a right perception of the action and interaction of the tissues and the bacillus. B. diphtherio in one person's throat (A) sets up diphtheria, in another person's throat 
(B) lies quiescent, producing no apparent disease. The cause of this extraordinary fact may be a question of different virulence in the two bacilli, but is much more likely to be due to the greater vigour and power of resistance of the mucous membrane of B's throat. Sewer air, as we shall see subsequently, does not contain many bacteria, and probably does not frequently convey germs of disease. But this does not prove that the inhalation of sewer air will not weaken the throat, and so form a favourable nidus for organisms resting there, or organisms shortly to be inhaled from dust or mucous particles from the throat of a diseased person. Which is the more important preventive method, to maintain the resistance of the individual or to waylay the infecting organism, is a nice point we need not attempt to decide. Obviously, both objects should be kept in view. Phthisis is another example. Thousands of persons inhale the tubercle bacillus who are not attacked by the clinical disease of consumption. This fortunate result is due to the resistant tissues of the healthy lung, and the lesson to be derived is to maintain such resistance at its maximum. This evidently is, in part, the scientific explanation of Koch's dictum, "It is the overcrowded dwellings of the poor that we have to regard as the real breeding places of tuberculosis; it is out of them that the disease always crops up anew, and it is to the abolition of these conditions that we must first and foremost direct our attention if we wish to attack the evil at its root and to wage war against it with effective weapons."* Part of the explanation of these words is doubtless that it is in such places that the tubercle bacillus breeds and passes from one person to another. But every sanitarian knows that the effect of such environment is to lower the natural resistance, to weaken the lung, impoverish the blood, and undermine the constitution, and thus a suitable nidus is supplied to the invading bacillus. "A perfectly healthy lung is seldom if ever primarily infected with the tubercle bacillus" (Woodhead).

But the evidence of bacteriology as to the part played by the soil is even stronger than at first sight appears. For we now know, by experiment, that micro-organisms which in some animals produce acute disease rapidly ending in death, result only in mild disease in other animals, and in yet a third group produce no apparent disease whatever. This is not due to variation in virulence but to variation in soil.

The advance of bacteriology has been so rapid and marked by such striking discoveries that there has been a tendency to over-rate altogether the potentiality of the bacillus apart from its medium. The latest findings in the study of comparative culture work, of immunity and of the production of antitoxins have, however, demon-

\footnotetext{
* Trans. Brit. Cong. of Tuberculosis, 1901, vol. i., p. 31.
} 
strated beyond all doubt the enormous part played by the medium or soil in which the micro-organism is growing.*

\section{Specificity of Bacteria}

A species may be defined as a group of individuals which, however many characters they share with other individuals, agree in presenting one or more characters of a peculiar and hereditary kind with some certain degree of distinctness. $\uparrow$ There is no doubt that separate species of bacteria occur and tend to remain as separate species. But it must be remembered that species are merely arbitrary divisions which present no deeper significance from a philosophical point of view than is presented by well-marked varieties, out of which they are in all cases believed to have arisen, and from which it is often a matter of individual opinion whether they shall be separated by receiving a specific label. What degree or character of variation shall be considered as sufficient for the demarcation of a species of bacteria? B. coli and B. typhosus have certain distinctive features, which are accepted as factors of provisional differentiation. But they have many points in common, the peculiarity and heredity of which are not as yet determined. And they have many allies, para-typhoid and para-colon organisms, in the same way as the tubercle bacillus possesses many allies, both bovine and human, among acid-fast species having similar characters but differing in degree of virulence. The fact is, that our present knowledge of these matters is very small, and it is impossible to dogmatise. The future may reveal some unlooked-for relationships, and organisms now classified as morphologically separate may ultimately prove to be nearly related. Further, it may be found that their respective action in the human body is not greatly dissimilar (the production, of diarrhœa, for example, by the colon group). Medium and tissue have their effect in the production of variations of greater or lesser mark in bacteria. B. typhosus may, in the course of subculture, become morphologically indistinguishable from $B$. coli, and its pathogenicity may also be reduced. The tubercle bacillus in old culture and in saprophytic existence becomes almost indistinguishable from the streptothrix family. Streptococcus conglomeratus on certain media simulates in a marked degree the Klebs-Löffler diphtheria bacillus, and by passage through a mouse loses its streptococcal

* The writer has been impressed in particular as to the truth of this view by observation of a number of epidemics, by the study of a long series of cultures of the same bacillus on different media, and by antitoxin production. But the same conclusion has been reached from other premises. See a suggestive paper by Sir W. J. Collins, M.D., in the Jour. of the Sanitary Institute 1902 (Oct.), xxiii., pt. iii., p. 335 .

† Darwin and After Darvin, G. J. Romanes, F.R.S., vol. ii., p. 231. 
form (Gordon). The Klebs-Löfller bacillus in its turn may be greatly modified in morphology and pathogenicity by environment. Nor is the change necessarily in descending order. Non-pathogenic organisms may possibly become pathogenic. We do not know. The subject is one full of difficulty in a transition period of knowledge in any branch of science. But there is no reason to suppose that bacteria are exceptional in nature and outside the influence of natural selection; and it is not improbable that the views of the early bacteriologists will have to be very much revised, and that eventually it will be found that many "species" of micro-organisms are in reality varieties of a single species showing involution and pleomorphic forms. At the same time it should be recognised that amongst the lowliest forms of life specific distinctions are, as a rule, less definite, and less permanent, than amongst forms of life much higher in the organic scale.

\section{The Association of Organisms}

At a later stage we shall have an opportunity of discussing Symbiosis and allied conditions. Here it is only necessary to draw attention to a fact that is rapidly becoming of the first importance in bacteriology. When species were first isolated in pure culture it was found that they behaved very differently under varying circumstances. This modification in function has been attributed to differences of environment and physical conditions. Whilst it is true that such external conditions must have a marked effect upon such sensitive units of protoplasm as bacteria, it has recently been proved that one great reason why modification occurs in pure artificial cultures is that the species has been isolated from amongst its colleagues and doomed to a separate existence. One of the most abstruse problems in the immediate future of the science of bacteriology is to learn what intrinsic characters there are in species or individuals which act as a basis for the association of organisms for a specific purpose. Some bacteria appear to be unable to perform their ordinary rôle without the aid of others.* An example of such association is well illustrated in the case of Tetanus, for it has been shown that if the bacilli and spores of tetanus alone obtain entrance to a wound the disease does not follow the same course as when with the specific organism the lactic acid bacillus or the common organisms of suppuration or putrefaction also gain entrance. There is here evidently something gained by association. Again the viru-

* The three different degrees of association have been expressed by the following terms : Symbiosis, the co-operation for a mutual advantage, not obtained otherwise; metabiosis, where one organism prepares the way for another; antibiosis (antagonism of bacteria), where one of the two associated organisms is directly or indirectly injuring the other. 
lence of other bacteria is also increased by means of association. The Bacillus coli is an example, for, in conjunction with other organisms, this bacillus, although normally present in health in the alimentary canal, is able to set up acute intestinal irritation, and various changes in the body of an inflammatory nature. It is not yet possible to say in what way or to what degree the association of bacteria influences their rôle. That is a problem for the future. But whilst we have examples of this association in Streptococcus and the bacillus of diphtheria, $B$. coli and yeasts, Tetanus and putrefactive bacteria, Diplococcus pneumonioe and Proteus vulgaris, and Streptococcus erysipelatis and Proteus vulgaris, we cannot doubt that there is an explanation to be found of many, hitherto unknown, results of bacterial action. This is the place in which mention should also be made of higher organisms associated for a specific purpose with bacteria. There is some evidence to support the belief that some of the Leptotricheæ (Crenothrix, Beggiatoa, Leptothrix, etc.) and the Cladotricheæ (Cladothrix) perform a preliminary disintegration of organic matter before the decomposing bacteria commence their labours. This occurs apparently in the self-purification of rivers, as well as in polluted soils.

Antagonism of Bacteria (Antibiosis).-Study of the lifehistory of many of the water bacteria will reveal the fact that they can live and multiply under conditions which would at once prove fatal to other species. Some of these water organisms can indeed increase and multiply in distilled water, whereas it is known that other species cannot even live in distilled water owing to the lack of pabulum. Thus we see that what is favourable for one species may be the reverse for another.

Further, we shall have opportunity of observing, when considering the bacteriology of water and sewage, that there is in these media in nature a keen struggle for the survival of the fittest bacteria for each special medium. In a carcase it is the same. If saprophytic bacteria are present with pathogenic, there is a struggle for the survival of the latter. Now whilst this is in part due to a competition owing to a limited food supply and an unlimited population, as occurs in other spheres, it is also due in part to the inimical influence of the chemical products of the one species upon the life of the bacteria of the other species. Moreover, in one culture medium, as Cast has pointed out, two species will often not grow. When Pasteur found that exposure to air attenuated his cultures, he pointed out that it was not the air per se that hindered growth, but it was the introduction of other species which competed with the original. The growth of the spirillum of cholera is opposed by Bacillus pyogenes foetidus. $B$. anthracis is, in the body of animals, opposed by either B. pyocyaneus or Streptococcus erysipelatis, and yet 
it is aided in its growth by $B$. prodigiosus. $B$. aceti is under certain circumstances antagonistic to $B$. coli.

In several of the reports of the late Sir Richard Thorne issued from the Medical Department of the Local Government Board, we have the record of a series of experiments performed by Dr Klein upon the subject of the antagonisms of microbes. From this work it is clearly demonstrated that whatever opposition one species affords to another it is able to exercise by means of its poisonous properties. These are of two kinds. There is, as is now widely known, the poisonous product named the toxin, into which we shall have to inquire in more detail at a later stage. There is also in many species, as several workers have pointed out, a poisonous constituent, or constivients, included in the body protoplasm of the bacillus, and which he therefore terms the intracellular poison. Now, whilst the former is different in every species, the latter may be a property common to several species. Hence those having a similar intracellular poison are antagonistic to each other, each member of such a group being unable to live in an environment of its own intracellular poison. Further, it has been suggested that there are organisms possessing only one poisonous property, namely, their toxin-for example, the bacilli of Tetanus and IJiphtheria-whilst there are other species, as above, possessing a double poisonous property, an intracellular poison and a toxin. In this latter class would be included the bacilli of Anthrax and Tubercle.

There can be no doubt that these complex biological properties of association and antagonism, as well as the parasitic growth of bacteria upon higher vegetables, are as yet little understood, and we may be glad that any light is being shed upon them. In the biological study of soil bacteria in particular may we expect in the future to find examples of association, even as already there are signs that this is so in certain pathogenic conditions. In the alimentary canal, on the other hand, and in conditions where organic matter is greatly predominating, we may expect to see further light on the subject of antagonism.

Attenuation of Virulence or Function.-It was pointed out by some of the pioneer bacteriologists that the function of bacteria suffered under certain circumstances a marked diminution in power. Later workers found that such a change might be artificially produced. Pasteur introduced the first method, which was the simple one of allowing cultures to grow old before sub-culturing. Obviously a pure culture cannot last for ever. To maintain the species in characteristic condition it is necessary frequently to sub-culture upon fresh media. If this simple operation be postponed as long as possible consistent with vitality and then performed, it will be found that the sub-culture is attenuated, i.e., weakened. Another mode is 
to raise the pure culture to a temperature approaching its thermal death-point. A third way of securing the same end is to place it under disadvantageous external circumstances, for example in a too alkaline or too acid medium. A fourth method is to pass it through the tissues of an insusceptible animal. Thus we see that, whilst the favourable conditions which we have considered afford full scope for the growth and performance of functions of bacteria, we are able by a partial withdrawal of these, short of that ending fatally, to modify the character and strength of bacteria. In future chapters we shall have opportunity of observing what can be done in this direction.

\section{Bacterial Diseases of Plants}

Reference has been made to the associated work of higher vegetable life and bacteria. The converse is also true. Just as we have bacterial diseases affecting man and animals, so also plant life has its bacterial diseases. Wakker, Prillieux, Erwin Smith, and others have investigated the pathogenic conditions of plants due to bacteria, and though this branch of the science is in its very early stages, many facts have been learned. Hyacinth discase is due to a flagellated bacillus. The wilt of cucumbers and pumpkins is a common disease in some districts of the world, and may cause widespread injury. It is caused by a micro-organism which fills the water-ducts. Wilting vines are full of the same sticky germs. Desiccation and sunlight have a strong prejudicial effect upon these organisms. Melon blight must not be confused with the bacterial wilt of cucumbers and melons. The blight disease is caused by Plasmopara cubensis, a sporulating fungus. Bacterial brown-rot of potatoes and tomatoes is another plant disease probably due to a bacillus. The bacillus passes down the interior of the stem into the tubers, and brown-rots them from within. There is another form of brown-rot which affects cabbages. It blackens the veins of the leaves, and a woody ring which is formed in the stem causes the leaves to fall off. This also is due to a micro-organism, which gains entrance through the water-pores of the leaf, and subsequently passes into the vessels of the plants. It multiplies by simple fission, and possesses a flagellum. Certain diseases of Sweet Corn have been investigated by Stewart, and traced to a causal bacillus possessing marked characters. Professor Potter believes that white-rot of the turnip is produced by Pseudomonas destructans, a liquefying, motile, aërobic bacillus. 


\section{CHAPTER II}

\section{BACTERIA IN WATER}

Quantity of Bacteria in Water-Quality of Water Bacteria: (a) Ordinary Water Bacteria ; (b) Sewage Bacteria ; B. coli communis ; (c) Pathogenic Bacteria in Water-Interpretation of the Findings of Bacteriology-Natural Purification of Water-Artificial Purification of Water-Sand Filtration-Domestic Purification of Water.

The collection of samples, though it appears simple enough, is sometimes a difficult and responsible undertaking. Complicated apparatus is rarely necessary, and fallacies will generally be avoided by observing two directions. In the first place, the sample should be chosen as representative as possible of the real water or conditions we wish to examine. Some authorities advise that it is necessary to allow the tap to run for some minutes previously to collecting the sample; but if we desire to examine chemically for lead or biologically for micro-organisms in the pipes, then such a proceeding would be injudicious.* If it is well water that is to be examined, the well should be pumped for some minutes before taking the sample. If it is river water which is to be examined, it is important to collect the sample without incorporating any deposit. In short, we must use common sense in the selection and obtaining of a sample, following this one guide, namely, to collect as nearly as possible a sample of the exact water, the quality of which it is desired to learn. In the second place, we must observe strict

* Water from a house cistern is rarely a fair sample of a town supply. It should be taken from the main. If taken from a stream or still water, the collecting bottle should be held about a foot below the surface before the stopper is removed. 
bacteriological cleanliness in all our manipulations. This means that we must use only sterilised vessels or flasks for collecting the sample, and in the manipulation required we must be extremely careful to avoid any pollution of air or any addition to the organisms of the water from unsterilised apparatus. A flask polluted in only the most infinitesimal degree will entirely vitiate all results. Vessels may be sterilised by heating at $150^{\circ} \mathrm{C}$. for two or three hours. If this is impracticable the vessel may be washed with pure sulphuric acid, and then thoroughly rinsed out in the water which is to be examined.

Accompanying the sample should be a more or less full statement of its source. There can be no doubt that, in addition to a chemical and bacteriological report of a water, there should also be made $a$ careful examination of its source. This may appear to take the bacteriologist far afield, but until he has seen for himself what "the gathering ground" is like, and from what sources come the feeding streams, he cannot judge the water as fairly as he should be able to do. The configuration of the gathering ground, its subsoil, its geology, its rainfall, its relation to the slopes which it drains, the nature of its surface, the course of its feeders, and the absence or presence of cultivated areas, of roads, of houses, of farms, of human traffic, of cattle and sheep-all these points should be noted, and their influence, direct or indirect upon the water, carefully borne in mind.

When the sample has been duly collected, sealed, and a label affixed bearing the date, time, and conditions of collection and full address, it should be transmitted with the least possible delay to the laboratory. Frequently it is desirable to pack the bottles in a small ice-case for transit. Miquel, Pakes, and others have constructed special forms of packing-cases, and these have their advantages. But the ordinary bottle of water may be quite satisfactorily conveyed, as a rule, packed in sawdust and ice. On receipt of such a sample of water the examination must be immediately proceeded with, in order to avoid, as far as possible, the fallacies arising from the rapid multiplication of germs.

Multiplication of Bacteria in Water.-In almost pure water, at the ordinary temperature of a room, Frankland found that organisms multiplied as follows:

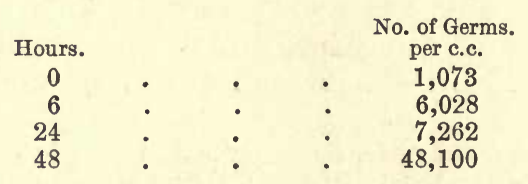

Another series of observations revealed the same sort of rapid 
increase of bacteria. On the date of collection the micro-organisms per c.c. in a deep-well water (in April) were seven. After one day's standing at room temperature the number had reached twenty-one per c.c. After three days under the same conditions it was 495,000 per c.c. At blood-heat the increase would, of course, be much greater, as a higher temperature is more favourable to multiplication. But this would depend in part also upon the degree of impurity in the water, a pure water decreasing in number of germs on account of the exhaustion of the pabulum, whereas, for the first few days at all events, an organically polluted water would show an enormous increase in bacteria.

It is desirable to remember that organisms, in an ordinary water, do not continue to increase indefinitely. Cramer, of Zurich, examined the water of the Lake after it had been standing in a vessel for different periods, with the following results:-

Hours and Days of
Examination.
0 hours
24 ,
3 days
8 ,"
17,
70,

No. of Micro-organisms
per c.c.
143
12,457
328,543
233,452
17,436
2,500

In a general way it may be said that foul waters, rich in putrescible animal matter, show a rapid increase of bacteria; surface waters, such as river water, show a slow and persistent multiplication of organisms; and deep-well waters and spring water show comparatively little increase in contained bacteria. Indeed it may be said that the condition of a water is partly indicated by the rapidity or slowness with which its bacteria increase. A low temperature $\left(5^{\circ}\right.$ C.) undoubtedly diminishes the multiplication, and there are other conditions such as exposure to air, movement, and antagonism of organisms which exert an indirect effect. As will be inferred from what has been said, the most important condition affecting the number of bacteria in a water is the organic matter contained in it.*

\section{The Bacteriology of Water}

In many natural waters there will be found varied contents even in regard to flora alone: algoe, diatoms, spirogyroe, desmids, and all

* For suggestions and hints on points of technique in the systematic examination of a water, see Delépine's Bacteriological Survey of Surface Water Supplies : Jour. of State Medicine, 1898, vol. vi., pp. 145, 193, 241, 289 ; and Bacteriological Examination of Water, by W. H. Horrocks. (See also present volume, pp. 463-473.) 
sorts of vegetable detritus. Many of these organisms are held responsible for certain disagreeable tastes and odours. The colour of a water may also be due to similar causes. Dr Garrett, of Cheltenham, has recorded the occurrence of redness of water owing to a growth of Crenothrix polyspora, and many other similar cases make it evident that not unfrequently great changes may be produced in water by contained microscopic vegetation.

With the exception of water from springs and deep wells, all unfiltered natural waters contain numbers of bacteria.* The actual number roughly depends, as we have seen, upon the amount of organic pabulum present, and upon certain physical conditions of the water. In some species multiplication does not appear to depend on the presence of much organic matter, and, indeed, some bacteria can live and multiply in almost pure water; e.g., Micrococcus aquatilis and Bacillus erythrosporus. Again, others depend not upon the quantity of organic matter, but upon its quality. And frequently in a water of a high degree of organic pollution it will be found that bacteria have been restrained in their development by the competition of other species monopolising the pabulum. It will be necessary to deal with the subject under the two subdivisions of (1) quantity and (2) quality of bacteria found in water.

\section{Quantity of Bacteria in Water}

Percy Frankland has quoted in his book $\uparrow$ a number of records of the quantity of organisms found in various waters. These tables give the returns for the rivers Seine (Miquel), Rhone, Saone (Roux), Spree (Frank), Isar (Prausnitz), Limmat (Schlatter), Rhine (Mœrs), etc. Here it is unnecessary to do more than give typical illustrations, and for comparative purposes English rivers may be taken. Prof. Frankland himself collected water from the river Thames at various times and seasons, and some of his results were as follow :-

* Bacteria, of course, exist in the water of the sea. Near land, as might be expected, the number is greatest, and diminishes rapidly further out to sea. Currents sometimes bring them to the surface from a depth of 596 fathoms (Fischer). At a depth of 100-200 fathoms bacteria have been found in large numbers. The comparative paucity at the surface is due to the germicidal effect of sunlight. Ocean bacteria vary widely in size and shape. Apparently, typical cocci and bacilli are never met with on the high seas. Spirilla and zoogloea masses are common. Most sea bacteria are motile and furnished with flagella ; some are anaërobes.

+ Miero-organisms in Water (1894), pp. 89-116. 


\section{River Thames Water Collected at Hampton.}

Number of Micro-organisms obtained from 1 c.c. of Water.

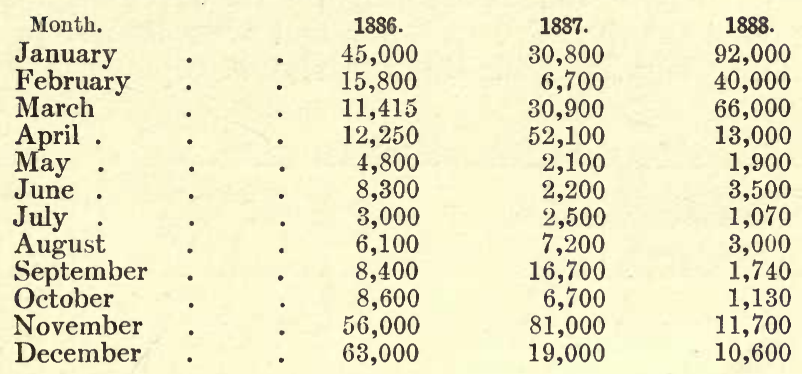

Another example from the river Lea was as follows:-

\section{River Lea Water Collected at Ching ford.}

Number of Micro-organisms obtained from 1 c.c. of Water.

\begin{tabular}{|c|c|c|c|c|}
\hline Month. & & 1886. & 1887. & 1888. \\
\hline January & - & 39,300 & 37,700 & 31,000 \\
\hline February & & 20,600 & 7,900 & 26,000 \\
\hline March & . & 9,025 & 24,000 & 63,000 \\
\hline April . & & 7,300 & 1,330 & 84,000 \\
\hline May . & & 2,950 & 2,200 & 1,124 \\
\hline June . & & 4,700 & 12,200 & 7,000 \\
\hline July . & & 5,400 & 12,300 & 2,190 \\
\hline August & . & 4,300 & 5,300 & 2,000 \\
\hline September & & 3,700 & 9,200 & 1,670 \\
\hline October & & 6,400 & 7,600 & 2,310 \\
\hline November & & 12,700 & 27,000 & 57,500 \\
\hline December & •• & 121,000 & 11,000 & 4,400 \\
\hline
\end{tabular}

"During the summer months these waters are purest as regards micro-organisms, this being due to the fact that during dry weather these rivers are mainly composed of spring water, whilst at other seasons they receive the washings of much cultivated land" (Frankland). Prausnitz has shown that water differs, as would be expected, according to the locality in the stream at which examination is made. His investigations wore made from the river Isar before and after it receives the drainage of Munich :-

Above Munich

Near entrance of principal sewer 13 kilometres from Munich . 
Frankland has shown that the river Dee affords another example, even more perfect, of pollution and restoration repeated several times until the river becomes almost bacterially pure.

Professor Boyce and his colleagues have recently made an examination of the river Severn before and after its waters pass the town of Shrewsbury.* Their findings may be represented briefly as follows :-

\begin{tabular}{|c|c|c|}
\hline Position of Examination. & $\begin{array}{c}\text { Average Total } \\
\text { No. of Bacteria } \\
\text { per c.c. }\end{array}$ & $\begin{array}{l}\text { Average } \\
\text { No. of } B . \text { coli } \\
\text { per c.c. }\end{array}$ \\
\hline $\begin{array}{l}\text { At Asylum, } 2 \text { miles above Shrewsbury } \\
\text { ", Waterworks, opposite Shrewsbury . } \\
\text { " Ferry i., } 0.6 \text { of a mile lower down } \\
\text { " English Bridge, } 1.6 \text { of a mile lower down } \\
\text { " Ferry iii., } 2.5 \text { miles lower down } \\
\text { "Uffington, } 4.7 \text { miles lower down } \\
\text { "Alcham, } 9 \text { miles lower down } \\
\text { " Cressage, } 16 \text { miles lower down }\end{array}$ & $\begin{array}{r}7,000 \\
13,000 \\
20,000 \\
23,000 \\
19,000 \\
17,000 \\
13,000 \\
5,000\end{array}$ & $\begin{array}{r}13 \\
46 \\
177 \\
321 \\
600 \\
142 \\
48 \\
36\end{array}$ \\
\hline
\end{tabular}

This table and that of Prausnitz-and many other workers have produced similar records-illustrate the effect of $(a)$ local pollution, and (b) river purification, upon the bacterial content of water, to which subsequent reference will be made. The record respecting the Severn includes also the indication of sewage pollution by the presence of $B$. coli. An elaborate examination has also been made of the water of the river Thames and the Thames estuary, by Houston, and the report dealing with it is full of information on the subject, to which reference should be made. $†$

Lastly, the accompanying table (pp. 39 and 40), for 1902 and 1903, deals with the London water supply as examined by Crookes and Dewar. $\ddagger$ It is concerned, it should be added, only with numerical results.

This record, compiled from the monthly reports respecting the three waters supplied to the metropolis, illustrates many interesting points upon which we have not space to dwell fully. A few notes, however, upon an actual example are more useful than much theoretical information, and therefore a brief study of these figures may be made. In the main the table illustrates two points more clearly than the preceding tables. The first is the effect of filtration, and the second is the effect of season, upon the number of bacteria in water. In respect to the former, comment is needless. It is only necessary to

* Royal Commission on Sewage Lisposal, Second Report, 1902, p. 99.

† Ilid., Fourth Report, 1904, vol. iii., pp. 1-75.

‡ Metropolitan Water Supply, 1902 and 1903 . 


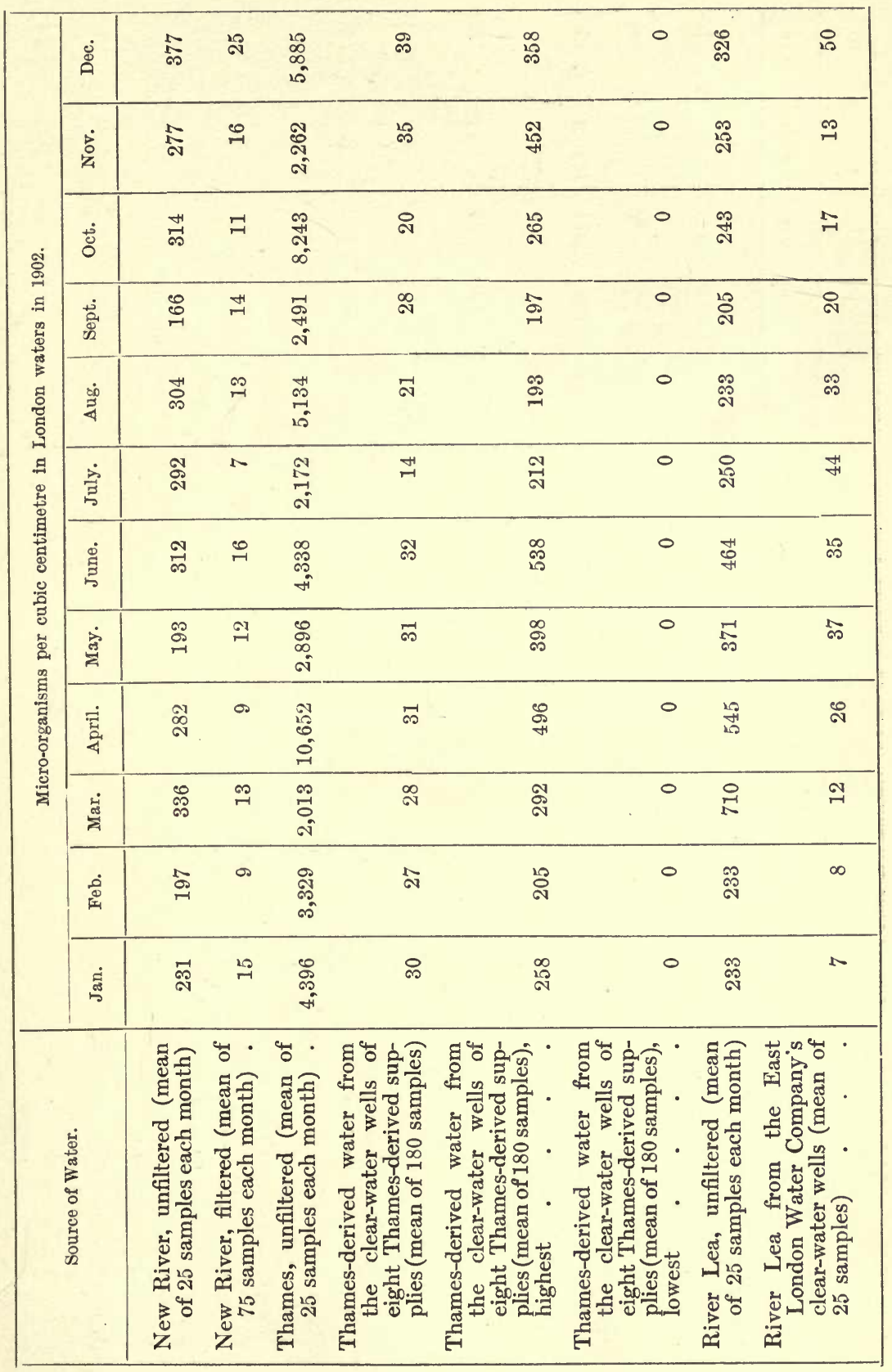




\begin{tabular}{|c|c|c|c|c|c|c|c|c|c|c|}
\hline \multirow{12}{*}{ 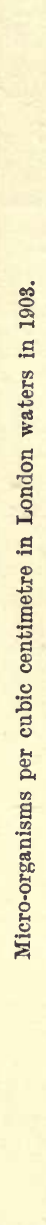 } & ळ் & $\vec{\Phi}$ & 아 & $\begin{array}{l}0 \\
\text { Nू } \\
\text { సे }\end{array}$ & ผิ & ผ̊ & 0 & b ผั & & $\stackrel{\infty}{\rightarrow}$ \\
\hline & 宽 & $\stackrel{19}{\infty}$ & เి & 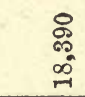 & $\stackrel{\text { ஜ }}{\sharp}$ & 胥 & 0 & 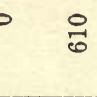 & & ๙ \\
\hline & $\stackrel{8}{\circ}$ & $\stackrel{9}{\infty}$ & ది & $\begin{array}{l}\mathbb{1} \\
0 \\
0 \\
0\end{array}$ & 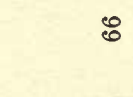 & $\begin{array}{l}\infty \\
10\end{array}$ & 0 & 용 & & เุ \\
\hline & 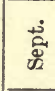 & ๓్ & 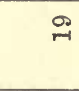 & $\begin{array}{l}\text { ๙ิ } \\
\text { ஸิ }\end{array}$ & శ్లి & \& & 0 & ఝ & & ని \\
\hline & $\ddot{g}$ & 온 & $\stackrel{20}{-10}$ & 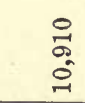 & ঞ & $\underset{c}{N}$ & 0 & $\rho \quad \stackrel{\dot{\delta}}{\sim}$ & & ๓ \\
\hline & 富 & $\begin{array}{l}\infty \\
\text { ลึ } \\
\text { ลิ }\end{array}$ & $\Rightarrow$ & $\begin{array}{l}\text { 10 } \\
\infty \\
\text { if } \\
\text { in }\end{array}$ & ๙ & ํㅗำ & 0 & $\overrightarrow{0}$ & & $\ddot{\circ}$ \\
\hline & $\stackrel{\dot{\Xi}}{5}$ & $\stackrel{\overrightarrow{3}}{-1}$ & . & 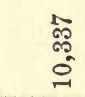 & $\underset{\sharp}{*}$ & $\begin{array}{l}\infty \\
\infty \\
\infty \\
\infty\end{array}$ & $=$ & $=\quad \frac{\pi}{4}$ & & న \\
\hline & $\dot{\leftrightarrows}$ & $\overrightarrow{\text { ติ }}$ & $\Rightarrow$ & 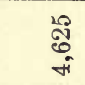 & $\stackrel{2}{-1}$ & $\stackrel{\stackrel{\Xi}{్}}{\mathbb{N}}$ & 0 & $\begin{array}{l}150 \\
\text { [0] }\end{array}$ & & $\stackrel{\leftrightarrow}{\sim}$ \\
\hline & 完 & $\underset{\sharp}{\sharp}$ & 0 & $\begin{array}{l}\text { के } \\
\text { o } \\
\text { ô }\end{array}$ & $\stackrel{10}{\sim}$ & $\because$ & 0 & $=\quad \stackrel{9}{\circ}$ & & 요 \\
\hline & 㟧 & ติ & $\Rightarrow$ & $\begin{array}{l}\hat{0} \\
0 \\
0 \\
0\end{array}$ & ลัง & $\underset{\mathrm{G}}{\sim}$ & 0 & $\begin{array}{lll}\infty \\
0 \\
0\end{array}$ & & ลి \\
\hline & ه. & 穼 & $\infty$ & $\begin{array}{l}0 \\
: 0 \\
0 \\
10 \\
10\end{array}$ & $\stackrel{\text { ڤ్ }}{\circ}$ & 今ึ & o & b 薄 & & 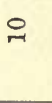 \\
\hline & ક్ & $\stackrel{9}{\infty}$ & $\stackrel{\oplus}{\sim}$ & 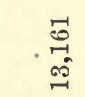 & \& & : & 0 & $\stackrel{20}{\omega}$ & & œ \\
\hline & & 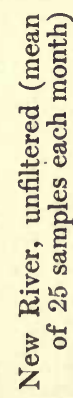 & 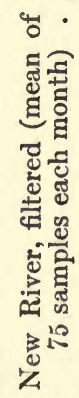 & 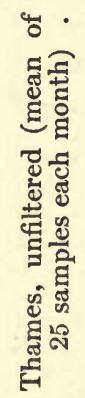 & 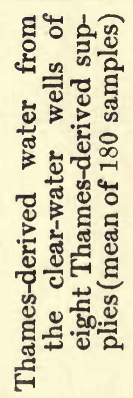 & 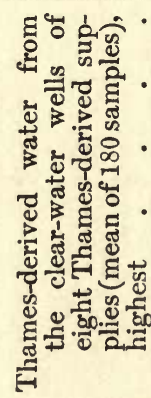 & 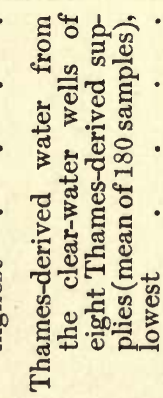 & 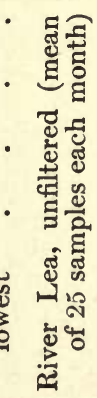 & 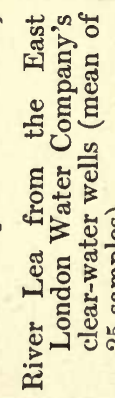 & · \\
\hline
\end{tabular}


examine the returns to recognise the marked reduction in the number of bacteria, in some cases amounting to 98 per cent., brought about by filtration. In respect to the latter, the effect of season, some note is required. It will be seen that during 1902 the figures are fairly uniform throughout the year, showing, on the whole, a rise in winter and spring, and a fall during summer. But in 1903 the returns show wide variation which calls for explanation, which is as follows:-

The water supply in December 1902, on the whole, maintained an equal microbic purity to that of November. This exceptional condition of the supply, the comparatively small number of bacteria, for the winter months is no doubt due to the absence of floods in the Thames Valley, and to the unusually mild character of the season. "If the large deficit in the rainfall is made up," wrote Crookes and Dewar, "no doubt there will be in the near future a period when the filtration of the London waters will require more than usual care. As the general filter-beds are, however, in good working order, we believe these difficulties, should they arise, will be overcome satisfactorily."

The standard of general organic purity during 1902, as defined by chemical methods, was maintained. As regards the month of December, the Thames-derived Companies showed decided differences among themselves, which, as the supply comes from the same source, were essentially connected with differences in storage capacity and variations in the structure of the filter-beds, although the latter is of less importance in removing soluble organic matter.

Crookes and Dewar add: "The longer our experience of the bacteriological method, as applied to the analysis of the filtered supply, and the wider its application, the more we are convinced of its primary importance as a safeguard to the public. It enables us to define in a much more delicate way than is possible by chemical analysis what is an efficiently filtered water, and thereby enables the chemist to warn the engineer the moment any one of his filters show signs of defective working. Whether the supply as regards the organic matter in solution varies more or less according to the season of the year, is of relatively small moment as compared with the knowledge that the microbic impurity is reduced to a minimum."

That was the position at the end of 1902. But at the turn of the year, owing to the great increase in the rainfall, the microbes in the unfiltered Thames water rose from about 6000 to 13,000 , that is, the bacteriological impurity about doubled, whereas the unfiltered New River water underwent little or no alteration. The result of this increase was that the filters of the Thames-derived Companies, which were not working at their best, furnished a larger number of samples from the filter wells, showing an increase in the number of bacteria which was the inevitable result of an increased rainfall.

Things remained thus until April, when in comparison with the month of March the bacteriological quality showed considerable improvement, a result which might have been anticipated from the advent of summer, and the improved natural conditions associated with vegetable growth; a state of things which generally improves the quality of the water obtained from such collecting areas as the valleys of the Thames and Lea. But in June, when the number of bacteria ought to have been low, as ordinarily there would be a small rainfall, an exceptional condition of things arose. The total excess of rainfall amounted to 49.9 per cent. on the thirty years' average, so that during the month of June actually 22.5 per cent., or an amount approaching one-half of the previous excess, of rain fell in the valley of the Thames. Such an amount of rain is altogether exceptional in twenty-two years' experience. The result was that the proportion of vegetable matter in solution, and therefore the colour of the water, were both quite exceptional for the summer months. Nevertheless, the general filtration was adequately and effectively performed, as is shown by the bacteriological results. Similar conditions prevailed in August and October. The exceptional rainfall, which amounted to sixty per 
cent. in excess of the average, kept the colour and amount of soluble vegetable matter in solution abnormally high. In December, owing to the continued rains, the New River and Thames unfiltered waters contained a maximum number of bacteria. In dry weather the number per c.c. had been as low as 149 and 2013 respectively, but owing to seasonal changes they had risen to 861 and 27,216 bacteria per c.c. respectively.

From these various records we find that in the result the number of bacteria in river water depends upon a variety of circumstances, amongst which the most important direct conditions are four, namely, (1) local pollution, (2) natural purification (to which subsequent reference will be made), (3) season and rainfall, and (4) sedimentation and filtration. Behind these direct conditions we have also seen that time, temperature, light, exposure to air, and the presence of organic matter play an essential part.

Bacteriological Examination of Water.-[See Appendix, p. 463.]

Quantitative Standard.-In arriving at a conclusion respecting the number of organisms in a water and their bearing upon its suitability for use, it should be remembered that a chemical report and a bacteriological report are desirable before forming an opinion. The former is able to tell us the quantity of salts and condition of the organic matter present: the latter the number and quality of microorganisms. Neither can take the place of the other, and, generally speaking, both are more or less useless until we can learn, by inspection and investigation of the source of the water, the origin of the organic matter or contamination. Hence a water report should contain not only a record of physical and microscopical characters, of chemical constituents, and of the presence or absence of microorganisms, injurious and otherwise, but it should also contain information obtained by personal investigation of the source. Only thus can a reasonable opinion be expected. Moreover, it is generally only possible to form an accurate judgment of a water by watching its history; that is to say, not from one examination only, but from a series of observations. The writer has examined a certain water supply for thirty-six consecutive months. In 1901 the average number of bacteria per c.c. was 93 , in 1902, 136, and in 1903, 57 . This shows a stable bacterial content which in itself is favourable. A water yielding a steady standard of bacterial content is a much more satisfactory water, from every point of view, than one which is unstable, one month possessing 50 bacteria per c.c. and another month 5000. It is obvious that rainfall and drought, soil and trade effluents, time and temperature, will have their influence in materially affecting the bacterial condition of a water.

Miquel and others have suggested standards which allow "very pure water" to contain up to 100 micro-organisms per c.c. Pure water must not contain more than 1000 , and water containing up to 
100,000 bacteria per c.c. is contaminated with surface water or sewage. Macé gives the following table:-

\begin{tabular}{|c|c|c|c|c|}
\hline \multirow{5}{*}{\multicolumn{2}{|c|}{$\begin{array}{l}\text { Very pure water } \\
\text { Good water } \\
\text { Passable (mediocre) water } \\
\text { Bad water. }\end{array}$}} & \multicolumn{3}{|c|}{ Bacteria per c.c. } \\
\hline & & 0 & to & 50 \\
\hline & & 50 & ," & 500 \\
\hline & & 500 & ," & 3,000 \\
\hline & & 3,000 & , & 10,000 \\
\hline Very bad water & - $\quad$ r & 10,000 & ," & 100,000 \\
\hline
\end{tabular}

Koch first laid emphasis on the quantity of bacteria present as an index of pollution, and whilst different authorities have all agreed that there is a necessary quantitative limit, it has been impossible to arrive at a settled standard of permissible impurity. Besson adopts the standard suggested by Miquel, and on the whole French bacteriologists follow suit. They also agree with him, generally speaking, in not placing much emphasis upon the numerical estimation of bacteria in water. In Germany and England it is the custom to adopt a stricter limit. Koch in 1893 suggested 100 bacteria per c.c. as the maximum number of bacteria which should be present in a properly filtered water. Miquel holds that not more than ten different species of bacteria should be present in a drinking water, and such is a useful standard. The presence of many rapidly liquefying bacteria or organisms associated with sewage or surface pollution would, even though present in fewer numbers than a standard, condemn a water. From a consideration of all the facts it will be seen that it is impossible to judge alone by the numbers. As the science of bacteriology advances less emphasis is laid upon quantitative estimation, for the reason that it is impossible to gauge the quality of a water only by such estimation. The character of the organisms present and the relative abundance of each species is of more importance than quantitative estimations. Such estimations of water bacteria, based upon the counting of colonies in plate cultures, are of little value, and are in no sense an adequate bacteriological examination of a water. It is such "examinations" which have brought bacteriology into disrepute, for it is certain that estimations of this kind are frequently not even approximately correct, nor do they furnish any final indication as to safety or otherwise of a water supply. At the same time it should not be forgotten that, other things being equal and constant, a low number of organisms tends to indicate that a water has not been contaminated with organic matter or the addition of foreign bacteria, and has not been in a condition to favour multiplication of bacteria, and vice vers $\hat{\text {. }}$. Broadly speaking, it must be true that a water containing a large degree of organic matter, the pabulum of bacteria, will contain a higher number of bacteria than a water containing a 
low degree, and this, of course, is the reason for quantitative estimations.

\section{Quality of Water Bacteria}

The species of bacteria found in water vary widely. Many of them are common in pure water, and may be strictly termed "water bacteria"; others are as clearly "sewage bacteria," with an allied group belonging to the soil and washed into rivers, or wells, by rain, and which may be described as "surface bacteria"; and a third group are the pathogenic bacteria, which have under exceptional conditions been isolated from water. Prof. Marshall Ward, in his fifth report to the Water Research Committee of the Royal Society, drew up a classification of water bacteria, which was adopted two years later by Boyce and Hill. + In 1899 Johnson and Fuller made other groups, + and many other workers have sug. gested classifications. The two most recent have been constructed by Horrocks of Netley§ and Jordan of Chicago.\|

Both authorities recognise that provisional classification is all that is at present possible. Their groups are as follows:-

CLASSIFICATION OF HORROCKS. GROUP

i. Fluorescent bacilli.

ii. B. aquatilis sulcatus.

iii. B. suhtilis and "Potato bacilli."

iv. B. liquefaciens.

v. Chromogenic (red) bacilli.

vi. Chromogenic (yellow) bacilli.

vii. Chromogenic (blue) bacilli.

viii. Chromogenic (milk-white) bacilli.

ix. Chromogenic (brown) bacilli.

x. Micrococci.

xi. Sarcinæ.

xii. Spirilla.

xiii. Denitrifying and nitrifying bacteria.

xiv. B. coli communis.

xv. B. enteritidis sporogenes.

xvi. Staphylococci.

xvii. Streptococci.

xviii. The Proteus group.

xix. Sewage bacteria.

xx. B. typhosus.

\section{CLASSIFICATION OF JORDAN.} GROUP

i. B. coli communis.

ii. B. lactis aërogenes.

iii. Proteus.

iv. B. enteritidis.

v. B. fluorescens liquefuciens.

vi. B. fluorescens non-liquefaciens.

vii. B. subtilis.

viii. Non-gas forming, non-fluorescent, non-sporulating, liquefy gelatine and acidify milk.

ix. Similar to Group viii., but milk rendered alkaline.

x. Similar to Group viii., but gelatine not liquefied.

xi. Similar to Group ix., but gelatine not liquefied.

xii. Similar to Group xi., but the reaction of milk not altered.

xiii. Chromogenic bacilli, not included in above groups.

xiv. Chromogenic.Staphylococci.

xv. Non-chromogenic Staphylococci.

xvi. Sarcinæ.

xvii. Streptococci.

* Proc. Roy. Soc., 1897, Ixi., p. 415.

† Jour. of Path. and Bact., 1899, vi., p. 32.

¥Jour. of Exp. Med., 1899, iv., p. 609.

$\S$ An Introduction to the Bacteriological Examination of Water, 1901, p. 42 et seq.

II Jour. of Hygiene, 1903, vol. iii., No. 1, p. 5. 
Both the above quoted authorities furnish a large body of facts illustrative of the characteristics of the various groups suggested, to which the reader is referred for further particulars. Broadly it may be said that the organisms classified in twenty groups by Horrocks are divisible into a few general divisions. Groups i.-xii. are the ordinary water bacteria ; Group xiii. is the denitrifying and nitrifying organisms found in soil, water, etc.; Groups xiv.-xix. are the sewage bacteria; and Group xx. represents the pathogenic group of organisms occurring occasionally in water. Brief reference will now be made to these four groups, with the exception of the second, which will be dealt with subsequently.

(a) Ordinary Water Bacteria.-These are organisms usually found in pure or approximately pure waters. They are common in well waters and unpolluted river water. They include the common fluorescent bacilli, liquefying and non-liquefying, and which create an iridescent green colour in the nutrient media. In this class also are $B$. aquatilis sulcatus, the "potato bacilli" (B. mesentericus, vulgatus, fuscus, et ruber), the "hay bacilli" (B. subtilis, B. mycoides, B. megatherium), the liquefying bacilli common in unfiltered waters, the chromogenic organisms (B. prodigiosus, B. lactis erythrogenes, B. rubescens, B. arborescens, B. aquatilis, B. aurantiacus, B. violaceus, etc.), and the micrococci, sarcinæ, and ordinary water spirilla.* The presence of these species of bacteria in water, unless in very exceptional numbers, indicates little of importance. They vary according to season, geological formation over or through which the water passes, surface washings, aëration of the water, forms of vegetation existing in the water, and many other similar natural conditions. The fluorescent and non-gas-producing and non-liquefying bacilli are generally less abundant in recently polluted waters than in purer waters, and non-chromogenic staphylococci more abundant.

(b) Sewage Bacteria.-This group includes B. coli communis and its allies, the Proteus family, B. enteritidis sporogenes of Klein, and certain streptococci and staphylococci. They will be treated of subsequently in a chapter devoted to the bacteriology of sewage (see pp. 152-157). Exception will, however, be made in the case of $B$. coli communis, as this organism is perhaps the most important in relation to water. It will, therefore, be considered here. In the first place the chief biological and cultural facts may be stated, and in the second place a general note may be added.

* The biological characters of these various groups of water bacteria will be found in Frankland's Micro-organisms in Water, pp. 399-508; Lehmann and Neumann's Bacteriology, vol. ii., pp. 133-381; Crookshank's Bacteriology and Infective Diseases ; Horrocks' Bacteriological Examination of Water, pp. 42-80 ; and in the systematic works of Sternberg, Flügge, Besson, Macé, etc. 


\section{BACILLUS COLI COMMUNIS (Escherich)}

Source and habitat-An organism of wide distribution, normally present in the excreta of man and animals. Abundant in crude sewage $(100,000$ per c.c. in London Sewage, Houston). In polluted water, milk, soil, etc.

Morphology-A short rod with round ends; size and shape may vary in same colony ; polymorphism, depending upon age of culture, products of culture, composition of medium, etc., 2 to $3 \mu$ long, 0.5 to $0.6 \mu$ broad; sometimes oval, hardly longer than broad. Usually single, but occasionally in pairs, bundles, or even chains and threads (Plate 3 ).

Staining reaction-Ordinary aniline dyes. Decolorised by Gram. (Schmidt states that $B$. coli from fatty stools of infants holds the Gram.)

Capsule-Present.

Flagella-3 or 4 in number, fragile, short, and not wavy. Sometimes only a terminal one; sometimes several long ones; but polar staining and vacuolation frequently present in old cultures, or cultures grown under unfavourable conditions.

Motility-Present, especially in young cultures, but not, as a rule, so active as B. typhosus; oscillatory rather than progressive. Sometimes apparently absent.

Spore formation-None.

Biology : cultural characters (including biochemical features)-Grows best at $37^{\circ} \mathrm{C}$. but will also grow at room temperature. Gordon showed that many varieties of B. coli exist with many minor modifications (Jour. of Path. and Bact., 1897).

In gelatine plate cultures the colonies appear generally within 24 hours at $20^{\circ} \mathrm{C}$. The deep colonies appear as small white dots, the surface colonies as delicate, slightly granular films of an irregularly circular shape. They are bluish-white by reflected, and amber colour by transmitted light. The diameter of the colony is 1 to $2 \mathrm{~mm}$. The colonies are transparent, and sometimes iridescent, especially towards the periphery, but at the centre and over the entire surface in old cultures an opacity due to a greater thickness of the bacterial growth is observed (Plate 4).

It has been observed that species derived from water grow in transparent colonies, whereas those from the alimentary canal or excreta may show opacity of the colony, which characteristic disappears if the culture be passed through milk. About the second or third day the surface colonies attain a diameter of 5 to $6 \mathrm{~mm}$., and become marked by concentric, or radiating, or irregular markings. The surrounding gelatine very frequently acquires a dull, cloudy, faded appearance, and the edges of the colony become more crenated and thinner. The whiteness of the colony turns to yellow. There is no liquefaction of the gelatine.

In gelatine stab-cultures the organism grows rapidly. On the surface, in twentyfour hours, the growth is often 2 to $3 \mathrm{~mm}$. in diameter, and closely resembles a surface colony in a plate culture, though more luxuriant. A thick white growth extends along the whole length of the track of the needle, and not infrequently gas bubbles or fissures appear. The gelatine is not liquefied, even in old cultures.

In gelatine streak cultures growth is also abundant. In twenty-four hours the elongated milky surface colony may be $5 \mathrm{~mm}$. in diameter. It consists of a delicate faintly-granular film with transparent and irregular margins. Down the centre longitudinally the growth is thicker and therefore more opaque. Irregular thickenings, foldings, and corrugations may occur in old cultures. Sometimes the film shows iridescence, and the medium, though not liquefied, becomes clouded. The growth, as on the plate cultures, is bluish-white by reflected, and yellowish-amber in colour by transmitted light.

In 25 per cent. gelatine at $37^{\circ}$ C.-In 48 hours the melted gelatine remains clear, but a thick pellicle forms on the surface (Klein).

Gelatine shake cultures become turbid, and within twenty-four hours at $20^{\circ} \mathrm{C}$. are riddled with bubbles of gas, which are generally more numerous and larger towards the foot of the tube. They increase in size by the second day, sometimes even forming fissures. The gas is mainly carbonic acid. The presence of a small per cent. of fermentable sugar in the medium increases the gas production (Plate 4.).

On potato-gelatine the colonies of $B$. coli are similar in appearance to those 


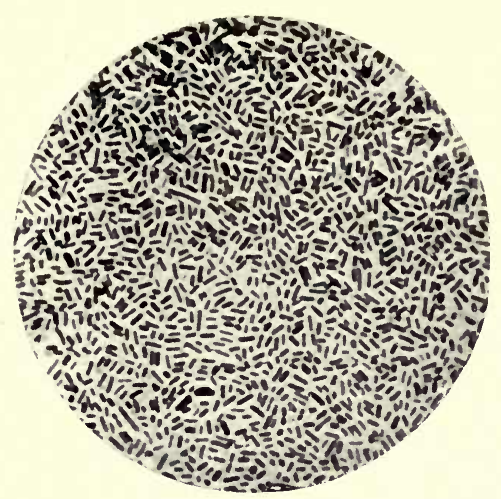

Bacillus coli communis. From agar culture, 48 hours at $37^{\circ}$ C. $\times 1000$.

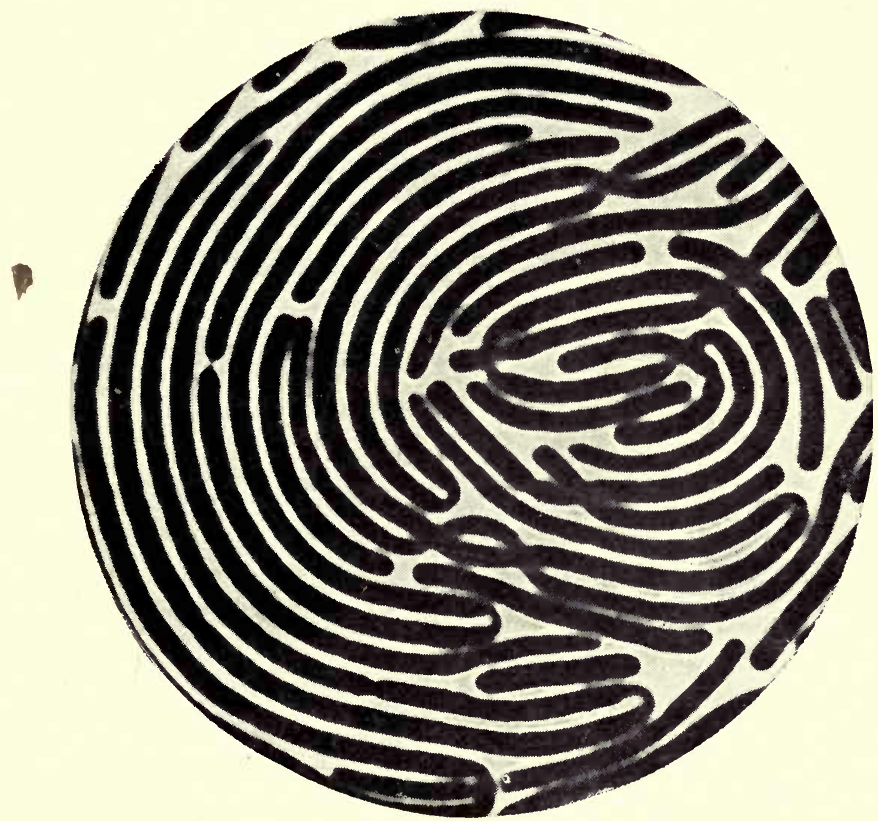

Proteus vulgaris. Impression preparation from "swarming islands" on gelatine, 20 hours at $20^{\circ} \mathrm{C} . \times 3000$. 
<smiles></smiles> 
occurring on ordinary gelatine, except that they grow more slowly, are more circumscribed, and of a characteristic brown colour (Houston).

On carbol-gelatine ( 05 per cent. of phenol), the growth does not differ from ordinary gelatine cultures except that it is delayed.

Broth-In less than twelve hours at $37^{\circ} \mathrm{C}$. the medium becomes uniformly turbid. It may be very pronounced. Frequently there is also at a later stage a marked amorphous flocculent sediment consisting of bacteria. Only a faint film forms on the surface, which rarely becomes a pellicle. There is a fotid odour, and sometimes gas formation. In glucose, lactose, saccharose broth (2 per cent.), and glucose-formate broth (Pakes), and bile-salt broth (M`Conkey), the growth is abundant, and gas is produced. In phenolated broth $(\cdot 05$ per cent. of phenol), and in broth containing formalin (1 to 7000), there is also growth.

On agar at $37^{\circ} \mathrm{C}$. the organism grows rapidly, producing thin, moist, translucent creamy greyish-white colonies of irregular shape and size. The colonies grow more rapidly on the surface than in the depth of the medium. The same appearances occur on agar at $20^{\circ} \mathrm{C}$., except that the growth is delayed. Gas bubbles frequently occur in the condensation fluid.

Litmus lactose agar (2 per cent.)-The medium is turned red in twenty-four hours,

B. Typhosus.

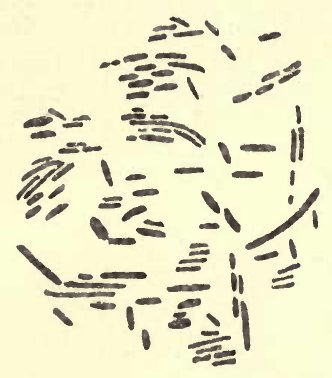

B. coli.

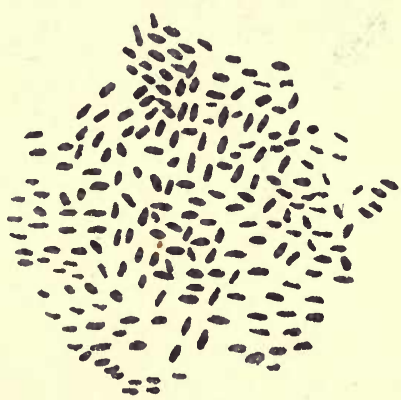

FiG. 9.-Diagrams of B. typhosus and B. coli.

and the surface growth becomes tinged slightly with the reddened litmus. Numerous gas bubbles are produced in the medium.

On potato at $37^{\circ} \mathrm{C}$. there is produced in twenty-four hours a thick, moist, yellowish-grey growth, becoming brown in old cultures. The colour varies widely in degree, sometimes being richer than at other times. The potato becomes changed in colour near the growth. If the potato is not fresh, or its reaction has been made alkaline, the growth of $B$. coli may be almost colourless. There are, of course, a very large number of bacteria which produce a growth on potato not readily distinguishable from $B$. coli.

Litmus milk-Usually an acid curdling of the milk occurs in twenty-four hours at $37^{\circ} \mathrm{C}$., though sometimes slightly delayed. The bluish-purple colour changes to pink, then the whole of the milk is turned into a solid compact coagulum, the milk itself becoming white. Later the redness extends from the surface downwards until the whole contents of the tube are bright red in colour.

On blood serum at $37^{\circ} \mathrm{C}$. an abundant white glistening layer is rapidly developed, somewhat similar to the growth on agar. There is no liquefaction.

Indol is produced in bouillon fluid cultures (e.g. peptone water). The reaction is frequently obtainable in forty-eight hours at $37^{\circ} \mathrm{C}$., but in any case is generally well-marked in bouillon cultures kept at $37^{\circ} \mathrm{C}$. for five days. The "red reaction" may be obtained by adding to such a culture 1 c.c. of a 0.02 per cent. solution of potassium nitrite, and 0.5 c.c. of strong sulphuric acid. If the colour (due to 
nitroso-indol) does not appear at once, the culture may be incubated for a brief period.

Reduction of nitrate. $-B$. coli is a vigorous denitrifying organism. In twentyfour hours at $37^{\circ} \mathrm{C}$. the reduction of nitrates to nitrites is well marked. (Bouillon 5 per cent., $\mathrm{KNO}_{3} 0 \cdot 1$ per cent., water $94 \cdot 9$ per cent.).

Aërobic or facultative anaërobic. bacillus.

Vitality and powers of resistance, not considerable, but more than the typhoid

The following table of comparative features of $B$. coli and B. typhosus is a provisional scheme of some of the differences between a typical $B$. coli and a typical typhoid bacillus. As is pointed out elsewhere, the Coli group is large and its characteristics vary according to origin, race, cultivation, and many other conditions. In some ways the table is misleading, as it is exceptional to find a bacillus which gives all these features, but the table is inserted for reference, because in a general way it states the broad differences between the types :-

\section{Comparative Features of $B$. coli and $B$. typhosus}

\section{B. typhosus.}

Morphology-Bacillus of unequal lengths; some filaments.

Flagella-Long, wavy, spiral, numerous (9 to 18$)$; movement very active.

Ongclatine and agar-Angular, irregular, slightly raised colonies; slow growth ; medium remains clear.

In gelatine-In ordinary gelatine and in lactose gelatine no gas is produced (at $20^{\circ} \mathrm{C}$.). No liquefaction.

Milk-Not curdled by the bacillus (at $37^{\circ}$ C.). No acid production.

Indol-In bouillon and Witte's peptone water, no production of indol.

Bouillon containing 0.3 per cent. Phenol, or Formalin (1 : 7000)-No growth.

Lactose-bouillon at $37^{\circ}$ C.-No gas production.

Neutral-red glucose-agar-No change.

Glucose or lactose media, shake culturesNo gas production.

Potato-An "invisible growth" if the potato is acid in reaction.

25 per cent. gelatine at $37^{\circ} \mathrm{C} .-$-Strongly and uniformly turbid (Klein). No pellicle.

Elsner's iodised potato-gelatine - Slow growth ; small transparent colonies.

Proskauer and Capaldi's Medium, No. 1 -No growth; no change in reaction.

Widal's reaction-Bacilli became motionless and agglutinated when suspended in blood serum from a typhoid patient. (See Appendix.)

$M$ 'Conkey's lactose agar-Surface colonies transparent; medium clear.

$V$ itality in water or sewage-B. typhosus soon ceases to multiply and more or less readily dies.

$P$ feiffer's inoculation test with anti-typhoid serum-Negative result.
B. coli.

Bacillus shorter and thicker; filaments rare.

Shorter, stiffer, few (average 3), movement less active, and sometimes almost absent.

Colonies with even margin, homogenous, much larger and quicker growth, medium becomes turbid or coloured.

Under the same circumstances abundant gas is produced. No liquefaction.

Milk is curdled, within 24 to 48 hours at $37^{\circ} \mathrm{C}$. Abundant acid production.

Indol is present as a rule.

Grows well and uniformly throughout medium.

Gas production occurs.

Marked green fluorescence.

Marked gas production.

Thick, yellowish-white growth, later becoming brown in colour.

Gelatine remains clear within 48 hours, but a thick pellicle forms on the surface.

Rapid growth; large brown colonies.

Growth ; acid reaction.

B. coli remains motile and not agglutinated.

Surface colonies white with yellow centre; haze on medium.

$B$. coli retains vitality and power of self-multiplication.

Positive result, variable symptoms according to virulence of bacillus. 
bicarbonate of lime, is converted into insoluble normal carbonate of lime by the addition of a suitable quantity of limewater. Carbonates of lime and magnesia are soluble in water containing free carbonic acid, but when fresh lime is added to such water it combines with the free $\mathrm{CO}_{2}$ to form the insoluble carbonate, which falls as a sediment:-

$$
\mathrm{CaCO}_{3}+\mathrm{CO}_{2}+\mathrm{CaH}_{2} \mathrm{O}_{2} \text { (limewater) }=2 \mathrm{CaCO}_{3}+\mathrm{H}_{2} \mathrm{O} \text {. }
$$

As the carbonate falls to the bottom of the tank it carries down with it the organic particles. Hence sedimentation is brought about by means of chemical precipitation. It is obviously a mechanical process as regards its action upon bacteria. Nevertheless its action is well-nigh perfect, and 400 bacteria per c.c. may be reduced to 4 or 5 per c.c. We shall refer to this same action when we come to speak of bacterial purification of sewage. Alum has been frequently used to purify water which contain much suspended matter. Five or six grains of alum are added to each gallon of water, plus some calcium carbonate by preference. Precipitation occurs, and with it sedimentation of the bacteria, as before. But, as Babés has pointed out, alum itself acts inimically on germs; in such treatment, therefore, we get sedimentation and germicidal action combined.

As a matter of actual practice, however, sedimentation alone is rarely sufficient to purify water. It is true that the collection of water in large reservoirs permits subsidence of suspended matters, affords time for the action of light, and the suicidal competition among the common water bacteria. But in small collections of water it is otherwise. Here filtration is the most important and most reliable method.

Sand filtration, as a means of purifying water, has been practised since the early part of last century. But it was not till 1885 that Percy Frankland first demonstrated the great difference in bacterial content between a water unfiltered and a water which had passed through a sand filter (only about 3 per cent. of the bacteria originally present being left in the water). Previous to this time the criterion of efficiency in water purification had been a chemical one only, and the presence or absence of bacteria in any appreciable quantity was described not in mathematical terms, but in indefinite descriptive words, such as "turbid," "cloudy," etc. It is needless to say that this difference in estimation was largely due to the introduction by Koch of the gelatine-plate method of examination. As a result of investigation Percy Frankland formulated the following conclusions as regards the chief factors influencing the number of microbes passing through the filter. The efficiency of filtration, he held, depended upon (a) the storage capacity for unfiltered water, by which it was possible to obtain the preliminary advantage of subsidence; (b) the thickness of fine sand through which the filtration is carried on; 
(c) rate of filtration; (d) the renewal of the filter-beds. After a certain time the filter-bed becomes worn out and inefficient, and at such times renewal is necessary. Not only may the age of the filter act prejudicially, but the extra pressure required will tend to force through it bacteria which ought to have remained in the filter.

In 1890 a special study of filtration was made by the Massachusetts State Board of Health, and in annual reports published from 1890, a number of experiments are recorded which have proved of classic importance, and which should be consulted by the student or practical worker desiring to acquire a thorough grasp of the principles of biological filtration. There it is shown that water can be filtered through sand filters at the rate of $3,000,000$ gallons per acre daily and 99.95 per cent. of the bacteria removed. In actual practice it was found that the finer sands were more effective than the coarser, and under moderate pressure 1 foot of sand was as effective as 5 feet. Over 80 per cent. of the bacteria removed were found in the upper inch of sand and 55 per cent. in the upper quarter inch. If the surface of the filter was scraped, it was shown that an increased number of bacteria passed through the filter, which was therefore much less effectual. Subsequently, Koch emphasised the importance of this vital layer. But it was the Massachusetts Board that first proved by experiment that the oxidation which occurs in a filter-bed was due to the nitrifying organisms in the surface or scum layer. When nitrification is established in a filter, the rate of filtration within certain limits was found to exert comparatively little effect upon the removal of the organic matter.

In 1893 Koch brought out his monograph upon Water Filtration and Cholera, and his work had a deservedly great influence upon the whole question. He showed how the careful filtration of water supplied to Altona from the Elbe saved the town from the epidemic of cholera which came upon Hamburg as a result of drinking unfiltered water, although Altona is situated several miles below Hamburg, and its drinking water is taken from the river after it has received the sewage of the latter.

Now, from his experience of water filtration, Koch arrived at several important conclusions. In the first place, he maintained that the portion of the filter-bed which really removed micro-organisms effectively was the slimy membranous organic layer upon the surface of the sand. This layer is produced by a deposit from the still unpurified water lying immediately above it. The most vital part of the filterbed is this organic layer, which, after formation, should not be disturbed until it requires removal owing to its impermeability. A filter-bed, as is well-known, consists of, say, 3 feet of sand and 1 foot of coarse gravel. The water to be filtered is collected into large reservoirs, where subsidence by gravitation occurs. From thence it 
is led by suitable channels to the surface of the filter-bed. Having passed through the 3 or 4 feet of the bed, it is collected in a storage reservoir and awaits distribution. Such being the principles of construction, it will be apparent that the action of the whole process is both mechanical and chemical. Mechanically by subsidence, much suspended matter is left behind in the reservoir. Again, mechanically, much of that which remained suspended in the water when it reached the filter-bed is waylaid in the substance of the sand and gravel of the filter-bed. The next change is a chemical one. Oxidation of the organic matter occurs to some extent as the water passes through the sand. Until recently this chemical action and the double mechanical action (sedimentation and straining) was believed to be the complete process, and its efficiency was tested by chemical oxidation and alteration, and absence of the suspended matter. Now, however, it is recognised that the second portion of the chemical action is vastly the more important, indeed, the only vital part of the process. This is the chemical effect of the layer of scum and mud on the surface of the sand at the top of the filterbed. The mechanical part of this layer is, of course, the holding back of the particulate matter which has not subsided in the reservoir; the vital action consists in what is termed nitrification of unoxidised substance, which is accomplished in this layer of organic matter. We shall deal at some length with the principles of nitrification when we come to speak of soil. But we may say here that by nitrification is understood a process of oxidation of elementary compounds of nitrogen, by which these latter are built up into stable bodies which can do little or no harm in drinking-water. The action of a filter-bed may, therefore, be summarised as follows:-There is (1) subsidence of the grosser particles of impurity in the settling tank; (2) mechanical obstruction to impurities in the interstices of the scum, sand, and gravel in the filter; (3) oxidation of organic matter by the oxygen held in the pores of the sand and gravel; (4) nitrification in the vital scum layer, which is accomplished by micro-organisms themselves. This latter is now considered to be incomparably the most important part of the filter. That being so, its removal, except when absolutely necessary, is to be avoided as detrimental to the efficiency of the filter. New filters have obviously but little of this action. Kümmel found that when a filter had new sand placed upon it the number of bacteria in the filtered water was as follows:

Before cleaning . $\quad$ - . $\quad 42$

One day after cleaning $\quad . \quad$. $\quad$. 1880

Two days after cleaning . $\quad$. $\quad 752$

Three days after cleaning . $\quad$ - 208

Four days after cleaning $\quad$. $\quad$. 156

$\begin{array}{lll}\text { Five days after cleaning } \quad \text {. } & 102\end{array}$

Six days after cleaning . $\quad$. $\quad 84$ 
Hence it is necessary to allow a new filter-bed to act for a short period (say four days) before the filtered water is used for domestic purposes, in order to allow a fresh film, the organic layer, to be formed. This must also be borne in mind after a filter-bed has been cleaned.*

To maintain this nitrifying action of a filter in efficiency, Koch suggested, in the second place, that the rate of filtration must not exceed four inches per hour. At the Altona water-works this rate of filtration was maintained, and the number of organisms always remained below 100 per c.c., which, as we have seen, is the standard. Thirdly, it is important that periodic bacteriological examinations should be made. Koch's emphasis upon this point is well known, and the cumulative experience of bacteriologists only further supports such a course being taken. Clark and Gage of the Lawrence Experimental Station, claim that the test for the presence of $B$. coli is a more delicate indication of filter efficiency when filtering polluted water than tests for the total number of bacteria present. Fourthly, Koch maintained that the thickness of the sand of the filter-bed should never be less than one foot. Fifthly, if it be true that efficient sand filtration is a safeguard against putrefactive and disease-producing germs, then there can bo but one criterion of efficiency, viz., their absence in the filtered water, which can only be ascertained by regular examination. But it is not alone for pathogenic germs that filtration is proposed. Hence Koch laid down that filtered water containing more than 100 microorganisms of any kind per c.c. is below the standard of purity, and should not, if possible, be distributed for drinking purposes. In this country chemical analysis, with a more or less cursory microscopic examination, has been almost invariably accepted as reliable indication of the condition of the water. But such an examination is not really any more a fair test of the working of the filter than it is of the actual condition of the water. It is true, the quantity of organic matter can be estimated and the condition in which it exists in combination obtained; but it cannot tell us what a bacteriological examination can tell us, viz., the quantity and quality of living micro-organisms present in the water. Upon this fact, after all, an accurate conclusion depends. There is abundant evidence to show that no valuable opinion can be passed upon a water except by both a chemical and a bacteriological examination, and further by a personal investigation, outside the laboratory, of the origin of the water and its liabilities to pollution.

So convinced was Koch of the efficiency of sand filtration as protection against disease-producing germs, that he advocated an adaptation of this plan in cases where it was found that a well yielded infected water. Such pollution in a well may be due to p. 228 . 
various causes; surface-polluted water oozing into the well is probably the commonest, but decaying animal or vegetable matter might also raise the number of micro-organisms present almost indefinitely. Koch's proposal for such a polluted well was to fill it up to its highest water level with gravel, and above that, up to the surface of the ground, with fine sand. Before the well is filled up in this manner it must, of course, be fitted with a pipe passing to the bottom and connected with a pump. This simple procedure of filling up a well with gravel and sand interposes an effectual filter-bed between the subsoil-water and any foul surface-water percolating downwards. Such an arrangement yields as good, if not better, results than an ordinary filter-bed, on account of there being practically no disturbance of the bed nor injury done to it by frost. The evidence that filter-beds remove pathogenic bacteria has not only been demonstrated by experiment but by actual experience. At Lawrence, Hamburg, Mount Vernon, and other towns, a marked decline in water-borne typhoid fever has occurred as a result of filtration.

The effect of filtration upon the number of bacteria was demonstrated in the results which Sir Edward Frankland arrived at in his investigation of London waters so long ago as 1887.*

\section{Mean of Monthly Examinations for the Year.}

\begin{tabular}{|c|c|c|c|c|c|}
\hline \multirow{2}{*}{ Name of Company. } & \multirow{2}{*}{$\begin{array}{l}\text { Source of } \\
\text { Supply. }\end{array}$} & \multicolumn{3}{|c|}{ Micro-organisms per c.c. } & \multirow{2}{*}{$\begin{array}{l}\text { Average \% of } \\
\text { Micro- } \\
\text { organisms } \\
\text { removed by } \\
\text { Filtration. }\end{array}$} \\
\hline & & $\begin{array}{c}\text { At } \\
\text { Source. }\end{array}$ & $\begin{array}{c}\text { After } \\
\text { Storage. }\end{array}$ & $\begin{array}{l}\text { After } \\
\text { Filtration. }\end{array}$ & \\
\hline The Chelsea Co. & $\left\{\begin{array}{l}\text { Thamesat } \\
\text { Hampton }\end{array}\right\}$ & 16,138 & 1,067 & 34 & $98 \cdot 96$ \\
\hline West Middlesex Co. . & (2) & 16,138 & 1,788 & 58 & $99 \cdot 40$ \\
\hline Southwark \& Vauxhall Co. & , & 16,138 & .. & - $\begin{array}{c}80 \\
(623)\end{array}$ & $97 \cdot 72$ \\
\hline Grand Junction Co. . & , & 16,138 & 2,500 & 100 & $98 \cdot 46$ \\
\hline Lambeth Co. . & , & 16,138 & 7,820 & 75 . & $99 \cdot 50$ \\
\hline
\end{tabular}

In 1899 the Massachusetts Board of Health found that by continuous filtration through 45 inches of sand (size $0.23 \mathrm{~mm}$.) 99.49 per cent. of the bacteria were removed; and by intermittent filtration 99.08 per cent. of the organisms were removed. In 1902 the intermittent filter removed 98.7 per cent. of the total bacteria, 99.9 per cent. B. coli, and 100 per cent. B. typhosus. The continuous 
filter removed 98.7 per cent. of the total bacteria, 99.8 per cent. of B. coli, and 99.9 of the typhoid bacillus.*

The teaching of these figures could, with great ease, be emphasised again and again if such was necessary; but sufficient has been said to show that sand filtration, when carefully carried out, offers a more or less absolute barrier to the passage of bacteria, whether nonpathogenic or pathogenic.

\section{Domestic Purification of Water}

Something may, however, be added, from a bacteriological point of view, relative to what is called domestic purification. There is but one perfectly reliable method of sterilising water for household use, viz., boiling. As we have seen, moist heat at the boiling-point maintained for a few minutes will kill all bacteria and their spores. The only disadvantages to this process are the labour entailed and the "flat" taste of the water. Nevertheless, in epidemics due to bad water, it is desirable to revert to this simple and effectual purification.

There are a large number of domestic filters on the market with, in many cases, but little difference between them. The materials out of which they are made are chiefly the following: carbon and charcoal, iron (spongy iron or magnetic oxide), asbestos, porcelain and other clays, natural porous stone, and compressed siliceous and diatomaceous earths. From an extended research in 1894 by Prof. Sims Woodhead and Dr Cartwright Wood, who repeated and extended experiments by Freudenreich, Schöfer, and others, our knowledge of the quality of these substances as protectives against bacteria has been largely increased. $†$ They concluded that a filter failed to act in one of two ways. It was either pervious to microorganisms, or its power of filtering became modified owing to $(a)$ structural alteration of its composition, or (b) to the growing through of the micro-organisms, which had been demonstrated by previous workers. The conditions which chiefly influence the growth of bacteria through a filter appear to be the temperature, the intermittent use of the filter, and the species of bacteria. The higher the temperature and the longer the organisms are retained in the filter the more likely is it that they will grow through, and in the next usage of the filter appear in the filtrate. As to the species, those multiplying rapidly and possessing the power of free motility will naturally appear earlier in a filtrate than others. Woodhead and Wood concluded that out of 18 different kinds of domestic filter, each of which had its supporter, the Pasteur-Chamberland candle filters

* Thirty-fourth Ann. Rep. State Bd. of Health, Massachusetts, 1903, pp. 224 and 269.

+ Brit. Medl. Jour., 1894, i., pp. 1053, 1118, 1182, 1375, 1486. 
(composed of porcelain formed by a mixture of kaolin and other clays) were the only filters out of the substances named above which were reliable and protective against bacteria. They tested over three dozen of the Pasteur filters, and "in every case these gave a sterile filtrate." Pure cholera bacillus in suspension (5000 bacilli to every c.c.) and typhoid bacillus in suspension ( 8000 per c.c.) were passed through these filters, and not a single bacillus was detectable in the filtrate. The Berkefeld filter (siliceous earth) came second on the list as an effective filter, and had but the one fault of not being a "continuous" steriliser. A certain Parisian filter ("Porcelaine d'Amiante"), made of unglazed porcelain, rendered water absolutely free from bacteria. Its action was, however, very slow. Setting aside these three efficient filters, we are face to face with the fact that most filters do not produce germ-free filtrates, even though they are nominally guaranteed to do so. It is professed for animal charcoal, which is widely used, that it absorbs oxygen, and so fully oxidises whatever passes through it. This may be so at first, but after a little use it does more harm than good. It appears to add nitrogen and phosphates to water, which are both nutritive substances on which bacteria grow, and it readily absorbs impurities from the air. As a matter of experiment and practice, it has been found by Frankland, Woodhead, and others, that charcoal actually adds to the number of germs after it has been in use for some time.

Subsequent experiments were made in this country by Lunt and Horrocks. Lunt working in 1897 investigated the power of the Berkefeld filter to intercept pathogenic bacteria, especially the typhoid bacillus. He concluded as a result

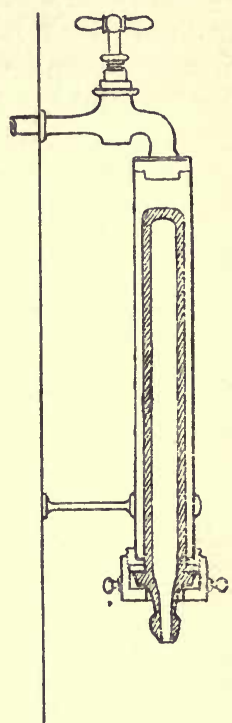

Fig. 10.-PASTEURChamberland Filter. Attached to Water Supply. of his inquiry that on the first day an efficiently sterilised Berkefeld filter gave an absolutely sterile filtrate, but that on the second or third day of using some water bacteria passed through. For thirtynine days the $B$. coli did not pass through, though the organism could be detected on the outside of the filtering candle. Lunt found that the action of the filter depended very much upon its method of use: forcing or intermittently pumping water through the filter resulted in a filtrate containing bacteria, whilst if the same filter was steadily used a germ-free filtrate was obtained. In short, the result of Lunt's work was to show the necessity for a frequent sterilisation of the filter, for though it allows ordinary water bacteria to 
pass on the second or third day, $B$. coli and the typhoid bacillus do not appear with them in the filtrate until a subsequent date. Probably, if reliance is to be placed upon such a filter from a bacteriological point of view, daily sterilisation is advisable.

Experiments of a similar nature have been done by Horrocks, * who arrives at the following conclusions. First, the B. typhosus is not able to grow through the walls of a Pasteur-Chamberland candle, and if proper care be taken to prevent the direct passage of organisms through flaws in the material and imperfections in the fittings, the Pasteur-Chamberland filter ought to give complete protection from water-borne disease. Secondly, typhoid bacilli can grow through the walls of Berkefeld candles, the time required for the passage being largely dependent on the nutriment supplied to the organisms by the filtering fluid. Possibly the weakness of the candle from a bacteriological point of view is due to the large size of the lacunar spaces, which cannot be avoided if a fair delivery is to be obtained, but which "appears to militate against the immobilising and devitalising influences which operate so strongly in filters made with very narrow lacunar spaces." Thirdly, Horrocks concluded that when a highly polluted liquid containing typhoid bacilli is filtered through a Berkefeld candle the bacilli may appear in the filtrate in four days. Consequently, it is necessary to sterilise these candles every third day. The method of sterilisation of filters is not washing or brushing or any other kind of cleansing or soaking in water, but by exposing them to steam or boiling them.

* Bacteriological Examination of Water, 1901, pp. 273-280. 


\section{CHAPTER III}

BACTERIA IN THE AIR

Methods of Examination of Air-Conditions of Bacterial Contamination of Air : (1) Dust and Air Pollution; (2) Moisture or Dampness of Surfaces : Bacteria in Sewer Air ; (3) the Influence of Gravity ; (4) Air Currents. The Relation of Bacteria to $\mathrm{CO}_{2}$ in the Atmosphere : in Workshops, in Bakehouses, in Railway Tubes, in the House of Commons.

THE basis of the usual methods in practice for bacterially examining air is to pass the air over or through some nutrient medium. By this means the contained organisms are waylaid, and finding themselves under favourable conditions of pabulum, temperature, and moisture, commence active growth, and thus reveal themselves in characteristic colonies. These are examined by the microscope and sub-culture. Returns of the number of bacteria in the sample taken may be made for the sake of information, but little or no conclusion of value can be drawn from such data. The standard recognised in Europe is the cubic metre or litre, and one may report, for example, of the air of a room containing 500 or more germs per cubic metre.

\section{Methods of Examination of Air}

1. The Plate Method.-Koch adopted the simplest of all the culture methods, viz $\}_{6}$ exposing a plate of gelatine or agar for a longer or shorter time to the air of which examination is desired. By gravity the suspended bacteria fall on the plate and start growth. As a matter of quantitative exactitude, this method is not to be recommended, but it frequently proves an excellent method for qualitative estimation. It will be found in practice that nutrient agar is better for the purpose than nutrient gelatine. Greater latitude is obtained both in point of temperature and length of incubation, and the result is uncomplicated by the, at times, very rapid liquefaction of the gelatine by liquefying organisms. Care should be taken in preparing the plates to allow them to cool on a level surface, and at least 15 c.c. 
of the medium should be employed for each Petri dish in order to ensure an even surface and sufficient depth of medium all over the plate. After exposure the plates are, under ordinary circumstances, best left at room temperature during the development of the colonies, but if it is desired to examine the bacteria alone it will be found well to favour the growth of these at the expense of the moulds, by first incubating the dish at a temperature of $37^{\circ} \mathrm{C}$. for, say, eighteen hours. In any case the plate should be shielded from light, or otherwise many of the chromogenic organisms will not assume their typical coloration. Should it be desired to photograph the plates in order to obtain a permanent record, the growth should be arrested, and the organisms killed about the third or fourth day of incubation. The best method of doing this is to reverse the dish, and to pour upon a piece of blotting paper placed on the inner surface of the lid, which will now be undermost, a sufficient quantity of Formalin to saturate it. The results of this method of examination may be expressed per square foot per minute, the area of the Petri dish being calculated $\left(=(\text { radius })^{2} \times \frac{22}{7}\right)$.

2. The Flask Method of Miquel.-Pasteur was the first to analyse air by the culture

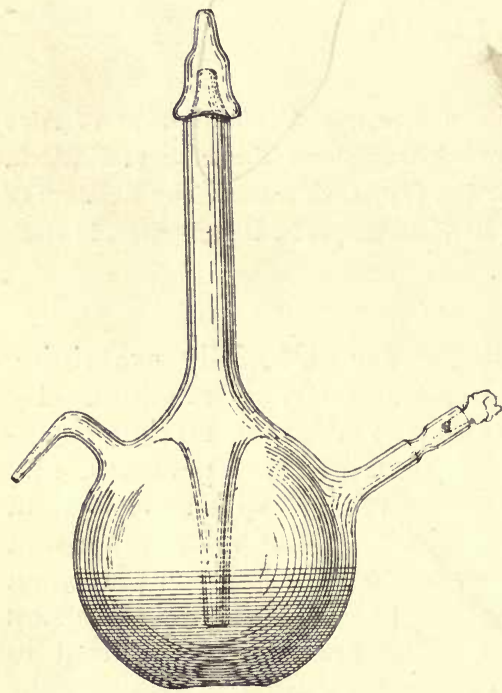

Fig. 11.-Miquel's Flask. method, and he adopted a plan which, in principle, is washing the air in some fluid culture medium which will retain all the particulate matter, which may then be cultured directly or sub-cultured into any favourable medium. Miquel has contrived a simple piece of apparatus for the carrying out of this principle. It consists of a flask with a central tube through its own neck for the entrance of the air. On one side of the flask is a tube to be connected with the aspirator, on the other side of the flask a tube through which to pour off the contained fluid at the end of the process. In the flask are placed 30 c.c. of sterilised water (or, indeed, if it be preferred, sterilised broth). The entrance tube is now unplugged, and the aspirator draws through a fair sample of the air in the room (say ten litres). This air perforce passes through the water, and by the exit tube to the aspirator, and is thereby washed, leaving behind in the water its bacteria. The aspiration is then stopped, and the entrance tube closed. The water (plus bacteria) is now poured out into test-tubes of media or plated out on Petri's dishes. Provided that the apparatus has been absolutely sterilised, and that only sterilised water is used, any colonies developing upon the Petri dish are composed of micro-organisms from the air examined.

3. The Method of Hesse. - This method is somewhat akin to Pouchet's aëroscope, but is in addition a culture method. Hesse's tube is $50-70 \mathrm{~cm}$. long and $3-5 \mathrm{~cm}$. bore throughout. At one end is an indiarubber stopper bored for a glass tube to the aspirator. The other end is open. Before using, the tube is sterilised, and 40 or 50 c.c. of sterilised gelatine are placed in it. The tube is now rapidly rotated in a groove on a block of ice or under a cold-water tap, and by this simple means the gelatine becomes fixed and forms a layer inside the tube throughout. We have therefore, so to speak, a tube of glass with a tube of gelatine inside it. The apparatus is now ready for use. It is fixed on the tripod, and 10-20 litres of air are drawn through, and the tube is properly plugged and incubated at room temperature. In two or three days the colonies appear upon the gelatine. 'They are most numerous generally in the first part of the tube. The disadvantages of 


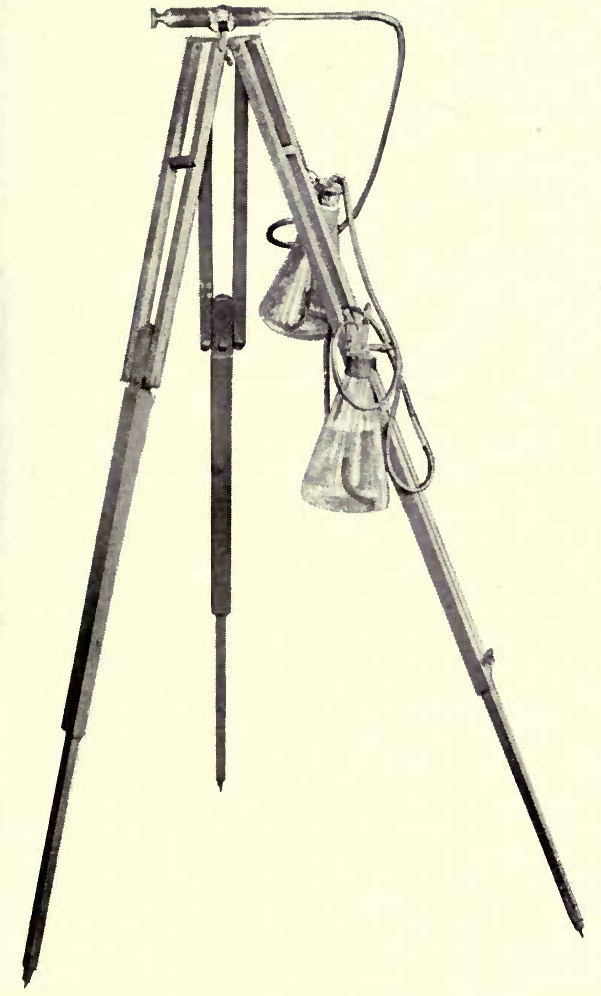

Sedgwick's Sugar Tube, in position on tripod, with siphon.

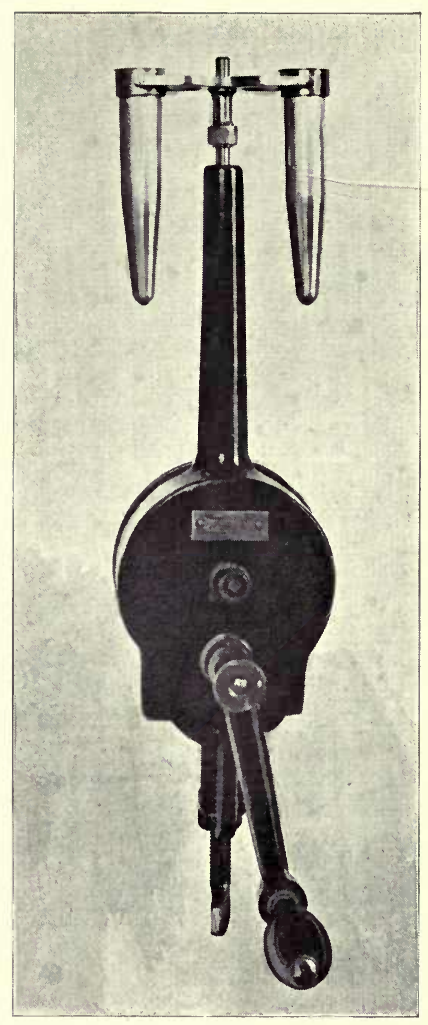

Small Hand Centrifuge. 
$\odot$ 
this process are that dried gelatine does not catch germs like the broth cultures of Pasteur or Miquel, and that many organisms are carried straight through the tube, and failing to be deposited, pass out at the aspirator exit, and thus are neither caught nor counted. The Hesse tube is generally used in practice with a pump consisting of two flasks and a double-way indiarubber tube. The flasks have a capacity for one litre of water. By a simple arrangement it is possible to securc syphon action, and hence measure with considerable exactitude the amount of air passing through the tube (Plate 5).

4. Methods of Filtration.-Frankland, Petri, Pasteur, Sedgwick, and others have suggested the adoption of methods of filtration. These depend upon catching the organisms contained in the air by filtering them through sterilised sand or sugar, and then examining these media in the ordinary way. Many different kinds of apparatus have been invented. Petri aspirates through a glass tube containing sterilised sand, which after use is distributed in Petri dishes and covered with gelatine. The principal objection to this method is the presence of the opaque particles of sand in and under the gelatine. Probably it was this which suggested the use of soluble filters like sugar. Pasteur introduced the principle, and Frankland and others have followed it out. Sedgwick's Tube consists of a comparatively small glass tube, about a foot long. Half of it has a bore of $2.5 \mathrm{~cm}$., and the other half a bore of $5 \mathrm{~cm}$. It is sterilised at $150^{\circ} \mathrm{C}$, after which the dry, finely granulated cane-sugar is inserted in such a way as to occupy an inch or more of the narrow part of the tube next the wide part. Next to it is placed a wool plug, and the whole is again sterilised. After sterilisation an indiarubber tube is fixed to the end of the narrow portion, and thus it is attached to the aspirator. The measured quantity (5-20 litres) of air is drawn through, and any particulate matter

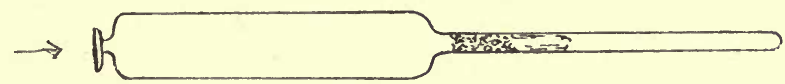

Fra. 12.--Sedgwick's Sugar-tube.

is caught in the sugar. Warm, nutrient gelatine (10-15 c.c.) is now poured into the broad end of the tube, and by means of a sterilised stilette the sugar is pushed down into the gelatine, where it quickly dissolves. We have now in the gelatine all the micro-organisms in the air which has been drawn through the tube. After plugging with wool at both ends, the tube is rolled on ice, or under a cold-water tap, in order to fix the gelatine all round the inner wall of the tube, which is incubated at room temperature. In a day or two the colonies appear, and may be examined.

Frankland used finely powdered sugar and glass wool as filtering-medium, and a tube with two constrictions. After passing sufficient air through, the tube is broken in halves and the wool and sugar are pushed by means of a sterile needle into liquefied gelatine. The sugar dissolves and the organisms are distributed in the medium. Andrewes has used a modification of this method, and the aspiration was carried out with a large brass syringe of known capacity, fitted with a two-way nozzle and cock, so that the requisite number of syringefuls could be aspirated without disturbance.*

Various other methods, including Miquel's filtration method, and the methods of Laveran, and Wiirtz and Strauss, have been used, but the principal are those mentioned above.

In respect of the results obtained in the examination of air bacteriologically, it may be said that they are twofold. First, a quantitative result is obtained by which we may arrive at the approximate number of bacteria and moulds. Secondly, the quality or species of organisms is determined. Reference will be made to both these points in the pages which follow. 1902.

* Brit. Med. Jour., 1902, ii., p. 1534; and Report to London County Council, 


\section{Conditions of Bacterial Contamination of Air}

There are, speaking in a general way, four chief external conditions affecting the occurrence of bacteria in air. They are as follow:-

1. The presence of dust and air pollution.

2. Dampness of surfaces.

3. Gravity.

4. Air currents.

1. Dust and Air Pollution.-Schwann was one of the first to point out that when a decoction of meat is effectually screened from the air, or supplied solely with calcined air, putrefaction does not set in. It is true that Helmholtz and Pasteur confirmed this, and greatly added to our knowledge of the subject, but on the whole it may be said that Schwann originated the germ-theory of air, and Lister applied it in the treatment of wounds. Lister believed that if he could surround wounds with filtered air free from dust and particulate or germ matter, the result would be as good as if the wounds were shut off from the air altogether.

It was Tyndall * who first laid down the general principles upon which our knowledge of organisms in the air is based. That the dust in the air was mainly organic matter, living or dead, was a comparatively new truth; that epidemic disease was not due to "bad air" and "foul drains," but to germs conveyed in the air, was a prophecy as daring as it was novel. From these and other like investigations it came to be recognised that putrefaction begins as soon as bacteria:from the air gain an entrance to the putrefiable substance, that it progresses in direct proportion to the multiplication of these bacteria, and that it is retarded when they diminish or lose vitality.

Tyndall made it clear that, both as regards quantity and quality of micro-organisms in the air there neither is nor can be any uniformity. The degree in either case will depend on air pollution and on dust particles. Bacteria may be conducted on particles of dust- " the raft theory" - but being themselves endowed with a power of flotation commensurate with their extreme smallness and the specific lightness of their composition, dust as a vehicle is not really requisite. Nevertheless the estimation of the amount of dust present in a sample of air may be a very good index of danger. It is to Dr Aitken that we are indebted for devising a method by which we can measure dust particles in the air, even though they be invisible. His ingenious experiments, reported in the Transactions of the Royal Society of Edinburgh (vol. xxxv.), have demonstrated that by supersaturation of air the invisible dust particles may become visible. As is now well known, Dr Aitken believes that fogs, mists,

* John Tyndall, F.R.S., Floating Matter of the Air, 1878. 


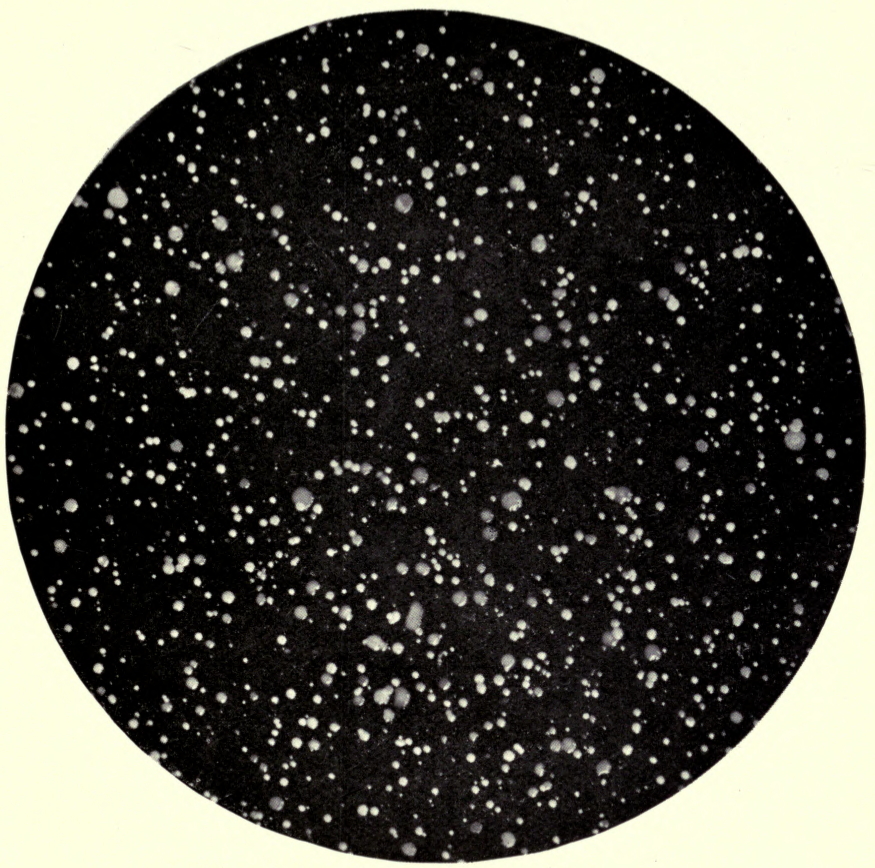

Air-Plate exposed in Labourer's Cottage in Buckinghamshire (30 minutes). Agar culture, 3 days at $22^{\circ} \mathbf{C}$.

(Grown_and photographed by Swithinbank). 


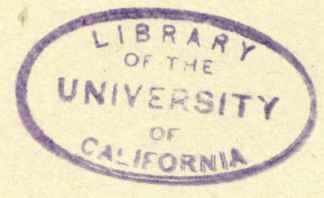

라 
and the like do not occur in dust-free air, and are due to condensation of moisture upon dust particles. And much the same has been found to be true in respect to dust and bacterial pollution. As a rule, when the former is abundant the latter is considerable. Haldane and Osborn (vide infra) found bacteria most numerous in a workplace where dust was most abundant, and their finding was merely confirmatory of many other previous researches. On the other hand it should be remembered that, though dust forms a vehicle for bacteria, dusty air is sometimes comparatively free from bacteria.

For the conditions which affect the number of bacteria in the air are various. In open fields, free from habitations, there are fewer, as would be expected, than in the vicinity of manufactures, houses, or towns. A dry, sandy soil or a dry surface of any kind will obviously favour the presence of organisms in the air. Frankland found that fewer germs were present in the air in winter than in summer, and that when the earth was covered with snow the number was greatly reduced. Miquel and Freudenreich have declared that the number of atmospheric bacteria is greater in the morning and evening between the hours of six and eight than during the rest of the day.

There is no numerical standard for bacteria in the air as there is in water. In houses and towns it would rise according to circumstances, and frequently in dry weather reach thousands per cubic metre. When it is remembered that air possesses no pabulum for bacteria, as do water and milk, it will be understood that bacteria do not live in the air. The quality and quantity of air organisms depend entirely upon environment and physical conditions. In some researches which the writer made into the air of workshops in Soho in 1896 , it was instructive to observe that fewer bacteria were isolated by Sedgwick's sugar-tube in premises which appeared to the naked cye polluted in a larger degree than in other premises apparently less contaminated. In the workroom of a certain skin-curer the air was densely impregnated with dust particles from the skin, yet scarcely a single bacterium was isolated. Macfadyen and Lunt have also found that the number of dust particles does not bear any relation to the number of bacteria. They found that air containing even millions of dust particles might be almost germ-free. In the polishing room of a well-known hat firm, in which the air appeared to the naked eye to be pure, and in which there was ample ventilation, there were found by the writer numerous bacteria belonging to four or five species of saprophytes. The public analyst for the city of Nottingham, estimated the bacterial quality of the air of the streets of that town during "the goose fair" held in the autumn. $\mathrm{He}$ used a modification of Hesse's apparatus, in which the gelatine is replaced by glycerine. The air was slowly drawn through and 
measured in the usual way. Sterilised water was then added to bring the glycerine to a known volume, the liquid thoroughly mixed, and a series of gelatine and agar plates made with quantities varying from 0.1 to 2 c.c. By this method a large number of bacteria were detected in this particular investigation, including Staphylococcus pyogenes aureus and albus, the common Bacillus subtilis, and, apparently, B. coli communis.* Carnelly, Haldane, and Anderson found 11 bacteria per litre of air in a classroom of the High School at Dundee with the boys at rest. But when the boys were instructed to stamp on the floor, and thus raise the dust, the number rose to 150 bacteria per litre.

During a six years' investigation the air of the Mont Souris Park yielded, according to Miquel, an average of 455 bacteria per cubic metre. In the middle of Paris the average per cubic metre was nearly 4000 . Flugge accepts 100 bacteria per cubic metre as a fair average. From this fact he estimates that "a man during a lifetime of seventy years inspires about $25,000,000$ bacteria, the number contained in a quarter of a litre of fresh milk." $\dagger$ Many authorities would place the average much below 100 per cubic metre, but even if we accept that figure it is at once clear how relatively small it is. This comparative freedom from bacteria is due to sunlight, rain, desiccation, dilution of air, moist surfaces, etc. So essentially does the bacterial content of air depend upon the facility with which certain bacteria withstand drying that Dr Eduardo Germano has addressed himself first to drying various pathogenic species and then to mixing the dried residue with sterilised dust and observing to what degree the air becomes infected.+ The typhoid bacillus appears to withstand comparatively little desiccation, without losing its virulence. Nevertheless it is able to retain vitality in a semi-dried condition, and it is owing to this circumstance, in all probability, that it possesses such power of infection. The bacillus of diphtheria, on the other hand, is capable of lengthened survival outside the body, particularly when surrounded by dust. The question of its power of resistance to long drying is an unsettled point. The power of surviving a rlrying process is, according to Germano, possessed by the Streptococcus pyogenes. This is not the case with the organisms of cholera or plague. Dr Germano classifies bacteria, as a result of his researches, into three groups: first, those like the bacilli of plague, typhoid, and cholera, which cannot survive drying for more than a few hours; second, those like the bacilli of diphtheria and streptococci, which can withstand it for a longer period; thirdly, those like the tubercle bacillus, which can very readily resist drying for months

* Public Health, vol. x., No. 4, p. 130 (1898).

† Flügge, Grundriss der Hygiene, 1897, pp. 161, 162.

¥Zeitschrift für Hygiene, vols. xxiv.-xxvi. 
and yet retain their virulence. It will be obvious that from these data it is inferred that Groups 1 and 2 are rarely conveyed by the air, whereas Group 3 is frequently so conveyed. Miquel has recently demonstrated that certain soil bacteria or their spores can remain alive in dried dust in hermetically sealed tubes for as long a time as sixteen years. Even at the end of that period such soil inoculated into a guinea-pig produced tetanus.

The presence of pathogenic bacteria in the air is, of course, a much rarer contamination than the ordinary saprophytes. The tubercle bacillus has been not infrequently isolated from dry dust in consumption hospitals, and in exit ventilating shafts at Brompton the bacillus has been found. From dried sputum, it has, of course, been many times isolated, even after months of desiccation. Indeed, a very large mass of experimental evidence attests the fact that the air in proximity to dried tubercular sputum or discharges may contain the specific bacillus of the disease. The bacillus of diphtheria in the same way, but in a lesser degree, may be isolated from the air, and from the nasal mucous membrane of nurses, attendants, and patients in a ward set apart for the treatment of the disease, and from the throats and nasal mucous membrane of persons who have been in contact with cases of the disease. Delalivesse, examining the air of wards at Lille, found that the contained bacteria varied more or less directly with the amount of floating matter, and depended also upon the vibration set up by persons passing through the ward and the heavy traffic in granite-paved streets adjoining. $B$. coli, staphylococci, and streptococci, as well as $B$. tuberculosis, were isolated by this observer. Other observers have found $B$. coli very rarely present in air (Chick, Andrewes, etc.).

2. Moisture or Dampness of Surfaces. - It is an interesting and important fact that except under special circumstances microorganisms do not leave moist surfaces, but remain adhering to them. A clear recognition of this fact is essential to a right understanding of the pollution of air by bacteria. They cannot leave the moist surface of fluids either under evaporation or by means of air currents.* Only when there is considerable molecular disturbance, such as splashing, can microbes be transmitted to the surrounding air. This is the reason why sewer gas and all air contained within moist perimeters is almost germ-free, whereas from dry surfaces the least air current is able to raise countless numbers of organisms.

This principle has been admirably illustrated in investigations made upon expired and inspired air. In a report to the Smithsonian Institute of Washington (1895) upon the composition of expired air,

* Flügge has lately attempted to demonstrate that an air current having a velocity of four metres per second can remove bacteria from surfaces of liquids by detaching drops of the liquid itself. 
it is concluded that "in ordinary quiet respiration no bacteria epithelial scabs or particles of dead tissue are contained in the expired air. In the act of coughing or sneezing such organisms or particles may probably be thrown out." The mucous membrane lining the cavity of the mouth and respiratory tract is a moist perimeter, from the walls of which no organisms can rise except under molecular disturbance. The popular idea that bacteria can be "given off by the breath" is therefore contrary to the laws of organismal pollution of air. The required conditions are not fulfilled, and such breath infection must be of extremely rare occurrence except in speaking, spitting, sneezing or coughing (Flügge). Air can only become infective when impregnated with organisms arising from dried surfaces.

Another series of investigations were conducted by Drs Hewlett and St Clair Thomson, and dealt with the fate of micro-organisms in inspired air and micro-organisms in the healthy nose. They estimated that from 1500 to 14,000 bacteria were inspired every hour. Yet, as we have seen, expired air contains practically none at all. It is clear, then, that the inspired bacteria are detained somewhere. Lister has pointed out, from observation on a pneumo-thorax caused by a wound of the lung by a fractured rib, that bacteria may be arrested before they reach the air cells of the lung, and other observations confirm this fact, although of course there are several well-known exceptions (e.g. tubercle of the lung). Hence it is at some intermediate stage that they are detained. Hewlett and Thomson examined the mucus from the wall of the trachea, and found it germ-free. It was only when they examined the mucous membrane and moist vestibules and vibrissæ of the nose that ithey found bacteria. Here they were present in abundance. The ciliated epithelium, the mucus, and the bactericidal influence of the wandering or "phagocyte" cells, probably all contribute to their final removal.*

There can be no doubt that the large number of bacteria present in the moist surfaces of the mouth is the cause of a variety of ailments, and under certain conditions of ill-health organisms may through this channel infect the whole body. Dental caries will occur to everyone's mind as a disease probably due in part to bacteria. As a matter of fact, acids (due to acid secretion and acid fermentation) and micro-organisms are two of the chief causes of decay of teeth. Defects in the enamel, inherent or due to injury, retention of débris on and around the teeth, and certain pathological conditions of the secretion of the mouth, are predisposing causes, which afford a

* Hewlett and Thomson graphically demonstrated the bactericidal power of the nasal mucous membrane by noting the early removal of Bacillus prodigiosus, which had been purposely placed on the healthy Schneiderian membrane of the nose. 
General Note.-Whilst the above description applies to the normal type of $B$. coli, it should be clearly understood that a large nuunber of bacilli have been described which possess some, but not all, of the above characters. Refik has described (Ann. de l'Inst. Pasteur, x., 1896, 242), five varying types very similar to the normal B. coli, but differing in one or more characters. Almost all forms, however, have some features in common, e.g., motility, few flagella, and characteristic growth on potato. Moreover, there are a group of organisms allied to $B$. coli, and often associated with it. Like it also, they are related, etiologically or otherwise, to similar pathological processes. Refik's types are briefly as follows:-

A. Ferments lactose, coagulates milk, but gives no indol reaction.

B. Ferments lactose, does not coagulate milk, gives indol reaction.

C. Ferments lactose, does not coagulate milk, does not give indol reaction.

D. Does not ferment lactose, coagulates milk, does not give indol reaction.

E. Does not ferment lactose, does not coagulate milk, does not give indol reaction.

Mervyn Gordon has made a careful study of the B. coli and its allies which he classified according to their reactions and their flagella. He differentiated 16 varieties.* Horrocks studied the cultural characters of 150 "varieties" of $B$. coli isolated partly from normal and partly from typhoid stools. $\dagger$ Other workers have observed an enormous variety of minor differences. The important point is the diagnosis of $B$. coli, and the following characters are now chiefly relied upon (see also p. 472). 1. The $B$. coli group is non-sporing and nonliquefying; 2. The members of the group rarely stain by Gram's method; 3. They produce acid and gas with both glucose and lactose; 4. They produce acid in milk and they usually also coagulate it; 5 . They produce acid and gas in bile-salt-glucose broth; 6. They grow well at a temperature of $42^{\circ} \mathrm{C}_{+}^{+}$Other fairly reliable features are motility, a small number of flagella, a fairly typical growth on potato, and more rapid development on all media than the typhoid bacillus. But there is not at the present time a complete unanimity of opinion as to the most reliable characters for diagnostic purposes. $\$$

* Jour. of Path. and Bact., 1897, vol. iv., p. 438.

† Bacteriological Examination of Water, 1901, p. 94 ; Jour. of Hyg., 1901, p. 202.

+ Roy. Com. on Sewage Disposal, Second Report, 1902, p. 101. See also Brit. Med. Jour., 1903, i. 418 (Klein), for summary of characters of B. coli.

$\S$ Houston considers the following the most useful tests for B. coli: (1) Gas formation in ordinary gelatine "shake" cultures; (2) indol in broth cultures ; (3) acid and clot in litmus milk-cultures; (4) greenish-yellow fluorescence in neutral-red broth cultures; (5) gas and acid in lactose-peptone cultures ; (6) gas, acid, and clot in peptone-lactose milk cultures; (7) gas and acid in glucose-peptone cultures; (8) reduction of nitrate to nitrite in nitrate broth cultures; (9) strong acid in Proskauer and Capaldi's medium No. 1, and no definite production of acid 
The significance of $B$. coli is of course its potential pathogenicity, and its similarity to the typhoid bacillus, but above all its relation to sewage. Roux, Rodet, and others have stated that B. coli, under certain circumstances, may assume a character not distinguishable from $B$. typhosus, both in its biological and cultural characteristics and in its pathogenic properties. Chantemesse, Widal, and others have held that polluted waters owe their power to produce typhoid fever to the presence of $B$. coli, and that possibly the organisms are transformable the one into the other. Klein and many other bacteriologists, as the result of very numerous experiments, have been unable to effect any transformation of one form into the other. Each organism has retained unimpaired its differential characters.

Certain strains of $B$. coli are distinctly pathogenic for lower animals, and there is some ground for considering the organism a cause of disease (epidemic diarrhœa and other conditions) in man, either by itself or in association with other organisms (Delépine). In the third place, as is pointed out elsewhere, B. coli is a sewage organism, and the chief importance of its detection in water is an indication of sewage pollution and therefore of possible contamination of the water with specific bacteria. It is therefore a most reliable test of pollution. Klein and Houston have emphasised the importance of the presence of $B$. coli and the B. enteritides sporogencs in water as indication of sewage pollution, and by this means a demonstration of the presence of sewage in water can be carried to an incomparably higher degree than by chemical examination. Chemistry is powerless to detect pollution by pathogenic germs or the small amount of organic pollution which can be detected by bacteriology, which is ten

in Proskauer and Capaldi's medium No. 2; (10) presence of motility; (11) non-liquefaction of gelatine; and (12) acidity in litmus whey cultures, varying from about 20-40 c.c. $\frac{\mathrm{N}}{10} \mathrm{Na}_{2} \mathrm{CO}_{3}$ per 100 c.c. of culture. In dealing with sewage, effluents, and non-drinking-water streams, Houston employs the first three tests, but in dealing with drinking-water, the first five tests (Fourth Report of Royal Commission Sewage Disposal, 1904, p. 106).

McWeeney relies chiefly upon ( $(a)$ the character of gelatin colony and nonliquefaction of that medium, even after a long time ; $(b)$ non-retention of Gram's stain ; $(c)$ fermentation of lactose with gas and acid formation ; $(d)$ coagulation of milk within four days at $37^{\circ} \mathrm{C}$; $(e)$ production of yellowish-green fluorescence in neutral-red-agar-shake culture; and $(f)$ production of indol in liquid peptone media. (Report of Local Government Board for Ireland, 1904). Klein describes B. coli as a motile, non-spore-bearing bacillus, possessing a limited number of flagella, capable of fermenting glucose and lactose, of curdling milk with the production of acid, of forming indol in broth culture, reducing neutral red with the production of a green fluorescence, producing gas-bubbles in nutrient jelly, of forming a more or less brownish growth on steamed potato, and of producing on the surface of gelatin a dry, translucent growth which does not liquefy the gelatin. The bacilli, under the microscope, appear as cylindrical rods, showing more or less pronounced motility, and they do not stain by the method of Gram (see also Appendix, pp. 466 and 472). 


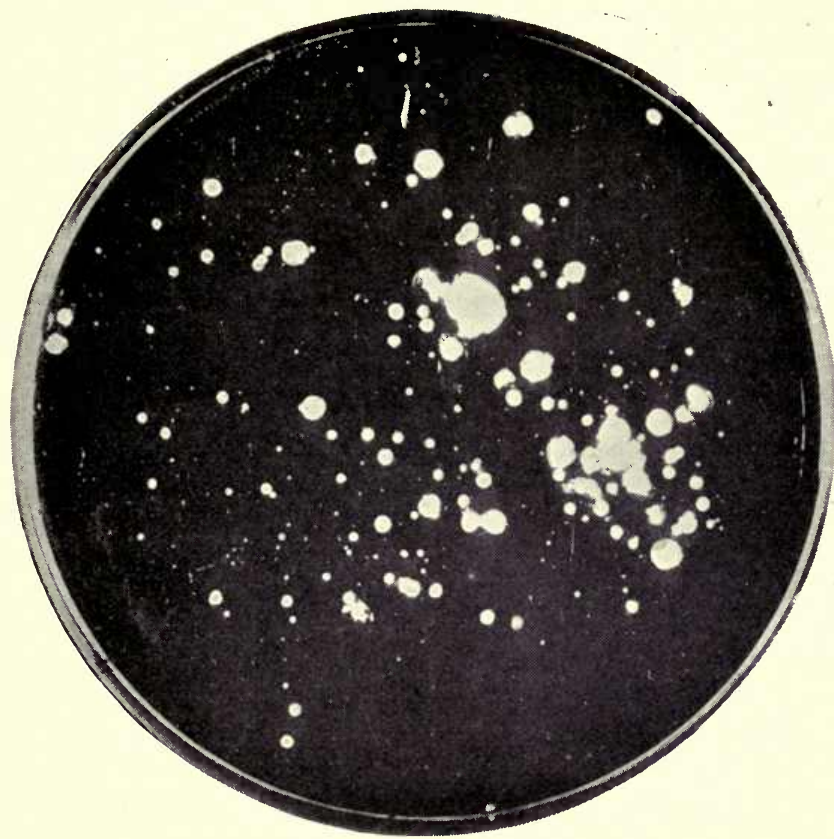

Bacillus coli communis. Surface gelatine plate culture, $0 \cdot 1$ c.c. of

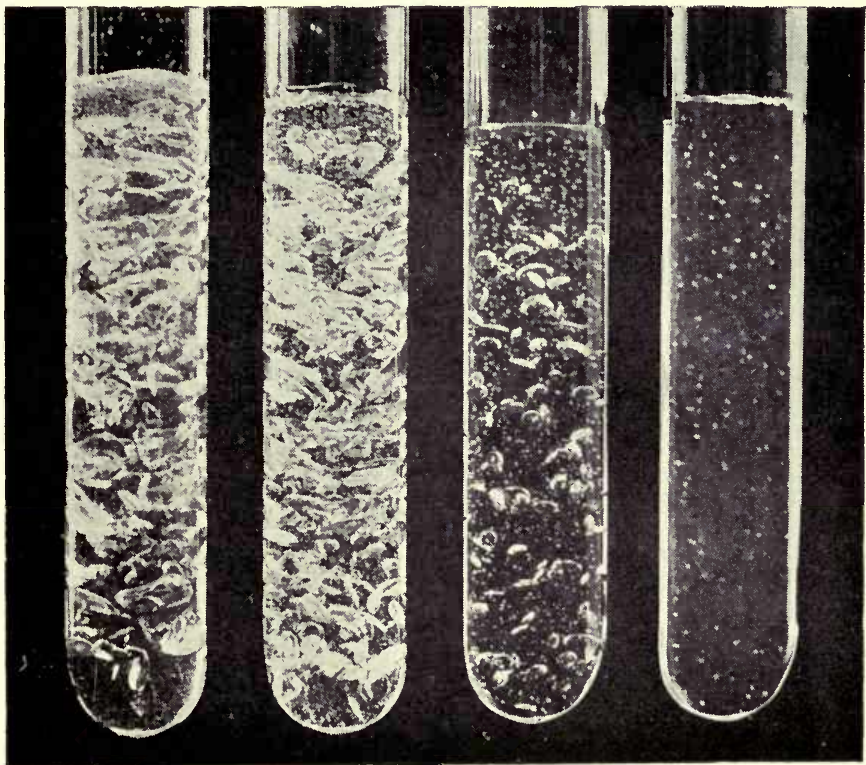

Gas in Gelatine Shake Ctruture, 24 hours at $20^{\circ} \mathrm{C}$.

From left to right the tubes represent 
? 
to one hundred times less than that detectable by chemistry.* It is, however, important to bear in mind that something more than the mere presence of $B$. coli must be ascertained. The comparative numbers present, the relative abundance, and the general character and source of the water must be considered. Waters containing no B. coli in 100 c.c. are of course of a high degree of purity. $\dagger$ In upland surface waters the presence of $B$. coli in such a small amount as 1 c.c., may be sufficient to condemn the waters. Certainly drinkingwater from a deep well should contain no $B$. coli. The presence in a water of $B$. coli in conjunction with streptococci or even the spores of $B$. enteritidis sporogenes, or both, would of course indicate serious pollution.

The differential diagnosis of $B$. coli from its allies or other organisms is not always a simple matter. An adherence to the characteristics set out above will generally prove safe guidance, but reliance should not be placed upon any single character or test. The tendency to adopt some rapid and easily-applied test for this organism is strongly to be deprecated, as likely to lead to error. Nothing can take the place of the careful study and sub-culture of the suspected organism in this and in all other species. At the same time, it has been found that diagnostic aid is obtained by a comparison of some of the biological characters of the colon and allied groups of bacteria. They may be divided into four divisions :(1) The proteus group, the members of which are motile, liquefy gelatine, produce gas in glucose and sucrose but not in lactose, curdle and acidulate milk very slowly, and usually produce indol; (2) the coli group include motile bacilli, producing gas in glucose and lactose, curdle milk rapidly, nearly always produce indol, but do not liquefy gelatine, and do not retain Gram's stain; (3) the group including $B$. lactis cerogenes are non-motile bacilli, which do not liquefy gelatine but which curdle and acidulate milk and ferment sugars other than. glucose; and (4) the enteritidis group contain bacilli which are motile, which only ferment glucose, and which do not liquefy gelatine or curdle milk, which is ultimately rendered alkaline. This group includes $B$. enteritidis of Gaertner, the para-colon and the paratyphoid bacilli.

Streptococci in Water.-Houston considers the presence of streptococci in water as indication of recent and dangerous pollution of water. They are absent even in large quantities of pure water and in virgin soils. + Streptococci, as a class, are delicate germs that readily lose their vitality and die when the physical conditions are unfavourable, and they comprise species highly pathogenic to human

* Medical Supplement to Report of Local Government Board, 1898-99, p. 498.

$\uparrow$ See also Jour. of Hyg., 1902, p. 339 (Savage).

$¥$ Report of Locul Government Board, 1899-1900, p. 483. 
beings. They are present in human fæces and in crude sewage in considerable number; and as we have said, they are absent from relatively large amounts of pure waters and virgin soils, but present in abundance in water and soil recently polluted with animal dejecta. It is not claimed that all streptococci are necessarily delicate germs, or pathogenic, or of recent animal outcome. It may be that certain streptococci are comparatively hardy germs, and that others may be capable of multiplying in Nature outside the animal body. Again, there may be streptococci in Nature which do not owe their origin to excremental matter, and doubtless many of them may be non-pathogenic, although this latter circumstance is no proof that at a stage prior to their isolation they were non-virulent, nor does it impair the value of the test as an indication of recent fouling with objectionable matters.

Houston found streptococci habitually present in crude sewage in $\frac{1}{1000}$ c.c., present in human fæces in one milligramme, and present in minimal quantities of soils and water recently polluted with matters of animal outcome. These results encourage the belief that the streptococcus test is one of the most delicate yet suggested for detecting recent, and therefore, presumably, specially dangerous, pollution.

The question of relative abundance in connection with the streptococcus test also deserves consideration. For if streptococci are absent from 10 c.c. or more of pure waters and present in $\frac{1}{1000}$ c.c. of crude sewage the distinctions as regards streptococci between water and sewage is sufficiently great to allow of considerable latitude being observed in framing a standard without seriously impairing the value of the test. What standard should be adopted is a matter of opinion, but as a rule it may be said that the presence of streptococci are to be thought of as indicating extremely recent, and $B$. coli less recent, but still not remote, pollution of animal sort (Houston). The presence of $B$. enteritidis sporogenes, however, cannot be considered to afford evidence of pollution bearing a necessary relation to the recent evacuations of animals. Streptococci and B. coli are either altogether absent or present in sparse amount in virgin soils, and may be absent even from polluted soils, unless the contamination is of comparatively recent sort. In soils recently polluted with animal matters streptococci and $B$. coli are of course present in abundance. $B$. enteritidis sporogenes may be present even in seemingly virgin soils, but in sparse proportion compared with the large number found in cultivated and polluted soils. Lastly, the presence of streptococci in any number in a water supply points not only to recent animal pollution, but also implies that the antecedent conditions-conditions intervening between the period of pollution of the water and the time of collection of the sample-could hardly have been of so un- 
favourable a character as to destroy the vitality of seemingly more hardy microbes-for example, the typhoid bacillus. The same cannot be said for the $B$. coli test, since $B$. coli is a more hardy germ than B. typhosus.

Broadly, therefore, it will be seen that the presence of $B$. coli or B. enteritidis sporogenes or Streptococci in a water is presumptive evidence of sewage pollution. But that in forming an opinion it is essential to bear in mind the relative abundance of organisms per c.c. and the relative abundance of certain species.

(c) Pathogenic Bacteria in Water.-The two chief types of disease-producing organisms found in water are the bacillus of typhoid fever and the bacillus of cholera. These diseases and their causal organisms are dealt with subsequently (see pp. 298 and 384). Here it will only be necessary to note one or two general facts as to the relation of pathogenic organisms to water supplies.

In sterilised water, and in very highly polluted water or sewage, pathogenic bacteria do not flourish. In the former case they die of starvation, although there are experiments on record which appear not to support this view; in the latter case they are killed by the enormous competition of common bacteria. Even in ordinary water there is a wide divergence of behaviour. Some bacteria are destroyed in a few hours; others appear to flourish for weeks. In all cases the spores are able to resist whatever injurious properties the water may have much more persistently than the bacilli themselves. These changes in the vitality of bacteria in water, partly due to the water and partly to the other micro-organisms, bring about two characteristics which it is important to remember, viz., that pathogenic germs in water are, as a rule, scanty and intermittent. It is these features in conjunction with the enormous quantities of common water bacteria which make the search for the bacillus of typhoid fever what Klein has called "searching for a needle in a rick of hay." Not that it cannot be detected, but its detection is one of the most difficult of investigations. In recent years the typhoid bacillus has been isolated from water which had given rise to cases of typhoid fever at Pierrefonds (Widal \& Chantemesse), Dijon (Vaillard), Chateaudun, Cuxhaven (Dunbar), and possibly one or two other instances.* Undoubtedly a large number of epidemics have been due to typhoid infected water, but for obvious reasons (long incubation of typhoid, the fact that the bacillus only lives in water for a few days, etc.), the cases where the bacillus has been actually isolated are very few. In the Milroy Lectures for 1902, Professor Corfield gives records of between 50 and 60 typhoid epidemics since 1864 . We shall refer to this matter subsequently when Bacillus typhosus is under consideration.

In artificial cultivation water bacteria respond very readily to

* Brit. Med. Jour., 1900, ii. p. 1198. 
external conditions. Increase of alkalinity ( 01 grams of sodium carbonate added to 10 c.c. of ordinary gelatine) causes the number of colonies to be five or six times greater than that revealed by using ordinary gelatine; on the other hand, very slightly increasing the acidity of a medium as markedly diminishes the number of bacteria. Advantage is taken of this in culturing the bacillus of typhoid, which is not inhibited by an acid medium.

Water may become contaminated with pathogenic bacteria in a variety of ways, as pollutions at the source, in the course, and at the periphery. Gathering grounds are frequently the source of the pollution. The Maidstone typhoid epidemic was an example. Here some of the springs supplying the town with water were contaminated by several typhoid patients. Frequently on the gathering ground one may find a number of houses the waste and refuse of which will furnish ample surface pollution, which in its turn may readily pass into a collecting reservoir or a well. On one occasion the writer investigated the cause of typhoid fever in a large country house in Oxfordshire, and traced it to pollution of the private well by surface washings from the stable quarters. Leakage of house drains into wells is not an infrequent source of contamination.

The same cause is generally operative in cases of pollution of a water supply in its course from the source to the cisterns or taps at the periphery, viz., a sewer or drain leaking into the water supply. Water companies and those responsible for water supply appear frequently to hold the opinion that so long as there is sand filtration or subsidence reservoirs, it is unnecessary to consider the gathering ground or possible contamination during transit. But it happens that a frost may completely dislocate the efficient action of a filter, and times of flood may prevent proper sedimentation; then our dependence for pure water is wholly upon the gathering ground and source. Hence we find water contaminated at its source by polluted wells, by sewage-infected rivers and streams, by drainage of manured fields, by innumerable excremental pollutions over the areas of the gathering grounds, and in transit by careless laying, bad construction and jointing of pipes, and close proximity of such drain pipes to the water supply.

In the third place, we may get a water infected at the periphery, in the house itself. Such cases are generally due to two causes: filthy cisterns and pipes or suction. Cisterns per se are more or less indispensable where a constant service does not exist, but they should be inspected from time to time and maintained in a cleanly condition. Suction into the tap has been emphasised by Dr Vivian Poore as a cause of pollution. It is liable to occur whenever a tap is left turned on, and a vacuum is produced in the supply pipe by inter- 
mission of the water supply, so that foul gas or liquid is sucked back into the house-pipe.

A further point has relation to bacterially polluted water when it has gained entrance to the body. It has been known for some time past that not all waters polluted with disease germs produce disease. As we have before said, this depends upon the infective agent, its quantity and quality, and upon the human body. The body is able in many cases to resist a small dose of poison. It is, however, necessary to infection, especially in water-borne disease, that the tissues shall be in some degree disordered, weakened, or injured. For instance, the perverted action of the stomach influences the acid secretion of the gastric juice, through which bacilli might then pass uninjured. Particularly must this be so in the bacillus of cholera, which is readily killed by the normal acid reaction of the stomach. Hence, in this disease at least, it is the opinion of bacteriologists that the condition of the mucous membrane of the stomach is of primary importance. Metchnikoff has indeed demonstrated the presence of the bacillus of cholera in the intestinal excretion of apparently healthy persons, which shows that they were protected by the resistance of their tissues to the bacilli. Further light has been thrown on this question by the researches of MacFadyen, who has pointed out that suspensions of cholera bacilli in water passed through the stomach untouched, and were thus able to exert their evil influence in other parts of the alimentary canal. When, however, cholera bacilli were suspended in milk, none appeared to escape the germicidal action of the gastric juice. The explanation of this is probably the simple one that the stomach reacted with its secretion of gastric juice only to food (milk), but passed the water on into the lower and more absorptive parts of the alimentary canal. Such a condition of affairs clearly increases the danger due to water-borne germs.

\section{The Interpretation of the Findings of Bacteriology}

Bacteriology is the most direct and delicate test of the safety of a water for drinking purposes. By it we obtain exact information not alone as to the constitution of a water, but as to its potentiality to cause disease. It is also a more delicate test than a chemical examination.* Klein and others have shown that by bacteriological methods it is possible to detect smaller degrees of sewage pollution than by chemistry. On the other hand, it is useless to expect to learn of the exact chemical constitution of a water by bacteriological methods. Bacteriology must be interpreted by what it can

* Clark and Gage state that polluted waters which might become unfit for drinking purposes are more plainly indicated by a single chemical analysis than by a single determination of $B$. coli. 
do and not by what it cannot; and in a general way it may be said that there are three groups of facts contained in a systematic bacteriological report of water. These findings are concerned with the number of bacteria per c.c., the presence of any organisms of contamination, and the presence of any specific organisms of disease.

(1) Number of Bacteria per c.c.-It would appear that in the past a great deal too much weight has been attributed to the number of bacteria per c.c. This fact is not of the first importance for two obvious reasons. In the first place there is no standard as to how many bacteria should be present in 1 c.c. of a potable water, and in the second place there is no known means by which this number can be accurately measured. In this country any number of bacteria under one hundred per c.c. is generally considered low. The metropolitan water supply, as consumed, usually contains less than twenty bacteria per c.c. Deep - well waters and spring waters frequently contain very few bacteria. Polluted or surface waters contain thousands of organisms per c.c. More than this, no standard exists. Nor would any numerical standard taken alone be of much value, for the reason that the number of bacteria in water is of comparatively little value apart from a knowledge of the species, and moreover a really accurate record of the number of bacteria per c.c. is not obtainable. Whether the organisms detected be many or few depends upon a variety of external circumstances, such as medium used for cultivation, temperature and period of incubation, length of time of cultivation before counting, or the use or not of a lens when counting. For these reasons it is evident that great reliance cannot be placed upon the number of bacteria per c.c. returned in bacteriological reports, and it is well that should be understood. The only circumstances under which such returns are valuable are $(\alpha)$ when used in a series of examinations of the same water supply, when such returns, if always obtained under the same conditions, are of great comparable value, and $(b)$ when used in the examination of water before and after filtration. In these two circumstances the number of organisms per c.c. is of great value in forming an opinion as to pollution or as to failure of filtration.

(2) Presence of Organisms of Contamination.-In the general bacteriological examination of water this point is perhaps the most important. Judgment must be formed on two facts, namely, the presence of any of the "bacteria of indication," such as $B$. coli, $B$. enteritidis sporogenes, streptococci, and the para-colon types (enteritidis, Gaertner, and the chologenes type), and the relative abundance of these species. The latter point is one of importance.

The chief organism of indication is $B$. coli, including under that 
term the typical bacillus and closely allied organisms. When this bacillus can be detected in a small measured quantity of water, that is to say, in 1,2 or 3 c.c., it is assumed $(a)$ that the organism has gained access to the water from sewage, and $(b)$ that recently. (c) It is further assumed that certain disease-producing bacteria which occur frequently in sewage may also be present in the water, though if present at all in the water, in considerably smaller numbers than B. coli. (d) Further, judging the matter broadly, the higher the number of $B$. coli the heavier will have been the recent sewage pollution, and the greater the probability of the presence of disease-producing bacteria. Conversely, if $B$. coli is not present, one may assume with some probability of being correct, that such disease-producing bacteria as the bacillus of typhoid fever will also be absent, and that the particular sample of water under examination might safely be used for drinking purposes.

There is difference of opinion as to the exact quantity of a water which must be free from a single specimen of $B$ coli in order that it may be said that the sample is a "safe" one; but many would in practice accept the standard 1 or 2 c.c.

It has already been stated that the presence of $B$. coli in a water is not of importance, because this organism itself, under the ordinary conditions, is likely to be harnful, but rather because it serves as an index of sewage or surface pollution. In this connection it may be said that a single examination of a water is of practically no value when the results of the bacteriological examination are favourable; it is only after repeated examination has shown that $B$. coli is absent from the water for a prolonged period, and after local inspection has shown that there are no possible sources of dangerous sewage contamination, that one is justified in giving a positive opinion as to the safety of a water. On the other hand, a single bacteriological examination with an unfavourable result will prove the actual occurrence, and suggest the possible recurrence, of sewage contamination, and will necessitate renewed inspection if no obvious source of contamination is known to exist.

B. coli is commonly considered as evidence of contamination by sewage, but it is possible for the bacillus to gain access to the water from other sources also. The bacillus is present in the excreta of mammals generally, and has been found in the excreta of birds, and in surface waters there will undoubtedly be a certain amount of contamination caused in this way. The question as to whether any contamination of this kind can be caused by various fishes, and other forms of aquatic life, is not fully established, though Eyre has recently found the $B$. coli in the excreta of fishes, as well as mammals and birds.* 
Most bacteriologists would condemn a water containing the typical $B$. coli in 1 c.c. as showing signs of sewage pollution. In the case of a recent pollution the presence of $B$. coli affords therefore a much more delicate test of pollution than any chemical examination which can be made.*

$B$. enteritidis sporogenes is another organism of indication as to sewage pollution, and its presence in bacillary form or as spores is now accepted as showing recent or remote contamination.

The presence of streptococcus is held by many bacteriologists to be a sign of sewage contamination, although some contend that the presence of streptococci does not indicate dangerous contamination unless accompanied by $B$. coli. The following table (p. 59), from the Thirty-fourth Annual Report of the Lawrence Sta., 1903, sets forth, in less space and with more accuracy than could be recorded in many words, the relative presence of the chief organisms of contamination, and it is therefore inserted.

Lastly, there are a number of organisms which appear to be frequently present in waters contaminated with sewage, and are rarely if ever found in pure supplies. The occurrence of such bacteria in a water should arouse suspicion as to its origin or contamination. Among this group of bacteria are $B$. fluorescens putridus, B. erythrospores, $B$. et $M$. urece, $B$. pyocyaneus, $B$. lactis cyanogenus, and $B$. megaterium.

(3) The presence of pathogenic species.-The presence of any pathogenic organisms, in however few numbers, is of course sufficient for the condemnation of a water. For instance, the presence of the bacillus of typhoid fever or the bacillus of cholera at once condemns a water. There are very few authentic records of such organisms being found, and it is therefore necessary to judge of waters by the presence of organisms of contamination.

Note.-A water may be considered safe and potable $(a)$ if it contains comparatively few organisms; $(b)$ an absence of organisms capable of fermenting glucose or lactose media; $(c)$ an absence of $B$. enteritidis sporogenes; and $(d)$ an absence of any pathogenic species, and especially if these conditions are found to exist as a result of several examinations or of periodic examinations. A water should be condemned, as a rule, $(a)$ if it contains a very large number of bacteria per c.c. of whatever kind; $(b)$ if it contains $B$. coli communis, or B. enteritidis sporogenes or streptococci in 1 c.c. or any such small quantity; $(c)$ if it gives the enteritidis change in milk cultures, or ferments glucose or lactose media. It should be condemned without hesitation if it contains B. coli and B. enteritidis sporogenes (or spores), and streptococci, or if it contains any pathogenic organism, in however small a quantity. But in condemning or

* See also Fourth Report Roy. Com. Sewage Disposal, 1904, pp. 106-109. 


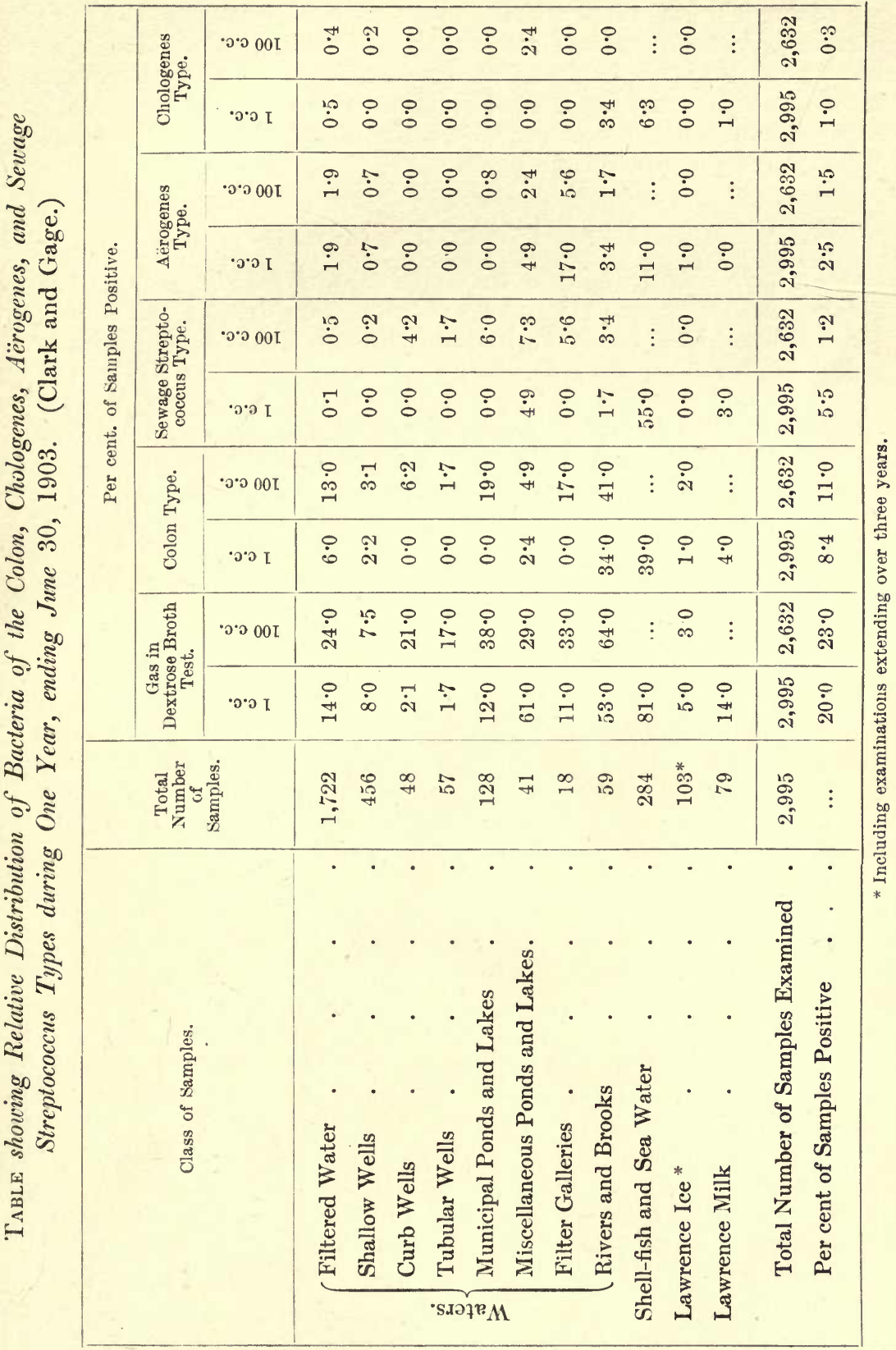


approving a water supply it is important to take all the findings of chemistry, bacteriology, and topography into consideration. The whole history of the sample must be considered, and too much reliance must not be placed upon the mere presence or absence of $B$. coli, or any single phenomenon or reaction. No ultimate reliance should, as a rule, be placed upon any single test.

\section{Natural Purification of Water}

We have already noticed that rivers purify themselves as they proceed. There are many excellent examples of such selfpurification. The Seine as it runs through Paris becomes highly polluted with every sort of filthy contamination. It receives daily about 250,000 c.m. of sewage. But 20 or 30 miles below the city it is found to be even purer than above the city before it received the sewage. In small rivers it is the same, provided the pollution is less in amount. The Thames and the Severn are excellent examples. Whilst authorities differ with regard to the means of self-purification which operate most effectually, all agree that in some way rivers receiving crude sewage are able in a marvellous degree to become pure again.

The chief conditions influencing this phenomenon are as follow :-

(a) The movement of the water.-It is probable that any beneficial result accruing from this cause is due not to any mechanical factor in the movement, but to the extra surface of water available for oxidation processes. Delépine has shown that the effect of agitation is an increase in the number of suspended bacteria which he attributes to the dislodgment of deposit and side adhesions. The greatest amount of purification in his experiments occurred when the rate of flow was about 8 c.m. per hour.*

(b) The pressure of the water.-It is believed that the volume of water pressing down upon any given area beneath it weakens the vitality of certain microbes. In support of this theory, it is urged that the number of bacteria capable of developing is less the greater the depth from the surface. Yet it must be remembered that mud at the bottom of a river, or at the bottom of shallow sea, is teeming with living organisms, and there is no evidence to show that pressure in river water ever reaches a degree capable of affecting the life of bacteria. Delépine found that in the Manchester mains increase of pressure did not reduce the number of bacteria. $\dagger$

(c) Light.-We have seen how prejudicial is light to the growth of organisms in culture media. This is so, though to a less extent, in water (see p. 18). Arloing held that sunlight could not pierce

* The Natural Purification of Running Water, Jour. of State Mell., 1901, p. 517.

† Report to the Manchester Water Works Committee, 1894. 
a layer of water an inch in thickness and still act inimically on micro-organisms. But Buchner found that the sun's rays could pass through 15 or 20 inches and yet be bactericidal. This evidence appears contradictory. On the whole, however, authorities agree that the influence of the sun's rays upon water is in some degree bactericidal and causes a diminution in the quantities of organisms after acting for some hours. Especially will this be so when the water is spread out over a wide area and is therefore shallow and stationary, or moving but slowly.* But taken as a whole it may be said that light does not exert a marked influence in water purification. There is, on the other hand, evidence to prove that water in its passage through dark mains of various sizes gradually becomes deprived of a great part of its bacterial contents.

(d) Vegetation in water.-Pettenkofer, in his observations upon the Iser below Munich, has shown how algæ bring about a marked reduction in the organic matters present in water. Boyce has pointed out that in the river Severn, in addition to the temperature and movement being unfavourable to $B$. coli and presumably pathogenic bacteria, that $(a)$ lack of pabulum, and $(b)$ antagonism due to the fauna and flora of the river exert an unfavourable influence upon these bacteria. The organic matter so abundant when the river becomes polluted at Shrewsbury is diluted and destroyed lower down stream, and therefore the water becomes purified of bacteria living on the organic matter. Fish, birds, rats, protozoa, and forms of river life generally contribute their share to the consumption of organic pabulum. The water Ranunculus, Sphœrotilus, Leptomitus lacteus, sewage fungi, chlorophyll containing protophytes, and river plants generally assist in the destruction of organic matter and bacteria.

(e) Dilution.-The pollutions passing into a flowing river are very soon diluted with the large quantities of comparatively pure water always forthcoming. And this, whilst it lowers the percentage of impurity, also raises the percentage of oxygenated water. Delépine has pointed out as a result of artificial experiments that dilution exerts a double effect on the bacterial content of water. In the first place it has the mechanical effect of increasing the space occupied by a definite number of bacteria, and in the second place it causes a diminution in the amount of pabulum present in a given bulk of the impure fluid. Dilution and deposition acting together exert a powerful influence as purifiers. Clark and Gage of the Lawrence station pointed out in 1903 that the number of B. coli in a polluted river varies in inverse ratio with the dilution of the entering sewage by the river water, and is affected by the temperature, the number of $B$. coli being larger during the warm weather than in the cold. In

* See also Spitta's work on the Spree at Berlin, Archiv für Hyg., vol. xxxviii.

† Roy. Com. on Sewage Disposal, Second Report, 1902, pp. 104-109. 
effluents from water filters the effect upon filter efliciency of dilution of the water in winter is less marked than the effect of high temperature in summer, the work of a filter in warm weather being, of course, more satisfactory than in cold.

(f) Sedimentation.-Whilst Pettenkofer attributes self-purification to oxygenation and vegetation, most authorities are now agreed that it is largely brought about by the subsidence of impure matters, and by their subsequent disintegration at the bottom of the river. Sedimentation and side-adhesion to the banks in rivers and streams of solids in suspension removes a large number of bacteria in the Severn (Boyce). Sedimentation obviously is greatest in still waters. Hence lake water contains as a rule very few bacteria. "The improvement in water during subsidence is the more rapid and pronounced the greater the amount of suspended matter initially present" (Frankland). Tils has pointed out that the number of micro-organisms was invariably smaller in the water collected from the reservoir than in that taken from the source supplying the latter. Percy Frankland has demonstrated the same effect of sedimentation by storage as follows:-

1. Intake from Thames, 25th June 1892

2. First small storage reservoir

3. Second small storage reservoir

4. Large storage reservoir

No. of Colonies in

1 c.c. of Water.

- 1991

- 1703

- 1156

- 464

The large reservoir would of course necessitate a prolonged subsidence, and hence a greater diminution than in the small reservoirs. Karlinski gives the following distribution of bacteria in the Borka Lake (Herzegovina) :-

$\begin{array}{lllr}\text { Surface water } & \text {. } & & 4000 \\ \text { Five inches below surface } & \cdot & & 1000 \\ \text { Ten inches below surface } & \cdot & & 600 \\ \text { Twelve to sixteen inches below surface } & \text {. } & 200 \\ \text { Bottom when mud was stirred up } & \text {. } & \text {. } & 6000\end{array}$

Delépine considers that bacteria die rapidly in the deposit, although their large numbers are evidence of the effect of sedimentation. He examined some water mains after the sediment had been disturbed and also with the sediment undisturbed. The results were as follows :-

Sediment undisturbed.

1. 51 living bacteria per c.c.

2. 356

3. 10

He concludes (1) that sedimentation is a very important factor of bacterial purification in flowing water, and (2) that the effects of
Sediment disturbed. 334 living bacteria per c.c. 3164

852

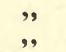


sedimentation are most manifest when the flow of water is rapid enough to prevent the accumulation at any point of the products of bacterial multiplication, but not so rapid as to interfere with a comparatively rapid action of gravity.*

In the case of a tidal stream the conditions are different, as recently pointed out by Foulerton. $\dagger$ In such rivers the diseaseproducing bacteria are deposited not only on the bed of the stream, but also on the mud, or sludge, on the banks, and are uncovered by water at low tide. It now requires only the agency of a fly, feeding first on the organic matter in the sewage-contaminated mud and then on some human food, milk for instance, to convey the bacillus of typhoid fever from the river to some human being. An additional way by which a bacillus of this kind may survive after it has been discharged into a river is by its being deposited on the bed of the stream where there are shell-fish layings. It has been proved that the typhoid bacillus can survive for a considerable time in the liquor contained in the shell of the oyster or the mussel, and in this way it may escape destruction by finding itself once more inside the consumer of the shell-fish. Therefore, in the case of sewage discharged into a tidal river, owing to lack of dilution and sedimentation, it is a menace to the inhabitants on the banks in one or both of these ways. The exact degree of danger depends first upon the extent to which the sewage is purified before its discharge into the 'stream, and secondly upon the distance from the source of pollution at which contamination of the water by special sewage bacteria is still appreciable.

This principle of sedimentation operates upon all bacteria, which are often carried down on gross particulate matter. The number of $B$. coli is reduced quite appreciably by storage of water (Clark and (Tage). Many species remain in the mud, sand, or other deposit at the bottom of the stream or reservoir. The parasitic organisms die on account of the unfavourable environment.

(g) Oxidation.-Many experiments and observations have been made to prove that large quantities of oxygen are used up daily in oxygenating the Thames water. Oxygenated water will come up with the tide and down with the fresh water from above London. There will also be oxygen absorption going on upon the surface of the water, and from these three sources enough oxygen is obtained to oxidise impurities and produce what is really an "effluent." In many smaller streams the opportunity for oxidation is afforded by weirs and falls.

Probably all these factors play a part in the self-purification of rivers, but we may take it that oxidation, dilution, and sedimentation are three of the principal agencies. The test of purification is in the

* Jour. of Stat. Med., 1901, p. 518.

+ Report on Pollution of Tidal Ouse, 1903, p. 11. 
number and character of the bacteria at different stages of the river (e.g., see Table of Bacteria in Severn, p. 38). Jordan has pointed out the peculiar value of the reduction of $B$. coli.*

We may here refer in passing to the facts obtainable from the late Sir Edward Frankland's report on Metropolitan water supply in 1894, as they will afford a connecting link between natural purification and artificial purification. First, judged by the relatively low proportion of carbon to nitrogen, the organic matter present in the water was, as usual, found to be chiefly of vegetable origin. Secondly, an immense destruction of bacteria was effected by storage in subsidence reservoirs. Thirdly, the bacterial quality of the water might differ widely from its chemical qualities. It is, of course, a much finer index of pollution. These three facts are of primary importance in the interpretation of water reports, and it will be well to bear them in mind. Sir E. Frankland also referred to the physical conditions affecting microbial life in river waters, and, as in previous reports, to the importance of changes of temperature, the effect of sunlight, and rate of flow. Respecting the relative proportion of these factors, he wrote: "The number of microbes in Thames water is determined mainly by the flow of the river, or, in other words, by the rainfall, and but slightly, if at all, by either the presence or absence of sunshine, or a high or low temperature. With regard to the effect of sunshine, the interesting researches of $\mathrm{Dr}$ Marshall Ward leave no doubt that this agent is a powerful germicide, but it is probable that the germicidal effect is greatly diminished, if not entirely prevented, when the solar rays have to pass through even a comparatively thin stratum of water before they reach the living organisms." Subsequent investigations have confirmed the importance of these broad principles, and from which it is clear that ovidence favours the effect of sedimentation and dilution. These two factors in conjunction with filtration are, practically speaking, the methods of artificial water purification, to which reference will now be made.

\section{Artificial Purification of Water}

Sedimentation and Precipitation.-In nature we see this factor in operation in lakes and reservoirs. For example, the water supply of Glasgow is the untreated overflow from Loch Katrine. Purification has been brought about by means of subsidence of impurities. Nothing further is needed. Much of the purification obtained in reservoirs supplying large towns is due to the same factor. Artificially we find it is this factor which is the mechanical purifier of biological impurity in such methods as Clark's process. By this mode "temporary hardness," or that due to soluble * Jour. of Hyg., 1901, p. 293. 
suitable nidus for putrefactive bacteria. The large quantities of bacteria which a decayed tooth contains are easily demonstrated.

From the two series of experiments which we have now considered we may gather the following facts :-

(a) That air may contain great numbers of bacteria which may be readily inspired.

(b) That in health those inspired do not, as a rule, pass beyond the moist surface of the nasal and buccal cavities, except in persons who practice oral instead of nasal respiration.

(c) That in the nose and mouth there are various influences of a bactericidal nature at work in defence of the individual.

(d) That expired air in normal quiet breathing contains, as a rule, no bacteria whatever.

The practical application of these things is a simple one. $\mathrm{To}_{*}$ keep air free from bacteria, the surroundings must be moist. Strong acids and disinfectants are not required. Moisture alone will be effectual. Two or three examples at once occur to the mind. Anthrax spores are conveyed from time to time from dried infected hides and skins to the hands or bodies of workers in warehouses in Bradford, Bermondsey, Finsbury, and other places. If the surroundings are moist and the hides moist, anthrax spores and other bacteria do not remain free in the air. As a matter of actual experience, it has been found that handling dried hair or dried skins leads to more anthrax infection than handling the same articles in a moist condition.*

Again, the bacilli (or "spores") of tuberculosis present in sputum in great abundance cannot infect the air until and unless the sputum dries. So long as the expectorated matter remains on the pavement or handkerchief wet, the surrounding air will derive from it no bacilli of tubercle. But when in the course of time the sputum dries, then the least current of air will at once infect itself with the dried spores or bacilli. It should, however, be remembered that the "cough-spray" and microscopic particles of saliva emitted in shouting, heavy breathing through the mouth, etc., have been shown by Flügge and others to carry the bacilli of tubercle. Such conveyance may, of course, prove a channel of infection between diseased and healthy persons. The typhoid bacillus, too, occupies the same position. Only when the excrement dries can the contained bacteria infect the air. It is of course well known that the common channel of infection in typhoid fever is, not the air, whereas the reverse holds true of tuberculosis. But if it happens that the excrement of patients suffering from typhoid dries, the air may become infected; if, on the other hand, it passes in a moist state into the sewer, even though untreated with disinfectants, all will be well as regards the surrounding air.

* Annual Reports of Medical Inspector of Factories and Workshops, 1902 and 1903. 
A still more remarkable illustration of the effect of a moist perimeter upon the contained bacteria is to be found in sewer air. For long it has been known that air polluted by sewage emanations is capable of giving rise to various degrees of ill-health. These chiefly affect two parts of the body; one is the throat and the other the intestine. Irritation and inflammation may be set up in both or either by sewer air. Such conditions are in all probability produced by a lowering of the resistance and vitality of the tissues, and not by a conveyance of bacteria in sewer air or by any stimulating effect upon bacteria exercised by sewer air. What evidence we have is against such factors. Several series of investigations have been made into the bacteriology of sewer air, amongst others by Uffelmann, Carnelly and Haldane, and Laws and Andrewes. From their labours we may formulate four simple conclusions:-

1. The air of sewers contains very few micro-organisms indeed, sometimes not more than two organisms per litre (Haldane), and generally fewer than the outside air (Laws and Andrewes).

2. There is not, as a rule, intimate relationship between the microbes contained in sewer air and those contained in sewage. Indeed, there is a marked difference which forms a contrast as striking as it is at first sight unexpected. The organisms isolated from sewer air are those commonly present in the open air. Micrococci and moulds predominate, whereas in sewage moulds and micrococci are rare, and bacilli are most numerous. Liquefying bacteria, too, which are common in sewage, are extremely rare in sewer air. Bacillus coli communis, which occurs in sewage from 20,000 to 200,000 per c.c., is altogether absent from sewer air.

3. As a rule it may be said that only when there is splashing in the sewage, or when bubbles are bursting (Carnelly and Haldane), is it possible for sewage to part with its contained bacteria to the air of the sewer. But under these conditions it may part with a considerable number.

4. Pathogenic organisms and those nearly allied to them are found in sewage, but are absent in sewer air. Uffelmann isolated the Staphylococcus pyogenes aureus (one of the organisms of suppuration), but such a species is exceptional in sewer air. Hence, though sewer air is popularly held responsible for directly conveying virulent micro-organisms of various diseases, there is up to the present no evidence of a substantial nature in support of such views. In 1894, Laws and Andrewes found an average of 2,781,650 bacteria per c.c. in fresh sewage, and in older sewage from $3,400,000$ per c.c., to $11,216,000$, and they pointed out that temperature and dilution of sewage were determining factors in the number of bacteria present. They consider that sewage may become a medium for the dissemination of the typhoid bacillus, and that sewage-polluted soil may possibly 
give up germs to the subsoil air, but they are satisfied that the air of sewers themselves does not play any part in the conveyance of the typhoid bacillus.*

In passing, mention may be made of some interesting observations recorded by Mr S. G. Shattock on the effect of sewer air upon the toxicity of lowly virulent bacilli of diphtheria. Some direct relationship, it has been surmised, exists between breathing sewer air and "catching" diphtheria. Clearly, it cannot be that the sewer air contains the bacillus. But some have supposed that the sewer air has had a detrimental effect by increasing the virulent properties of bacilli already in the human tissues. Two cultivations of lowly virulent $B$. diphtherioe were therefore grown by Mr Shattock in flasks upon a favourable medium over which was drawn sewer air. This was continued for two months in the one case, and five weeks in the other. Yet no increased virulence was secured. $\dagger$ Such experiments require ample confirmation, but even now it may be said that sewer air does not necessarily have a favouring influence upon the virulence of the bacilli of diphtheria. Such experiments do not affect the contrary question of the possibility of sewer air depressing the vitality of the individual, and so allowing even lowly virulent bacilli to do mischief. Of such depression caused by breathing sewer air there is clinical proof, and although sewer-men do not appear to be affected, persons freshly breathing sewer air may be.

It should be noted that the bacilli of diphtheria are capable of lengthened survival outside the body, and are readily disseminated by very feeble air - currents. The condition necessary for their existence outside the body for any period above two or three days is moisture. Dried diptheria bacilli soon lose their vitality. It is possible, owing to this fact, that the disease is not as commonly conveyed by air as, for example, tubercle.

3. The Influence of Gravity upon bacteria in the air may be observed in various ways, in addition to its action within a limited area like a sewer or a room. Miquel found in some investigations in Paris that, whereas on the Rue de Rivoli 750 germs were present in a cubic metre, yet at the summit of the Pantheon only 28 were found in the same quantity of air. Frankland found that air at the top of Primrose Hill contained 9 organisms per ten litres, and air at the bottom 24. On the spire of Norwich Cathedral (310 feet), ten litres of air yielded 7 organisms, on the tower (180 feet) 9 , and on the ground 18. At the level of the golden gallery of St Paul's Cathedral he found in every ten litres 11 bacteria, at the stone gallery 34, and in St Paul's Churchyard 70. As Tyndall has pointed out,

Report to the London County Council on the Result of Investigation on the Microorganisms of Sewage, by J. Parry Laws and F. W. Andrewes, 1894, p. 14.

$\dagger$ Pathological Society of London, Transactions, 1897. 
even ultra-microscopic cells obey the law of gravitation. This is equally true in the limited areas of a laboratory or warehouse, and in the open air. At high altitudes, the air may be looked upon as practically germ-free, although here again the lighter spores of the mould fungi may cause them to be carried by air currents to a very great height. In the recent researches of Dr Jean Binot of the Pasteur Institute,* 100 litres of air taken at the summit of Mont Blane did not contain a single microbe, and the total number of organisms varied between 4 and 11 per metre cube (1000 litres). An examination of the air of the interior of M. Janssen's Observatory, situated on the highest point of Mont Blanc, and taken in two different rooms, gave, on the other hand, 540 and 260 organisms per metre cube. The gradual increase of the number of organismis as descent to lower level takes place is of interest. Thus 6 per metre cube were found in the Grand Plateau, 8 at the Grand Mulet, and 14 at the Plon de l'Aiguille. Upon the Mer de Glace 23 organisms were found, and 49 at Montanvert. Graham Smith found that at the top of the Clock Tower of the Houses of Parliament in London there was only about one-third of the number of bacteria found at the ground level. $\dagger$

4. Air Currents.-Miquel, Pasteur, Cornet, and other workers have shown that the presence of micro-organisms in air depends in part upon air currents, winds, etc. In the month of August, with the wind from the south, i.e. blowing from the country citywards, the number of organisms was found by Miquel to be 40 in the Mont Souris Parc around the Observatory, while at the same moment a record of 14,800 was obtained in the 4 th Arrondissement, which may be taken as the centre of Paris, and comprises the surroundings of Notre Dame and of the Hotel de Ville. In the month of June, on the other hand, with the wind blowing from the N.E., i.e. across the city towards Mont Souris, the numbers were, in the 4th Arrondissement, 10,000 per metre cube, and in the Park of Mont Souris itself, 1180 per metre cube.

The seasonal variations of the organisms present in the air are also worthy of note, and depend chiefly upon dust and air currents. The following table shows the mean over a period of ten years in the air taken at Mont Souris :-

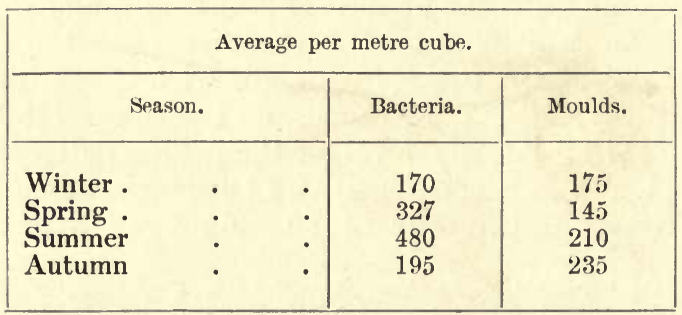

* Communication à l'Académie des Sciences de Paris, 17 Mars 1902.

† Jour. of Hyg., 1903, p. 513. 
Similar experiments have been carried out by Frankland, Flïgge, Delalivesse, Neisser, Chick, Andrewes, and others.* The last named conducted some experiments in London streets in 1902, and reported his results to the Pathological Society. He found the number of organisms varied greatly, but no pathogenic species were detected. The four species he isolated were staphylococci, sarcinæ, streptothriceæ, and moulds.

Carnelly, Haldane, and Anderson found the ratio of organisms in the air increased according to whether the air was examined on still damp days, windy damp days, still dry days, and windy dry days, and in brief this expresses the findings of most investigators.

Some new light has been thrown upon the subject of pathogenic organisms in air by Neisser in his investigations concerning the amount and rate of air currents necessary to convey certain species through the atmosphere. He states that the bacteria causing diphtheria, typhoid fever, plague, cholera, and pneumonia, and possibly the common Streptococcus pyogenes, are incapable of being carried by the molecules of atmospheric dust which the ordinary insensible currents of air can support, whilst Bacillus anthracis, $B$. pyocyaneus, and the bacillus of tubercle are capable of being aërially conveyed. This work will require further confirmation before being entirely accepted.

Finally, some mention may be made of the relationship alleged to exist between the presence of a considerable degree of carbonic acid gas in an atmosphere and the number of bacteria contained in the same atmosphere. As far as may be judged, it would appear that the relationship is but slight. But to illustrate the subject as well as other points of importance in the bacteriology of air, four separate investigations may be mentioned.

(i.) Haldane and Osborn, in their inquiry into the ventilation of factories and workshops, made a number of bacteriological examinations. $\uparrow$ The determinations of bacteria were made by a slightly modified form of Frankland's method. The air was drawn through a sterilised plug of glass wool by means of a brass syringe of known capacity. The glass tubes containing the glass wool plugs were each enclosed in a separate outside sterilised glass tube, with an asbestos plug. In taking the sample of air the inside tube was attached directly to the pump by means of a short piece of stout rubber tubing. The plug was afterwards transferred with the necessary precautions to a shallow, flat-bottomed flask, containing a small quantity of liquefied gelatine, which was shaken so as to disintegrate and spread the glass

* See also Jour. of Sanitary Institute (Oct. 1902), vol. xxiii., pt. iii., p. 209-236. The Dusi Problem, by Sir J. Crichton-Browne, F.R.S.

+ First Report of the Departmental Committee appointed to inquire into the Ventilation of Factories and Workshops, 1902. J. S. Haldane, M.D., F.R.S., and E. H. Osborn. 
wool. The gelatine having set, the flask was incubated at $20^{\circ} \mathrm{C}$. till no further colonies of bacteria or moulds developed. Some of the chief results were as follows:-

\begin{tabular}{|c|c|c|c|c|c|c|}
\hline & & \multirow{2}{*}{$\begin{array}{l}\text { Cub. } \\
\text { Content. }\end{array}$} & \multicolumn{2}{|c|}{$\begin{array}{c}\mathrm{CO}_{2} \\
\text { per } 10,000 \text { parts. }\end{array}$} & \multicolumn{2}{|c|}{$\begin{array}{l}\text { Bacteria and Moulds } \\
\text { per Litre of Air. }\end{array}$} \\
\hline & & & Inside. & Outside Air. & Bacteria. & Moulds. \\
\hline Tailor, Whitechapel. & - & 67,500 & $35 \cdot 8$ & $3 \cdot 5$ & 17 & 22 \\
\hline , , $\quad$, & . & 21,953 & $9 \cdot 2$ & $3 \cdot 5$ & 8 & 1 \\
\hline ," & . & 2,750 & $4 \cdot 6$ & $3 \cdot 5$ & 16 & 2 \\
\hline, & & 18,636 & $10^{\circ} 0$ & $3 \cdot 5$ & 9 & 8 \\
\hline London, $\mathrm{E}^{*} \cdot$ & & 9,800 & $7 \cdot 4$ & $3 \cdot 5$ & 9 & 0 \\
\hline ", London, E. & & 27,265 & $14 \cdot 6$ & $3 \cdot 5$ & 10 & 2 \\
\hline $\begin{array}{l}\text { London, E.C. } \\
\text { Capmaker, London, E. }\end{array}$ & & 26,460 & $14^{\circ} 6$ & $3 \cdot 5$ & 25 & 3 \\
\hline $\begin{array}{l}\text { Capmaker, London, E. } \\
\text { Dressmaker, London, W. }\end{array}$ & & $\begin{array}{r}4,296 \\
01\end{array}$ & $23 \cdot 0$ & $\begin{array}{l}3 \cdot 5 \\
3.5\end{array}$ & $\begin{array}{l}9 \\
8\end{array}$ & 2 \\
\hline Boot Workshop, London & E. & $\begin{array}{r}21,600 \\
8,688\end{array}$ & $\begin{array}{r}13 \cdot 2 \\
8 \cdot 8\end{array}$ & $\begin{array}{l}3 \cdot 5 \\
3 \cdot 5\end{array}$ & $\begin{array}{r}8 \\
25\end{array}$ & $\begin{array}{l}0 \\
6\end{array}$ \\
\hline Railway Works, Wilts. & .. & 93,786 & $4 \cdot 6$ & $3 \cdot 5$ & 20 & 2 \\
\hline Chocolate Factory, Berm & ondsey & 12,000 & $6 \cdot 2$ & $3 \cdot 5$ & 8 & 0 \\
\hline Newspaper Printer, Lond & I., E.C. & 24,098 & $16 \cdot 5$ & $3 \cdot 5$ & 9 & 0 \\
\hline ," . , & ," & 45,259 & $15 \cdot 2$ & $3 \cdot 5$ & 6 & 6 \\
\hline '" '", & ," & 23,562 & $25 \cdot 4$ & $3 \cdot 5$ & 10 & 2 \\
\hline Ropemaker, Chatham * & - & $\cdots$ & $\cdots$ & $\cdots$ & 20 & $\begin{array}{l}6 \\
8\end{array}$ \\
\hline ," & - & $\cdots$ & $\cdots$ & $\cdots$ & 850 & $18^{\circ}$ \\
\hline " & $\cdot$ & $\cdots$ & $\cdots$ & . & & \\
\hline
\end{tabular}

* The ventilation of this large room was considerable, but having the effect of keeping dust in suspension rather than expelling it from the room. Three tests made here were all in the same workplace, differing only in degree of dust present.

(ii.) In 1902 the writer made some observations in Finsbury on the number of bacteria to be found in the air of underground bakehouses. Four were selected, and the degree of carbonic acid gas was estimated by Pettenkofer's method, and examinations were made as follow of the bacteria pollution. In each of these bakehouses, whilst work was going on, three agar-plates (of $9 \cdot 6$ inches area each) were exposed for thirty minutes. One plate was placed on the floor, one on the table or trough where the bread was being made, and one on a shelf near the ceiling. After exposure for thirty minutes the plates were re-covered and incubated at blood-heat $\left(37^{\circ} \mathrm{C}\right.$.), for exactly twentytwo hours. All the plates then showed abundant growth. Doubtless if the plates had been incubated for forty-eight hours, or three or four days, there would have been a greater growth of colonies, and it is probable also that if some of the plates had been placed at room temperature certain bacteria would have grown which did not appear at blood-heat in twenty-two hours. It is not suggested that these plates provide an adequate record of the bacteria present in the air of these bakehouses. The object was merely to obtain a comparative 


\section{AIR-PLATES EXPOSED IN BAKEHOUSES (30 minutes).}

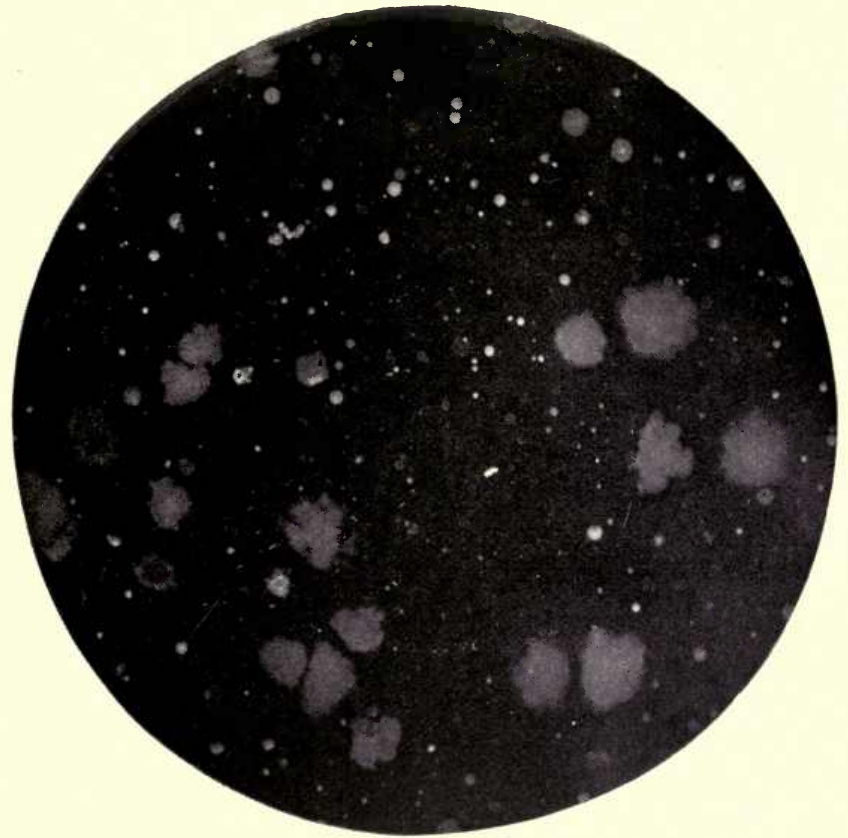

(i.) Above-ground Bakehouse (Z.) Agar culture, 22 hours at $37^{\circ} \mathrm{C}$.

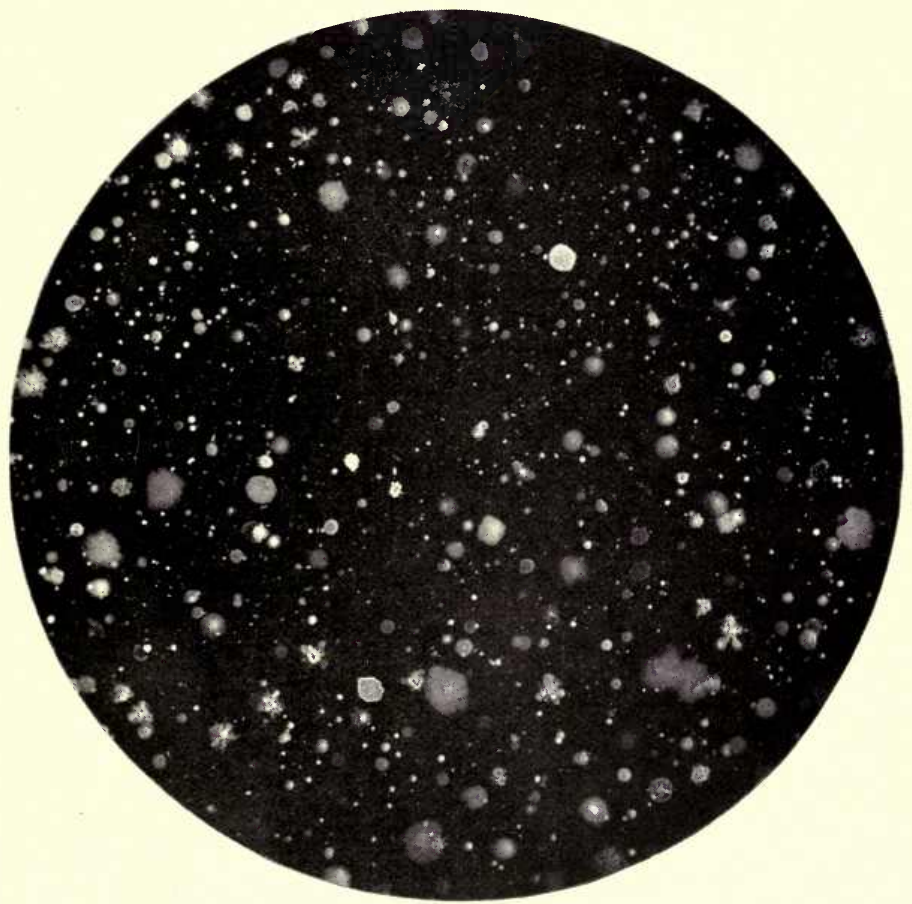

(ii.) Under-ground Bakehouse (C.) Agar culture, 22 hours at $37^{\circ} \mathrm{C}$ 

idea of the air of underground bakehouses and above-ground bakehouses in Finsbury. Accordingly, the whole of the 30 plates used in this examination were treated exactly the same in every way, the medium, exposure, and temperature and period of incubation being precisely similar. The results, therefore, whilst of little value as a complete examination of the air, are useful and reliable for comparison with each other.

The results were as follow:-

\begin{tabular}{|c|c|c|c|c|c|}
\hline & & & & $\begin{array}{c}\text { Carbonic Acid Gas } \\
\text { in parts } \\
\text { per } 10,000 \text { (Colwell). }\end{array}$ & $\begin{array}{c}\text { Average No. of } \\
\text { Bacteria } \\
\text { on each Plate. }\end{array}$ \\
\hline $\begin{array}{c}\text { Underground Bakehouse } \\
\qquad, ", \\
\text { ", } \\
\text { Above-ground Bakehouse } \\
\text { Outside Air in street }\end{array}$ & $\begin{array}{l}\mathrm{B} \\
\mathrm{C} \\
\mathrm{D} \\
\mathrm{E} \\
\mathrm{Z} \\
\text { (C) }\end{array}$ & 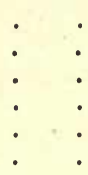 & $\begin{array}{l}\dot{ } \\
\dot{.} \\
\dot{.}\end{array}$ & $\begin{array}{r}12 \cdot 0 \\
17 \cdot 5 \\
16 \cdot 9 \\
13 \cdot 6 \\
4 \cdot 9 \\
4 \cdot 5\end{array}$ & $\begin{array}{l}800 \\
680 \\
600 \\
600 \\
200 \\
160\end{array}$ \\
\hline
\end{tabular}

Inside the bakehouses there was also an interesting distribution of bacteria as follows:-

\begin{tabular}{|lc|c|c|c|}
\hline & $\begin{array}{c}\text { No. of Bacteria } \\
\text { per Plate on } \\
\text { Shelf. }\end{array}$ & $\begin{array}{c}\text { No. of Bacteria } \\
\text { per Plate on } \\
\text { Table. }\end{array}$ & $\begin{array}{c}\text { No. of Bacteria } \\
\text { per Plate on } \\
\text { Floor. }\end{array}$ \\
\hline Underground Bakehouse & $\mathbf{C}$ & 490 & $720^{*}$ & 850 \\
Above-ground Shop of & $\mathrm{C}$ & 130 & 150 & 720 \\
Above-ground Bakehouse & $\mathrm{Z}$ & 150 & $170^{*}$ & 300 \\
\hline
\end{tabular}

* Illustrations of these two plates are attached (Plate 7).

From these figures it will be seen $(a)$ that underground bakehouse air contained at least four times more bacteria than street air around it; $(b)$ at least three times more bacteria than the air of the shop over it; and $(c)$ at least three times more bacteria than the aboveground bakehouse. The general result of the investigations was that the air of the typical underground bakehouses examined (1) contained 14.8 volumes per 10,000 of carbonic acid gas, $\mathrm{CO}_{2}$ (as compared with 4.9 in above-ground bakehouses and 4.3 in the streets of Finsbury); (2) that it contained between 10 and 24 per cent. less moisture than outside air surrounding the bakehouses; and (3) that it contained at least four times more bacteria than surrounding street air, and three 
times more bacteria than the air of a typical above-ground bakehouse.*

Dr Scott Tebb has also made a somewhat parallel examination of the air of London streets as compared with the railway tube of the City and South London railway. $\dagger$ As the result of a large number of investigations carried out in a similar way to the writer's examinations in bakehouses, the following figures were arrived at:-

\begin{tabular}{|c|c|c|c|}
\hline Air of & & $\begin{array}{c}\mathrm{CO}_{2} \\
\text { per } 10,000 \text { parts. }\end{array}$ & $\begin{array}{l}\text { No. of } \\
\text { Micro-organisms } \\
\text { per Plate. }\end{array}$ \\
\hline $\begin{array}{l}\text { The open streets } \\
\text { Platforms in Railway Tube } \\
\text { Railway Carriages in Tube }\end{array}$ & $: \quad:$ & $\begin{array}{r}3 \cdot 8 \\
7 \cdot 9 \\
11 \cdot 6\end{array}$ & $\begin{array}{l}459 \\
114 \\
218\end{array}$ \\
\hline
\end{tabular}

(iii.) Thirdly, some of the results of the investigations of Graham Smith into the condition of the atmosphere of the House of Commons may be mentioned. + He used a modification of Frankland's method of filtering the air to be examined (4:5 litres in each case) through glass wool. An air-pump and a rubber tube of 10 feet in length were used for drawing the air through, and gelatine was used as the medium, the cultures being incubated at $20^{\circ}$ C. for five days or longer. The results may be expressed in tabular form in three series :-

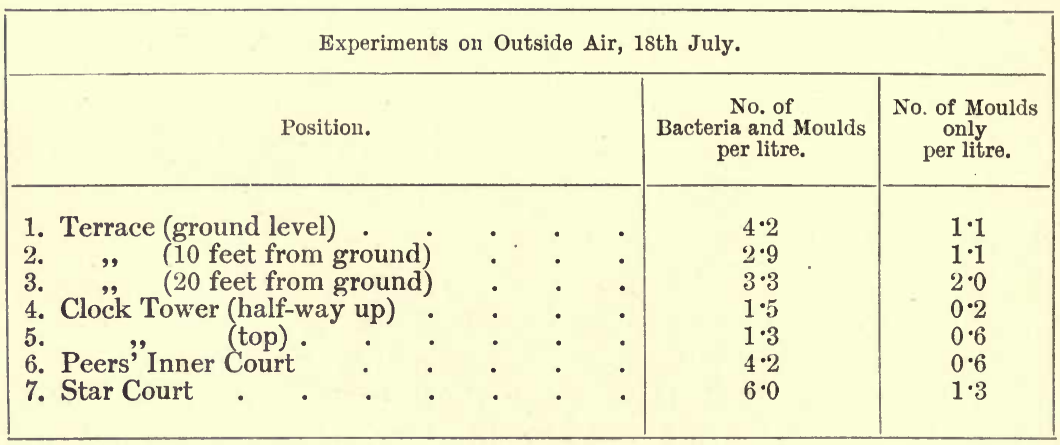

Similar experiments were performed in the House itself during a

* Report on Bakeloouses in Finsbury (Newman), 1902, p. 51.

+ Report of Public Analyst of Southwark on Condition of Air on City and South London Railway, 1903. W. Scott Tebb, M.D.

‡ Jour. of Hyg., 1903, pp. 498-513. 
sitting. The air as it entered the House contained 2.6 bacteria and moulds per litre.

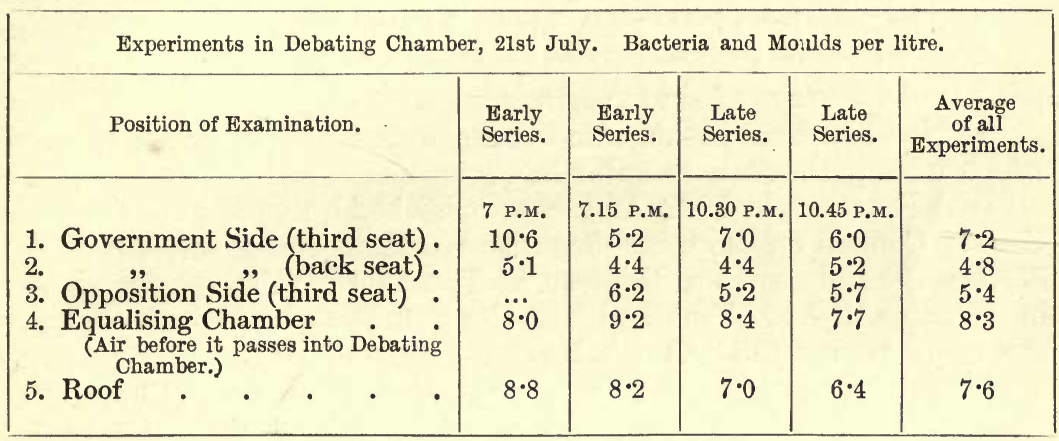

A third series of examinations was made by Graham Smith of the air in certain committee rooms, etc., as follows :-

\begin{tabular}{|c|c|c|}
\hline \multicolumn{3}{|c|}{ Experiments in Committee, Dining, and Smoking Rooms. } \\
\hline Position of Examination. & $\begin{array}{l}\text { No. of } \\
\text { Bacteria and Moulds } \\
\text { per litre. }\end{array}$ & $\begin{array}{c}\text { No. of Moulds } \\
\text { only } \\
\text { per litre. }\end{array}$ \\
\hline 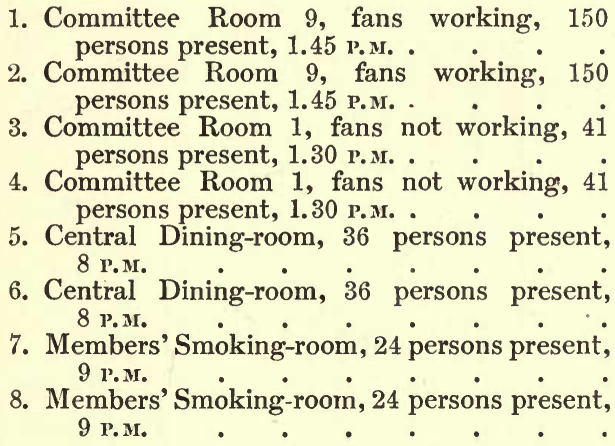 & $\begin{array}{l}20 \cdot 9 \\
35 \cdot 5 \\
33 \cdot 7 \\
41 \cdot 3 \\
44 \cdot 2\end{array}$ & $\begin{array}{l}4 \cdot 6 \\
5 \cdot 3 \\
4 \cdot 2 \\
8 \cdot 4\end{array}$ \\
\hline
\end{tabular}

Separate investigation as to the degree of $\mathrm{CO}_{2}$ present in the Debating Chamber of the House of Commons revealed between 3-4 parts per 10,000 .

Dr Graham Smith, as a result of his investigations, arrived at the following conclusions:-

1. The number of micro-organisms in the open space surrounding the House of Parliament is comparatively small ( $4 \cdot 2$ per litre). 
2. The air in the debating chamber is from a bacteriological point of view remarkably pure (5.8 per litre as average of eleven experiments).

3. The number of bacteria found in the committee, dining, and smoking rooms was several times greater than in the chamber $(32.3$ per litre as average of six experiments).

4. No organisms pathogenic to man were isolated, and only a few which were pathogenic to animals.

(iv.) Fourthly, in 1902 Andrewes furnished a report to the London County Council on the micro-organisms present in the air of the tube of the Central London Railway.* The method he employed was in principle that of Frankland, viz., the aspiration by means of a brass syringe (capacity 425 c.c.) of a known volume of air (5 litres), through a plug of glass wool and finely-powdered cane-sugar. The latter retains the micro-organisms, which can be subsequently distributed through a suitable cultivating medium (such as gelatine) in a Petri dish. The gelatine plate-cultures were incubated at $20^{\circ} \mathrm{C}$. for four days, when the colonies were counted, examined, and sub-cultured. Special control experiments were made, and search was also made for the presence of anaërobic organisms. The twelve series of experiments yielded results which may be abstracted and tabulated as follow :-

\begin{tabular}{|c|c|c|c|c|c|c|c|}
\hline- & 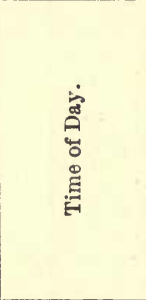 & 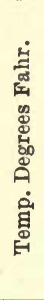 & 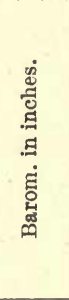 & 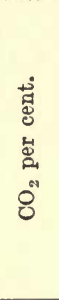 & 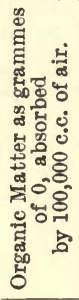 & 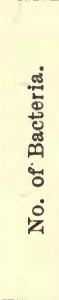 & 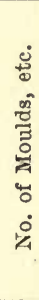 \\
\hline $\begin{array}{l}\text { 1. Platform : } \\
\text { 2. Lift } \\
\text { 3. Carriage (smoking) } \\
\text { 4. Tunnel } \\
\text { 5. Carriage (non-smoking) } \\
\text { 6. Platform : } \\
\text { 7. Platform : } \\
\text { 8. Carriage (non-smoking) } \\
\text { 9. Tunnel } \\
\text { 10. Lift : } \\
\text { 11. Staircase, Passage : } \\
\text { 12. Staircase, Passage }\end{array}$ & $\begin{array}{r}11.30 \text { a.m. } \\
11.45 \text { a.m. } \\
11.45 \text { a.m. } \\
11.45 \text { a.m. } \\
2.45 \text { p.m. } \\
5.10 \text { p.m. } \\
11.40 \text { a.m. } \\
11.20 \text { a.m. } \\
11.15 \text { a.m. } \\
11.0 \text { a.m. } \\
11.0 \text { a.m. } \\
11.0 \text { a.m. }\end{array}$ & $\begin{array}{l}66 \\
61 \\
70 \\
67 \\
68 \\
67 \\
68 \\
72 \\
70 \\
66 \\
64 \\
68\end{array}$ & $\begin{array}{l}30 \cdot 3 \\
30 \cdot 5 \\
29 \cdot 5 \\
30 \cdot 2 \\
30 \cdot 5 \\
30 \cdot 1 \\
30 \cdot 4 \\
30 \cdot 2 \\
30 \cdot 2 \\
29 \cdot 9 \\
29 \cdot 8 \\
30 \cdot 0\end{array}$ & $\begin{array}{l}\cdot 09 \\
\cdot 109 \\
\cdot 108 \\
\cdot 082 \\
\cdot 111 \\
\cdot 103 \\
\cdot 094 \\
\cdot 134 \\
\cdot 104 \\
\cdot 152 \\
\cdot 078 \\
\cdot 102\end{array}$ & $\begin{array}{l}\cdot 0019 \\
\cdot 0028 \\
\cdot 0026 \\
\cdot 0010 \\
\cdot 0027 \\
\cdot 0012 \\
\cdot 0010 \\
\cdot 0016 \\
\cdot 0018 \\
\cdot 0042 \\
\cdot 0013 \\
\cdot 0023\end{array}$ & $\begin{array}{r}14 \\
20 \\
51 \\
10 \\
13 \\
30 \\
106 \\
90 \\
10 \\
64 \\
13 \\
17\end{array}$ & $\begin{array}{r}13 \\
19 \\
10 \\
8 \\
14 \\
11 \\
6 \\
13 \\
4 \\
9 \\
3 \\
7\end{array}$ \\
\hline
\end{tabular}

* Examination of the Atmosphere of the Central London Railway, London County Council, 1902. No. 615. F. W. Andrewes, M.D., F.R.C.P. 
By way of summary, it may be said that Andrewes found that miero-organisms were present in the air of the Central London Railway in a somewhat greater proportion (as 13 to 10) than in the fresh air outside. The number was high in proportion to the concentration of human traffie, being highest in carriages, platforms, and lifts. The tube air does not eompare unfavourably with that known to exist in ordinary dwelling-rooms. No pathogenic germs were discovered, though the number of organisms eapable of growing at body temperature was greater in the tube air than in the outside air, and the number of organisms in the tube air was found generally proportional to the degree of chemical contamination, but this rule was subject to striking exceptions. It is evident that bacterial contamination of air, though, as a rule, parallel to chemical contamination, may yet vary quite independently as the result of special conditions, such as air eurrents, which indicates that chemieal examination alone cannot always be taken as a trustworthy guide to the eontamination of air.

The speeies of bacteria which Andrewes found in the railway air were in the main identical with those oecurring in fresh air, and included Staplylococcus cereus flavus et albus, Micrococcus candicans, M. flavus, M. citreus, M. lactis, $M$. albicans tardissimus, Sarcina lutea, S. flava, S. alba, Bacillus luteus, B. lactis innocuus, Streptothrix Försteri, S. chromogenes, S. albido-flava, Torula alba, and Saccharomyces cerevisice.

\section{Interpretation of Reports on Bacterial Content of Air}

In the present position of our knowledge of the baeteriology of air, reports are only of comparative value. Mere numbers of saprophytie bacteria in air are not of great service. Up to the present it has not been possible to isolate pathogenie organisms, though such must inevitably oceur in air under eertain cireumstances, though even then probably only in very small numbers. To deteet pathogenie organisms, it will probably be necessary to examine large volumes of air, and by methods which will eliminate the common saprophytes. The truth is that the foundations of our knowledge eoneerning the bacterial flora of the air are only beginning to be laid, and until we ean detect, by bacteriological examination, organisms of disease, the bacteriology of air ean only be a subject of relative importanee. 


\section{CHAPTER IV}

\section{BACTERIA AND FERMENTATION}

Early Work-Kinds of Fermentation: (1) Alcoholic Fermentation, Ascospores, Pure Cultures, Films ; (2) Acetous Fermentation ; (3) Lactic Acid Fermentation ; (4) Butyric Fermentation ; (5) Ammoniacal Fermentation-Diseases of Wine and Beer: Turbidity, Ropiness, Bitterness, etc.-Industrial Applications of Bacterial Ferments.

IT was Pasteur who, in 1857, first propounded the true cause and process of fermentation. The breaking down of sugar into alcohol and carbonic acid gas had been known, of course, for a long period. Since the time of Spallanzani (1776) the putrefactive changes in liquids and organic matter had been prevented by boiling and subsequently sealing the flask or vessel containing the fluid. Moreover, this successful preventive practice had been in some measure correctly interpreted as due to the exclusion of the atmosphere, but wrongly credited to the exclusion of the oxygen of the air. It was not until the beginning of the present century that authorities modified their view and declared in favour of yeast cells as the agents in the production of fermentation. That this process was due to oxygen per se was disproved by Schwann, who showed that so long as the oxygen admitted to the flask of fermentable fluid was sterilised no fermentation occurred. It was thus obvious that it was not the atmosphere or the oxygen of the atmosphere, but some fermenting agent borne into the flask by the admission of unsterilised air. It was but a step further to establish this hypothesis by adding unsterilised air plus some antiseptic substance which would destroy the fermenting agent. Arsenic was found by Schwann to have this germicidal property. Hence Schwann supported Latour's theory 
that fermentation was due to something borne in by the air, and that this something was yeast.

Passing over a number of counter-experiments of Helmholtz and others, we come to the work of Liebig. He viewed the transformation of sugar into alcohol and carbonic acid gas simply and solely as a non-vital chemical process, depending upon the dead yeast communicating its own decomposition to surrounding elements in contact with it. Liebig insisted that all albuminoid bodies were unstable, and if left to themselves would fall to pieces-i.e. ferment-without the aid of living organisms, or any initiative force greater than dead yeast cells. It was at this juncture that Pasteur intervened to dispel the obscurities and contradictory theories which had been propounded.

As in all the conclusions arrived at by Pasteur, so in those relating to fermentation, there were a number of different experiments which were performed by him to elucidate the same point. We will choose one of many in relation to fermentation. If a sugary solution of carbonate of lime is left to itself, it begins after a time to effervesce, carbonic acid is evolved, and lactic acid is formed; and this latter decomposes the carbonate of lime to form lactate of lime. This lactic acid is formed, so to speak, at the expense of the sugar, which little by little disappears. Pasteur demonstrated the cause of this transformation of sugar into lactic acid to be a thin layer of organic matter consisting of extremely small moving organisms. If these be withheld or destroyed in the fermenting fluid, fermentation will cease. If a trace of this grey material be introduced into sterile milk or sterile solution of sugar, the same process is set up, and lactic acid fermentation occurs.

Pasteur examined the elements of this organic layer by aid of the microscope, and found it to consist of small short rods of protoplasm quite distinct from the yeast cells which previous investigators had detected in alcoholic fermentation. One series of experiments was accomplished with yeast cells and these bacteria, a second series with living yeast cells only, a third series with bacteria only, and the conclusions at which Pasteur arrived as the result of these labours he expressed in the following words:-

"As for the interpretation of the group of new facts which I have met with in the course of these researches, I am confident that whoever shall judge them with impartiality will recognise that the alcoholic fermentation is an act correlated to the life and to the organisation of these corpuscles, and not to their death or their putrefaction, any more than it will appear as a case of contact action in which the transformation of the sugar is accomplished in the presence of the ferment without the latter giving or taking anything from it." 
Pasteur occupied six years (1857-1863) in the further elucidation of his discovery of the potency of these hitherto unrecognised agents, and the establishment of the fact that "organic liquids do not alter until a living germ is introduced into them, and living germs exist everywhere." It must not be supposed that to Pasteur is due the whole credit of the knowledge acquired respecting the cause of fermentation. He did not first discover these living organisms; he did not first study them and describe them; he was not even the first to suggest that they were the cause of the processes of fermentation or disease. But nevertheless it was Pasteur who "first placed the subject upon a firm foundation by proving with rigid experiment some of the suggestions made by others."

\section{Kinds of Fermentation}

Although fermentation is nearly always due to a living agent, as proved by Pasteur, the process is conveniently divided into two kinds.* (1) When the action is direct, and the chemical changes involved in the process occur only in the presence of the cell, the latter is spoken of as an organised ferment; (2) when the action is indirect, and the changes are the result of the presence of a soluble material secreted by the cell, acting apart from the cell, this soluble substance is termed an unorganised soluble ferment, or enzyme. The organised ferments are bacteria or vegetable cells allied to the bacteria; the unorganised ferments, or enzymes, are ferments found in the secretions of specialised cells of the higher plants and animals. It will be sufficient to illustrate the enzymes by a few of the more familiar examples, such as the digestive agents in human assimilation. This function is performed, in some cases, by the enzyme combining with the substance on which it is acting and then by decomposition yielding the new "digested" substance and regenerating the enzyme; in other cases, the enzyme, by its molecular movement, sets up molecular movement in the substance it is digesting, and thus changes its condition. These digestive enzymes are as follow: in the saliva, ptyalin, which changes starch into sugar; in the gastric juice of the stomach, pepsin, which digests the proteids of the food and changes them into more soluble forms; the pancreatic ferments, amylopsin, trypsin, and steapsin, capable of attacking all classes of food stuffs; and the intestinal ferments, which have not yet been separated in pure condition. In addition to these, there are ferments in bitter almonds, mustard, etc. Concerning these unorganised ferments we have little further to say. Perhaps the commonest of them all is diastase, which occurs in malt, and to which some reference will be made later. Its function is to convert the starch, which occurs in

* E. A. Schäfer, F. R.S., Text-book of Physiology, vol. i., p. 312, 
barley, into sugar. These unorganised ferments act most rapidly at a high temperature.*

We may preface our consideration of the organised ferments by an axiom by which Professor Frankland sums up the vitalistic theory of fermentation, which was supported by the researches of Pasteur: "Nofermentation without organisms, in every fermentation a particular organism." From these words it is to be inferred that there is no one particular organism or vegetable cell to be designated the microorganism of fermentation, but that there are a number of fermentations each started by some specific form of agent. It is true that the chemical changes, induced by organised ferments, depend on the life processes of micro-organisms which feed upon the sugar or other substance in solution, and excrete the product of the fermentation. Fermentation always consists of a process of breaking down of complex bodies, like sugar, into simpler ones, like alcohol and carbonic acid. Of such fermentations we may mention at least five: the alcoholic, by which alcohol is produced; the acetous, by which wine absorbs oxygen from the air and becomes vinegar; the lactic, which sours milk; the butyric, which out of various sugars and organic acids produces butyric acid; and the ammoniacal, which is the putrefactive breaking down of compounds of nitrogen into ammonia. We shall have occasion to refer at some length to this process when considering denitrifying organisms in the soil.

There are four chief conditions common to all these five kinds of organised fermentation. They are as follow :-

1. The presence of the special living agent or organism of the particular fermentation under consideration. This, as Pasteur pointed out, differs in each case.

2. A sufficiency of pabulum (nutriment) and moisture to favour the growth of the micro-organism.

3. A temperature at or about blood-heat $\left(35-38^{\circ}\right.$ C., $98 \cdot 5^{\circ}$ F.).

4. The absence from the solution or substance of any obnoxious or inimical substances which would destroy or retard the action of the living organism and agent. Many of the products of fermentation are themselves antiseptics, as in the case of alcohol; hence alcoholic fermentation always arrests itself at a certain point.

The causal micro-organisms of particular fermentations are of various kinds, belonging, according to botanical classification, to

* The unorganised ferments are frequently otherwise classified than as above, according to function. The chief are these :-amylolytic, those which change starch and glycogen (amyloses) into sugars, e.g., ptyalin, diastase, amylopsin (organisms of the subtilis group and the micrococcus of mastitis are said to produce amylolytic ferments); proteolytic, those which change proteids into proteoses and peptones, e.g., trypsin, pepsin ; inversive, those which change maltose, sucrose, and lactose into glucose, e.g., invertin (various species of bacteria produce inversive ferments); coagulative, those which change soluble proteids into insoluble, e.g., rennet; steatolytic, those which split up fats into fatty acids and glycerine, e.g., steapsin. 
various different subdivisions of the non-flowering portion of the vegetable kingdom. A large part of fermentation is based upon the growth of a class of microscopic plants termed yeasts. These differ from the bacteria in but few particulars, mainly in their method of reproduction by budding (instead of dividing or sporulating, like the bacteria). Their chemical action is closely allied to that of the bacteria. Secondly, there are special fermentations and modifications of yeast fermentation due to bacteria. Thirdly, a group of somewhat more highly specialised vegetable cells, known as moulds, make a perceptible contribution in this direction. According to Hansen, these latter, so far as they are really alcoholic ferments, induce fermentation, that is, inversion of sugar, not only in solutions of dextrose, but also in maltose. Mucor racemosus is the only member that is capable of inverting a cane-sugar solution; Mucor erectus is the most active fermenter, yielding 8 per cent. by volume of alcohol in ordinary beer wort. Both of these will be referred to as they occur in considering the five important fermentations already mentioned.

The general microscopic appearance of yeast cells may be shortly stated as follows: They are round or oval cells, and by budding become "daughter" yeast cells. Each consists of a cellulose membrane and clear homogeneous contents. As they perform their function of fermentation, vacuoles, fat-globules, and granules make their appearance in the enclosed plasma. Just as in many vegetable cells a nucleus was detected by Schmitz by means of special methods of staining, so Hansen has found the nucleus in old-yeast cells from "films" without any special staining.

\section{Alcoholic Fermentation}

Cause, yeast ; medium, sugar solutions ; result, alcohol and carbonic acid.

It was Caignard-Latour who first demonstrated that yeast cells, by their growth and multiplication, set up a chemical change in sugar solutions which resulted in the transference of the oxygen in the sugar compound from the hydrogen to the carbon atoms, that is to say, in the evolution of carbonic acid gas and the production, as a result, of alcohol. Expressed in chemical formula, the change is as follows:-

$$
\mathrm{C}_{6} \mathrm{H}_{12} \mathrm{O}_{6} \text { (plus the fermenting agent) }=2 \mathrm{C}_{2} \mathrm{H}_{6} \mathrm{O}+2 \mathrm{CO}_{2} \text {. }
$$

A natural sugar, like grape-sugar, present in the fruit of the vine, is thus fermented. The alcohol remains in the liquid; the carbonic acid escapes as bubbles of gas into the surrounding air. If we go a step further back, to cane-sugar (which possesses the same elements as grape-sugar, but in different proportions), dissolve it in water, 
and mix it with yeast, we get exactly the same result, except that the first stage of the fermentation would be the changing of the canesugar into grape-sugar, which is accomplished by a soluble ferment secreted by the yeast cells themselves. If now we go yet one step further back, to starch, the same sort of action occurs. When starch is boiled with a dilute acid it is changed into a gum-like substance, dextrin, and subsequently into maltose, which latter, when mixed with these living yeast cells, is fermented, and results in the evolution of carbonic acid gas and the production of alcohol. In the manufacture of fermented drinks from cereal grains containing starch there is, therefore, a double chemical process: first, the change of starch into sugar by means of conversion, a chemical change obtained by the action of sulphuric or some other acid, or by the influence of diastase; and secondly, the change of the sugar into alcohol and carbonic acid gas by the process of fermentation, an organic change brought about by the living yeast cells.

In all these three forms of alcoholic fermentation the principal features are the same, viz., the sugar disappears; the carbonic acid gas escapes into the air; the alcohol remains behind. Though it is true that the sugar disappears, it would be truer still to say that it reappears as alcohol. Sugar and alcohol are built up of precisely the same elements: carbon, hydrogen, and oxygen. They differ from each other in the proportion of these elements. It is obvious, therefore, that fermentation is really only a change of position, a breaking down of one compound into two simpler compounds. And this redistribution of the molecules of the compound results in the production of some heat. Hence, we must add heat to the results of the work of the yeasts.*

It will be necessary subsequently to consider a remarkable faculty which bacteria possess of producing products inimical to their own growth. In some degree this is true of the yeasts, for when they have set up fermentation in a saccharine fluid there comes a time when the presence of the resulting alcohol is injurious to further action on their part. It has become indeed a poison, and, as we have already mentioned, a necessary condition for the action of a ferment is the absence of poisonous substances. This limit of fermentation is reached when the fermenting fluid contains 13 or 14 per cent. of alcohol.

The Biology of Yeast.-Having briefly discussed the "medium" and the results, we may now turn to the other side of the mitter, and enumerate some of the chief forms of the yeast plant. Jörgensen

* When alcohol is pure and contains no water it is termed absolute alcohol. If, however, it is mixed with 16 per cent. of water, it is called rectified spirit, and when mixed with more than half its volume of water ( 56.8 pey cent.) it is known as proof spirit. 
gives more than a score of different members of this family of Saccharomycetes.* But before mentioning some of the chief of these, it will be desirable to consider a number of properties common to the genus. The yeast cell is a round or oval body of the nature of a fungus, composed of granular protoplasm surrounded by a definite envelope, or capsule. It reproduces itself, as a general rule, by budding, or gemmation. At one end of the cell a slight swelling or protuberance appears, which slowly enlarges. Ultimately there is a constriction, and the bud becomes partly and at last completely separated from the parent cell. In many cases the capsules of the daughter cell and the parent cell adhere, thus forming a chain of budding cells. The character of the cell and its method of reproduction do not depend merely upon the particular species alone, but are also dependent upon external circumstances. There are differences in the behaviour of species towards different media at various temperatures, towards the carbohydrates (especially

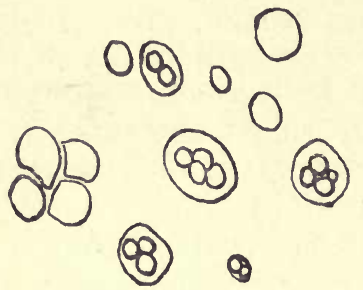

Fig. 13.-Diagram of Ascospore Formation.

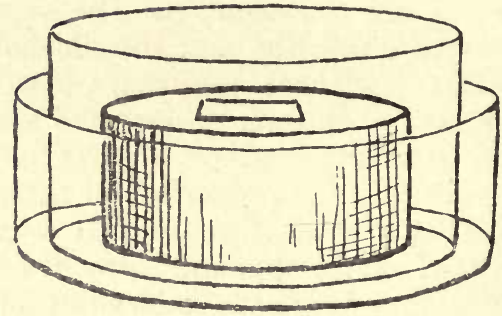

Fia. 14.-Gypsum Block.

maltose), and in the chemical changes which they bring about in nutrient liquids. In connection with these variations Professor Hansen has pointed out that, whilst some species can be made use of in fermentation industries, others cannot, and some even produce "diseases" in beer. $\dagger$

One of the most remarkable evidences of the adaptability of the yeasts to their surroundings; and a specific characteristic, occurs in what is termed ascospore formation. If a yeast cell finds itself lacking nourishment or in an unfavourable medium, it reproduces itself not by budding, but by forming spores out of its own intrinsic substance, and within its own capsule. To obtain this kind of spore formation Hansen used small gypsum blocks as the medium on which to grow his yeast cells. Well-baked plaster-of-Paris is mixed with distilled water, and made into a liquid paste. The moulds are made by pouring this paste into cardboard dishes, where it hardens

* Micro-organisms and Fermentation.

$\dagger$ E. C. Hansen, Studies in Fermentation (Copenhagen), p. 98. 


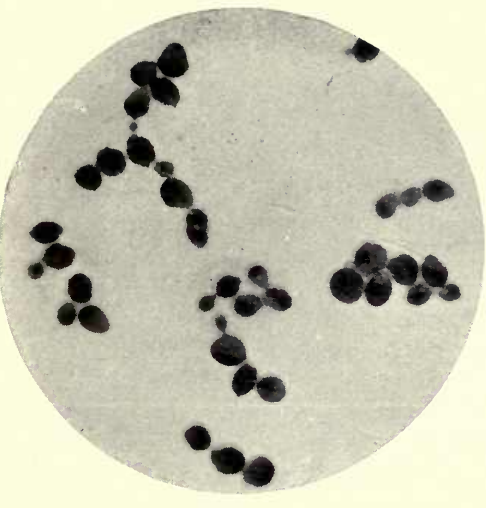

Saccharomyces cerevisice.

Film preparation. $\times 1000$.

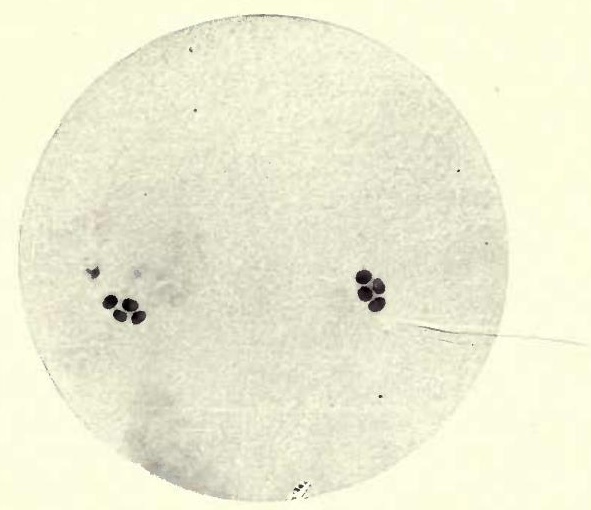

Ascospore Formation in Yeast. The capsule of the parent cell around the spores is invisible. $\times 1000$.

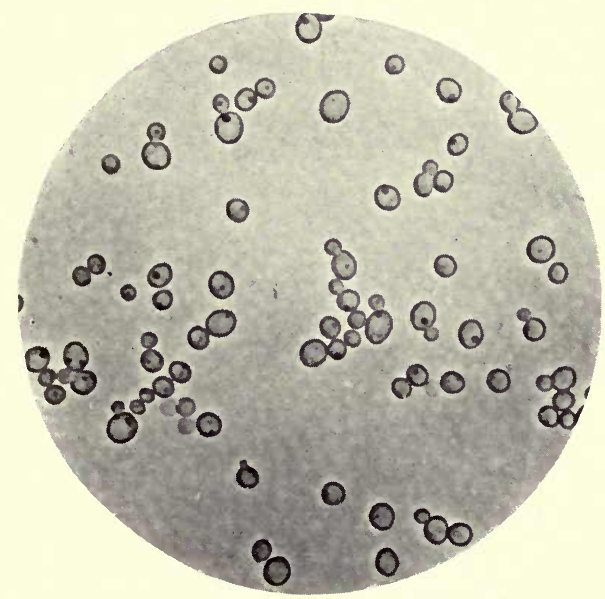

Pathogenic Yeast, (Fonlerton). $\times 1000$. 

again. The mould is then sterilised by heat, a few cells of yeast are placed on its upper surface, and the whole is floated in a small vessel of water and covered with a bell-jar. Under these conditions of limited pabulum the cell undergoes the following changes: it increases in size, loses much of its granularity, and becomes homogeneous, and about thirty hours after being sown on the gypsum there appear several refractile cells inside the parent cell. These are the ascospores. In addition to the gypsum, it is necessary to have a plentiful supply of oxygen, some moisture (gained from the vessel of water in which the gypsum stands), a certain temperature, and a young condition of the protoplasm of the parent yeast cells. Hansen found that the lowest temperature at which these ascospores were produced was $5-3^{\circ} \mathrm{C}$., and at the other extreme up to $37^{\circ} \mathrm{C}$. The rapidity of formation also varies with the temperature, the favourable degree of warmth being about $22-25^{\circ} \mathrm{C}$. (Plate 8 ).

Hansen pointed out that it was possible by means of sporulation to differentiate species of yeasts. For it happens that different species show slight differences in spore formation, e.g.:-

(a) The spores of Saccharomyces cerevisio expand during the first stage of germination, and produce partition walls, making a compound cell with several chambers. Budding can occur at any point on the surface of the swollen spores. To this group belong $S$. pastorianus and S. ellipsoideus.

(b) The spores of Saccharomyces Ludwigii fuse in the first stage, and afterwards grow out into a promycelium, which produces yeast cells.

(c) The spores of Saccharomyces anomalus are different in shape from the others in that they possess a projecting rim round the base.

Another point in the cultivation of yeasts has been elucidated by a number of workers, among whom is Hansen, namely, the methods of obtaining pure cultures. Only by starting with one individual cell can it be hoped to secure a pure culture of yeasts. For the study of the morphology of yeasts under the microscope the problem was not a difficult one. It was comparatively easy to keep out foreign germs from a cover-glass preparation sufficiently to perceive germination of spores and the growth of yeasts. But when pure cultures are required for various physiological purposes then a different standard and method is necessary.

Hansen employed dilution with water in the following manner:Yeast is diluted with a certain amount of sterilised water. A drop is carefully examined under the microscope, a single cell of yeast is taken, and a cultivation made upon wort. When it has grown abundantly a quantity of sterilised water is added. From this, again, a single drop is taken and added to say 20 c.c. of sterilised water in a fresh flask. This flask will contain, let us suppose, ten 
cells. It is now vigorously shaken, and the contents are divided into twenty portions of 1 c.c. each, and added to twenty tubes of sterilised water. It is highly probable that half of these tubes have received one cell each. In the course of a few days it can be seen how far the culture is pure. If only one colony is present, the culture is a pure one, and as this grows we obtain an absolutely pure culture in necessary quantity. Even when the gelatine plate method is used, it is desirable to start with a single cell (Hansen). The advantage of Hansen's yeast method over Koch's bacterial plate method is that it has a certain definite starting-point. This is obviously impossible when dealing with such microscopic particles as the bacteria proper.

A third point in the differentiation of yeast species is the question of films. Hansen set to work, after having obtained pure eultures and ascospores, to examine films appearing on the surface of liquids undergoing fermentation. The object of this was to ascertain whether all yeasts produced the same mycelial growth on the surface of the fermenting fluid. To produce these films the process is as follows: Drop on to the surface of sterilised wort in a flask a very small quantity of a pure culture of yeast; secure the flask from movement, and protect it, not from air, which is necessary, but from falling particles in the air. In a short time small colonies appear, which coalesce and form patches, and finally a film or membrane which covers the liquid and attaches itself to the sides of the flask. By the differences in the films and the temperatures at which they form it is possible to obtain something of a basis for classification. The further advances in yeast culture and in our knowledge of the agencies of fermentation have, however, tended to show that no strict dividing lines can be drawn. Hansen's researches have, notwithstanding, been of the greatest moment to the whole industry of fermentation. What has been found true in bacteriology has also been demonstrated in fermentation, namely, that, though many yeasts differ but little in structure and behaviour, they may produce very different products and possess very different properties. Industrial cultivation of these finer differences in fermentative action has to a large extent revolutionised the brewing industry. The formation of films is not a peculiarity of certain species, but must be regarded as a phenomenon occurring somewhat commonly amongst yeasts. The requisites are a suitable medium, a yeast cell, a free, still surface, direct access of air, and a favourable temperature. The wort loses colour, and becomes pale yellow. Microscopic differences soon appear between the sedimentary yeast and the film yeast of the same species, the latter growing out into long mycelial forms, the character of which depends in part upon the temperature. This often varies from $3^{\circ}$ to $38^{\circ} \mathrm{C}$. 
A fourth point helpful in diagnosis is the temperature which proves to be the thermal death-point. Saccharomyces cerevisio is killed by an exposure to $54^{\circ} \mathrm{C}$. for five minutes, and $62^{\circ} \mathrm{C}$. kills the spores. As a rule, yeasts can resist a considerably higher temperature when in a dry state than in the presence of moisture.

It should be noted that yeasts may be cultivated on solid media. Hansen employed wort-gelatine (5 per cent. gelatine), and found that at $25^{\circ} \mathrm{C}$. in a fortnight the growths which develop show such microscopic differences as to aid materially in diagnosis. Saccharomyces ellipsoideus $I$. exhibits a characteristic network which readily distinguishes it.

There is one other feature to which reference must be made. The process of fermentation may be set up by a "high" or a "low" yeast. These terms apply to the temperature at which the process commences. "High" yeasts rise to the surface as the action proceeds, accomplish their work rapidly, and at a comparatively high temperature, say about $16^{\circ}$ C.; "low" yeasts, on the contrary, sink in the fermenting fluid, act slowly, and only at the low temperature of $4^{\circ}$ or $5^{\circ} \mathrm{C}$. This is maintainable by floating ice in the fluid. Formerly all beer was made by the "high" mode, but on the continent of Europe "low" yeast is mostly used, whilst the " high" is in vogue in England. This latter method is more conducive to the development of extraneous organisms, and therefore risky in all but well-ordered brewing establishments.

Before proceeding to mention shortly some of the commoner forms of yeast we must again emphasise Hansen's method of analysis in separating a species. The shape, size, and appearance of cells are not sufficient for differentiation, because it is found that the same species, when exposed to different external conditions, can occur in very different forms. Hence Hansen established the analytical method of observing (1) the microscopic appearance, (2) the formation of ascospores, and (3) the production of films. In addition, the temperature limits, cultivation on solid media, and behaviour towards carbohydrates, are characters which aid in the separation of yeasts. In well-grown cultures on wort-gelatine, a broad division can be made of yeasts according as they produce $(a)$ a dry, hard, cohesive growth; (b) a soft, moist growth with liquefaction of gelatime; and (c) those producing pigment. By basing differentiation of species upon these features, the following can be distinguished:-

Saccharomyces Cerevisia. -Oval or ellipsoidal cells; reproduction by budding ; ascospores, rapidly at $30^{\circ} \mathrm{C}$, , slowly at $12^{\circ} \mathrm{C}$, not formed at all at lower temperatures ; film formation, seven to ten days at $22^{\circ} \mathrm{C}$.; an active alcoholic ferment, producing in a fortnight in beer wort from 4 to 6 per cent. by volume of alcohol (Jörgensen). This species is a typical English high yeast, possessing the power of "inverting" cane sugar previous to producing alcohol and carbonic acid. It is said to have no action on milk-sugar. It is the "true brewing yeast" (Plate 8 ). 
Saccharomyces Ellipsoidus I.-Round, oval, or sausage-shaped cells, single or in chains; ascospores in twenty-four hours at $25^{\circ} \mathrm{C}$. (not above $30^{\circ} \mathrm{C}$., not below $4^{\circ}$ C.). Grown on the surface of wort gelatine, a network is produced by which they can be recognised (in eight to twelve days at $33^{\circ}$ C.). At $13-15^{\circ}$ C. a characteristic branching mass is produced. It is an alcoholic ferment as active as

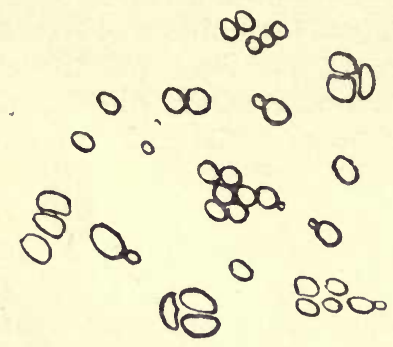

Fia. 15.-Diagram of $S$. cerevisice.

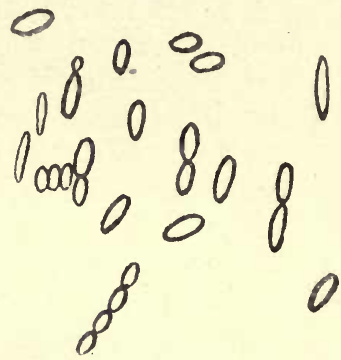

Fia. 16.-Diagram of $S$. ellipsoideus.

S. cerevisice. S. ellipsoideus 11 . - Round and oval, rarely elongated, a widely distributed yeast, causing " muddiness" in beer and a bitter taste. It is essentially a "low" yeast, and one of the so-called " wild yeasts" injurious to beer.

Saccharomyces Conglomeratus is a round cell, often united in clusters, and occurring in rotting grapes, and at the commencement of fermentation.

Saccharomyces Pastorianus I.-Oval or club-shaped cells, occurring in afterfermentation of wine, etc., and producing a bitter taste, unpleasant odour, and

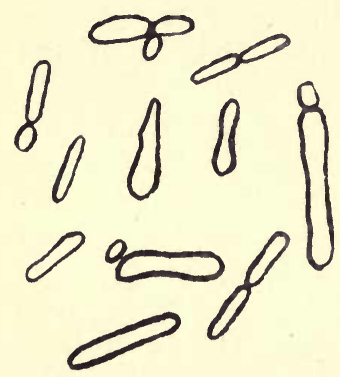

Fig. 17.-Diagram of $S$. pastorianus. turbidity. The spores frequently occur in the air of breweries.

S. Pastor. 1I.-Elongated cells, possessing an invertose ferment. They do not, like S. pastor. I., produce disease in beer.

S. Pastor. 111.-Oval or elongated cells, producing turbidity in beer. Grown on yeast-water gelatine; the colonies show after sixteen days crenated hairy edges.

Saccharomyces Apiculatus. - Lemon-shaped cells. They give rise to a feeble alcoholic fermentation, and produce two kinds of spores-round and oval; they appear at the onset of vinous fermentation, but give way later on to $S$. cerevisioe.

Saccharomyces Mycoderma.-Oval or elliptical cells, often in branching chains. They form the so-called " mould" on fermented liquids, and develop on the surface without exciting fermentation. When forced to grow submerged, they produce a little alcohol. beer.

Saccharomyces Exiguus.-Conical cells, appearing in the after-fermentation of

Saccharomyces Pyriformis._Oval cells, converting sugary solutions containing ginger into ginger-beer. alcohol.

Saccharomyces lllicis, Hansenii, and Aquifolii produce a small percentage of

\section{Acetous Fermentation}

Cause, Mycollerma aceti; medium, wine and other alcoholic liquids ; result, the formation of vinegar.

If alcohol be diluted with water, and the specific ferment mixed with it and exposed to the air at $22^{\circ} \mathrm{C}$., it is rapidly converted into 
vinegar. The change is accompanied by the absorption of oxygen, one atom of which combines with two of hydrogen to form water, and a substance remains termed aldehyde, further oxidation of which produces the acetic acid. We may express it chemically thus :-

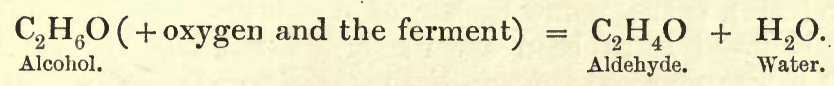

The aldehyde becomes further oxidised:-

$$
\mathrm{C}_{2} \mathrm{H}_{4} \mathrm{O}+\mathrm{O}=\mathrm{C}_{2} \mathrm{H}_{4} \mathrm{O}_{2} \text { (acetic acid). }
$$

This method of simply oxidising alcohol to obtain acetic acid may be carried out chemically without any ferment. If slightly diluted alcohol be dropped upon platinum black, the oxygen condensed in that substance acts with energy upon the spirit, and union readily occurring, acetic acid results. Here the whole business of the platinum sponge is to persuade the oxygen of the air and the hydrogen of the alcohol to unite. In the ordinary manufacture this is accomplished by the vegetable cells of Mycoderma aceti.

There are two chief methods adopted in the commercial manufacture of vinegar, both of which depend upon the presence of the mycoderma. The method in vogue at Orleans when Pasteur (about 1862) commenced his studies of the vinegar organism, was to fill vats nearly to the brim with a weak mixture of vinegar and wine. Where the process is proceeding the surface is covered with a fragile pellicle, "the mother of vinegar," which is produced by, and consists of, certain micro-organisms whose function is to convey the oxygen of the air to the liquor in the vats, thus oxidising the alcohol into vinegar. This oxidation may be carried on even beyond the stage of acetic acid (when no more alcohol remains to be oxidised), resulting in carbonic acid gas, which escapes into the air. But as in the alcoholic, so in the acetic, fermentation there comes a time when the presence of an excess of the acid inhibits the further growth of the organism. This point is approximately when the acetic acid has reached a percentage as high as 14 . But if the acid be removed, and fresh alcohol added, the process recommences.

The second method, sometimes called by the Germans the "quick vinegar process," is to pour the weakened alcohol through a tall cylinder filled with wood-shavings, having first added some warm vinegar to the shavings. After a number of hours the resulting fluid is charged with acetic acid. What has occurred? Liebig maintained that a chemical and mechanical change had brought about the change from the alcohol put into the cylinder and the vinegar drawn off at the exit tube. It was reserved for Pasteur to demonstrate by experiment that the addition of the warm vinegar to the shavings was in reality an addition of a living micro-organism, which, forming a film 
upon the shavings, became "the mother of vinegar," and oxidised the alcohol which passed over it, inducing it to become aldehyde and then acetic acid.

Mycoderma aceti (described by Persoon 1822, Kützing 1837, and Pasteur 1864), is the name rather of a family than an individual. Pasteur believed it to be a specific individual, but Hansen pointed out that it was composed of two distinctly different species (Bacterium accti and $B$. Pasteurianum), and subsequently other investigators have added members to the acetic fermentation group of which $M$. acet $i$ is the type. This bacterium is made up of small, slightly elongated cells, with a transverse diameter of 2 or $3 \mu$, sometimes united in short chains of curved rods. They frequently show a central constriction, are motile, and produce in old cultures involution forms. The way in which the cells act and are made to perform their function is as follows: A small quantity, taken from a previous pellicle, is sown on the surface of an aqueous liquid, containing 2 per cent. of alcohol, 1 per cent. of vinegar, and traces of alkaline phosphates. Very rapidly indeed the little isolated colonies spread, and becoming confluent, form a membrane or pellicle over the whole area of the fluid. When the surface is covered the alcohol is converted to acid. After this it is necessary to add, each day, small quantities of alcohol. When the oxidation is completed the vinegar is drawn off, and the membrane is collected and washed, and is then again ready for use. It ought not to remain long out of fermenting liquid, nor ought it to be allowed to over-perform its function, for thus having oxidised all the alcohol it will commence oxidation of the vinegar.

In wort-gelatine Bacterium Pasteurianum develops as round colonies with a smooth or wavy border, whilst $B$. acet $i$ has a tendency towards stellate arrangement. Spores have not been observed, and from a morphological point of view the two species behave alike. Neither produces any turbidity in the liquid containing them. In order to flourish, $B$. aceti requires a temperature of about $33^{\circ} \mathrm{C}$. and a plentiful supply of oxygen. In a cool store or cellar there is, therefore, nothing to fear from $B$. aceti. Frankland has isolated a Bacillus ethaceticus, which is a fermentative organism producing ethyl-alcohol and acetic acid. By oxidation the ethyl-alcohol may be converted into acetic acid.

\section{3.-Lactic Acid Fermentation}

Cause, Bacillus acidi lactici; medium, milk-sugar, cane-sugar, glucose, dextrose, etc.; result, lactic acid.

The process set up by the lactic ferment is simply a decomposition, an exact division of one molecule of sugar into two molecules of lactic acid, there being neither oxidation nor hydration. The con- 
ditions under which the ferment acts are very similar to those we have already considered (see also p. 196). There is frequently carbonic acid gas formed; there is a cessation of fermentation when the medium becomes too acid; there is the same method of starting the process by inoculation of milk or cheese or any such substance with the specific bacillus. It is probable that such inoculated matter will contain a mixture of micro-organisms, but if the lactic bacillus is present, it will grow so vigorously and abundantly that the fermentation will be readily set up.*

In 1877 Lister was able, by means of the "dilution method," to isolate from sour milk, in a form of pure culture, an organism to which he gave the name B. lactis, and which he believed gained access to milk from the air of dairies and similar places. $†$ For some time this organism was held to be causally related to lactic fermentation. But in 1884, by means of culture on solid media, as introduced by Koch, Hueppe was able to isolate a bacillus which he named the Bacillus acidi lactici. This was probably identical with Lister's bacillus, and is now a term used to cover a whole family of organisms having somewhat similar characters, and possessing the property of setting up lactic fermentation. + In 1894 Günther and Thierfelder published the result of their work on lactic acid fermentation, from which they concluded that Lister and Hueppe had discovered one and the same species, and that it was the causal agent of lactic acid production in Europe. Esten found a similar organism to be the cause of lactic acid fermentation in America, and Conn holds that three organisms, or rather types of species, are the chief agents in the production of lactic fermentation, namely B. acidi lactici, Nos. i. and ii., and B. lactis cerogenes. The first named forms between 75 to 90 per cent. of the bacteria present. No. ii. is also very abundant. $B$. lactis cerogenes is found almost universally, although never in large numbers. It is a type of a species which produces intense acid on litmus gelatine cultures, produces much gas in milk or milk-sugar broth, curdles milk at high temperatures, and produces a distinctive odour in the milk, which it ferments. According to Escherich, the formation of lactic acid by this organism prevents fermentation in the stomach and intestines.

It was Hueppe who made the important discovery that many

* For full discussion of the subject of lactic fermentation of milk, see Bacteriology of Milk, 1903 (Swithinbank and Newman), pp. 149-159.

+ Trans. of Path. Soc., 1878, p. 437.

¥ Hueppe isolated five forms of his lactic acid bacillus, and Flügge described eleven forms. Maddox, Beyer, Fokker, Krueger, Grotenfeld, and other workers isolated lactic acid organisms.

\$ Storr's Agricultural Expt. Sta. Rep., 1899, p. 22. Others than those named are $B$. acidi lactici of Günther, B. acidi lactici of Leichmann, Bacillus XIX. of Adametz, Bacillus a of Freudenreich, $B$. and $M$. acidi lavolactici of Leichmann, Grotenfeld's B. acidi lactici(Nos. i. and ii.), No. 8 of Eckles, and B. casei. 
different species of bacteria are capable of setting up lactic fermentation, and what we have now said amply supports that view. Indeed, it has been estimated that upwards of 100 different bacteria possess this property.*

Bacillus acidi lactici (Hüppe) consists of rods about $2 \mu$ long and $\cdot 4 \mu$ wide, occurring singly or in pairs, chains or threads. Its habitat is sour milk.

It grows best at blood-heat, but much above that it fails to produce its fermentation. It ceases to grow under $10^{\circ} \mathrm{C}$. It inverts milk-sugar and changes it to dextrose, from which it then produces lactic acid. Sugars do, however, differ considerably in the degrees to which they respond to the influence of the lactic: ferment, and some which are readily changed by the alcoholic ferment are untouched by Bacillus acidi lactici.

Staining reaction-Ordinary stains and slightly by Gram's method.

Motility - No flagella ; non-motile.

Spore formation-Absent.

Biology: cultural characters (including biochemical features)-Good growth at room temperature and blood-heat.

Bouillon-Diffuse turbidity ; abundant sediment. white.

Gelatine plates and tubes-Colonies similar to B. coli; small, smooth, round,

Agar plates and tubes-Colonies similar to B. coli; small, smooth, white growths, moist and shiny.

Potato-A wavy, smooth-edged growth, elevated ; greyish-white or yellowishwhite in colour, sometimes turning brown.

Milk-Solid coagulation, leaving clear fluid; occasionally some gas-bubbles. Lactic and acetic acids are produced. Powers of acid coagulation of milk are gradually lost after long cultivation upon gelatine or agar.

Anaềrobic or aërotic-Grows- well aërobically, and if sugar present in medium anaërobically also.

Non-pathogenic.

The lactic fermentation bacteria are short rods, do not liquefy gelatine, nor do they form spores. They grow readily on gelatine at room temperature, forming as a rule small circular colonies, white or grey in colour, with sometimes a tinge of yellow, and the surface of the colony is smooth and glistening. The lactic acid organisms produce appreciable amounts of lactic acid only at somewhat elevated temperatures. If the amount of acid rises much above 2 per cent., the growth of the lactic acid bacteria is inhibited. Many other substances, as we have seen, are produced in addition to lactic acid (e.g. acetic and ferric acids, alcohol, methane, $\mathrm{CO}_{2} . \mathrm{H}$. N., etc.). Lactic acid organisms (as non-spore bearers) are readily killed by pasteurisation.

* Delbrück, Zopf, Krause, Peters, Lindner, Weigmann, Storch, and Marpmann, are amongst those who, in addition to workers we have named, have described bacteria possessing the power of setting up lactic fermentation. Only provisional classifications are possible at present, as, owing to variations in biology and terminology, it is probable that certain lactic organisms are described under several different terms. Generally, it may be said that some grow well in the presence of oxygen, and others do not. The latter group, facultative anaërobes, are perhaps the most common. They sour milk best in deep vessels, and produce a righthanded lactic acid. They are widely distributed in nature, and may form 90 per cent. of the total bacteria in milk. Some produce gas, others liquefy gelatine, and yet others produce spores. 
The economic function of the lactic ferments concerns, of course, the manufacture of butter and cheese.

Van Laer has described a saccharo-bacillus which produces lactic acid amongst other products, and brings about a characteristic disease in beer, named tourne. The liquid gradually loses its brightness and assumes a bad odour and disagreeable taste. The bacillus is a facultative anaërobe. A number of workers have separated organisms having a lactic acid effect, which diverge considerably from the ordinary type of lactic acid bacillus.

\section{Butyric Acid Fermentation}

Cause, Bacillus butyricus and other forms; medium, milk, butter, sugar and starch solutions, glycerine; result, butyric acid.

When sugars are broken down by Bacillus acidi lactici the lactic acid resulting may, under the influence of the butyric ferment become converted into butyric acid, carbonic acid, and hydrogen. Neither butyric acid nor lactic acid is as commonly used as alcohol or vinegar. Both, like vinegar, can be manufactured chemically, but this is rarely practised. Butyric acid is a common ingredient in stale milk and butter, and its production by bacteria was historically one of the first bacterial fermentations understood. Moreover, in its investigation Pasteur first brought to light the fact that certain organisms acted only in the absence of oxygen. In studying a drop of butyric fermenting fluid, it was observed that the organisms at the edge of the drop were motionless and apparently dead, whilst in the central portion of the drop the bacilli were executing those active movements which are characteristic of their vitality. To Pasteur's mind this at once suggested what he was able later to demonstrate, namely, that these bacilli were paralysed by contact with oxygen. When he passed a stream of air through a flask containing a liquid in butyric fermentation, he observed the process slacken and eventually cease. So were discovered the anaërobic micro-organisms. The aërobic ferments give rise to oxidation of certain products of decomposition; the anaërobic organisms, on the other hand, only commence to grow when the aërobic have used up all the available oxygen. Thus in such fermentations certain bodies (carbohydrates, fatty acids, etc.) undergo decomposition, and by oxidation become carbonic acid gas, and the remainder is left as a "reduced" product of the whole process. Hence sometimes this is termed fermentation by reduction. The chemical formula of this butyric reaction may be expressed thus :-

$$
\underset{\substack{\mathrm{C}_{6} \\ \text { Glucose. }}}{\mathrm{H}_{12} \mathrm{O}_{6}} \text { (by simple decomposition) }=\underset{\text { Lactic acid. }}{2 \mathrm{C}_{3} \mathrm{H}_{6} \mathrm{O}_{3}} \text {, }
$$


which is followed by the fermentation of the lactic acid:-

$$
\underset{\text { Lactic acid. }}{2 \mathrm{C}_{3} \mathrm{H}_{6} \mathrm{O}_{3}}=\underset{\substack{\text { Butyric acid. } \\ \mathrm{C}_{4} \mathrm{H}_{8} \mathrm{O}_{2}}}{2}+\underset{\substack{\text { Carbonic } \\ \text { acid gas. }}}{2 \mathrm{CO}_{2}}+\underset{\text { Free hydrogen. }}{2 \mathrm{H}_{2} .}
$$

Previously to 1880 , the only work which had been done in the elucidation of the bacterial origin of butyric fermentation had been accomplished by Prazmowski and Pasteur; the former designating the organism he found Clostridium butyricum, and the latter naming his "infusoires" Vibrion butyrique. Prazmowski emphasised the motility and resistance of the bacillus, and found that the latter was due to the spores produced by the organism. These spores were able to withstand boiling for several minutes. Fitz went so far as to say that butyric spores could resist boiling for twenty minutes. Prazmowski was unable to obtain pure cultures. Clostridium butyricum grows most readily at a temperature of about $40^{\circ} \mathrm{C}$., and is very widely distributed in nature. It is capable of dissolving cellulose, and therefore plays a part in the cellulose fermentation, which is employed in various maceration industries. It is generally held that in such fermentations there is symbiotic action between the butyric bacillus and an organism incapable of causing "retting" by itself. The organisms discovered by Prazmowski and Pasteur were anaërobic. But Fitz and Hueppe isolated an aërobic butyric bacillus. This fact was confirmed by Gruber, working with a pure culture in 1887, and it was at the same time demonstrated that the Clostridium butyricum of Prazmowski consists of a number of closely allied, but distinct, species. Lafar states that nearly related to this is a ferment isolated by Liborius from old cheese, and introduced into literature under the name of Clostridium foetidum. This organism liberates foul-smelling gases, in addition to producing butyric acid, and forms one of the many connecting links between the butyric acid bacteria and the so-called "potato" bacilli. No sharply defined limit can be drawn between these two groups.

The following are the three chief organisms of butyric acid:-

\section{Bacillus Butyricus (Botkin)}

Source and habitat-Widely distributed in milk, water, soil, dust.

Morphology-Rods, 1 to $3 \mu$ long, $0.5 \mu$ thick. Sometimes in threads, sometimes in chains.

Staining reaction-Stains by Gram's method.

Motility-Motile.

Spore formation-Spores in middle or at end of bacillus; about $1 \mu$ thick (provisional); sporulation not proved (Botkin).

Biology: cultural characters (including biochemical features)-Favourable temperature $37^{\circ} \mathbf{C}$. ; organism contains starch granules.

Bouillon-Slight growth; at $18^{\circ}$ C. involution forms may occur. Vigorous growth if glucose present, with opaque turbidity.

Grelatine plates and tubes-Round or oval colonies with sinuous edges; medium 
is rapidly liquefied ; gas development ; slight undulating colonies, as if consisting of mass of felted threads. No odour.

Agar plates and tubes-A luxuriant growth with gas development and ramifications in medium. Odour present.

Potato-Growth extends into potato substance; smell of alcohol.

Milk-At $37^{\circ} \mathrm{C}$, after fifteen hours casein precipitated and butyric acid is formed without intermediate formation of lactic acid. Coagulum eventually dissolves ; before that stage the appearance is very characteristic; there is a spongy fatty layer on surface, then clear fluid, and then a white deposit. The presence of this organism is readily proved in almost all milk. Fill a half litre flask with milk, and steam at $100^{\circ} \mathrm{C}$. for half an hour. Incubate at $37^{\circ} \mathrm{C}$. In less than twenty-four hours the characteristic changes will occur, with strong odour of butyric acid. Care must be taken that the gas pressure does not burst the flask. There is a marked odour of butyric acid. Other acids present are acetic, formic, and lactic.

Anaërobic or aërobic-Facultative anaërobe.

Non-pathogenic-It has been suggested that the $B$. enteritidis sporogenes of Klein is a pathogenic form of this bacillus.

\section{Bacillus Butyricus (Hüppe)}

Source and habitat-Milk.

Morphology-Slender rods ; 1.2 to $4 \mu$ long, $0.5 \mu$ thick; round ends. May grow into filaments ; rods slightiy bent ; $2 \cdot 1 \mu$ long, $0.3 \mu$ broad.

Staining reaction-Stains by Gram's method.

Flagella; motility-Many flagella; actively motile.

Spore formation-Oval spores at $37^{\circ} \mathrm{C}$. ; mesially situated.

Biology: cultural characters (including biochemical features).

Bouillon-A pellicle is formed; bouillon remains clear. No indol.

Gelatine plates and tubes - Small whitish-yellow colonies with crater-shapea depression; liquefaction; whitish-grey wrinkled pellicle produced in liquid cultures in tubes; liquefied medium cloudy and yellowish in colour.

Agar plates and tubes-A thin yellow layer, similar to B. mesentericus.

Potato-A fawn-coloured transparent layer, sometimes wrinkled. Somewhat similar to $B$. megatherium.

Milk-Is coagulated. Precipitated casein subsequently dissolved. Bitter taste. Butyric acid produced from salts of lactic acid; also from milk-sugar when it is previously hydrated.

Facultative anaërobe.

Non-pathogenic.

\section{Bacillus Butyricus (Pasteur). (Vibrion Butyrique)}

Source and habitat-Air, and thence to milk.

Morphology-Cylindrical rods with rounded extremities; $3 \mu$ to $5 \mu$ long by $.6 \mu$ to $8 \mu$ broad. Isolated or in chains; at times in long filaments indistinctly articulated.

Staining reaction-Ordinary aniline stains.

Motility-Feebly motile; motility ceases at once in presence of free oxygen.

Spore formation-Ovoid spores.

Biology: cultural characters (including biochemical features).

Bouillon-Grows freely under strictly anaërobic conditions in bouillon containing lactate of lime.

Agar plates and tubes-In agar "shake" cultures free from oxygen the medium becomes clouded in the lower portion, and is soon broken up, with copious gas formation accompanied by strong smell of butyric acid.

Gelatine plates and tubes-As upon agar, but in a less degree, the medium liquefying in the neighbourhood of the forming colonies.

Anaërobic.

Non-pathogenic.

Several other butyric acid organisms have been isolated, of which a few notes may be added :- 
Bacillus acidi butyrici-(Kedrowski's Butyric acid bacillus). Anaërobic. Kedrowski (Z. 16. 3) has isolated from mixtures of sugar solution with bad cheese or rancid cream-butter which has been placed in the incubator, two organisms which only show small deviations from one another. ( $C f$. the B. saccharo-butyricus of Klencki from cheese). Kedrowski's B. acidi butyrici is a motile bacillus, which towards one end produces ellipsoidal spores. The staining of the spores is readily accomplished. The colonies in gelatine show rays - those in agar partly reticulatedand interlaced spurs. Liquefaction of gelatine is more or less marked. Milk is coagulated with separation of serum on the surface (acid reaction). There is gradual peptonisation and simultaneous gas development.

Although, according to Pasteur's researches, the butyric acid ferment performs its function anaërobically, many butyric organisms can act in the presence of oxygen, and yield somewhat different products.

All of them, however, ferment most actively at a temperature at or about blood-heat, and the spores are able to withstand boiling for from three to twenty minutes (Fitz). It will be observed that as in lactic acid fermentation so in butyric, the results are not due to one species only.

\section{Ammoniacal Fermentation (see under Soil).}

From what has now been said, it is obvious that although we learn many important facts by a study of these different forms of fermentation, we may also learn on the one hand how to prevent or correct those conditions constantly occurring in fermented beverages, which are known as "diseases," and on the other, the opportunities which occur in industrial processes for the application of fermentation. We will first deal with the former.

\section{Diseases in Beer and Wines}

We have seen how the knowledge of fermentation has been compiled by a large number of workers. Spallanzani, Schwann, Pasteur, and Hansen all contributed epoch-making researches. In the same way the investigations of diseases in beers and wines were carried on by many observers, and were, at all events in the early stage, closely connected with those relating to spontaneous generation and mixed cultures of bacteria, or of yeasts occurring in fermentation. These so-called "diseases" are analogous to the taints occurring in milk. It was in 1883 that Hansen demonstrated that the universally dreaded yeast turbidity and the disagreeable changes in taste, odour, or colour of beer were caused not by the water or malt or particular method of brewing, as was commonly believed, but that these diseases had their origin in micro-organisms or in the yeast itself.* A clue had been given by Scheele and Appert, who had prevented these diseased conditions by physical agents which had destroyed the

\footnotetext{
* Practical Studies in Fermentation, E. C. Hansen, pp. 156-231.
} 
organisms able to produce the diseases. The demonstration by experiment of the cause of these diseases was worked out by Pasteur, who, as we have seen, established the fact that there are different micro-organisms inducing different kinds of fermentation, and therefore if it be desired to procure a pure fermentation, a pure and not a mixed ferment must be used at the commencement; and immediately after the primary fermentation the wine must be "pasteurised" to destroy the disease-producing organisms. In short, disease-producing organisms must either be excluded from, or killed in, the wine.

By carrying out a large number of experiments, partly with single species of yeast, and partly with mixtures, Hansen was able to declare that many of these diseases were due to particular yeasts. The number of such yeasts is by no means small. Hence we have two groups of yeasts, namely, "culture" or " brewery yeasts," those that are employed in brewing; and "wild yeasts," occurring widely distributed in nature, and which on gaining entrance to breweries set up diseases in the fermentations. The development of wild yeasts is promoted by vigorous aëration of the beer whilst it is being drawn off, and also through the bottles being badly corked. Beer which has undergone a feeble fermentation, and which has a high extract, is more subject to contamination than a beer which has not. When beer which has remained sound in the larger casks is attacked after it has been drawn off, it is clear that the agent of the disease obtained entrance into the beer from the surrounding air or from unclean vessels. If the infection is not great in amount, it may, particularly in a good beer, have practically no effect. There can be no doubt that some of the Saccharomycetes can live for months in soil and dust, even atmospheric dust, and amongst these may be various disease-yeasts.

The diseases of wines and beers are various. Generally speaking, the chief forms are comprised in the following simple classification:-

1. Turbidities.-(a) Gluten turbidities, or albuminous scud, due to precipitation of albuminoids.

(b) Chemical suspension and deposits, e.g. calcium tartrate, reduced sulphur scud, resins, essential oils, etc.

(c) Starch turbidity, due to the presence of unsaccharified starch.

(d) Yeast turbidity, due to a high content of yeast cells.

(e) Bacterial turbidity, brought about by fission fungi.

2. Ropiness, which may be thus classified separately, although doubtless frequently due to a high degree of turbidity. This condition of ropiness in wine, formerly attributed to a coagulation of the albuminoids, was traced by Pasteur to a number of organisms of which he described two chief forms, namely, a streptococcus and 
Bacillus viscosus vini. This latter organism occurs in the form of small rods, frequently united in pairs, and capable of producing ropiness in white wines in the absence of air. The presence of sugar is a sine quâ non for the occurrence of the malady, since it forms the material from which the strings of mucus are produced. Nessler maintains that wines containing over 10 per cent. of alcohol are proof against ropiness.

Pasteur also investigated ropiness in beer, and traced it to Micrococcus viscosus. But in all probability there are a number of the Schizomycetes possessing the power of rendering beer and wine viscid. The so-called Sarcina turbidity of beer has been traced to the Pediococcus cerevisice. But it should be borne in mind that such conditions may be easily mistaken for turbidities set up in other ways.

3. Changes in Colour.-The browning of wines-changing of colour with turbidity and unpleasant flavour, sometimes occurring in white wines-is said to be due to oxydaxes, enzymes produced by some of the yeasts and setting up an oxidation.

4. Alteration of Flavour, Bitterness, Acidity, etc.-Bitterness of wine almost exclusively affects red wines. The wine decolorises and develops a strange odour and a bitter after-taste. Pasteur attributed the disease to bacteria, but up to the present no species has been isolated able to bring about this condition upon inoculation in healthy wines. Bittering of beer may be occasioned by a diseaseyeast (Saccharomyces pastorianus $I$.) introduced at the commencement of the primary fermentation, even in such small quantity as one-fifth of the pitching yeast. This organism, according to Hansen, not only injuriously affects the taste and odour of the beer, but also its stability. It is of very frequent occurrence in breweries.

The turning (tourne) of wines is by no means a clearly-defined or uniform phenomenon. The most frequent form, perhaps, is that due to the vinegar taint (caused by Mycoderma aceti). But the condition may be set up by the lactic acid bacteria. It mostly attacks young vintages. The wine becomes turbid, eventually having an appearance of diluted milk, and even later it may assume a condition of brown or inky-black liquid.

The turning of beer, on the other hand, is a simple malady due to lactic acid fermentation, set up by the Saccharo-bacillus pastorianus III. The beer at first loses its brightness, then becomes turbid, and ultimately, according to some authorities, of unpleasant smell and taste. If the sample be shaken delicate waves or films of the organism are apparent to the naked eye, and eventually the beer becomes muddy. Hansen has shown that there are two species of yeast, $S$. pastor. III. and S. ellipsoideus II., which produce the disease when they are present in the pitching yeast, and are, therefore, introduced at the commencement of the primary fermentation. Both species are 
injurious when present at this stage, and indeed only at this stage. $S$. ellipsoideus $I I$. is the stronger of the two species. Whilst upon this particular subject, we may add that in 1883 Hansen demonstrated that these much-dreaded turbidities and other beer diseases may be due to mixtures of two yeasts, even though each of them by itself gives a faultless product.

\section{The Industrial Application of Bacterial Ferments}

We may commence our brief category of the industrial application of bacteria by referring the reader to fermentations, like the acetous (which results in the manufacture of vinegar), the alcoholic (alcoholic beverages), the lactic acid (souring of milk for dairying purposes, checse, etc.), the butyric (resulting in butyric acid), and those fermentations occurring in the soil and improving the fertility of land for farming purposes. With the principal facts concerning each of these applications of bacteria to industrial processes we have dealt elsewhere. It remains for us to mention other spheres of industry where bacteria are, whether we recognise it or not, playing a leading rôle. Their industrial effects are often secondary to vital processes. For instance, in securing their food bacteria break down organic material and bring about chemical and physical change. Now this power which organisms have of chemically destroying compounds may, or may not, be of primary importance, but there can be no doubt that many of the products which arise as a result are of an importance in the world which it is difficult to over-estimate. Perhaps the most remarkable examples occur in soil and in milk. But other illustrations which will at once occur to the reader are the maccration industries. For example, linen, as is well known, is produced from flax. The flax stem is made up of cellular substance, flax fibres and wood fibres; the latter are of no service in the making of linen, but the whole is bound together by a gummy, resinous substance termed "the central lamellæ" (an intermediate inter-cellular substance consisting probably not of pectose, but of calcium pectate). The solution of this cementing substance can be brought about by chemical means by treating the plant with very dilute sulphuric acid and then neutralising the adherent acid by a weak alkali bath (Bura). But it can also be solved by the process known as retting. There is dew-retting and water-retting. The former is practised in Russia, and consists in spreading the flax on the grass and exposing it to the influence of dew, air, rain, and light. The result is a soft and silky fibre. Water-retting is the method more commonly adopted, and is accomplished by means of steeping the flax in bundles, roots downwards, in tanks or ponds, with appliances so arranged as to keep the flax below water. In ten to fourteen days, according to 
the warmth of the weather, fermentation is completed by the breaking away of the "shore" or "shive" (the woody core) from the flax fibres. This decomposition and eventual breaking-down is due to bacteria, which, under favourable circumstances, multiply rapidly and set up the decomposition of the pectin resinous substance. Winogradsky, in 1895, proved that the process was due to a large bacillus (10-15 $\mu$ long, $1 \mu$ broad). It is an anaërobe, growing not in gelatine, but in the presence of nitrogenous food will ferment saccharose, lactose, and starch.

A precisely similar process is used in the preparation of jute and hemp. The former is of course used in various fabric industries, the chief centre of such manufactures being at Dundee. Jute fibre is obtained from the bark of at least two species of plants allied to the lime-tree order. The fibre, which is the inner bark, is separated from the stem by retting, either in rivers or tanks. The retting lasts for different periods, from two days to three weeks, and when the cementing substance between the fibres and the stem is sufficiently decomposed to allow of it, the jute fibre is separated, and may bo woven into sacking, carpets, curtains, etc. It is said that many of the brightly-dyed prayer-carpets used in the East by Moslems are made of this material in Dundee, and exported. Hemp also is cultivated in Poland and European Russia for the sake of its fibre, which is used for sail-cloth and other coarse material. This fibre is also separated by retting. Another example of the same putrefactive process is the preparation of cocoanut fibre for matting, etc. Sometimes retting for as long as twelve months is necessary to separate the fibres from the unripe husk of the cocoanut. Sponges are cleared in much the same manner by the putrefaction and softening of the organic matter in their interstices, set up by micro-organisms. The preparation of indigo from the indigo plant is brought about by a special bacterium found on the leaves. If the leaves are sterilised no fermentation occurs, and no indigo is formed. If, however, some of the specific bacteria are added to the mass, the fermentation soon begins, and the blue colour of the indigo makes its appearance. In the treatment of ox-hides for the production of certain kinds of leather the first object of the tanner is to clean and soften the hide, which is accomplished by washing. The unhairing and removal of the scarf-skin is the next operation, and this is achieved in America by "sweating" the hides, or artificially heating them till incipient putrefactive fermentation is set up by means of bacteria. Even in the subsequent tanning bacteria probably play an important part. But little is known at present of their work in this respect.

In the production of tobacco, the leaves, when gathered, are allowed to become somewhat withered, and are then arranged in moderatesized heaps, where they undergo a so-called "sweating," after which 
they are tied in bundles and arranged in huge heaps, containing sometimes 50 tons of tobacco. Hereupon active decomposition rapidly ensues, and the temperature rises to $50^{\circ}$ or $60^{\circ} \mathrm{C}$. This fermentation is due to bacteria, and was studied by Schloesing and Suchslan, who used pure cultures of bacteria for the purpose of favourably influencing the fermentation of tobacco, and producing a definite aroma. There is some evidence to show that certain of the family of Aspergillus co-operate with the bacteria in this process. Throughout the needful operations in tobacco-curing the producer has to contend with a number of micro-organisms which may produce disease in the tobacco.

The fermentation of cellulose is an example of bacterial action which has been more or less widely applied to industry. The process is due to Bacillus amylobacter, which acts, it is supposed, in symbiotic relationship with some other organism incapable of fermenting cellulose by itself. In relation to these so-called industrial symbioses it will be remembered by some that Calmette drew attention at the British Association Meeting at Dover (1899) to the application of bacteria to various processes carried out in the East. For example, the Japanese manufacture their sake with a form of aspergillus described by Ahlburg in 1879, and the cau de vie and vins de riz of the Chinese and Javanese have their source in symbiotic fermentations. Thus, in many cases, without the manufacturer even knowing it, micro-organic ferments are utilised in industrial operations.

In all these applications it is obvious we have advanced only the first stage of the journey. Nevertheless, here, as in nature on a large scale in the formation of fertile soils and coal measures, we find bacteria or their allies silently at work achieving great ends by co-operating in countless hordes. 


\section{CHAPTER V}

BACTERIA IN THE SOIL,

Methods of Examination-Methods of Anaërobic Culture-Place and Function of Micro-organisms in Soil-Denitrification, Nitrification, Nitrogen-fixation, Bacterial Symbiosis - Saprophytic and Pathogenic Organisms in Soil Tetanus - Quarter-Evil - Malignant CEdema - The Relation of Soil to Bacterial Diseases, such as Typhoid Fever.

SuRface soils and those rich in organic matter supply a varied field for the bacteriologist. Indeed, it may be said that the introduction of the plate method of culture and the improved facilities for growing anaërobic micro-organisms have opened up possibilities of research into soil micro-biology unknown to previous generations of workers.

From the nature of bacteria it will be readily understood that their presence is affected by physical conditions of the soil, and in all soils they occur only within a few feet of the surface. As we go down below 2 feet, bacteria become less, and below a depth of 5 or 6 feet we only find a few anaërobes. At a depth of 10 feet, and in the "ground water region," bacteria are scarce or absent. This is held to be due to the porosity of the soil acting as a filtering medium. Regarding the numbers of micro-organisms present in soil, no very accurate standard can be obtained. Ordinary earth may yield anything from 10,000 to $5,000,000$ per gram, whilst from polluted soil even $100,000,000$ per gram have been estimated. These figures are obviously only approximate, nor is an exact standard of any great value. Nevertheless Fränkel, Beumer, Miquel, and Maggiora have, as the result of experiments, arrived at a number of conclusions respecting bacteria in soil which are of practical use. From these results it appears that, in addition to the "ground water region" being free, or nearly so, virgin soils contain much fewer than cultivated lands, and these latter, again, fewer than made soils and 

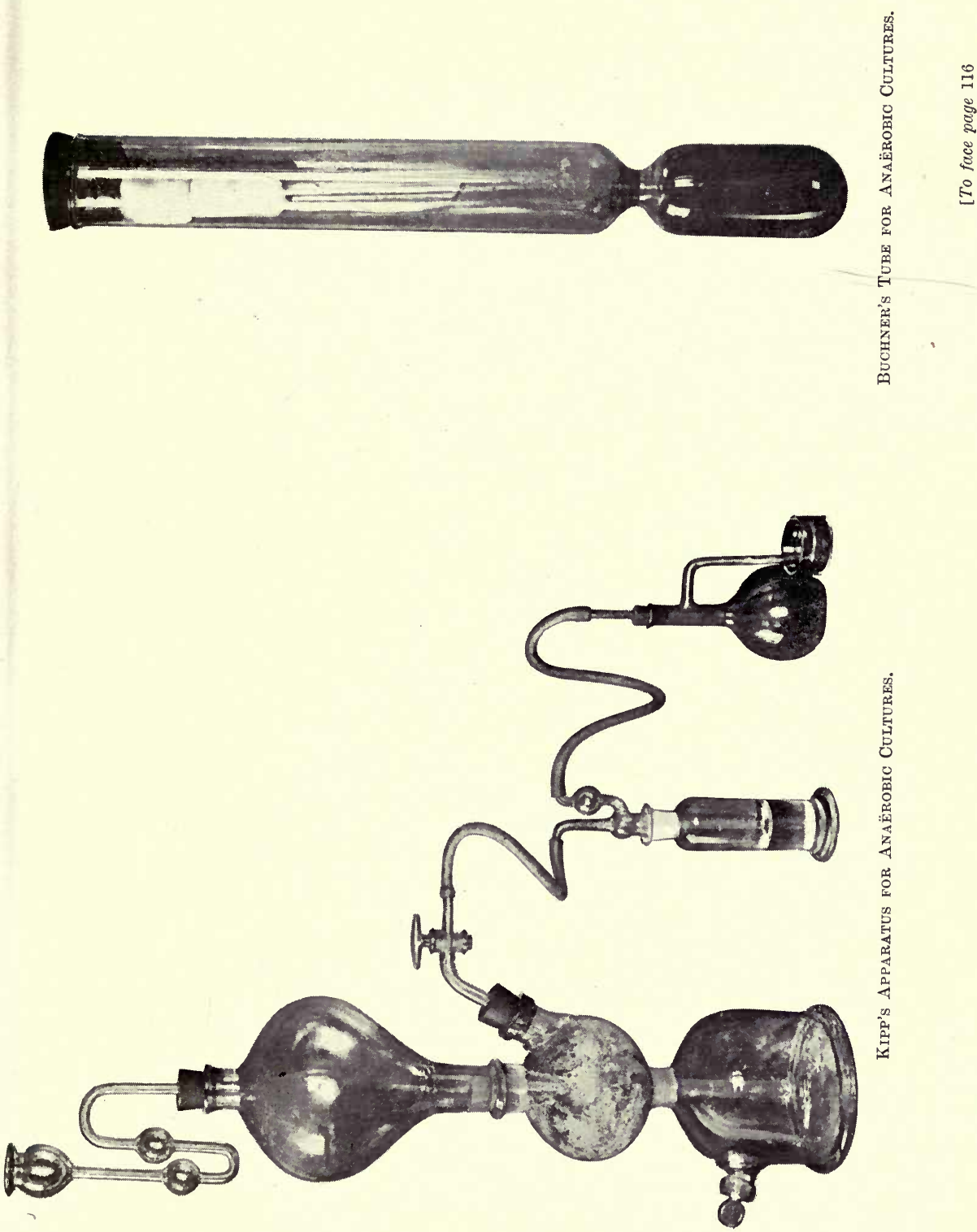

inhabited localities. In cultivated lands the number of organisms augments with the activity of cultivation and the strength of the fertilisers used. In all soils the maximum occurs in July and August.

But the condition which more than all others controls the quantity and quality of the contained bacteria is the degree and quality of the organic matter in the soil. The quantity of organic matter present in soil having a direct effect upon bacteria will be materially increased by placing in soil the bodies of men and animals after death. Dr Buchanan Young two or three years ago performed some experiments to discover to what degree the soil bacteria were affected by these means. "The number of micro-organisms present in soil which has been used for burial purposes," he concludes, "exceeds that present in undisturbed soil at similar level, and that this excess, though apparent at all depths, is most marked in the lower reaches of the soil."* The numbers were as follows :-

Virgin soil, $4 \mathrm{ft} .6$ in. $=53,436$ m.o. per gram of soil.

Burial soil ( 8 years), $4 \mathrm{ft.} 6$ in. $=363,411 \mathrm{~m} .0$. per gram of soil.

, (3 , $), 6 \mathrm{ft} .6$ in. = 722,751 , ,

Methods of Examination of Soil.-Two simple methods are generally adopted. The first is to obtain a qualitative estimation of the organisms contained in the soil. It consists simply in adding to test-tubes of liquefied gelatine or broth a small quantity of the sample, finely broken up with a sterile rod. The test-tubes are now incubated at $37^{\circ} \mathrm{C}$. and $22^{\circ} \mathrm{C}$., and the growth of the contained bacteria observed in the test-tube, or after a plate culture has been made on gelatine, agar, or glucoselitmus agar. The second plan is adopted in order to secure more accurate quantitative results. One gram or half-gram of the sample is weighed on the balance, and then added to 100 c.c. or 1000 c.c. of distilled sterilised water in a sterilised flask, in which it is thoroughly mixed and washed. From either of these two different sources it is now possible to make sub-cultures and plate cultures. The procedure is, of course, that described under the examination of water (p. 463 et seq.), and Petri's dishes, Koch's plates, or Esmarch's roll cultures are used. $\dagger$ Many of the commoner bacteria in soil will thus be detected and cultivated. Spores may be isolated, as is described under Examination of Sewage. But it is obvious that this by no means covers the required ground. It will be necessary for us here to consider the methods generally adopted for growing anaërobic bacteria, that is to say, those species which will not grow in the presence of oxygen. This anaërobic difficulty may be overcome in a variety of ways.

\section{Methods of Anaërobic Cultivation}

1. The oxygen may be displaced by some other gas, and though coal-gas, nitrogen, and carbon dioxide may all be used for this purpose, it has become the almost universal practice to grow anaërobes in hydrogen. The hydrogen is readily obtained by Kipp's or some other suitable apparatus for the generation of hydrogen

* Proc. Royal Soc. of Edin., xxxvii., pt. iv., p. 759.

+ See also Report of the Medical. Officer to the Local Government Board (1897-98), A. C. Houston, pp. 251-307. 
from zinc and dilute sulphuric acid, or it may be provided in a cylinder. The free gas is passed through various washbottles to purify it of any contaminations ; e.g. lead acetate (1-10 of water) removes any traces of sulphuretted hydrogen, silver nitrate (1-10) doing the same for arseniated hydrogen; whilst a flask of pyrogallate of potash will remove any oxygen. It is not necessary to have these three purifiers if the zinc used in the Kipp's apparatus is pure. Occasionally a fourth flask is added of distilled water, and this, or a dry cotton-wool stopper in the exit tube, will ensure germ-free gas. From the further end of the exit tube of the Kipp's apparatus an indiarubber tube will carry the hydrogen to ils desired destination. With some it is the custom to place anaërobic cultures in test-tubes, and the test-tubes in a large flask, tube, or desiccator, having a two-way tube for entrance and exit of the hydrogen, or Petri dishes may be used and placed in well-sealed jar or desiccator ; others prefer to pass the hydrogen immediately into a large test-tube containing the culture (Fränkel's method). Either method, if properly carried out, will be found effectual, and the growth of the culture in hydrogen is readily observed. Another

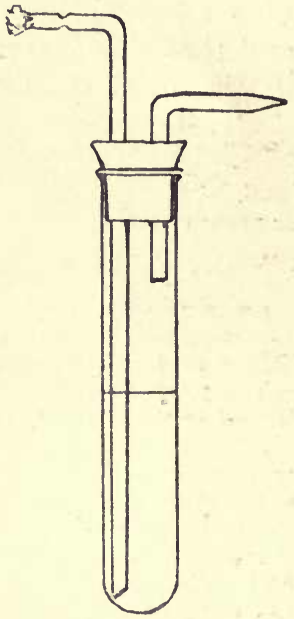

Fra. 1S.-Fränkel's Tube. For Cultivation of Anaërobes. plan is to use a yeast flask, and after having passed the hydrogen through for about half an hour, the lateral exit tube is dipped into a small capsule containing mercury (as in Plate 9). The entrance tube is now sealed, and the whole apparatus placed in the incubator. The interior of the flask containing the culture is filled with an atmosphere of hydrogen. No oxygen can obtain entrance through the sealed entrance tube, or through the exit tube immersed in mercury Yet through this latter channel any gases produced by the culture may escape.

2. The Absorption Method.-Instead of adding hydrogen to the tube or flask containing the anaërobic culture, it is feasible to add to the medium substances, such as glucose or pyrogallic acid, which will absorb the oxygen which is present, and thus enable the anaërobic requirement to be fulfilled. To various media-gelatine, agar, or broth (the latter used for obtaining the toxins of anaërobes)-2 per cent. of glucose may be added. Pyrogallic acid, or pyrogallic acid one part and 20 pcr cent. caustic potash one part, is also readily used for absorptive purposes. A large glass tube of 25 c.c. height, termed a Buchner's cylinder, having a constriction near the bottom, is taken; and about two drachms of the pyrogallic solution are placed in the bulb. A test-tube containing the culture is now lodged in the upper part above the constriction, and the mouth of the Buchner tube is carefully sealed. The apparatus is then placed in the incubator at the desired temperature, and the contained culture grows under anaërobic conditions. As the pyrogallic solution absorbs the oxygen it assumes a darker tint.

3. Mechanical Methods. -These include various ingenious methods for preventing an admittance of oxygen to the culture. An old-fashioned one was to plate out the culture and protect it from the air by covering it with a plate of mica. A more serviceable mode is to inoculate, say, a tube of agar with the anaërobic organism, and then pour over the culture a small quantity of melted agar, which will readily set, and so protect the culture itself from the air. Oil or vaseline may be used instead of melted agar. Another mechanical method is to make a deep inoculation, and then melt the top of the medium over a Bunsen burner, and thus close the entrance puncture and seal it from the air.

4. Absorption of Oxygen by an Aërobic Culture.-This method takes advantage of the power of absorption of certain aërobic bacteria, which are planted over the culture of the anaërobic species. It is not practically satisfactory, though occasionally good results have been obtained.

5. Lastly, there is the Vacuum Method.-By this means it is obviously intended to extract air from the culture and seal it in vacuo. The culture tubes are connected 


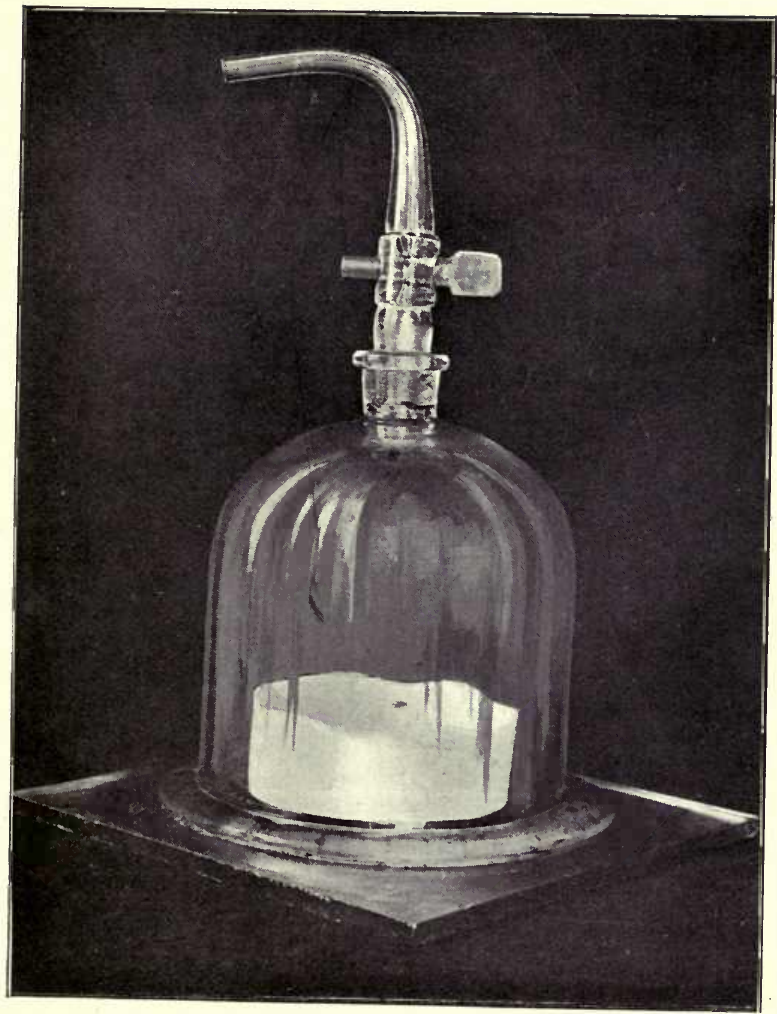

A Vacuum Method of Anä̈robic Culture. 
 
with the air-pump, and exhausted as much as possible. The method can be applied in many different ways (for example, with pyrogallic solution, as in Bullocl's apparatus).

Of these various methods it is on the whole best to choose either the hydrogen method, the vacuum, or the plan of absorption by grape-sugar or pyrogallic. In anaërobic plate cultures grape-sugar agar plus 0.5 per cent. of formate of soda may be used. The poured inoculated plate should be placed over pyrogallic solution under a sealed bell-glass and incubated at $37^{\circ} \mathrm{C}$. Pasteur, Roux, Joubert, Chamberland, Esmarch, Kitasato, and others have introduced special apparatus to facilitate anaërobic cultivation, but the principles adopted are those which have been mentioned.

\section{The Qualitative Examination of Bacteria in the Soil.-We} may now turn to consider the species of bacteria found in the soil. They may be classified in five main groups; the division is somewhat artificial, but convenient:-

1. The Denitrifying Bacteria.-This group, whose function has been elucidated largely by the investigations of Professor Warington, is held responsible for the breaking down of nitrates. With its members may be associated the Decomposition or Putrefactive Bacteria, which break down complex organic products other than nitrates into simpler bodies.

2. The Organisms of Nitrification.-To this group belong the two chief types of nitrifying bacteria, viz., those which oxidise ammonia into nitrites, and those which change nitrites into nitrates.

3. The Nitrogen-fixing Bacteria, found mainly in the nodules on the rootlets of certain plants.

4. The Common Saprophytic Bacteria, whose function is at present but imperfectly known. Many are putrefactive germs.

5. The Pathogenic Bacteria.-This division includes three types, the bacilli of tetanus, malignant œdema, and quarter evil. Under this heading we shall also have to consider in some detail the intimate relation between the soil and such important bacterial diseases as tubercle and typhoid.

To enable us to appreciate the work which the "economic bacteria" perform, it will be necessary to consider shortly the place they occupy in the economy of nature. This may be perhaps most readily accomplished by studying the scheme shown on p. 120 .

The threefold function of ordinary plant life is nutrition, assimilation, and reproduction, i.e., the food of plants, the digestive and storage power of plants, and the various means they adopt for multiplying and increasing their species. With the two latter we have little concern in this place. Respecting the nutrition of plant life, it is obvious that, like animals, plants must feed and breathe to maintain life. Plant food is of three chief kinds, viz., water, inorganic salts, and gases. Water is an actual necessity to the plant as a direct food and as a food-solvent, i.e. as the vehicle of important inorganic materials. The hydrogen, too, of the organic compounds is obtained 
from the decomposition of the water which permeates every part of the plant, and is derived by it from the soil and from the aqueous vapour in the atmosphere. The chief inorganic salts of which protoplasm is constituted are composed in part of potassium, magnesium, calcium, iron, phosphorus, or sulphur. These inorganic elements do not enter the plant as such, but combined with other substances or dissolved in water. Potassium, calcium, and magnesium are absorbed chiefly as nitrates, phosphates, and carbonates. Iron contributes to the formation of the green colouring-matter of plants, indeed, is essential to it, and is also derived from the soil. Phosphorus, one of the chief constituents of seeds, generally occurs as nucleo-albumin.

\section{A SCHEME SHOWING THE PLACE AND FUNCTION OF THE ECONOMIC MICRO-ORGANISMS FOUND IN SOIL}

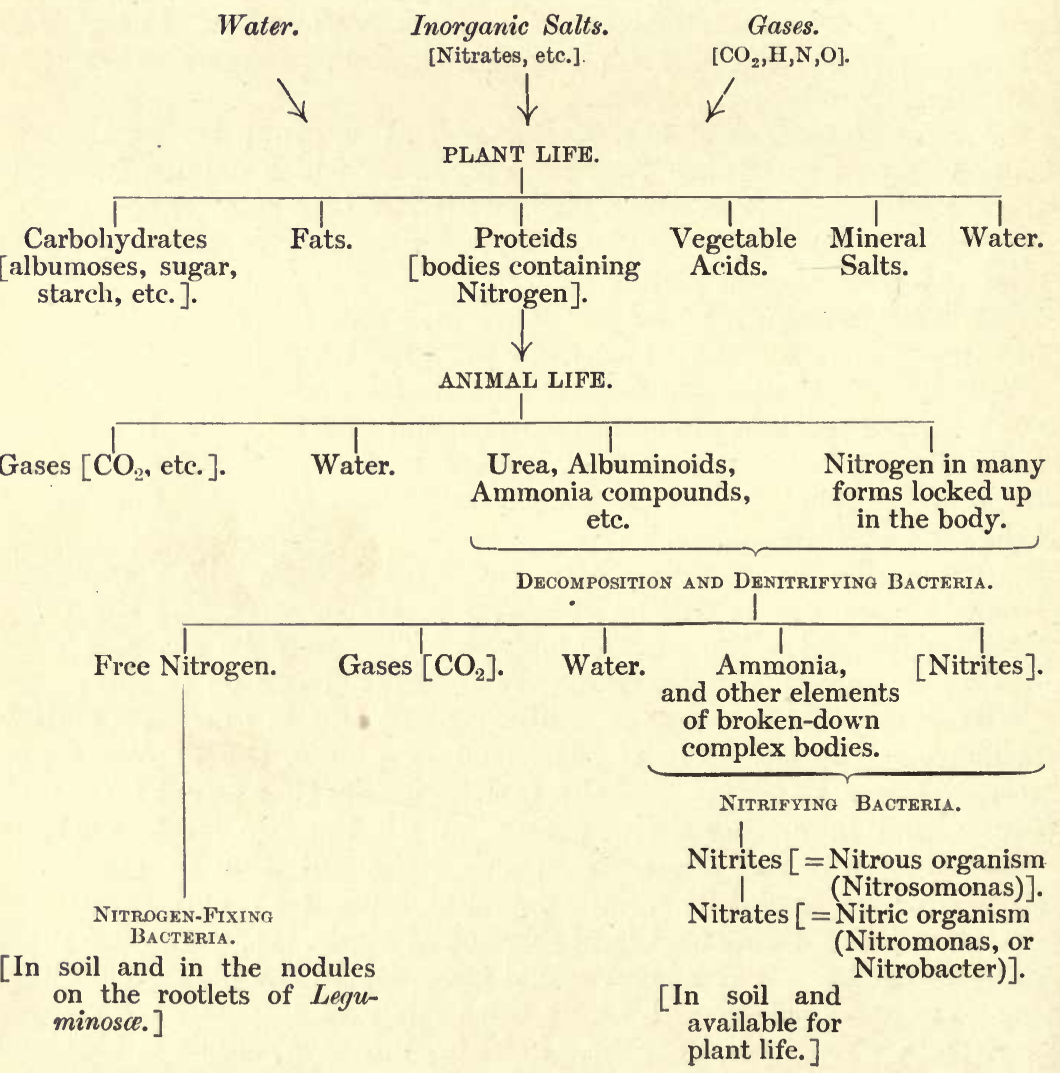


Sulphur, which is an important constituent of albumen, is derived from the sulphates of the soil. In addition to the above, there are other elements, sometimes described as non-essential constituents of plants. Amongst these are silicon (to give stiffness), sodium, chlorine, iodine, bromine, etc. All these elements contribute to the formation or quality of the protoplasm of plants.

The gases essential to plants, and absorbed as such, are two: Carbon dioxide (carbonic acid) and Oxygen; the necessary hydrogen and nitrogen being absorbed in the form of salts. By the aid of the green chlorophyll corpuscles, and under the influence of sunlight, we know that leaves absorb the carbon dioxide of the atmosphere, and effect certain changes in it. The hydrogen, as we have seen, is obtained from the water. Oxygen is absorbed through the leaves and through the root from the interstices of the soil. Each of these gases contributes vitally to the existence of the plant. The fourth gas, nitrogen, which constitutes more than two-thirds of the air we breathe (79 per cent. of the total volume and 77 per cent. of the total weight of the atmosphere), is also an absolutely necessary food required by plants. Yet, although this is so, the plant cannot absorb or obtain its nitrogen in the same manner in which it acquires its carbon-viz., by absorption through the leaves-nor can the plant take nitrogen into its own substance by any means as nitrogen. Hence, although this gas is present in the atmosphere surrounding the plant, the plant will perish if nitrogen does not exist in some combined form in the soil. Nitrates and compounds of ammonia are widely distributed in nature, and it is from those bodies that the plant obtains, by means of its roots, the necessary nitrogen.

Until comparatively recently it was held that plant life could not be maintained in a soil devoid of nitrogen or compounds thereof. But it has been found that certain classes of plants (the Leguminoso for example), when they are grown in a soil which is practically free from nitrogen at the commencement, do take up this gas into their tissues. One explanation of this fact is that free nitrogen becomes converted into nitrogen compounds in the soil through the influence of micro-organisms present there. Another explanation attributes this fixation of free nitrogen to micro-organisms existing in the rootlets of the plant. These two classes of organisms, known as the nitrogen-fixing organisms, will require our consideration at a later stage. Here we merely desire to make it clear that the main supply of this gas, absolutely necessary to the existence of vegetable life upon the earth, is drawn not from the nitrogen of the atmosphere, but from that contained in nitrogen compounds in the soil. The most important of these are the nitrates. Here then we have the necessary food of plants expressed in a sentence: water, inorganic salts, gases; some of the salts containing nitrogen in the form of nitrates. 
Plant life seizes upon its required constituents, and by means of the energy furnished by the sun's rays builds these materials up into its own complex forms. Its many and varied forms fulfil a place in beautifying the world. But their contribution to the economy of nature is, by means of their products, to supply food for animal life. These products of plant life are chiefly sugar, starch, fat, and proteids. Animal life is not capable of extracting its nutriment from soil, but it must take the more complex foods which have already been built up by vegetable life. Again, the complementary functions of animal and vegetable life are seen in the absorption by plants of one of the waste materials of animals, viz., carbonic acid gas. Plants abstract from this gas carbon for their own use, and return the oxygen to the air, which in its turn is of service to animal life.

By animal activity some of these foods supplied by the vegetable kingdom are at once decomposed into carbonic acid gas and water, which goes back to nature. Much, however, is built up still further into higher and higher compounds. The proteids are converted by digestion into more soluble forms, such as albumoses and peptones; these in their turn are reconverted into less soluble proteids, and become assimilated as part of the living organism. In time they become further changed into carbonic acid, sulphuric acid, water, and certain not fully oxidised products, * which contain the nitrogen of the original proteid. In the table these bodies have been represented by one of their chief members, viz., urea.

It is clear that there is in all animal life a double process continually going on; there is a building up (anabolism, assimilation), and there is a breaking down (katabolism). These processes will not balance each other throughout the whole period of animal life. We have, as possibilities, elaboration, balance, degeneration; and the products of animal life will differ in degree and in substance according to which period is in the predominance. These products we may subdivide simply into excretions during life and final materials of dissolution after death, both of which may be used more or less immediately by other forms of animal or vegetable life, or immediately after having passed to the soil. We may shortly summarise the final products of animal life as carbonic acid, water, and nitrogenous remnants. These latter will occur as urea, new albumens, compounds of ammonia, and nitrogen compounds of great complexity stored up in the tissues and body of the animal. The carbonic acid, water, and other simple substances like them will return to nature and be of immediate use to vegetable life. But otherwise the cycle cannot be completed, for the more complex bodies are of no service as such to plants or animals.

* E. A. Schäfer, Text-book of Physiology, vol. i., p. 25 (W. D. Halliburton). 


\section{Decomposition and Denitrification}

In order that this complex material should be of service in the economy of nature, and its constituents not lost, it is necessary that it should be broken down again into simpler conditions. This prodigious task is accomplished by the agency of two groups of organisms, the decomposition and denitrifying* bacteria. The organisms associated with decomposition processes are numerous; some denitrify as well as break down organic compounds. This group will be referred to under "Saprophytic Bacteria." The reduction by the denitrifying bacteria may be simply from nitrate to nitrite, or from nitrate to nitric or nitrous oxide gas, or indeed to nitrogen itself. In all these processes of reduction the rule is that a loss of nitrogen is involved. How that free nitrogen is brought back again and made subservient to plants and animals we shall understand at a later stage.

Professor Warington has set forth the chief facts known of this decomposition process. $\dagger$ That the action in question only occurs in the presence of living organisms was first established by Mensel in 1875 in natural waters, and by Macquenne in 1882 in soils. If all living organisms are destroyed by sterilisation of the soil, denitrification cannot take place, nor can vegetable life exist. "Bacteria reduce nitrates," says Professor Warington, "by bringing about the combustion of organic matter by the oxygen of the nitrate, the temperature distinctly rising during the operation." The reduction to a nitrite is a common property of bacteria. But only a few species have the power of reducing a nitrate to gas. These few species are, however, widely distributed. In 1886 Gayon and Dupetit first isolated the bacteria capable of reducing nitrates to the simplest element, nitrogen. They obtained their species from sewage, but ten years later denitrifying bacteria were isolated from manure. That soil contains a number of these reducing organisms is proved by introducing a particle of surface soil into some broth, to which has been added 1 per cent. of nitre. During incubation of such a tube gas is produced, and the nitrate entirely disappears.

Whenever decomposition occurs in organic substances there is a reduction of compound bodies, and in such cases the putrefying substances obtain their decomposing and denitrifying bacteria from the air. The chief conditions requisite for bringing about a loss of nitrogen by denitrification are enumerated by Professor Warington as follows:-(1) the specific micro-organism; (2) the presence of a nitrate and suitable organic matter; (3) such a condition as to

* “Denitrifying” means reducing nitrates.

† R. Warington, M.A., F.R.S., Jour. Roy. Agric. Soc. Eng., series iii., vol. viii., part. iv., p. 577 et seq. See also Trans. Chem. Soc., 1884, 1888, etc. 
aëration that the supply of atmospheric oxygen shall not be in excess relatively to the supply of organic matter; (4) the usual essential conditions of bacterial growth. "Of these," he says, "the supply of organic matter is by far the most important in determining the extent to which denitrification will take place." The necessarily somewhat unstable condition facilitates its being split up by means of bacteria. The bacteria in their turn are ready to seize upon any products of animal life which will serve as their food. Thus, by reducing complex bodies to simple ones, these denitrifying organisms act as the necessary link to connect again the excretions of the animal body, or after death the animal body itself, with the soil.

In a book of this nature it has been deemed advisable not to enter into minute description of all the species of bacteria mentioned. Some of the chief are described more or less fully. We cannot, however, do more than name several of the chief organisms concerned in reducing and breaking down compounds. As we shall find in the bacteria of nitrification, so also here, the entire process is rarely, if ever, performed by one species. There is indeed a remarkable division of labour, not only between decomposition bacteria and denitrification bacteria, but between different species of the same group. Bacillus fluorescens non-liquefacions, Mycoderma ureo, and some of the staphylococci break down nitrates (denitrification), and also decompose other compound bodies. Amongst the group of putrefactive bacteria found in soil may be named $B$. coli, B. mycoides, B. mesentericus, B. liquidus, B. prodigiosus, B. ramosus, $B$. vermicularis, $B$. liquefaciens, and many members in the great family of Proteus. Some perform their function in soil, others in water, and others, again, in dead animal bodies. Dr Buchanan Young, to whose researches in soil we have referred, has pointed out that in the upper reaches of burial soil, where these bacteria are most largely present, there is as a result no excess of organic carbon and nitrogen. Even in the lower layers of such soil it is rapidly broken down.

It will be observed, from a glance at the table (p. 120), that the chief results of decomposition and denitrification are as follow: free nitrogen, carbonic acid gas, and water, ammonia bodies, and sometimes nitrites. The nitrogen passes into the atmosphere, and is "lost"; the carbonic acid and water return to nature, and are at once used by vegetation. The ammonia and nitrites await further changes. These further changes become necessary on account of the fact, already discussed, that plants require their nitrogen to be in the form of nitrates in order to use it. Nitrates obviously contain a considerable amount of oxygen, but ammonia contains no oxygen, and nitrites very much less than nitrates. Hence a process of oxidation 
is required to change the ammonia into nitrites and the nitrites into nitrates.

\section{Nitrification}

This oxidation is performed by the nitrifying micro-organisms, and the process is known as nitrification. It should be clearly understood that the process of nitrification may, so to speak, dovetail with the process of denitrification. No exact dividing line can be drawn between the two, although they are definite and different processes. In a carcase, for example, both processes may be going on concomitantly, so also in manure. There is no hard-and-fast line to be drawn in the present state of our knowledge. Other organisms beside the true nitrification bacteria may be playing a part, and it is impossible exactly to measure the action of the latter, where they began and where the preliminary attack upon the nitrogenous compounds terminated. In all cases, however, according to Professor Warington, the formation of ammonia has been found to precede the formation of nitrous or nitric acid.

It was Pasteur who (in 1862) first suggested that the production of nitric acid in soil might be due to the agency of germs, and it is to Schlösing and Müntz that the credit belongs for first demonstrating (in 1877) that the true nature of nitrification, the conversion of ammonia into nitric acid, depended upon the activity of a living micro-organism.* Partly by Schlösing and Müntz and partly by Warington (who was then engaged in similar work at Rothamsted), it was later established (1) that the power of nitrification could be communicated to substances which had not hitherto nitrified by simply seeding them with a nitrified substance, and (2) that the process of nitrification in garden soil was entirely suspended by the vapour of chloroform or carbon disulphide. The conditions for nitrification, the limit of temperature, and the necessity of plant food, have furnished additional proof that the process is due to a living organism. These conditions, according to Warington, are as follows:-

1. Food (of which phosphates are essential constituents). "The nitrifying organism can apparently feed upon organic matter, but it can also, apparently with equal ease, develop and exercise all its functions upon purely inorganic food" (J. M. H. Munro). W Winogradsky prepared vessels and solutions carefully puritied from organic matter, and these solutions he sowed with the nitrifying organism, and found that they flourished. Professor Warington has employed the acid carbonates of sodium and calcium with distinct success as ingredients of an ammoniacal solution undergoing nitrification.

2. The next condition of nitrification is the presence of oxygen.

* C'ompt. Rend., 1877, pp. 84, 301.

+ Truns. Chem. Soc., 1886, etc. 
Without it the reverse process, denitrification, occurs, and instead of a building up we get a breaking down, with an evolution of nitrogen gas. The amount of oxygen present has an intimate proportion to the amount of nitrification, and with 16 to 21 per cent. of oxygen present the nitrates are more than four times as much as when the smallest quantity of oxygen is supplied. The use of tillage in promoting nitrification is doubtless in part due to the aëration of the soil thus obtained.

3. A third condition is the presence of a base with which nitric acid when formed may combine. Nitrification can only take place in a feebly alkaline medium, but an excess of alkalinity will retard the process.

4. The last requirement is a favourable temperature. As low as $37^{\circ}$ or $39^{\circ} \mathrm{F}$. $\left(3-4^{\circ}\right.$ C.) will suffice, but at a higher temperature it becomes much more active. According to Schlösing and Müntz, at $54^{\circ} \mathrm{F}$. $\left(12^{\circ} \mathrm{C}\right.$.) nitrification becomes really active, and it increases as the temperature rises to $99^{\circ} \mathrm{F}$. $\left(37^{\circ} \mathrm{C}\right.$.), after which it falls. A high temperature or a strong light are prejudicial to the process.

We are now in a position to consider shortly some of the characters of these nitrification bacteria. They may readily be divided into two chief groups, not in consideration of their form or biological characteristics, but on account of the duties which they perform. Just as we observed that there were few denitrifying organisms which could break down ammonia compounds to nitrogen gas, so is it also true that there are few nitrifying bacteria which can build up from ammonia to the nitrates. Nature has provided that this shall be accomplished in two stages, viz., a first stage from ammonia bodies to nitrites (nitrosification), and a second stage from nitrites to nitrates. The agent of the former is termed the nitrous organism, the latter the nitric organism. Both are contributing to the final production of nitrates which can be used by plant life.*

The Nitrous Organism (Nitrosomonas). Prior to Koch's gelatine method the isolation of this bacterium proved an exceedingly difficult task. But even the adoption of this isolating method seemed to give no better results, and for an excellent reason: the nitrifying organisms will not grow on gelatine. To Winogradsky $\dagger$ and Percy Frankland $\ddagger$ belongs the credit of separately isolating the nitrous organism on the surface of gelatinous silica containing the necessary

* The saltpetre beds of Chili and Peru are an excellent example of the application of these facts. Nitrates are there produced from the fæcal evacuations of seafowl in such quantities as to form an article of commerce. A like form of utilisation of the action of these bacteria was once practised on the continent of Europe. Considerable nitrate deposits have recently been discovered in California. Economic application is also seen in the treatment of sewage referred to elsewhere.

$\dagger$ Ann. de l'Inst. Pasteur, 1890, p. 213.

$\ddagger$ Phil. T'rans. Roy. Soc., 1890, B. 107. 
inorganic food. Professor Warington, in his lectures under the Lawes Agricultural Trust, has described this organism as follows:-

"The organism as found in suspension in a freshly nitrified solution consists largely of nearly spherical corpuscles, varying extremely in size. The largest of these corpuscles barely reaches a diameter of one-thousandth of a millimetre, and some are so minute as to be hardly discernible in photographs. The larger ones are frequently not strictly circular, and are sometimes seen in the act of dividing.

"Besides the form just described, there is another, not universally present in solutions, in which the length is considerably greater than its breadth. The shape varies, being occasionally a regular oval, but sometimes largest at one end, and sometimes with the ends truncated. The circular organisms are probably the youngest.

"This organism grows in broth, diluted milk, and other solutions without producing turbidity. When acting on ammonia it produces only nitrites. It is without action on potassium nitrite. It is, in fact, the nitrous organism which, as we have previously seen, may be separated from soil by successive cultivations in ammonium carbonate solution."**

The elongated forms appear to be a sign of arrested growth. Normally, the organism is about $1.8 \mu$ long, or nearly three times as long as the nitric organism. It possesses a gelatinous capsule. "The motile cells, stained by Löffler's method, are seen to have a flagellum in the form of a spiral." When grown on silica jelly the nitrous organism appears in the same two forms-zooglea and free cells-as when cultivated in a fluid. It commences to show growth in about four days, and is at its maximum on about the tenth day. Upon gypsum, to which 1 per cent. of magnesium carbonate has been added, the organism grows in the same form of small brown colonies, but more rapidly. Winogradsky found that there were considerable differences in the morphology of the organism according to the soil from which it was taken. The solution used by him consisted of water containing 1 per 1000 ammonium sulphate, 1 per 1000 potassium phosphate, and 1 per 100 magnesium carbonate.

As we have already seen, an astonishing property of this organism is its ability to grow and perform its specific function in solutions absolutely devoid of organic matter (Munro). Some authorities hold that it acquires its necessary carbon from carbonic acid. The mode of culturing it was as follows:- To sterilised flat-bottomed flasks add 100 c.c. of a solution made of two grams of ammonium sulphate, one gram of potassium phosphate, and 1000 c.c. of distilled water. To this was added half a gram of magnesium sulphate, two grams of common salt, and 0.4 of a gram of ferrous sulphate. Now the flask

* U.S.A, Dept. of Agriculture: Lectures under the Lawes Agricultural Trust. By Robert Warington, 'F.R.S., 1891, pp. 58, 59. 
was inoculated with a small portion of the soil under investigation, and after four or five days sub-cultured on the same medium in fresh flasks, and repeated half a dozen times. Now, as this inorganic medium was unfavourable to the ordinary bacteria of soil, it was supposed that after several sub-cultures the nitrous organism was isolated in pure culture. Winogradsky employed for sub-culturing upon a solid medium a mineral gelatine, silica jelly.* Upon this medium it is possible to sub-culture a pure growth from the film at the bottom of the flasks in which the nitrous organism is first isolated. In 1899 Winogradsky showed that the nitrous organism (nitroso-bacterium) was able to grow in the presence of large amounts of organic matter, and since that date Fremlin has carried this branch of work to a further stage of advancement. He has shown that cultures developed in inorganic solutions become eventually pure cultures of this species of nitrifying organism, and when inoculated into solutions containing small quantities of organic matter they were able to oxidise the ammonia present. Fremlin has also demonstrated that the nitrous organism grows well on silica jelly and ammonia agar, and colonies from these media transferred to beef-broth agar and gelatine also grew well. From these experiments he concluded "that the nitroso-bacterium grows well on any ordinary medium" but "that in the presence of large percentages of organic matter the nitrosobacterium, although growing very profusely, loses for a time the power of converting ammonia into nitrites." +

The Nitric Organism.-It was soon learned that the nitrous organism, even when obtainable in large quantities and in pure culture, was not able entirely to complete the nitrifying process. As early as 1881 Professor Warington had observed that some of his cultures, though capable of changing nitrites into nitrates, had no power of oxidising ammonia. . These he had obtained from advanced sub-cultures of the nitrous organism, and somewhat later Winogradsky isolated and described this companion of the nitrous organism. It develops freely in solutions to which no organic matter has been added; indeed, much organic matter will prevent it growing:* The temperature for incubation is $30^{\circ} \mathrm{C}$. Winogradsky con-

* Two per cent. of dialysed silicic acid mixed with neutral salts and magnesium carbonate in order to solidify it.

$\dagger$ Jour. of Hyg., 1903, pp. 378, 379.

¥ Compt. Rend., 113 (1891) p. 89-Winogradsky isolated it from soils from various parts of the world on the following medium :-Water, $1000^{\circ} 0$; potassium phosphate, $1 \cdot 0$; magnesium sulphate, 0.5 ; calcium chloride, a trace; sodium chloride, $2 \cdot 0$. About 20 c.c. of this solution was placed in a flat-bottom flask, and a little freshly washed magnesium carbonate was added. 'The flask was closed with cotton-wool, and the whole sterilised. To each flask 2 c.c. of a 2 per cent. solution of ammonium sulphate was subsequently added. Recently, the following medium has been used for cultivation of the nitric organism:- Sodium nitrite, $10^{\circ}$; sodium carbonate, $1{ }^{\circ} 0$; sodium chloride, 0.5 ; potassium phosphate, 0.5 ; magnesium sulphate, 0.3 ; ferrous sulphate, $0 * 4$, in 1000 parts of distilled water. 


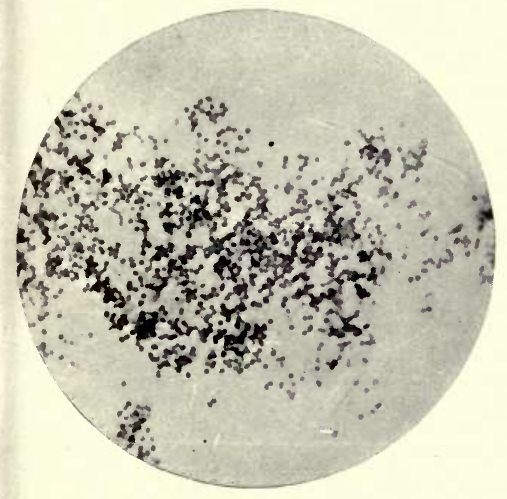

Nitrous OrganisM.

$\times 1000$.

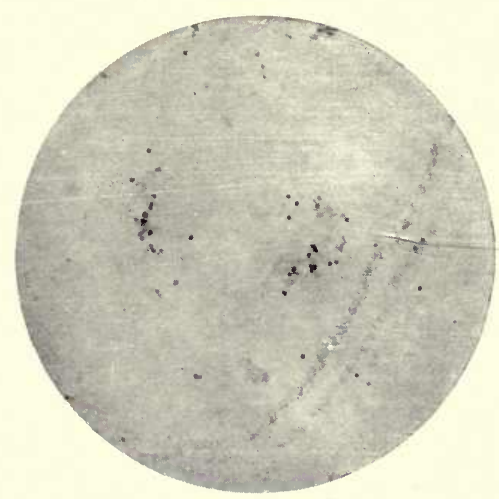

NITRIC ORgANISM. $\times 1000$.
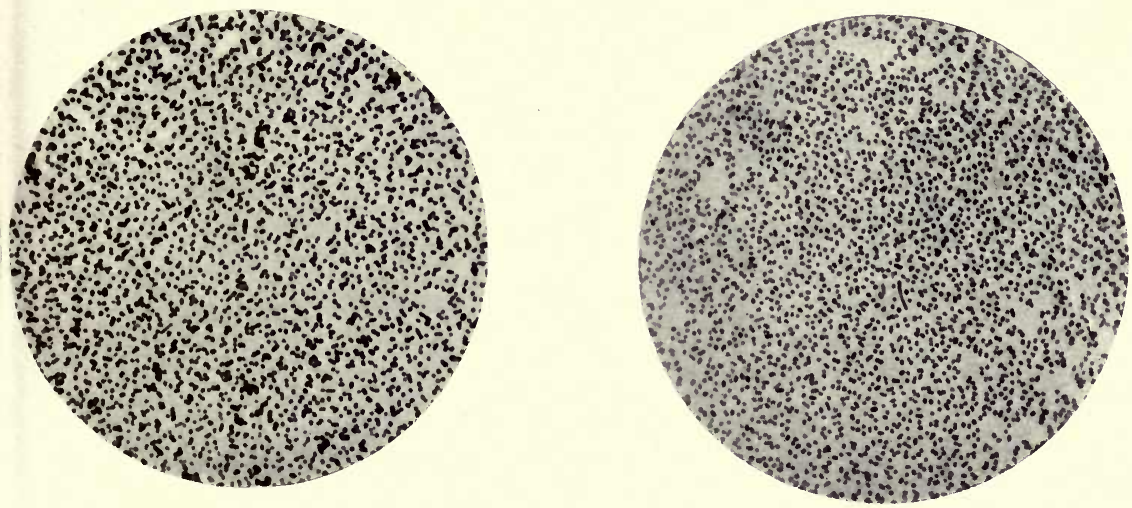

Nitrogen-Fixing Organisms from Secretions of Root-Nodules taken from Leguminose.

Film preparations, $\times 1000$. 
cluded that the oxidation of nitrites to nitrates was brought about by a specific organism independently of the nitrous organism. He successfully sub-cultured it from his inorganic medium on to silica jelly and also on to purified agar. He believes the organism, like its companion, derives its nutriment solely from inorganic matter, but this is not finally established.

The form of the nitric organism (or nitromonas, as it was once termed) is allied to the nitrous organism. The cells are elongated, rarely oval, but sometimes pear-shaped. They are more than half a micromillimetre in length, and somewhat less in thickness. The cells have a gelatinous membrane. Like the other nitrifying bacteria, its. development and action are favoured by the presence of the acid carbonates of calcium and sodium. Of the latter, six grams per litre or even a smaller quantity gives good results. The sulphate of calcium can be used, but the organism prefers the carbonates. The differences between these two bacteria are small, with the exception of their chemical action. The nitric organism has no action upon ammonia, and its presence in very small amount (five parts per million) hinders its development, and in sixty-four parts per million prevents its action on a nitrite.*

We may here summarise the general facts respecting nitrification. Winogradsky proposes to term the group nitroso-bacteria, and to classify thus:-

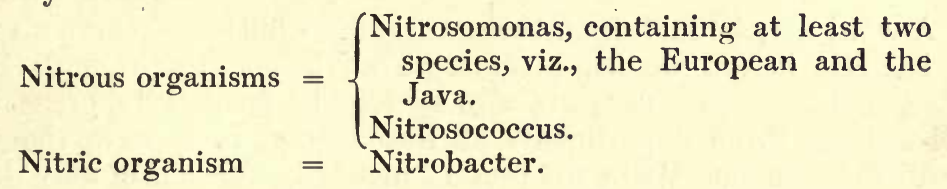

Nitrification occurs in two stages, each stage performed by a distinct organism. By one (nitrosomonas) ammonia is converted into nitrite; by the other (nitrobacter) the nitrite is converted into nitrate. $\dagger$ Both organisms are widely and abundantly distributed in

* The course of nitrification may be followed by means of chemical tests. 1 . The disappearance of ammonia. 2. The appearance of nitrite. 3. Its disappearance. 4. Appearance of nitrate.

t Professor Warington, in Report IV. (p. 526) of his admirable series of papers on the subject, draws attention to Müntz's criticism that the nitrifying organisms only oxidise from nitrogenous matter to nitrites, and not from nitrites to nitrates. Müntz held that the conversion of nitrite into nitrate is brought about by the joint action of carbonic acid and oxygen. Professor Warington's experiments, however, clearly illustrate that the production of nitrates from nitrites in an ammoniacal solution can be determined by the character of the bacterial culture with which the solution is seeded, and that in a solution of potassium nitrite conversion into nitrate can be determined by the introduction of the nitric organism. Professor Warington still adheres to the opinion, in favour of which he has produced so much evidence, that the formation of nitrates in the soil is due to the nitric organism which soil always contains. 
the superficial soils. They act together and in conjunction, and for one common purpose. They are separable by employing favourable media. "If we employ a suitable inorganic solution containing potassium nitrite, but no ammonia, we shall presently obtain the nitric organism alone, the nitrous organism feeding on ammonia being excluded. If, on the other hand, we employ an ammonium carbonate solution of sufficient strength, we have selected conditions very unfavourable to the growth of the nitric organism, and a few cultivations leave the nitrous organism alone in possession of the field"* (Warington). Adeney has summarised conclusions respecting nitrification as follows: (1) In organic solutions containing ammonia nitrous organisms thrive, but nitric organisms gradually lose their vitality; (2) nitrous organisms cannot oxidise nitrites to nitrates in inorganic solutions; (3) nitric organisms thrive in inorganic solutions containing nitrites; (4) the presence of peaty or humous matter appears to preserve the vitality of nitric organisms during the fermentation of ammonia, and establishes conditions whereby it is possible for the nitric organisms to thrive simultaneously in the same solution as the nitrous organisms. Other conditions necessary for nitrification are, of course, the presence of ammonia preceding the appearance of nitrous or nitric acid, the presence of a fixed base, not too high a degree of alkalinity, and darkness and free admission of air.

A word may be said upon the natural distribution of these nitrifying bacteria before we leave them. They belong to the soil, riverwater, and sewage. They are also said to be frequently present in well-water. From experiments at Rothamsted it appears that the organisms occur mostly in the first 12 inches, in subsoils of clay down to 3 or 4 feet, and in sandy soils probably at even a greater depth. These facts are of the first importance in relation to the biological treatment of sewage.

We have now given some consideration to the chief events in the life-cycle of nature depicted in the table (p. 120). There is but one further process in which bacteria play a part, and which requires some mention. It will have been noticed that at certain stages in the cycle there is a more or less appreciable "loss" of free nitrogen. In the process of decomposition brought about by the denitrifying bacteria, a very considerable portion of the nitrogen is dissipated into the air in the form of a free gas. This is the last stage of all proteid decomposition, so that wherever putrefaction is going on there is a continual "loss" of an element essential to life. Thus it would appear at first sight that the sum-total of nitrogen food must be diminishing.

But there are other ways also in which nitrogen is being set free. * Laves Agricultural Trust Lectures, 1891, p. 63. 
In the ordinary processes of vegetation there is a gradual draining of the soil and a passing of nitrogen into the sea; the products of decomposition pass from the soil by this drainage, and are "lost" as far as the soil is concerned. Many of the methods of sewage disposal are in reality depriving the land of the return of nitrogen, which is its necessity. Again, nitrogen is freed in explosions of gunpowder, nitroglycerine, and dynamite, for whatever purpose they are used. Hence the great putrefactive "loss" of nitrogen, with its subsidiary losses, contributes to reduce this essential element of all life, and if there were no method of bringing it back again to the soil, it would seem that plant life, and therefore animal life, would speedily terminate.

\section{Nitrogen-Fixing Bacteria}

It is at this juncture, and to perform this vital function, that the nitrogen-fixing bacteria play their wonderful part: they help to recover the free nitrogen and fix it in the soil. Excepting a small quantity of combined nitrogen coming down in rain and in minor aqueous deposits from the atmosphere, the great source of the nitrogen of vegetation is the store in the soil and subsoil, whether derived from previous accumulations or from recent supplies by manure.

Sir William Crookes has pointed out the vast importance of using all the available nitrogen in the service of wheat production.* The distillation of coal in the process of gas-making yields a certain amount of its nitrogen in the form of sulphate of ammonia, and this, like other nitrogenous manures, might be used to give back to the soil some of the nitrogen drained from it. But such manuring cannot keep pace, according to Sir W. Crookes, with the present loss of fixed nitrogen from the soil. We have already referred to several ways in which "loss" of nitrogen occurs. To these may well be added the enormous loss occurring in the waste of sewage when it is passed into the sea. As the President of the British Association pointed out, the more widely this wasteful system is extended, recklessly returning to the sea what we have taken from the land, the more surely and quickly will the finite stocks of nitrogen, locked up in the soils of the world, become exhausted. Let us remember that the plant creates nothing in this direction; there is no combined nitrogen in wheat which is not absorbed from the soil, and unless the abstracted nitrogen is returned to the soil, its fertility must be ultimately exhausted. When we apply to the land sodium nitrate, sulphate of ammonia, guano, and similar manurial substances, we are drawing on the earth's capital, and our drafts will not be

* The Wheat Problem, 1899. 
perpetually responded to.* We know that a virgin soil cropped for several years loses its productive powers, and without artificial aid becomes unfertile. For example, through this exhaustion forty bushels of wheat per acre have dwindled to seven. Rotation of crops is an attempt to meet the problem, and the four-course rotation of turnips, barley, clover, and wheat witnesses to the fact that practice has been ahead of science in this matter. It is unnecessary to add that rotation of crops and the use of the Leguminosæ does not absolvo the agriculturist from maintaining the land in ripe condition by manuring and ordinary tillage.

The store of nitrogen in the atmosphere is practically unlimited, but it is fixed and rendered assimilable only by organic processes of extreme slowness. We may shortly glance at these, for it is upon these processes, plus a return to the soil of sewage, that we must depend in the future for storing nitrogen as nitrates.

1. Some combined nitrogen is absorbed by the soil or plant from the air, for example, fungi, lichens, and some algæ, and the absorption is in the form of ammonia and nitric acid. This is admittedly a small quantity.

2. Some free nitrogen is fixed within the soil by the agency of porous and alkaline bodies.

3. Some, again, may be assimilated by the higher chlorophyllous plants themselves, independently of bacteria (Frank).

4. Electricity fixes, and may in the future be made to fix more, nitrogen. If a strong inducive current be passed between terminals, the nitrogen from the air enters into combination with the oxygen, producing nitrous and nitric acids.

5. Abundant evidence has now been produced in support of the fact that there is considerable fixation by means of bacteria.

Bacterial life in several ways is able to reclaim from the atmosphere this free nitrogen, which would otherwise be lost. The first method to which reference may be made is that involving symbiosis. This term signifies "a living together" of two different forms of life, generally for a specific purpose. Marshall Ward has recently defined it as the co-operation of two associated organisms to their mutual advantage, each symbiont being incapable of carrying on alone the work which the symbiotic association is able to perform. It is convenient to restrict the term symbiosis to complementary partnerships such as exist between algoid and fungoid elements in lichens, or between unicellular algæ and Radiolarians, +

* Sir John Lawes and Sir Henry Gilbert (Times, 2nd December 1898) have pointed out that the addition of nitrates only would be of no permanent use to the wheat crop. They rely upon thorough tillage and proper rotation of crops as the means of improving the nitrogen value of the soil.

+ British Association for the Advancement of Science Report, 1899, p. 693.

‡ Geddes, Nature, xxv., 1882. 
or between bacteria and higher plants. The partnerships between hermit crabs and sea-anemones and the like are sometimes defined by the term commensalism (joint diet), which is applied to such associations having negative results, neither partner gaining much advantage from the association. Symbiosis and commensalism must be distinguished from parasitism, which indicates that all the advantage is on the side of the parasite, and nothing but loss on the side of the host. Association of organisms together for increase of virulence and function should be distinguished from symbiosis, and mere existence of two or more species of bacteria in one medium is not, of course, symbiosis. Most frequently such a condition would result in injury and the subsequent death of the weaker partner, an effect precisely opposite to that defined by this term.

The example of bacteriological symbiosis with which we are concerned here is that partnership between bacteria and some of the higher plants (Leguminosæ) for the purpose of fixing nitrogen in the plant and in the surrounding soil.*

The nitrogen-fixing bacteria, the third group of micro-organisms connected with the soil, exist in groups and colonies situated inside the nodules, appearing, under certain circumstances, on the rootlets of the pea, bean, and other Leguminosæ. It was Hellriegel and Wilfarth who first pointed out that, although the higher chlorophyllous plants could not directly

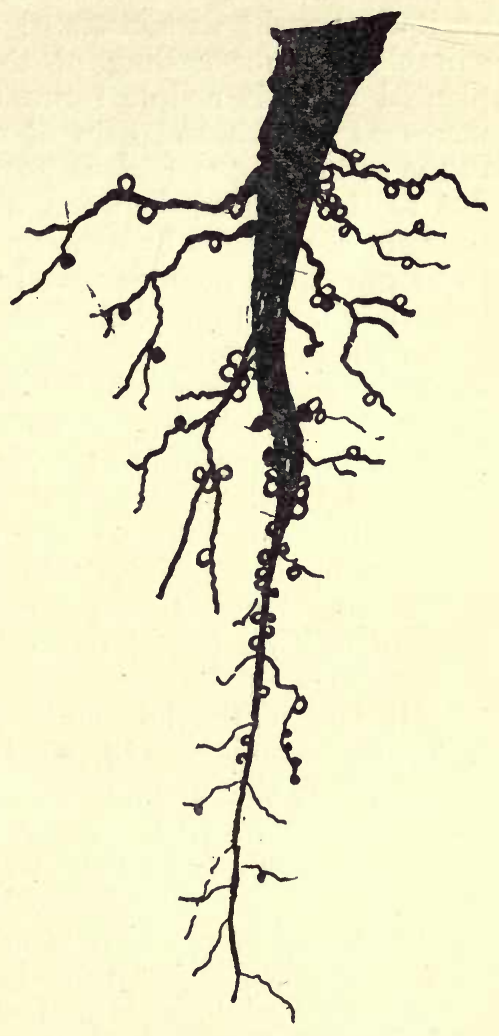

Fig. 19.-Rootlet of Pea with Nodules. obtain or utilise free nitrogen, some of them at any rate could acquire nitrogen brought into combination under the influence of bacteria. Hellriegel found that the gramineous, polygonaceous, cruciferous, and other orders depended

* Examples of bacteria symbionts are numerous ; e.g. the dissolution of cellulose (Van Senus); the decomposition of sound potato in water exhausted of air (Ward); the reduction of sulphates; the oxidation of sulphuretted hydrogen; the iron bacteria, etc. 
upon combined nitrogen supplied within the soil, but that the Leguminosæ did not depend entirely upon such supplies.

It was observed that in a series of pots of peas to which no nitrogen was added most of the plants were apparently limited in their growth by the amount of nitrogen locked up in the seed. Here and there, however, a plant, under apparently the same circumstances, grew luxuriantly, and possessed on its rootlets abundant nodules. The experiments of Sir John Lawes and Sir Henry Gilbert at Rothamsted ${ }^{*}$ demonstrated further that under the influence of suitable microbe-seeding of the soil in which Leguminosæ were planted there is nodule formation on the roots, and coincidentally increased growth and gain of nitrogen beyond that supplied either in the soil or in the seed as combined nitrogen. Presumably this is due to the fixation, in some way, of free nitrogen. Nobbe proved the gain of nitrogen by non-leguminous plants (Eloagnus, etc.) when these grow root nodules containing bacteria, but to all appearances bacteria differing morphologically from the Bacillus radicicola of the leguminous plants.

These facts being established, the question naturally arises, How is the fixation of nitrogen to be explained, and by what species of bacteria is it performed? In the first place, these matters are simplified by the fact that there is very little fixation indeed by bacteria in the soil apart from symbiosis with higher plants. Hence we have to deal mainly with the work of bacteria in the higher plant. Sir Henry Gilbert concludes t that the alternative explanations of the fixation of free nitrogen in the growth of Leguminosæ seem to be:

" 1 . That under the conditions of symbiosis the plant is enabled to fix the free nitrogen of the atmosphere by its leaves;

" 2 . That the nodule organisms become distributed within the soil and there fix free nitrogen, the resulting nitrogenous compounds becoming valuable as a source of nitrogen to the roots of the higher plant;

" 3 . That free nitrogen is fixed in the course of the development of the organisms within the nodules, and that the resulting nitrogenous compounds are absorbed and utilised by the host. "Certainly," he adds, "the balance of evidence at present at command is much in favour of the third mode of explanation." If this is finally proved to be the case, it will furnish another excellent example of the power existing in bacteria of assimilating an elementary substance.

Experiments at Rothamsted have confirmed those of others, in showing that, by adding to a sterilised sandy-soil growing leguminous

* Sir Henry Gilbert, F.R.S., The Lawes Agricultural Trust Lectures, 1893, p. 129 .

† Ibid., p. 140. 


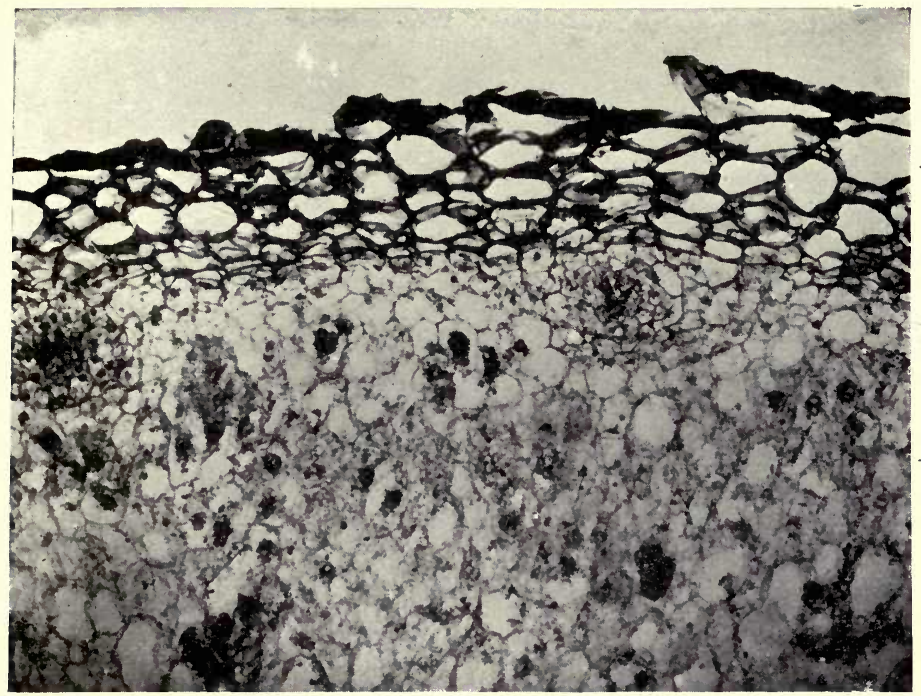

-Cellular

sheath of

Rootlet

forming

capsule

of nodule.

-Colonies of

bacteria

in situ.

Nitrogen-Fixing Bacteria in situ in Nodule on Rootlet of Pea. $\times 400$.

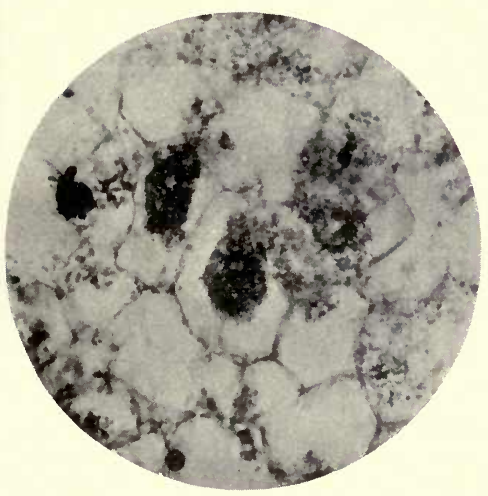

Nitrogen-Fixing BaCteria in situ in Root-Nodule OF PEA. (Section of Nodule). $\times 500$.

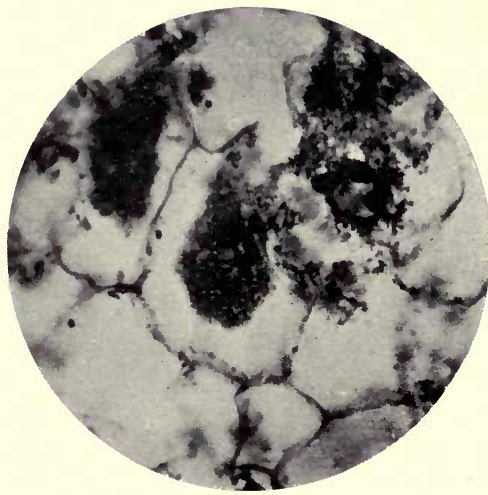

Nitrogen-Fixing Bacteria in situ in Root-Nodule of Pea. (Section of Nodule). $\times 600$. 
LIBRART

UNIVERSITY

CA OF

CALIFOREN 
plants, a small quantity of the watery extract of a soil containing the appropriate organisms, a marked development of the so-called leguminous nodules on the roots is induced, and that there is coincidently increased growth, and gain of nitrogen. There is no evidence that the leguminous plant itself assimilates free nitrogen; the supposition is, that the gain is due to the fixation of nitrogen in the course of development of the lower organisms within the root-nodules, the nitrogenous compounds so produced being taken up and utilised by the higher plant.

It would seem, therefore, that in the growth of leguminous crops, such as clover, vetches, peas, beans, sainfoin, lucerne, etc., at any rate some of the large amount of nitrogen which they contain, and of the large amount which they frequently leave as nitrogenous residue in the soil for future crops, may be due to atmospheric nitrogen brought into combination by the agency of lower organisms. It has yet to be ascertained, however, under what conditions a greater or less proportion of the total nitrogen of the crop will be derived-on the one hand from nitrogen-compounds within the soil, and on the other from such fixation. It might be supposed, that the amount due to fixation would be the less in the richer soils, and the greater in soils that are poor in combined nitrogen, and which are open and porous. On the other hand, recent results obtained at Rothamsted indicate that, at any rate with some leguminous plants, there may be more nodules produced, and presumably more fixation, with a soil rich in combined nitrogen, than in one poor in that respect.

Most authorities would agree that all absorption of free nitrogen, if by means of bacteria, must be through the roots. As a matter of fact, legumes, especially when young, use nitrogen, like all other plants, derived from the soil. It has been pointed out that, unless the soil is somewhat poor in nitrogen, there appears to be but little assimilation of free nitrogen and but a poor development of root nodules.* The free nitrogen made use of by the micro-organism is in the air contained in the interstices of the soil. For in all soils, but especially in well-drained and light soils, there is a large quantity of air. Although it is not known how the micro-organisms in legumes utilise free nitrogen and convert it into organic compounds in the tissues of the rootlet or plant, it is known that such nitrogen compounds pass into the stem and leaves, and so make the roots really poorer in nitrogen that the foliage. But the ratio is a fluctuating one, depending chiefly on the stage of growth or maturity of the plant.

If the nodules from the rootlets of Leguminosæ be examined, the nitrogen-fixing bacteria can be readily seen. They may be isolated

* This has been denied in the official report by the chemist of the Experimental Farm to the Minister of Agriculture at Ottawa (Report, 1896, p. 200). 
and grown in pure culture as follows:-The nodules are removed, if possible, at an early stage in their growth, and placed for a few minutes in a steam steriliser. This is advisable in order to remove the various extraneous organisms attached to the outer covering of the nodule. The latter may then be washed in antiseptic solution, and their capsules softened by soaking. When opened with a sterilised knife, thick creamy matter exudes. On microscopic examination this is found to be densely crowded with small roundended bacilli or oval bodies, known as bacteroids. By a simple process of hardening and using the microtome, excellent sections of the nodules can be obtained which show these bacteria in situ. In the central parts of the section may be seen densely crowded colonies of the bacteria, which in some cases invade the cellular capsule of the nodule derived from the rootlet.

The organisms are of various shapes, sometimes rod-like, and at other times assuming a V or $\mathrm{Y}$ shape. Probably these latter forms are due either to circumflex arrangement, branching or pleomorphism. At the end of the summer most nodule-bearing roots, being annuals, perish, and the nitrogen-fixing bacteria are liberated in the surrounding soil. Probably they are able to exist for long periods in the soil, and re-infect other rootlets.

Other Bacterial Symbioses.-As we have already pointed out, incidental association of organisms must not be mistaken for symbioses. The decomposition organism, $B$. ramosus, may be found associated with Nitrosomonas and Nitrobacter in the processes of denitrification and nitrification, but this does not necessarily fulfil the conditions of symbiosis, even though each of the three produces substances which provide pabulum for the other two. True symbiosis involves a much closer relationship than this, namely, the inability of each symbiont to produce its effect apart from its partner.

Now, in addition to the case of the nitrogen-fixing bacteria we have other bacterial examples, and brief reference must be made to them. Van Senus, for instance, found an anaërobic bacillus capable of dissolving cellulose if associated with another organism, also incapable by itself of attacking cellulose. Winogradsky, too, found that a certain anaërobic organism (Clostridium Pasteurianum), if supplied with abundance of dextrose but no oxygen, could fix atmospheric nitrogen. This capacity was found to be due to the organism being surrounded with aërobic bacteria acting in partnership with it. Probably, also, the bacteria concerned in the reduction of sulphates, and the oxidation of sulphuretted hydrogen, as also the iron bacteria, are further examples of symbiosis. Kephir and the so-called gingerbeer plant must also be named in the same category. Kephir is a common beverage amongst the Caucasians. The "Kephir grains" are in reality composed of three separate organisms. The first is a fila- 
mentous bacterium forming "zooglea." The second is a lactic-acidproducing bacillus, and the third is a yeast. By these agents a fermentation is set up in the milk of cows, goats, or sheep. "The yeast and the bacteria, either jointly or separately, split up the lactose or milk-sugar into two other sugars, galactose and glucose. The yeast then forms alcohol from the latter, and the bacterium lactic acid from the former" (Green). This filamentous bacillus probably affects the casein. The outline is, it is true, only the probable course of action, as full details as to the whole function of the separate factors are not yet known.

The gingerbeer plant is the agent of fermentation in the so-called "stone gingerbeer," and is composed essentially of two organisms, one a yeast, Saccharomyces pyriformis, the other a bacillus, Bacterium vermiforme. It rarely happens that these two forms are found pure, there being as a rule an admixture of other organisms with them. Professor Green describes B. vermiforme as growing in two different ways, namely, as long rods or convoluted threads, invested by a translucent wrinkled sheath, and as constituent microbes contained within the sheath, yet able to escape from it. The sheathing form of the organism can only be produced when oxygen is replaced by carbon dioxide. In the symbiotic association the yeast absorbs the oxygen, and during its fermentative activity produces carbon dioxide, thus providing the necessary conditions for the formation of the sheath. The bacterium benefits by substances excreted by the yeast, and the latter profits in its turn by the removal of these matters through the agency of the former. The yeast sets up the usual fermentation of cane-sugar.

A third organism manifesting symbiosis occurs in Madagascar as a curious gelatinous substance found attacking the sugar-cane, and consisting again of a yeast and a bacterium associated together in very much the same way as are the organisms in the gingerbeer ferment.

Before leaving this subject of symbiosis as illustrated in the lichens, in.Winogradsky's Clostridium, in the nodule-bacteria, in the gingerbeer plant, and in Kephir, we may suitably inquire whether anything is at present known as to how the symbionts are related to each other. Obviously the matter presents many difficulties, and the problem is by no means solved. There are, however, three chief hypotheses. First, the provision of definite food materials by the one symbiont for the other may be an important factor; e.g. an alga supplies a fungus with carbo-hydrates, or a fungus converts starch into the fermentable sugars which the associated yeast needs. In other cases the advantage derived is one of protection from some injurious agent; e.g., the aërobic bacterium prevents the access of oxygen to the anaërobic one. Thirdly, there is some evidence, 
according to Professor Marshall Ward, in support of the hypothesis that one symbiont may stimulate another by exciting some body which acts as an exciting drug to the latter-just as truly as certain drugs act as stimulants to some cell or organ of a higher animal, and probably in a fundamentally similar manner.

Before we leave the subject of the economic bacteria present in the soil, it may be well to refer briefly to the application of the new knowledge to agriculture. Whilst many of the details of our knowledge concerning "the living earth" have not passed beyond the experimental stage, it is not to be wondered at that the New Soil Science has been received with some caution, and possibly in some quarters even scepticism. This is neither surprising, nor, as regards the details, altogether undesirable. A number of the cardinal principles, however, are now obtaining very general acceptance amongst practical agriculturists. Briefly, these may be stated as follows. That a soil which has been sterilised, or is otherwise not occupied by soil bacteria, is necessarily an unfertile soil; that the disintegration and oxidation of organic matter in the soil are the result of bacterial life and work; that the sowing, growing, and feeding of the desirable soil germs are of as much importance to the agriculturist to-day as is the sowing of seeds, or the growing and feeding-by manuring-of plants; that the physical and chemical conditions of soil favourable to these bacteria are of as much value to the agriculturist as the requisite physical and chemical conditions for the growth of the yeast cell are to the brewer; and indeed that one of the essential functions of manure in the soil is to provide favourable pabulum and conditions for the operation of these soil ferments.

In the further elucidation of these principles various series of experiments, in addition to those at Rothamsted (under Sir J. B. Lawes and Sir J. H. Gilbert) and at Woburn (under Dr Voelcker), have been designed and carried out. Of such a nature are the wellknown Dalmeny Experiments originated by Lord Rosebery some years ago. The chemists engaged in this series were aware that though large doses of caustic lime would kill outright certain of the economic soil bacteria, annual or biennial dressings of mild lime added to the culture media, that is the soil, would materially assist the process of nitrification. Five acres of land have been worked as a miniature farm, each division being divided into sixteen plots. The soil is of very uniform character, and is of the usual loamy kind met with in the low grounds of the Lothians. Each plot has been manured, or left unmanured as the case may be, on a regular system, so that the residual values of the different manures, as well as the yield of crop, may be accurately dealt with. The crops grown are regularly analysed in order to determine the feeding value. Concern- 
ing results, it may be said that the wheat plot points to the fact that where the soil is in good condition, through the application of farmyard manure, the artificials that may be most profitably applied are lime (4 parts), superphosphate (3 parts), and sulphate of ammonia (1 part). On the other hand, where the land is not in such high condition, this dressing should be supplemented by a dressing of potash salt. The analyses show that by the application of these dressings the value and quality of the crop are increased because the operations of the nitrifying organisms have been thus favoured.

\section{The Saprophytic Bacteria in Soil}

This group of micro-organisms is by far the most abundant as regards number. They live on the dead organic matter of the soil, and their function appears to be to break it down into simpler constitution. Specialisation is probably progressing among them, for their name is legion, and the struggle for existence keen. After we have eliminated the economic bacteria, most of which are obviously saprophytes, the group is greatly reduced. It is also needless to add that of the remnant little beyond morphology is known, for as their function is learned they are classified otherwise. It is probable, as suggested, that many of the species of common saprophytes normally existent in the soil act as auxiliary agents to denitrification and putrefaction. At present we fear they are disregarded in equal measure, and for the same reasons, as the common water bacteria. An excess of either, in soil or water, is not of itself injurious as far as we know; indeed, it is probably just the reverse. It is, however, frequently an index of value as to the amount and sometimes condition of the contained organic matter. The remarks made when considering water bacteria apply here also, viz., that an excess of saprophytes acts not only as index of increase of organic matter, but as at first auxiliary, and then detrimental, to pathogenic organisms. It will require accurate knowledge of soil bacteria generally to be able to say which saprophytic germs, if any, have no definite function beyond their own existence. It may be doubted whether the stern behests of nature permit of such organisms. However that may be, we may feel confident, though at present there are many common bacteria in soil, as also in water, the life object of which is not ascertained, that as knowledge increases and becomes more accurate, this special provisional group will become gradually absorbed into other groups having a part in the economy of nature, or in the production of disease. At present the decomposition, denitrifying, nitrifying,* and nitrogen-fixing organisms are the only saprophytes

* It has already been pointed out that the nitrifying bacteria, though able to live on organic matter, do not require such either for existence or for the performance of their function. 
which have been rescued from the oblivion of ages, and brought more or less into daylight. It is but our lack of knowledge which requires the present division of saprophytes, whose business and place in the world is unknown.

\section{The Pathogenic Organisms found in Soil}

In addition to these saprophytes and the economic bacteria, there are, as is now well known, some disease-producing bacteria finding their nidus in ordinary soil. The three chief members of this group are the bacillus of Tetanus, the bacillus of Quarter-Evil, and the bacillus of Malignant Edema.

\section{Tetanus}

The pathology of this disease has, during recent years, been considerably elucidated. It was the custom to look upon it as "spontaneous," and arising no one knew how; now, however, after the experiments of Sternberg and Nicolaier, the disease is known to be due to a micro-organism common in the soil of certain localities, existing there either as a bacillus or in a resting stage of spores. Fortunately, Tetanus is comparatively rare, and one of the peculiar biological characters of the bacillus is that it only grows in the absence of oxygen. This fact contributed not a little to the difficulties which were met with in securing its isolation.

Tetanus occurs in man and horses most commonly, though it may affect other animals. There is usually a wound, often an insignificant one, which may occur in any part of the body. The popular idea that a severe cut between the thumb and the index finger leads to tetanus is without scientific foundation. As a matter of fact, the wound is nearly always on one or other of the limbs, and becomes infected simply because the limbs come more into contact with soil and dust than does the trunk. It is not the locality of the wound nor its size that affects the disease. A cut with a dirty knife, a gash in the foot from the prong of a gardener's fork, the bite of an insect, or even the prick of a thorn, have before now set up tetanus. Wounds which are jagged, and occurring in absorptive tissues, are those most fitted to allow the entrance of the bacillus. The wound forms a local factory, so to speak, of the bacillus and its secreted poisons; the bacillus always remains in the wound, but the toxins may pass throughout the body, and are especially absorbed by the cells of the central nervous system, and thus give rise to the spasms which characterise the disease. Suppuration generally occurs in the wound, and in the pus thus produced may be found a great variety of bacteria, as well as the specific agent itself. After a few days or, 
$\odot$ 
it may be, as much as a fortnight, when the primary wound may be almost forgotten, general symptoms occur. Their appearance is often the first sign of the disease. Stiffness of the neck and facial muscles, including the muscles of the jaw, is the most prominent sign. This is rapidly followed by spasms and local convulsions, which, when affecting the respiratory or alimentary tract, may cause a fatal result. Fever and increased rate of pulse and respiration are further signs of the disease becoming general. After death, which results in the majority of cases, there is very little to show the cause of fatality. The wound is observable, and patches of congestion may be found on different parts of the nervous system, particularly the medulla (grey matter), pons, and even cerebellum. Evidence has recently been forthcoming at the Pasteur Institute to support the theory that tetanus is a "nervous" disease, more or less allied to rabies, and is best treated by intra-cerebral injection of antitoxin, which then has an opportunity of opposing the toxins at their favourite site. The toxins diffuse throughout the tissues of the body, but particularly affect the spinal cord. The long incubation period indicates that the toxins are probably produced by a ferment of some kind. Whatever its exact nature, it is undoubtedly a most powerful poison.

Tetanus bacilli spores have been found in considerable quantities in the dust of dry jute fibre (Andrewes), and various cases are on record where the disease has been contracted by workers in jute mills in Dundee and elsewhere. Legge attributes the presence of the bacilli in the jute (Corchorus) to the soil in which it is grown in Bengal.

The Bacillus of Tetanus.-In the wound the bacillus is present in large numbers, but mixed up with a great variety of suppurative bacteria and extraneous organisms. It is in the form of a straight short rod with rounded ends, occurring singly or in pairs or threads, and slightly motile. It has been pointed out that by special methods of staining flagella may be demonstrated. These are both lateral and terminal, thin and thick, and are shed previously to sporulation. Branching also has been described. Indeed, it would appear that, like the bacillus of tubercle, this organism has various polymorphic forms. Next to the ordinary bacillus, filamentous forms predominate, particularly so in old cultures. Clubbed forms, not unlike the bacillus of diphtheria, may often be obtained from agar cultures. Without doubt the most peculiar characteristic of this bacillus is its sporulation. The well-formed round spores occur readily at incubation temperature. They occupy a position at one or other pole of the bacillus, and have a diameter considerably greater than the organism itself. Thus the well-known "drumstick" form is produced. In practice the spores frequently occur free in the medium and in 
microscopical preparation. Like other spores, they are extremely resistant to heat, desiccation, and antiseptics.*

As we have seen, this bacillus is a strict anaërobe, growing only in the absence of oxygen. The favourable temperature is $37^{\circ} \mathrm{C}$., and it will only grow very slowly at or below room temperature. The organism is readily stained by the ordinary stains and by Gram's method.

An excellent culture is generally obtainable in glucose gelatine. The growth occurs only in the depth of the medium, and appears as fine threads passing horizontally outwards from the track of the needle. At the top and bottom of the growth these fibrils are shorter than at the middle or somewhat below the middle. For extraction of the soluble products of the bacillus, glucose broth may be used. (For isolation and detection of the B. tetani, see Appendix, p. 481.)

In some countries, and in certain localities, the bacillus of tetanus is a very common habitant of the soil, and when one thinks how frequently wounds must be more or less contaminated with such soil, the question naturally arises, How is it that the disease is, fortunately, so rare? Probably we must look to the advance of bacteriological science to answer this and similar questions at all adequately. Much has recently been done in Paris and elsewhere to emphasise the relation which other organisms have to such bacteria as those of typhoid and tetanus. In tetanus, Kitasato, Vaillard, and others have pointed out that the presence of certain other bacteria, or of some foreign body, is necessary to the production of the disease. The common organisms of suppuration in particular appear to increase the virulence of the bacillus of tetanus. How these auxiliary organisms perform this function has not been fully elucidated. Probably, however, it is by damaging the tissues and weakening their resistance to such a degree as to afford a favourable multiplying ground for the tetanus bacillus. Some authorities hold that they act by using up the surrounding oxygen, and so favouring the growth of the germ of tetanus. In any case it is now generally held that in natural infection the presence of some foreign body or suppurative bacteria is necessary to produce the disease.

\section{Quarter-Evil and Malignant Edema}

Quarter-Evil (or symptomatic anthrax) is a disease of animals, produced in a manner analogous to tetanus. It is characterised by a rapidly-increasing swelling of the upper parts of the thighs, sacrum, etc., which, beginning locally, may attain to extraordinary size and

* Atlas and Principles of Bacteriology, by Lehmann and Neumann, part ii., pp. $330-337$. 
extent. The swelling may assume a dark colour, and crackles on being touched. There is high temperature, and secondary motor and functional disturbances. The disease ends fatally in two or three days.

Slight injuries to the surface of the skin or mucous membrane are sufficient for the introduction of the causal bacillus. This organism is, like the bacillus of tetanus, an anaërobe, existing in the superficial layers of the soil. From its habitat it readily gains entrance to animal tissues. It has spores, but though they are of greater diameter than the bacillus itself, they are not absolutely terminal. Hence they merely swell out the capsule of the bacillus, and produce a clubshaped rod. The bacillus forms gas while growing in the tissues and in artificial culture. External physical conditions have little effect upon this organism, and dried and even buried flesh retains infection for a long period of time.

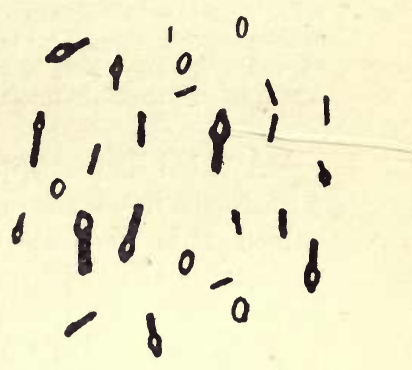

FIG. 20.-Diagram of Bacillus of Symptomatic Anthrax.

\section{Quarter-Ill, Quarter-Evil, or Black-Leg}

Quarter-ill may be said to lack much of the importance and interest which is attached to anthrax, inasmuch as it is confined to two domestic animals-sheep and cattle-and is not communicable to man. It, however, resembles anthrax, in so far as they are both caused by the introduction into the blood of the healthy animal of specific bacilli. Both diseases have a tendency to recur on farms or premises on, or in, which animals affected with these diseases have been previously kept. On the other hand neither anthrax nor quarter-ill is communicable by association of the affected with the healthy animal, and in that respect they differ from most of the contagious diseases which are legislated for in this country. Another peculiar feature of quarter-ill is that while it is very fatal to sheep at any age, cattle over two years may be said to have an immunity against the disease.

The symptoms of quarter-ill in young cattle are so strikingly different from any other disease that an error in diagnosis is almost impossible. The first indication of an animal being affected with quarter-ill is a marked stiffness or lameness of one of the limbs; it is exceedingly dull, and presents a most anxious and dejected appearance, does not feed, and it is with extreme difficulty that it can be forced to move. Very soon after the limb is attacked a swelling appears beneath the skin, usually upon one of the hind quarters, which is extremely hot, increases in size rapidly, and is most painful to the animal when touched. This swelling has a disposition to extend down the leg, or perhaps along the loins and back, and when pressed gives a peculiar crackling sensation to the fingers. In almost every instance death supervenes within a few hours after the swelling has appeared.

In the case of sheep the symptoms are not of so marked a character. The first indication is lameness, but the swelling is not so observable in sheep as in cattle, being hidden to a great extent in the case of the former by the fleece.

There is no doubt that the disease exists to a greater extent among the sheep in certain counties in England than has been generally known, and from the rapidity with which sheep frequently die it is often locally called "strike."

Should any doubt exist as to whether a sheep has died from quarter-ill, the difficulty can easily be solved by making an incision through the skin of the dead 
animal into the tumour or swelling, which contains a large quantity of dark coloured fluid, which emits a very strong and peculiarly offensive odour. Any fluid that may thus escape should be carefully collected and destroyed.

The carcases of animals which have died of quarter-ill should be buried as in anthrax, or, still better, cremated on or in the place where the animal died. All dung, fodder, litter, or other materials of a like character which may have been on or about places or sheds where animals have died should be burnt, or thoroughly mixed with some powerful disinfectant, and buried in a part of the premises to which cattle and sheep do not have access. The sheds, particularly the flooring and mangers, should be thoroughly washed and scrubbed with a 5 per cent. solution of carbolic acid, and it would be prudent to repeat the process before they are again used for cattle or sheep.

A third disease-producing microbe found naturally in soil is that which produces the disease known as Malignant Edema. Pasteur called this disease gangrenous septiccemia, and the bacillus vibrion septique. Unlike quarter-evil, malignant œdema may occur in man in cases where wounds have become septic. It is usually described as a spreading inflammatory œedema, with emphysema, and followed by gangrene. Man and animals become inoculated with this bacillus from the surface of soil, straw-dust, upper layers of garden-earth, or decomposing animal and vegetable matter.

The bacillus occurs in the blood and tissues as a long thread (3 $\mu$ to $10 \mu$ in length), composed of slender segments of irregular length. It is motile and anaërobic, and readily stained by aniline stains but not by Gram's method, in this way differing from the anthrax bacillus. The spores are larger than the diameter of the bacillus, and usually centrally placed. The organism produces gas, and so much is this the case in artificial culture, that the medium itself is frequently split up. The bacillus liquefies gelatine. The most suitable medium for cultivation is glucose agar at $37^{\circ} \mathrm{C}$.

Both malignant œedema and symptomatic anthrax are similar in some respects to anthrax itself. There are, however, a number of points for differential diagnosis. The enlargement of the spleen, the enormous numbers of bacilli throughout the body, the square ends of the bacillus, its non-motility, its equal inter-bacillary spaces, its aërobic growth, and its characteristic staining, afford ample evidence of the anthrax bacillus.

Fränkel and Pasteur have both demonstrated the possible presence in soil of the bacillus of anthrax itself. Frïnkel maintained that it could not live there long, and at 10 feet below the surface no growth occurred. This may have been due to the low temperature of such a depth. Pasteur held that earthworms are responsible for conveying the spores of anthrax from buried carcases to the surface, and thus bringing about re-infection. In any case it is well-known that the spores of anthrax may infect a soil for months. The bacillus of cholera, too, has been successfully grown in soil, except during winter. The presence of common saprophytes in the soil is prejudicial 
to the development of the cholera spirillum, and under ordinary circumstances it succumbs in the struggle for existence. Other species of bacteria have also been isolated from soil from time to time.

Now whilst since the early days of bacteriology the three organisms we have described have been looked upon as the typical bacteria of soil, modern research has brought to light a new relationship between soil and disease, which has greatly enhanced the importance of our knowledge of the subject. Directly, it has been shown that soil may harbour germs of disease, acting sometimes as a favourable and at other times as an unfavourable nidus. Indirectly, it has been shown that a right understanding of the bacteriology of water and its potentiality of disease production, depends upon a knowledge of bacteria in the soil over, or through which, the water has passed. The matter must, therefore, be briefly considered here.

\section{The Relation of Soil generally to certain Bacterial Diseases}

It is now some years since Sir George Buchanan, for the English Local Government Board, and Dr Bowditch, for the United States, formulated the view that there is an intimate relationship between dampness of soil and the bacterial disease of Consumption (tuberculosis of the lungs). The matter was left at that time sub judice, but the conclusion has since been drawn, and it is surely a legitimate one, that the dampness of the soil acted injuriously in one of two ways. It either lowered the vitality of the tissues of the individual, and so increased his susceptibility to the disease, or in some unknown way favoured the life and virulence of the bacillus. That is one fact. Secondly, Pettenkofer traced a definite relationship between the rise and fall of the ground water with pollution of the soil and enteric (typhoid) fever.* A third series of investigations concluded in the same direction, viz., the researches by Dr Ballard respecting summer diarrhœa. This, it is generally held, is a bacterial disease, although no single specific germ has been isolated as its cause. Ballard demonstrated that the summer rise of diarrhœe mortality does not commence until the mean temperature of the soil, recorded by the 4 -foot thermometer, has attained $56.4^{\circ} \mathrm{F}$, and the decline of such diarrhœa coincides more or less precisely with the fall in soil temperature. This temperature $\left(56 \cdot 4^{\circ} \mathrm{F}\right.$.) is, therefore, considered

* The conditions requisite for an outbreak of enteric fever were, according to Pettenkofer, $(a)$ a rapid fall (after a rise) in the ground water, $(b)$ pollution of the soil with animal impurities, $(c)$ a certain earth temperature, and lastly $(d)$ a specific micro-organism in the soil. These four conditions have not, particularly in England, always been fulfilled preparatory to an epidemic of typhoid. Yet the observations necessary for these deductions were a definite step in advance of the idea of the significance of mere dampness of soil. 
as the "critical" 4-foot earth temperature, that is to say, the temperature at which certain changes (putrefactive, bacterial, etc.) take place either, primarily, in the earth, or secondarily, in the atmosphere, with the consequent development of the diarrhœal poison.

After prolonged investigation on behalf of the Local Government Board, Dr Ballard formulated the causes of diarrhoa in the following conclusions:-*

(a) The essential cause of diarrhœa resides ordinarily in the superficial layers of the earth, where it is intimately associated with the life processes of some micro-organism not yet detected or isolated.

(b) That the vital manifestations of such organism are dependent, among other things, perhaps principally upon conditions of season and the presence of dead organic matter, which is its pabulum.

(c) That on occasion such micro-organism is capable of getting abroad from its primary habitat, the earth, and having become airborne, obtains opportunity for fastening on non-living organic material, and of using such organic matter both as nidus and as pabulum in undergoing various phases of its life-history.

(d) That from food, as also from contained organic matter of particular soils, such micro-organism can manufacture, by the chemical changes wrought therein through certain of its life processes, a substance which is a virulent chemical poison.

Here, then, we have a large mass of evidence from the data collected by Buchanan, Bowditch, Pettenkofer, and Ballard. But much of this work was done anterior to the time of the application of bacteriology to soil constitution. Recently the matter has received increased attention from various workers abroad, and in this country from Robertson, Martin, Houston, and others, and we must now consider the new facts brought forward by these investigators.

From the first, experiments on this subject have been more or less confined to the behaviour of the typhoid bacillus than any other pathogenic organism. This has been partly due to the importance of this organism in relation to soil, and partly because it is more convenient than, say, the tubercle bacillus for experimental work. In 1888 Grancher and Deschamps showed that the typhoid bacillus was able to survive in soil for more than twenty weeks, $\uparrow$ and Karlinski arrived at a similar conclusion. + In 1894 Dempster published the results of his work on the same subject, in which he obtained the typhoid bacillus from sand after twenty-three days, from garden soil after forty-two days, and from peat not later than twenty-four hours. $§$ Four years later came Dr Robertson's valuable researches into the

* Supplement to the Report of the Medical Officer of the Local Government Board, 1887, p. 7 .

† Arch. de Med. Exp. et d'Anat. Path., 1889, 7th January.

$\ddagger$ Arch. f. Hyg., Bd. xiii., Heft 3.

$\$$ Brit. Med. Jour., 1894, i., p. 1126. 
growth of the bacillus of typhoid in soil of an ordinary field. By experimental inoculation of the soil with broth cultures, he was able to isolate the bacillus twelve months after, alive and virulent. He concluded that the typhoid organism is capable of growing very rapidly in certain soils, and under certain circumstances can survive from one summer to another. The rains of spring and autumn, or the frosts and snows of winter, do not kill it off so long as there is sufficient organic pabulum. Sunlight, the bactericidal power of which is well known, had, as would be expected, no effect except upon the bacteria directly exposed to its rays. The bacillus typhosus quickly died out in the soil of grass-covered areas.*

Next came the experiments of Dr Sidney Martin, which were undertaken to inquire into the extra-corporeal existence of the bacillus of typhoid fever in soil. $\mathrm{He}$ found, after a prolonged research, that certain cultivated soils, especially garden soils, when sterilised are favourable to the vitality and growth of this bacillus, whether the soil was kept at room temperature $\left(19^{\circ} \mathrm{C}\right.$.) or blood-heat $\left(37^{\circ}\right.$ C. $)$. In such soils the $B$. typhosus was still alive after four hundred and four days, and remained alive, though not for a long period, if the soil were dried and reduced to dust. If, however, the bacillus is added to a well-moistened but not sloppy cultivated soil, it rapidly dies, and is usually not obtainable two days after being sown in it, and its disappearance appears to be more rapid the higher the temperature, which is probably due to the rapid growth of ordinary soil bacteria. If the cultivated soil is not made very moist when the $B$. typhosus is added, the organism can be recovered from the soil up to twelve days after it has been added. Lastly, if this bacillus is added to natural uncultivated soils which have not been sterilised, it ceases to exist within twenty-four hours. Martin holds that the reason of the rapid disappearance of the typhoid bacillus from natural unsterilised soils is probably twofold. First, there is the antagonism of the soil bacteria, many of which are putrefactive; and secondly, the typhoid bacillus requires for its growth nitrogenous substances, usually in the form of proteids. Cultivated soil is distinguished from uncultivated soil by containing more nitrogenous organic matter in the form of nitrates and ammonia, and also more partially changed proteid substances. Hence it is a more favourable environment for the typhoid bacillus. As a general result of these investigations, it may be concluded that the typhoid bacillus has, commonly, only a short existence in the soil, being destroyed by the products of the putrefactive bacteria which exist in most cultivated soils. $\uparrow$

* Brit. Med. Jour., 1898, i., pp. 69-71.

+ Reports of Medical Officer to the Local Government Board, 1898-99, pp. 382-412 ; 1899-1900, pp. 525-548; 1900-01, pp. 487-510. 
Lastly, we have the results of the investigations of Firth and Horrocks, who conclude that the typhoid bacillus is able to assume a vegetative existence in ordinary soils and in sewage-polluted soils for as long as seventy-four days. They further maintain that the controlling factor is an excess or deficiency of moisture in the soil rather than organic nutritive material. From dry fine sand the bacillus was recovered after twenty-five days; from moist fine sand, after twelve days; from damp (rain-water) ordinary soil, after sixtyseven days; from damp (sewage) ordinary soil, after fifty-three days; and from ordinary soil dried to the state of dust, after twenty-four days. In peat the bacillus lives apparently only a few days.* Firth and Horrocks, therefore, arrive at a different conclusion from Martin, namely, that the typhoid bacillus is able to assume a vegetative or saprophytic existence for considerable periods outside the body; that it can survive ordinary earth for over two months, whether the soil be virgin or polluted with sewage, or frozen hard; and that, therefore, it follows that outbreaks of enteric fever may be due to the dissemination (for example by wind or flies) of infective soil dust. Pfuhl of Berlin has arrived at results confirmatory of these experiments. From moist garden earth he recovered the typhoid bacillus eighty-eight days after inoculation, from dry sand after twenty-eight days, and from moist peat twenty-one days. $\uparrow$

On the whole it would appear that whilst much valuable research has been accomplished, it cannot be said that the relation of the typhoid bacillus to soil is understood. Some further light on the subject is obtained from researches carried out in relation to the bacterial condition of sewage-treated land and made-up refuse soil, and brief reference may be made to such work.

Various workers have carried out experiments with the object of ascertaining whether in the surface layers of soil, after it has had sewage upon it, certain microbes characteristic of sewage retain their vitality for any considerable length of time; what, in short, was the fate of such organisms as $B$. coli, $B$. enteritidis sporogenes, etc., when sown broadcast on soil. For if their fate be known, not only would light be thrown upon questions of sewage treatment, and the pollution of soil which might in turn affect water supplies, but indication would be obtained as to the possibility of disease germs maintaining their existence in soil, and eventually infecting man. Chief among such investigations in England have been those of Dr A. C. Houston, + whose conclusions are briefly as follows:-

(1) The addition of sewage to an ordinary garden soil does not

* Brit. Med. Journ., 1902, ii., pp. 936-943.

+ Zeit. f. Hyg., 1902, Bd. xl., Heft 3, p 555.

¥ Report of Medical Officer to Local Government Board, 1900-01, App. No. 4, p. 405 ; 1901-02, App. No. 6, p. 455. 
seemingly lead to other than a temporary increase of the sewage microbes at the expense of the soil microbes, the ordinary soil bacteria ousting the sewage microbes in the struggle for existence. But the addition of sewage to a sandy soil leads to an enormous increase in the total number of microbes as compared with the number originally present in the soil, which does not revert to its original state for some months.

(2) The addition of sewage to garden soil tends primarily to increase the ratio of total number of bacteria to spores of aërobic bacteria, though the alteration is apt to be soon lost.

(3) The addition of sewage to a soil leads to an increase for a time in the number of certain kinds of bacteria, namely: $(a)$ indolproducing bacteria; $(b)$ gas-producing organisms; $(c)$ the spores of B. enteritidis sporogenes; $(d) B$. coli communis and its allies; and $(e)$ streptococci. The occurrence of true streptococci in soil indicates, in Houston's opinion, extremely recent contamination. Whatever interpretation be placed on these facts, it is evident that they indicate that pathogenic organisms such as the typhoid bacillus do not maintain their vitality in the surface layers of soil for more than a brief period. Further, it is evident that some kinds of soils heavily polluted with excremental matter tend to purify themselves, so far as non-sporing bacilli of intestinal origin are concerned.

On the bacterial content of made-soil Dr Savage of Colchester has carried out some work. The samples of such soil were collected with a sterilised Fränkel's borer, and the samples transmitted to the laboratory in sterile Petri dishes. Each sample was then thoroughly broken up and uniformly mixed in the Petri dishes by means of sterile spatulas. Ten grammes were weighed on sterile glazed paper, and added to 100 c.c. of sterile water in a large flask, and thoroughly mixed. The contents of the flask were allowed to settle for five minutes, and without disturbing the sediment 1 c.c. of the water was taken up and added to further quantities of sterile water for dilution purposes. The examination was then carried out in the ordinary way, with a view of determining $(a)$ total number of organisms present; (b) number of B. coli; (c) number of B. mycoides, and of Bismarkbrown Cladothrix; and $(d)$ the smallest quantity of soil producing indol in one week at $37^{\circ} \mathrm{C}$. grown in peptone water solution.

As a result of these experiments, Dr Savage reports that at a depth of two feet in mounds of tip-refuse deposited on damp impervious clay, putrefaction and concurrent purification takes place fairly rapidly for the first two or three years. The organisms present in the refuse as deposited rapidly decrease at the same time, but after two to three years increase, apparently due to the invasion of ordinary soil organisms. After two to three years, purification at this depth takes place extremely slowly, and samples nine to ten years old give 
results very little different from four to five year old samples. The $B$. coli readily dies out in such refuse heaps, from which Dr Savage infers that the $B$. typhosus, being a less resistant organism, would still more rapidly die out, and that therefore "the danger of specific typhoid contamination from building on such made-soil can be neglected." *

From what has been said, it will be seen that though a considerable amount of knowledge has been obtained respecting bacteria in the soil, it may be conjectured that actually there is still a great deal to ascertain before the micro-biology of soil is in any measure complete or even intelligible. The mere mention of the bacilli of tetanus and typhoid in the soil, and their habits, nutriment, and products therein, not to mention the work of the economic bacteria, is to open up to the scientific mind a vast realm of possibility. It is scarcely too much to say that a fuller knowledge of the part which soil plays in the eulture and propagation of bacteria may suffice to modify many views in preventive medicine. True, our knowledge at the moment is rather a heterogeneous collection of isolated facts and theories, some of which, at all events, require ample confirmation; yet still there is a basis for the future which promises much constructive work.

* Jour. of Sanitary Institute, 1903, vol. xxiv., pt. iii., pp. 442-458. 


\section{CHAPTER VI}

\section{THE BACTERIOLOGY OF SEWAGE AND THE BACTERIAL TREATMENT OF SEWAGE}

Composition of Sewage-Quantity and Quality of Bacteria in Sewage-Treatment of Sewage: (1) Disposal without Purification; (2) Chemical Treatment; (3) Bacterial Treatment-Evolution of Bacterial Methods - Septic Tank Method-Contact Bed Method-Manchester Experiments-Effect of Bacterial Treatment on Pathogenic Organisms.

THE relation of bacteria to sewage has during the last twenty-five years assumed a position of the first importance. This is due, generally speaking, to three causes. In the first place, our knowledge of the economic function of bacteria present in sewage has increased in a very large measure in recent years. Secondly, as the population has tended to gravitate to cities, the problem of a pure water supply, free from sewage pollution, has become infinitely more complicated than was the case in rural communities in the past. How often sewage, from sewage or cesspools, gains access by means of direct connection or percolation to drinking water, the history of typhoid epidemics and similar outbreaks in this country only too fully records. And thirdly, practical issues have now arisen in connection with the bacterial treatment of sewage. In order to understand the bacteriology of sewage and its practical lessons, we may first briefly consider the quality and constitution of sewage as regards its bacterial content, and then proceed to discuss its biological treatment.

\section{The Constitution of Sewage}

It is impossible to lay down any exact standard of the chemical and bacterial quality of sewage. The quality will differ according to the size of the community, the inclusion or otherwise of trade-effluents and waste products, the addition of rain and storm water, and other 
similar physical conditions.* Moreover, the sewage itself is constantly undergoing rapid changes owing to fermentation, and the competition of micro-organisms and the effect of their products. It is clear that they are the chief agents in setting up fermentative and putrefactive changes, for if sewage be placed in hermetically sealed flasks and sterilised by heat it will be found that these changes do not occur. Hence it will be at once apparent that no exact or hardand-fast formula can be laid down. Respecting the chemical condition, with which we have but little to do here, we may shortly say that the chief characteristic of sewage is its enormous amount of contained organic matter (yielding saline and albuminoid ammonia, etc.) in suspension or in solution. But there are in addition various inorganic substances, and hence it is customary to subdivide the chemical constituents into (a) organic matter in suspension; excreta, etc.; (b) organic matter in solution; (c) inorganic matter in suspension, such as sand, grit, street and road washings, gravel, etc.; and (d) inorganic matter in solution; which is not great in amount, but includes phosphates, one of the favouring agencies of sewage fungus. We may summarily classify the constituents of sewage as follows :-

(a) Excretory substances, composed of solid excreta and urine. The former consist of nitrogenous partly-digested matter, together with vegetable non-nitrogenous residues of food. The former are easily broken.down; but the latter are only gradually attacked (chiefly by the anaërobic bacteria), and broken down into soluble compounds fœetidly odorous, and into small black masses, which slowly deposit as black sludge.

(b) Household waste, solid substances, washings, etc.

(c) Rain and storm water of varying amount, according to season.

(d) Grit, gravel, sand, etc.

(e) Manufacturing waste products in certain localities.

Turning to the bacterial content, it will at once occur to us that such a large quantity of organic matter as sewage contains, and in which decomposition is constantly taking place, will afford an almost ideal nidus for micro-organic life. There is, indeed, but one reason why such a medium is not absolutely ideal from the microbe's point of view, and that reason is that in sewage the vast numbers of bacteria present make the struggle for existence exceptionally keen. The source of the organisms is most largely the organic dejecta chiefly constituting the sewage, but there are in addition the organisms of the air and extraneous fluids and substances found in sewage. The result of Jordan's † investigations into sewage gave an average of 708,000 living bacteria per c.c., his highest result being 3,963,000

* Analyst, 199, xxiii., 1898.

$\dagger$ Report of State Board of Ilealth, Massachusetts, 1890. 
per c.c. He obtained higher figures during the summer: months than at other times; but in any case his average was extremely low. Laws and Andrewes* found that London crude sewage varied from $2,781,650$ to $11,216,666$ micro-organisms per c.c. "It will thus be seen," they conclude, "that very wide variations exist in the total number of micro-organisms present in sewage at different times and in different places. Temperature is one important factor in determining the rapidity of their reproduction, and hence their increase in numbers; dilution of the sewage by rainfall must also exert a marked influence." Houston + has also examined the sewage of London, and found, in 1898, that the Barking crude sewage contained an average of nearly four millions of organisms per c.c. and the Crossness crude sewage three and a half millions per c.c. In 1899 the same observer + reported 7,357,692 bacteria per c.c. as the average in the sewage at the Crossness outfall. On one occasion he records $19,500,000$ micro-organisms as present in one cubic centimetre. In 1900, Houston reported similar figures, and on occasion as many as $1,900,000$ B. coli per c.c. in crude sewage. He further added some records as to the number of bacteria from crude sewage growing at blood-heat and room temperature. In the former case he found as many as 6,830,000, and in the latter $11,170,000$ per c.c. $\S$

Not only are the numbers incredibly large, but we also find an extensive representation of species, including both saprophytes and parasites, non-pathogenic and pathogenic. Many of these are known as "liquefying" bacteria (from the power which they possess of liquefying or peptonising nutrient gelatine used as a culture medium), and this is one of the features of putrefactive bacteria. Bacilli preponderate over micrococci in actual numbers, and in numbers of species present. There are also many spores. Dr Houston has tabulated these results in his Third Report (1900) from which it appears that there are about 340 spores per c.c., and 1,076,923 liquefying bacteria per c.c. Moulds are but rarely found in sewage, though common in sewer air.

It is probable that the investigations made into the contained bacteria of sewage have up to the present, excellent though they have been, only revealed those species of bacteria which occur in considerable abundance. So though it is impossible to make any very complete record as regards the species of bacteria present in

* Report on the Result of Investigations on the Micro-organisms of Sewage, London County Council, 1894.

+ "Filtration of Sewage," Report on the Bacteriological Examination of London Crude Sewage (First Report), London County Council, 1898.

¥ "Bacterial Treatment of Crude Sewage" (Second Report), London County Council, 1899.

$\S$ Ibid. (Third Report), 1900, p. 59. 
sewage, we may attempt a provisional list of normal types of sewage bacteria * as follows:-

1. Bacillus coli communis and all its varieties and allies. Houston reports as many as $600,000 \mathrm{~B}$. coli per c.c. in London sewage.

2. The Proteus family-Proteus vulgaris, P. Zenkeri, P. mirabilis, and $P$. cloacinus, first isolated from putrid meat by Hauser, isolated from sewage by Jordan, etc. Houston also reports that frequently there may be 100,000 "sewage proteus" present in one c.c. This is an aërobic, non-chromogenic, actively motile, and rapidly liquefying bacillus with round ends, one flagellum, and no spore formation. ' It differs in essential particulars from the $P$. vulgaris. Some of the cultures were pathogenic to guinea-pigs (Plate 14).

3. Bacillus enteritidis sporogenes of Klein. The number of spores of this organism found in London sewage by Houston varied from 10 to 1000 per c.c., thus often exceeding in number the total number of spores of aërobic bacilli. The relative numbers of $B$. coli and the spores of $B$. enteritidis sporogenes in crude sewage have been demonstrated by Klein and Houston in the following table:-

\begin{tabular}{|c|c|c|c|}
\hline Sample of Crude Sewage. & $\begin{array}{l}\text { No. of Bacteria } \\
\text { per c.c. }\end{array}$ & $\begin{array}{l}\text { No. of } B . \text { coli } \\
\text { per c.c. }\end{array}$ & $\begin{array}{l}\text { No. of Spores } \\
\text { of } \\
\text { B. ent. sporog. } \\
\text { per c.c. }\end{array}$ \\
\hline $\begin{array}{l}\text { 1. Chiefly domestic sewage } \\
\text { 2. Mixed sewage } \\
\text { 3. Chiefly domestic sewage: } \\
\text { 4. Mixed sewage and trade-effluent } \\
\text { 5. Hospital sewage } \\
\text { 6. Domestic sewage and trade-effluent } \\
\text { 7. Domestic sewage } \\
\text { 8. Mixed sewage. }\end{array}$ & $\begin{array}{r}14,240,000 \\
7,800,000 \\
4,800,000 \\
36,000,000 \\
2,800,000 \\
4,100,000 \\
28,100,000 \\
21,100,000\end{array}$ & $\begin{array}{r}260,000 \\
180,000 \\
500,000 \\
1,100,000 \\
200,000 \\
500,000 \\
2,000,000 \\
1,000,000\end{array}$ & $\begin{array}{r}2000 \\
200 \\
2000 \\
400 \\
30 \\
56 \\
50 \\
35\end{array}$ \\
\hline
\end{tabular}

* The methods adopted for making a quantitative and qualitative examination of sewage are precisely analogous to those used in milk research. Dilution with sterilised water previous to plating out on gelatine in Petri dishes is essential (1 c.c. to 10,000 c.c. of sterile water, or some equally considerable dilution), otherwise the large number of germs would rapidly liquefy and destroy the film. The plate should be incubated at a definite temperature, which is usually $20^{\circ} \mathrm{C}$. Special methods must be used for the isolation of special organisms, phenol-gelatine ( ${ }^{-1}$ c.c. of a 5 per cent. solution of phenol to every 10 c.c. of gelatine). Elsner medium, Parietti broth, indol-reaction, and "shake" cultures in gelatine (for testing gasproduction) must often be restored to for certain species. Spores in sewage may be isolated by adding 1 c.c. of diluted sewage (1-10) to 10 c.c. of melted gelatine in a test-tube, and heating the mixture to $80^{\circ} \mathrm{C}$. for ten minutes before pouring out into the Petri dish. This temperature kills all the bacilli, but leaves the spores untouched. The same plan is adopted in principle for separating $B$. enteritidis sporogenes : a small quantity of sewage is added to 15 c.c. of fresh sterile milk, which is heated at $80^{\circ} \mathrm{C}$. for ten minutes, and then incubated at $37^{\circ} \mathrm{C}$. anaërobically in a Buchner tube. B. coli communis is grown in phenol-broth for twenty-four hours, and then plated out on phenol-gelatine. 


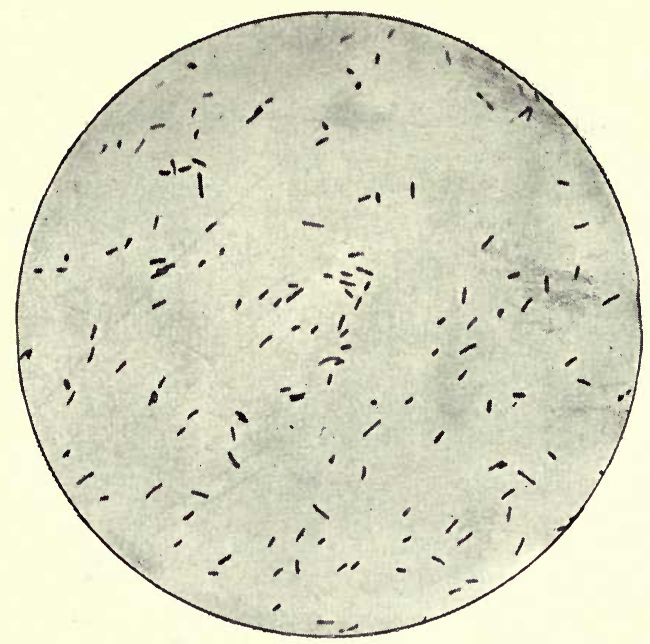

Sewage Proteus (Houston).

Film preparation from agar culture, 24 hours at $20^{\circ} \mathrm{C}$. $\times 1000$.

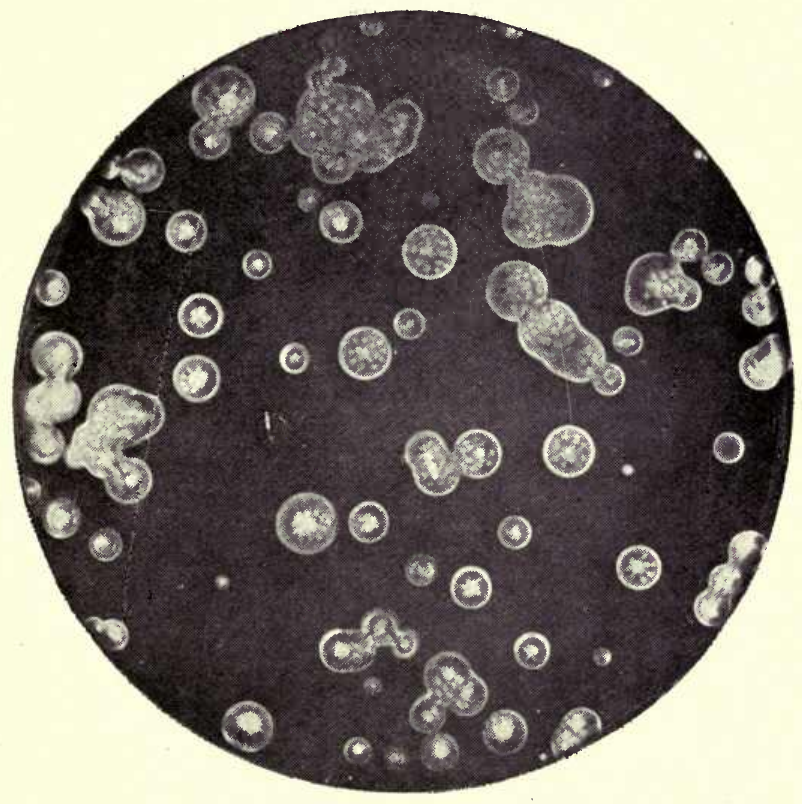

Sewage Proteus (Houston).

Gelatine plate culture, 48 hours' growth at $20^{\circ}$ C. (Natural size). 


$$
\begin{aligned}
& \text { LIBRART } \\
& \text { OF THE } \\
& \text { UNIVERSITY }
\end{aligned}
$$

of

$C_{d}-1 F O R N A$ 
4. Sewage Streptococci.-Laws and Andrewes, Houston, Horrocks, and others have isolated streptococci from crude sewage, which appear to be normal sewage organisms, and as such may be taken, when present in water, to indicate contamination, and, if accompanied by $B$. coli, recent and dangerous contamination. Staphylococci have also been frequently isolated. Houston has described some twenty streptococci as present in London sewage. They are generally present in crude sewage in numbers not less than 1000 per c.c. These sewage streptococci are delicate, and readily lose their vitality and die. They are probably little prone to enter on a saprophytic phase or to multiply to any great extent, if at all, under such conditions as prevail in sewage. They are present in the intestinal discharge of animals, and comprise highly pathogenic organisms. They are usually absent in pure waters and virgin soils, and waters recently polluted with excremental matters. They stain well by Gram's method. The majority form short chains, which sometimes cohere in masses. They grow well at blood-heat in the ordinary media, producing acid in milk without clotting it.* Some streptococci from sewage coagulate milk (Plate 15).

5. Liquefying bacteria, e.g. Bacillus superficialis (Jordan), $B$. frondosus (Houston), B. hyalinus (Jordan), B. delicatulus (Jordan), B. cloacce (Jordan), B. fluorescens stercoralis, B. membraneus patulus, B. capillareus (Houston), B. cloaco fluorescens (Laws and Andrewes), various forms of Clostridium and the typical B. mesentericus (Plate 16).

6. Non-liquefying bacteria, e.g. Bacillus subtilissimus, B. fusiformis (Houston), B. rubescens (Jordan), B. pyogenes cloacinus (Klein †).

We have not included in the above classification any bacteria virulently pathogenic to man.+ Doubtless, such species (e.g. Bacillus typhosus) not infrequently find their way into sewage. But they are not for various reasons normal habitants, and though they struggle for survival, the keenness of the competition among the dense crowds of saprophytes makes existence for a continuous period in sewage almost impossible for them. In the investigation to which reference has already been made, Laws and Andrewes devoted some attention to the behaviour of $B$. typhosus in sewage. They found that this bacillus was unable to grow, indeed quickly perished, in sewage sterilised by filtration and heat, whereas the $B$. coli is able to increase and multiply in such a medium. Sewage, therefore, even in the absence of the normal micro-organisms which it contains, is an

* Bacterial Treatment of Crude Sewage-Third Report to the London County Council, by Dr Houston, 1900, pp. 60-68 ; Royal Commission on Sewage Disposal, Second Report, 1902, p. 25.

+ See British Medical Journal, 1899, vol. ii., p. 69.

$¥$ Bacillus enteritidis sporogenes, B. pyogenes cloacinus, and other organisms have been held responsible for diarrhœea, abscess formation, etc., but they cannot yet be compared with:B. typhosus as regards pathogenic effect. 
unfavourable medium for the growth of the typhoid bacillus, which in all probability would die out in a few days' time. In crude unsterilised sewage it is clear that owing to competition and the inimical effect some of the non-pathogenic species have upon $B$. typhosus,* that the death of that organism is, in sewage, "probably only a matter of a few days or at most one or two weeks." MacConkey found that in sterilised crude sewage inoculated with the B.typhosus, this bacillus is recoverable in seventeen days, though it does not appear to multiply. In ordinary crude sewage so inoculated, the bacillus was recoverable after thirteen days. $\dagger$

Of the organisms which we have named as normally present in sewage, it is unnecessary to speak in detail, with the exception of the Bacillus enteritidis sporogenes of Klein.+ This bacillus is credited with being a causal agent in diarrhœa, and has been isolated by Dr Klein from the intestinal contents of children suffering from autumnal diarthœa, and from adults having "English cholera." It has readily been detected in sewage from various localities, and also in some sewage effluents. It has been separated from ordinary milk, even from what was termed by the trade "sterilised" milk. The biological characters of this bacillus are briefly as follows. It is in thickness about equal to the bacillus of quarter-evil, thicker and shorter than the bacillus of malignant œdema, and standing therefore between the latter and the bacillus of anthrax. It is motile and possesses flagella, but does not assume a thread form. It readily forms spores, which develop, as a rule, near the ends of the rods, and are thicker than the bacilli. They can withstand a temperature of $80^{\circ} \mathrm{C}$. for fifteen minutes. The bacillus takes the Gram stain. In various media it produces gas rapidly. Particularly is this so in milk. It is an anaërobe, and may be isolated by the following method. A small quantity of the suspected matter is added to a tube of fresh sterilised milk, which is then heated in a water-bath to $80^{\circ} \mathrm{C}$. for fifteen minutes. It is then cooled and incubated at blood-heat in a Buchner's tube (see p. 478). In twentyfour hours the milk is coagulated into white stringy masses and small casein coagula, whilst a large portion of the test-tube is filled with gas or a thick, watery, slightly-turbid whey. It is necessary to differentiate the $B$. enteritidis sporogenes from the bacilli of malignant œdema and symptomatic anthrax and the Bacillus butyricus of Botkin. For such differentiation it is important to remember that the enteritidis organism $(a)$ stains by Gram's method, (b) in gelatine culture

* Klein reports that although $B$. typhosus can live in crude sewage, it is only for a short period. When sewage is diluted with large quantities of water the case is different.

$\dagger$ Royal Commission on Sewage Disposal, Second Report, 1902, p. 62.

‡Annual Report of the Medical Officer of the Local Government Board, 1897-98, pp. $210-250$. 


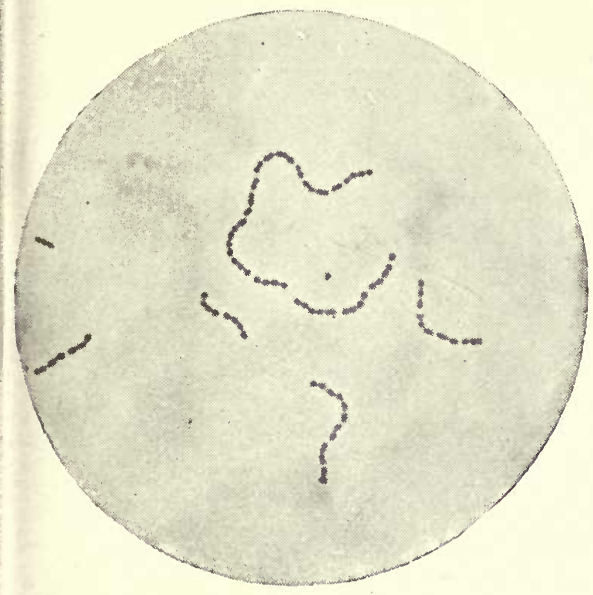

Sewage Streptococcus, from Eflluent. (Houston.) From broth culture, 48 hours at $38^{\circ} \mathrm{C}$. Stained by Gram's method. $\times 1000$.

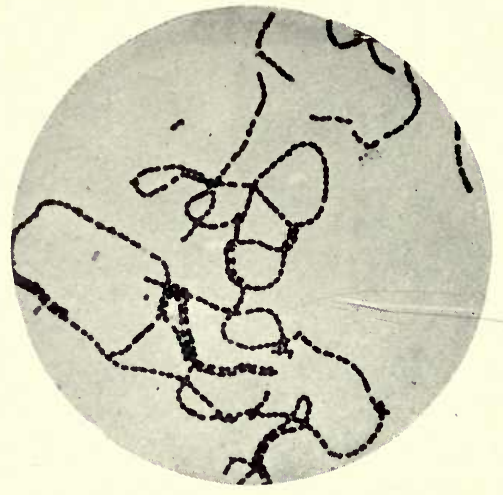

Streptococcus pyogenes.

Film culture from broth culture. Stained by Gram method. $\times 1000$.

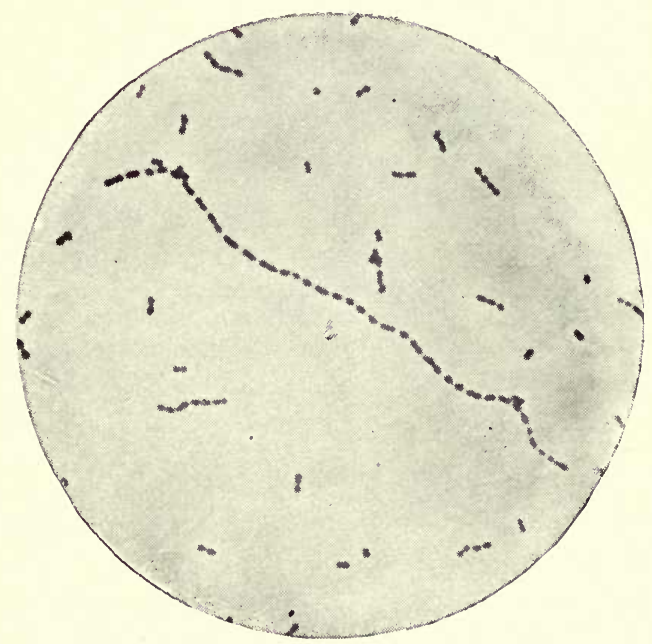

Sewage Streptococcus, from Crude Sewage. (Houston.)

From broth culture, 48 hours at $33^{\circ} \mathrm{C}$. Stained by Gram's method. $\times 1000$. 
() 
shows no lateral offshoots, and (c) possesses different pathological characters on inoculation. If 1 c.c. of whey from a milk culture be inoculated into a guinea-pig (200-300 grammes) a swelling appears in the groin after six hours, which extends to the abdomen and thigh. The animal is usually dead in eighteen to twenty-four hours with gangrene of the subcutaneous tissue and offensive sanguineous exudation. These characteristics, coupled with the morphological and biological features, are sufficient for differentiation purposes (see also p. 307).

Houston has shown that the cholera bacillus, B. pyocaneus, and Staphylococcus pyogenes aureus are capable of retaining their vitality in crude sewage in competition with the very numerous bacteria normally present.* The bacillus of anthrax, and still more so its spores, can also live in sewage and sewage effluents (Houston). +

Whilst we cannot here enter more fully into an account of the bacteria found in sewage or their functions, it is necessary to remark ipon one important feature. A large number of these organisms which we have named as normal inhabitants of sewage fulfil as their main function the process of decomposition and denitrification, that is to say, their rôle is to break down, by means of putrefaction, the organic compounds constituting sewage. For example, urea which is abundantly present in sewage is thus transformed with extraordinary rapidity by several different forms of bacteria.

By way of summary we may quote Houston's account of the "standard" of crude sewage. Crude sewage usually contains, at least, in one cubic centimetre-

(a) 1-10 million bacteria.

(b) $100,000 \mathrm{~B}$. coli or closely allied forms.

(c) 100 spores of $B$. enteritidis sporogenes.

(d) 1000 streptococci.

(e) $\frac{1}{1000}$ c.c. is usually sufficient to produce "gas" in gelatine shake cultures in twenty-four hours at $20^{\circ} \mathrm{C}$.

(f) The inoculation of animals with crude sewage always leads to a local reaction, and not uncommonly results in death.+

As we have already said, when dealing with the Bacteria of the Soil, Nature is dependent upon the services of the "economic" organisms. Dead organic matter is broken down as the result of the vital activity of putrefactive bacteria (decomposing and denitrifying). The ammonia which is thus liberated becomes oxidised first to nitrous and then to nitric acid by the agency of the nitrifying bacteria, and the acids by their action upon bases, always present, produce nitrites and then nitrates. It is upon these substances that

* Bact. Treatment of Crude Sevoage, Third Report, 1900, p. 75.

$\dagger$ Royal Commission on Sewage Disposal, Second Report, 1902, p. 31.

† Ibid., 1902, p. 126 . 
plant life finds nutriment. That the carbon is converted into $\mathrm{CO}_{2}$, the hydrogen into water $\left(\mathrm{H}_{2} \mathrm{O}\right)$, and the "lost" nitrogen refixed in the soil, we have already seen.

Now just as soil contains these Economic Organisms, whose rôle is to complete the cycle of nature, removing the dead remains of plants and animals, and assimilating them in such a way as to add to the fertility of the soil and recommence the cycle of life, so also in sewage we have all the required organisms normally present, whose business it is to render soluble the solid matters, and to split up the organic compounds into their simple elements, and then as a final stage in the process to oxidise these elements and so produce an effluent free from putrescible matter, but containing nitrates and other mineral substances.* For practical purposes these two main groups of bacteria, the breakers-down and the builders-up, are looked upon as anaërobic or aërobic. The former are active in the absence of air, and their activity effects a decomposition of complex organic matter and allied substances. The aërobes are most active in the presence of oxygen, and part of their business is to convert urea into ammonia and ammonia into nitrate.

From this brief recital of the functions of many of the sewage bacteria we learn that they have important operations to perform, and that their presence in sewage, even in very large numbers, is not matter for regret, but far otherwise. We see also a remarkable adaptation of those fermentations discovered by Schloesing and Müntz, in 1878, to be of such inestimable economic value in soil.

We are now in a position to consider the treatment, especially the biological treatment, of sewage.

\section{The Biological Treatment of Sewage}

Almost from time immemorial there has been adopted one of three great methods of disposal of sewage:-

1. Disposal without purification.

2. Mechanical and chemical separations.

3. Biological methods.

It may be convenient to add here that the complete purification of sewage involves three processes:-First, the process of clarification, that is to say, the removal of suspended solid matters; secondly, an alteration of the chemical constitution of organic putrescible

* The following have been considered as the general conditions which an effluent ought to fulfil : (a) It must contain practically no solids in suspension; $(b)$ it must not contain in solution a quantity of organic matter sufficient to seriously absorb the oxygen from the stream water into which it is discharged; $(c)$ it must not be liable to putrefaction or secondary decomposition; $(d)$ it must contain nothing inimical to microbial growth and activity, therefore it must not be treated with strong antiseptics; $(e)$ it must not contain pathogenic organisms. 


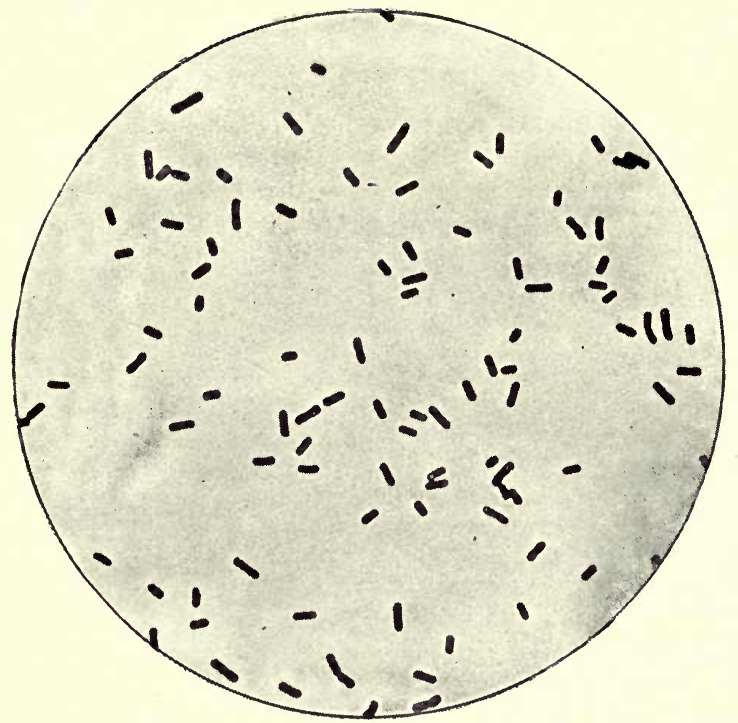

Bacillus mesentericus, Sewage variety (No. i.) (Houston.)

Film preparation from agar culture, 20 hours at $20^{\circ} \mathrm{C} . \times 1000$.

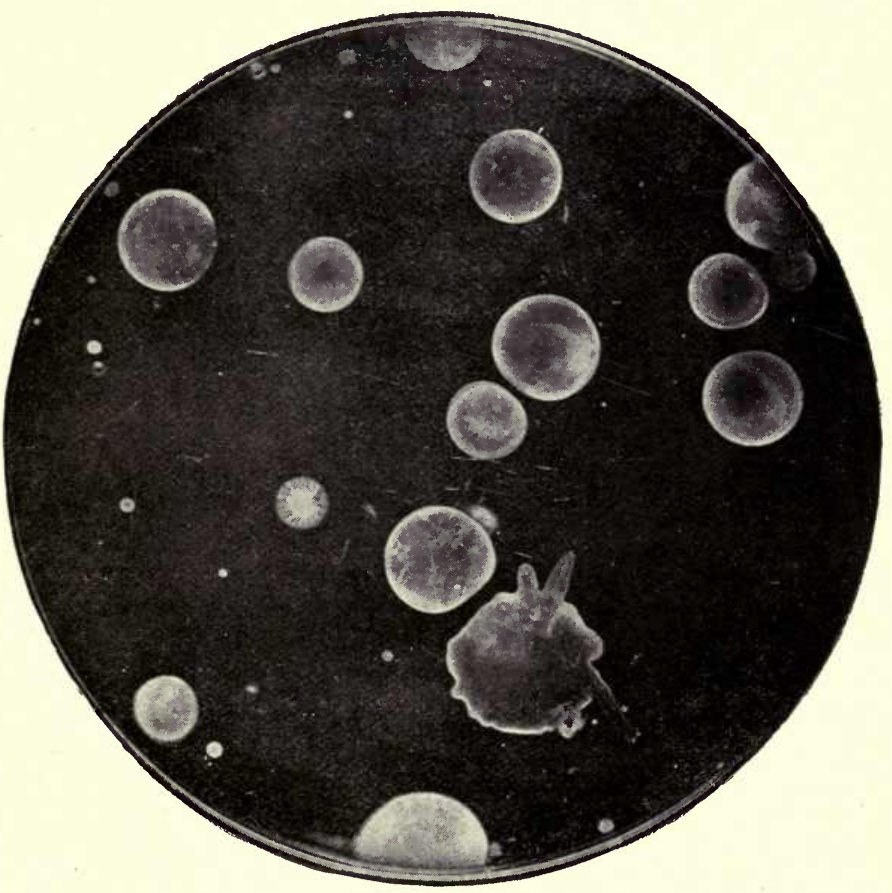

Bacillus mesentericus, Sewage variety (No. i.) (Houston.)

Gelatine plate culture, $20^{\circ}$ C. (Natural size.) 
 
matter in solution in the sewage, so that such putrescible matter appears in the effluent in a form which will not undergo any further putrefactive change; and thirdly, the removal of disease-producing bacteria, which will be present in practically all crude town sewage.

These results are not obtained equally by the various methods employed, but it will be best to consider each of these separately.

\section{Disposal without Purification.}

Various antiquated forms of carrying out this mode have been used. Seaside places have often been content to carry their untreated sewage out to sea. Towns situated on the banks of rivers have frequently by means of a conduit conveyed sewage into the running stream. There is nothing necessarily objectionable in this mode of disposal, for both in the sea and in running river water the sewage matter will become disintegrated and dissolved. Yet the method is liable to give rise to very serious nuisance, unless the conditions requisite for solution are carefully studied. Nuisances may arise in respect to the pollution of bathing grounds, or actually injurious effect upon the health of the population on the banks of the river, or by injury to fish (by reducing the oxygen in the water, destroying the food of fish, admitting poisonous matters into the water, or by suspended matters clogging the gills of fish). In a general way it may be said that before the admission of sewage into any body of water is permissible, two points require consideration, namely, the removal as far as practicable of the matters in suspension in the sewage, and the sufficiency of dissolved oxygen in the water completely to prevent any putrefaction. Broadly, also, it may be said that for towns situated on non-tidal streams some form of bacterial treatment is preferable. Towns on tidal rivers require as a rule a chemical precipitation process.*

* Foulerton has recently drawn attention to a modified chemical precipitation process treatment of sewage which is to be discharged into a tidal water, which may be carried out as follows:- The effluent from an ordinary chemical precipitation process is distributed continuously over a coarse "filter-bed" by means of a sprinkler. In this way a thorough aëration of the effluent before its discharge into the stream is ensured, and provision is made for the complete removal of all traces of solid suspended matter. As the effluent from the sedimentation tanks trickles slowly through the coarse interstices of the filter-bed, any solid suspended matter which has escaped precipitation in the previous part of the process will be deposited, and then dealt with by bacteria. And in the result an effluent, fully oxygenated, free from the solid suspended matter of the crude sewage, and with the bacteria originally present in the crude sewage considerably decreased in numbers, will be discharged into the stream. The somewhat higher proportion of dissolved organic putrescible matter in the effluent from such a chemical process, as compared with the proportion which may obtain in a good bacterial process, is probably not a matter of considerable importance in the case of tidal waters.-Report on Pollution of Tidal Fishing Waters by Sewage, 1903, p. 8. 


\section{Mechanical and Chemical Separations.}

Methods in which this principle is applied are numerous; they have generally been of the nature of a "precipitation" process. Six to twelve grains of quicklime have been added to each gallon of sewage, forming a precipitate of carbonate of lime, which carries down with it the light, flocculent suspended matter of the sewage. The process is simple and cheap; it does not, however, remove the organic matter in solution, but merely the solid matters in suspension. On the one hand it does not produce a valuable manure; on the other it fails to purify the effluent. A score of other methods have been tried (e.g. the lime and ferrous sulphate treatment, Hanson's process, "ferozone," amines, electrolysis, etc.), but with the exception of electrolysis, all based on the addition of chemical substances able to precipitate or otherwise change the organic matter of the sewage. All these methods produce large quantities of sludge, the removal of which, by pressing, digging into the land, or sending out to sea, presents many difficulties. But the chemical processes have this advantage, that they remove disease-producing organisms more perfectly than the bacterial process, though the latter carries further the purification of dissolved organic putrescible matter.

\section{Biological Methods.}

The biological methods, though very various, all have two common features. In the first place, the injurious and putrescible substances in the sewage are not merely "disposed of " nor yet only "separated." They are destroyed. There is a destruction of sewage as sewage, and a building-up of new substance in its place. Secondly, this desired effect is achieved, not by adding anything to the sewage, but by the organisms normally present in the sewage or in the mediumthe land or the "filtering" agent-upon which the sewage is treated. In short, all biological processes depend upon the employment of bacteria in some shape or form. Hence each is a bacterial treatment of sewage. It may appear at first sight that such a process, involving, as it does, encouragement to the growth of putrefactive bacteria, is not without danger. But we shall be satisfied that this is not really so, when it is remembered that the bacterial treatment of sewage is under control, and may be regulated at will. Moreover, the processes of decomposition and nitrification ultimately destroy the pabulum upon which the organisms in question depend for their existence, and hence lead to their death when they have fulfilled their function.

Two applications of this principle have long been in vogue, namely, the intermittent downward filtration and broad irrigation. 
The former may be defined as "the concentration of sewage at short intervals, on an area of specially-chosen porous ground as small as will absorb and cleanse it, not excluding vegetation, but making the product of secondary importance" (Metropolitan Sewage Commission). The intermittency is essential, and the process is partly mechanical and partly bacterial, that is to say, due in part to the nitrification set up by the bacteria in the superficial layers of soil. For successful filtration a porous soil is requisite, a proper inclination of the land to allow of distribution, and a division into areas, in order that each part may receive sewage for, say, six hours, and then have eighteen hours' rest. Soil pipes carry off the effluent. Broad irrigation (sewage-forms) is the "distribution of sewage over a large surface of ordinary agricultural ground, having in view a maximum growth of vegetation (consistently with due purification) for the amount of sewage supplied." To ensure success, the area must be large (say, about one acre to every 100 of the population), the sewage passed on intermittently to allow of aëration of the soil, and the soil itself must be light and porous. Like the former, there is a bacterial influence at work here. Both of these methods are much to be preferred to chemical treatment (and were recommended by the Sewage Commission of 1865); yet, on account of space and management, as well as on account of the tendency of the land to clog or become, as it is termed, "sewage sick," their success has not been all that could be desired.

In 1868, a Commission was appointed to inquire into the best means of preventing the pollution of rivers. They made several reports, the fifth and last being made in 1874. The opinion of this Commission on the comparative merits of the three classes of processes for the treatment of sewage, viz :-chemical precipitation, intermittent filtration, and broad irrigation, may be stated thus:-(1) All these processes are to a great extent successful in removing polluting organic matter in suspension. But intermittent filtration is best, broad irrigation ranks next, and the chemical precipitation processes are less efficient. (2) But for removing organic matters in solution the processes of downward intermittent filtration and broad irrigation are greatly superior to upward filtration and chemical processes.

The last Commission was appointed in 1882. They were directed to inquire into and report upon the system under which sewage was discharged into the Thames by the Metropolitan Board of Works, whether any evil effects resulted therefrom, and if so, what measures could be applied for remedying or preventing the same. In November 1884 they issued their final Report. They found that evils did exist "imperatively demanding a prompt remedy," and that by chemical precipitation a certain part of the organic matter of the sewage would be removed. They reported, however, "that the liquid 
so separated would not be sufficiently free from noxious matters to allow of its being discharged at the present outfalls as a permanent measure. It would require further purification, and this, according to the present state of knowledge, can only be done effectually by its application to the land."

The present Royal Commission has recorded its view in a preliminary report on land treatment, "that peat and stiff clay lands are generally unsuitable for the purification of sewage, that their use for this purpose is always attended with difficulty, and that where the depth of top soil is very small, say six inches or less, the area of such lands which would be required for efficient purification would in certain cases be so great as to render land treatment impracticable." On the subject of effluents they state in the same preliminary report:-

"We may, however, even at this stage, point out that as a result of a large number of examinations of effluents from sewage farms and from artificial processes, we find that while in the case of effluents from land of a kind suitable for the purification of sewage, there are fewer micro-organisms than in the effluents from most artificial processes, yet both classes of effluents usually contain large numbers of organisms, many of which appear to be of intestinal derivation, and some of which are of a kind liable, under certain circumstances at least, to give rise to disease.

"We are of opinion, therefore, that such effluents must be regarded as potentially dangerous, and we are considering whether means are available and practicable for eliminating or destroying such organisms, or, at least, those giving rise to infectious diseases."

Until comparatively recent years, the above methods of treating sewage were the only ones available, or, at all events, practised. But now, as is well known, some new applications of the biological treatment of sewage have been introduced, which call for consideration. Their popularity has been due to it being possible to adopt them where suitable soil did not exist for the other biological methods, and to the fact they have been on the whole less expensive to work. These new departures depend upon bacteria contained in the sewage. The process may depend mainly upon anaërobes (Cameron's Septic Tank or Scott-Moncrieff's process) or aërobes (Duckett's filter or Dibdin's filter). These may be conveniently dealt with in more or less chronological order.

\section{The Bacterial Treatment of Sewage}

In 1872 the Berlin Sewerage Commission reported that sewage matter was converted into nitrates, not simply by molecular processes but by organisms present in sewage and soil. Muntz, Mueller, and others demonstrated this in various ways. Mueller, indeed, had shown 
this to be so in 1865 , naming spirilla, vibrios, and a "protococcus" as the organisms in question. Then in $1881 \mathrm{M}$. Louis Mouras, of Vesoul (Haute-Saône), published an account of an hermetically-sealed, inodorous, and automatically discharging cesspool, in which sewage was anaërobically broken down by "the mysterious agents of fermentation." The effluent, a homogeneous and scarcely turbid fluid, was produced in a tolerably short time and without any addition of chemical ingredients. It was surmised that the agents of fermentation might possibly be the "anaërobes of M. Pasteur." This, it would seem, is the first record we have of the treatment of sewage by simply allowing Nature to fulfil her function by means of bacteria.

The next step-and indeed as regards the problem in England the first step-in the new bacterial treatment of sewage was inaugurated by the workers (Jordan, and others) under the State Board of Health of Massachusetts, who have carried out a laborious series of experiments upon sewage purification during the last fifteen years. The work undertaken at this station may be briefly divided into three main classes: first, purification of unfiltered crude sewage by means of intermittent filtration through sand filters; secondly, rapid filtration of sewage, from which a certain amount of sludge has been removed, by different methods and through different materials; thirdly, purification by dependence upon rapid oxidation or burning of sludge either by forced aëration or other method of introducing air into the filter. Various methods have also been devised with the object of getting rid of the insoluble matter in sewage. The result of this extremely valuable work by the Massachusetts Board clearly demonstrated the accuracy of the fundamental principle that on prepared beds or "intermittent downward filtration" the contained bacteria were the potent agency.*

Whilst this work opened up a new prospect for the bacterial treatment of sewage, it still left the question in an experimental stage, and then it was that Scott-Moncrieff, Dibdin, Cameron, Ducat, and others carried forward the work. $†$ It was in 1892 that $\mathrm{Mr}$ Scott-

* In his evidence before Lord Bramwell's Commission, 1883, Dr Sorby had pointed out that the destruction of the organic sewage matter in the Thames was due to bacteria, and that it was only when these were unable to exert their functions to the full extent by reason of deficient aëration of the water that putrefaction set in.

+ The following general classification will serve to show the nature of the processes adopted by various workers :-

Closed septic tank and contact beds.

Open septic tank and contact beds.

Chemical treatment, subsidence tanks, and contact beds.

Subsidence tanks and contact beds.

Contact beds alone.

Closed septic tank followed by continuous filtration.

Open septic tank followed by continuous filtration.

Chemical treatment, subsidence tanks, and continuous filtration.

Subsidence tanks followed by continuous filtration.

Continuous filtration alone. 
Moncrieff introduced his cultivation beds filled with flint, coke, and gravel. In this system the crude sewage passes into the bottom of the bed, the liquid portion rises through the bed, and the suspended matter is kept back at the bottom, where it undergoes solution by the action of bacteria present upon the surfaces of the flints, etc. In order to complete the process highly oxygenated water was added to the effluent, which was then passed down a "nitrification channel," where further oxidation was secured. The results of this process were superior to anything previously obtained in this country.

Between the years 1891-95 Mr Dibdin experimented with sewage from which solid organic matter, had been previously removed by screens or chemical sedimentation. Such sewage he passed through filter-beds of coke breeze, and was able by intermittent filtration through such beds (i.e. allowing rest periods between charging the filters) to obtain a purification of more than 70 per cent.

Dr Clowes has carried on this work on behalf of the London County Council, and he reports that the sewage is allowed to flow into large tanks which contain fragments of coke about the size of walnuts. As soon as the level of the liquid has reached the upper surface of the coke-bed, its further inflow is stopped, and it is allowed to remain in contact with the bacteria coke surface for about three hours. It is then allowed to flow slowly away from the bottom of the coke-bed. After an interval of about seven hours, the processes of emptying and filling the coke-bed are repeated with a fresh portion of sewage. The coke-bed is usually filled in this way twice in every twenty-four hours. The aëration of even the lowest portions of a deep coke-bed seems to be satisfactory in the above method of working, since the air present in the interstices of the coke, between two fillings with sewage, usually contains 75 per cent. of the amount of oxygen present in the open air.

In dealing with the sewage of the metropolis, Dr Clowes holds that it is best to allow the roughly-screened raw sewage to undergo a somewhat rapid process of sedimentation, in order to permit these matters to subside; and then to pass the sewage direct into the cokebeds. The dissolved matters and the small amount of suspended matters which are still present in the sewage are then readily dealt with by the bacteria of the coke-bed, and practically no choking of the bed occurs. The sewage effluent from the coke-bed is entirely free from offensive odour, and remains inoffensive and odourless even after it has been kept for a month.

The chemical character of this effluent may be briefly indicated by stating that on an average 51.3 per cent. of the dissolved matter of the original sewage, which is oxidisable by permanganate, has been removed by the bacteria, and that the portion which has been removed is evidently the matter which would become rapidly 
offensive, and would rapidly lead to deoxygenation of the river water if it were allowed to pass into the river. The above percentage removal (51:3) was effected by coke-beds varying from 4 to 6 feet in depth. A similar bed, 13 feet in depth, has proved more efficient, and has for some time produced a percentage purification of 64 per cent., while an old bed, 6 feet in depth, has given a percentage purification of 86 per cent. A repetition of the treatment of the effluent in a second similar coke-bed has produced an additional purification of 19.3 per cent., giving a total purification of 70.6 per cent. Effluents from chemical treatment would show a total purification of under 20 per cent. It should be noted that the above purification is reckoned on the dissolved impurity of the sewage; the suspended solid matter is not taken into account. The bacteriological condition of the effluent corresponds in the main with that of the raw sewage. The total number of bacteria undergoes some reduction in the coke-beds, but the different kinds of bacteria which were present in the sewage are still represented in the effluent.

From these and many other similar experiments, it has come to be understood that the bacterial purification depends, as we have seen, upon two main groups of organisms, namely, those that are able to break down and liquefy solid organic matter, and those that deal with it when in solution. Of the former group, some act best under anaërobic conditions. No strict line of demarcation can be drawn as to where one group begins and the other absolutely ends. It is a complex co-operation, shared in by a large variety of organisms classified roughly into these two groups. Systems may be introduced in which the anaërobes are encouraged (as at Exeter), or systems may be established on an aërobic basis (as at Sutton and Manchester). Hence it may be accepted as finally settled that the bacterial treatment may be mainly an anaërobic one (Cameron, ScottMoncrieff, and others), or mainly an aërobic one (Dibdin, Fowler, and others), or a mixture of the two. Whatever system is used, the two great agencies of breaking down and oxidation must be allowed ample opportunity. Probably we shall most clearly recount the application of these principles by considering in some detail two examples of the two typical methods of bacterial treatment. These two examples are furnished in Cameron's Septic Tank Installation (anaërobic), and at the Davyhulme Works, Manchester, in the Multiple Contact Bacteria-beds (ä̈robic).

1. Septic Tank and Cultivation-bed Method (Cameron).-This method has been adopted at Exeter and other places. The plant is twofold, namely, a septic tank and several cultivation or bacteria beds.

The septic tank is a large underground vault constructed of concrete, cemented on exposed surfaces, and having a capacity of thousands of gallons, according to the population. That at Exeter 
has a capacity of 53,800 gallons, and takes the average sewage of 1500 inhabitants in twenty-four hours. Near the entrance is a submerged wall, forming a grit chamber for the arrest of gravel and coarser detritus. The remaining solid matter passes into the tank itself. The inlet and outlet being below the level of the sewage, light and air are excluded as far as possible. Both in the sediment at the bottom of the tank and in the thick scum on the surface the organic compounds are broken down and made soluble. In the former position this is accomplished by anaërobic bacteria, in the latter, on the surface, by aërobic bacteria. It need hardly be added that these are denitrifying and putrefactive bacteria, and that those at the bottom of the tank perform greater service than those at the top. What are the changes taking place in the tank? On every side throughout the tank innumerable small masses of organic matter may be observed rising and falling. At first the masses fall to the bottom by gravity; here they are attacked by countless bacteria which generate numerous gases in the small masses which are thus caused to rise again to the surface; the pressure being then reduced, the gases expand and burst in bubbles, leaving the particles to sink again and commence a similar cycle. Thus the sewage is rapidly broken down by a process of peptonisation and digestion (anaërobic hydrolysis) until all the organic matter is in solution (soluble nitrogenous compounds, phenol derivatives, gases, ammonia, nitrites, etc.). No rest is necessary, for the supply of organisms is unlimited, being perpetually replenished by incoming sewage. It is contended, and probably with some truth, that most pathogenic organisms would not be able to survive in the competition which must be present in the septic tank. When the liquid sewage passes out of the tank, it differs from the crude sewage entering the tank, in the following particulars:- $(\alpha)$ The gravel and particular débris have been removed; (b) the organic solids in suspension are so greatly diminished that they are almost absent; $(c)$ there is an increase of organic matter in solution; $(d)$ the sewage is darker in colour and more opalescent; (e) compounds like albuminoids, urea, etc., have been more or less completely broken down, reappearing in more elementary conditions, like ammonia, methane, carbonic acid gas, and sulphuretted hydrogen. These latter bodies may be in solution, or may have escaped as gas.

The cultivation beds are five or six "filters," to which the sewage from the tank flows, and by an automatic arrangement is distributed to each bed in turn. Each filter may thus be full, say, about six hours, and has from ten to twelve hours' rest. The depth of the filtering medium is 4 or 5 feet, and is composed as follows from the bottom upwards :-

(a) About 1 foot in depth of broken furnace clinker, which will pass a 3 -inch mesh, but not a 1 -inch. 


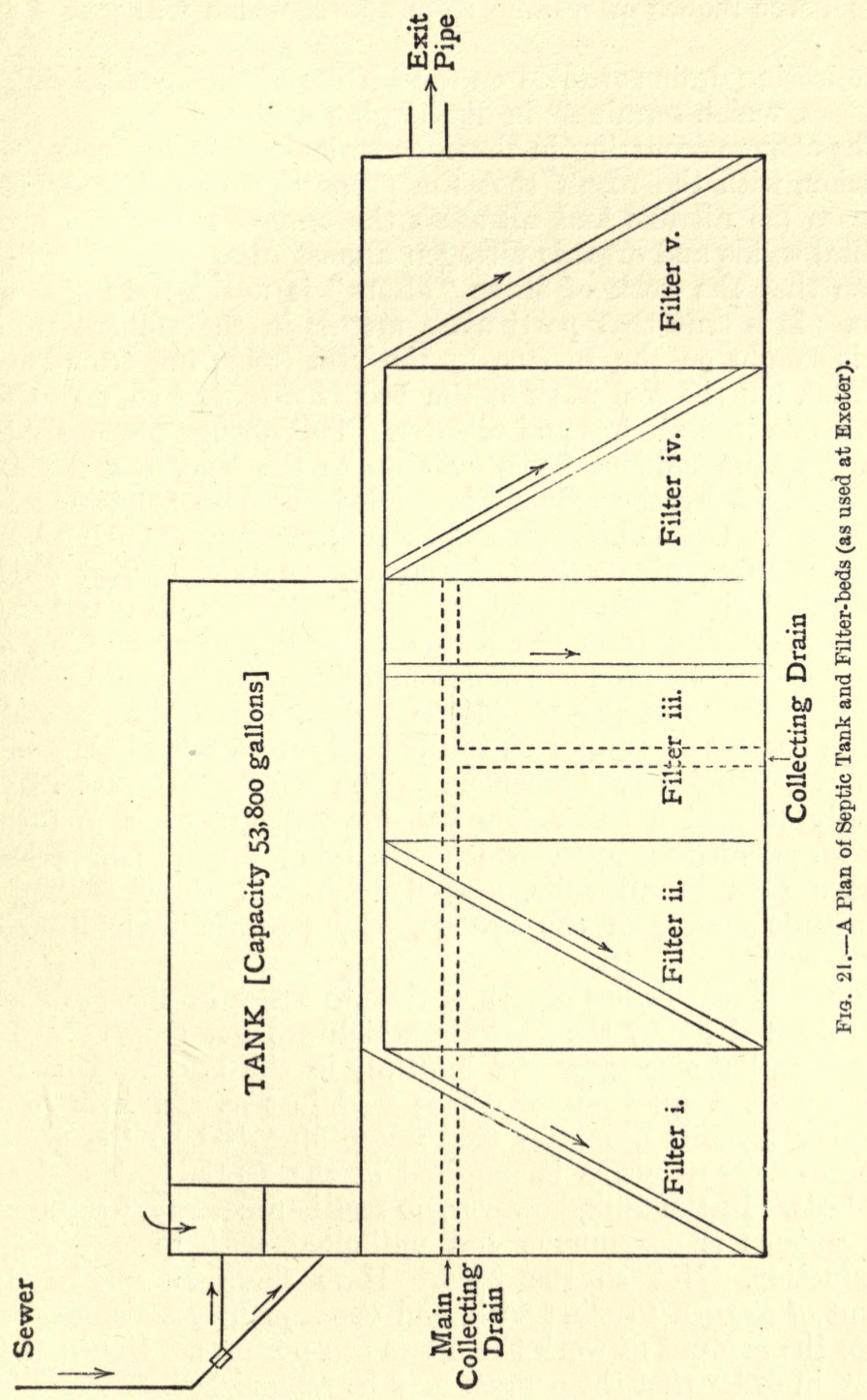


(b) Two feet or so of screened clinkers to pass a $\frac{3}{4}$-inch mesh, but not a $\frac{1}{8}$-inch mesh. mesh.

(c) Three inches of residue from above, which will pass a $\frac{1}{8}$-inch

Collecting drains are laid on the bottom of the beds, joining main collectors, which terminate in discharging wells.*

The change occurring in these bacteria beds is of the nature of oxidation, with the result that the proportion of oxidised nitrogen increases (as nitrites and nitrates), the ammonia becomes less and the total solids and organic nitrogen almost disappear. It will thus be seen that the work of these "filters" is not merely a straining action. It is true that particulate matter in the effluent from the tank is caught on the surface by the film (resulting from previous effluents), but the real work of the bed is nitrification, an oxidation of ammonia into nitrites and nitrates. This change obviously begins when the tank effluent flows over on to the beds and the oxygen then obtained by the effluent is carried down in solution into the coke breeze. Upon the surfaces of the filtrant are oxidising bacteria. When the effluent is on the bed they oxidise its contained products; when the bed is empty and "resting" they oxidise carbon. An advantage arising from the periodical emptying and filling of the "filter" is that the products of decomposition which would eventually inhibit the action of the aërobic bacteria are washed away and pass into the nearest stream, or on to the land direct, where they become, of course, absolutely innocuous. The "filter" is perhaps more correctly termed a cultivation bed, for its purpose is to furnish a very large surface upon which nitrifying organisms, present as we have seen in all soils, may flourish, and these feeding upon the organic matter of the sewage, may perform their function of oxidation.

The solid matter has plenty of time to settle in the tank and be fully operated on by the bacteria, which are not only contained in the sewage, but also grow and multiply in the tanks. This growth is, of course, a question of time; and just as the growth of the nitrifying layer is necessary to a water filter-bed, so the growth of the necessary organisms is required in the septic tank and on the filter-beds. In the tank, however, no rest is necessary, for the supply of organisms is continuous and unlimited, both in supply and in reproduction. Not so the beds. Here there is only a limited amount of oxygen to start with, and consequently a definite limitation to the amount of work the filters can perform. Hence the need of rest, in order that the oxygen may be replenished.

The amount of sludge in the chemical processes has always been

* An account of the Septic Tank method will be found in the Brit. Med. Jour., 1900 , i., pp. $83-86$. 
a difficulty. In the bacterial processes it is reduced to one-third, and often is so little as to be a negligible quantity.

It is not possible to lay down exact limits as to where denitrification ends and oxidation begins. To a certain extent, and in varying degree, they overlap each other. But, generally speaking, we may say that in the tank there is a breaking-down (denitrification and decomposition) and in the filter-beds a building-up (nitrification). The case is precisely parallel to similar changes occurring in soil,

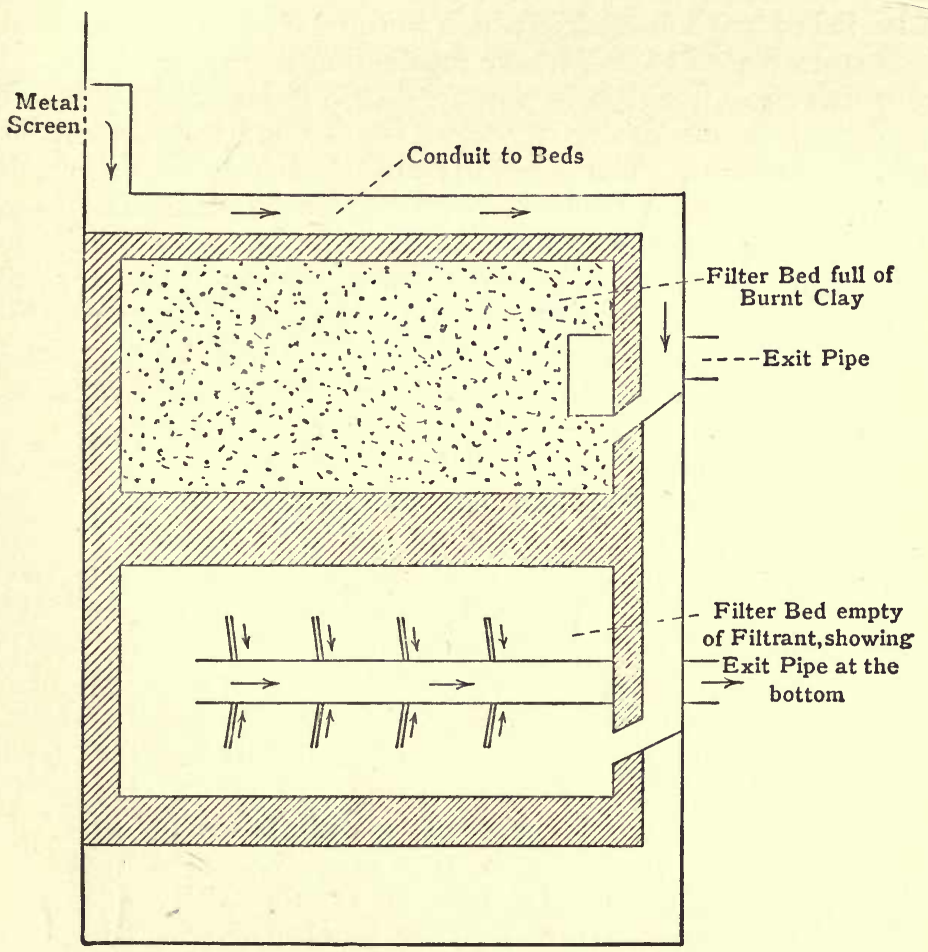

Fic. 22.-Contact Beds (as used at Sutton).

and with which we have already dealt. It is hardly necessary to add that there is a marked reduction in the number of bacteria present in the crude sewage, and the tank and cultivation-bed effluents. One investigation has shown that a sample of crude sewage contained $4,084,827$ bacteria per c.c.; the sewage precipitate, $1,344,925$; the tank effluent, 398,695 ; and the cultivation-bed effluent, 45,755 bacteria per c.c.

2. Multiple Contact Bacteria Beds. - This method in its simplest form has been applied, for instance, at Sutton, and in a 
more advanced form at Davyhulme, Manchester. At Sutton there is no tank. A metal screen holds back part of the solids from being carried on to the beds. The filtrant is burnt clay, and it is forked over occasionally to let in oxygen. The beds are $3 \frac{1}{2}$ feet deep. The bottom of the bed is provided with a 6 -inch main drain with tributary drains. The crude sewage, after passing through a roughing screen to intercept floating paper, etc., is run directly upon the filter without the addition of any chemicals. The filter is charged to within 6 inches from the surface, and the sewage remains in contact for a period of two hours, after which the outlet valve is opened and the filtrate is drawn off to be further purified on fine-grain bacteria beds, after which the effluent is in a fit condition to be discharged into the stream, and is uniformly superior to the effluent obtainable by chemical treatment. The sludge is absorbed by bacterial agency in the beds, and does not accumulate or manifest itself. The beds are free from any offensive odour. At first the beds were seeded with Micrococcus candicans, but it is now known that the necessary bacteria are in the sewage, and seeding is not required. For more effective screening of the sewage an automatic rotary screen may be fixed. This screen may be driven by a Poucelet water-wheel, actuated by the sewage.

Experiments seem to prove that coarse-grain beds worked on the contact principle may be constructed of a numerous class of materials, and that different districts may adopt materials which are obtainable locally, and often at a small cost, although it may be observed that porous coarse-grained materials such as coke and burnt ballast effect a greater degree of purification than do fine-grained impervious material such as granite, slate, etc. The cost of such a system would be in many cases one-quarter of a chemical precipitation and irrigation system, and yet more effective. It will be understood that the absence of a septic tank does not mean an entire absence of the anaërobic action of the process. It simply takes for granted that this portion of the process has been in part performed in the sewers.

The Manchester bacterial system is practically the same in principle as at Sutton. But there are two important and interesting differences. First, the quantity of sewage to be dealt with is very much greater. Secondly, there is the added complication that the Manchester sewage contains large quantities of trade-effluent from breweries, dyeing and bleaching works, galvanising works, tanneries, and derivatives from coal-tar, colour, and naphthalene works. It has been frequently suggested that such chemicals in the sewage would prevent the contained bacteria from fulfilling their rôle in the purification. Consequently, the trial of the two chief methods of bacterial treatment of Manchester sewage has been followed with much interest. In 1898 three experts were appointed, and requested to 
furnish a report as to the system best adapted for Manchester. Various points demanded elucidation which had previously escaped. These were chiefly (1) whether trade-refuse in the sewage impaired the efficiency of bacterial purification; (2) whether a portion, at any rate, of the sludge can be destroyed by bacterial agency; (3) whether chemical precipitation, as in Dibdin's first method, before bacterial treatment could be dispensed with; (4) whether an aërobic process alone or a combination of anaërobic and aërobic processes is the more effective. With these objects in view a septic-tank (Cameron) method and a filter-bed method were installed, and under the superintendence of Dr G. J. Fowler, the superintendent and chemist of the Corporation Sewage Works, the observations were carried out.*

That the experts' view of the bacterial treatment of sewage was similar to that set forth above may be gathered from a preliminary note: "The bacteria already existing in the sewage," they state, "are brought by it on to the bacteria beds, or into the septic tank. The former, by providing an enormously extended surface for the development of bacterial growths, furnish an ideal habitat for the aërobic micro-organisms which require air for the display of their powers, whilst the septic tank, by confining a large volume of liquid which is but superficially in contact with air, enables the anaërobic micro-organisms to work to great advantage. It will be understood that some time must elapse before the bacterial life attains its maximum, either in the bacteria bed or in the septic tank, and consequently the amount of sewage which can be purified therein will gradually increase as time goes on." $†$

The contact beds at Manchester were five in number, and of different area. In principle they were of similar construction to those described already. The filtering medium was clinkers laid to a depth of 3 feet, and of varying size in the five beds, but uniform in each bed. Clinkers which passed $\frac{3}{4}$-inch mesh, rejected by $\frac{1}{8}$-inch mesh, were found to be the best grade.+

Arrangements are made by which it is possible to admit sewage to the beds in three different conditions, namely, raw, screened sewage; sewage which has undergone settlement in a small settling tank; sewage which has undergone both settlement and anaërobic action. Eventually a plan was adopted by which the sewage was

* A full record of the work done at Manchester will be found in the Rivers Department Reports for 1901, 1902, 1903, and 1904.

† City of Manchester, Rivers Department. Experts' Report on Trealment of Manchester Sevage, 1899, p. 12. (Mr Baldwin Latham, Professor Percy Frankland, F.R.S., and Professor W. H. Perkin, Junr., F.R.S.).

$\ddagger$ It appears that the initial capacity of a contact bed is uninfluenced by the grade of clinker. At first there is a rapid decrease in capacity, due in part to sinking of the surface and in part to bacterial growth on the clinkers, which must necessarily occupy some space, even though relatively little. In a comparatively short time the beds acquire a constant average capacity. 
passed through a settling tank prior to its being brought on to the contact beds. In short, the method resolved itself into one of an open septic tank and multiple contact following the settlement or screening out of grosser solids. The results surpassed even the most sanguine expectations, even though the beds were filled four times daily. If the two methods-namely, the closed septic tank and single contact, and the open tank and multiple contact-are compared, it is the opinion of the three experts named that "where both systems are dealing with the same volume of sewage on the same area, the advantage as regards efficiency belongs indisputably to the double contact system." Boyce subsequently confirmed this conclusion in favour of a combination of the anaërobic and aërobic processes, provided that the septic process was perfected, and the suspended sludge did not pass over on to the beds.*

Before summarising the main conclusions which may now be legitimately drawn from the Manchester experiments, a word or two may be said concerning the characters of an efficient bacteria bed and the management of storm-waters in sewage treatment.

The material, or filtrant, of which the bed is composed may vary within wide limits. Burnt clay, coke, clinkers, cinders, or various forms of gravel may all be efficient, provided there is ample aëration and porosity. The required organisms exist, of course, mainly in the sewage, but they require abundant oxygen in order to perform their function. To assist in maintaining this aëration the surface should be raked over from time to time. It has been suggested that in times of frost a layer of ice would prevent the action of the bed. But in point of fact such obstruction would rarely occur, the temperature of the sewage being sufficient both to prevent such stoppage of the beds and also to maintain the necessary activity of the bacteria, which, as we have already seen, require for their vitality nutriment, oxygen, moisture, and a favourable temperature. From December to April the average daily temperature of the Manchester sewage was $55.5^{\circ} \mathrm{F}$., whilst the average temperature of the surrounding air was $45.3^{\circ} \mathrm{F}$. Hence the ice difficulty is naturally overcome. Another point of importance in connection with aëration is the allowance of sufficiently frequent and prolonged periods of rest. Without such intervals the beds would of course become clogged, and eventually inactive, because lacking in aërobic bacteria. Though not absolutely a character of the beds, there is one further point always to be borne in mind in securing their efficiency. It is, that the sewage being applied to the bed should be as far as possible uniform in consistence and freed from suspended matters by sedimentation. Any suspended matter not so removed should be retained as far as possible on the surface of the bed.

* Royal Commission on Sewage Disposal, 1902, p. 11. 
A moment's refiection will make it evident that the problem may be seriously complicated at short notice by the great increase in volume of the sewage following rain-storms. To this matter the experimenters at Manchester have also directed their attention. They draw the necessary distinction between the first flush of a storm and the highly-diluted sewage which follows, designating the latter only as "storm water." They decide that provision must be made for the storage or separate treatment of "first flush" of sewage at the beginning of a storm, and that about two hours after the augmented flow is the time to commence accelerated treatment, the exact procedure varying according to the character and duration of the storm. Short double contacts, or even a single contact, is sufficient to purify storm water, and there is no decrease in the purifying capacity of the bed.

Summarily, the final conclusions arrived at by Latham, Frankland, and Perkin were as follows:-

"1. That the bacterial system is the system best adapted for the purification of the sewage of Manchester.

"2. That any doubts which may have arisen in the first instance as to its suitability, owing to the presence in Manchester sewage of much manufacturing refuse, have, through the convincing results of our experimental inquiry, been entirely banished.

" 3 . That inasmuch as a bacterial contact bed can only effect a definite amount of purification in a single contact, it becomes necessary, in order to carry the purification beyond this limit, to apply the effluent to a second bed, in which again a further definite amount of purification can be effected. Hence, for obtaining a high degree of efficiency in bacterial purification of sewage, a system of multiple contact is generally necessary. Thus it may be taken broadly that in the first contact 50 per cent. of the dissolved impurity is removed, and that in the second contact 50 per cent. of the impurity still remaining in the effluent is disposed of, and so on." *

In subsequent experiments these conclusions were amply confirmed, and the Manchester Corporation eventually extended their sewage works, laying down five additional tanks, and a large number of contact beds (primary and secondary). These included 92 half-acre primary beds, with 26 acres storm-water filter-beds at Davyhulme,

* Rivers Department of Manchester, Experts' Report, 1899, p. 53. The Borough Surveyor of Leicester (Special Report, 1900) examined various bacterial systems for the disposal of the sewage of the Belgrave district, and finally recommends as the best method the following: (1) Crude sewage passed into an open or closed detritus tank to remove suspended mineral matters ; (2) then on to clarifying bacteria beds of 4 feet 6 inches depth, and containing crushed and screened clinkers (coarse and fine) from the refuse destructors; three fillings a day ; (3) finally, land purification of the effluent on old pasture. (Total purification of suspended matter of sewage $99 \cdot 12$ per cent. ; of albuminoid ammonia, 86.76 per cent.; and of oxygen absorbed 91.08 per cent.). 
and 46 acres of secondary contact beds on land at Flixton.* Dr Fowler, the superintendent and chemist, concludes that the bacterial process is best conducted in three stages:- $(a)$ Settlement and screening out of the grosser solids; (b) Anaërobic decomposition in the septic tank; and $(c)$ Oxidation on bacteria beds. He concludes in respect of the septic tanks:-

That the effluents from closed and open septic tanks are practically indentical in composition, and that with a tank space equal to half the daily flow of Manchester sewage, it is possible to digest about 25 per cent. of the total suspended matter in the sewage. The suspended matter in the septic-tank effluent is of a granular character, and readily separates out on standing, and when arrested on the surface of a bacteria bed does not seriously impede the free flow of the water into the bed. The organic matter in solution is much more easily nitrified than that present in fresh sewage, so that it is possible with one contact to constantly obtain non-putrefactive filtrates. The blending which takes place in the septic tank is of value in minimising the effect of excessive amounts of manufacturing refuse, and in producing an effluent of fairly constant composition.

In respect to contact beds he points out that the capacity of contact beds suffers a rapid initial decrease, but afterwards, with careful working, the rate of decrease is very much less.

The causes of loss of capacity appear to be five, namely - (a) Settling together of the material; (b) Growth of organisms; (c) Impaired drainage; (d) Insoluble matter entering bed; and $(e)$ Breaking down of material. These matters will be found fully discussed in the annual reports of the Rivers Department, and we have not space to enter into them here. We may, however, briefly refer to the conditions of the successful working of contact beds as arrived at as a result of the Manchester experience :-

(1) The bed should be worked very slowly at first, in order to allow it to settle down and the bacterial growths to form. In this way there will be less danger of suspended matter finding its way into the body of the bed, while the material is still loose and open. (2) The burden should not be increased till analysis reveals the presence of surplus oxygen, either dissolved or in the form of nitrates in the effluent. (3) Analyses of the air in the bed may usefully be made from time to time during resting periods. (4) The variations in capacity should be carefully recorded. If the capacity is found to be rapidly decreasing, a period of rest should be allowed. (5) Long periods of rest should be avoided during winter, as when deprived of the heat of the sewage the activity of the organisms decreases. If necessary, the burden on the bed should then be decreased by reducing the number of fillings per day, rather than by giving a long rest at one time. (6) The insoluble suspended matter should be retained on the surface by covering the latter with a layer of finer material not more than three inches in depth. The suspended matter thus arrested should not be raked into the bed, but when its amount becomes excessive it should be scraped off. This should be done if possible in dry, warm weather, after the bed has rested some days. By placing the inlet and outlet penstocks as close together as possible, the suspended matter will tend to concentrate in their vicinity, and its removal will be facilitated. $\dagger$

How far the various applications of the bacterial agency in purification will pass the scrutiny of the Royal Commission on Sewage Treatment, now sitting, it is impossible to say. But there can be no

* The particulars as to these new works, their construction, inaterials, capacities, etc., will be found in the Manclester Rivers Department Report, 1902, with plates and charts, pp. 18-24, and more recent extensions in subsequent Reports (1903-4).

$\dagger$ City of Manchester Rivers Department, Annual Report, 1902, p. 15. 
longer any doubt that some form of such agency is the only efficient, because the only natural, means of disposing of sewage.

\section{The Effect of the Bacterial Treatment upon Disease- Producing Organisms}

It has been urged from time to time by the advocates of the various methods of bacterial treatment, that pathogenic organisms are destroyed during the purification in many of these processes. It is clearly a matter of importance to know how far an effluent, in addition to being non-putrescible and fully nitrified, also possesses no disease-producing eapacity. We have already seen, from the researches of Klein, and Laws and Andrewes, that sewage is not a favourable medium for B. typhosus. The bacillus of Asiatic cholera is known to be but little less favoured by sewage. The spread of diphtheria by sewage is at least a matter of doubt, and Shattock's experiments tend to prove that in any case the virulence of the Bacillus diphtherioe is not increased by sewer air.

Anything like exhaustive researches into the effect of the septic tank or cultivation beds upon pathogenic germs has not been undertaken up to the present, and we can only conjecture as to their fate. Dr Houston has made a cautious declaration upon this matter, and at present we have not evidence to justify a more certain statement. "The balance of evidence," he says, "points to the probability that some, at all events, of the pathogenic organisms are crowded out in the struggle for existence in a nutritive medium containing a mixed bacterial flora, their vitality being weakened or destroyed by the enzymes of the saprophytic species." * He further adds: "It must be distinctly understood that I do not imply that such organisms as the typhoid bacillus or the cholera vibrio would necessarily lose their vitality, or even suffer a diminution in virulence under the conditions prevailing in a biological filter. In the absence of actual experiments, I am not prepared to say more than that I believe that if these germs did gain access to the sewage they would suffer a diminution in numbers primarily in the sewers [or septic tank], and secondarily in the coke-beds [or cultivation beds]." Subsequently, as a result of further experience of the effluent from the Crossness Sewage Works, Houston wrote, "However satisfactory the process may be from the chemical and practical point of view, the effluents from the bacterial beds cannot reasonably be assumed to be more safe in their possible relation to disease than raw sewage." $†$

Indirectly connected with this point, a word or two may be added concerning some recent investigations made by Dr Houston upon the

* Bacterial Treatment of Crude Seroage, 1899 (Second Report), p. 19.

† Eddin. Med. Jour., Feb. 1901. 
deposit which accumulates on coke fragments used in the beds at Barking and Crossness.* The coke was found to be coated with a black-coloured slimy deposit, free from objectionable smell, and almost odourless. On examination of the deposit, diluted with sterile water, and making cultures, it was found that the number of bacteria per gramme of the deposit was $1,800,000$. This number, large as it may seem, would weigh only a minute fraction of a gramme, $\uparrow$ so that it is evident that the number of living bacteria do not in themselves account in any way for the deposit. As to the nature of these organisms, Dr Houston adds: "The character of the microbes appearing in the cultures differed somewhat from those found in crude sewage. For example, there was an increase in the number of spores of Bacillus enteritidis sporogenes (Klein), and a decrease in the number of $B$. coli. Proteus-like germs were present in abundance, many being of $P$. mirabilis type. Further, $B$. arborescens and an allied form were present in considerable numbers. An organism apparently identical with $B$. prodigiosus was also isolated." + The deposit also contained a number of bacilli with precisely similar staining properties as those of tubercle bacilli (acidfast). They were also morphologically indistinguishable from the tubercle bacillus. In one instance Houston isolated a virulent tubercle bacillus from a sewage effluent. Such facts are of evident practical importance in relation to the final disposal of the effluent, whether it is discharged into a stream used for drinking purposes or otherwise.

Many of the researches having for their object the fate of pathogenic organisms in sewage have been based upon the typhoid bacillus as a type. Laws and Andrewes, in addition to demonstrating that this organism could only live in sewage a short time, showed that one sewage bacillus (B. fuorescens stercoralis) possessed the chief powers of antagonism, and it is probable that the contained bacteria rather than the chemical products of sewage act as unfavourable conditions for the typhoid bacillus. Horrocks found that in sterilised sewage the typhoid bacillus could live for sixty days. $\$$ Houston has thrown light upon the fate of $B$. typhosus by his work on the occurrence of $\bar{B}$. coli and $B$. enteritidis sporogenes in effluents, $\|$ and Miss Chick has furnished evidence in respect of $B$. coli, tending in the direction of showing that after sewage had passed over double contact beds about 75 per cent. of the $B$. coli were removed, and

* Bacterial Treatment of Crude Sewage (Supplement to Second Report), 1899,

$+\mathrm{Dr}$ Houston finds that $1,800,000$ typhoid bacilli weigh only 0.0000147 gramme.

$\ddagger 1 b i d$., p. 4.

\& Jour. of Sanitary Institute, January 1900.

|| First, Second, and Third Reports to the London County Council (vide supra). 


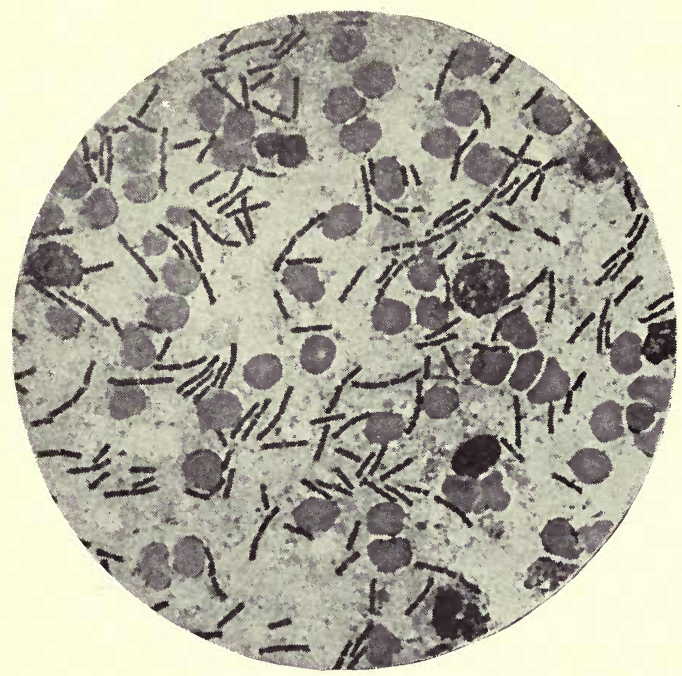

Bacillus anthracis. Smear preparation from splenic juice of guinea-pig that died after inoculation with 2 c.c. of Yeovil septic tank liquor. $\times 500$.

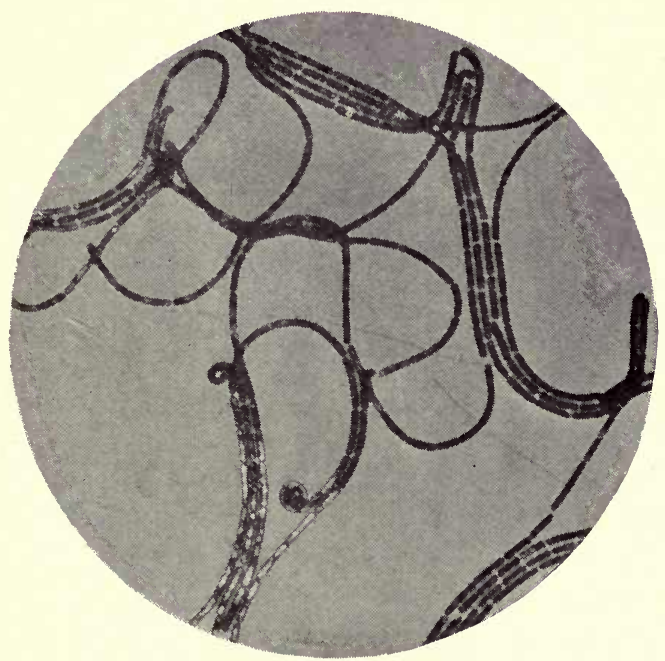

Bacillus anthracis. "Impression" preparation from a surface gelatine plate culture, 24 hours at $20^{\circ}$ C. Stained with methylene blue. $\times 1000$. 
O 
after land filtration practically an effluent might be free from $B$. coli.* Pickard has recently shown that the typhoid bacillus vanishes from crude sewage in about fourteen days. If the percentage of original amount of typhoid bacillus introduced into the sewage for experimental purposes be 100 ; in twenty-four hours it has fallen to 76 per cent., in thirty-two hours to 71 per cent., in forty-eight hours to 60 per cent., in seven days to 8 per cent., and in fourteen days to 0.73 per cent. He also demonstrated that a large proportion (90 per cent.) of typhoid bacilli are actually destroyed in filter-beds such as are used in the bacterial treatment of sewage. $\dagger$

Houston likewise has found the $B$. anthracis in septic-tank liquor and sludge, and in the secondary beds and general effluent. $\mathrm{He}$ also found the anthrax bacillus in the mud of the banks of the river Yeo at Yeovil within 150 feet of the main sewer. The spores of anthrax are peculiarly resistant, and it is in this form that the bacillus can pass through sewage unaffected $\ddagger$ (Plate 17). The same author has demonstrated that the B. pseudo-tuberculosis of Pfeiffer may be present in the effluent of various sewage processes, and the same is true of $B$. pyocyaneus which, however, occurs more rarely. Both these organisms are highly pathogenic to lower animals, and are also related to morbid processes occurring in the human subject. $§$ Houston, who has made extended inquiries on this question of the effect of bacterial treatment of sewage on pathogenic organisms, summarises his conclusions by stating that biological treatment on land, or by artificial processes, does not necessarily remove pathogenicity from the sewage effluent; that the absence of pathogenic result when sewage has been filtered shows that the products of pathogenic bacteria in sewage are not of a markedly poisonous nature; and that the pathogenicity of sewage may depend on spores rather than bacilli.\| These conclusions must, however, be accepted with reserve, and in a relative sense only, at present. Broadly, it may be said that if sewage contains pathogenic bacteria, and is then treated by bacterial methods, the effluent cannot be certainly assumed to be safer in this respect than the raw sewage slightly diluted; or, expressed in other words, Houston's work indicates " the inadvisability of relying on septic tanks, contact beds, or continuous filters to remove altogether the element of potential danger to health associated with the discharge of effluents from these processes of sewage treatment into drinking-water streams." - "

\footnotetext{
* Thompson-Yates Laboratory Report, vol. iii., part i., 1900.

† Jour. of State Medicine, 1903, pp. 203-210.

† Royal Commission on Sewage Disposal, Second Report, 1902, p. 39.

$\S 1 b i d$. , p. 54 .

It Ibid., Fourth Report, 1904, vol. iii., pp. 77-96.
} 


\section{CHAPTER VII}

\section{BACTERIA IN MILK AND MILK PRODUCTS}

General Principles-Sources of Pollution-Number of Bacteria in Milk-Influence of Time and Temperature-Species of Bacteria found in Milk-Fermentations of Milk-Pathogenic Organisms in Milk-Milk-borne Disease : Tuberculosis, Typhoid Fever, Scarlet Fever, Sore-Throat Illnesses, Cholera, Epidemic Diarrhœa-Preventive Measures-Protection of Milk Supply-Control of Milk Supply : Refrigeration, Straining, Sterilisation, Pasteurisation-Specialised Milk-Bacteria in Milk Products-Cream-Ripening-Butter-MakingCheese-Making-Abnormal Cheese-Ripening-Poisonous Cheese.

INJURIOUs micro-organisms in foods are, fortunately for the consumers, usually killed by cooking. Vast numbers are, as far as we know, of no harm whatever. Alarming reports of the large numbers of bacteria which are contained in this or that food are generally as irrelevant as they are incorrect. Bacteria, as we have seen, are ubiquitous. In food we have abundance of the chief thing necessary to their life and multiplication-favourable nutriment. Hence we should expect to find in uncooked or stale food an ample supply of saprophytic bacteria. There is much wholesome truth in the assertion that good food as well as bad frequently contains large numbers of bacteria, and often of the same species. It is well that we should become familiarised with this idea, for its accuracy cannot be doubted, and its acceptance at the present time may not be without beneficial effect.

Nevertheless, it is well we should know the bacterial flora of good and bad foods, for at least two reasons. First, there is no doubt whatever that a considerable number of cases of poisoning can be traced every year to food containing harmful bacteria or their products. To several of the more illustrative cases we shall have occasion to refer in passing. Secondly; we may approach the study of the bacteriology of foods with some hope that therein light will be found 
upon some important habits and effects of microbes. There can be little doubt that food-bacteria afford an example of association and antagonism of organisms to which reference has already been made. Any information that can be gleaned to illumine these abstruse questions would be very welcome at the present time. But there is a still further, and possibly an equally important, point to bear in mind, namely, the economic value of microbes in food. In a short account like the present it will be impossible to enter into hypotheses of pathology, but we shall at least be able to consider some of those interesting experiments which have been conducted in the sphere of beneficial bacteria.

The injurious effects of organisms contained in foods has been elucidated by the excellent work of the late Dr Ballard. From the careful study of a number of epidemics due to food poisoning, he was able, without the aid of modern bacteriology, to arrive at a simple principle which must not be forgotten. Food poisoning is due either to bacteria themselves or to their products, which are contained in the substance of the food. In cases of the first kind, bacteria gaining entrance to the human alimentary canal set up their specific changes and produce their toxins, and by so doing in course of time bring about a diseased condition, with its consequent symptoms. On the other hand, if the products, sometimes called ptomaines, are ingested as such, the symptoms set up by their action in the body tissues appear earlier. From these facts Dr Ballard deduced the simple principle that if there is no incubation period or, at all events, a comparatively short space of time between eating the poisoned food and the advent of disease, the agents of the disease are products of bacteria. (If, on the other hand, there is an incubation period, the agents are probably bacteria. )

It is necessary to mention two other facts. Dr Cautley has isolated from poisoned foods some of the different species of bacteria present.* It would appear that these are limited, as a rule, to two or three kinds. As regards disease, the organisms of suppuration are the most common. Liquefying or fermentative bacteria are frequently present, the Protens family being well represented. In addition there are, according to circumstances, a number of common saprophytes. Now, as we have pointed out, these organisms may act injuriously by some kind of co-operation, or they may by themselves be harmless, and pathological conditions be due to the occasional introduction of pathogenic species.

The other fact requiring recognition from any one who proposes to study the bacteriology of milk, or indeed of other foods, is, that a not inconsiderable amount of the evil results of food poisoning depends upon the tissues of the individual ingesting the foed.

* Report of Medical Officer to Local Government Board, 1895-96, Appendix. 
There is ample evidence in support of the fact that not all the persons partaking of infected milk suffer equally, and occasionally some escape altogether. We know little or nothing of the causes of such modification in the effect produced. It may be due to other organisms, or chemical substances already in the alimentary canal of the individual, or it may be due to some insusceptibility or resistance of the tissues. Be that as it may, it is a matter which must not be neglected in estimating the effects of food contaminated with bacteria or their products.

There are few liquids in general use which contain such enormous numbers of germs as milk. To begin with, milk is in every way, physical and physiological, admirably adapted to be a favourable medium for bacteria. It is constituted of all the chief elements of the nutriment upon which bacteria live.

Briefly, we may summarise the full diet of bacteria as:-nitrogenous matter (proteids); non-nitrogenous matter containing carbon and hydrogen (carbohydrates); calcium, potassium, phosphates, etc. (salts); and, for some species, oxygen. When we turn our attention to milk as a medium for bacteria, we find a complete bacterial diet -proteids represented by casein and lactalbumin, 4 per cent. in total-carbohydrates represented by lactose, the most readily affected of all the sugars by bacteria; fat as palmitin and olein; salts, potassium and calcium largely as phosphates, the calcium phosphate being united with casein. Even the normal reaction of milk, neutral or amphoteric, is favourable to the growth of bacteria, most of which find a definitely acid or a definitely alkaline reaction inimical to their growth. It is true that changes, mostly of a fermentative nature, rapidly set in, which affect milk as a medium for bacteria. But in its fresh, normal, untreated condition we have theoretically an almost ideal medium for both saprophytic and parasitic bacteria. Notwithstanding the truth of this general statement, we must not pass over the experiments of Fokker, Freudenreich, Cunningham, and others, which appear to demonstrate that freshly-drawn milk possesses for certain species of bacteria a germicidal power.

In the healthy condition of animals we have, generally speaking, no micro-organisms whatever in their secretions, whatever may be the condition of their excretions. Hence, though milk affords, from its constitution, such an ideal nidus for the growth and multiplication of bacteria, it is, as secreted, a perfectly sterile fluid. This was demonstrated more than twenty years ago by Lister, who states that " unboiled milk as coming from a healthy cow, really contains no material capable of giving rise to any fermentative change, or to the development of any kind of organism which we have the means of 
discovering." * Subsequent experiment has only confirmed the general truth of this statement. $\dagger$ With efficient precautions it is possible to draw from the udder of a healthy cow perfectly sterile milk, which retains its sterility unchanged for long periods of time in a sterilised and sealed flask. Yet we know by practical experience as well as by ultimate changes in the milk, that, generally speaking, the presence therein of bacteria is very marked.

\section{Sources of Pollution of Milk}

These are various, and depend upon many minor circumstances and conditions. For all practical purposes there are four chief opportunities between the cow and the consumer when milk may become contaminated with bacteria :-

1. At the time of milking and during manipulation at the farm.

2. During transit to the town, or dairy, or consumer.

3. At the milkshop.

4. In the home of the consumer.

Pollution at the time of milking arises from the animal, the milker, or unclean methods of milking. It is now well known that in tuberculosis of the cow affecting the udder the milk itself shows the presence of the bacillus of tubercle. In a precisely similar manner all bacterial diseases of the cow which affect the milksecreting apparatus must inevitably add their quota of bacteria to the milk. To this matter we shall have occasion to refer again. There is a further contamination from the animal when it is kept unclean, for it happens that the unclean coat of a cow will more materially influence the number of micro-organisms in the milk than the popularly supposed fermenting food which the animal may eat. It is from this external source rather than from the diet that organisms occur in the milk. The hairy coat offers many facilities for harbouring dust and dirt. The mud and filth of every kind that may be habitually seen on the hinder quarters of cattle all contribute largely to polluted milk. Nor is this surprising. Such filth at or near the temperature of the blood is an almost perfect environment for many of the putrefactive bacteria.

The milker is also a source of risk. His hands, as well as the clothes he is wearing, can and do readily convey both innocent and pathogenic germs to the milk. Clothed in dust-laden garments, and frequently characterised by dirty hands, the milker may easily act as an excellent purveyor of germs. Not a few cases are also on record where it appears that milkers have conveyed germs of

* Transactions of Pathological Society of London, 1878, p. 440.

$\uparrow$ See also Rotch, Pediatrics, the Hygiene and Medical Treatment of Children, London, 1896. 
disease from some case of infectious disease, such as scarlet fever, in their homes. But under the more efficient registration of such disease, which has recently characterised many dairy companies, the danger of infection from this source has been reduced to a minimum.

Professor Russell recounts a simple experiment, which clcarly demonstrates these simple but effective sources of pollution: " $\mathrm{A}$ cow that had been pastured in a meadow was taken for the experiment, and the milking done out of doors, to eliminate as much as possible the influence of the germs in the barn air. Without any special precaution being taken, the cow was partially milked, and during the operation a covered glass dish, containing a thin layer of sterile gelatine, was exposed for sixty seconds underneath the belly of the cow, in close proximity to the milk-pail. The udder, flank, and legs of the cow were then thoroughly cleaned with water, and all of the precautions referred to before, were carried out, and the milking then resumed. A second plate was then exposed in the same place for an equal length of time, a control also being exposed at the same time at a distance of ten feet from the animal and six feet from the ground to ascertain the germ contents of the surrounding air. From this experiment the following instructive data were gathered. Where the animal was milked without any special precautions being taken, there were 3250 bacterial germs per minute deposited on an area equal to the exposed top of a ten-inch milk-pail. Where the cow received the precautionary treatment as suggested above, there were only 115 germs per minute deposited on the same area. In the plate that was exposed to the surrounding air at some distance from the cow there were 65 bacteria. This indicates that a large number of organisms from the dry coat of the animal can be kept out of milk if such simple precautions as these are carried out."*

The influence of the byre air, and the cleanliness or otherwise of the byre, is obviously great in this matter. As we have seen, moist surfaces retain any bacteria lodged upon them; but in a dry barn, where molecular disturbance is the rule rather than the exception, it is not surprising that the air is heavily laden with microbic life derived from dust, dried manure, hay, straw, fodder, etc. Here again many improvements have been made by sanitary cleanliness in various well-known dairies. Still there is much more to be done in this direction to ensure that the drawn milk is not polluted by a microbe-impregnated atmosphere. Lastly, it should not be forgotten that during the straining and cooling of milk there are many opportunities of contamination.

The risks in transit differ according to many circumstances. * H. L. Russell, Dairy Bacteriology, p. 46. 
Probably the commonest source of contamination is in the use of unclean utensils and milk-cans. Any unnecessary delay in transit affords increased opportunity for multiplication; particularly is this the case in the summer months, for at such times all the conditions are favourable to an enormous increase of any extraneous germs which may have gained admittance at the time of milking. Thus we have (1) the milk itself affording an excellent medium and supplying ideal pabulum for bacteria; (2) a more or less lengthened railway journey or period of transit giving ample time for multiplication; (3) the favourable temperature of summer heat. We shall refer again to the rate of multiplication of germs in milk. It has been shown that milk brought into large cities, such as London, Paris, or New York, has been travelling often for as long as two to ten hours, often under conditions favourable to pollution or at least under conditions of temperature favourable to the multiplication of bacteria.

Pollution at the Milkshop.-Many are the advantages given to bacteria when milk has reached its commercial destination. In milkshops there are not a few risks to be added to the already imposing category. Water is occasionally, if not frequently, added to milk to increase its volume, either at the farm or the milkshop. Such water of itself will make its own contribution to the flora of the milk, unless indeed, which is unlikely, the water has been recently and thoroughly boiled before addition to the milk. Again, it is impossible to suppose that in small milkshops, perhaps of a general nature-where the milk stands for several hours, pollution is avoidable. From a hundred different sources such milk runs the risk of being polluted. The dust of the shop and the street gain access to the pan of milk on the counter, which, commonly, is uncovered. The "dipper" and the vendors' hands and clothes contribute bacteria. Flies also increase the pollution.

Pollution in the Home.-Lastly, there is pollution from dust and dirt, inorganic and organic, in the home. More than a million of the population of London live in tenements of two rooms or less. Cooking, eating, sleeping, cleaning, and sometimes even trade employments in the form of "home-work," are all conducted under conditions of overcrowding and lack of space. Often there is no pantry or larder, and consequently the days' supply of milk stands in a dirty uncovered vessel in the midst of dirty surroundings. It is evident that this is but one more opportunity for pollution.

Fore-Milk.-Before proceeding, a word must be said respecting the first milk which flows from the udder in the process of milking, and which is known as the fere-mille. This portion of the milk is always rich in bacterial life, on account of the fact that it has remained in the milk-ducts since the last milking. However thorough 
the manipulation, there will always be a residue remaining in the ducts, which will, and does, afford a suitable nidus and incubator for organisms. The latter obtain their entrance through the imperfectly closed teat of the udder, and pass readily into the milk-duct, sometimes even reaching the udder itself and setting up inflammation (mastitis). Professor Russell states that he has found 2800 germs in the fore-milk, in a sample of which the average was only 330 per c.c. Schultz found 83,000 micro-organisms per c.c. in the fore-milk, and only 9000 in the mid-milk. As a matter of fact, most of this large number belong to the lactic acid fermentation group, and the fore-milk rarely contains more than two or three species, and still more rarely any disease-producing bacteria. Still, bacteria occur in such enormous numbers that their addition to the ordinary milk very materially alters its quality. Bolley and Hall, of North Dakota, report sixteen species of bacteria in the fore-milk, twelve of which produced an acid reaction. Dr Veranus Moore, of the United States Department of Agriculture,* concludes from a large mass of data that freshly-drawn fore-milk contains a variable but generally enormous number of bacteria, but only a few species, the last milk containing, as compared with the fore-milk, very few micro-organisms. The bacteria which become localised in the millk-ducts, and which are necessarily carried into the milk, are for the greater part acidproducing organisms, i.e., they ferment milk-sugar, forming acids. They do not produce gas. Nevertheless their presence renders it necessary to "pasteurise" as soon as possible. Dr Moore holds that much of the intestinal trouble occurring in infants fed with ordinarily "pasteurised" milk arises from acids produced by these bacteria between the drawing of the milk and the pasteurisation. Prof. MacFadyean has given a full account of the ways in which milk becomes pathogenic, and his views have received further support from Prof. Delépine. $†$

\section{The Number of Bacteria in Milk}

From all that has been said respecting the sources of pollution and the favourable nidus which milk affords for bacteria, it is not surprising that a very large number of germs are almost always present in milk. The quantitative estimation of milk appears more alarming than the qualitative. It is true some diseases are conveyed by bacteria in milk, but on the whole most of the species are nonpathogenic. Nor need the numbers, though serious, too greatly alarm us, for, as we shall see at a later stage, disease is due to other agencies and conditions than merely the bacteria, which may be the

* Bureau of Animal Industry Reports, 1895-96.

† Jour. of Comp. Path., 1897, vol. x., pp. 150-189. 
vera causa. In addition to the fact that the high numbers have but a limited significance, we must also remember that there is no uniformity whatever in these numbers. The conditions which chiefly control them are (1) time, and (2) temperature.

The Influence of Time and Temperature.-We have already noticed, when considering the general conditions affecting bacteria, how potent an agent in their growth is the surrounding temperature. Generally speaking, temperature at or about blood-heat favours bacterial growth. Freudenreich has drawn up the following table which graphically sets forth the effect of temperature upon bacteria in milk:-

\begin{tabular}{|c|c|c|c|c|}
\hline & 3 hours. & 6 hours. & 9 hours. & 24 hours. \\
\hline $\begin{array}{l}59^{\circ} \mathrm{F} . \\
77^{\circ} \mathrm{F} . \\
95^{\circ} \mathrm{F} .\end{array}$ & $\begin{array}{l}1+ \\
2 \\
4\end{array}$ & $\begin{array}{r}2 \cdot 5 \\
18 \cdot 5 \\
1290\end{array}$ & $\begin{array}{r}5 \\
107 \\
3800\end{array}$ & $\begin{array}{r}163 \\
62,100 \\
5,370\end{array}$ \\
\hline
\end{tabular}

It will be noticed that at $59^{\circ} \mathrm{F}$. there is very little multiplication. That may be accepted as a rule. At $77^{\circ} \mathrm{F}$. the multiplication, though not particularly rapid at the outset, results finally, at the end of the twenty-four hours, in the maximum quantity. These were probably common species of saprophytic bacteria, which increase readily at a comparatively low temperature. During the subsequent hours, after the twenty-four, we should expect a temporary decline rather than an increase in 62,000 , owing to the keen competition consequent upon the limitation of the pabulum. From a consideration of these facts, we conclude that a warm temperature, somewhat below blood-heat, is most favourable to multiplication of bacteria in milk; that the common saprophytic organisms multiply the most rapidly; that, in the course of time, competition kills off a large number.

Another example may be taken from Professor Conn:-

Number of Bacteria per cubic centimetre in milk kept at different temperatures.

\begin{tabular}{|c|c|c|c|c|c|c|}
\hline $\begin{array}{l}\text { No. at } \\
\text { outset. }\end{array}$ & $\begin{array}{l}\text { In } 12 \mathrm{hrs} \text {. } \\
\text { at } 50^{\circ} \text {. }\end{array}$ & $\begin{array}{l}\text { In } 12 \mathrm{hrs} \text {. } \\
\text { at } 70^{\circ} \text {. }\end{array}$ & $\begin{array}{l}\text { In } 50 \mathrm{hrs} \text {. } \\
\text { at } 50^{\circ} .\end{array}$ & $\begin{array}{l}\text { In } 50 \mathrm{hrs.} \text {, or at } \\
\text { time of curdling, } \\
\text { at } 70^{\circ} .\end{array}$ & $\begin{array}{l}\text { No. of hrs. } \\
\text { to curdling } \\
\text { at } 50^{\circ} \text {. }\end{array}$ & $\begin{array}{l}\text { No. of hrs. } \\
\text { to curdling } \\
\text { at } 70^{\circ} \text {. }\end{array}$ \\
\hline 46,000 & 39,000 & 249,500 & $1,500,000$ & $542,000,000$ & 190 & 56 \\
\hline 47,000 & 44,800 & 360,000 & 127,500 & $\begin{array}{c}792,000,000 \\
36 \text { hours }\end{array}$ & 289 & 36 \\
\hline 50,000 & 35,000 & 800,000 & 160,000 & $\begin{array}{c}2,560,000,000 \\
42 \text { hours }\end{array}$ & 172 & 42 \\
\hline
\end{tabular}


So strongly convinced is Conn of the exceptional influence of temperature on the increase of bacteria in milk, and the subsequent souring, that he holds that " the keeping of milk is more a matter of temperature than of cleanliness." The cooling of milk immediately after milking, and keeping it at a low temperature, will do more for its preservation than any other practical device. Conn has also pointed out that lactic organisms flourish in milk when it is kept at temperatures above $50^{\circ} \mathrm{C}$. He summarises the influence of temperature as follows:-

(1) Variations in temperature have a surprising influence upon the rate of multiplication of bacteria. At $50^{\circ} \mathrm{F}$. these organisms may multiply only five-fold in twenty-four hours, while at $70^{\circ}$ they may multiply seven hundred and fifty-fold. (2) Temperature has a great influence upon the keeping property of milk. Milk kept at $95^{\circ}$ (heat of the cow's body) will curdle in eighteen hours, while the same mills kept at $70^{\circ}$ will not curdle for forty-eight hours, and if kept at $50^{\circ}$, the temperature of an ice-chest, may sometimes keep without curdling for two weeks or more. (3) So far as the keeping property of milk is concerned, the matter of temperature is of more significance than the original contamination of the milk with bacteria. (4) Milk preserved at $50^{\circ}$ or lower will keep sweet for a long time, but it becomes filled with bacteria of a more unwholesome type than those that grow at higher temperatures*

The influence of time is not less marked than that of temperature, as the following table will show:-

\begin{tabular}{|c|c|c|c|c|c|}
\hline Milk & Arav & n at $59^{\circ} \mathrm{F}$. & $=$ & 153,000 & m.o. per cub. inch. \\
\hline After & 1 & hour & $=$ & 616,000 & , \\
\hline ," & 2 & ,, & $=$ & 539,000 & ," \\
\hline ," & 4 & ," & $=$ & 680,000 &, \\
\hline ," & 7 & ,, & $=$ & $1,020,000$ & ,, \\
\hline , & 9 & , & $=$ & $2,040,000$ & ," \\
\hline ," & 24 & ,, & $=$ & $85,000,000$ & , \\
\hline
\end{tabular}

Freudenreich gives another example, as follows:-

$\begin{array}{llll}\text { Milk drawn at } 15 \cdot 5^{\circ} \mathrm{C} . & = & 27,000 \text { m.o. per c.c. } \\ \text { After } 4 \text { hours } & = & 34,000 & , \\ , " 9, & = & 100,000 & , \\ , 24, & = & 4,000,000\end{array}$

Concerning these figures little comment is necessary. But here again, also, we may remember that this rapid multiplication only continues up to a certain point, after which there is a marked reduction owing to products of activity.

Quite recently further investigations have been made in milk maintained at a standard temperature by various workers. For the

* Storr's Agric. Expt. Sta. Conn. Bull. 26 (H. W. Conn). 
sake of comparison with other statistics, we may take two series recorded by Park. In the first the temperature was $90^{\circ} \mathrm{F}$, a temperature common in New York in hot summer weather, and the samples of milk were of three degrees of quality, namely, fresh and good, fair, and bad. The result was as follows:-

\begin{tabular}{|c|c|c|c|c|c|}
\hline & & & \multicolumn{3}{|c|}{ Number of Bacteria per 1 c.c. } \\
\hline & & & Good Fresh Milk. & $\begin{array}{l}\text { Fair Milk from } \\
\text { Store. }\end{array}$ & $\begin{array}{l}\text { Bad quality from } \\
\text { Store. }\end{array}$ \\
\hline \multicolumn{3}{|c|}{ Original number of Bacteria. } & 5,200 & 92,000 & $2,600,000$ \\
\hline After 2 & ours & .. & 8,400 & 184,000 & $4,220,000$ \\
\hline, 4 & , . & . & 12,400 & 470,000 & $19,000,000$ \\
\hline, 9 &, & . & 68,500 & $1,260,000$ & $39,000,000$ \\
\hline , 8 & , . & . $\quad$. & 654,000 & $6,800,000$ & $124,000,000$ \\
\hline
\end{tabular}

The second series of Park was milk taken from cows in common dirty stalls, twenty-four, thirty-six, and forty-eight hours after milking. The milk was cooled to $52^{\circ} \mathrm{F}$., three hours after milking, and maintained at that temperature, for the forty-eight hours of the experiment. The result, therefore, shows the effect of time even more exactly than the first series:-

\begin{tabular}{|c|c|c|c|}
\hline \multicolumn{3}{|c|}{ Average Number of Bacteria per 1 c.c. of Milk at $52^{\circ} \mathrm{F}$. (six samples). } \\
\hline After 3 hours. & After 24 hours. & After 36 hours.* & After 48 hours. \\
\hline 30,366 & 69,433 & 348,883 & $1,668,333$ \\
\hline
\end{tabular}

* The figures at 36 hours were estimated from the test of one sample only.

Even a cursory examination of these figures with those already given will have shown how intimately the two influences of time and temperature act and interact in relation to the multiplication of micro-organisms in milk. They are scarcely separable, and no hardand-fast line can be drawn by way of comparison of these two influences.

Reference may also be made to two investigations made, one by Park of New York, and the other carried out by Swithinbank and the writer.

The following figures obtained by Park show the development of bacteria in two samples of milk maintained at different temperatures for twenty-four, forty-eight, and ninety-six hours respectively. The first sample was obtained under the best conditions possible, the second in the usual way (the figures of this sample are underlined). 
When received, Specimen No. 1 contained 3000 bacteria per c.c., and Specimen No. 2, 30,000 per c.c.

\begin{tabular}{|c|c|c|c|c|c|}
\hline \multirow{2}{*}{ Temperature. } & & \multicolumn{4}{|c|}{ Time which elapsed before making the test. } \\
\hline & & 24 hours. & 48 hours. & 96 hours. & 168 hours. \\
\hline$\pi^{32^{\circ} \mathbf{F} .\left(0^{\circ} \text { C. }\right)}$ & - & $\begin{array}{r}2,400 \\
30,000 \\
\end{array}$ & $\begin{array}{r}2,100 \\
27,000 \\
\end{array}$ & $\begin{array}{r}1,850 \\
24,000\end{array}$ & $\begin{array}{r}1,400 \\
19,000 \\
\end{array}$ \\
\hline $39^{\circ} \mathrm{F} .\left(4^{\circ} \mathrm{C}.\right)$ & - & $\begin{array}{r}2,500 \\
38,000 \\
\end{array}$ & $\begin{array}{r}3,600 \\
56,000\end{array}$ & $\begin{array}{r}218,000 \\
4,300,000 \\
\end{array}$ & $\begin{array}{r}4, \overline{200,000} \\
38,000,000 \\
\end{array}$ \\
\hline $42^{\circ} \mathrm{F} .\left(5 \cdot 5^{\circ} \mathrm{C}.\right)$ & - & $\begin{array}{r}2,600 \\
43,000\end{array}$ & $\begin{array}{r}\overline{3,600} \\
210,000\end{array}$ & $\begin{array}{r}500,000 \\
5,760,000\end{array}$ & \\
\hline $46^{\circ} \mathrm{F} .\left(6^{\circ} \mathrm{C}.\right)$ & • & $\begin{array}{r}3,100 \\
42,000\end{array}$ & $\begin{array}{r}12,000 \\
360,000\end{array}$ & $\begin{array}{r}1,480,000 \\
12,200,000\end{array}$ & \\
\hline $50^{\circ} \mathrm{F} .\left(10^{\circ} \mathrm{C}.\right)$. & . & $\begin{array}{l}\overline{11,600} \\
89,000\end{array}$ & $\begin{array}{r}\overline{540,000} \\
1,940,000\end{array}$ & & \\
\hline $55^{\circ}$ F. $\left(13^{\circ}\right.$ C. $)$ & • & $\begin{array}{r}18,800 \\
187,000\end{array}$ & $\begin{array}{r}\overline{3,400,000} \\
38,000,000\end{array}$ & & \\
\hline $60^{\circ} \mathrm{F} .\left(16^{\circ} \mathrm{C}.\right)$ & . & $\begin{array}{l}180,000 \\
900,000\end{array}$ & $\begin{array}{r}28,000,000 \\
168,000,000\end{array}$ & & \\
\hline $68^{\circ} \mathrm{F} .\left(20^{\circ}\right.$ C. $)$ & . & $\begin{array}{r}450,000 \\
4,000,000\end{array}$ & $\begin{array}{l}25, \overline{000,000,000} \\
25,000,000,000\end{array}$ & & \\
\hline $86^{\circ} \mathrm{F} .\left(30^{\circ} \mathrm{C}.\right)$ & - & $\begin{array}{r}1,400,000,000 \\
14,000,000,000\end{array}$ & & & \\
\hline $94^{\circ} \mathrm{F} .\left(35^{\circ} \mathrm{C}.\right)$ & . & $\begin{array}{l}25,000,000,000 \\
25,000,000,000\end{array}$ & · & & \\
\hline
\end{tabular}

There are two points in this table which may be noted. First, it may be seen that at $32^{\circ} \mathrm{F}$. $\left(0^{\circ} \mathrm{C}\right.$.) there is a decline in the number of organisms both in good and bad milk during the first 168 hours. At all the other temperatures, to which there is no exception, there is a rise in the number of organisms. Secondly, the numbers of bacteria at $20^{\circ} \mathrm{C}$. in forty-eight hours are equal to the numbers at $35^{\circ} \mathrm{C}$. in twenty-four hours, and in both instances the number is phenomenally high.

In 1900, Mr Swithinbank and the writer conducted a series of experiments as part of an inquiry into the behaviour of bacteria in milk, during which careful observation was made of a certain milk from the time it was drawn from the udder up to thirty days, and then subsequently after two years. Further, the observations were made at three different temperatures. Broadly speaking, the conclusions were as follow:-

First, there was an extremely rapid increase in the number of organisms in the first four hours, particularly at $37^{\circ} \mathrm{C}$. At the 
commencement the milk contained 812,000 bacteria per c.c. After four hours it contained 2,066,000 (at $5^{\circ}$ C.), $3,650,000$ (at $15^{\circ} \mathrm{C}$.), and $6,116,000$ (at $37^{\circ}$ C.).

Secondly, speaking in a general way, the following great principle became evident, namely, that there is at each temperature $(a)$ a sudden rise, $(b)$ a sudden fall, $(c)$ a steady rise to maximum, and $(d)$ a steady fall ultimately to sterility. In other words, there are tides of organisms, and this was found to occur invariably in our study of "natural" milks. It is a variable phenomenon in ordinary milks, but is the rule in respect to "natural" milk examined immediately after milking. It is obvious that if we had commenced our examination, as is frequently the case in the study of town milks, twelve or twenty hours after milking, we should, even if we had obtained the same figures, have drawn very different deductions, because the initial rise and initial fall would have been lost sight of. The sudden fall occurred in forty-eight hours at $5^{\circ} \mathrm{C}$., in twelve hours at $15^{\circ} \mathrm{C}$. and $37^{\circ} \mathrm{C}$.

Thirdly, the maximum number of bacteria occurred in ten days at $5^{\circ}$ C. $\left(406,400,000\right.$ bacteria per c.c.), in six days at $15^{\circ} \mathrm{C}$. $(84,000,000)$, and in seventy-two hours at $37^{\circ} \mathrm{C} .(8,360,000)$. The maximum was lowest at blood-heat and highest at $5^{\circ} \mathrm{C}$. It is evident, therefore, that what occurs in a short time at a high temperature occurs in a longer period at a low temperature, but at a low temperature the bacteria eventually become most numerous. These facts are of great importance in relation to the time which milk is kept before use, and to the injurious properties which it may acquire during such a period in the direction of increased bacterial toxin production.

Fourthly, marked acidity commenced between the twelfth and sixteenth hours in the sample at $37^{\circ}$; between the twentieth and twenty-fourth hours at $15^{\circ}$; and between the seventy-second and ninety-sixth hours at $5^{\circ} \mathrm{C}$. At the end of these particular stages it will be noticed that there is a rising tide following the "low-water mark" of organisms at each temperature. The relation which the degree of acidity bears to the bacterial content is an intimate one. As far back as 1878 Lister pointed out the marked inhibitory effect which the presence of a high degree of lactic acid had upon common moulds and ordinary saprophytic bacteria.* When the lactic acid declines, these other forms commence growth, and eventually enormously preponderate.

Fifthly, as the flasks of milk were kept intact, we were able to repeat the experiment in every particular after the lapse of exactly two years from the commencement. The milk was the same milk, 
and the experiment was repeated as at thirty days. During the intervening period the flasks had been kept, hermetically sealed, at the three temperatures. The result was that the flasks were found to be germ-free, with the exception of an abundant growth of Oidium lactis and other moulds.

Mr Swithinbank and the writer came to the conclusion that the explanation of the results of this investigation could only be found in a glance at the life-history of such a milk as that under consideration.

"At the time of milking there is, as we have seen, an introduction into the warm milk of vast numbers of common saprophytic and parasitic bacteria. Finding themselves in an ideal nidus, they multiply with almost incredible rapidity. Hence the first rise in numbers of bacteria. Competition and exhaustion of pabulum soon produce inevitable effects, and we obtain the first decline. At this stage it may be said that the common extraneous bacteria, whether putrefactive or simple saprophytes, practically die out, and that for a very simple reason, namely, that they cannot live in the presence of the new tide of acid-forming bacteria. Although the lactic acid group of organisms do not multiply as rapidly as ordinary saprophytes, they reach a much higher maximum in the end. It is to this family of bacteria that the second and maximum rise is due. In time, also, the same inimical conditions begin to act, and the lactic acid bacteria decline owing to the acidity and to the lack of pabulum. Eventually, the medium, which twenty days before was an ideal one for any organism, and mostly so for those which came first, and which ten days before was favourable to lactic acid organisms, is now favourable to no bacteria at all. Accordingly, bacteria of all descriptions gradually die out, and the medium is eventually left in possession of Oidium lactis and the common moulds. That the destruction of large quantities of solid albuminous substances may occur simply through bacterial agencies has been conclusively shown in the so-called septic tank method of sewage disposal. The death of bacteria under these circumstances always follows shortly after their enormous multiplication, and how much is due to starvation or how much to poisoning by the products of their own activity it is impossible to say. It is, however, clear that the decomposition of large quantities of albuminous substance is first accompanied by great bacterial reproduction, and this is invariably followed by a season of speedy and extreme mortality of bacteria. In a general way that represents, we believe, the changes taking place as represented in the record we have considered. That there are two rises and two falls in the number of bacteria, the first rise being due to extraneous organisms, and the second rise to lactic acid organisms, we believe to be the almost universal rule in untreated 'natural ' milk." *)

The effect of temperature and time has been illustrated by Dr Buchanan Young's researches into the numbers of bacteria in milk according to season, and the results of which were laid before the Royal Society of Edinburgh. He estimated in the Edinburgh milk supply that three hours after milking there were 24,000 micro-organisms per c.c. in winter; 44,000 in spring; 173,000 in late summer and autumn. Again, he found that five hours after milking there were 41,000 micro-organisms per c.c. in country milk, and more than 350,000 micro-organisms per e.c. in town milk. Many London milks would

* Bacteriology of Milk (Swithinbank and Newman), 1903, p. 135. 
exceed 500,000 per c.c.* In summer the writer has found as many as 4,000,000 organisms per e.c. in fresh London milk obtained at firstrate milkshops. $\dagger$

There is no standard or uniformity in the numerical estimation of bacteria in milk. A host of observers have recorded widelyvarying returns due to the widely-varying circumstances under which the milk has been collected, removed, stored, and examined, and due also to the two dominating influences of time and temperature. Nor is it possible to establish any standard which may be accepted as a normal or healthy number of bacteria, as is done in water examination. Bitter has suggested 50,000 micro-organisms per c.c. as a maximum limit for milk intended for human consumption, but actual experience shows that, at present at all events, such standards are impracticable.

Owing to differences of nomenclature and classification, in addition to differences in mode of examination, at present existing in various countries, it is impossible to state even approximately how many bacteria, and how many species of bacteria, have been isolated from milk. Until some common international standard is established, mathematical computations are practically worthless. They are needlessly alarming and sensational. And it should be remembered that great reliance cannot be placed upon these numerical estimations, for they vary from day to day, and even hour to hour. Furthermore, vast numbers of bacteria are economic in the best sense of the term, and the bacteria of milk are chiefly those of a fermentative kind, and not disease-producers. ${ }^{+}$

The effects of time and temperature upon the bacteria of milk do not only concern the numbers of organisms present. As long ago as 1897 Delépine showed that toxicity of milk was increased by a rise in temperature, and this is as we should expect, for it stands to reason that conditions favourable to the multiplication of bacteria in milk must of necessity tend to increase the products of bacteria in milk, and is likely to increase its virutence. $\$$

The following tables summarise the more detailed figures given in 1897, and in which the effects which length of keeping and of temperature have upon the noxious effects of the milk are indicated.

* Brit. Med. Jour., 1895, ii., p. 322.

† Report on Milk Supply of Finsbury, 1903.

\# At the same time it is important to remember that comparative series of estimations as to the number of bacteria per c.c. in milk may be of value as indication of unclean dairying. Leighton of Montclair, U.S.A., has shown that numbers increase in direct proportion to unclean management. See The Milk Supply of 'Two IIundred Cities and Towns (Alvord \& Pearson), U.S. Dep. of Agriculture, Bull. 46, 1903, p. 117.

\$ Jour. of Comp. Path. and Therapeutics, 1897. 
Delépine showed that mixed milks coming from a distance of over 40 miles, and generally kept for from twenty-four to sixty hours, and even more in a few cases (tuberculous samples excluded), gave the following returns:-

\begin{tabular}{|c|c|c|c|c|}
\hline $\begin{array}{c}\text { Mean Temperature in the } \\
\text { Shade (Manchester) } \\
\text { during Time the Specimens } \\
\text { were kept. }\end{array}$ & $\begin{array}{c}\text { Specimens } \\
\text { producing } \\
\text { no Noxious } \\
\text { Effects. }\end{array}$ & $\begin{array}{c}\text { Noxious } \\
\text { Specimens. }\end{array}$ & Totals. & $\begin{array}{c}\text { Percentage } \\
\text { of Good } \\
\text { Specimens. }\end{array}$ \\
\hline Deg. Fahr. & 7 & 5 & 12 & $58 \cdot 0$ \\
30 to 35 & 7 & 11 & 18 & $38 \cdot 5$ \\
35 to 40 & 2 & 3 & 5 & $40 \cdot 0$ \\
40 to 45 & 1 & 4 & 5 & $20 \cdot 0$ \\
45 to 50 & $\ldots$ & 2 & $\ldots$ & $\ldots \cdot 0$ \\
50 to 55 & 0 & 2 & \\
55 to 60 & 17 & 25 & 42 & $39 \cdot 0$ \\
& & & & \\
\hline
\end{tabular}

Mixed milks coming from a short distance (generally under 20 miles), most of them kept for less than ten hours (with the exception of five out of the seven bad specimens, and four out of the twenty-two good specimens, which had been kept somewhat longer), (tuberculous samples excluded), gave the following results :-

\begin{tabular}{|c|c|c|c|c|}
\hline $\begin{array}{l}\text { Mean Temperature in the } \\
\text { Shade (Manchester) } \\
\text { during Time the Specimens } \\
\text { were kept. }\end{array}$ & $\begin{array}{c}\text { Specimens } \\
\text { producing } \\
\text { no Noxious } \\
\text { Effects. }\end{array}$ & $\begin{array}{c}\text { Noxious } \\
\text { Specimens. }\end{array}$ & Totals. & $\begin{array}{l}\text { Percentage } \\
\text { of Good } \\
\text { Specimens. }\end{array}$ \\
\hline $\begin{array}{l}\text { Deg. Fahr. } \\
50 \text { to } 55 \\
55 \text { to } 60 \\
60 \text { to } 65 \\
65 \text { to } 70 \\
70 \text { to } 75\end{array}$ & $\begin{array}{r}1 \\
8 \\
11 \\
\cdots \\
2\end{array}$ & $\begin{array}{r}0 \\
1 \\
4 \\
\dddot{2}\end{array}$ & $\begin{array}{r}1 \\
9 \\
15 \\
\cdots \\
4\end{array}$ & $\begin{array}{r}100 \cdot 0 \\
88 \cdot 8 \\
73 \cdot 2 \\
\ldots \\
50 \cdot 0\end{array}$ \\
\hline & 22 & 7 & 29 & $75 \cdot 68$ \\
\hline
\end{tabular}

Whilst unmixed, milks kept for various lengths of time, but collected from the udder in sterilised vessels (tuberculous samples excluded), resulted as follows :- 


\begin{tabular}{|c|c|c|c|c|}
\hline $\begin{array}{c}\text { Mean Temperature in the } \\
\text { Shade (Manehester) } \\
\text { during Time the Specimens } \\
\text { were kept. }\end{array}$ & $\begin{array}{c}\text { Specimens } \\
\text { producing } \\
\text { no Noxious } \\
\text { Effects. }\end{array}$ & $\begin{array}{c}\text { Noxious } \\
\text { Specimens. }\end{array}$ & Totals. & $\begin{array}{c}\text { Percentage } \\
\text { of Good } \\
\text { Specimens. }\end{array}$ \\
\hline \begin{tabular}{c|c} 
Deg. Fahr. \\
35 to 40
\end{tabular} & 6 & 0 & 6 & $100 \cdot 0$ \\
40 to 45 & 3 & 2 & 5 & $60 \cdot 0$ \\
45 to 50 & $\ldots$ & 2 & 7 & $71 \cdot 5$ \\
50 to 55 & $\ldots$ & $\cdots$ & $\cdots$ & $\ldots$ \\
55 to 60 & 0 & 3 & 3 & 0.0 \\
60 to 65 & 14 & 7 & 21 & $67 \cdot 2$ \\
\hline
\end{tabular}

"The influence of time," Prof. Delépine adds, "is well shown by the number of specimens remaining good, even at a high temperature, when the milk had been kept only half a day. On the other hand, the influence of temperature is still more. evident, for in every category the number of good specimens is almost inversely proportional to the height of the temperature. Still, it is important to keep the two factors of time and temperature in mind. What is produced in a few hours in summer may also occur in winter, when the milk has been kept a long time.")

The converse is also true, namely, that f the temperature of milk be reduced by refrigeration, the toxicity of the milk is lessened Professor Delépine has shown that the mortality from all causes in guinea-pigs inoculated with refrigerated milk is considerably less than it is if unrefrigerated milk be inoculated :-

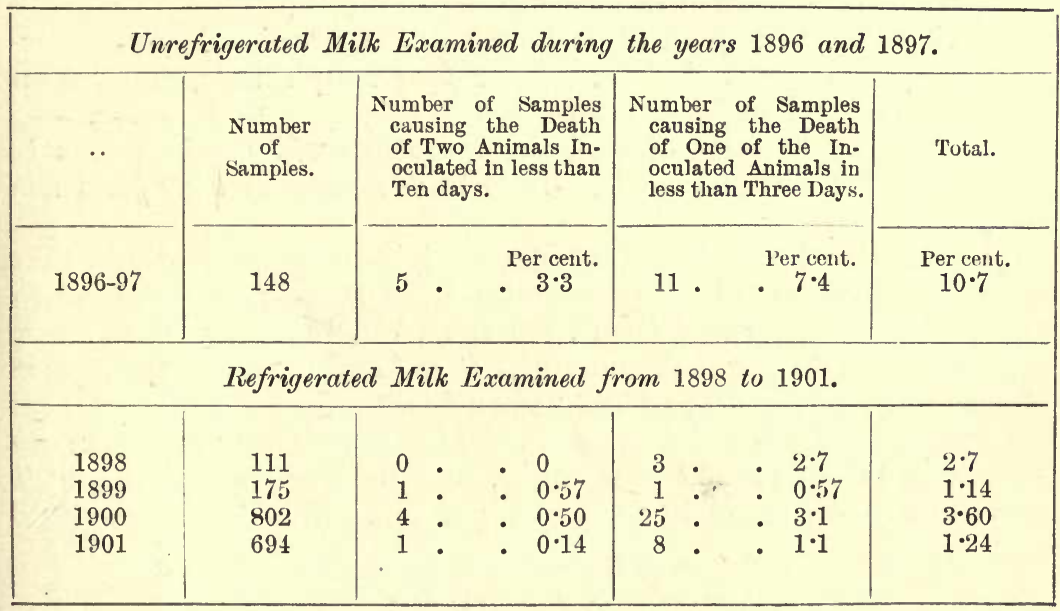


"The inference to be drawn from these gross results is clear: a certain proportion of the samples of milk contained bacteria which, under favourable circumstances, gave to the milk noxious properties, the development of which could be checked in many cases by preventing the growth of these bacteria. (The difference between refrigerated and non-refrigerated milk would have been very much greater, if the milk had invariably been cooled immediately after the milking of the cows' (Delépine).

Therefore, it may be said that to refrigerate milk immediately after drawing it from the cow is to reduce the number of bacteria and to diminish the potential toxicity of the milk. Finally, Professor Delépine writes :-

"When the clear relation existing between time of keeping, plus temperature and the noxious properties of a certain number of samples of milk, is contrasted with the ambiguous results obtained when an attempt is made to connect these noxious properties with disease of the udder (tuberculosis being excluded), it is difficult not to feel convinced that infection of the milk outside the udder, and the conditions under which milk is kept, are the most important factors causing it to acquire infective properties."*

\section{Species of Bacteria found in Milk}

The kinds of bacteria occurring in milk may for purposes of convenience be classified in the following four divisions; though of the first two groups it is not necessary to say much here:-

1. Ordinary bacteria of air, soil, or water.

-2. Bacteria of sewage or intestinal origin.

3. Bacteria concerned in fermentation.

4. Pathogenic bacteria, in particular those associated with tuberculosis, enteric fever, cholera, scarlet fever, diphtheria, sore-throat illnesses, and epidemic diarrhœa.

1. Ordinary bacteria of soil, air, or water readily gain access to milk from their natural media. It is unnecessary to consider them here.

2. Bacteria of sewage and intestinal origin occur from time to time in milk. The two chief representatives are B. coli and B. enteritidis sporogenes. In Liverpool, from 1900-1902, 788 " country" milks were examined, and 55 per cent. contained $B$. coli and 9 per cent. contained B. enteritidis sporogenes; of 722 "town" milks, 23 per cent. contained the former bacillus, and 4 per cent. the latter.† Chick found B. coli present in 17 out of 239 new milks, and Balfour Stewart found $B$. enteritidis sporogenes in 49 samples out of 213 . When it is considered 
how filthily many cows are kept, it is not to be wondered that many intestinal organisms find their way to milk.

3. Bacteria concerned in fermentations in milk cannot well be understood without some appreciation of the different elements of milk which are most affected by the changes of fermentation. It is therefore necessary, before proceeding, to consider shortly what are the constituents of milk upon which living ferments of various kinds exert their action, for without these facts the action of fermentation bacteria is not evident. A tabulation of the chief constituents of milk may be stated as follows:-

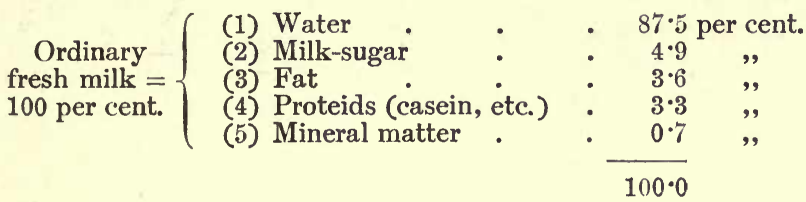

Or the average milk constitution may be expressed thus :-

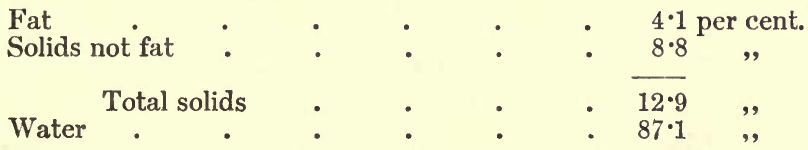

It is not necessary to remark that milks vary in standard, and the above figures can only be taken as fair averages.

Milk-sugar, or Lactose $\left(\mathrm{C}_{12} \mathrm{H}_{24} \mathrm{O}_{12}\right)$, is an important and constant constituent of milk. It forms the chief substance in solution in whey or serum, and is a member of the cane-sugar group. Milk-sugar is found in varying quantities in the milk of mammals. About 5 per cent. is present in human milk, and somewhat less in that of the cow. It is very resistant to fermentation by yeast, and therefore undergoes alcoholic fermentation very slowly. It is not acted upon by rennet, pepsin, or trypsin. But of all the sugars it is most readily acted upon by micro-organisms.

Fat occurs in milk as suspended globules of varying size. It forms the cream, and by churning is, of course, made into butter, though both cream and butter contain other constituents besides fat. Lloyd has shown that it is the large globules that form the cream, and he has also made observations-upon the size of fat globules in relation to breed of cattle. The decomposition and breaking down of milk-fat by fermentation is the chief cause of gross abnormalities of cream and the rancidity of butter.

The Proteids of Milk include casein, lactalbumin, and lactoglobulin. Casein is by far the most abundant and the most important. When milk separates naturally into its constituent parts the fat rises and the casein falls, leaving a clear fluid, the milk plasma or serum, between the two substances. The changes set up in casein by bacteria are various, and furnish a means of diagnosis.

Mineral Matter.-The ash of milk, obtained by careful ignition of the solids, contains calcium, magnesium, potassium, sodium, phosphoric acid, sulphuric acid, chlorine, and iron-phosphoric acid and lime being present in the largest amounts.

We may now consider the fermentations of milk and the pathogenic organisms associated with milk. 


\section{The Fermentations of Milk}

(1) Lactic Acid Fermentation: the Souring of Milk.-If milk is left undisturbed, it is well known that eventually it becomes sour. The casein is coagulated, and falls to the bottom of the vessel; the whey or serum rises, carrying to the surface flakes or lumps of fat. In fact, a coagulation analogous to the clotting of blood has taken place. In addition to this, the whole has acquired an acid taste. Now this double change is not due to any one of the constituents we have named above. It is, in short, a fermentation set up by a living ferment introduced from without. The constituents most affected by the fermentation are $(a)$ the milk-sugar, which is broken down into lactic acid, carbonic acid gas, and other products, and (b) the casein, which is curdled and becomes suspended in a semi-colloidal form.

For many years it has been known that sour milk contained bacteria. Pasteur first described the Bacillus acidi lactici, which Lister isolated in 1877, and obtained in pure culture by the dilution method. In 1884 Hueppe contributed still further to what was known of this bacillus, and pointed out that there were a large number of varieties, rather than one species, to be included under the term $B$. acidi lactici. We have already dealt with the chief characters of this family of organisms. When a certain quantity of lactic acid has been formed, the fermentation ceases. It will recommence if the liquid be neutralised with carbonate of lime, or if pepsine be added. Since Pasteur's discovery of a causal bacillus for this fermentation, other investigators have added a number of bacteria to the lactic acid family. Some of these in pure culture have been used in dairy industry, as we shall subsequently have occasion to notice.

We have already seen that milk as it leaves the healthy udder is generally sterile, and immediately gains bacteria from air, dust, etc. Whilst the exact origin of lactic acid bacilli is not known, many bacteriologists hold that they gain entrance to the milk from the surrounding air of byre or dairy. Others maintain that some species, at any rate, are soil bacteria, and associated with certain geographical localities. Russell states that, under ordinary conditions, the organisms found in the teat of the udder are those which produce lactic fermentation. He quotes Bolley and Hall as finding twelve out of sixteen species in the teat of the udder to be lactic acid producers.* Veranus Moore has arrived at very similar results. $\dagger$ Rollin Burr has recently investigated this subject with a different

* Outlines of Dairy Bacteriology, H. L. Russell, 1898, p. 43.

+ Twelfth and Thirteenth Reports of the Bureau of Animal Industry, U.S.A., 1895 and 1896, p. 265. 
result.* He finds that when milk is drawn from the cow in such a manner as to exclude from it dirt and dust from the air, the stalls, and the cow, such milk may contain none of the organisms capable of producing a normal souring of milk. This also has been the experience of the writer. The lactic acid organisms are a secondary contamination of the milk from some external source. None of the species of lactic organisms characteristic of the locality in which Burr worked, could be found in the udder. This is in accordance with the results of others who have had the opportunity of examining the udder or milk ducts for lactic bacilli. Out of 300 examinations made of fore-milks drawn directly from the udder into sterile flasks, Burr found only 2 per cent. contained ordinary lactic acid bacteria, and in these cases the origin was probably outside contamination. Conn found the acid organisms present in 5 cases out of 200 examinations, involving 75 cows. He also maintains that the origin of lactic acid bacteria is in external conditions. + Further, there is the recognised fact which has been pointed out by Conn and Esten, and frequently met with by Swithinbank and the writer, namely, that lactic acid organisms are not the predominant species in freshlydrawn milk, as they undoubtedly would be were they organisms of the udder. Hence there can, we think, be little doubt that the origin of lactic acid organisms is to be found in some external condition or conditions.

It follows from what has been said that cleanliness of byre, dairy, and general manipulation is an important factor in the presence, both actual and in degree, of lactic acid organisms.

(2) Butyric Acid Fermentation.-This form of fermentation is also one which we have previously considered. Both in lactic and butyric fermentation we must recognise that in the decomposition of milk-sugar there are almost always a number of minor products occurring. Some of the chief of these are gases. Hydrogen, carbonic acid, nitrogen, and methane occur, and cause a characteristic effect which is frequently deleterious to the flavour of the milk and its products. Most of the gas-producing ferments are members of the lactic acid group, and are sometimes classified in a group by themselves. In butyric fermentation of milk the three chief products are butyric acid (which causes the bitterness), hydrogen, and carbonic acid gas.

(3) Coagulation Fermentations without Acid Production.--Of these there are several, caused by different bacteria. What happens is that the milk coagulates, but no acid is produced, the whey being sweet to the taste rather than otherwise. The condition is in the

* Storr's Agricultural Expt. Sta. Rep. for 1900, pp. 66-81. Centralb. f. Bakt., Abth. ii., 1902, p. 236.

† Storr's Agricultural Expt. Sta. Rep., 1899, p. 23. 
main one of milk-clotting rather than milk-curdling. The two chief examples are the rennet fermentation of milk and the production of casease.

(4) The Alcoholic Fermentations of Milk.-Lactose is not readily acted upon by yeasts though they have the power of breaking it up and producing alcohol and carbonic acid gas. When it does occur the percentage of alcohol is very small. The first change is the inversion of the milk-sugar into dextrose and galactose, and the second is fermentation of these sugars.

Occasionally, alcohol is present in the milk of a dairy, as a sort of by-product accompanying lactic fermentation, and alcoholic fermentation may, under exceptional circumstances, cause serious trouble to the dairyman. But the chief illustrations of this fermentation in milk are the well-known examples of the artificial beverages known as koumiss (or kumiss, kumys) and kephir (or kefyr, kefr), the former a fermentation of mare's milk, the latter of cow's milk. Matzoon and Leben are two other examples of similar changes.

Koumiss is made on the Steppes of South-Western Siberia and European Russia, by nomadic Tartars. It is not a simple process nor a single fermentation. There is first a lactic fermentation producing lactic acid, and secondly, a vinous fermentation resulting in alcohol. The former is produced by bacteria, the latter by yeasts. In neither case is the process set up by a pure culture. "The net change which has taken place in the original milk may be summed up by saying that the sugar has been to a large extent replaced by lactic acid, alcohol, and carbonic acid gas; the casein has been partly precipitated in a state of very fine division, and partly predigested and dissolved, while the fat and salts have been left much as they were." * The total proteid in koumiss is hardly less than in mare's and cow's milk; the fat is practically the same as in mare's milk, and the sugar is reduced from about 5 per cent. to 1.5 per cent. The amount of alcohol in koumiss is as little as 1.7 per cent., and there is not as much as 1 per cent. of lactic acid.

Kephir, the second example named, is an effervescent alcoholic sour milk prepared by inhabitants of the Caucasus from the milk of goats, sheep, and cows. The process of fermentation is a double one, and precisely parallel to that occurring in the production of koumiss. Its method of manufacture is simply to add to milk a few "kephir grains," allow the milk to stand for twenty-four hours at a temperature of $17^{\circ}$ to $19^{\circ} \mathrm{C}$., pour off the milk and mix with fresh volumes, and so on. Fermentation is complete in two or three days' time, and the resultant fluid contains about 2 per cent. of alcohol, being slightly more than in koumiss. p. 136 .

* Food and the Principles of Dietetics, by R. Hutchison, M.D., F.R.C.P., 1902, 
(5) Anomalous Fermentations of Mill. - There are a number of changes, mostly due to fermentation, which occur in milk, and to which reference must be made. These conditions have been termed "diseases" of milk, but it is not altogether a satisfactory term.

(a) Bitter Fermentation.-Some bitter conditions of milk are due to irregularity of diet in the cow. Similar changes occur in conjunction with some of the acid fermentations and proteid decompositions. Weigmann and Conn have, however, shown that there is a specific bitterness in milk due to bacteria, which appear to produce no other change. Hueppe suggests that it may be due in part to a proteid decomposition resulting in bitter peptones. Such bodies are produced by bacteria from the albuminoids of milk, and hence the bitterness does not appear immediately after milking, but only after an incubation period. Some nine or ten different micro-organisms have been credited with this power, and such organisms may infect a farm, a byre, or a dairy, for months or even years, contaminating the milk. In all probability, most outbreaks of this bitter fermentation are due to Weigmann's bacillus of bitter milk or Conn's micrococcus. There seems to be evidence for supposing that some of the "bitter" bacilli produce very resistant spores, which make them resistant against conditions in the milk itself or externally.

(b) Slimy Fermentation.-This graphic but inelegant term is used to denote an increased viscosity in milk, and its tendency when being poured to become ropy and fall in strings. Such a condition deprives the milk of its use in the making of certain cheeses, whilst in other cases it favours the process. In Holland, for example, in the manufacture of Edam cheese, this "slimy" fermentation is desired. Toettemoelk, a popular beverage in Norway, is made from milk that has been infected with the leaves of the common butterwort, Pinguicula vulgaris, from which-Weigmann separated a bacillus possessing the power of setting up slimy fermentation. There are, perhaps, as many as a dozen species of bacteria which have in a greater or less degree the power of setting up this kind of fermentation. In 1882, Schmidt-Mühlheim isolated the Micrococcus viscosus, which occurs in chains and rosaries, affecting the milk-sugar. It grows at blood-heat, and is not easily destroyed by cold. Its effect on various sugars is the same. M. Freudenreichii, one of the specific micro-organisms of "ropiness" in milk, is a large, non-motile, liquefying coccus, which can produce its result in milk within five hours. On account of its resistance to drying, it is difficult to eradicate when once it makes its appearance in a dairy. The organism used in making Edam cheese is the Streptococcus Hollandicus, and in hot milk it can produce ropiness in one day. A number of bacilli have been detected by several observers, and classified as slime fermenta- 
tion bacteria. The Bacillus lactis pituitosi, a slightly curved, nonliquefying rod, which is said to produce a characteristic odour, in addition to causing ropiness, brings about some acidity. B. lactis viscosus of Adametz, B. actinobacter of Duclaux, B. Hessii of Guillebeau, and other bacilli are similar agents. Many of the above organisms, with others, produce "slimy" fermentation in alcoholic beverages as well as in milk.

(c) Soapy Mitk is another form of fermentation, the etiology of which has been elucidated by Weigmann. The Bacillus lactis saponace i imparts to milk a peculiar soapy flavour. It was detected in the straw of the bedding and hay of the fodder, and from such sources may infect the milk. There is little or no coagulation, but a certain amount of sliminess and ropiness, with a peculiar soapy taste to the milk.

(d) Chromogenic Changes.-We have already remarked that colour is the natural and apparently chief product of many of the innocent bacteria. They put out their strength, so to speak, in the production of bright colours. The chief colours produced by germs in milk are as follows:-

Red Milk.-Bacillus prodigiosus, in the presence of oxygen, causes a redness, particularly on the surface of milk. It was possibly the work of this bacillus that caused "the bleeding host" which was one of the superstitions of the Middle Ages. B. lactis erythrogenes produces a red colour only in the dark, and in milk that is not strongly acid in reaction. When grown in the light this organism produces a yellow colour. There is a red sarcina (Sarcina rosea) which also has the faculty of producing red pigment. One of the yeasts is another example. It must not be forgotten that redness in milk may actually be due to the presence of blood from the udder of the cow.

It is of importance clearly to differentiate between milk reddened by the admixture of blood from the mammary gland, and that produced by the organism isolated and studied by Hueppe and Grotenfeldt-Bacillus lactis erythrogenes - the presence of which in the milk is now looked upon as the active causation of the disease. In the former case the coloration is apparent immediately after milking, is uniform, and if the milk is allowed to rest the flocculent blood coagulum causing the coloration will gradually sink and deposit itself in the form of a precipitate at the bottom of the milk receptacle. In the latter the red spots do not appear until later, the infection of the milk is comparatively slow, and the milk serum is alone affected, the cream layer not taking the red coloration. This is probably due to the simple fact that the cream layer being at the surface is exposed to the light, which inhibits the coloration. A general coagulation of the milk takes place accompanied by a 
distinctive sickly sweetish odour. The red coloration will not occur if the milk is exposed to light or has an acid reaction. The Bacillus lactis erythrogenes of Hueppe (Bacterium erythrogenes of Grotenfeldt) is an aërobic, liquefying, non-motile, non-sporing, chromogenic bacillus of 1 to $1.5 \mathrm{~mm}$. in length by 3 to $4 \mathrm{~mm}$. in breadth, at times attaining, especially in broth cultures, a length of 4 to $5 \mathrm{~mm}$. in the form of filaments. It takes readily all ordinary stains and holds the Gram. In sterile milk a gradual precipitation of the casein takes place with a neutral or slightly alkaline reaction of the medium. The resultant serum, in the absence of light, absorbs the red colouring matter produced by the organisms, taking a deep red tint provided the medium has no acid reaction. The coagulation by rennet of milk infected with the organism has the effect of producing a marked dirty red coloration, changing to a reddishbrown and finally to blood red.

Blue Milk is due to the growth of the Bacillus cyanogenes (Bacterium syncyaneum of Ehrenberg), or as Hueppe originally termed it, the Bacillus lactis cyanogenus, an anaërobic, non-liquefying bacillus, motile, bi-polar, flagellated, chromogenic, and round-ended, with a varying average length of from 1 to $4 \mathrm{~mm}$. by 3 to $5 \mathrm{~mm}$. in breadth. Spore formation has been claimed by Hueppe, but denied by Heim, who describes the so-called spores of Hueppe as involution forms only. In liquid cultures curious involution forms are often observed, which are especially noticeable if the organism is grown in mineral media, as those of Conn and Nægeli. The organism does not liquefy gelatine and grows freely on all the usual laboratory media at room temperature, the dark purplish blue or in some cases brownish coloration of the medium being very characteristic, but this freedom of growth becomes less as the temperature advances to $37^{\circ} \mathrm{C}$., and the cultures themselves die at $40^{\circ}$. The reaction is invariably alkaline, although the medium itself may have been in the first place acid. It stains well with all the ordinary stains but does not hold the Gram. In milk the bluish tint would appear to be dependent upon certain unknown conditions, and in the sterile milk used for the laboratory purposes it is not easy to obtain it.

Yellow Milk.-Bacillus synxanthus is held responsible for curdling. the milk, and then at a later stage, in redissolving the curd, produces a yellow pigment.

In addition to the bacteria of fermentation occasionally present in milk, there is a group of Various Unclassified Bacteria. In milk this is a comparatively small group, for it happens that those bacteria in milk which cannot be classified as fermentative or pathogenic are few. B. coli communis occurs here as elsewhere, and might be grouped with the gaseous fermentative organisms on account of its extraordinary power of producing gas and breaking up 
the medium (whether agar or cheese) in which it is growing. What its exact rôle is in milk it would be difficult to say. It may act, as it frequently does elsewhere, by association in various fermentations. Some authorities hold that its presence in excessive numbers may cause epidemic diarrhœa in infants (Delépine).

Several years ago a commission was appointed by the British Medical Journal to inquire into the quality of the milk sold in some of the poorer districts of London. Every sample was found to contain B. coli, and it was declared that this particular microbe constituted 90 per cent. of all the organisms found in the milk.* We record this statement, but accept it with some reserve. The diagnosis of $B$. coli eight or nine years ago was not such a strict matter as to-day. Still, undoubtedly, this particular organism is not uncommonly found in milk, and its source is uncleanly dairying. In the same investigation, Proteus vulgaris, B. fluorescens, and many liquefying bacteria were frequently found. Their presence in milk means contamination with putrefying matter, surface water, or a foul atmosphere.

A number of water bacteria find their way into milk in the practice of adulteration, and foul byres, and dirty dairies and-milk shops, afford ample opportunity for aërial pollution.

Another unclassified group occasionally present in milk is represented by moulds, particularly Oidium lactis, the mould which causes a white fur, possessing a sour odour. It is allied to the Mycoderma albicans (O. albicans), which also occurs in milk, and causes the whitish-grey patches on the mucous membrane of the mouths of infants $(t h r u s h)$. These and many more are occasionally present in milk.

\section{The Disease-Producing Power of Milk}

The general use of milk as an article of diet, especially by the younger and least resistant portion of mankind, very much increases the importance of the question as to how far it acts as a vehicle of disease. Recently, considerable attention has been drawn to the matter, though it is now a number of years since milk was proved to be a channel for the conveyance of infectious diseases. During the last twenty years, particular and conclusive evidence has been deduced to show that milch cows may themselves afford some measure of infection. The extensive work on tuberculosis by three Royal Commissions has done much to obtain new light on the conveyance of that disease by milk and meat. The enormous strides in the knowledge of the bacteriology of diphtheria and other germ diseases have also placed us in a better position respecting the conveyance of such diseases by milk. Generally speaking, for reasons already given,

* Brit. Med. Jour., 1895, vol. ii., p. 322. 
milk affords an ideal medium for bacteria, and its adaptability therefore for conveying pathogenic organisms is undoubted. We shall speak shortly of the outstanding facts of the chief diseases carried by milk.

\section{Milk-borne Tuberculosis}

It is a well-known fact that tuberculosis is a common disease of cattle. Probably not less than 20 to 30 per cent. of milch cows in this country are affected with it. Therefore, at first sight it might appear that the consumption of milk from such animals would lead to considerable spread of the disease. But in point of fact there are two limiting conditions. The first has relation to the question of the communicability of the disease from the cow to man. The second concerns the degree of disease in the cow which can affect the milk. It is necessary that both points should be discussed somewhat fully. But the former question will be discussed in the chapter dealing with Tuberculosis (see pp. 338-346). The latter condition only will be discussed here. It has reference to that which limits the transmissibility of tuberculosis from the cow to man by means of milk has relation to the well-established fact that the milk of tuberculous cows is only certainly infective when the tuberculous disease affects the udder.* This is not necessarily a condition of advanced tuberculosis. The udder may become infected at a comparatively early stage. The presence or absence of tubercle bacilli in the milk of cows

* This is the generally accepted view, but it should be added that various workers have shown that cows having generalised tuberculosis, but apparently unaffected udders, may yield tuberculous milk. Quite recently (1903) Mohler, as the result of a long series of experiments, arrives at the following important conclusions :-(1) That the tubercle bacillus may be demonstrated in milk from tuberculous cows when the udders show no perceptible evidence of disease either macroscopically or microscopically; (2) that the bacillus of tuberculosis may be excreted from such an udder in sufficient numbers to produce infection in experimental animals, both by ingestion and inoculation ; (3) that in cows suffering from tuberculosis the udder may, therefore, become affected at any moment; (4) that the presence of the tubercle bacillus in the milk of tuberculous cows is not constant, but varies from day to day; (5) that cows secreting virulent milk may be affected with tuberculosis to a degree that can be detected only by the tuberculin test; $(6)$ that the physical examination or general appearance of the animal cannot foretell the infectiveness of the milk; (7) that the milk of all cows which have reacted to the tuberculin test should be considered as suspicious, and should be subjected to sterilisation before using; and (8) that it would be better still that tuberculous cows should not be used for general dairy purposes.* Experiments by Lydia Rabinowitsch have given somewhat similar results. She relies on tuberculin as a test of infectivity, and the animal experiment as proof of tuberculosis in milk. $\dagger$ Ravenel also maintains that cows which show no evidence of tuberculous udders, but which react to tuberculin, may yield tubercle bacilli in their milk. J. H. Young of Aberdeen maintains, on the other hand, that cows free from udder disease though reacting to tuberculin yield milk free from tuberculosis. +

* Bureau of Animal Industry, Washington, U.S.A., Bulletin 44, 1903, p. 93.

+ See also Zeitschrift fïr Thiermedicin, 1904, p. 202.

$¥$ Brit. Med. Jour,, 1903, i., p. 816 . 
with udder tuberculosis is greatly dependent on the extent of the disease in the udder. But to make the milk infective the udder must be affected, and milk from such an udder possesses a considerable degree of virulence. When the udder is thus itself the seat of disease, not only the derived milk, but the skimmed milk, butter-milk, and even butter, may all contain tuberculous material. Furthermore, tubercular disease of the udder spreads in extent and degree with extreme rapidity. From these facts it will be obvious that it is of first-rate importance to be able to diagnose udder disease. This is not always possible in the early stage. The signs upon which most reliance may be placed are the enlargement of the lymph-glands lying above the posterior region of the udder; the serous, yellowish milk which later on discharges small coagula; the partial or total lack of milk from one quarter of the udder (following upon excessive secretion); the hard, diffuse nodular swelling and induration of a part or the whole wall of the udder; and the detection in the milk of tubercle bacilli. The whole organ may increase in weight as well as size, and on postmortem examination show an increase of connective tissue, a number of large nodules of tubercle, and a scattering of small granular bodies, known as "miliary" tubercles.

The udder is affected in about 2 per cent. of the cows in the milking herds in this country (MacFadyean).* In London about 0.2 per cent. of the cows are so affected, as judged by clinical observation. It will be remembered that in the country generally between 20-30 per cent. of the cows suffer from tuberculosis. In London 15-20 per cent. are tuberculous. The higher standard in London is due to better-class animals being housed in London, to more thorough inspection, and to the fact that there is no inbreeding. But let us take the generally-accepted figure of 2 per cent. of the cows in the United Kingdom as having tuberculous udders, and therefore yielding, in greater or less degree, tuberculous milk, leaving altogether out of account cows which may be tuberculous but are not affected in the udder. In the United Kingdom in 1901 there were $4,102,000$ milch cows. $†$ If we take 2 per cent. of these as having tuberculous udders, it gives us 80,000 . The average annual yield of milk per cow may be taken as, at least, 400 gallons, ++ which means that from these 80,000 tuberculous udders $32,000,000$ gallons of milk are obtained. It is not asserted that this large amount of milk is actually virulent with tuberculous matter, but it will be admitted that the entire amount of it is open to grave suspicion, if not absolute condemnation.

* Trans. of Brit. Congress on Tuberculosis, 1902, vol. i., p. 84.

+ In the United States of America there were in 1901, 18,112,000 milch cows in actual dairy use.

$¥ 420$ gallons is the generally accepted figure. 


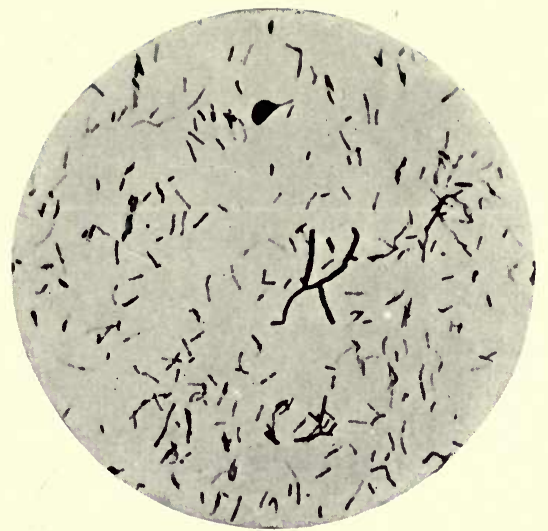

Bacillus tuberculosis. Film preparation from glycerine glucose, agar culture. Fuur months old2 months at $37^{\circ} \mathrm{C}$, and 2 months at $20^{\circ} \mathrm{C}$. Stained with carbol fuchsin. $\times 1000$.

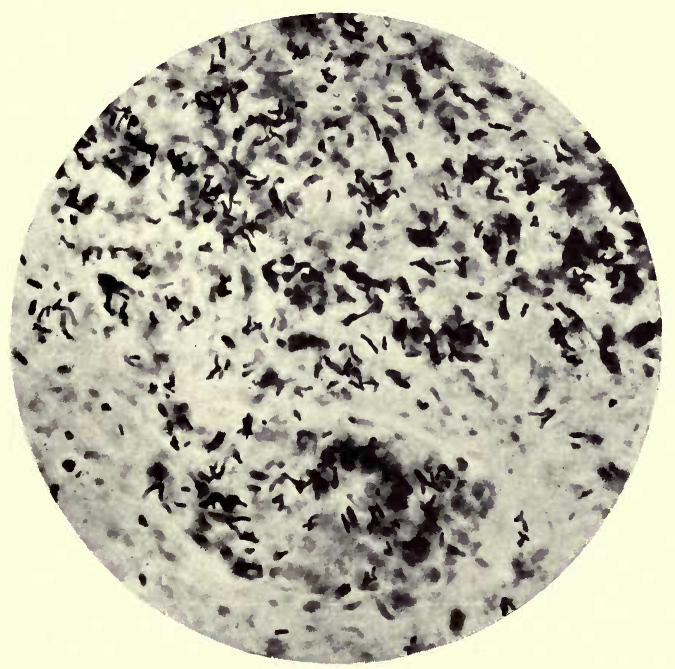

Bacillus tuberculosis, in Udder of Cow. Stained with carbol fuchsin and methylene blue. $\times 1000$. 


\section{LIBRAR T
OF THE \\ UNIVERSITY}

OF

CALIFORNIA 
There are a variety of conditions in addition to the vera causa, the presence of the bacillus of tubercle, which make the disease common amongst cattle. Constitution, temperament, age, work, food, inbreeding, and prolonged lactation, are the individual features which act as predisposing conditions; they may act by favouring the propagation of the bacillus or by weakening the resistance of the tissues. To this category must further be added conditions of environment. Bad stabling, dark, ill-ventilated stalls, high temperature, prolonged and close contact with other cows, all tend in the same direction.

The danger from drinking raw tuberculous milk only exists for persons who use it as their sole or principal food, that is to say, young children and certain invalids. With adults in normal health, the danger is greatly minimised, as the healthy digestive tract is relatively insusceptible. Moreover, dairy milk is almost invariably mixed milk; that is to say, that if there is a tubercular cow in a herd yielding tubercle bacilli in her milk, the addition of the milk of the rest of the herd so effectually dilutes the whole as to render it in some degree innocuous.

It should not be forgotten that milk may become tubercular through the carelessness or dirty habits of the milker. Such a common practice as moistening the hands with saliva previously to milking may, in cases of tubercular milkers, effectually contaminate the milk. Again, it may become polluted by dried tubercular matter getting into it from dust or infected dried excreta. Such conveyances must be of rare occurrence, yet their possibility should not be forgotten.

The Tubercle Bacillus in Market Milk.-Investigations have been made in many cities as to the actual occurrence of the tubercle

TABLE shouing the Total Number of Milks Examined Bacteriologically for Tubercle Bacilli from August 1896 to 31st December 1903.

(Liverpool.)

\begin{tabular}{|c|c|c|c|c|c|c|c|}
\hline \multirow{2}{*}{ Year. } & \multirow{2}{*}{$\begin{array}{c}\text { Total } \\
\text { number } \\
\text { of Samples } \\
\text { taken. }\end{array}$} & \multicolumn{3}{|c|}{ Town Samples. } & \multicolumn{3}{|c|}{ Country Samples. } \\
\hline & & $\begin{array}{l}\text { Number } \\
\text { taken. }\end{array}$ & Tubercular. & $\begin{array}{l}\text { Percentage } \\
\text { Tubercular. }\end{array}$ & $\begin{array}{l}\text { Number } \\
\text { taken. }\end{array}$ & Tubercular. & $\begin{array}{l}\text { Percentage } \\
\text { Tubercular }\end{array}$ \\
\hline 1896 & 119 & 83 & 4 & $4 \cdot 8$ & 36 & 5 & $14 \cdot 0$ \\
\hline 1897 & 150 & 63 & 4 & $6 \cdot 3$ & 87 & 5 & $5 \cdot 7$ \\
\hline 1898 & 112 & 84 & 7 & $8 \cdot 3$ & 28 & 5 & $17 \cdot 9$ \\
\hline 1899 & 352 & 167 & 1 & 0.6 & 185 & 15 & $8 \cdot 1$ \\
\hline 1900 & 560 & 255 & 4 & $1 \cdot 5$ & 305 & 5 & $1 \cdot 6$ \\
\hline 1901 & 566 & 254 & 2 & 0.7 & 312 & 20 & $6 \cdot 4$ \\
\hline 1902 & 595 & 213 & 1 & 0.4 & 382 & 32 & $8 \cdot 3$ \\
\hline 1903 & 582 & 231 & 2 & 0.8 & 351 & 19 & $5 \cdot 6$ \\
\hline
\end{tabular}

bacillus in milk as placed on the market. It has been found in 
varying percentage, ${ }^{*}$ but, as a rule, the milk coming in to the cities from the country has contained more tubercle bacilli than the milk obtained from the town cows. This characteristic has been found to occur in London, Manchester, Liverpool, and other cities.

It should also be added that there are a number of cases on record where the tubercle bacillus has been found in butter.

The Virulence of the Tubercle Bacillus in Milk.-Martin and Woodhead concluded as the result of their investigations for the Royal Commission on Tuberculosis that tuberculous milk possessed a high degree of virulence for man. Sir Richard Thorne held that tabes mesenterica (alimentary tuberculosis) of children had not declined as phthisis (pulmonary tuberculosis) had done in recent years on account of the conveyance of the virus of tubercle in milk.t If the occurrence of primary lesion in the intestine is indication of infection through the alimentary tract, then it is instructive to notice that of all the tuberculosis in children in this country about 25 per cent. is alimentary in origin, and in 60 to 70 per cent. of the cases the mesenteric glands are affected. Both of these figures deal with deaths only, but as Raw has pointed out, no doubt a number of cases of alimentary tuberculosis recover, the infection having been mild. Amongst 269 tuberculous children under twelve years of age whom Dr Still examined post mortem, he found it possible to determine the channel of infection with some degree of certainty in 216 cases. In 138 (63.8 per cent.) infection entered through the lung; in $63(29 \cdot 1$ per cent.) primary infection occurred, in all probability through the intestine. Of children up to two years of age he found 65 per cent. contracted infection through the lung, and 22 per cent. through the intestine. In infants under one year of age apparently only 13 per cent. contracted tuberculosis through the intestine. +

It is recognised that, owing to the great tendency to generalisation of tuberculosis in children, it is a matter of extreme difficulty to determine which was, in fact, the primary channel of infection, and this must be taken into consideration in estimating the significance of the frequency in the above figures. It should also be remembered that the tubercle bacillus may, and probably

* In 1898, 14 per cent. of the milks examined in Berlin by Petri contained the tubercle bacillus. In 1899 , in Islington, the percentage was $144^{\circ}$; in 1893 , St Petersburg, 5 per cent. ; in 1901, in London, 7 per cent. (Klein); in 1901, at Croydon, 6.7 per cent., and Manchester, 9.5 per cent.; in 1902, at Woolwich, 10 per cent. ; in Camberwell, 11 per cent. ; in the City of London and in Finsbury, nil. These percentages must not be accepted as anything but passing figures and illustrations of what various investigators have found under varying conditions.

$\dagger$ The Administrative Control of Tuberculosis (Harben Lectures), 1899, pp. 5-7 and 28-32.

$\ddagger$ Practitioner, 1901 (July), p. 94. 
does, pass through the intestinal wall into the nearest lymphatic glands, leaving no visible trace on the intestine. Further, owing to the fact that children swallow their pulmonary expectoration, secondary infection of the intestine may rapidly follow primary infection of the lungs. Hence it comes about that, in many cases, the intestine and mesenteric glands are affected, and yet such a condition cannot be taken as evidence of the infection by food. Dr Still concludes that $(a)$ the commonest channel of infection with tuberculosis in childhood is through the lung; (b) infection through the intestine is less common in infancy than in later childhood; (c) milk, therefore, is not the usual source of tuberculosis in infancy; and $(d)$ inhalation is much the commonest mode of infection in the tuberculosis of childhood, and especially in infancy. Dr Still has placed on record 5 cases of tuberculous ulcer of the stomach in children.

Taking a broad view of the facts, it would appear that whilst tuberculosis is not chiefly spread by means of milk, there is unmistakable evidence, derived from pathological and clinical experience, proving that tuberculous milk can, and does on occasion, set up some form of tuberculosis (bovine or human) in the bodies of man and other animals consuming the milk.

\section{Milk-borne Typhoid Fever}

Dr Michael Taylor of Penrith was the first to establish the now well-known fact that milk may act as a vehicle of the virus of enteric fever. That was in 1857.* Since that date more than 160 epidemics of this disease have been traced to a polluted milk supply. Schuder states that 17 per cent. of all typhoid epidemics are due to the consumption of infected milk.

The steps in the process of infection are briefly as follow. Enteric fever affects the intestine, and hence the excreta, especially in the early stages of the disease, are charged with large numbers of the causal bacilli. It is now known that the sweat, expectoration from the lungs, and the urine of a typhoid patient may also contain the typhoid bacillus. Indeed, the urine in 25 per cent. of the cases generally contains large numbers of the bacillus (Horton Smith). $\dagger$ There is also evidence to show that the bacilli may remain in the urine for long periods after convalescence, even for months and possibly for years. The bowel discharges and the urine are, therefore, the two chief channels by which the typhoid bacillus is excreted. It, therefore, readily gains access to the soil, to drains, and eventually

* Edin. Med. Jour., 1858, pp. 993-1004.

+ Lectures on Typhoid Fever, 1900. 
on occasion to the water supply, and thus into milk and back again to man. The virus does not always pass in the discharges to water and milk, but may reach them by becoming dried dust. A small pollution may in this way set up widespread disease. (For the behaviour of the typhoid bacillus in soil, see pp. 145-150.)

The most common way for milk to become infected by the typhoid bacillus is through infected water. Such water may be added to the milk by way of adulteration or by accident; or the milk vessels may have been "cleansed" with polluted water (in 29 per cent. of milk-borne outbreaks according to Schuder). Another source of infection of the milk is when persons suffering from a mild attack of typhoid fever continue to work a dairy or otherwise deal with milk, and this has proved a frequent means of infection. Flies doubtless convey the germ of the disease not infrequently, as was shown in the Spanish-American War of $1898^{*}$ and the Boer War of 1900-1901.†

Though the typhoid bacillus appears not to have the power of rapid multiplication in milk, it has the faculty of existing in milk for a considerable time (twenty days or longer) even when milk has curdled or soured, and may thus infect milk products, such as butter and cheese. But infection by milk products may be eliminated as of too rare occurrence to deserve attention. The bacillus does not coagulate milk like its ally the $B$. coli communis, which is a much more frequent inhabitant of milk. It flourishes in milk at room temperature and blood-heat, and does not produce acid or alter the appearance of the milk.

Several typical milk-borne outbreaks of typhoid fever may be cited :-

1. Infection from Personal Contact with Typhoid Patients.-At Penrith, it appears that about the beginning of September, 1857, a young servant girl, E. O., returned home to Penrith from Liverpool suffering fom typhoid fever. The family of which she was a member consisted of father, mother, and five children, of whom she was the eldest. The cottage in which they lived consisted of two ill-ventilated and ill-lighted rooms, a kitchen or sitting-room, and a bedroom opening out of it. The father possessed three cows, and carried on a small milk business dealing with some fourteen families. The mother milked the cows, and the milk was brought into the kitchen, direct from the byre, and in due course distributed in tin measures amongst the customers. After her return home the girl continued ill for about a fortnight, during which period she was nursed by her mother in the kitchen or common

* American War Department, Official Report, 1900.

† Brit. Med. Jour., 1901, i., p. 642 et seq. ; ibid., 1902, ii., pp. 936-941 (Firth and Horrocks). 
sitting-room. At the end of the fourth week in September she was convalescent, and began to help at once in the distribution of the milk. Two other children of the family sickened and passed through the fever. The mother nursed all three patients, and continued to milk the cows and attend to the distribution of the milk. In October and November some 13 cases of typhoid fever occurred in seven families dealing with the infected cottage, and from these primary cases a number of persons, over a somewhat wide area, were infected by contact. By most careful observation and reasoning, Dr Taylor arrived at the conclusion that the milk became contaminated in the kitchen of this cottage, from the typhoid patients there being nursed.*

2. Infection from Washing Milk Vessels with Polluted Water.-At Clifton, Bristol, in October 1897, an outbreak affected 244 persons, 31 of whom died. Ninety-six per cent. of the patients consumed suspected milk. It happened that a brook received the sewage of thirtyseven houses, the overflow of a cesspool serving twenty-two more, the washings from fields over which the drainage of several others was distributed, and the direct sewage from at least one other, and then flowed directly through a certain farm. In September it seems that some excreta from a man suffering from typhoid fever gained access to the brook. The water of this stream supplied the farm pump, and the water itself, it is scarcely necessary to add, was highly charged with putrescent organic matter and micro-organisms. This water was used for washing the milk-cans from this particular farm, otherwise the dairy arrangements were efficient. Part of the milk was distributed to fifty-seven houses in Clifton; in forty-one of them cases of typhoid occurred. Another part of the milk was sold over the counter; twenty households so obtaining it were attacked with typhoid fever, and a number of further infections arose in the course of a third delivery. $\dagger$

3. Infection from Water added to Milk.-At Moseley, in 1873, 96 persons in fifty families contracted typhoid fever from milk. Boy at milkman's house fell ill of typhoid fever, suffered there for a fortnight and died. Two wells were polluted from a privy into which typhoid excreta had been thrown. The water of the well was added accidentally or intentionally to the milk. Dr Ballard summed up his view of the causation in this outbreak as follows:-(1) Two wells upon adjoining premises occupied by milk sellers became infected early in November with the infectious matter or virus of enteric fever, through the soakage from a privy into them of excremental matters containing that matter of infection. Through the medium of water drawn from these wells the milk

* Edin. Med. Jour., 1858, pp. 993-1004.

† Trans. Epidem. Soc. of London, vol. xvii., pp. 78-103 (Dr D. S. Davies). 
supplied by these milk sellers became infected, and many of their regular customers who drank the milk suffered from the disease. (3) The same infected milk having been sold to two other milk purveyors, some of the persons using the milk supplied by these milkmen also suffered in a similar manner. (4) There is no evidence that the disease spread in these districts in any other way than through this milk.*

4. Infection from the Air by Dried Typhoid Excreta. - At Millbrook, in Cornwall, in 1880 (July-September), an outbreak occurred having a total number of cases of 91 . In this instance part of a slaughter-house not used as such but as a wash-house was boarded off to constitute a dairy. On a shelf of this dairy the milk was habitually set in pans, exposed to the air. In one corner of the slaughter-house, nearest the dairy, was a badly trapped and very offensive drain inlet. Close to this inlet ran the wooden partition between the slaughter-house and the dairy, which near the inlet had been long broken away. The drain was in communication with an old square drain which had received typhoid excreta, so that the infected sewer air from the inlet had free access to the dairy and the exposed milk which stood in the dairy. There was evidence to show that the drain was in a dry and "waterless" condition. Six cases of typhoid occurred in the butcher's family. $\dagger$ A similar outbreak occurred in county Durham in 1893.

5. Infection from Contaminated Cloths and Clothes.-At Barrowford in Lancashire there occurred a typhoid epidemic in 1876. The total number of cases was 57, all of whom drank the suspected milk. The farmer had had typhoid fever in his house for two or three weeks before the outbreak, and no precautions had been taken to prevent the spread of the disease. The milk was left for some time in the farm-house before being sold. The milk-tins were wiped with the same "dish cloth" as that used among the fever patients. The farmer himself nursed his children, and then went immediately without disinfection amongst his cattle and milked them in the same clothes he had worn whilst nursing his children. The cases occurred within a very short space of time, and every one of them without exception drank the milk from this farm. Twenty-five of the patients were under ten years of age. There was no other typhoid in the district.

6. Infection owing to Cooling Milk in Water.-At Springfield, Mass., U.S.A., in 1892, an outbreak affecting 150 persons (25 of whom died). Upon the farm supplying the implicated milk there was one, and probably more than one, case of typhoid fever. The farmer submerged his sealed milk-cans when full of milk, in the well

* Report of Local Government Board, 1874, p. 92.

† Brit. Med. Jour., 1881, i., p. 20. 
adjoining the cow yards, with the object of keeping the milk cool. The water in this was polluted, and it was found that four of nine milk-cans leaked when inverted. Hence it became evident that water could gain access if the cans were submerged as they had been. The investigators suggest that as the typhoid excreta of the patient were placed, undisinfected, in the privy, and the contents of the latter spread over the tobacco field, the germs of typhoid may have gained access to the well by dirt from the labourers' boots, who both worked in the field and at the milk. Coliform organisms were found in the well water.*

\section{Milk-borne Diphtheria}

Milk is a favourable medium for the $B$.diphtherioe. The organism both lives and multiplies in ordinary sterilised milk, but it thrives better in milk at comparatively low temperatures than at $37^{\circ} \mathrm{C}$. In ordinary milk, unsterilised and unprepared, the commoner organisms multiply much more rapidly, and so the diphtheria bacillus is in all probability soon crowded out.

The cases, however, in which the $B$. diphtherioe has been actually isolated from market milk are extremely few. In the outbreak of diphtheria at Senghenydd in South Wales, in 1899, Bowhill $\dagger$ isolated a diphtheria bacillus from the suspected milk. The culture of the bacillus in broth proved fatal to a guinea-pig in two days. In the same year, Eyre + isolated a virulent diphtheria bacillus from some milk implicated in an outbreak of diphtheria in a school. In 1900, Klein $§$ also reported the isolation of a genuine diphtheria bacillus in an examination of 100 samples of milk in London. Lastly, Dean and Todd, isolated the B. diphtherioe from cow's milk in 1901.\| These are the only four authentic cases of actual detection of the $B$. diphtherioe in ordinary milk with which we have met.

There is a question which must now be considered, viz.: the relationship existing between diphtheria in man and animals and the milk supply. How does the milk become infected?

(1) In the first place, it is now generally held that the $B$. diphtherioe has a comparatively wide distribution in nature; whilst it appears not to be conveyed by water, it is believed that certain conditions of soil favour its growth as a saprophyte. But this is not proved. (2) In the second place, it has been proved that persons

* Boston Med. and Surg. Jour., 1893, ii., p. 485 (Sedgwick and Chapin).

+ Veterinary Record, 8th April 1899, No. 561. Jour. of State Medicine, 1899, pp. $705-710$.

$\ddagger$ Brit. Med. Jour., 1899, vol. ii., p. 586.

$\S$ Jour. of Hygiene, 1901, vol. i., p. 85 .

|| Ibid., 1902, vol. ii., pp. 194-205. 
suffering from diphtheria are foci of infection. The exact channels of infection differ under varying circumstances; but, in general, the source of infection is the throat and mouth of the patient. Anything which comes into contact with the mucous membrane becomes infected. Thus handkerchiefs, sweets, children's toys, etc., may act as the vehicles of contagion. The mucus and saliva may also be infective, and in speaking, kissing, coughing, or expectorating such mucus (probably rich in bacilli) may be disseminated in very fine particles, and so carry the disease. It is by such means that the disease is spread. Moreover, there is the fact of the long period during which the human throat may remain infective. Professor Sims Woodhead has recently stated that the persistence of the diphtheria bacillus for periods up to eight weeks is of very common occurrence. (3) Richardière and Tollemer* and others have proved that the dust floating in the air of a diphtheria ward may contain large numbers of diphtheria bacilli, and in this way milk and other foods may become contaminated.

Between 1878 and 1883, certain outbreaks of diphtheria due to milk appeared to suggest that the cow itself might suffer from diphtheria. The discovery of the Klebs-Löffler bacillus in 1883 furnished the basis for experimental work, and in $1886 \mathrm{Dr}$ Klein undertook some experiments to ascertain whether or not diphtheria was inoculable into cows. He took for the experiment two healthy milch cows which had calved some three or four weeks previously. One c.c. of broth culture of $B$. diphtheria (derived from human diphtheritic membrane) was injected under the skin into the subcutaneous tissue of the left shoulder in each of the two cows. Two or three days after the inoculation $(a)$ the temperature rose (to $40.6^{\circ}$ ), and the animals suffered from slight general malaise. On the third day $(b)$ a tumour appeared at the site of inoculation, which steadily increased in size to the seventh day. On the fifth day $(c)$ a papular eruption appeared on the udder and hind teat. In addition to the papules there were half a dozen vesicles, and some round patches covered with brown crusts. The process of eruption was mature by the eighth day. In the lymph of the vesicles and pustules the $B$. diphtherioe could be demonstrated, according to Klein, both microscopically and by culture. He therefore concluded that the $B$. diphtherioe, as such, inoculated into the shoulder of the cow, was received into the general system of the cow, and produced its effects not in the viscera, but on the udder as a specific eruption, and that before the end of five days after inoculation, was finally excreted in the cow's milk. "The presence of the diphtheria bacillus," he wrote, "could with certainty, by microscopic and culture observations, be demonstrated in the milk of the cow collected under all precautions;

* Gazette des Maladies Infantiles, 1899, No. 10. 
the number of bacilli present on that day in the milk amounted to 32 per c.c. Scrapings from vesicles on the sixth day were inoculated into two calves, which then suffered from a like disease." *

During 1890 and 1891, Dr Klein repeated these experiments on milch cows, and in two further instances, out of six cows, an eruption was produced on the udder and teats, and in one of these positive cases the $B$. diphtherioe was found in the milk about a week after inoculation.

It must be admitted that positive results did not always follow these experimental researches. Löffler, Abbott, Ritter, and others, including many veterinarians, criticised the experiments, and held that there was no conclusive evidence that diphtheria was a bovine disease. Since that time some twenty milk-diphtheria outbreaks have been investigated, with the result that, with one or two exceptions, the infectivity of the milk was certainly derived from human sources and not from bovine. In the Croydon outbreak in 1890 , at Worcester in 1891, and at Glasgow in 1892, evidence was obtained which appeared to support Klein's views.

Up to the present it may, however, be said that the evidence forthcoming points in the direction of human rather than bovine infection as the origin of the diphtheria bacillus in milk.

An interesting investigation has recently been made by Dean and Todd, respecting a small outbreak of diphtheria occurring in 1901.† In this outbreak several individuals suffered from diphtheria, and several others in the same households suffered from sore throat, probably diphtheritic. These individuals obtained their milk from two cows suffering from a contagious eruptive disease of the udder, from which Dean and Todd isolated a bacillus indistinguishable from Klebs Löffler bacillus of diphtheria. The case was a very interesting one. But the whole matter of bovine diphtheria is sub judice.

It was then in 1878, that evidence was forthcoming in support of the view that diphtheria, like typhoid fever, might on occasion be spread by means of milk. In that year, Mr W. H. Power made an inquiry into an outbreak of diphtheria in North London, chiefly in Kilburn and St John's Wood. There were as many as 264 persons attacked, and 38 died. The infection invaded some 118 different households. The epidemic was most severe in May (first four weeks), when about 190 cases occurred. The outbreak terminated abruptly. The area infected, and time of infection, clearly showed that there was some factor at work over a circumscribed area, and operative

* See A Treatise on Hygiene and Public Health (Stevenson and Murphy), vol. ii., pp. 161-164 (Klein). Also Local Government Board Report, 1889, p. 167 et seq.

$\dagger$ Jour. of Hygiene, April 1902 (vol. ii., No. 2, p. 194). Experiments on the relation of the Cow to Milk Diphtheria, by George Dean, M.B., and Charles Todd, M.D. 
during a limited time. There was no antecedent throat illness, and no school infection or contact contagion traceable. The houses were sanitarily good, and had a good water supply. There was but one thing common to most of the cases, and this was the milk supply. It was found that within the area of the greatest prevalence of throat illness, about one-fifth of the households were supplied by a common milk supply. The incidence of the disease fell, actually and relatively, upon consumers of the suspected milk.

Inquiry into the milk supply elicited no evidence of human disease pollution, nor contamination by water or air. Nor was there any definite disease of cows present at the time as far as could be judged. But by a process of exclusion, Mr Power suggested that "there may have been risk of specific fouling of milk by particular cows suffering, whether recognised or not, from specific disease."

Since that time there have been some 30 outbreaks of milk-borne diphtheria. In most cases the milk appears to have been infected directly by persons suffering from the disease, recognised or unrecognised.

\section{Milk-borne Scarlet Fever}

There are some seventy milk-borne epidemics of scarlet fever on record, and yet comparatively little is known as to the bacteriology of the disease (see p. 296). In almost all the outbreaks which have occurred there is evidence, more or less conclusive, that persons suffering from scarlet fever have been concerned in milking or in the distribution of milk. But in $1882 \mathrm{Mr}$ W. H. Power, in investigating an outbreak of milk-borne scarlet fever in North London, came to the conclusion that the cow had been the exciting cause of the epidemic, and was suffering from some diseased condition which could convey to the milk the virus of scarlet fever.* In 1885 occurred the "Hendon outbreak" of scarlet fever, which affected North London districts and Hendon. After inquiry, it was believed that the scarlet fever in these districts followed the consumption of milk from a particular farm at Hendon. Further, in these four districts wherein scarlatina had shown an extravagant incidence upon the milkman's customers, the disease had begun its peculiar incidence about the end of November or beginning of December. In one of those districts (South Marylebone), scarlatina continued day by day, and with increasing force up to the date of the inquiry, to attack the customers of the retail business. In two other districts (Hampstead and St Pancras), after attacking in some numbers, for a few days at the end of November and beginning of December, the customers of the business, the disease showed no fresh attacks for about ten days (a short but clearly defined intermission), and then about the middle of

\footnotetext{
* Supplement to the Report of Local Government Board, 1882, p. 65.
} 
December attacked them again in larger numbers, and continued to do so up to date of inquiry.

The chief facts concerning the distribution of the milk may be set out as follows: (a) The Marylebone customers suffered at the end of November and up till the end of the third week in December.

The Hampstead cases occurred in two groups, one small group at the end of November and a larger group in the latter part of December. (c) The St Pancras customers suffered like the Hampstead ones, but in a less degree. They obtained milk from the same vendors. The St John's Wood customers did not suffer until after the end of the year. (e) The few persons affected at Hendon suffered early in December, having consumed milk which had been returned from Marylebone, and at the same time new cases of scarlet fever ceased to occur in Marylebone. Examination was then made to ascertain if there had been any possible infection of the milk to explain this incidence and intermission.

When Mr Power came to inquire as to the movements of the cows, he learned that on 15th November three newly-calved cows arrived at the Hendon farm from Derbyshire, this event shortly preceding the first attack of scarlatina. It happened that these three animals were placed in a shed by themselves, and their milk was distributed in part to South Marylebone, Hampstead, and St Pancras, immediately preceding the outbreak of scarlatina in those districts. On examination it was found that the implicated cows were suffering from some kind of disease of the udders, which had spread to other cows in the herd. It would appear that the diseased condition, whatever it was, had been introduced by one of the Derbyshire cows, and had then spread through various sheds at the Hendon farm. Mr Power was able by the most minute inquiry to trace the movements of those cows and the various sheds in which they were placed from time to time, and he held that the various recrudescences of the outbreak in North London corresponded with the movements of the affected cows.

The exciting cause, then, of this outbreak was believed by $\mathrm{Mr}$ Power to be a condition of certain milch cows which had for its outward manifestation an eruption on teats and udders, and which was communicable from cow to cow. Subcultures of the ulcerous discharges of the affected animals inoculated into calves produced a disease having unmistakable affinities, under some conditions, with the disease in the milch cows, and under other conditions with scarlet fever in the human subject (Klein). Now, it must be added, that scarlet fever appeared simultaneously in all but one of the five localities to which the milk was distributed. The exception received none of the milk from the affected cows until later, when the disease also appeared in this district, owing to some of the milk from the affected cows being sent there. When the sale of the milk was pro- 
hibited in London, some of it was clandestinely distributed amongst the poor of Hendon. Amongst those served, half a dozen families were invaded by scarlatina at a time when the disease had ceased to exert its influence in the London districts. The intermission which had occurred in the scarlatina in Hampstead and St Pancras during the ten days referred to above, was at the time when the infective cows had been moved into a shed sending milk only to Marylebone. A few days later they were again moved into a shed from which milk was distributed to the two former districts.

Thus the investigation showed the Hendon farm to be the main source, and, as far as could be judged, the cows referred to, the particular source, of the implicated milk, for the disease followed the distribution of their milk. The further inquiry was with regard to the nature of the disease or influence appertaining to these cows.*

Sir George Buchanan summarised for the Local Government Board his interpretation of the facts, and concluded that the Hendon disease was "a form occurring of the very disease that we call scarlatina when it occurs in the human subject." + His views found acceptance with a large number of persons, but most veterinarians and certain pathologists were not prepared to accept the matter as proved. In consequence, further investigations and inquiries were instituted, and a controversy arose. Sir George Brown, then head of the Privy Council's Agricultural Department, held (1) that the Hendon disease was not confined to the Hendon farm from which the implicated milk was derived, but occurred elsewhere, and was followed by no scarlet fever; (2) that the probable source of infection at Hendon was human; and (3) that the alleged bovine scarlet fever was cowpox.+

As a matter of fact, the exact origin of the London epidemic at that time has not yet been, and now probably never will be, demonstrated. Even at the present time the specific micro-organism which is the causal agent of human scarlet fever is not established or proved. That is to say, no micro-organism has yet been isolated in human scarlet fever which fulfils the postulates of Koch. Much less was this the case sixteen years ago, when bacteriological methods were less perfect than they are even to-day. From this it follows that the vera causa was obscure, and yet without this link it was impossible to complete the chain of evidence by which it could be definitely known that any disease of the cow was responsible for the epidemic. The probabilities might be strong or weak, but proof was

* Local Government Board Report, 1885, pp. 73-111 (W. H. Power).

$\dagger$ Seventeenth Annual Report of the Local Government Board, 1887-88 (Medical Officer's Supplement), pp. 13, 14 .

$\mp$ For a discussion of the whole subject, see Bacteriology of Milk (Swithinbank and Newman), 1903, pp. 279-304. 
wanting. The inoculation experiments, in so far as they yielded positive results, were also open to the same unreliability. Unfortunately, too, there was, on the other hand, circumstantial evidence of various kinds, which, while it proved little, opened up a variety of possibilities by which the milk consumed in London might have become infected. The case was therefore unproved. Nevertheless, it raised many important questions and stimulated much valuable inquiry. When milk becomes infected with scarlet fever the infection is almost invariably derived directly from some person suffering from the disease, recognised or unrecognised.

Scarlet Fever, in not a few milk-epidemics, has shown certain modifications of a more or less marked character. The disease is generally mild, and simultaneously with an outbreak of the specific disease due to milk, there will not infrequently be found a large number of "ordinary sore throats." Even in the scarlatinal cases, the disease has a tendency to remain localised to the throat (Power). The rash may be evanescent, and the desquamation is scanty (Parsons). There is also a marked absence of post-scarlatinal nephritis or any other kidney complication (Parsons, Buchanan, and others). A characteristic which has been frequently noted, and is readily to be understood, is the frequency of vomiting and diarrhœa, rather particularly at the commencement of the disease (the Fallowfield epidemic, 1879, is an illustration). It is probable that these signs of alimentary irritation or poisoning are due to poisonous organismal products contained in the milk. On more than one occasion they have led to an appearance of intoxication rather than infection. Finally, there is a clinical feature, to which reference has already been made, and which may bear a significance not at first appreciated, namely, the comparative indisposition of the disease to spread by contagion. This may be attributable to the mildness of the disease, to the small amount of skin eruption and desquamation commonly present, and possibly to the fact that the poisonous properties of the milk are to a certain extent eliminated from the system by the vomiting and purging. Every clinical sign which has been noted leads to the conclusion that the disease as conveyed by milk is frequently mild, and therefore has both a small mortality, and no tendency to spread by contact. There is one other point deserving of mention. Sir George Buchanan noticed, in a scarlet fever epidemic with which he had to deal, that in persons who had had scarlet fever at some previous time, and who drank the implicated milk, almost the only symptom of ill-health which they presented was a sore throat. There was no rash, no vomiting, no pyrexia, although other members of the family under precisely similar circumstances suffered from typical scarlet fever. Many other workers have confirmed the occurrence of aberrant forms of milk-borne scarlatina. 


\section{Characteristics of Milk-borne Epidemics}

The following are the chief characteristics of infectious disease carried by milk:-

(a) There is a special incidence of disease upon the track of the implicated milk supply. It is localised to such areas.

(b) Better-class houses and persons generally suffer most.

(c) Milk drinkers are chiefly affected, and those suffer most who are large consumers of raw milk.

(d) Women and children suffer most, and frequently adults suffer proportionately more than children.

(e) Incubation periods are shortened.

$(f)$ There is a sudden onset and a rapid decline.

(g) Multiple cases in one house occur simultaneously.

(h) Clinically, the attacks of disease are often mild, contact infectivity is reduced, and the mortality rate is lower than usual.

\section{Other Diseases Conveyed by Milk}

In addition to the above, there are other diseases spread by means of polluted milk. From time to time exceptional cases have occurred in which diseases like anthrax, or some forms of foot-andmouth disease have been spread by this means. But it is not to such rare cases that we refer. There are three very common diseases in which milk has been proved to play a not inconsiderable part, viz., thrush, sore throat, and diarrhoea.

Thrush.-The mould which gives rise to the curd-like patches in the throats of children, and which is known as Oidium albicans, frequently occurs in milk. Soft, white specks are seen on the tongue and mucous membrane of the cheeks and lips, looking not unlike particles of milk curd. If a scraping be placed upon a glass slide with a drop of glycerine, and examined by means of the microscope, the spores and mycelial threads of this mould will be seen. The spores are oval, and possess a definite capsule. The threads are branched and jointed at somewhat long intervals. Milk affords an excellent medium for the growth of this parasite. Thus undoubtedly we must hold milk partly responsible for spreading this complaint. Penicillium, Aspergillus, and Mucor are also frequent moulds in milk.

Sore - Throat Illnesses.-The obscure milk-borne epidemics of which sore throat has been the chief symptom, are among the most interesting of all the diseases conveyed by milk. The usual symptoms are congestion of the tonsils and mucous membrane of the throat, with sometimes ulceration, enlargement of the cervical glands, and some pyrexia, and general malaise. In not a few 
instances there has been observed various kinds of rash which have generally been of an evanescent character. Where the throat illnesses have occurred contemporaneously with outbreaks of scarlet fever or diphtheria, it is not unlikely that the condition was in reality scarlet fever or diphtheria. In South Kensington, in 1875, there was an outbreak of disease which attracted much attention at the time, and was officially investigated.* The illness was traced to some cream consumed at a dinner party, and in all twenty persons suffered, some of whom had scarlet fever, and others only sore throat. But the investigation showed that in the district from which the cream was obtained 119 cases of sore throat had occurred. Dr Darbishire described an outbreak of 18 cases of sore throat at Oxford in 1882, the condition being characterised by marked severity of throat symptoms and a disproportionate amount of constitutional symptoms. $†$

Similar outbreaks occurred in 1881 at Aberdeen (300 persons affected), and Rugby school (90 boys) in three school-houses supplied by one milkman, who did not supply any other houses in the school. But he supplied houses in the town, of which nearly 50 per cent. were attacked with sore throat. Inquiry showed that some of the milk used had been obtained from a cow suffering from mastitis. + A similar outbreak took place in Edinburgh in 1888, and was investigated by Cotterill and Woodhead; and another at Dover in 1884, where there was a sudden and severe outbreak of sore throat in a localised area of good-class houses, affecting 205 persons, who all obtained milk from one particular farm. The chief symptoms were local inflammation of the throat, enlargement of lymphatic glands in neck, and vesicular eruptions preceding and accompanying the inflammation. The dairyman obtained his supply from 12 cows of his own, and from three farms in the country. On one of these latter apthous fever had broken out, and it was from this farm that the dairyman obtained his implicated milk and cream. Moreover, when the supply from this farm was diverted temporarily, it set up a simultaneous second outbreak of sore throat. $\$$ In 1890 there occurred an epidemic of sore throat at Craigmore, which was referred to milk infection. The number of cases was 80 . The disease manifested chiefly in the form of severe sore throat, but in a number of the cases erysipelas developed in addition. The milk appears to have been infected by two milkmaids who were suffering from sore throat. Those attacked most severely had drunk most of the implicated milk. A dog and cat which had a good

* Report of Medical Officer of Local Government Board, 1875, vol. vii., p. 80.

† St Bartholomew's Hospital Reports, vol. xx.

$¥$ Brit. Med. Jour., 1881, vol. i., p. 657 ; vol. ii., p. 415.

$\S$ Practitioner, 1884, vol. i., p. 467 (Dr M. K. Robertson). 
deal of the milk became very ill with "severe inflammation of the throat." *

In 1892 there was an extensive outbreak of sore throat in Upper Clapton. Dr King Warry, describing the symptoms in the Practitioner, at the time held that the pathological condition was scarlet fever in a mild form. His reasons for this view were three: (a) scarlet fever attacked one member of a family, and the sore throat disease other members who had previously had scarlet fever; (b) both scarlet fever and sore throat patients were isolated together, but those suffering from sore throats did not contract the scarlet fever; and $(c)$ some of the patients suffering from sore throat had at the same time certain symptoms simulating scarlet fever, such as desquamation of the skin, kidney disease, and rheumatic symptoms. With this view of the specificity of throat illness under similar circumstances Dr Parsons agrees. $t^{\dagger}$ In the Upton and Macclesfield scarlet-fever outbreak of 1889 , there were 40 cases of sore throat which were apparently related to scarlet fever for the following reasons:- $(a)$ The sore throat occurred in older persons in whom rashes are less prone to occur, and who had had scarlet fever; (b) in some cases there was skin desquamation; $(c)$ when the children suffered from scarlet fever the adults in the same house suffered from sore throat; $(d)$ two cases of diphtheria at the same period showed symptoms simulating scarlet fever; and $(e)$ pyrexia and delirium were present in the worst cases.

Two comparatively small milk-borne outbreaks of "follicular tonsillitis" were reported in 1897, one in Anglesey ${ }_{+}^{+}(15$ cases), and the other at Surbiton $\S(30$ cases). The milk was bacteriologically examined, and Staphylococcus pyogenes and Streptococcus pyogenes were found, but no $B$. diphtherio. Bacteriological examinations of the patients' throats yielded precisely similar results. A man whose business it was to milk the cows was found to be out of health, with well-marked tonsillitis and suppurating whitlows on both hands.

In April and May 1900 an outbreak of septic sore throat occurred in North Hackney affecting 151 persons in eighty-eight households, 85 per cent. of which were supplied by one milkman.

A sore-throat outbreak at Brighton in November 1901 was investigated by Dr Newsholme. Out of a total of 29 persons in a private girls' school, 18 were affected. The chief symptoms were high temperature, rapid pulse, tonsillitis with fibrinous exudation locally except on the soft palate. In two cases there was an evanescent rash. Dr Newsholme was able, after minute inquiry, to

* Glasgow Med. Jour., 1890, vol. xxxiv., pp. 241-258.

+ Report to Local Government Board, 1889.

$\ddagger$ Brit. Med. Jour., 1897, vol. ii., p. 339 (Dr C. Grey-Edwards of Beaumauris).

$\S$ Annual Report of Medical Officer of Health, 1897 (Dr Coleman). 
trace the cases at the school to one of their number, who had come into the way of infection derived from a milk supply contaminated by infectious disease in three families connected with the dairy.*

In 1902 an outbreak of milk-borne sore throat occurred at Lincoln (199 cases). Of the total 168 or 85 per cent. had consumed the suspected milk. The outbreak commenced suddenly, lasted a few days, and then suddenly terminated. The majority of the victims were adults or persons over twelve years of age. Females were much more affected than males. The symptoms of the disease simulated scarlet fever. There was marked sore throat and swelling of the tonsils, which were in many cases furred. On the third or fourth day of the disease there was enlargement of the cervical glands, rash (like rötheln), and fever. The commonest complications were gastritis and rheumatism, but there were a number of irregular conditions and varieties of rash. The poison in the milk seems to have existed in the highest degree in the cream, and Klein isolated a yeast which he considers related to a yeast known heretofore to have been associated with throat illness and thrush. It has been suggested that some relationship may exist between this yeast and the spores of rusts, smuts, and mushroom fungi consumed by the cows. The whole circumstances of the case of this outbreak furnish one of the most interesting modern chapters in milk epidemiology. $\dagger$ In 1903 another outbreak occurred (56 cases) at Lincoln of a somewhat similar kind. In 1902 an outbreak occurred at Bedford (42 cases) consisting of sore throat, malaise, headache, giddiness, etc. Here also the cream seemed more infective than the milk. Indeed, in several families only those who had taken the cream suffered. The incidence was chiefly upon young adults.+

A somewhat similar outbreak occurred in October and November 1903, at Woking, in which persons were infected in ninety-eight different houses. The illness was sore throat, with glandular enlargement and general symptoms. Of the ninety-eight households affected, seventy-six obtained their milk directly from a source open to criticism. Dr Pierce, the medical officer of health, examined four cows yielding the milk, and a bacteriological examination was made of the milk. In the result it was found that two of the cows suffered from suppurative mammitis, and the liquid yielded by these two cows "consisted of the most part of pus such as would be contained in an abscess." This was the character of the milk which had been consumed by the persons suffering from the illness. $§$

* Jour. of Hygiene, 1902, vol. ii., pp. 150-169. Annual Report of Medical Officer of Health of Brighton, 1901. Mair).

+ Report to the Local Government Board, No. 190, Oct. 1903 (Dr L. W. Darra

$¥$ Report of Medical Officer of Bedfordshire County Council, 1902, pp. 60-62.

$\$$ Brit. Med. Jour., 1903, ii. p. 1492. 
The most recent sore-throat outbreak due to consumption of infected milk occurred in Finchley in 1904. Some 500 cases came to the knowledge of the medical officer of health (Prof. Kenwood) of the district, and another fifty occurred in the outlying neighbourhood. The incubation period was twenty-four to forty-eight hours, followed by enlargement of submaxillary glands, sore throat, fever, and general malaise. In a few cases there was a measles-like eruption on the lower limbs. Professor Kenwood formed the opinion that the epidemic was due to disease in the cows furnishing the millk, but no specific organism was discovered.* A somewhat similar outbreak occurred in the same district in 1894.

Pus in Milk.-It may here be stated that not infrequently milk contains pus cells, and there can be little doubt that such milk may set up disease in persons consuming it.

Stokes and Wegefarth made an inquiry into the subject some years ago, counting the number of pus cells in the field of the microscope in milk from cows kept under various conditions of insanitation. Taking one pus cell in the field as a standard, Stokes and Wegefarth found 25 per cent. of the milks of country cows, kept under good conditions, and 88 per cent. of town cows, kept under bad conditions, contained pus cells. Eastes, who made an examination of 186 London milks, found pus cells present in 30 per cent., muco-pus in 48.7 per cent., and streptococci in 75.2 per cent. $\dagger$ An investigation of milk in St Pancras in 1899 yielded 24 per cent. of samples containing pus cells. + Foulerton, examining a series of milks from Finsbury in 1903, found pus and allied cells in 32 per cent. of them, staphylococci in 28 per cent., and streptococci in 32 per cent. Forty per cent. of the samples examined contained "foreign dirt." \$ Mucous threads are commonly found in milk containing pus. Such threads probably consist of nucleo-albumin, and when occurring with pus cells, the condition of "muco-pus" is present. This is held to indicate inflammatory lesion of the ducts of the udder, and not abscess formation in the substance of the gland. Blood corpuscles are not rare in milk, particularly soon after lactation. The last and least important kind of cell is that of the epithelium. Such scales may be derived, either from the hand of the milker or from the teats of the udder. Epithelial cells are large and nucleated. Milk containing many blood cells, mucous threads, and leucocytes, and milk containing any pus cells, should be looked upon as unfit for human consumption. Eastes, Holst, Niven, Stokes, Bergey, Hirsch, and others, have drawn attention to the ill effects which streptococcal

\footnotetext{
Special Report of Medical Officer of Stoke Newington, 1904.

+ Brit. Med. Jour., 1899, vol. ii., p. 1342.

† Report on Health of St Pancras, 1899, pp. 61-66 (Dr Sykes).

$\S$ Report on Milk Supply of Finsbury, 1903, p. 44.
} 
milk has upon persons consuming it. In the main these are twofold, namely, gastro-intestinal diseases and sore throats. The evidence implicating streptococcal milk is empirical and circumstantial, and yet it appears to be growing in force and volume. On the other hand, streptococcus has been found in the fresh milk derived from healthy udders (Reed and Ward).

\section{Milk-borne Cholera}

The cholera bacillus is unable to live in an acid medium. Hence its life in milk is a limited one, and generally depends upon some alkaline change in the milk. Heim found that the organism of cholera would live in raw milk from one to four days, depending upon the temperature. D. D. Cunningham, from the results of a large number of investigations in India, concludes that the rapidly developing acid fermentations normally or usually setting in, connected with the rapid multiplication of other common bacteria and moulds, tend to arrest the multiplication of cholera bacilli, and eventually to destroy their vitality. Boiling milk appears, on the contrary, to increase the suitability of the milk as a nidus for cholera bacilli, partly by its germicidal effect upon the acid-producing microbes, and partly that it removes from the milk the enormous numbers of common bacteria, which in raw milk cause such keen competition that the cholera bacillus finds existence impossible.

Professor W. J. Simpson, sometime the Medical Officer of Health for Calcutta, has placed on record an interesting series of cholera cases on board the Ardenclutha, in the port of Calcutta, which arose from drinking milk which had been polluted with one quarter of its volume of cholera-infected water. This water came from a tank into which some cholera dejecta had passed. Of the ten men who drank the milk, four died, five were severely ill, and one, who drank but very little of the milk, was only slightly ill. There was no illness whatever among those who did not drink the milk. In 1894, a milk-borne outbreak of cholera occurred in the Gaya Gaol, affecting some twenty-six persons.

\section{Milk-borne Epidemic Diarrhœa}

In 1892, Gaffky recorded an instance in which three men connected with the Hygienic Institute at Giessen were suddenly taken ill. They had chills, fever, diarrhoea, and general symptoms. The only article of diet of which they had all partaken was milk, which was traced to a cow suffering from enteritis. The milk of this cow as it left the udder contained no bacteria. But bacteria gained access during the milking from the dried particles of fæcal matter on the 
posterior portion of the udder. In these particles was found a bacillus which proved very pathogenic for mice and guinea-pigs, and which corresponded to an organism isolated from the stools of the patients.*

In 1894 an outbreak occurred at Manchester, $\uparrow$ characterised by diarrhæa, sickness, and abdominal pains. The cases numbered 160 in forty-seven houses, or just 50 per cent. of the houses served by one and the same milk-seller. Raw-milk drinkers were the chief sufferers, and those not drinking the implicated milk did not suffer. Dr Niven visited the farm whence the milk came, and found that it was the milk from the farm itself, and not the added milk from a more distant farm, which supplemented the farmer's stock that had caused the epidemic, the home-farm milk only being sent into the affected district. Near the farm were 40,000 tons of privy-midden refuse. Two streams ran near the farm, meeting below, one fouled by the drainage of the tip, and the other being contaminated with sewage and with matter from a tripe-boiling place. The water used for washing the milk-pails was tepid, and kept in a foul cistern. The cows drank from a pool which received the drainage from the cowshed midden. The stored milk could be readily contaminated from emanations from the cowshed. Professor Delépine examined the milk, and found $B$. coli communis abundantly present, and Dr Niven elicited the fact that a cow affected with inflamed udder ("garget") had been removed from the farm and slaughtered. The outbreak was attributed to milk in any case, and to the probable infection of it by the diseased cow. But Delépine has pointed out that it is more probable that the milk was contaminated with fæcal pollution rather than infectious disease of the cow.+

In $1895 \S$ and $1898 \|$ three outbreaks of epidemic diarrhœa occurred amongst the patients at St Bartholomew's Hospital, London, traceable in the first two instances to milk, and in the third, to rice pudding made with milk. I On Sunday night, 27th October 1895, an outbreak of diarrhoea affected 59 in-patients, all of whom had recently taken milk, and from the evacuations the spores of $B$. enteritidis sporogenes was isolated by.Klein. The patients suffered quite irrespective of whether or not the milk had been boiled. Some milk also, derived from the same source as the milk which had caused the poisoning, was examined by Klein, and found to contain the spores of the same organism. On Sunday, 6th March 1898, a second outbreak of severe diarrhœa occurred in this hospital, affect-

* Deut. Med. Woch., vol. xviii., p. 14.

† Annual Report of Medical Officer of Health of Manchester, 1894 (Dr Niven).

$\ddagger$ Jour. of Hygiene, 1903, vol. iii., No. 1, pp. 76, 77.

$\S$ Report of the Medical Officer of Local Government Board, 1895-96, pp. 197-204.

|| Ibid., 1897-98, p. 235.

ๆ Ibid., 1898-99, p. 336. Lancet, 7th January 1899. 
ing 146 patients, and there was evidence on this occasion also that the medium of infection had been milk. On 5th August 1898, a third outbreak affecting 84 patients and 2 nurses took place at the same hospital, the vehicle of infection in this instance being some rice pudding made with milk, also said to contain an organism similar or identical with the $B$. enteritidis sporogenes. There can be no doubt that milk was the agent of infection in each of these three outbreaks. It was in these outbreaks that the $B$. enteritidis sporogenes of Klein was isolated and held to be the specific organism. Dr Klein has produced evidence in behalf of this bacillus being the true cause of epidemic diarrhœa.*

Other similar outbreaks are on record traceable to contaminated milk. Nor is the evidence on this subject derived only from epidemics. Newsholme has shown that of the fatal cases of diarrhoea in children only $9 \cdot 4$ per cent. occur in children which have been breast-fed. $\dagger$ In Finsbury 20 per cent., in Kensington 35 per cent., and in Croydon 12 per cent., were breast-fed. From such figures it is evident that most of the deaths of infants from diarrhœa occur in children who have been hand-fed. This fact is now widely accepted. In one of his official reports + Dr Hope, of Liverpool, states that "the method of feeding plays a most important part in the causation of diarrhœa: when artificial feeding becomes necessary, the most scrupulous attention should be paid to feeding-bottles." Careless feeding, in conjunction with a warm, dry summer, invariably results in a high death-rate from this cause. These two causes interact upon each other. A warm temperature is a favourable temperature for the growth of the poisonous micro-organism; a dry season affords ample opportunity for its conveyance through the air. Unclean feeding-bottles are obviously an admirable nidus for these injurious bacteria, for in such a resting-place the three main conditions necessary for bacterial life are well fulfilled, viz., heat, moisture, and pabulum. The heat is supplied by the warm temperature, the moisture and food by the dregs of milk left in the bottle; and the dry air of summer assists in transit. It becomes clear that diarrhœa is, in part at all events, due to polluted milk, polluted by dust or flies, directly or indirectly, at the farm or in the home.

Delépine has urged that milk is infected at the farm or in transit, as many of the milks which he examined and proved to be virulent had not been exposed to any influence attributable to a consumer's home, but were in fact infective before they reached the consumer. $§$

* Reports of Medical Officer of Local Government Board, 1895-96, 1896-97, 1897-98,

+ Report on Health of Brighton, 1902, p. 50.

\# Report of Health of Liverpool, 1899, p. 41.

$\S$ Jour. of Hygiene, 1903, p. 86. 
He considers the injurious properties of such milk is due to frecal pollution and the action of $B$. coli (in particular those coliform bacilli which produce a large amount of acid and do not coagulate milk). Newsholme considers such contamination may be responsible for setting up epidemics of diarrhœa occurring in connection with a particular milk supply, as in the analogous case of epidemics of infectious diseases, such as typhoid. But he holds that the ordinary sporadic cases of diarrhœa, which carry off single children in large numbers in urban districts, are due "chiefly to domestic infection of milk or other foods, or to direct swallowing of infective dust."** Probably, we have a double pollution of milk in actual practice, one originating at the farm, one brought about subsequently. The latter may be produced by flies, or from manure heaps (Waldo), or from dust in roads and yards of towns (Richards), or from the generally filthy manipulation of the milk from the time when it becomes the property of the milk-seller to the moment of consumption. It should not be forgotten in this relation that stale milk contains toxic properties altogether apart from, and in addition to, actual bacteria. It is possible that the products of organismal action have a much greater effect in the causation of diarrhœa than is generally supposed. $\dagger$

\section{Preventive Measures}

It is not possible in the present state of our knowledge in respect of milk bacteriology to lay down very exact limits as to what is, and what is not, unsatisfactory milk. A numerical standard of contained organisms is not practicable at present. But we think, it may be said, that, in any case, milk should not be considered as marketable if it contains (a) numerous pus cells; $(b)$ pathogenic organisms; or $(c)$ "organisms of indication" of contamination. The presence of vast numbers of bacteria, such as millions per cubic centimetre, also indicates unclean manipulation.

1. Among the preventive measures which these conditions indicate, cleanliness of cows, dairy-hands, and milk-cans or other milk vessels, stands first in importance. Refrigeration of the milk, being more easily obtained than cleanliness, should be insisted upon without delay. Similar measures are also needed with regard to all things or persons coming in contact with the milk. Absolute bacterial cleanliness is most difficult to obtain, if not practically impossible. Occasional infection must, therefore, occur.

2. To guard against the effects of accidental fæcal infection, milk should be consumed fresh, when possible. When it cannot be con-

* Report on Health of Brighton, 1902, p. 50.

$\dagger$ For a fuller discussion of the whole question of the disease-producing power of milk, see Bacteriology of Milk, 1903, pp. 210-391. 
sumed fresh it should be refrigerated, i.e. kept at a temperature below $4^{\circ} \mathrm{C}$., for this inhibits the rapid multiplication of bacteria. When milk cannot be used fresh or refrigerated, it should be steritised by heat.

3. Greater domestic and municipal cleanliness is an essential requirement. This must include the cleanly preparation of food, and particularly the handling and storage of milk; the cleansing of milk-bottles; reduction of dust in houses, courts, and streets, and protection of milk from dust in shops and houses; impervious roads and paving; and the substitution of wet-cleansing for dry cleansing, and frequent cleansing for infrequent.

4. Lastly, there is needed "a crusade against the domestic fly, which is most numerous at the seasons and in the years when epidemic diarrhoa is most prevalent, and probably plays a large part in spreading infection" (Newsholme).

\section{Methods of Protecting and Purifying Milk}

After the consideration of the somewhat extensive category of diseases which may be milk-borne, it will be desirable to make brief reference to some of the means at our disposal for obtaining and preserving good, pure milk.

We considered at the commencement of this chapter the most frequent channels of contamination. If these be avoided or prevented, and if the milk be derived from cows in good health and well kept, the risk of infection is reduced to a minimum. The two things necessary are clean, healthy cows and clean methods of milling and manipulation. What the Danes can do, other dairy workers can do. The cow byre, the udder, the milk vessel,* and the milkers should each be thoroughly clean. $\dagger$ But we have seen that much,

* Probably the best method of cleansing dairy utensils is by using steam or boiling water and soda. The advantage of boiling water is obvious. The addition of soda enhances its value, as the soda unites with the lactic acid present, forming a soluble lactate of soda, and also with grease, a fat forming an easily soluble soap. Nor does it injure or rust the metal with which it comes into contact.

$\dagger$ It may be well to add in a footnote an account of the Danish method as carried out in England, for it illustrates in concrete form the practical way of reducing pollution of milk to a minimum :-

The principles and practice of the Copenhagen Milk Supply Company have been introduced into England, and are being carried out by $\mathrm{Mr} \mathrm{C}$. W. Sorensen at the White Rose Dairy, West Huntington, York. Mr Sorensen is a nephew of Mr Busck, of the Copenhagen Company, and has been trained in the Danish methods. His dairy farm at York is carried on in a similar manner to the Copenhagen Company's work, with this difference, that whilst the latter obtain their milk from contributory farms, Mr Sorensen works his own farm, and the control and management of the cows is under his direct and immediate supervision. The writer had an opportunity recently of visiting this dairy farm near York, and a brief description of the most important points may be added here.

1. The health of the cows is secured by a special monthly inspection by the 
if not most, of the pollution of milk arises after the milking process and during transit and storage preparatory to use. Bacteria are so ubiquitous that to prevent the entrance of any at all is futile. It is, therefore, well to bear in mind the extreme importance of careful straining and immediate cooling. Straining or screening milk removes the grosser particles of dust, dirt, hairs, etc., and these, it will be remembered, are the "rafts" and "vehicles" of bacteria. If they are at once removed therefore, many bacteria will be removed with them.*

York Corporation Veterinary Officer, Mr William Fawdington, M.R.C.V.S., who has authority to order the disposal of any unhealthy or even suspected animal, and whose reputation and experience affords a guarantee of efficiency in this important point. There are about 50 cows in all, 10 of which are Jerseys. The feeding of the cows is scientifically carried out. No brewers' grains, turnip-tops, or other unsuitable foods are used, and especial care is exercised in the selection and feeding of the cows supplying "Table or Nursery Milk," so as to maintain a high standard of richness and flavour. To ensure an abundant supply of pure water for the cows to drink, as well as for cleansing purposes, the farm has been connected with the York City Water Supply, which is provided in a continuous trough at the head of the stalls. The cleanliness and ventilation of the cow-houses receives special attention, and is in every way excellent.

2. While no money has been wasted on fancy fittings (which make the milk no better, but simply increase the cost), the proprietor's aim has been to keep everything sweet and clean from the cows themselves down to the smallest utensil. A high-pressure boiler has been put in for sterilising all utensils, cans, etc., with steam.

The udders of the cows are cleansed before milking. The milkers are clothed in over-alls, and wash before, and if necessary, during milking. The operation of milking is carried out under cleanly conditions and with clean utensils. After milking the milk is strained by a "Ullax" strainer.

3. Immediately after the milk is strained, prompt and efficient cooling is obtained by allowing it to flow in a thin layer over a corrugated copper cylinder, inside which cold water and ice are passed, thus reducing the temperature in a few seconds to a point at which germ life cannot develop. Clean milk, so treated, needs no " preservation." If kept in a cool place it will remain perfectly sweet for several days, even in the hottest weather. Therefore, no preservation or sterilisation is necessary.

4. The usual practice of slopping milk about from one can to another in the street-exposed to contamination from clouds of dust, the not always clean hands, or, in wet weather, the dripping garments of the driver-is one so objectionable that only long usage and the absence of anything better has made it tolerated. The ideal system, without doubt, is delivery in glass bottles, filled and sealed at the dairy, and placed straight on the table without the intervention of jugs, basins, or what not. Next comes delivery in cans, likewise filled and sealed at the dairy. After that comes the system of drawing the milk by tap from a sealed can, which, however, is much preferable to the plan of dipping into an open can. The entire system at this dairy farm is so arranged as to supply a clean whole milk from healthy cows kept under hygienic conditions, and protected from dust and infection. This desirable object is attained by $(a)$ clean milking, $(b)$ straining, $(c)$ cooling, and (d) bottling promptly, efficiently, and at the dairy farm. On the whole, Mr Sorensen's methods appear to represent the high tide of dairy farm work in England. But nothing is done by him which could not be done by every dairy farmer in the country.

* One of the most satisfactory strainers in the market is that known as the "Ulax." This apparatus consists of a metal sieve through which the milk is first passed. Then a finer double sieve with a thin layer of sterilised cotton-wool placed between the two metal sieves acts as a secondary filter (see Fig. 23). 
Low temperatures, it is true, do not easily destroy life, but they have a most beneficial effect upon the keeping quality of milk. It has been suggested that at the outset of the process of cooling, currents of air, inimical to bacteria, are started in the milk. If, however, the temperature be lowered sufficiently, the contained bacteria become inactive and torpid, and eventually are unable to multiply or produce their characteristic fermentations. At about $50^{\circ} \mathrm{F}$. $\left(10^{\circ} \mathrm{C}\right.$.) the activity ceases, and at temperatures of $45^{\circ} \mathrm{F}$. $\left(7^{\circ} \mathrm{C}\right.$.) and $39^{\circ} \mathrm{F}$. $\left(4^{\circ} \mathrm{C}\right.$.) organisms are practically deprived of their injurious powers. If it happens that the milk is to be conveyed long distances, then even a lower temperature is desirable. The most important point with regard to the cooling of milk is that it should take place immediately. Various kinds of apparatus are
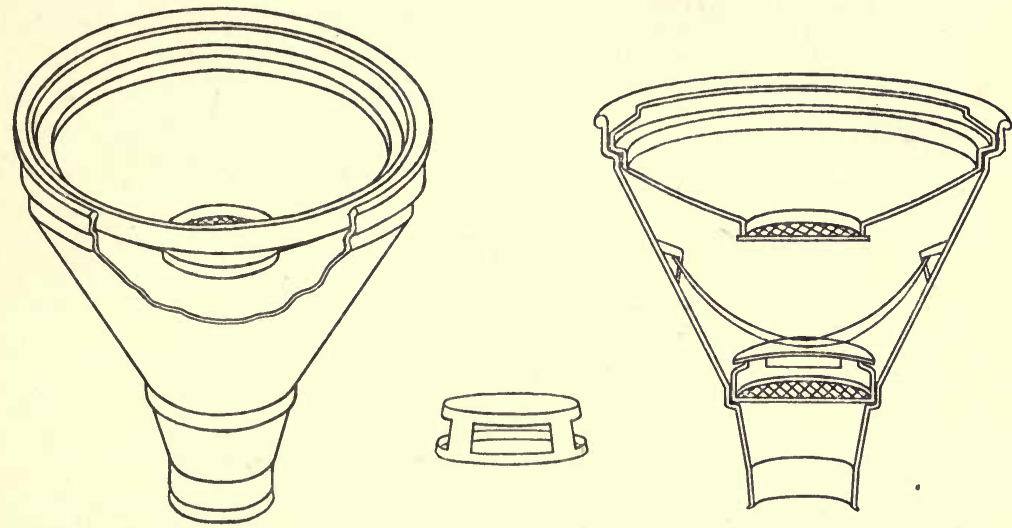

FIg. 23.-" Ulax" Filter.

effective in accomplishing this. Perhaps those best known are Lawrence's cooler and Pfeiffer's cooler, the advantage of the latter being that during the process the milk is not exposed to the air. It must not be forgotten that cooling processes are not sterilising processes. They do not necessarily kill bacteria; they only inhibit activity, and under favourable circumstances torpid pathogenic bacteria may again acquire their injurious faculties. Hence, during the cooling of milk greater care must be taken to prevent aërial contamination than is necessary during the process of sterilising milk. No cooling whatever should be attempted in the stable; but, on the other hand, there should be no delay. Climate makes little or no difference to the practical desirability of cooling milk, yet it is obvious that less cooling will be required in the cold season. The final treatment of milk has in practice comprised the addition of preservatives, filtration, and sterilisation. 
Preservatives are widely used, especially in town milks. They do not, as a rule, kill bacteria in milk, but merely stifle them, and prevent rapid multiplication and increasing acidity. They disguise the true condition of the milk in which they exist. It is to be feared that their systematic addition to milk tends to place a premium on uncleanly and improper dairying. There is evidence, also, to show that by a cumulative process preservatives may be injurious to persons consuming the milk. The most commonly used antiseptics in milk are borax, formalin,* carbonate of soda, and salicylic acid. $\dagger$

Secondly, it is possible to remove in part the pollution of milk by filtration. Filtration has been practised for some time by the Copenhagen Dairy Company, by Bolle, of Berlin, and various milk companies. The filters used consist of large cylindrical vessels divided by horizontal perforated diaphragms into five superposed compartments, of which the middle three are filled with fine sand of three sizes. At the bottom is the coarsest sand, and at the top the finest. The milk enters the lowest compartment by a pipe under gravitation pressure, and is forced upwards, and finally is run off into an iced cooler, and from that into the distribution cans. By this means the number of bacteria are reduced to one-third. The difficulty of drying and sterilising enough sand to admit a large turnover of milk is a serious one. This, in conjunction with the belief that filtration removes some of the essential nutritive elements of milk, has caused the process to be but little adopted. Dr Seibert states that if milk be filtered through half an inch of compressed absorbent cotton, seven-eighths of the contained bacteria will be removed, and a second filtration will further reduce the number to one-twentieth. One quart of milk may thus be filtered in fifteen minutes.

* S. Rideal and A. G. R. Foulerton conclude from a series of experiments that boric acid (1-2000) and formaldehyde (1-50,000) are effective preservatives for milk for a period of twenty-four hours, and that these quantities have no appreciable effect upon digestion or the digestibility of foods preserved by them (Public Health, 1899, pp. 554-568).

$\uparrow$ The Departmental Committee on Preservatives and Colouring Matters in Food, 1901 (Report, pp. xxiv.-xxv.) recommend :-

1. That the use of Formaldehyde in food and drink is absolutely prohibited, and the Salicylic Acid be not used in greater proportion than one grain per pint or pound respectively for liquid or solid food, its presence in all cases to be declared.

2. That the use of any preservatives or colouring matter in milk be made an offence under the Sale of Food and Drugs Acts.

3. That Boric Acid preservatives only be allowed in cream, the amount not to exceed $0 \cdot 25$ per cent., and be notified on a label.

4. That Boric Acid preservatives only be allowed in butter, the amount not to exceed 0.5 per cent.

5. That chemical preservatives be prohibited in all dietetic preparations for the use of children and invalids.

6. That the use of Copper Salts for " greening" be prohibited.

7. That a Court of Reference be established to supervise the use of preservatives and colouring matters in foods. 


\section{Sterilisation and Pasteurisation}

Sterilisation means the use of heat at or above boiling-point, or boiling under pressure. This may be applied in one application of one to two hours at $212^{\circ}-250^{\circ} \mathrm{F}$., or it may be applied at stated intervals at a lower temperature. The milk is sterilised-that is to say, contains no living germs-is altered in chemical composition, and is also boiled or "cooked," and hence possesses a flavour which to many people is unpalatable.

Now such a radical alteration is not necessary in order to secure non-infectious milk. The bacteria causing the diseases conveyable by milk succumb at much lower temperatures than the boilingpoint. Advantage is taken of this in the process known as pasteurisation. By this method the milk is heated to $167-185^{\circ} \mathrm{F}$. $\left(75-85^{\circ}\right.$ C. $)$. Such a temperature kills harmful microbes, because $75^{\circ} \mathrm{C}$. is decidedly above their average thermal death-point, and yet the physical changes in the milk are practically nil, because $85^{\circ} \mathrm{C}$. does not relatively approach the boiling-point. There is no fixed standard for pasteurisation, except that it must be above the thermal death-point of pathogenic bacteria, and yet below the boiling-point. As a matter of fact, $158^{\circ} \mathrm{F}$. $\left(70^{\circ} \mathrm{C}\right.$.) will kill lactic acid bacteria as well as most disease-producing organisms found in milk. If the milk is kept at that temperature for ten or fifteen minutes, we say it has been "pasteurised." If it has been boiled, with or without pressure, for half an hour, we say it has been "sterilised." The only practical difference in the result is that sterilised milks have a better keeping quality than pasteurised, for the simple reason that in the latter some living germs have been unaffected.

Sterilisation may, of course, be carried out in a variety of modifications of the two chief ways above named. When the process is to be completed in one event an autoclave is used, in order to obtain increased pressure and a higher temperature. Milk so treated is physically changed in greater degree than in the slower process. The slow or intermittent method is, of course, based on Tyndall's discovery that actively growing bacteria are more easily killed than their spores. The first sterilisation kills the bacteria, but leaves their spores. By the time of the second application the spores have developed into bacteria, which in turn are killed before they can sporulate.

The application of sterilisation to milk is now very widely adopted by corporations, dairy companies, etc. Recently the writer has had the opportunity of studying an excellent system in vogue in Essex,* and which may be mentioned because it seems to emphasise

\footnotetext{
* J. Carson, Crystalbrook Farm, Theydon Bois, Essex.
} 
principles which might be practised all over England. Briefly, it may be said that Mr Carson's system lays emphasis on five chief points :-

1. The cows used are carefully selected for milking purposes, are regularly inspected, and have all been tested with the tuberculin test. Their feeding is also kept under strict control, no brewers' grains being used. In summer the cows feed on grass, linseed oil cake, and a small quantity of cotton cake and bran; in winter they have hay, mangolds, maize, germ meal, and linseed and cotton cake. The farm is well kept, and maintained under strict sanitary control, the health and cleanliness of the cows being the one thing aimed at.

2. The cows are milked in the byre adjoining the sterilising plant. Both cows and cowsheds are continually maintained in cleanliness. The udders are cleansed before milking, and it is required that milkers also shall be clean in person and management of milking.

3. Immediately after milking, the milk is removed into an adjoining room, strained through a metal screen, and at once separated by an ordinary Laval separator. This separation is adopted for purposes of purification only. The milk and cream are again mixed, and poured by means of a mechanical automatic bottle-filler into bottles.

4. The milk in bottles is then, within a few minutes of leaving the udder, placed in the steriliser and maintained at $212^{\circ} \mathrm{F}$. for twenty minutes. The bottles have been previously sterilised at $220^{\circ} \mathrm{F}$. for sixty minutes.

5. After sterilisation the milk is cooled to $53^{\circ} \mathrm{F}$., and kept at that temperature till required for delivery.

We have examined this milk chemically and bacteriologically, and have found it to be of excellent quality. It is unquestionably an advantage to have milk which is to be sterilised brought under treatment at once, after milking. This cannot always be done, and hence it is the custom of some dairy companies and institutions to sterilise milk on its delivery. But it is of extreme importance to avoid this if practicable. Whatever treatment milk receives, be it refrigeration or sterilisation, such treatment should be applied immediately after the milk is drawn from the udder. There are a large number of appliances and different forms of apparatus now on the market, having for their object the sterilisation of milk. Our object has not been the recommendation of any apparatus or process, but the principles underlying the efficient pasteurisation and sterilisation of milk.

The methods of pasteurisation are continually being modified and improved, especially in Germany, Denmark, and America. Most of the variations in apparatus may be classed under two 
headings. There are, first, those in which a sheet of milk is allowed to flow over a surface heated by steam or hot water. This may be a flat, corrugated surface or a revolving cylinder. The milk is then passed into coolers. Secondly, milk is pasteurised by being placed in reservoirs surrounded by an external shell containing hot water or steam. Dr H. L. Russell * has described one apparatus consisting of a pasteuriser, a water cooler, and an ice cooler. The pasteuriser is heated by hot water in the outside casement. To equalise rapidly the temperature of the water and milk, a series of agitators must be used. These are suspended on movable rods, and hang vertically in the milk and water chambers. By this ingenious arrangement, the heat is diffused rapidly throughout the whole mass, and as the temperature of the milk reaches the proper point, the steam is shut off and the heat of the whole body of water and milk will remain constant for the proper length of time. The somewhat difficult problem of drawing off the pasteurised milk from the vat without reinfecting it by contact with the air is solved by placing a valve inside the chamber, and by means of a pipe leading the pasteurised milk directly and rapidly into the coolers. These are of two kinds, which may be used separately or conjointly. In one set of cylinders there is cold circulating water, in the other finely-crushed ice.

In England, many methods (including a number of patents) are in common use where milk is pasteurised. For instance, at the Hospital for Sick Children in Great Ormond Street, which is in advance of other London hospitals in this respect, milk is received from a well-known metropolitan dairy company in quantities of 200 quarts daily, some being delivered in the morning, and a smaller quantity in the evening. The milk is derived from healthy cows, and sanitary cowsheds, the farms being placed under strict supervision. On receipt, the milk is filtered through muslin, by downward and upward filtration, and passed directly into a bottle-filling machine. Clean, stoppered bottles are kept in readiness. When filled, the bottles are placed in circles in the cage at the bottom of the pasteuriser. Into the centre of the apparatus is placed the thermometer. The lid is closed down and clamped, and the steam is admitted from below. The temperatures used are $160^{\circ} \mathrm{F}$. (or $71^{\circ} \mathrm{C}$.) for twenty minutes in winter, and $180^{\circ} \mathrm{F}$. (or $82^{\circ}$ C.) for twenty minutes in summer. After the elapse of this period, the lid is removed, the stoppers of the bottles are fixed down, and hot water is admitted into the floor of the apparatus. To this hot water is slowly added cold water, and in about forty minutes the pasteurised milk has been cooled down, and is ready for use in the wards. The apparatius is readily cleansed after use, and the various parts, including the bottles, stoppers, etc., are cleaned daily. A somewhat

\footnotetext{
* Report from Wisconsin Agricultural Experiment Station, 1896.
} 
similar apparatus is in use by a Health Association at York,* which has recently started (1903) the York Infants' Milk Depôt, after the manner of the Liverpool and Battersea system. The apparatus provided for this work is one of the latest construction. It consists of an ordinary oval cylinder disinfecting chamber, having doors at both ends. The apparatus is lagged, and with outside steel casing, provided with a steam distributor inside, steam gauge, safety valve, thermometers, ready for steam supply from boiler. In connection with this apparatus there is also provided a convenient size trolley, upon three wheels, together with a steel frame holding three separate platforms, which can be taken apart to suit bottles lor vessels of larger sizes. This frame is mounted also upon wheels rumning in grooves, and channels are fitted inside steriliser to correspond. The steam rises around the bottles from the bottom of the cylinder. The trolley is fitted for both ends, and when duplicated, a "charge" can be taken from one end of the apparatus, and a fresh one inserted at the other. This apparatus can be used as a steriliser or a pasteuriser.

Domestic pasteurisation can be accomplished readily by heating the milk in vessels in a water-bath raised to the required temperature for half an hour, or Aymard's milk sterilisers may be used.

Without entering into a long discussion upon the various pasteurising methods adopted, we may summarise the chief essential conditions. It need scarcely be said that the operation must be efficiently conducted, and in such a way as to maintain absolute control over the time and temperature. The apparatus should be simple enough to be easily cleaned and sterilised, and economical in use. Arrangements must always be made to protect the milk from reinfection during and after the process. The entire preparation of pasteurised milk for market may be summed up in four items :-

1. Pasteurisation in heat reservoir.

2. Rapid cooling in water or ice coolers.

3. All cans, pails, bottles, and other utensils to be thoroughly sterilised in steam before use.

4. The prepared milk to be placed in sterilised bottles, and sealed up.

The quality of the milk to be pasteurised is an important point. All milks are not equally suited for this purpose, and those containing a large quantity of contamination, especially of spores, are distinctly unsuitable. Such milks, to be purified, must be sterilised. Dr Russell has laid down a standard test for the degree of contamination which may be corrected by pasteurisation by estimating the degree of acidity, a low acidity (e.g. 0.2 per cent.) usually indicating

* The York Health and Housing Reform Association, established 1901. Secretary, Miss Hutchinson, 63 Gillygate, York. Apparatus by Wyttenbach : a central depôt in Gillygate, and branch depôts elsewhere in the city. 
a smaller number of spore-bearing germs than that which contains a high percentage of acid.

Lastly, while the heating process is, of course, the essential feature of efficient pasteurisation, it must not be forgotten that rapid and thorough cooling is almost equally important. As we have seen, pasteurisation differs from complete sterilisation in that it leaves behind a certain number of microbes or their spores. Cooling inhibits the germination and growth of this organismal residue. If after the heating process the milk is cooled and kept in a refrigerator, it will probably keep sweet from three to six days, and may do so for three weeks.

Results of Pasteurisation.-Before leaving this subject, we may glance for a moment at the bacterial results of pasteurisation and sterilisation. The two chief of these are the enhanced keeping quality and the removal of disease-producing germs. The former is due in part to the latter, and also to the removal of the lactic acid and other fermentative bacteria. As a general rule, these bacteria do not produce spores, and hence they are easily annihilated by pasteurisation. True, a number of indifferent bacteria are untouched, and also some of the peptonising species. The cooling itself contributes to the increased keeping power of the milk, especially in transit to the consumer.

Pasteurised milks have the following three economical and commercial advantages over sterilised milks, namely $(a)$ they are more digestible, $(b)$ the flavour is not altered, and $(c)$ the fat and lactalbumen are unchanged. Professor Hunter Stewart, of Edinburgh, compiled from a number of experiments the following instructive and comprehensive table:-

\begin{tabular}{|c|c|c|c|c|c|c|}
\hline $\begin{array}{l}\text { No. of } \\
\text { Experi- } \\
\text { ments. }\end{array}$ & $\begin{array}{l}\text { Average No. } \\
\text { of Microbes } \\
\text { per c.c. in } \\
\text { Milk befort } \\
\text { Treatment. }\end{array}$ & $\begin{array}{l}\text { Temperature } \\
\text { and } \\
\text { Duration of } \\
\text { Pasteurisation } \\
\text { in minutes. }\end{array}$ & $\begin{array}{c}\text { No. of Microbes } \\
\text { per c.c. in } \\
\text { Pasteurised Milk } \\
\text { after } 24 \text { hours. }\end{array}$ & $\begin{array}{l}\text { Soluble } \\
\text { Albumen } \\
\text { in } \\
\text { Fresh Milk } \\
\text { per cent. }\end{array}$ & $\begin{array}{c}\text { Soluble } \\
\text { Albumen } \\
\text { in } \\
\text { Pasteurised } \\
\text { Milk } \\
\text { per cent. }\end{array}$ & $\begin{array}{c}\text { Taste of } \\
\text { Pasteurised Milk. }\end{array}$ \\
\hline 5 & 136,262 & $10^{\prime} 60^{\circ} \mathrm{C}$. & 1722 average & 0.423 & 0.418 & Unaffected \\
\hline 4 & 53,656 & $30^{\prime} 60^{\circ} \mathrm{C}$. & $\begin{array}{l}1 \text { sterile } \\
3 \text { averaged } 955\end{array}$ & 0.435 & 0.427 & , \\
\hline 12 & 78,562 & $10^{\prime} 65^{\circ} \mathrm{C}$. & $\begin{array}{l}6 \text { sterile } \\
6 \text { averaged } 686\end{array}$ & $0 \cdot 395$ & 0.362 & $\begin{array}{l}\text { Not appreci- } \\
\text { ably affected }\end{array}$ \\
\hline 12 & 132,833 & $30^{\prime} 65^{\circ} \mathrm{C}$. & $\begin{array}{l}9 \text { sterile } \\
3 \text { averaged } 233\end{array}$ & 0.395 & 0.333 & " \\
\hline 13 & 49,867 & $10^{\prime} 70^{\circ} \mathrm{C}$. & sterile & 0.422 & $0 \cdot 269$ & Slightly bciled \\
\hline 9 & 38,320 & $30^{\prime} 70^{\circ} \mathrm{C}$. & , & 0.421 & 0.253 & 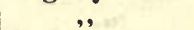 \\
\hline 2 & 77,062 & $10^{\prime} 75^{\circ} \mathrm{C}$. & ,, & 0.38 & 0.07 & Boiled \\
\hline 3 & 48,250 & $30^{\prime} 75^{\circ} \mathrm{C}$. & ,, & $0 \cdot 38$ & 0.05 & ," \\
\hline 1 & $1,107,000$ & $10^{\prime} 80^{\circ} \mathrm{C}$. & ," & 0.375 & 0.00 & ,, \\
\hline 1 & $1,107,000$ & $30^{\prime} 80^{\circ} \mathrm{C}$. & ," & $0 \cdot 375$ & 0.00 & ", \\
\hline
\end{tabular}


It will be admitted that this table exhibits much in favour of pasteurisation; yet the crucial test must ever be the effect upon pathogenic bacteria. Flügge has conducted a series of experiments upon the destruction of bacteria in milk, and he states that a temperature of $158^{\circ} \mathrm{F}$. $\left(70^{\circ}\right.$ C.) maintained for thirty minutes will kill the specific organisms of tubercle, diphtheria, typhoid, and cholera. MacFadyen and Hewlett have demonstrated,* by sudden alternate heating and cooling, that $70^{\circ} \mathrm{C}$. maintained for half a minute is generally sufficient to kill suppurative organisms, and such virulent types of pathogenic bacteria as B. diphtheric, B. typhosus, and B. tuberculosis.

Respecting the numerical diminution of microbes brought about by pasteurisation and sterilisation respectively, we may take the following series of experiments. Dr H. L. Russell $\dagger$ tabulates the immediate results of pasteurisation as follows:

\begin{tabular}{|c|c|c|c|c|c|c|}
\hline & \multicolumn{6}{|c|}{ Number of Micro-organisms per c.c. } \\
\hline & \multicolumn{3}{|c|}{ Unpasteurised. } & \multicolumn{3}{|c|}{ Pasteurised. } \\
\hline & Minimum. & Maximum. & Average. & Min. & Max. & Av. \\
\hline $\begin{array}{l}\text { Full cream } \\
\text { milk. }\end{array}$ & 25,300 & $18,827,000$ & $3,674,000$ & 0 & 37,500 & 6,140 \\
\hline Cream . . & 425,000 & $32,800,000$ & $8,700,000$ & 0 & 57,000 & 24,250 \\
\hline
\end{tabular}

As regards the later effect of the process, he states that in fifteen samples of pasteurised milk examined from November to December, nine of them revealed no organisms, or so few that they might almost be regarded as sterile; in those samples examined after January, the lowest number was 100 germs per c.c., while the average was nearly 5000. With the pasteurised cream a similar condition was to be observed. Other workers hold that from 95 to 99 per cent. of all bacteria are removed by pasteurisation.

\section{Summary of Practical Control of Milk Supply}

Briefly, it may be said that the requirement is a pure milk supply, that is:tion;

(1) A clean, whole milk, unsophisticated and without preserva-

Jenner Institute of Preventive Medicine (First Series Transactions).

$\uparrow$ Centralblatt fiir Bakteriologie, etc., Abth. ii. 
(2) To be derived from healthy cows, guaranteed free from tuberculosis by the tuberculin test, and living under clean and sanitary conditions;

(3) To be obtained by clean methods of milking, to be strained, and to be protected from contamination by dust or dirt, or from infection by disease of milker;

(4) To be kept cool by means of refrigeration from the time it leaves the cow to the time it reaches the consumer, and not to be exposed to dust or uncleanliness in any way from the vessels in which it is placed or from the persons by whom it is handled.*

Specialised Milk Supplies for Infants. - The movement for the supply of modified milk for the use of infants, particularly of the artisan class, has now become a considerable one, both in Europe and America. Broadly speaking, the systems represented in England are (i.) the Municipal Milk Depôt (Liverpool, Battersea, Bradford, etc.), and (ii.) the Rotch system (Walker-Gordon Laboratories). (i.) There can be little doubt that this kind of milk supply may be of great service for the children of the poor, in the reduction of infantile mortality due to the use of contaminated or infected milk, and in special cases calling for special treatment. It is not, however, of the nature of control of the milk supply, but rather, of a specialised supply, to meet special needs. There is evidence to show that at Liverpool, Battersea, and other places, it has had beneficial results in this special direction. It has, however, several limitations, unless properly managed. Its object being the saving of life and prevention of infant diseases, it is necessary that the system should be individualised. Each mother must be separately advised, each infant inspected and weighed periodically, each home supervised, the condition of the milk regularly tested, and the source of the milk kept under control, the cows and cowsheds from which the milk is derived being supervised by a veterinary surgeon and the Medical Officer of Health. And here, in any event, the quality of the milk used must reach a high standard, chemically and bacteriologically. If these conditions are not fulfilled, it would appear that a municipal sterilised milk supply can only be a palliative measure of transient usefulness. The chief desideratum is a naturally pure milk supply, rather than an artificially purified and humanised supply. The latter question is one certainly requiring careful consideration, but of a different nature to the former. If undertaken by a Local Authority, it would appear desirable to do it very thoroughly, after the manner of Budin and Variot in Paris, each case being under strict medical supervision.

A typical municipal milk depôt in this country is described in the following words :-

The milk is supplied by a local dairyman, and arrives in the early morning. It is guaranteed free from chemical preservatives, and to contain not less than $3 \cdot 25$ per cent. butter fat, and 8.75 per cent. of solids not fat, and cream which must contain not less than 50 per cent. of butter fat. The milk must be drawn from healthy cows, stabled and milked under clean and sanitary conditions. Utensils, etc., used must be thoroughly clean. These and other requirements are set out in the contract. The first process is the modification or humanisation. Three modifications are employed. The first contains one part milk to two of water, seven ounces of cream and seven of lactose being added to each gallon of the mixture. This modification is given to infants under three months old. The second modification, which is given to infants between three and six months old, consists of equal parts of milk and water, with five ounces of cream and lactose added per gallon. 'The third consists of two parts

* For details respecting control of milk supply, see Bacteriology of Milk, pp. 452-599; also, Report on Health of City of Manchester, 1902; Report on Milk Supply of Finsbury, 1903; The Milk Supply of 200 Cities and Towns (U.S.A. Depart. of Agric., 1903, Bulletin 46); Brit. Med. Jour., 1904, vol. ii., pp. 421-429 (Newman). 
milk to one of water, with three ounces of cream and lactose added per gallon, and is given to infants over six months old. *

The milk having been modified, it is bottled, and the number of bottles and the quantities contained are set out below :-

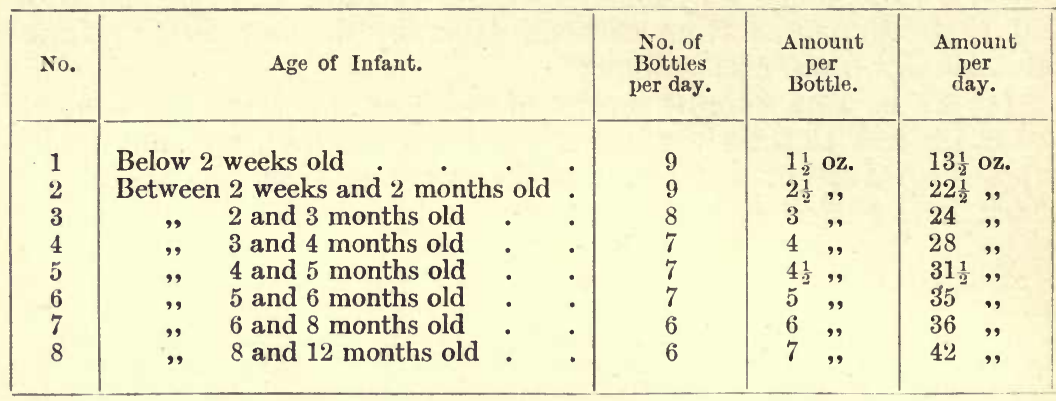

After bottling, the milk is heated by steam under pressure for some 20-30 minutes, and kept at a temperature of $212^{\circ} \mathrm{F}$. for from five to ten minutes. It is allowed to cool, and then is supplied to the consumers. Each mother, on first coming to the depôt, is given a leaflet of instructions as to the proper method of using the milk. The method is very simple. When feeding time arrives, all she has to do is to place the bottle, unopened, in some warm water till the milk has reached body temperature. The bottle is then opened, a small teat put on the mouth of the bottle, and the baby takes its milk from the sterilised bottle direct. The use of the long-tube feeding-bottle is obviated.

This process of humanisation adapts the milk to the infant's digestive organs, the sterilisation kills the germs, and as the bottle is not opened-or should not be opened from the time it enters the steriliser until the infant is ready to take milk from it direct (no feeding-bottle should be used)-home contamination, unless it is wilful or due to extreme carelessness, is prevented.

It may be convenient briefly to summarise the chief advantages and disadvantages of this system :-Advantages.-(1) Infants fed on depôt milk receive a modified milk suited to their digestive capacities. (2) The milk is free from injurious and other bacteria. (3) The mother is saved labour, and mistakes are prevented, as the preparation of food for infants, etc., is not necessary if modified and sterilised milk is obtained ready prepared. (4) Home contamination of milk is avoided. These four advantages are, in my judgment, of great value. Disadvantages. - (1) The object being the saving of life and the prevention of infant disease, it is necessary that such a system should be individualised and placed under direct medical supervision. Indiscriminate use of such milk is undesirable, and the hand-feeding of infants requires so much intelligent care that it should not be generally recommended. (2) There must inevitably result from any success of this system a tendency or risk for mothers to feed their infants on such milk, instead of nursing them in the natural way. Therefore, such milk should only be provided for those infants which for some adequate reason cannot be nurtured on human milk. Such infants are the exception and not the rule, and it is undesirable to adopt any method which tends to lessen maternal feeding or maternal responsibility. There are cases, and these not a few, where depôt milk solves the problem of infant feeding, especially in large towns. But anything which tends in the direction of placing the responsibility of rearing infants on the municipality is to be deprecated. It is not a question of sentiment, but of fact, to say that the great need of the present time in respect of this infant problem is a better home life, more maternal care and feeding rather than less, and a more

* These quantities are of course dependent on the proportions of fat, sugar, and albuminoid substances originally present in the milk dealt with. 
intelligent, cleanly, and simple mode of rearing infants. Infants should be breast-fed, and anything which relieves the healthy mother from this duty should not be looked upon favourably until it has absolutely justified its worth. (3) The cost is at present in many places prohibitive. (4) The evidence of benefit is not yet of a conclusive or sufficient nature to form an opinion as to how far the use of depôt milk reduces the infant death-rate. But there can be no doubt that, indirectly, benefit is derived.

Infantile mortality has a definite relationship to $(a)$ the feeding of infants ; $(b)$ personal care of infants by parents; $(c)$ housing accommodation ; and $(d)$ certain meteorological conditions affecting temperature and the dissemination of dust. Other elements enter into the problem, but, so far as municipal action is concerned, those are the four main elements. If we can succeed in raising the quality, as regards purity, of the milk on which infants are fed, we shall at the same time educate and improve the sense of duty towards their infants on the part of parents. The mischief lies in polluted milk. The sources of the pollution are not only in unsatisfactory methods of milking, and in storing and conveying the milk supplied, but also in dirty domestic conditions, and particularly in carelessness in the use of feedingbottles. Successfully to attack, by municipal administration, all the sources of pollution, is at present impossible, but the ideal of public health administration in respect of infant feeding is a pure milk supply which needs no sterilisation; and towards that end all our efforts should be directed. Modification of such cow's milk for infant use will still be necessary. Meanwhile we must do the best possible under existing conditions, and that involves sterilisation, modification, and protection from home contamination. It is also essential that such a milk supply should be under medical supervision, and adopted only in suitable cases. Without entering into unnecessary details, it would appear that there are five possible means of supplying such a suitable and pure milk for infants :-(1) By means of municipal milk depôts (vide supra); (2) by one or more milk-vendors or dairymen undertaking, by private enterprise, to furnish such modified milk (certified) under medical supervision ; (3) by obtaining such a supply from some central institution, company, or society, such, for example, as the Walker-Gordon Laboratory; (4) by means of medical milk commissions, as is done, in part, by the milk commissions established in the United States of America; and (5) by means of a voluntary health society supplying such milk under necessary supervision and control of sources and usage, as is done, in part, by the York Health and Housing Association.

The essential points requiring attention are such modification of the cow's milk as will make it, like human milk, suitable for infant consumption, absolute control of its source and handling prior to its modification, and prevention of home contamination by delivery in sealed bottles. At present it would also be necessary to pasteurise or sterilise such milk.

(ii.) The Rotch system was introduced by Dr Thomas Morgan Rotch of Harvard University, and is now in operation in some eighteen or twenty cities in the United States and Canada, and has also a centre in London. The system, which has for its object the betterment of infant feeding, consists in controlling the milk supply by controlling the farms, and establishing a chain of protection from the time the milk leaves the cow until it arrives at the mouth of the infant. But in addition to this scheme of protection, there is also combined with it a scheme of modification of the milk to make it meet more exactly the requirements of infant feeding. The twofold function of the Rotch system may be briefly referred to:-(a) Protection of the Milk. - With this object in view Rotch made a number of recommendations similar to those laid down by various Milk Commissions, which latter indeed took many of Rotch's proposals for their model. At the farms supplying milk under this system, the breed of the cow and its food are matters which receive primary attention. In America the Holstein has been found to be the best for its adaptability for infant feeding. The cow itself must be regularly and wisely fed on the basis to which reference has been made. There is regular grooming and good housing. The cowhouse has cemented walls, ceilings, and floors, and is properly drained and frequently cleansed. A most careful supervision of the cow's health is maintained, and if in any way abnormal, the cow is isolated until in normal health. Careful tuberculin testing is made of each cow used, and the milk of each cow also undergoes microscopical examination for the purposes of detecting pus cells, colostrum 
cells, bacteria, etc. The milkers are under strict medical supervision, and regulations are enforced in respect of "cleanliness." Cows are milked in their own stalls, but immediately after milking the milk is taken in closely-covered vessels to the milk-room, where it is cooled and screened. The milk-room is a specially prepared chamber, having smooth surfaces of polished cement, and specially constructed ventilators with cold-water sprays to moisten the air and prevent dust gaining access, asepsis being the requirement. The milk is now ready for the laboratory. (b) Modification.-The object of the Walker-Gordon Laboratories is first to insure and distribute the naturally pure milk; and secondly, to provide a place where different combinations of milk may be put up, according to the prescriptions of medical men, with accuracy, and under such conditions of cleanliness and asepsis as to insure the best possible food for infant feeding. The necessity for modification arises from two facts, namely, that milk varies in constituent percentages, and to obtain a regular and uniform constitution, modification is necessary ; and secondly, some children require, for one reason or another, a milk containing certain percentages of the various constituents. Thus the patient can receive on the physician's order a mixture of the percentages calied for, made up of either separated cream or gravity cream, separated milk or whole milk. Twelve years' experience in Boston, U.S.A., seem to indicate the practicability of this system in preventing the summer diarrhœea of infants due to contaminated milk.

In addition to these two types, there are various similar methods in vogue, each of which has its points of advantage.*

\section{Bacteria in Milk Products}

Cream is generally richer in bacteria than milk. Set cream contains more bacteria than separated cream, but germs are abundant in both. The number of organisms found in eream is enormous. Probably no other natural medium contains more, We have frequently examined fresh cream in the country, and found it to contain more than $100,000,000$ bacteria per c.c. It is not only a favourable medium. It is the filter, so to speak, of milk. For, as the cream rises, the milk parts with more than 90 per cent. of its contained bacteria. Conn and Esten + found 110,000,000 of bacteria per c.c. in unripened cream (average of four examinations) and $284,000,000$ in the same cream ripened (average of four examinations). Cream obtained from a creamery gave an average on eight examinations of 56,000,000 organisms per c.c. unripened, and 350,000,000 organisms per c.c. ripened. Other examples of unripened cream averaged more than $90,000,000$, but when ripened averaged over $300,000,000$. Normally ripened cream probably averages four or five hundred millions of bacteria per e.c., which is greatly in excess of any other natural media. The number of organisms in unripened cream varies widely.

The most characteristic feature of cream-ripening is the growth of the acid-producing organisms, chiefly $B$. acidi lactici, and the decline of the liquefying and extraneous organisms. B. acidi lactici is found in very small numbers in fresh milk, as we have already pointed out,

* For full account, see Jour. of Hygiene, 1904, p. 329 (McCleary).

† Thirteenth Annual Report of the Storr's Agricultural Expt. Stu., Connecticut, 1900, pp. 13-33. 
and also in cream. But as the ripening process proceeds with uniform regularity, the numbers of this organism rapidly increase. Rollin Burr considers these lactic bacteria gain access to the milk from outside sources.* Buttermilk and whey vary much in their bacterial content.

Butter necessarily follows the standard of the cream. But, as the butter fat is not well adapted for bacterial food, the number of bacteria in butter is usually less than in cream. Butter, when first made, may contain many million bacteria per gramme. After a few days only two or three million may be found, and if butter is examined after it is several months old, it is often found to be almost free from germs; yet in the intervening period a variety of conditions are set up directly or indirectly through bacterial action. $\dagger$ Rancid butter is largely due to organisms. Putrid butter is caused, according to Jensen, by various putrefactive bacteria, one form of which is named Bacillus foetidus lactis. This organism is killed at a comparatively low temperature, and is, therefore, completely removed by pasteurisation. Ill-flavoured butter may be due to germs or an unsuitable diet of the cow and a retention of the bad quality of the resulting milk. Lardy and oily butters have been investigated by Storch and Jensen, and traced to bacteria. Lastly, bitter butter occasionally occurs, and is due to fermentative changes in the milk, or the presence of acid-producing organisms in the butter such as B. fluorescens liquefaciens, Oidium lactis, and Cladosporium butyri (Jensen). Butter may also contain pathogenic bacteria, like tubercle. The $B$. coli can live for a month in butter.

Cheese suffers from very much the same kind of "diseases" as butter, except that chromogenic conditions oceur more frequently. Most of the troubles in cheese originate in the milk.+ The number of bacteria in cheese is naturally less than that present in milk or cream. The closer texture and consistence of cheese, coupled with the lessened degree of moisture, are sufficient factors to account for this. Nevertheless, cheese contains a considerable number of organisms. Adametz found that freshly precipitated curd, moulded in the press and freed from excess of whey, contained between 90,000 and 140,000 micro-organisms per gramme, a comparatively large number of them having the power of liquefying gelatine, or, in other words, they possessed a peptonising ferment. During the period of ripening, the bacterial content of the cheese gradually rose to 850,000 in Emmenthaler cheese, and 5,600,000 per gramme,

* Thirteenth Annual Report of Storr's Agricultural Expt. Sta., Connecticut,

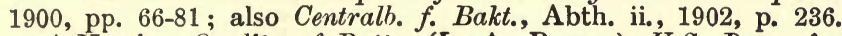

† Keeping Quality of Butter (L. A. Rogers), U.S. Dep. of Agriculture, 1904, Bull. 57.

$\ddagger$ Board of Agriculture Report on Cheddar Cheese Making (F. J. Lloyd), 1899, pp. $78,103$. 
in a soft household cheese. Only a small percentage of these are of the peptonising species. Tides of organisms occur in cheese, as in butter and milk.

Method of Examination of Butter and Cheese.-Several grams of the butter or cheese should be placed in a large test-tube, which is then two-thirds filled with sterilised water and placed in a waterbath at about $45^{\circ} \mathrm{C}$. until the butter or cheese is melted or "washed." A small quantity may then be added to gelatine or agar and plated out on Petri dishes, or in flat-bottomed flasks, in the usual way. After which the tube may be well shaken and returned to the bath inverted. In the space of twenty or thirty minutes the butter or cheese has separated from the water with which it has been emulsified. It is then placed in the cold to set. The water may be now either centrifugalised or placed in sedimentation flasks, and the deposit examined for bacteria.

\section{The Uses of Bacteria in Dairy Produce}

In considering the relation of bacteria to milk, we found that many of the species present were injurious rather than otherwise, and when we come to consider bacteria in dairy products, like butter and cheese, we find that the dairyman possesses in them very powerful allies. Within recent years almost a new industry has arisen owing to the scientific application of bacteriology to butter and cheese making.

\section{Bacteria in Butter-Making}

As a preliminary to butter-making, the general custom in most countries is to subject the cream to a process of "ripening." As we have seen, cream in ordinary dairies and creameries invariably contains some bacteria, a large number of which are in no sense injurious. Indeed, it is to these bacteria that the ripening and flavouring processes are due. They are perfectly consistent with the production of the best quality of butter. The aroma of butter, as we know, controls in a large measure its price in the market. This aroma is due to the decomposing effect upon the constituents of the butter of the bacteria contained in the cream. In the months of May and June the variety and number of these types of bacteria are decidedly greater than in the winter months, and this explains in part the better quality of the butter at these seasons. As a result of these ripening bacteria the milk becomes changed and soured, and slightly curdled. Thus it is rendered more fit for buttermaking, and acquires its pleasant taste and aroma. It is then churned, after which bacterial action is reduced to a minimum or absent altogether. Sweet-cream butter lacks the flavour of ripened 
or sour-cream butter. The process is really a fermentation, the ripening bacteria acting on each and all of the constituents of the milk, resulting in the production of various bye-products. This fermentation is a decomposition, and just as we found when discussing fermentation, so here also the action is only beneficial if it is stopped at the right moment. If, for example, instead of being stopped on the second day, it is allowed to continue for a week, the cream will degenerate and become offensive, and the pleasant ripening aroma will be changed to the contrary. Speaking generally, about 25 per cent. of cream bacteria exert a favourable effect on butter, and 10-15 per cent. a deleterious effect. Many of the former are acid-producers, and are widely distributed.

Bacteriologists have demonstrated that butters possessing different flavours have been ripened by different species of bacteria. Occasionally, one comes across a dairy which seems to be impregnated with bacteria that improve cream and flavour well. In other cases the contrary happens, and a dairy becomes impregnated with a species having deleterious effects upon its butter. Such a species may be favoured by unclean utensils and dairying, by disease of the cow, or by a change in the cow's diet. Thus it comes about that the butter-maker is not always able to depend upon good ripening for his cream. At other times he gets ripening to occur, but the flavour is an unpleasant one, and the results correspond. It may be bitter or tainted, and just as certainly as these flavours develop in the cream, so is it certain that the butter will suffer. Fortunately, the bacterial content of the cream is generally either favourable or indifferent in its action. Thus it comes about that the custom is to allow the cream simply to ripen, so to speak, of its own accord, in a vat exposed to the influence of any bacteria which may happen to be around. This generally proves satisfactory, but it has the great disadvantage of being indefinite and uncertain. Occasionally it turns out wholly unsatisfactory, and results in financial loss. Shortly, it may be said that cream-ripening assists the making of butter in four ways :-

1. Churning is easier and more effectual.

2. The yield of butter is increased.

3. The butter has better keeping qualities.

4. The flavour and aroma are more satisfactory.

Control of Ripcning Process.-There are various means at our command for improving the ripening process. Perfect cleanliness in the entire manipulation necessary in milking and dairying, combined with freedom from disease in the milch cows, will carry us a long way on the road towards a good cream-ripening. Recently, however, a new method has been introduced, largely through the 
work and influence of Professor Storch in Denmark, which is based upon our new knowledge respecting bacterial action in creamripening. We refer to the artificial processes of ripening set up by the addition of pure cultures of favourable germs.* If a culture of organisms possessing the faculty of producing in cream a good flavour be added to the sweet cream, it is clear that advantage will accrue. This simple plan of starting any special or desired flavour by introducing the specific micro-organism of that flavour, may be adopted in two or three different ways. If cream be inoculated with a large, pure culture of some particular kind of bacteria, this species will frequently grow so well and so rapidly that it will check the growth of the other bacteria which were present in the cream at the commencement and before the "starter" was added. That is, perhaps, the simplest method of adding an artificial culture. But secondly, it will be apparent to those who have followed us thus far, that if the cream is previously pasteurised at $70^{\circ} \mathrm{C}$., these competing bacteria will have been mostly or entirely destroyed, and the pure culture, or "starter," will have the field to itself. There is a third modification, which is sometimes termed ripening by natural starters. A "natural starter" is a certain small quantity of cream taken from a favourable ripening-from a clean dairy or a good herd-and placed aside to sour for two days until it is heavily impregnated with the specific organism which was present in the whole favourable stock of which the "natural starter" is but a part. It is then added to the new cream, the favourable ripening of which is desired. Of the species which produce good flavours in butter, the majority are found to be members of the acid-producing class; but probably the flavour is not dependent upon the acid. The aroma of good ripening is also probably independent of the acid production.

Artificial Ripening.-Of all the methods of ripening-natural ripening, the addition of "natural starters," the addition of pure cultures with or without pasteurisation-there can be no doubt that pure culture after pasteurisation is the most accurate and reliable. The use of "natural starters" is a method in the right direction; yet it is, after all, a mixed culture, and therefore not uniform in action. In order to obtain the best results with the addition of pure cultures, Professor Russell has made the following recommendations :-

1. The dry powder of the pure culture must be added to a small amount of milk that has been first pasteurised, in order to develop an active growth from the dried material.

2. The cream to be ripened must first be pasteurised, in order to

* Such pure cultures for such purposes are in the United States termed "starters," because they start the process of special ripening. For the sake of convenience the term will be used here. 
destroy the developing organisms already in it, and thus be prepared for the addition of the pure culture.

3. The addition of the developing "starter" to the pasteurised cream, and the holding of the cream at such a temperature as will readily induce the best development of flavour.

4. The propagation of the "starter" from day to day. A fresh lot of pasteurised milk should be inoculated daily with some of the pure culture of the previous day, not with the ripening cream containing the culture. In this way the purity of the "starter" is maintained for a considerable length of time. Those "starters" are best which grow rapidly at a comparatively low temperature $\left(60-75^{\circ} \mathrm{F}\right.$.), which produce a good flavour, and which increase the keeping qualities of the butter. Now, whilst it is true that the practice of using pure cultures in this way is becoming more general, very few species have been isolated which fulfil all the desirable qualities above mentioned. In America, "starters" are preferred which yield a "high" flavour, whereas in Danish butter a mildaroma is more common. In this country, as yet, very little has been done, and that on an experimental scale rather than a commereial one. In 1891 it appears that only 4 per cent. of the butter exhibited at the Danish butter exhibitions was made from pasteurised cream plus a culture "starter"; but in 1895 , 86 per cent. of the butter was so made. Moreover, such butter obtained the prizes awarded for first-class butter with preferable flavour. Different cultures will, of course, yield differently flavoured butter. If we desire, say, a Danish butter, then some-species like "Hansen's Danish starter" would be added; if we desire an American butter, we should use a species like that known as "Conn's Bacillus, No. 41." But whilst these are two common types, they are not the only suitable and effective "starters." On many farms in England there are equally good cultures, which, placed under favourable temperatures in new cream, would immediately commence active ripening. A good lactic acid culture for dairy use (a) should sour cream strongly in a short time, $(b)$ should be able to thrive at low temperatures, and $(c)$ should produce a favourable taste and flavour in the butter (Jensen).

Professor H. W. Conn, who, with Professor Russell, has done so much in America for the advancement of dairy bacteriology, reports a year's experience with the bacillus to which reference has been made, and which is termed No. 41.* It was originally obtained from a specimen of milk from Uruguay, South America, which was exhibited at the World's Fair in Chicago, and proved the most successful flavouring and ripening agent among a number of cultures that were tried. The conclusions arrived at after a considerable period of testing and experimentation appear to be on the whole * Report of Storr's Agricultural Expt. Sta., State of Connecticut, 1895. 
satisfactory. A frequent method of testing has been to divide a certain quantity of cream into two parts: one part inoculated with the culture, and the other part left uninoculated. Both have then been ripened under similar conditions, and churned in the same way; the differences have then been noted. It is interesting to know that, as a result of the year's experience, creameries have been able to command a price varying from half a cent to two cents a pound more for the "culture" butters than for the uninoculated butters. The method advised in using this pure culture is to pasteurise (by heating at $155^{\circ} \mathrm{F}$.) six quarts of cream, and after cooling, to dissolve in this cream the pellet containing bacillus No. 41. The cream is then set in a warm place $\left(70^{\circ} \mathrm{F}\right.$.), and the bacillus is allowed to grow for two days, and is then inoculated into twenty-five gallons of ordinary cream. This is allowed to ripen as usual, and is then used as an infecting culture, or "starter," in the large cream vats in the proportion of one gallon of infecting culture to twenty-five gallons of cream, and the whole is ripened at a temperature of about $68^{\circ} \mathrm{F}$. for one day. The cream ripened by this organism needs to be churned at a little lower temperature (say $52-54^{\circ} \mathrm{F}$.), but to be ripened at a little higher temperature than ordinary cream to produce the best results. Cream ripened with No. 41.has its keeping power much increased, and the body or grain of the butter is not affected. More than 200 creameries in America used this culture during 1895, which proves that its use for the production of flavour in butter is feasible in ordinary creameries, and in the hands of ordinary butter-makers, provided they will use proper methods and discretion. More recently, pasteurisation has fallen into abeyance, and the use of artificial cultures is said to have declined in America. In England, with few exceptions, practically nothing has been done in a commercial way in the direction of artificial "starters."

\section{Bacteria in Cheese-Making}

The cases where it has been possible to trace bacterial disease to the consumption of butter and cheese have been rare. Notwithstanding this fact, it must not be supposed that therefore cheese contains few or no bacteria. On the contrary, for the making of cheese bacteria are not only favourable, but actually essential, for in its manufacture the casein of the milk has to be separated from the other products by the use of rennet, and is then collected in large masses and pressed, forming the fresh cheese. In the course of time this undergoes ripening, which develops the peculiar flavours characteristic of cheese, and upon which its value depends.

We have said that the casein is separated by the addition of 
rennet, which has the power of coagulating the casein. But this precipitation may also be accomplished by allowing acid to develop in the milk until the casein is precipitated, as in some sour-milk or cottage cheeses. The former method is; of course, the usual one in practice. It has been suggested that the bacteria contained in the rennet exert a considerable influence on the cheese, but this, although rennet contains bacteria, is hardly established. It is not here, however, that bacteria really play their rôle. After this physical separation, when the cheese is pressed and set aside, is the period for the commencement of the ripening process.

That bacteria perform the major part of this ripening process, and are essential to it, is proved by the fact that when they are either removed or opposed the curing changes immediately cease. If the milk be first sterilised (Freudenreich), or if antiseptics, like thymol, be added (Adametz), the results are negative. It is not yet known whether this ripening process is due to the influence of a single organism or not. The probability, however, is that it is to be ascribed to the action of that group of bacteria known as the lactic acid organisms. Nor is it yet known whether the peptonisation of the casein and the production of the flavour are the results of one or more species. Freudenreich believes them to be due to two different forms.

However that may be, we meet with at least four common groups of bacteria more or less constantly present in cheese-ripening, either in the early or late stages. First, there are the lactic acid bacteria, by far the largest group, and the one common feature of which is the production by fermentation of lactic acid; secondly, there are the casein-digesting bacteria, present in relatively small numbers; thirdly, the gas-producing bacteria, which give to cheese its honeycombed appearance; lastly, an indifferent or miscellaneous group of extraneous bacteria, which were in the milk at the outset of cheese-making, or are intruders from the air or rennet. All these four groups may bring about a variety of changes, beneficial and otherwise, in the cheese-making.

Russell divides the ripening process into three divisions :-

1. Period of Initial Bacterial Decline in Cheese.-Where the green cheeses were examined immediately after removing from the press, it was usually found that a diminution in numbers of bacteria had taken place. This period of decline lasts but a short time, not beyond the second day. Lower temperature and expulsion of the whey would account for this general decline in all species of bacteria.

2. Period of Bacterial Increase.-Soon after the cheese is removed from the press a most noteworthy change takes place in green cheese. A very rapid increase of bacteria occurs, confined almost exclusively 
to the lactic acid group. This commences in green cheese about the eighth day, and continues more or less for twenty days. In Cheddar cheese it commences about the fifth day, reaches its maximum about the twentieth day, declines rapidly to the thirtieth day, and gradually for a hundred following days. During the first forty days of this period the casein-digesting and gas-producing organisms are present, and at first increasing, but relatively to only a very slight degree. With this rapid increase in organisms the curd begins to lose its elastic texture, and before the maximum number of bacteria is reached the curing is far advanced. Freudenreich has shown that acid inhibits the growth of the casein-digesting microbes, and vice versâ.

3. Period of Final Bacterial Decline.-The cause of this decline can only be conjectured, but it is highly probable that it is due to a general principle to which reference has frequently been made, viz., that after a certain time the further growth of any species of bacteria is prevented by its own products. We may observe that the gas-producing bacteria in Cheddar cheese last much longer than the peptonising organisms, for they are still present up to eighty days. Professor Russell aptly compares the bacterial vegetation of cheese with its analogue in a freshly-seeded field. "At first multitudes of weeds appear with the grass. These are the casein-digesting organisms, while the grass is comparable to the more native lactic acid flora. In course of time, however, grass, which is the natural covering of soil, 'drives out' the weeds, and in cheese a similar condition occurs." In milk the lactic acid bacteria and peptonising organisms grow together; in ripening cheese the former eliminate the latter.

Artificial Ripening.-We have seen that the conclusion generally held respecting these lactic acid bacteria is that they are the main agents in curing the cheese. Upon this basis a system of pure "starters" has been adopted, the characteristics of which must be as follows:- (a) The organism should be a pure lactic-acid-producing germ, incapable of producing gaseous products; $(b)$ it should be free from any undesirable aroma; $(c)$ it should be especially adapted for vigorous development in milk. The "starter" may be propagated in pasteurised or sterilised milk from a pure culture from the laboratory. The advantages accruing from the uses of this lactic acid culture, as compared with cheese made without a culture, are that with sweet milk it saves time in the process of manufacture; that with tainted milk, in which acid develops imperfectly, it is an aid to the development of a proper amount of acid for a typical Cheddar cheese; and that the flavour and quality of such cheese is preferable to cheese which has not been thus produced. Professor Russell is of opinion that the lactic acid organisms are to be credited with greater 
ripening powers than the casein-digesting organisms, but it must not be forgotten that these two great families of bacteria are still more or less on trial, and it is not yet possible finally to decide on either of them. Lloyd holds that though " the greater the number of lactic acid bacilli in the milk the greater the chance of a good curd," still "this organism alone will not produce that nutty flavour which is so much sought after as being the essential characteristic of an excellent Cheddar cheese."

There are three difficulties to be encountered by dairymen "starting" a ripening by the addition of a pure culture. First, there is the initial difficulty of not being able to use pasteurised milk for cheese, as such milk is uncoagulable by rennet (Lloyd). Hence it is impossible to avoid some contamination of the milk previous to the addition of the culture. Secondly, the continual uncontaminated supply of pure culture is by no means an easy matter. Thirdly, the maintenance of a low-temperature cellar to prevent the rapid multiplication of extraneous bacteria will, in some localities, be a serious difficulty. These difficulties have, however, not proved insurmountable, and by various workers in various localities and countries culture-ripening of cheese is being carried on.*

* As regards the Cheddar cheese industry in this country, Lloyd arrives at the following five conclusions as a result of investigation :-

1. To make Cheddar cheese of excellent quality, the Bacillus acidi lactici alone is necessary ; other germs will tend to make the work more rather than less difficult. Hence scrupulous cleanliness should be a primary consideration of the cheese-maker.

2. No matter what system of manufacture be adopted, two things are necessary. One is that the whey be separated from the curd, so that when the curd is ground it shall contain not less than 40 per cent. of water, and not more than 43 per cent. ; the other point is that the whey left in the curd shall contain developed in it before the curd is put in the press at least 1 per cent. of lactic acid if the cheese is required for sale within four months, and not less than 8 per cent. of lactic acid if the cheese is to be kept ripening for a longer period.

3. The quality of the cheeses will vary with the quality of the milk from which they have been made, and proportionately to the amount of fat present in that milk.

4. "Spongy curd" is produced by at least five organisms, and one of these is responsible for a disagreeable taint found in curd. They occur in water. Hence the desirability of securing clean water for all manipulative purposes, and also for the drinking purposes of the milch cow.

5. The fact that certain bacteria are found in certain localities and dairies is due more to local conditions than to climatic causes.

It is needless to remark that these conclusions once more emphasise the fact that strict and continual cleanliness is the one desideratum for bacteriologically good dairying. That being secured in the cow at the milking, in the transit, and at the dairy, it is a comparatively simple step, by means of pasteurisation and the use of good pure cultures of flavouring bacteria, to the successful application of bacteriology to dairy produce. 


\section{Abnormal Cheese-Ripening}

Unfortunately, from one cause or another, faulty fermentations and changes are not infrequently set up. Many of these may be prevented, being due to lack of cleanliness in the process or in the milking; others are due to the gas-producing bacteria being present in abnormally large numbers. When this occurs we obtain what is known as "gassy" or inflated cheese, on account of its substance being split up by innumerable cavities and holes containing carbonic acid gas or sometimes ammonia or free nitrogen. Some twenty-five species of micro-organisms have been shown by Adametz to cause this abnormal swelling. In severe cases of this gaseous fermentation the product is rendered worthless, and even when less marked the flavour and value are much impaired. Winter cheese contains more of this species of bacteria than summer. Acid and salt are both used to inhibit the action of these gas-producing bacteria and yeasts, and with excellent results. The character of the gas holes in cheese is not of import in the differentiation of species. If a few gas bacteria are present, the holes will be large and less frequent; if many, the holes will be sinall, but numerous. (Swiss cheese having this characteristic is known as Nissler cheese.) Many of these gasproducing germs belong to the lactic acid group, and are susceptible to heat. A temperature of $140^{\circ} \mathrm{F}$. maintained for fifteen minutes is fatal to most of them, largely because they do not form spores.

The sources of the extensive list of bacteria found in cheese are of course varied, more varied indeed than is the case with milk. For there are, in addition to the organisms contained in the milk bronght to the cheese factory, the following prolific sources, viz., the vats and additional apparatus; the rennet (which itself contains a great number); the water that is used in the manufacture.

In addition to the abnormalities due to gas, there are also other faulty types. The following chromogenic conditions occur: red cheese, due to a micrococcus; blue cheese, produced, according to Vries, by a bacillus; and black cheese, caused by a copious growth of low fungi. Bitter cheese is the result of Tyrothrix geniculatus (Duclaux), or the Micrococcus casei amari of Freudenreich, a closely allied form of Conn's micrococcus of bitter milk. Sometimes cheese undergoes a putrefactive decomposition, and becomes more or less putrid. At other times it becomes "tainted." These latter conditions, like the gassy cheeses, are due to the intrusion of bacteria from without, or from udder disease of the cow.* Healthy cows, clean milking, and the introduction of pure cultures, are the methods

* For a discussion on the duration of life of the tubercle bacillus in cheese, see Nineteenth Ann. Rep. Bureau of Animal Industry, 1902, p. 217. 
to be adopted for avoiding "diseases" of cheese and obtaining a well-flavoured article which will keep.

Finally, there is poisonous cheese which is of more importance to the public health than all the other abnormal conditions of cheese put together. In 1883 and 1884 there occurred in Michigan, U.S.A., an outbreak of cheese-poisoning. Three hundred persons in all were affected, and the illness was traced by Professor V. C. Vaughan to a poisonous ptomaine present in the cheese, and to which he gave the name tyro-toxicon. It is not improbable that this ptomaine is a product of bacterial fermentation. It is one of a large class of substances said to be formed by the action of bacteria upon nitrogenous compounds. It is unstable, and easily destroyed by the action of heat and moisture, and even by exposure to the air. Being present in small quantities only, it has never been isolated in sufficiently large quantities to allow of its composition being definitely determined. Tyro-toxicon has been proved to be a violent poison both to man and the lower animals. A minute portion consumed by a child produced sickness and diarrhœa in a manner almost identical with cholera infantum (Vaughan). Similar symptoms were obtained with cats and dogs. Vaughan found that three months are required for the formation of tyro-toxicon in milk kept in tightlystoppered bottles; but under certain circumstances, and in the presence of butyric fermentation in milk, the poison is produced in about eight or ten days. Similar, and possibly identical, poisons occasionally occur in cream, rancid butter and milk (lacto-toxicon and diazo-benzol). They have the same poisonous effects. Vaughan has isolated a microbe growing readily on ordinary culture media and upon fruit and vegetables. This micro-organism, it is considered, may be the agent producing tyro-toxicon, but the bacteriology of the subject has not been worked out.

The writer investigated a similar outbreak due to tyro-toxicon in Dutch cheese in London in 1901.* Seventeen persons were affected. The symptoms of illness in all these 17 cases occurred in from two to eight hours after eating the cheese in question, which came from the same consignment. Moreover, the symptoms were similar, namely, epigastric pain, rigors, vomiting, diarrhœa, prostration, and some fever. The degree of sickness does not appear to have depended upon the amount of cheese eaten. There was no death attributed to the poisoning, and in general the symptoms appear to have passed off in the course of forty-eight hours. A short incubation period suggests that the poison was "available" in the cheese, as a product of previous changes, possibly bacterial, set up therein. A long incubation period between eating the food and symptoms of poisoning would suggest that the persons affected had consumed, not

* Report on the Pullic Health of Finsbury, 1901, pp. 110-116. 
the products of bacteria, but the bacteria themselves, which had then taken some little time to produce their injurious effects in the persons eating the food. In the present case the incubation period was comparatively short, and the acuteness of the symptoms did not appear to have a direct relationship to the amount of cheese eaten.*

* For a general discussion and bibliography of this subject, see Die Milch und ihre Bedeutung für Volkswirtschaft und Volksgesundheit, Hamburg, 1903, pp. $345-357$. 


\section{CHAPTER VIII}

BACTERIA IN OTHER FOODS

1. Shell-fish : Oysters, Cockles, Clams, and their Relation to Disease; Symptoms of Oyster-borne Disease; Channels of Infection; Preventive Methods-2. Meat Poisoning; Tuberculous Meat-3. Ice-cream and Ice-4. Bacterial Infection of Bread-5. Miscellaneous Foods, Watercress, etc.

In this chapter the occurrence and significance of bacteria in shellfish, meat, ice-cream, and bread will be considered.

\section{Shell-fish}

Shell-fish have recently claimed the attention of bacteriologists, owing to the outbreak of typhoid and other epidemics apparently traceable to oysters.

Oysters.-It was not till 1880 (Cameron) that any substantial evidence was forthcoming to establish the view, which had previously been promulgated (by Pasquier in 1816), that oysters and other shell-fish might convey the infection of typhoid fever. In 1893 oysters came under the suspicion of Sir Richard Thorne Thorne as concerned in the diffusion of scattered cases of cholera in England, and he reported on the risks of consuming shell-fish cultivated at sewage outfalls.* In the spring of 1894 Dr Newsholme reported to the Corporation of Brighton the particulars of a number of cases of typhoid fever which were apparently attributable to the consumption of oysters obtained from layings grossly contaminated by sewage. At the end of the same year an outbreak of typhoid fever occurred at the Wesleyan University, in the State of Connecticut, U.S.A., and an investigation was made by Professor $\mathrm{H}$.

* “ On Cholera in England in 1893," Local Government Board Report, 1894. 
W. Conn, who found that the only channel of infection was the consumption of oysters served at certain college suppers. These oysters had been obtained from dealers at Middletown, and had been cultivated on oyster-beds in the region of a sewage outfall. The facts briefly were these: Two cases of typhoid fever occurred in a house discharging into a certain sewer; the outfall of the sewer was in immediate proximity to an oyster-bed, from which oysters were taken for consumption at the college suppers; 23 cases of typhoid fever followed among the students who attended the suppers at which the oysters were eaten, but these cases were limited to three out of seven fraternities; the only article of food used by the three implicated fraternities, and not by the other four, was raw oysters from the polluted consignment; and lastly, some of the same consignment were consumed at Amherst College, and an outbreak of typhoid fever occurred among those who consumed them.*

This outbreak furnished evidence almost equal to a series of experiments designed with the object of proving the possibility of the transmission of typhoid fever by oysters. It served also to stimulate inquiry, and since its occurrence a number of outbreaks have been traced to a similar source. Sir William Broadbent described several such cases in 1895, and Dr Newsholme continued to follow the matter up, and reported that in 189438.2 per cent., in $1895,33.9$ per cent., in $1896,31.8$ per cent., and in $1897,30.7$ per cent., of the total cases of typhoid fever originating in Brighton were caused by sewage-contaminated shell-fish. Between midsummer 1893 and the end of 1902, 630 cases of enteric fever occurred at Brighton, of which Dr Newsholme states 226 or 36 per cent. were caused by sewage-polluted shell-fish, 152 cases being traced to oysters and the remainder to other kinds of shell-fish. $\dagger$ In $1896 \mathrm{Dr}$ Bruce Law reported an outbreak of typhoid fever at Southend, in which certain cases had apparently been due to the same vehicle of infection. In the same year Chantemesse described to the Academy of Medicine an outbreak at Saint-André, in the Mediterranean Department of Hérault, which was caused by a barrel of oysters derived from contaminated oyster-beds at Cette. Fourteen persons eating these oysters in an uncooked condition contracted typhoid fever, or a disease simulating it. Evidence of the same character as that recorded in the above cases was forthcoming from Brightlingsea (Buchanan), Chichester (Theodore Thomson), Belfast (Jaffé), Southend-on-Sea (Foulerton, Nash), Yarmouth,

* Seventeenth Annual Report of the State Board of Health of Connecticut, U.S.A., 1894 ; New York Medical Record, 1894; Report of Medical Officer to Local Government Board, 1894-95.

+ Report on Health of Brighton, 1902, p. 45. 
Exeter, Blackpool, and other places. In 1902 occurred the outbreaks of typhoid fever and similar illnesses at Winchester and Southampton, following upon the consumption (at mayoral banquets) of oysters derived from some oyster-beds at Emsworth. From the same beds at the same time oysters were obtained which apparently caused cases of the disease at Portsmouth, Brighton, Ventnor, Hove, and Eastbourne. The matter was inquired into by Dr Timbrell Bulstrode, who found that at Winchester, out of a total of 134 guests at the banquet, 62 or 46.3 per cent. were attacked with illness; and at Southampton mayoral banquet, out of 132 guests, 55 or $41 \cdot 6$ per cent. were attacked with illness. Eleven of these cases were enteric fever, and 44 were cases of gastro-enteritis. In the two outbreaks 266 persons were guests, 21 (or 7.8 per cent.) were attacked with enteric fever, and 118 (or 44.3 per cent.) suffered from other illness. All those who had no oysters escaped enteric fever. After a minute inquiry Dr Bulstrode came to the following conclusion:- $(a)$ Two mayoral banquets occur on the same day in separate towns several miles apart; $(b)$ in connection with each banquet there occurs illness of analogous nature attacking, approximately speaking, the same percentage of guests and at corresponding intervals; $(c)$ at both banquets not every guest partook of oysters, but all those who suffered enteric fever, and approximately all those who suffered other illness, did partake of oysters, the exceptions to this rule appearing insignificant when all the facts are marshalled; $(d)$ oysters derived directly from the same source constituted the only article of food which was common to the guests attacked; and $(e)$ oysters from this source were at the same time and in other places proving themselves competent causes of enteric fever. It may be added that the oyster-beds at Emsworth from which the implicated oysters were obtained are in immediate proximity to the outfall of the Emsworth sewers, and had for several years been known to be contaminated beds.*

In 1902 there also occurred an outbreak of typhoid fever at Mistley and Bardfield in Essex, which was shown to be due to oysters, in which only a small portion of the oysters appears to have been capable of causing illness, and the nature of the illness varied from a mere feeling of nausea and weakness to a fatal attack of typhoid. $\dagger$

In 1902 and 1903 further evidence was forthcoming from various sources which went to show the intimate and apparently causal relationship between the consumption of polluted shell-fish and typhoid fever. Dr Nash, of Southend-on-Sea, states that in 1902 only 0.4 per cent. of the cases (501) of notifiable infectious diseases

* Special Report to the Local Government Board, 14th May 1903, by Dr H. Timbrell Bulstrode. See also Foulerton's Report on the Pollution of Tidal Fishing Waters by Sewage, 1903, pp. 31-37.

+ Report of Medical Officer of Eissex County Council, 1902, pp. 53-60. 
other than typhoid fever occurred in persons who had eaten shell-fish, whereas 54 per cent. of the cases of typhoid fever occurred in persons who had recently eaten shell-fish. In 1903 the comparative figures were 2 per cent. and 90 per cent. respectively.*

Evidence necessary to prove Contamination of Oysters.-Evidence of contamination of oyster-layings by sewage must be sought in three directions :-

(1) There must be personal inspection of the neighbourhood and surroundings of the layings and storage ponds. The immediate sanitary circumstances may be such that a definite conclusion can be come to that the locality is dangerously unfit for the purposes of the oyster industry, and no further examination by chemical or bacteriological methods will be necessary. On the other hand, local inspection may not reveal any probable source of dangerous sewage contamination; and to test the matter, it may be necessary to make further examination in order to detect traces of pollution which may have arisen from sources of contamination which were not obvious to the eye.

(2) In the event of the results of inspection being satisfactory, the next step is to examine the water in which the oysters are laid, in order to ascertain whether the chemical or bacteriological evidence of sewage contamination is sufficiently strong to enable one to say, in spite of the local inspection, that the sewage is not sufficiently diluted and purified to obviate all possible danger.

(3) A bacteriological examination of the molluses themselves must be made, in order to ascertain whether they contain those bacteria which are ordinarily associated with contamination by sewage.

It is hardly needful to add that, in order to establish the fact that infection has occurred or may occur from the consumption of polluted oysters, it is necessary to prove disease in persons or animals who have eaten some of the oysters. In any inquiry of this kind it is essential to take into consideration, $(a)$ clinical evidence, $(b)$ the history and circumstances of each case, and $(c)$ the exclusion of all other possible causes.

Symptoms of Oyster-Poisoning.-Obviously, the diseased conditions set up by the consumption of polluted oysters will vary according to circumstances. If the pollution be the specific infection of typhoid fever, the clinical disease of typhoid fever will supervene. The same applies to cholera. But in many cases on record the illness resulting has been of a less specific character, and has simulated gastroenteritis, colic, certain nervous conditions, and so on. Hence it may be desirable to make a provisional classification as follows :-

(a) Nervous Conditions, which Mosny likens to curare poisoning. This type is rare, always severe, and generally fatal.

(b) Gastro-Enteritis.-This group, which is one of the most common and least 
fatal, includes colic, nausea, vomiting, with more or less prostration. The onset is generally sudden, and the attack lasts a comparatively short time.

(c) Iysenteric Symptoms may occur which simulate the symptoms of group (b), but are more severe. In this group, between it and (b), may be classified the choleralike conditions which sometimes occur.

(d) Specific Disease, such as typhoid fever or cholera.*

In all cases there is, of course, an incubation period, which is usually longer in duration than that occurring in ptomaine poisoning.

Infection of Oysters. - The mode of infection of oysters by pathogenic bacteria is briefly as follows:-The sewage of certain coast towns is passed untreated into the sea. At or near the outfall, oyster-beds are laid down for the purpose of "fattening" oysters. Thus they become contaminated with saprophytic and pathogenic germs contri the sewage. It will be at once apparent that seve ary questions require attention before any deductions .w. as to whether or not oysters convey virulent disease isumers.

The precise conditions which render one locality more favourable than another in respect to oyster culture are not fully known. But it has been observed that they do not flourish in water containing less than 3 per cent. of salt. Hence they are absent from the Baltic Sea, which, owing to the fresh river-water flowing into it, contains a small percentage of salt. Oysters appear, in addition, to favour a locality where they find their chosen food of small animalcula and particles of organic matter. Such a favourable locality is the mouth of a river, where tides and currents also assist in bringing food to the oyster. Unfortunately, however, in a crowded country like England, such localities round our coast are frequently contaminated by sewage from outfalls. Thus the oysters and the sewage come into intimate relation with each other.

Professor Giaxa carried out some experiments in 1889 at Naples which appeared to show that the bacilli of cholera and typhoid rapidly disappeared in ordinary sea-water. Other observers at about the same time, notably Foster and Freitag, arrived at an opposite conclusion. Klein also found the cholera bacillus four days after the removal of the oysters from water purposely contaminated with them. In 1894. Professor Percy Frankland, in a report to the Royal Society, declared "that common salt, whilst enormously stimulating the multiplication of many forms of water bacteria, exerts a directly and highly prejudicial effect on the typhoid bacilli, causing their rapid disappearance from the water, whether water bacteria are present or not." Boyce and Herdman found that up to a certain point oysters could render clear sewage-contaminated

* The general question of mollusc poisoning is treated of by Mosny in the Revue d'Hygiene, December 1899 to March 1900. Mosny also furnished the French Government with reports on the subject. 
water, and could live for a prolonged period in water rendered opaque by the addition of fæcal matter. They also proved that the number of organisms in the pallial cavity and rectum of oysters which had been in clear water was much less than occurred in oysters laid down in proximity to a sewer outfall (10 bacteria in the former case as against 17,000 in the latter). It was found that more organisms were present in the pallial cavity than in the rectum. B. typhosus could be identified in cultures taken from the water of the pallial cavity and rectum fourteen days after inspection, but in diminishing numbers.* Several important links in the chain of evidence remained in obscurity. It was at this time, when the matter was admittedly in an unsatisfactory stage, that Dr Cartwright Wood made his experiments. We have not space here to enter into this work. But his conclusions seem to have been amply established, and were to the effect that typhoid and cholera bacilli could, as a matter of fact, exist over very lengthened periods in ordinary seawater. The next step was to demonstrate the length of time the bacilli of cholera remained alive in the pallial cavity and body of the oyster. Dr Wood found they did so for eighteen days after infection, though in greatly diminished numbers. This diminution was due to one or all of three reasons: $(a)$ the effect of the seawater already referred to as finally prejudicial to bacilli of typhoid; (b) the vital action of the body-cells of the oyster; $(c)$ the washing away of bacilli by the water circulating through the pallial cavity.

Broadly it may be said that the same principles apply to the typhoid bacillus. It can live in sea-water, probably, for three to five weeks (Klein, Boyce) although it does not appear to multiply in this medium. Oysters infected with the typhoid bacillus can retain their infective properties for two to three weeks, and even if placed in running sea-water, may not lose their infective properties for some days. Mosny suggested any period from one to eight days. In cockles there is evidence to show that the typhoid bacillus thrives and even multiplies, and these shell-fish are not rendered free from infection by being laid in pure water.

It will have been noticed that up to the present we have learned that typhoid bacilli can and do live in sea-water, and also inside oysters up to eighteen days, but in ever-diminishing quantities. The question now arises: What is the influence of the oyster upon the contained bacilli? Under certain conditions of temperature organisms may multiply with great rapidity inside the shell of the oyster. Yet, on the other hand, the amœboid cells of the oyster,

* Report of British Association for Advancement of Science, 1895 ; and ThompsonYates Laboratory Report, vol. ii.

† Brit. Med. Jour., 1896, ii., p. 760 et seq. 
the acid secretion of its digestive glands, or the water circulating through its pallial cavity, may act inimically on the germs. Proof can be produced in favour of the third and last-named mode by which an oyster can cleanse itself of germs. So far, then, we have met with no facts which make it impossible for oysters to contain for a lengthened period the specific bacteria of disease. Let us now turn to their opportunity for acquiring such disease germs. It is afforded them during the process of what is termed "fattening." By this process the body of the oyster acquires a plumpness and weight which enhances its commercial value. This desired condition is obtained by growing the oyster in " brackish" water, for thus it becomes filled out and mechanically distended with water. But if this water contain germs of disease, what better opportunity could such germs have for multiplication than within the body-cavity of an oyster? "The contamination of seawater, therefore, in the neighbourhood of oyster-beds may undoubtedly lead to the molluscs becoming infected with pathogenic organisms" (Wood). Yet we have seen that, apart altogether from the individual susceptibilities or otherwise of the consumer, there are in the series of events necessary to infection many occasions when circumstances would practically free the oysters from infection. This explains the absence of uniformity in degree of contamination, and, coupled with individual susceptibility and degree of cooking, the absence of uniformity in causing outbreaks of disease.

The sources of pollution of oysters are not the fattening beds alone. The native beds also may afford opportunity for contamination. Then, in packing and transit, and in storage in shops and warehouses, there is frequently abundant facility for putrefactive bacteria to gain entrance to the shells of oysters.

Dr Klein's researches into this question have been largely confirmatory of the facts elicited by Dr Cartwright Wood.* Despite the tendency of the bacilli of cholera and typhoid to die out quickly in crude sewage, the sewage is sufficiently altered or diluted at the outfall for these organisms to exist there in a virulent state. We may give Dr Klein's conclusions:-

1. That the cholera and typhoid bacilli are difficult of demonstration in sewage known to have received them.

2. That both organisms may persist in sea-water tanks for two or more weeks, the typhoid bacillus retaining its characteristics unimpaired, the cholera bacillus tending to lose them.

3 . That oysters from sources free from sewage pollution contained no bacteria of sewage (e.g. B. coli communis). Subsequently to these experiments, Klein examined 172 oysters from various layings to which no sewage gained access, and $B$. coli was absent

* Special Report of the Medical Officer to the Local Government Board on Oyster Culture, etc., 1896. 
in all cases.* The Massachusetts State Board of Health have recently arrived at similar results, and conclude that the presence of $B$. coli in shell-fish is abnormal and due to contamination either by sewage or by uncleanly handling. The presence of $B$. coli is therefore looked upon as "invariable aid" in determining the occurrence of pollution. $\dagger$

4. That oysters from sources exposed to risk of sewage contanination did contain colon bacilli and other sewage bacteria.

5. That in one case Eberth's typhoid bacillus was found in the body and liquor of the oyster. Nor do typhoid bacilli lose activity or virulence by passing through an oyster.

In 1902 Dr Klein had occasion to examine a number of oysters in connection with the Winchester outbreak of typhoid fever, to which reference has already been made. In all 18 oysters were examined with the following results:- $(a)$ Every one of the 18 contained B. coli, (b) 3 out of the 18 contained a bacillus belonging to the Gærtner-typhoid group, and (c) 3 out of 15 contained the spores of $B$. enteritidis sporogenes. All these oysters came from the Emsworth layings, and all showed contamination with excrementitious matter. At the end of 1902 and beginning of 1903, Dr Klein examined 25 different sets of oysters, only 7 sets of which showed no signs of pollution.+

Boyce examined 140 samples of shell-fish at Liverpool in 1902, and found $B$. coli present in 104, and B. enteritidis sporogenes in 10 cases. The former was more frequently present in oysters and mussels, and the latter in cockles. $\oint$

In $1903 \mathrm{Mr}$ Foulerton examined a number of oysters derived from suspicious oyster-beds, with the object of detecting the presence or absence of bacteria characteristic of sewage. The two sewage organisms which he selected as "indication" bacteria were B. coli and $B$. enteritidis sporogenes, and his results were as follows: Out of 65 oysters examined, in 48 neither bacillus was found; in $5, B$. enteritidis sporogenes was present alone; in $8, B$. coli was present alone; and in 4, both organisms were present. Foulerton attaches most importance, as indication of recent sewage contamination, to the presence of $B$. coli, and he therefore concludes that out of 65 oysters 12 or 19.4 per cent. showed evidence of recent sewage pollution.| In a second series of 27 oysters $B$. coli was found in 4 instances, or a percentage of $14 \cdot 7$.

* Brit. Med. Jour., 1903, i., p. 419.

+ Thirty-fourth Annual Report of the State Board of Health of Massachusetts, 1903 , pp. 260-264, and 280.

$¥$ Report of Medical Officer of Health, City of London, 1902, pp. 150-157.

$\S$ Report on Health of Liverpool, 1902, p. 173.

II The Pollution of Tidal Fishing Waters by Sewage, 1903. A special report by A. G. R. Foulerton, F.R.C.S., D.P.H., pp. 42-49. 
Lastly, the results of the investigations carried out by Dr Houston for the Royal Commission on Sewage, tend to prove that the contamination of oysters by $B$. coli is widespread and not altogether dependent on sewage contamination of the oyster. $\mathrm{He}$ examined over 1000 oysters, and nearly all, from whatever laying they were taken, contained $B$. coli or coliform organisms. This did not hold good as regards deep-sea oysters which were free from this organism (and spores of $B$. enteritidis sporogenes) as was also deep-sea water. Houston found the number of $B$. coli in an oyster varied from 10 to 10,000 (in 10-15 c.c.), and the contents of the stomach of the oyster contained more $B$. coli than the liquor in the shell. Fewer B. coli were found, as a rule, in oysters stored in pure waters, but instances occurred where the number of such bacilli was as great as in oysters from contaminated sources.*

Such is the bacteriological evidence down to recent date, and whilst some of it may appear to be of a conflicting nature, there are certain conclusions which may be drawn. First, the presence of $B$. coli in oysters must be judged relatively. Secondly, topographical evidence as to pollution must be taken in conjunction with bacterial evidence. Thirdly, there is the broad general fact that oysters ordinarily grown on oyster-beds contaminated with bacteria may, and do on occasion, contain the virulent specific bacillus of typhoid, which can live both in sea-water and within the shell of the oyster. This being so, the risk of infection of typhoid by oysters is a real one. Yet in actual occurrence many conditions have to be fulfilled. For, in addition to the fact that the oysters must be consumed, as is usual, uncooked, the following conditions must also be present:-

(a) Each infective oyster must contain infected sewage, which presupposes that typhoid excreta from patients suffering from the disease have passed into that particular crude sewage, and have not been disinfected.

(b) The infective oyster must have fed upon infected sewage, and still contain the virus in its substance.

(c) There must have been no period of natural cleansing after "fattening."

(d) The oyster must then be eaten, uncooked or undercooked, by a susceptible person.

Even to this formidable list of conditions we must add the further remark that, owing to the vitality of the body-cells of the oyster or to the lessened vitality of the bacilli of cholera and typhoid, it is generally the case that the tendency of these organisms is rather to decrease and die out than live and multiply.

* Royal Commission on Sevage Disposal: Fourth Report on Pollution of Tidal Waters and Contamination of Shell-fish, 1904, vols. i. and iii. The latter volume contains a large amount of information as to bacteriological technique, etc. 
We shall probably maintain a satisfactory balance of truth if we place alongside these facts the summary of the Local Government Board Report. "There can be no doubt," wrote Sir Richard Thorne, "that oysters which have been brought into sustained relation with the typhoid bacillus are liable to exhibit that microbe within the shell contents, and to retain it for a while under circumstances not only permitting its rapid multiplication when transferred again to appropriate media, but conserving at the same time its ability to manifest its hurtful properties." * The Royal Commission on Sewage Disposal concludes, that at the present time it is undesirable to condemn oysters only on bacteriological evidence of the presence of $B$. coli. At present topography must stand before bacteriology, and the condemnation or otherwise of oysters must be judged on broad, common-sense lines. It should be borne in mind that the oyster trade is a considerable industry, which should not be injured except on proved and substantial evidence.

Means of Prevention. - In the special report issued by the Local Government Board in 1896, on oyster culture, which had been drawn up by $\mathrm{Dr}$ Timbrell Bulstrode, accounts are given with diagrams of the layings, fattening beds, and storage ponds used in oyster cultivation in various counties round the coast of England and Wales. Various proposals were made by Dr Bulstrode for the control of this industry. In some cases, particularly the larger layings, altering the position of the fattening beds was eonsidered sufficient, but in other cases nothing short of a complete diversion of the sewers and drains, or withdrawal of existing layings, could be regarded as sufficient. $\dagger$ We may repeat that the Royal Commission on Sewage Disposal urge that topographical conditions shall be taken along with bacteriological evidence in arriving at a decision as to any oyster layings, and under the present circumstances of our limited knowledge of the bacteriology of the subject, this is the right course and should assist in indicating preventive methods.

From what has been said, such preventive treatment is obvious :(1) All oyster layings and shell-fish beds round the coast should be registered, superintended, and inspected by the sanitary authority or the Government. (2) Local Sanitary Authorities should have power of control over oyster layings situated in their district, and should be enabled to prevent the sale in their district of oysters and other molluses derived from sewage - contaminated sources. (3) The importation of foreign oysters, grown on uncontrolled beds, should,

* Special Report to Local Government Board on Oyster Culture, etc., 1896. This report by Dr Timbrell Bulstrode is probably the fullest statement yet written on the question as it affects England.

+ For a brief record of the attempts at legislation on this subject, see Brit. Med. Jour., 1903, ii., p. 296 (Newsholme). 
if possible, be restricted or supervised. (4) Further, as a protective measure of the first importance, oysters should be cleansed, after fattening on a contaminated bed, by being deposited for several weeks at some point along the coast which is washed by pure seawater. (5) Retention in dirty-water tanks, in uncleanly shops and warehouses, should also be prohibited.

Other shell-fish than oysters do, from time to time, cause epidemics or individual cases of gastro-intestinal irritation, and probably contain various germs. These they acquire in all probability from their food, which by their own choice is frequently of a doubtful character.

In a preliminary inquiry into "Cockles as agents of Infectious Diseases," Dr Klein detected the B. coli in 3 out of 8 cockles which had been taken from a foreshore polluted with the discharge from a sewer outfall, and also $B$. enteritidis sporogenes in 4 of them. No typhoid bacilli were detected. In 8 raw cockles in their shells bought from a street hawker, Dr Klein found no typhoid organisms, but $B$. coli was found in 5 out of the 8 cockles and $B$. enteritidis sporogenes in 4 out of the $8{ }^{*}$ In subsequent experiments Dr Klein came to the conclusion that a mussel immersed for twenty-two hours in cholera-infected water retained the bacilli of cholera for forty-eight hours after immersion in clean sea-water, and the same may be said in respect of typhoid infection. Indeed, evidence was obtained showing that the typhoid bacillus could multiply in cockles. He also showed that merely pouring boiling water over a heap of shell-fish did not necessarily destroy either cholera or typhoid infection contained in them. $†$ Since the time of these investigations, a number of outbreaks of disease, including enteric fever, have been traced to the consumption of mussels and cockles, and it has been shown that the cooking which these shell-fish undergo is not sufficient to rid them of poisonous pollution.

In 1902 several cases of typhoid occurred in Wandsworth due to infected cockles, and a number of similar cockles being examined by Dr Klein showed the presence of $B$. coli and other allied forms, and by other workers (Lister Institute) during the same year the typhoid bacillus itself is stated to have been isolated from cockles derived from a sewage-polluted laying: +

In 1903 an outbreak of typhoid fever occurred in Glasgow, which was traced to the consumption of sewage-polluted shell-fish at a neighbouring seaside town. An examination of a number of shell-fish from this particular locality was made by $\mathrm{Dr} \mathrm{R}$. M. Buchanan, the Corporation bacteriologist, who reported that-(1) All

* Report of Medical Officer to Local Government Board, 1899-1900, p. 574.

† Ibid., 1900-01, pp. 564-71.

$\mp$ Report of Medical Officer of Health of City of London, 1902, pp. 134-49. 
the edible shell-fish within the area of sewage contamination showed by the presence of virulent $B$. coli excremental pollution, and their consumption must therefore be regarded as highly prejudicial to health. Cultures of the species of $B$. coli killed guinea-pigs within eighteen hours. (2) The shell-fish beyond the range of sewage contamination were found to be normal and perfectly safe for edible purposes. (3) Certain shell-fish, cockles and "muscins" (My'a arenaria) within the area of sewage contamination showed, according to Buchanan, the presence of the bacillus of typhoid fever in great number, and in some cases almost in pure culture, and the consumption of similarly infected shell-fish by holiday visitors in the end of July would sufficiently explain the outbreak of typhoid fever which occurred amongst them after return to their own homes in Glasgow and elsewhere.

As regards the specificity of this bacillus, Buchanan reports that it had all the microscopical and cultural characteristics of the typhoid bacillus. Further, it gave the characteristic reaction with human typhoid serum and with serum obtained from a typhoid immunised guinea-pig. The finding of this bacillus in such numbers, and in so many individual shell-fish, is so exceptional that it was repeatedly subjected to reliable culture tests and to repeated serum tests, and always with the result of proving its general identity with the typhoid bacillus obtained from a case of typhoid fever.

A third apparent instance of finding the typhoid bacillus may be quoted. In 1902-1903 Klein examined ten samples of Leigh cockles, and found every one of them showing evidence of sewage pollution, though six had been "cooked." One of the uncooked ones contained B. typhosus, a typical typhoid bacillus agglutinating with typhoid blood (Klein). The cooking to which some of these cockles were submitted must have been perfunctory, as it is fairly well established that $60-61^{\circ} \mathrm{C}$. kills $B$. coli. Yet this organism was found in two instances where the cockles in question had been "boiled continuously for one minute," or "put in boiling water and taken out when the water boiled over, time in water three and a quarter. minutes." *

These facts reflect a new and not reassuring light upon the possibility of cockle infection. But they must be accepted with great reserve until very fully confirmed. Dr Bulstrode in his report on Oyster Culture in 1896 suggested that the infection by cockles was a remote contingency, because "in the first place these molluscs are, as a rule, only eaten after being cooked; and in the second place it is seldom that extensive cockle industries are carried on in other localities than those where large stretches of sand are exposed at low tide, and such stretches are found chiefly on the actual seashore

* Report of Medical Officer of Health of City of London, 1902, pp. 134-149. 
or quite at the mouth of estuaries far away from sources of sewage contamination." The experience at Leigh-on-Sea, Southend, and other places seems to tell a different tale, and it is evident that the shell-fish may be grown on polluted beds. After growth, it is true, they are raked into hand-nets, and taken to the cockle-sheds, and here are plunged into coppers of boiling water in the nets, after which they are riddled through wide-meshed sieves, which allow the soft parts to pass through, retaining the shells, which are deposited in heaps for sale to oyster cultivators. The cockles themselves are then washed in about five changes of water, to the last of which a certain quantity of salt is added. Not infrequently, the same water is used in all the washings. The so-called boiling is evidently misleading. Though the water is actually at the boiling point, the cockles are plunged in in a mass, and for a short time, and it by no means follows that every part is exposed to a temperature of $212^{\circ} \mathrm{F}$.

Dr Klein has shown that the usual method of cooking only amounts to scalding, and cannot be relied on to sterilise microorganisms. The live fish, with shells tightly closed, are held in a net and plunged en masse into a vessel containing boiling water. The immersion of the cold mass immediately lowers the temperature, and when in the course of two or three minutes it begins to boil again, the net is lifted out. The scalding kills the fish and causes the shells to open, but it does not sterilise the contents. Dr Klein found that the temperature of the water fell, on the immersion of the fish, from $100^{\circ} \mathrm{C}$. to $65^{\circ}$; and that cooking for the usual time was totally inadequate to kill the micro-organisms. Fish that had been kept in typhoid polluted water were tested, and were found to be swarming with live bacilli after cooking. Prolonged boiling would, no doubt, be effective, but it causes the fish to shrivel up and spoils them for sale.

Dr Klein then suggested that cooking by steam might be found an efficient steriliser without spoiling the fish as food. It is well established that current steam is much more penetrating than boiling water for purposes of disinfection, and it is always used in preference. The question was whether an exposure sufficient to sterilise would amount to over-cooking, and recent experiments carried out in the kitchen at Fishmongers' Hall were intended to settle that point. Cockles and mussels were cooked in a steamer under the direction of Dr Klein in the presence of several representatives of the trade, who examined them afterwards. Two batches were cooked, one for ten minutes and the other for five. The steamer used was a fixed vessel some 2 feet deep, into which steam is introduced by a pipe about an inch from the bottom. A layer of cockles was placed at the bottom, and two other layers on 
trays above it. In the top tray mussels were also placed. Some of the fish were spread out and others heaped up. The results were:Ten minutes-mussels pronounced spoilt, and useless to the trade; cockles "all right" in upper layers, but the bottom layer overcooked. Five minutes-mussels "all right," and cockles better than the ten minutes' batch; the upper layers "could not be better" in appearance and flavour, but the bottom layer was again pronounced somewhat overcooked, or at any rate less satisfactory than the others. No doubt the steam was hotter at the bottom of the vessel and the exposure greater. The bacterial results were as follows:The cockles were found to be sterile in all cases: the mussels were also found to be sterile, except in the case of those placed in heap on the top layer and steamed for five minutes. Some of these still retained living spores. It is probable that if exposed to the more direct action of the steam even the heaped mussels would be completely sterilised by five minutes' cooking, without impairing their trade value. As a result of these experiments the Fishmongers' Company was reported as recommending to the trade the substitution of steaming for boiling.

Many other similar foods have been implicated in the spread of disease. Dr Hamer investigated outbreaks of typhoid fever in London in 1900 and 1903, in which he showed the extreme probability of fried fish acting as the vehicle of infection.* In $1900 \mathrm{Dr}$ Plowright traced similar infection in thirty persons to polluted clams, shell-fish comparatively little known in this country as an article of diet. $\uparrow$ Derived from sewage-polluted layings, clams may readily become contaminated, and if uncooked may convey disease to the consumer.

* Ninth Annual Report of Medical Officer of Health of Administrative County of London, 1900, p. 37, and Appendix ; and Special Report, No. 719, issued 1904.

$\dagger$ The clam is a shell-fish comparatively little known in this country as an article of diet except to the dwellers near those of our coasts on which it occurs. Belonging to the Siphonidæ division of the Conchiferæ, the clam (Mya arenaria), like its ally the cockle, is found abundantly round our shores. It has, however, a wider geographical distribution, being found in the Arctic Regions, where it constitutes an important article of food. In America it is largely consumed in Boston and along the Massachusetts seaboard. The clam of New York is a different species (Venus marcenaria). It has a remarkably developed syphon, the inhalent and exhalent tubes being joined into a trunk-like body 3 or 4 inches in length, which the animal protrudes in an upward direction towards the surface of the mud. The clam itself lies buried in the mud, into which it has worked itself by the aid of its muscular foot to a depth varying from 8 to 18 inches. The currents of water passing in and out the syphon keep open the vertical burrow the creature has made, while the surface of the mud is covered by the tide, but where this recedes and the mud becomes dry, the position of the clam is shown by a small round depression on the surface. In Great Britain it is regarded as a kind of inferior oyster, and like the last-named is preferred uncooked by those persons who are really fond of it and to whom it is a luxury. More generally, clams are cooked by having boiling water poured over them, and being allowed to remain in it until the shells open. 


\section{Meat}

Since 1880 , more than fifty outbreaks of disease have been traced to the consumption of unwholesome or diseased meat. In 1880 occurred the well-known "Welbeck disease" epidemic. A public luncheon was followed by severe and, in some cases, fatal illness. Seventy-two persons were affected and four died. A specific bacillus was isolated by Klein from the cold hams, the consumption of which caused the outbreak. The incubation period varied from twelve to forty-eight hours. This epidemic drew marked attention to the whole question of food-poisoning, and subsequent epidemics were very thoroughly investigated by the aid of bacteriology. Some of the better-known outbreals may be tabulated as follows:-

\begin{tabular}{|c|c|c|c|c|}
\hline $\begin{array}{l}\text { Date of } \\
\text { Occurrence. }\end{array}$ & Place of Occurrence. & $\begin{array}{l}\text { No. of } \\
\text { Cases. }\end{array}$ & $\begin{array}{l}\text { Period of } \\
\text { Incubation in } \\
\text { Hours. }\end{array}$ & $\begin{array}{l}\text { Probable Source of } \\
\text { Infection. }\end{array}$ \\
\hline 1880 & Welbeck & 72 & $12-48$ & Cold boiled hams \\
\hline 1881 & Nottingham & 15 & $12-34$ & Pork \\
\hline 1882 & Oldham & 9 & 4 & $\begin{array}{c}\text { American Tinned } \\
\text { Pig's Tongue }\end{array}$ \\
\hline 1882 & Bishop Stortford & 6 & 24 & Beef \\
\hline 1882 & Whitchurch & 20 & $1-5$ & Brawn \\
\hline 1886 & Carlisle & 20 & $6-40$ & Ham and game pie \\
\hline 1886 & Ironbridge & 12 & $6-12$ & Veal pies \\
\hline 1887 & Retford & 80 & $8-36$ & Pork brawn \\
\hline 1888 & Middlesborough & 114 & $\ldots$ & American bacon \\
\hline 1889 & Carlisle & 25 & $\ddot{24}$ & Pork pies \\
\hline 1891 & Portsmouth & 13 & $14-17$ & Cold meat pie \\
\hline 1896 & Mansfield & 265 & $18-24$ & Potted meat \\
\hline 1898 & Oldham & 54 & 48 & Veal pies \\
\hline 1899 & Nuneaton & 42 & $12-48$ & Pork chitterlings \\
\hline 1902 & Derby & 221 & $4-24$ & Pork pie \\
\hline
\end{tabular}

In nearly all these cases the general symptoms have been usually one of two kinds, namely, conditions simulating gastro-enteritis, or conditions simulating nervous disease. Each of the outbreaks have shown more or less clearly the characters common to these epidemics :-

1. Simultaneous attacks.

2. Similarity of symptoms and post-mortem signs.

3. A history of infection and collateral circumstances.

The common symptoms have included rigors, faintness, vomiting, diarrhoa, abdominal pain, and occasionally skin eruptions. As a rule, certain nervous conditions have supervened, such as giddiness, headache, paralyses, mental depression, etc., and occasionally these symptoms have been predominant. 
Meat-poisoning appears to depend not upon the number of bacteria present in the meat, but upon the particular species and their products. As we have already stated, a long incubation period generally indicates poisoning by bacteria, and a short incubation period poisoning by products (ptomaines, toxins, etc.). In 1888, Gaertner of Jena investigated an outbreak of disease affecting 58 persons who had eaten uncooked meat. One of the unfortunate victims died, and from his body, as well as from the meat, Gaertner isolated a bacillus which he called the $B$. enteritidis, an organism allied to the coli group. This was practically the starting-point of accurate bacteriological investigation into this group of epidemics (fleischvergiftung, Ger.; and intoxications alimentaires, Fr.). Since that period, the $B$. botulinus of Ermengem, and certain putrefactive bacteria, have been held responsible for causing such illnesses. More than twenty different species of bacteria have been isolated from tinned meats and hams. As is pointed out elsewhere in the present volume, there is evidence that the infectious properties which food acquires frequently in summer, and which give rise to the ordinary type of epidemic diarrhœa, are due to bacilli belonging to the colon group, of which the B. coli communis of Escherisch and the B. enteritidis of Gaertner are the two extreme types. According to Delépine, the varieties of those bacilli which are the most important sources of infection are those which resemble the bacillus of Gaertner, and which, therefore, produce no permanent acidity, coagulation, or distinct smell when grown in milk. Very few infectious samples of milk give a distinct acid reaction, so that absence of acidity in milk is not, as generally believed, an index of safety. It is probable that the most dangerous kind of fæcal infection is that produced by matter containing bacilli resembling Gaertner's bacillus. Such an infection is probably connected with the existence of an infectious diarrhœal disease liable to occur in the lower animals as well as in man.

It is certain that bacilli presenting the characters of the ordinary $B$. coli communis are seldom capable of producing such a rapid infection as that produced by the B. enteritidis, or by closely-allied bacilli, such as the $B$. cnteritidis Derbiensis.

The last-named organism is a member of the Gaertner group isolated by Delépine from pork pies, the consumption of which caused the Derby illness in 1902. He considered the presence of this bacillus in the pork pies was due to fæcal pollution of the meat before it was cooked, and that the central parts of the pies were not thoroughly cooked.* It frequently happens in these cases

* Report on Outbreak of Food-Poisoning in Derby, 1902 (Howarth and Delépine). In this reference, and in Brit. Med. Jour., 1898, ii.pp. 1456-58 and 1797-1801, and ibid., 1899, vol. ii., pp. 791, 1367, will be found many particulars with regard to meat-poisoning, its symptoms, prevention, investigation, etc. 
that some constituent part (such as jelly) of the manufactured article or prepared dish is really the polluted portion.

\section{Bacteria associated with Meat-Poisoning}

The chief organisms, therefore, which have been considered as causally related to meat (and " ptomaine") poisoning are $B$. coli communis, B. enteritidis sporogenes, B. enteritidis of Gaertner, and B. botuliuus. The main facts respecting these organisms must be mentioned here.

(a) B. coli communis (see p. 46).

(b) B. enteritidis sporogenes (see pp. 156 and 307).

(c) B. enteritidis of Gaertner. Isolated by Gaertner in 1888 from flesh of diseased cow which had caused illness in persons eating it. Characters similar to B. typhosus (morphology, motility, and staining properties), but grows more rapidly in gelatine; fewer flagella; ferments lactose and sometimes dextrose; does not produce indol or coagulate milk; positive neutral-red reaction; in litmus whey or litmus broth, acid is first produced, and then the medium becomes distinctly alkaline. Virulent to rodents and small animals (gastro-intestinal symptoms, hæmorrhagic enteritis, and swelling of lymph follicles). B. enteritidis Derbiensis of Delépine is one of the members of the Gaertner group of enteritidis bacilli. B. enteritidis possesses no spores, and therefore cannot stand very high temperatures. It produces agglutinating properties in the blood of the patient.

(d) B. botulinus (Ermengem). This bacillus is held to be responsible for setting up botulism. Van Ermengem describes, under the name of botulism, a state brought about by the ingestion of various articles of food, such as ham, tinned or preserved foods, oysters, mussels, etc., and which is characterised by comparatively slow onset (twelve to twenty-four hours after infection), secretory troubles, paralysis of certain muscles, particularly tongue and pharynx, dilatation of pupil, aphonia, dysphagia, constipation, retention of urine, absence of unconsciousness and of fever, etc. Van Ermengem has found that these symptoms were produced by a bacillus, to which he has given the name of bacillus botulinus. Botulism differs considerably from the more common form of food-poisoning with which we are acquainted in England, and which is characterised by practically the same symptoms as those of epidemic diarrhoea. B. botulinus is 4-9 $\mu$ long and $9-12 \mu$ broad; round, slowly motile, 4-9 flagella. Polar spores; killed in thirty minutes at $80^{\circ} \mathrm{C}$. Liquefies gelatine; does not coagulate milk; anaërobic ; in cultures often produces gas and a sour, rancid odour. Pathogenic for guinea-pigs, rabbits, and the other small animals (botulism).

Preventive methods. - Experience of meat-poisoning outbreaks leads to the conclusion that the meat has contracted its poisonous properties in either or both of two ways- $(\alpha)$ putrefaction or unsoundness in the meat itself; (b) unclean manipulation or storage in insanitary conditions. Generally there has also been insufficient cooking. The methods of prevention are therefore obvious. Occasionally tinned foods cause poisoning owing to metallic absorption, and this must be differentiated from bacterial poisoning.

There is another class of meat conditions related to disease, to which reference must now be made, viz., certain conditions occurring in fresh meat, joints, or carcases. It is well known that the meat substance itself does not frequently contain injurious bacteria. They may nevertheless occur in the organs, glands, and tissues 
other than muscular, and when present set up during life the bacterial diseases of animals, or after death putrefactive changes. It is for these conditions that meat is "seized" under the Public Health Acts as unfit for food of man. Such conditions may be broadly divided into two kinds:-

1. Specific Diseases in Meat, such as tuberculosis, anthrax, swine fever, actinomycosis, milk fever, etc.

2. Decompositions of Meat due to invasion by putrefactive organisms after death. These conditions may be disposed of at once by saying that they arise commonly as a result of keeping meat too long under conditions likely to lead to putrefaction. Unclean storage, insufficient preservation, summer weather, and similar circumstances afford the opportunity for putrefactive organisms to perform their function. The signs of decomposing meat do not require explanation or elaboration. They are mainly three:- (a) Smell of putrefaction; (b) discoloration; and $(c)$ loss of elasticity of tissue which becomes doughy, pits on pressure, or may even become slimy or soapy.

The chief specific diseases which occur in meat are dealt with briefly in the section treating of the relation between bacteria and disease. It will, therefore, be unnecessary to make more than a passing reference in this place.

Tuberculosis.-This disease is set up in animals by the tubercle bacillus, which is either identical with or closely allied to the $B$. tuberculosis of Koch. It may set up a generalised disease affecting the body of the animal more or less completely, or it may set up only a local disease.

The Royal Commission on Tuberculosis, in the report which they made in 1898, referred to the degree of tubercular disease which should cause a carcase, or part thereof, to be seized, and which may be accepted broadly as indicative of general and local tuberculosis. They stated as follows:

"We are of opinion that the following principles should be observed in the inspection of tuberculous carcases of cattle:-

(a) When there is miliary tuberculosis of both lungs .

(b) When tuberculous lesions are present on the pleura and peritoneum

(c) When tuberculous lesions are present in the muscular system, or in the lymphatic glands embedded in or between the muscles

Generalised tuberculosis is present, and the entire carcase and all the organs may be seized.

(d) When tuberculous lesions exist in any part of an emaciated carcase . 
(a) When the lesions are confined to the lungs and the thoracic lymphatic glands

(b) When the lesions are confined to the liver

(c) When the lesions are confined to the pharyngeal lymphatic glands .

(d) When the lesions are confined to any combination of the foregoing, but are collectively small in extent

Localised tuberculosis is present, and the carcase, if $o$ therwis e healthy, shall not be condemned, but every part of it containing tuberculous lesions shall be seized.

"In view of the greater tendency to generalisation of tuberculosis in the pig, we consider that the presence of tubercular deposit in any degree should involve seizure of the whole carcase and of the organs.

"In respect of foreign dead meat, seizure shall ensue in every case where the pleura have been 'stripped.'"

The tubercle bacilli are most easily found in the glands. They are scarce in the caseating nodules. In the pig it is difficult to detect the bacilli as a rule. The Royal Commission on Tuberculosis emphasised the absence of bacilli in the meat substance:- "In tissues which go to form the butcher's joint, the material of tubercle is not often found even where the organs (lungs, liver, spleen, membranes, etc.) exhibit very advanced or generalised tuberculosis; indeed, in muscle and muscle juice it is very seldom that tubercle bacilli are to be met with; perhaps they are somewhat more often to be discovered in bone, or in some small lymphatic gland embedded in intermuscular fat." * The chief way in which such meat substance becomes infected with tubercle appears to be through carelessness of the butcher, who perchance smears the meat substance with a knife that has been used in cutting the organs, and so has become contaminated with infected material. Very instructive also are the results at which Dr Sims Woodhead arrived in furnishing evidence for the same Commission on the effect of cooking upon tuberculous meat:"Ordinary cooking, such as boiling and more especially roasting, though quite sufficient to sterilise the surface, and even the substance for a short distance from the surface of a joint, cannot be relied upon to sterilise tubercular material included in the centre of rolls of meat, especially when these are more than three pounds or four pounds weight. The least reliable method of cooking for this purpose is roasting before a fire; next comes roasting in an oven, and then boiling." + From this statement it will be understood that

* Royal Commission on Tuberculosis, Report, 1895, part i., p. 13.

$\dagger$ 1bid., p. 18. 
rolled meat may be a source of infection to a greater degree than the ordinary fresh joint, and this is borne out by the experience derived from epidemics due to meat-poisoning.

Tuberculous meat also finds its way occasionally into sausages, and other similarly prepared meat foods.

Swine Fever is not an uncommon disease of pigs, and makes the meat unfit for food. Schütz and others have isolated a bacillus from this disease. The chief post-mortem signs are the red punctiform rash, generally becoming confluent on the back, extremities, and ears; the ulceration of the intestine, and the characteristic mottling of the lymph glands.

Anthrax (see p. 315), Actinomycosis (see p. 321), and other conditions are described elsewhere. Many parasitic diseases also make meat unfit for food.

\section{Ice-cream}

In 1894 Dr Klein had occasion bacteriologically to examine icecream sold in the streets of London. In all six samples were analysed, and in each sample the conclusions resulting were of a nature sufficiently serious to support the view that the bacterial flora was not inferior to ordinary sewage. The water in which the ice-cream glasses were washed was also examined, and found to contain large numbers of bacteria.

Since that date many investigations have been made into icecream. It appears that this luxury is frequently manufactured under extremely objectionable circumstances, and with anything but sterilised appliances. Little wonder, then, that the numbers of bacteria present run into millions per c.c. (varying from two to twenty millions or more). In nearly all recorded cases, the quality of the germs as well as the quantity has been of a nature to cause some concern. $B$. coli communis has been very commonly found, and in considerable abundance. The Proteus family, which also possesses a putrefactive function, is common in ice-creams. The common water bacteria are nearly always present. B. typhosus itself, it is said, has been isolated from some ice-cream which was held responsible for an outbreak of enteric fever. The material had become infected during process of manufacture in the house of a person suffering from unnotified typhoid fever.

The Manufacture of Ice-cream.-There are, practically speaking, three methods of manufacture :-

(1) The real ice-cream, which cannot be sold at a low price, and which is made simply by mixing cream (with a small proportion of milk), fruit or fruit pulp and sugar. This mixture is then at once frozen.

(2) Milk is flavoured with fruit, or fruit essence and sugar, and has then added to it a small quantity of dissolved gelatine, and at once frozen. In these two processes there is no boiling, and both are frozen immediately after mixture. 
(3) Skimmed milk is boiled, either with or without the addition of some form of starch (generally corn flour) with a certain number of eggs, sugar and flavouring. Practically, the number of eggs used varies inversely with the amount of starch, the effect of both being to thicken the mixture, and in some cases the practice has been to at once freeze the mixture ; in others, in order to economise the consumption of ice, the heated liquid has been allowed to cool naturally. Usually the number of eggs is three or four to the quart. The whole is again boiled (for perhaps twenty minutes), after which it is set aside to cool until the following morning, when it is placed in the "freezers" and frozen. In short, the mixture contains no cream, and is, in fact, a frozen custard. The milk and eggs are generally obtained locally, and are usually of good quality. It is stated that if such were not the case the ice-cream would be unpalatable, owing to ill-flavour, and therefore unsaleable. The eggs used to be blown, but of late year's that practice has fallen into disuse, and they are now broken and mixed in the ordinary way. The boiling is carried out in various utensils over the open fire. The mixture stands for cooling purposes in the living room, or in any out-of-the-way corner, sometimes in the open yard or area. The freezing takes place early on the following morning in the "freezers." A "freezer" consists of a tin or galvanised iron cylinder. or container, in which the mixture is placed. The cylinder fits into an outer vessel of wood or metal so loosely as to leave an inch or two of space all round. In this space is placed broken ice and salt, and the inner cylinder is rotated from time to time in this ice medium. No ice is added directly or indirectly to the mixture itself, nor are colouring agents used as a rule. The utensils and materials appear, as a general rule, to be clean.

From this description, which applies, generally speaking, to the street ice-cream industry in London, it will be seen that the "ice-cream " is boiled for some time, and in all probability sterilised, and in due course it undergoes, at least approximate, freezing. These facts, added to the generally wholesome condition of the elementary materials used, would appear, at first sight, to place the substance beyond risk of contamination. But the critical period is the time of exposure between boiling and freezing. Boiling sterilises, but freezing does not sterilise. Hence, if in the long cooling process the substance is exposed to contaminated surroundings, the result may be, in effect, a contaminated ice-cream. Such, in fact, frequently occurs.* Hence it would appear that it is not the process of manufacture that needs supervision so much as the general condition of the houses in which the substance is made, and of the persons who make it, and the manufacture so far as length of time between boiling and freezing is concerned.

The important stage of the operation is, therefore, that between the boiling and freezing. Attention has been drawn to the fact that the majority of the specific pathogenic bacilli discovered in ice-cream are of a non-sporing variety, and that, therefore, if originally present in the material, would have been destroyed by boiling, and, if found subsequently, must have gained access to the material while cooling. The subsequent freezing, while it might inhibit such bacilli, would certainly not destroy them, and on ingestion and melting their growth and development would again commence.

Some dozen outbreaks of disease have been attributed to the consumption of ice-creams. A typhoid epidemic occurred in Liverpool (27 cases) in 1897 due to ice-cream, and an earlier epidemic of the same disease traceable to the same cause occurred at Deptford in 1891 (Turner). Recently, a small outbreak occurred in the city of London affecting 16 telegraph boys. The symptoms were colic and diffuse abdominal pains, headache, vomiting, diarrhœa, and nervous depression. Dr Collingridge's inquiry resulted in the following conclusions:-

* See investigations by Klein, Cook, Wilkinson, Foulerton, and others. 
(1) That in a number of cases of illness occurring among young persons of a susceptible age, the symptoms were strictly identical, and were characteristic of poisoning by ingestion of toxic material.

(2) That the cases reported followed the ingestion of ice-creams.

(3) That ice-creams subsequently obtained at shops frequented by the patients contained bacilli of a virulent character.

(4) That the symptoms observed were those generally following the ingestion of material containing such bacilli.

(5) That where pathogenic bacilli were found, the ices had been manufactured under insanitary conditions. The majority of the manufacturers are aliens, and although the premises may be kept in a fairly sanitary condition, their personal habits unfortunately leave much to be desired where the preparation of food is concerned.

Dr Klein examined 24 samples of ice-cream from the same locality, and found 13 (or 54 per cent.) to be poisonous to guineapigs.* The writer traced 18 cases of typhoid fever in 1902 to the consumption of contaminated ice-cream. + Owing to outbreaks of this nature the London County Council (General Powers) Act, 1902 (sects. 42-45), has given powers for controlling this trade:-

(a) Ice-cream must be made and stored in sanitary premises.

(b) It must not be made or stored in living rooms.

(c) Strict precautions must be taken as to protection from contamination.

(d) Cases of infectious disease must be reported.

(e) The name and address of the maker must appear on street barrows.

These regulations are new for London, though they have practically been in existence in Glasgow since 1895, and in Liverpool since 1898.

It should not be forgotten that ice-cream may have deleterious effects on the consumer owing to its low temperature or to the presence of alkaloidal poisons of the nature of tyro-toxicon which have been detected in such substance as well as in milk (Mount Morgan outbreak) and cheese (Michigan and Finsbury outbreaks).

Ice contains bacteria in varying quantities, from 20 per c.c. to 10,000 or more. Nor is variation in number affected alone by the conditions of the water, for samples collected from one and the same place differ widely. The quality follows in large measure the standard of the water.

Water bacteria, B. coli, putrefactive and even pathogenic bacteria, have been found in ice. Many organisms can live without much difficulty, and are most numerous in ice containing air-bubbles.

* Report of Medical Officer of City of London, 1902, pp. 116-26.

+ Report on Health of Finsbury, 1902, p. 67. 
Dr Prudden, of New York, performed a series of experiments in 1887 to show the relative behaviour of bacteria in ice. Taking half a dozen species, he inoculated sterilised water and reduced it to a very low temperature for a hundred and three days, with the following results: $-B$. prodigiosus diminished from 6300 per c.c. to 3000 within the first four days, to 22 in thirty-seven days, and vanished altogether in fifty-one days; a liquefying water bacillus numbering 800,000 per c.c. at the commencement, had disappeared in four days; Staphylococcus pyogenes aureus and B. fluorescens showed large numbers present at the end of sixty-six and seventy-seven days respectively; B. typhosus, which was present 1,000,000 per c.c. after eleven days, fell to 72,000 after seventy-seven days, and 7000 at the end of one hundred and three days. - Anthrax bacilli are susceptible to freezing, but their spores are practically unaffected (Frankland). From these facts it will be seen that bacteria live, but do not multiply, in ice.

Hutchings and Wheeler recently examined some ice suspected of conveying typhoid fever at the St Lawrence State Hospital on the river St Lawrence. The fragments were melted in a clean vessel at room temperature, after which a considerable black sediment deposited itself in the vessel. Cultures and plates were made in the usual way, and B. coli and B. typhosus were both isolated.* The lastnamed had the following characters:-On nutrient agar it grew readily, in broth growth without pellicle, in lactose media no fermentation occurred, on potato the "invisible" growth, litmus milk became alkaline without coagulation, and the bacillus was morphologically identical with $B$. typhosus. With the serum of typhoid patients characteristic agglutination occurred. Blumer found the number of bacteria in some of the same ice was 30,400 per c.c. (agar) and 50,400 per c.c. (gelatine). Many colon bacilli were present.

Sedgwick and Winslow have also carefully studied the influence of natural and normal conditions of eold upon the typhoid bacillus in particular. The experiments were earried out with special reference to the danger of conveyance of the disease in question by polluted ice, and with reference to the seasonal distribution of the disease. The matter was undoubtedly one that called for investigation, and notably so in America where ice and iced drinks are in such universal demand.

The apparent purity of ice is deceptive. It is true that water in freezing undergoes a certain amount of purification. It loses, on conversion into ice, saline constituents, contained air, and a certain proportion of organic suspended matter. At the same time, it is not entirely freed from microbes. The figures quoted by Sedgwick and Winslow show that snow-ice may contain an average of more than 600 bacteria per cubic centimetre.

* American Jour. of Medical Sciences, Oct. 1903, p. 683. 
Laboratory. experiments have confirmed the conclusion that a freezing process is not necessarily fatal to bacterial life (see p. 18). We have instances of bacteria multiplying at zero, and of their survival after six months' exposure to the temperature of liquid air. It would appear that about 90 per cent. of the ordinary water bacteria are eliminated by the process of freezing. In the case of a specific pathogenic organism such as the $B$. typhosus, less than 1 per cent. survive simple freezing for a period of fourteen days. Complete sterility does not occur even at the end of three months, whilst a process of alternate thawing and freezing, if on the whole more fatal to the typhoid germs than a simple freezing, is equally unsuccessful in effecting an absolute sterilisation of the infected water. The reduction in the number of typhoid bacilli in chilled water is approximately as great as occurs in ice. Cold exercises an inhibitory action as regards the typhoid bacillus, and in natural ice there is a supplementary purifying influence to be taken into account, as, at the time of freezing, 90 per cent. of the germs are thrown out by a process of physical exclusion. Therefore, the danger of infection in the case of ice, if it is minimised, is not abolished. A certain number of typhoid bacilli do remain alive, and these may, on rethawing, undergo a rapid multiplication outside as well as inside the human body. And it has likewise to be remembered that it is notoriously difficult to trace the exact channels of infection in sporadic cases of typhoid fever. Sedgwick and Winslow have rightly drawn attention to the unfavourable conditions furnished by natural ice for the propagation of the typhoid organism.

In making a bacterial investigation into the flora of ice and icecream, it is necessary to remember that considerable dilution with sterilised water is required. The usual methods of examining water and milk are adopted.

\section{Bread}

Bread forms an excellent medium for moulds, but unless specially exposed the bacteria in it are few. Waldo and Walsh have, however, demonstrated that baking does not sterilise the interior of bread. These observers cultivated numerous bacteria from the centre of newly-baked London loaves.* The writer has recently made a series of examinations of the air of some nine or ten underground bakehouses in central London. The general result of these investigations was that the air of the typical underground bakehouses examined-(1) contained 14.8 volumes per 10,000 of carbonic acid gas, $\mathrm{CO}_{2}$ (as compared with 4.9 in above-ground bakehouses, and 4.3 in the street); (2) that it contained between 10 and

* Brit. Med. Jour., 1895, vol, ii., p. 519. 
24 per cent. less moisture than outside air surrounding the bakehouses; and (3) that it contained at least four times more bacteria than surrounding street air, and three times more bacteria than the air of a typical above-ground bakehouse.* (See also p. 86.)

The normal fermentation of bread with which all bakers are familiar is due to the energy of the yeast plant growing in the dough. Any other fermentation going on at the same time as the normal one, or arising after the bread has left the oven, must be looked upon as abnormal.

Flour or dough is open to infection by bacteria, and scrupulous cleanliness is absolutely necessary to avoid unfavourable fermentations. Bacteria are especially numerous in low-grade flours; in fact, the poorer the flour the larger the number of injurious organisms. Prescott has lately shown that flour may contain bacilli indistinguishable from the $B$. coli. This organism is more liable to be found in poor than in high-class flours.

1. Sour Bread.-The commonest abnormal fermentation of bread produces what is known as "sour bread," which means that the odour and flavour of the bread are "sour" to the senses of smell and taste. Lactic and butyric germs are commonly found in poor flours, where they remain in a dormant condition until provided with the essentials necessary for their growth-moisture, a sufficient temperature, and proper and adequate food supply. The food supply naturally surrounds them, and when water is added to the flour, and the temperature is raised to between $70^{\circ}$ and $90^{\circ} \mathrm{F}$., they are able to reproduce and rapidly manifest their presence by the products they form. Dough, with considerable moisture present, or, as it is termed, "slack," gives bacteria a better environment, and consequently sourness is more apt to increase rapidly in such doughs.

Acid-producing germs are also present in many samples of yeast. Analyses of a large number of yeast samples used for bread-making purposes, have shown that many of them contain injurious bacteria which may lessen the alcoholic fermentation. If, on the other hand, the normal alcoholic fermentation is at first vigorous, and then diminishes, it gives bacteria opportunity to grow. Hence, overproved dough is especially liable to become sour. Dirty utensils, tubs or troughs, harbour injurious bacteria which are able to reproduce when given favourable conditions. All cracks and crevices which harbour food are teeming with life, usually undesirable from the bakers' standpoint, and, therefore, absolute cleanliness should be the rule in every detail.

Acetic bacteria, which are often present in flours, sometimes cause trouble, and as these bacteria require a plentiful supply of oxygen, it has been suggested that all dough should be kept as much

* Special Report on Bakehouses in Finsbury, 1902. 
as possible out of contact with the air. It is doubtful if such a remedy is practical, as the lowering of the temperature follows the removal of covers on the dough troughs and retards the whole course of fermentation.

2. Sticky, Slimy, or Viscous Bread.-This affection is not nearly as common as the preceding, yet the number of cases recorded is quite large, and this abnormal fermentation is frequently met with in country districts. As the name implies, the bread, usually the crumb near the centre of the loaf, is slimy or sticky. The stringiness increases with age, a proof of the living nature of the trouble. Cases of sticky bread usually occur in the warm summer months, the high temperature favouring the growth of the bacteria which produce the trouble. From this sticky bread it is comparatively easy to isolate an organism which, when placed in sterilised bread, is able to produce the stickiness met with under natural conditions, thus proving the relation of bacteria to the trouble. The specific germ causing stickiness, known as the "potato bacillus" on account of the frequency with which it is met with on potatoes, is also formed in yeast cake. Harrison has repeatedly found this germ present in both dried and compressed yeast cake. Given favourable conditions for rapid growth, this organism might produce epidemics of slimy bread at any time. The bacillus forms spores able to resist unfavourable conditions. This germ is occasionally met with in milk. Slimy bread may be controlled by exercising absolute cleanliness in the yeast tubs and kneading troughs, and by the proper sterilisation of the brew or ferment by the use of a certain quantity of hops. In a number of experiments made with hop extracts it has been found that even a small quantity of good hops (one half-ounce to the gallon) has some antiseptic power and hinders the development of the potato bacillus, without injuring the activity of the yeast. The bread should be kept in a cool place after baking, for this stickiness is most prevalent during hot weather, and a cool temperature prevents the rapid growth of the organism.

3. Musty or Mouldy Bread.-Musty or mouldy bread is, as a rule, only met with after the bread has been cut and allowed to stand several days. Occasionally, however, we find bread only one day old affected with mustiness. The specific organism is the mould Mucor mucedo, which has action on bread, producing a musty odour without decomposing the bread. But the chemical composition of the bread is changed by the growth of mould, and this change favours the subsequent growth of any bacteria that may be present. Flours which have become damp, or even very low-grade flours, may have this mould present in large amount, and although the organism is killed by the baking process, yet the musty flavour persists and is present in the baked loaf. 
4. Red or "Bloody" Bread.-Bloody, or red bread, is not an affection which often troubles bakers, but it sometimes makes its appearance in the household. The microbe which produces this affection is of great historical interest. Livy refers to its occurrence in the Roman army, and it is said to have appeared during the siege of Troy. There are various records of its occurrence in England during the Middle Ages, and early in the nineteenth century a large quantity of red spotted bread occurred in the province of Padua, in North Italy. It is possibly due to B. prodigiosus or other similar chromogenic organism, and is traceable to contamination of the bread.

\section{Miscellaneous Foods}

Watercress has frequently been found to be the vehicle of bacteria if grown in polluted water. There are several instances on record where the consumption of such contaminated watereress has caused disease. In June and July 1903 an outbreak of enteric fever occurred in Hackney, in N.E. London, in which there were 110 cases of the disease, of whom 55.5 per cent. had consumed watercress which was shown to have been grown in polluted water. The latter contained $50 \mathrm{~B}$. coli $\mathrm{per}$ c.c., and the cresses themselves were markedly contaminated with sewage organisms of intestinal type. Altogether, 17 samples of watercress were examined, and every one of them revealed the bacteria of sewage. This was a fairly clear case of conveyance of enteric infection, as (1) the excess of enteric fever corresponded with the season for watercress, viz., June to September; (2) the excess of cases of enteric fever was amongst watercress eaters, viz., 55 per cent. for the whole period; (3) watercress eaters suffered more than three times as much as non-watercress eaters, who constituted only 27.5 per cent. of the entire population; (4) samples of watercress, taken from the places where infected persons market, were found on bacteriological examination, to be sewagepolluted; and, (5) a large proportion of the polluted samples were found to be cultivated in beds fed by almost undiluted sewage.*

Other foods and beverages (including aërated waters) have from time to time been contaminated with bacteria to the injury of the consumer, but the above represent the chief foods infected. Sausages have frequently been found to be contaminated. In Liverpool, Boyce found $B$. coli present in all 17 samples examined, and $B$. enteritidis sporogenes in 2 out of 17 . Pork pies, tinned meats and pastes, chicken, jellies, etc., have been shown to harbour injurious organisms. $\dagger$

* Report on Outbreak of Enteric Fever at Hackney, 1903 (Dr King Warry).

$\uparrow$ Report on Health of Liverpool, 1902, p. 172. 


\section{CHAPTER IX}

\section{BACTERIA AND DISEASE}

Growth of Knowledge of Bacteria as Disease Producers-Channels of InfectionHow Bacteria cause Disease-Diphtheria: Conditions of Infection-Scarlet Fever, Typhoid Fever, Epidemic Diarrhœa: Conditions of InfectionSuppuration and Abscess Formation-Anthrax-Pneumonia-InfluenzaActinomycosis-Glanders.

Probably the most universally known fact respecting bacteria is that they are related in some way to the production of disease. Yet we have seen that it was not as disease-produeing agents that they were first studied. Indeed, it is only within comparatively the latest period of the two centuries during which they have been more or less under observation that our knowledge of them as causes of disease has assumed any exactitude or general reeognition. Nor is this surprising, for although an intimate relationship between fermentation and disease had been hinted at in the middle of the seventeenth century, it was not till the time of Pasteur that the bacterial cause of fermentation was experimentally, and finally, established.

In the middle of the seventeenth century men learned, through the eyes of Leeuwenhoek, that drops of water contained "moving animaleules." A hundred years later Spallanzani demonstrated the fact that decomposition and fermentation were set up in boiled vegetable infusions when outside air was admitted, but when it was withheld from these boiled infusions no such change oecurred. Almost a hundred years more passed before the epoch-making work of Tyndall and Pasteur, who separated these putrefactive germs from the air. Quickly following in their footsteps came Davaine 
and Pollender, who found in the blood of animals suffering from anthrax the now well-known specific bacillus of that disease. Improvements in the microscope and in methods of cultivation (Koch's plate method in particular) soon brought an army of zealous investigators into the field, and during the last thirty years one disease after another has been traced to a bacterial origin. We may summarise the vast collection of historical, physiological, and pathological research extending from 1650 to 1904 in three great periods : The period of detection of living, moving cells (Leeuwenhoek and others in the seventeenth century); the period of the discovery of their close relationship to fermentation and putrefaction (Spallanzani, Schulze, Schwann, in the eighteenth century); and, thirdly, the period of appreciation of the rôle of bacteria in the economy of nature and in the production of disease (Tyndall, Pasteur, Lister, Koch, in the nineteenth).

But we must look less cursorily at the growth of the idea of bacteria as the cause of disease. More than two hundred years ago Robert Boyle (1627-91), the philosopher who did so much towards the foundation of the present Royal Society, wrote an elaborate treatise on The Pathological Part of Physic. He was one of the earliest scientists to declare that a relationship existed between fermentation and disease. When more accurate knowledge was attained respecting fermentation, great advance was consequently made in the etiology of disease. The preliminary discoveries of Fuchs and others between 1840 and 1850 had relation to the existence in diseased tissues of a large number of bacteria. But this was no proof that these germs caused disease. It was not till Davaine had inoculated healthy animals with bacilli from the blood of an anthrax carcase, and had thus reproduced the disease, that reliance could be placed upon that bacillus as the vera causa of anthrax. Too much emphasis cannot be laid upon the idea, that unless a certain organism produces in healthy tissues the disease in question, it cannot be considered as proven that the particular organism is related to the disease as cause to effect. In order to secure a standard by which all investigators should test their results, Koch introduced four postulates. Until each of the four has been fulfilled, the final conclusion respecting the causal agent in any bacterial disease must be considered sub judice. The postulates are as follows:-

(a) The organism must be demonstrated in the circulation or tissues of the diseased animals.

(b) The organism thus demonstrated must be cultivated in artificial media outside the body, and successive generations of a pure culture of that organism must be obtained.

(c) Such pure cultures must, when introduced into a healthy and susceptible animal, produce the specific disease. 
(d) The organism must be found and isolated from the circulation or tissues of the inoculated animal.

It is evident that there are some diseases-for example, cholera, leprosy, and typhoid fever-which are not communicable to lower animals, and therefore their virus cannot be made to fulfil postulate (c). In such cases there is no choice. They cannot be classified along with tubercle and anthrax. Bacteriologists have little doubt that Hansen's bacillus of leprosy is the cause of that disease, yet it has not fulfilled postulates $(b)$ and $(c)$. Nor has the generally accepted bacillus of typhoid fever fulfilled postulate $(c)$, yet by the majority it is provisionally accepted as the agent in producing the disease. Hence it will be seen that, though there is an academical classification of causal pathogenic bacteria according as they respond to Koch's postulates, yet nevertheless there are a number of pathogenic bacteria which are looked upon as causes of disease provisionally. The bacilli of anthrax and tubercle, with perhaps the organisms of suppuration, tetanus, plague, and actinomycosis, stand in the first order of pathogenic germs. Then comes a group awaiting further confirmation, which includes the organisms related to typhoid fever, cholera, malaria, leprosy, epidemic diarrhœa, and pneumonia. Then comes in a third category, a long list of diseases, such as scarlet fever, small-pox, measles, rabies, and others too numerous to mention, in which the nature of the causal agent is still unknown. Hence it must not be supposed that every disease has its germ, and without a germ there is no disease. Such universal assertions, though not uncommonly heard, are devoid of accuracy.

In the production of bacterial disease there are two factors. First, there is the body tissue of the individual; secondly, there is the specific organism.

Whatever may be said hereinafter with regard to the power of micro-organisms to cause disease, we must understand one cardinal point, namely, that bacteria are never more than causes, for the nature of disease depends upon the behaviour of the organs or tissues with which the bacteria or their products meet (Virchow). Fortunately for a clear conception of what "organs and tissues" mean, these have been reduced to a common denominator, the cell. Every living organism, of whatever size or kind, and every organ and tissue in that living organism, contains and consists of cells. Further, these cells are composed of organic chemical substances which are not themselves alive, but the mechanical arrangement of which determines the direction and power of their organic activity and of their resistance to the specific agents of disease. With these facts clearly before us, we may hope to gain some insight into the reasons for departure from health.

The normal living tissues have an inimical effect upon bacteria. 
Saprophytic bacteria of various kinds are normally present on exposed surfaces of skin or mucous membrane. Tissues, also, which are dead or depressed in vitality from injury or previous disease, but which are still in contact with the living body, afford an excellent nidus for the growth of bacteria. Still these have not the power, unless specific, to thrive in the normal living tissue. It has been definitely shown that the natural fluids of the body have in their fresh state protective substances (alexines) which prevent bacteria from flourishing in these tissues. Such protection depends in measure upon the number of invading germs as well as their quality, for the killing power of blood and lymph must be limited. Buchner has pointed out that the antagonistic action of these fluids depends in part possibly upon phagocytosis, but largely upon a chemical condition of the serum. The blood, then, is no friend to intruding bacteria. Its efforts are to a certain extent seconded by the lymphoid tissue throughout the body. Rings of lymphoid tissue surround the oral openings of the trachea and cesophagus, and the tonsils are masses of lymphoid tissue. Composed as it is of cells having a germicidal influence when in health, the lymphoid tissue may afford formidable obstruction to invading germs.

All the foregoing points in one direction, namely, that if the tissues are maintained in sound health, they form a very resistant barrier against disease-producing germs. But we know from experience that a full measure of health is not often the happy condition of human tissues. There are a variety of circumstances which predispose the individual to disease. One of the commonest forms of predisposition is that due to heredity. Probably it is true that what are known as "hereditary diseases" are due far more to a hereditary predisposition than to any transmission of the virus itself in any form. Again, antecedent disease predisposes the tissues to form a nidus for bacteria, and conditions of environment or personal habits act powerfully in the same way. Damp soils must be held responsible for many disasters to health, not directly, but indirectly, by predisposition; dirty houses and insanitary houses, dusty trades and injurious occupations, have a similar effect. Any one of these different influences may in a variety of ways affect the tissues and increase their susceptibility to disease. Not infrequently we may get them combined. For example, the following is not an unlikely series of events terminating in consumption (tuberculosis of the lungs):-(a) The individual is predisposed by inheritance to tuberculosis; (b) an ordinary chronic catarrh, which lowers the resisting power of the lungs, may be contracted; $(c)$ the epithelial collections in the air vesicles of the lung-i.e. dead matter attached to the body-afford an excellent nidus for bacteria; $(d)$ owing to occupation, or personal habits, or surroundings, the patient comes 
within a range of tubercular infection, and the specific bacilli of tubercle gain access to the lungs. The result will be a case of consumption more or less acute according to environment and treatment.

\section{Channels of Infection}

The channels of infection by which organisms gain the vantageground afforded by the depressed tissues are various, and next to the maintenance of resistant tissues they call for most attention from the physician and surgeon. It is in this field of preventive medicine-that is to say, preventing infective matter from entering the tissues at all-that science has triumphed in recent years. It is, in short, applied bacteriology.

1. Pure Heredity.-This term is to be understood in this connection as concerned with actual transmission of germs of disease from the mother to the child in utero. That such conveyance may occur is admitted, but it is certainly not frequent, nor is bacterial disease widely spread by this means. The transmission of tendency (diathesis) is, of course, another matter, and there can be little doubt that antenatal conditions exert an influence on bacterial diseases of infancy.

2. Inoculation, or inserting virus directly through a broken surface of skin, is a method of producing diseases in animals commonly used in experimental work. Such inoculations may be subcutaneous, intravenous, intracerebral, intraperitoneal, etc. In the natural production of disease, inoculation is also a not uncommon channel of infection. Injuries of the skin caused by instruments, gunshot wounds, broken glass or china, etc., may serve as the point of introduction of specific virus. Tetanus is commonly an inoculated disease. Malaria must now also be so considered. Local tuberculosis is not infrequently produced by inoculation through a broken skin surface.

3. Contagion indicates that a disease is transmitted by personal contact, through unbroken skin surfaces. Small-pox, measles, ringworm, and other diseases may be thus contracted. It is not unlikely that as our knowledge grows, the diseases to be defined as spread by contagion will become less.

4. The Alimentary System.-Many diseases are spread by the consumption of infected food or water, and in children the sucking of dirty objects may introduce germs of disease into the alimentary canal. Milk, cream, butter, cheese, ice-cream, oysters, shell-fish, meats of various kinds, vegetables, water-cress, ice, and a large variety of foods, have been the means of introducing pathogenic organisms into the body, and in this way enteric fever, cholera, dysentery, and a large number of acute and chronic diseases are originated. Waterborne disease furnishes a large percentage of such cases.

5. The Respiratory 'Tract.-The air may become infected with 
pathogenic organisms, which may be inhaled, and thus gain entrance to the body and set up disease. Diphtheria and pulmonary tuberculosis are two examples. In this channel of infection pathogenic bacteria must, as a rule, be present in large numbers, or must meet with devitalised and non-resisting tissues, to set up disease.

These, then, are the five possible ways in which germs gain access to the body tissues. The question now arises, How do bacteria, having obtained entrance, set up the process of disease? For a long time pathologists looked upon the action of these microscopic parasites in the body as similar to, if not identical with, the larger parasites sometimes infesting the human body. Their work was viewed as a devouring of the tissues of the body. Now it is well known that, however much or little of this may be done, the specific action of pathogenic bacteria is of a different nature. It is twofold. We have the action of the bacteria themselves, and also of their products or toxins. In particular diseases, now one and now the other property comes to the front. In bacterial diseases affecting or being transmitted mostly by the blood, it is the toxins which act chiefly. The convenient term infection is applied to those conditions in which there has been a multiplication of living organisms after they have entered the body, the word intoxication indicating a condition of poisoning brought about by their products. It will be apparent at once that we may have both these conditions present, the former before the latter, and the latter following as a direct effect of the former. Until intoxication occurs, there may be few or no symptoms; but directly enough bacteria are present to produce in the body certain poisons in sufficient amount to result in more or less marked tissue change, then the symptoms of that tissue change appear. This period of latency between infection and the appearance of the disease is known as the incubation period. Take typhoid, for example. A man drinks a typhoid-polluted water. For about fourteen days the bacilli are making headway in his body without his being aware of it. But at the end of that incubation period the signs of the disease assert themselves. Professor Watson Cheyne and others have maintained that there is some exact proportion between the number of bacteria gaining entrance and the length of the incubation period.

Speaking generally, we may note that pathogenic bacteria divide themselves into two groups: those which, on entering the body, pass at once, by the lymph or blood-stream, to all parts of the body, and become more and more diffused throughout the blood and tissues, although in some cases they settle down in some spot remote from the point of entrance, and produce their chief lesions there. Tubercle and anthrax would be types of this group. On the other hand, there is a second group, which remain almost 
absolutely local, producing only little reaction around them, rarely passing through the body generally, and yet influencing the whole body eventually by means of their ferments or toxins. Of such, the best representatives are tetanus and diphtheria. The local site of the bacteria is, in this case, the local factory of the disease.

Whilst the mere bodily presence of bacteria may have mechanical influence injurious to the tissues (as in the small peripheral capillaries in anthrax), or may in some way act as a foreign body and be a focus of inflammation (as in tubercle), the real diseaseproducing action of pathogenic bacteria depends upon the chemical poisons (toxins) formed directly or indirectly by them. Though within recent years a great deal of knowledge has been acquired about the formation of these bodies, their exact nature is not at present known. They are allied to the proteoses, and are frequently described as tox-albumens. It may be found, after all, that they are not of a proteid nature. Sidney Martin has pointed out that there is much that is analogous between the production of toxins and the production of the final bodies of digestion. Just as ferments are necessary in the intestine to bring about a change in the food by which the non-soluble albumens shall be made into soluble peptones, and thus become absorbed through the intestinal wall, so also a ferment may be necessary to the production of toxins. Such ferments have not as yet been isolated, but their existence in diphtheria and tetanus is, as we have seen, extremely likely. However that may be, it is now more or less established that there are two kinds of toxic bodies, differing from each other in their resistance to heat. It may be that the one most easily destroyed by heat is a ferment and possibly an originator of the other. A second division which has been suggested for toxic bodies, and to which reference will be made, is intracellular and extracellular, according to whether or not the poison exists within or without the body of the bacillus.

Lastly, we may turn to consider the action of the toxins on the individual in whose body-fluids they are formed. It is hardly necessary to say that any action which bacteria or toxins may have will depend upon their virulence, in some measure upon their number, and not a little upon the channel of infection by which they have gained entrance. It could not be otherwise. If the virulence is attenuated, or if the invasion very limited in numbers, it stands to reason that the pathogenic effects will be correspondingly small or absent. The influence of the toxins is twofold. In the first place, (i.) they act locally upon the tissues at the site of their formation, or at distant points by absorption. There is inflammation with marked cell-proliferation, and this is, more or less rapidly, followed by a specific cell-poisoning. The former change may be 
accompanied by exudation, and simulate the early stages of abscess formation; the latter is the specific effect, and results, as in leprosy and tubercle, in infective nodules. The site in some diseases, like typhoid (intestinal ulceration, splenic and mesenteric change) or diphtheria (membrane in the throat), may be definite and always the same. But, on the other hand, the site may depend upon the point of entrance, as in tetanus. The distant effects of the toxin are due to absorption, but what controls its action it is impossible to say. We only know that we do find pathological conditions in certain organs at a distance from the site of disease, and without the presence of bacteria. We have a parallel in the action of drugs; for example, a drug may be given by the mouth, and yet produce a rash in some distant part of the body. In the second place, (ii.) toxins produce toxic symptoms. Fever and many of the nervous conditions resulting from bacterial action must thus be classified. We have, it is true, the physical signs of the pathological tissue change, for example, the large spleen of anthrax or the obstruction from diphtheritic membrane. But, in addition to these, we have general symptoms, as fever, in which after death no tissue change can be found.

We may now consider briefly some of the more important forms of disease produced by bacteria.*

\section{Diphtheria}

Diphtheria is an infective disease characterised by a variety of clinical symptoms, including a severe inflammation usually followed by a fibrinous infiltration (constituting a membrane) of certain parts. The membrane ultimately breaks down. The parts affected are the mucous membrane of the fauces, larynx, pharynx, trachea, and sometimes wounds, or the inner wall of the stomach. Diphtheritic conjunctivitis may also occur. The common sign of the disease is the membrane in the throat; but muscle

* Bacterial diseases may be classified as follows :-

(1) Diseases common to man and certain animals, and presumably transmissible from animals to man, and vice versâ, e.g. bubonic plague and tuberculosis.

(2) Diseases common to man and animals, but not known to be directly transmissible, e.g. actinomycosis, tetanus. Diphtheria, belongs to this class, or Group (1) or (5).

(3) Diseases transmitted from animals to man, but not as a rule communicated from man to man owing to interfering conditions, e.g. anthrax, glanders, rabies, vaccinia, foot-and-mouth disease, meat-poisoning, psittacosis, and possibly infections due to pus bacteria.

(4) Certain specific symbiotic relations requiring two hosts for the complete cycle of life of the micro-organisms, e.g. malaria, trichinosis, tape-worm infection.

(5) Diseases occurring in man, but not, as far as known, in animals, e.g. typhoid fever, gonorrhœa, leprosy. 
weakness, syncope, albuminuria, post-diphtheritic paralyses, convulsions, and many other symptoms guide the physician in diagnosis and the course of the disease. It begins as a local disease, and the greyish-white membranous deposit, already referred to, is produced. The toxins or poisons resulting from the growth and multiplication of the bacillus are absorbed into the blood stream, and general symptoms follow. The incubation period is from two to seven days.

Although diphtheria owes its name to the false membrane seen in the throats of typical cases, it is now almost universally recognised that in many cases of undoubted diphtheria no membrane is formed. The occurrence of a nasal form of diphtheria has, too, in recent years been recognised, and as such cases are not easily recognisable without a bacteriological examination, they are very liable to remain undiagnosed and be left free to spread the infection.

The fons et origo of the disease is the specific bacillus. Without the presence of that organism it is not possible to have diphtheria. Yet that organism may exist in the healthy throat without producing the recognised clinical symptoms of diphtheria. It may be conveyed to the human throat in a variety of ways, for example, by kissing and other forms of contact, or by drinking milk and other contaminated foods. In a perfectly healthy throat it may do no mischief. But in a sore throat or in the throat of a weakly person, it might readily set up severe and even fatal disease. Anything, therefore, which tends to lower the vitality of the individual may play an important part in propagating diphtheria, and must be as carefully considered as any agency which might directly or indirectly introduce the bacillus to the human throat. Some epidemics have been due to school influence; other epidemics have been brought about through an infected milk supply; and yet other outbreaks are due to the introduction of a case of diphtheria into a susceptible community, weakened by insanitary surroundings or the prevalence of previous sore throat.

Further, there is reason to suppose that Bacillus diphtherioe may retain its virulence-and possibly spend a stage of its cycle of existence as a saprophyte-in the soil, in dust, and even in the throat for months. Three or four weeks is the average length of time for its presence in the throat, but, as a matter of fact, all the conditions in the throat-mucous membrane, blood-heat, moisture, and air-are extremely favourable to the bacillus, and it may linger there far beyond the time of disappearance of clinical symptoms of the disease.

The Bacillus diphtherio was isolated from the many bacteria found in the membrane by Löffler (1884). Klebs had previously identified the bacillus as the cause of the disease (1883). It is a 


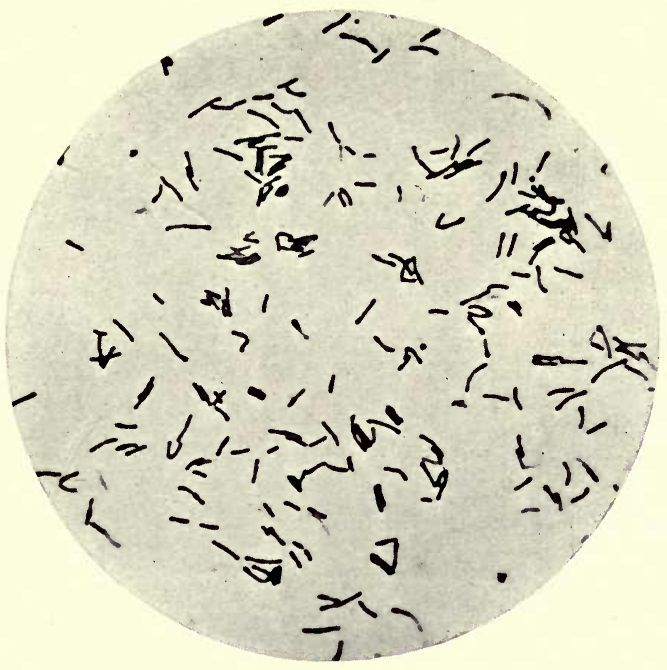

Bacillus diphtherice.

Film preparation from serum culture, 24 hours at $37^{\circ} \mathrm{C}$. $\times 1000$.

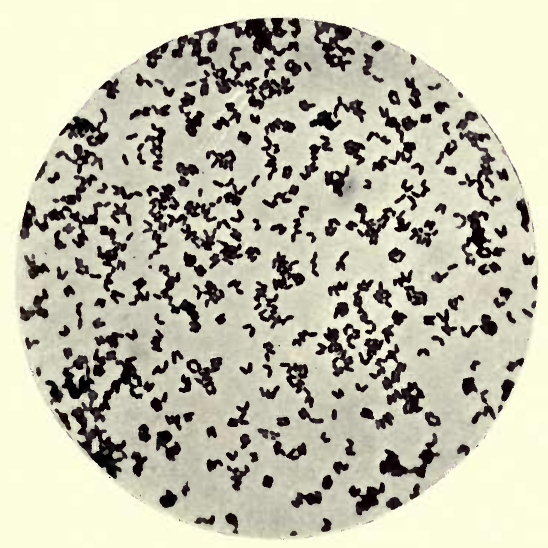

Bacillus von Hofmann.

Film preparation from serum culture, 48 hours at $37^{\circ} \mathrm{C}$. Roux's stain. $\times 1000$. 

slender rod, straight or slightly curved, and remarkable for its bearled appearance; there are also irregular and club-shaped forms. It differs in size according to its culture medium, but is generally 3 or $4 \mu$ in length. Cobbett and Graham-Smith recognise five morphological types of diphtheria bacilli on young serum cultures: -(1) Oval bacilli, with one unstained septum; (2) long, faintly-stained, irregularly-beaded bacilli; (3) long, regularlybeaded bacilli-“streptococcal" forms; (4) segmented bacilli; and (5) uniformlystained bacilli. All these forms are longer than the pseudo-diphtheria bacilli, more curved, and generally with clubbed ends. Their arrangement to each other

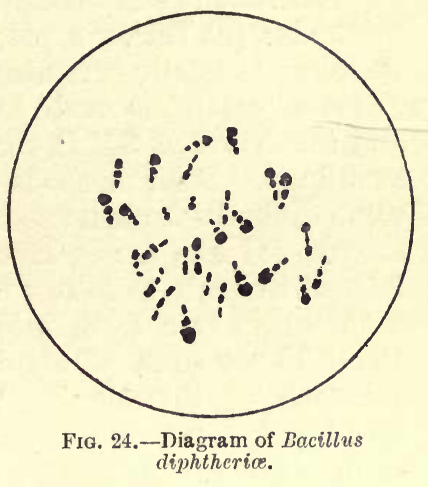
is generally likened to Chinese characters. In the membrane which is its strictly local habitat in the body-indeed, with the exception of the secretions of the pharynx and larynx, and occasionally in lymph-glands and the spleen, the bacillus is found practically nowhere else in the body-it sometimes shows parallel grouping, lies on the surface of the exudation, and is separated from the mucous membrane by the fibrin. It is mixed with other organisms, which are performing their part in complicating the disease, or are normal inhabitants of the mouth. The bacillus possesses five negative characters: namely, it has no spores, no threads, and no power of motility; it does not liquefy gelatine; nor does it produce gas. It is stained with Löffler's methylene blue, and shows metachromatic granules and polar staining. It is generally best to use the stain in dilute form. The favourable temperature is blood-heat, though it will grow at room temperature. It is aërobic, and, indeed, prefers a current of air. Löffler contrived a medium for cultivation which has proved most successful. It is made by mixing three parts of ox-blood serum with one part of broth containing 1 per cent. of glucose, 1 per cent. of peptone, and $\frac{1}{2}$ per cent. of common salt; the whole is coagulated. Upon this medium the Klebs-Löffler bacillus grows rapidly in eighteen or twenty hours, producing scattered, "nucleated," round, white colonies, becoming yellowish. Horse serum is used by some bacteriologists instead of ox serum. Lorrain Smith and Marmorek also devised excellent serum media. The bacillus grows well in broth, but without producing either a pellicle or turbidity; it can grow on the ordinary media, though its growth on potato is not readily visible; on the white of egg it flourishes extremely well. In a moist condition, as a rule, the bacillus has a low degree of 
resistance, but when in a dry condition it can survive for long periods.

The bacillus is secured for diagnostic purposes by one of two methods: (a) Either a piece of the membrane is detached, and after washing carefully examined by culture as well as the microscope; or (b) a "swab" is made from the infected throat, cultured on serum, and incubated at $37^{\circ} \mathrm{C}$. for eighteen hours, and then microscopically examined. Both methods-and there is no further choice-present some difficulties owing to the large number of bacteria found in the throat. Hence a negative result must be accepted with reserve. Indeed the rule to follow is three examinations before deciding that a throat is free from infectivity, and it should be remembered that about 20 per cent. of all cases of diphtheria offer no bacteriological evidence of infection.* It therefore comes back to the point of broad judgment and common-sense. The clinical condition is the main fact for guidance, and the bacteriological must not usurp it. $†$

Locally, the bacillus produces inflammatory change with fibrinous exudation and some cellular necrosis. In the membrane a ferment is probably produced which, unlike the localised bacilli, passes throughout the body, and by digestion of the proteids, produces albumoses and an organic acid which have the toxic influence. The toxins act on the blood-vessels, the nerves, the muscle fibres of the heart (hyaline degeneration and even fatty changes), and many of the more highly specialised cells of the body. Thus we get degenerative changes in the kidney (cloudy swelling, and, clinically, albuminuria) in cells of the central nervous system, in the peripheral nerves (post-diphtheritic paralysis), and elsewhere, these pathological conditions setting up, in addition to the membrane, the symptoms of the disease. The bacillus is pathogenic for the horse, ox, rabbit, guineapig, cat, and some birds. Cases are on record of supposed infection of children by cats suffering from the disease. Roux and Yersin in 1888-89 showed that the diphtheria bacillus is capable of producing the various phenomena associated with the disease, including the formation of false membrane and diphtheritic paralysis. They also succeeded in separating and studying the toxin, which they found to be capable of producing all the effects produced by the bacillus. In 1890 appeared the great work by Behring, to which reference will be made subsequently; and the observations in regard to diphtheria made in that work were extended and strengthened in a paper by Behring and Wernicke in 1892. At the Medical Congress at Buda-Pesth in 1894, Roux read a paper on the treatment of diphtheria by diphtheria antitoxin, which first proved to the medical

* Jour. of Hygiene, 1903, p. 217.

† See also Brit. Med. Jour., 1900, ii., p. 907 (Andrewes). 
world that this was the one method of successfully combating the disease. The experimental and clinical data, and the favourable statistics brought forward by Roux, at once put this method in a secure position from the practical standpoint.*

\section{Conditions Favourable to the Spread of Diphtheria.-} Sir Richard Thorne Thorne held that soil, and especially surface soil, when considered in comnection with relative altitude, slope, aspect, and prevailing winds, plays a part in the maintenance and diffusion of diphtheria, and possibly has relation with its beginnings. $\mathrm{He}$ believed that where a surface soil retained moisture and organic refuse, and where buildings founded on such soil were exposed to cold wet winds, there you had conditions likely to foster diphtheria. $\dagger$ Dr Newsholme of Brighton considers that such conditions act as "vital depressants," favouring sore throat and catarrh, and thus preparing the way for the inroads of the diphtheria bacillus. $\mathrm{He}$ concludes as the result of extended investigations that "diphtheria only becomes epidemic in years in which the rainfall is deficient, and the epidemics are on the largest scale when three or more years of deficient rainfall immediately follow each other. Occasionally, dry years are unassociated with epidemic diphtheria, though usually in these instances there is evidence of some rise in the curve of diphtheritic death-rate. Conversely, diphtheria is nearly always at a very low ebb during years of excessive rainfall, and is only epidemic during such years when the disease in the immediately preceding dry years has obtained a firm hold of the community and continues to spread presumably by personal infection." $\neq$ Newsholme thinks the specific micro-organism has a double cycle of existence: one phase passed in the soil, saprophytic; another in the human organism, parasitic.

Insanitary surroundings necessarily act prejudicially. Damp and ill-constructed houses and bad drainage, have undoubtedly played a part, and that not a small part, in diphtheria outbreaks. The position of many houses must inevitably lead to dampness; there is also the dampness arising from undrained and unpaved curtilages; and lastly, there is damp and steamy atmosphere. Now these conditions cannot but affect the health of children and give rise to sore throats and similar complaints. When to this dampness of houses is added the pollution of the soil, the undrained condition of towns, and the nuisances readily arising from ash-pits, cess-pits, and similar methods of refuse removal in close proximity to the houses, there is

* See also Brit. Med. Jour., 1900, ii., pp. 658-62 (Marsden) ; Metropolitan Asylums Board Reports, 1869-1902. See also pp. 425-431 of present volume.

$\dagger$ The Natural History of Diphtheria, p. 17.

‡ Epidemic Diphtheria (Newsholme), p. 157. 
ample ground for concluding that the sanitary circumstances of a town may be such as to depress the physical vitality of children, and lessen their powers of resistance to infectious disease once introduced among them. Thus the insanitary conditions named weaken the physique of the children, as well as preparing favourable external circumstances for the growth and multiplication of the germs of disease. Hence it must come about that from time to time a disease like diphtheria will take on an increased virulence as well as a higher measure of epidemicity.

But general conditions do not wholly account for the occurrence of diphtheria. Apart from these general conditions personal infection is the chief means by which diphtheria is spread.

Infection has been proved to be conveyed by nasal discharge of infected persons, or by kissing infected persons, or by sucking sweets, pencils, pens, slates, and other articles in schools. School influence as an agency in the dissemination of diphtheria was shown as far back as 1876 by Mr W. H. Power, and since that date abundant confirmatory evidence has been forthcoming. In $1894 \mathrm{Mr}$ Shirley Murphy, medical officer to the London County Council, reported that there had been an increase in diphtheria mortality in London at school ages (three to ten) as compared with other ages since the Elementary Education Act became operative in 1871; that the increased mortality from diphtheria in populous districts, as compared with rural districts, since 1871 , might be due to the greater effect of the Education Act in the former; and that there was a diminution of diphtheria in London during the summer holidays at the schools in 1893, but that 1892 did not show any marked changes for August. In 1896 Professor W. R. Smith, the medical officer to the London School Board, furnished a report* on this same subject of school influence, in which he produced evidence to show that the recrudescence of the disease in 1881-90 was greatest in England and Wales at the age of two to three years, and in London at the age of one to two years, in both cases before school age; that age as an absolute factor in the incidence of the disease is enormously more active than any school influence, and that personal contact is another important source of infection.

Although it is said that "statistics can be made to prove anything," there can be little doubt that both of these reports contain a great deal of truth; nor are these truths wholly incompatible with each other. They both emphasise age as a great factor in the incidence of the disease, and whatever affects the health of the child, population, like schools, must play, directly or indirectly, a not unimportant part in the transmission of the disease.

* Jour. of State Metl., 1896, vol. iv., p. 169; see also L.C.C. Education Report, 1904, No. 718. 
Infection can also be conveyed by means of milk (see p. 211). Clothing and other articles which have been in contact with infected persons may carry the bacillus. Birds and cats also have been, as far as can be judged, channels of infection (Klein, Bruce Low, and others). But there is from a bacteriological point of view another body of facts altogether which affect the spread of the disease, namely, the behaviour of the bacillus in the throat.

\section{The Diphtheria Bacillus in the Human Throat.-Since 1896} it has been known that the diphtheria bacillus may remain for long periods in the throat.

"The persistence of the diphtheria bacillus for periods up to eight weeks is of very common occurrence whether antitoxin be given or not; indeed, the majority of cases appear to retain bacilli in the throat for from two to nine weeks." * After the ninth week, the number falls off very rapidly, but not infrequently the bacillus remains in the throat for 100 days, and it has been known to remain more than 200 days. This persistence of diphtheria bacilli in the throat may play an important part in determining the spread of the disease by means of such cases which are supposed to be no longer infective. "For it is now a matter of common experience that so long as these diphtheria bacilli, even the less virulent forms, remain in the crypts of the tonsils, etc., so long is the patient a centre of infection, the diphtheria bacilli present resuming, under favourable conditions, their more virulent form" (Woodhead). In this way diphtheria bacilli can be readily transmitted by patients who are apparently no longer suffering from the effects of the disease, to those who have weak or ulcerated throats. In precisely a similar manner, may the bacillus be conveyed to articles of attire and articles of food, such as milk (as at Leeds in 1903).

Further, whilst in 72 per cent. of notified definite cases of diphtheria the bacillus may be found, it has been shown that in apparently healthy persons who have not suffered from diphtheria, the $B$. diphtherioe may be present. Löffler found diphtheria bacilli in the throats of 4 out of 160 healthy children, and Park and Beebe found similar virulent bacilli in 8 out of 330 "healthy" throats. Hewlett and Murray found 15 per cent. of the children in a general hospital had diphtheria bacilli in their throats. $\dagger$ Kober $\ddagger$ examined diphtheria cultures from two series of healthy persons. The first series comprised 128 individuals known to have been in recent contact with patients suffering from diphtheria. The diphtheria bacillus was

* Report on the Bacteriological Diagnosis and Antitoxic Serum Treatment of Cases admitted to the Hospitals of the Metropolitan Asylums Board, 1895-96, by Professor Sims Woodhead, sect. 2, 1902, pp. 14, 28, 31.

$\dagger$ Brit. Med. Jour., 1901, vol. i., p. 1474 .

¥ Revue des Maladies de l'Enfance, Juillet, 1900. 
found in the throat of 8 per cent. of these. The second series comprised 600 individuals who had not recently come into contact with any diphtheria cases - from 5 of these a bacillus similar to diphtheria was isolated. It was rather short, with swollen ends, and was not pathogenic to guinea-pigs. Denny of Brooklyn examined 235 healthy persons, and found the diphtheria bacillus in one case. Biggs met with 32 cases out of 330 healthy persons, and the Committee of the Massachusetts Board of Health reported that 1-2 per cent. of healthy persons amongst the general public are infected with diphtheria bacilli. Only 17 per cent. of such bacilli appear, however, to be virulent. Goadby examined 100 healthy school children, and found 18 with diphtheria bacilli, but the disease was prevalent in the neighbourhood.*

It is certain that, as a rule, "healthy" throats do not yield the true $B$. diphtherice unless those examined have been in contact with infected persons. But that raises the real difficulty in practical public health work. The definitely diseased and the definitely healthy persons can be arranged for. It is the apparently healthy person, who coming into contact with the infected person acts as a "carrier" of infection, that creates the problem. The actual percentage of such persons varies widely. In infected families it may be 50 per cent. (Park and Beebe), or 100 per cent. (Goadby). In schools and institutions the percentage is, of course, lower. Goadby found it to be 34 per cent. in the Poplar Union Schools, but it may be as low as 7 (Thomas). Aaser found that 19 per cent. of the "contacts" in a soldiers' barracks contained the bacillus in their throats, and Denny found in a truant school that the percentage was 11 . In hospital wards it is commonly above 20, and among the general public above 10 per cent. $†$ These figures at once explain the spread of diphtheria. They also suggest the methods of prevention.

In the ordinary diphtheria epidemic, whether large or small, these methods are mainly five. First, the actual cases of diphtheria must be effectually and promptly isolated. Secondly, the throats of contact persons should be bacteriologically examined, and those persons in whose throats the bacillus is found-c "carriers"- should be isolated, and their throats treated. Thirdly, sore throats in the immediate vicinity of the diphtheria infection should be similarly examined. Fourthly, thorough disinfection should take place in respect of infected houses, and inquiry made as to school influences, social habits, etc., of infected households. Fifthly, antitoxin should be used as prophylactic in infected families.

The new facts respecting the persistence of the bacillus in the

* See Jour. of Hygiene, 1903 (Graham-Smith), p. 216 ; and 1904, p. 255.

† See Jour. of Hygiene, 1904, pp. 258-328 (Graham-Smith); and Practitioner, 1903, vol. ii., pp. 715-21 (Newman). 
throat indicate the importance of throat treatment which there has been a tendency to ignore on account of the increased use of antitoxin. But antitoxin has little direct effect upon the bacilli in the throat, which should, therefore, be treated by painting with perchloride of mercury (1-500), or washed with chlorine water or permanganate of potash (1-300). The methods to adopt in order to clean the throat of the diphtheria bacillus are three, namely, $(\alpha)$ complete isolation of the patient, coupled with open-air treatment; $(b)$ application of antiseptics to throat; and (c) antitoxin.

The Pseudo-diphtheria Bacillus.*-In this group should not be included non-virulent forms of the diphtheria bacillus, but allied forms. $\dagger$ Löffler and Hofmann described a bacillus having similar morphological characters as the true $B$. diphtherice, except that it had no virulence. It is frequently found in healthy throats, and is believed by some to be a common inhabitant of the mouths of the poorer classes, especially children. The chief differences between the real and the psendo-bacillus are-

1. The pseudo-bacillus is thicker in the middle than at the poles, and not so variable as the $B$. diphtherice. Polar staining is generally less marked. It appears as an oval bacillus of variable length, generally having one narrow unstained septum. In broth cultures it often more closely resembles $B$. diphtherioe, but under all other conditions is shorter. It forms no toxins (Cobbett).

2. Hofmann's bacillus forms no acid in glucose culture media.

3. It does not give Neisser's reaction (see pp. 476 and 481) when grown for twenty-four hours on Löffler's ox serum.

4. The colonies produced by Hofmann's bacillus on blood serum usually become after a few days larger, more opaque, and whiter than those of the diphtheria bacillus. They also grow a little more rapidly than the true bacillus.

5. No pathogenic change is produced in guinea-pigs inoculated with this bacillus (1 c.c. of a twenty-four hours' broth culture), and it appears to be innocuous to man. In forming an opinion, all the facts, including the clinical, if possible, must be taken into consideration. But on the whole, recent evidence appears to support the view that Hofmann's bacillus possesses little, if any, pathological significance in man. It does not agglutinate like $B$. diphtherioe.

Attempts, followed by some degree of success, have been made to convert a virulent diphtheria bacillus into Hofmann's bacillus, and Hofmann's bacillus into a virulent form (Roux and Yersin,+

* For a fuller statement, see Trans. Jenner Inst. (First Series), pp. 7-32.

$\dagger$ For a discussion of the three forms $(a)$ the true virulent bacillus, $(b)$ the true non-virulent bacillus, and (c) the pseudo-bacillus, see Report of Metropolitan Asylums Board, 1901 (Gordon Pugh).

$\ddagger$ Ann. de l'Inst. Past., 1890, vol. iv. 
Hewlett and Knight, ${ }^{*}$ Richmond and Salter, $\uparrow$ Ohlmacher, ${ }_{+}^{+}$and others). Evidence in support of the view that Hofmann's bacillus is an attenuated variety of the true diphtheria bacillus has been brought forward. But it can only be accepted provisionally. Graham-Smith, Thomas and others consider the pseudo-bacillus to be absolutely innocuous. In practice, it is the right course at present to look upon the presence of the Hofmann bacillus as indicating a suspicious throat.

It should not be forgotten that there are a number of other bacilli from which the true diphtheria bacillus must be differentiated. $\$$ These include the B. coryzoe segmentosus, the bacillus of Hofmann, B. xerosis, and a number of diphtheroid bacilli, and organisms from nasal and aural discharge. Similar organisms occur in birds and other animals. There are, as summarised by Gordon, five chief characters by which the true diphtheria bacillus may be known:- $(a)$ The macroscopic and microscopic appearance of the growth on blood serum; $(b)$ the behaviour of the bacillus to Löffler's blue, Gram's stain, and Neisser's stain for granules; $(c)$ the reaction to litmus of a culture in alkaline broth, containing 2 per cent. of dextrose after 48 hours at $37^{\circ} \mathrm{C}$.; (d) the pathogenic test-1 c.c. of broth culture, 48 hours' growth at $37^{\circ}$ C., injected subcutaneously into 200-300 gramme guinea-pig, produces death generally in 48 hours, whilst post-mortem hæmorrhagic necrosis and odema are found locally, the internal organs are congested, the pleural, pericardial, and peritoneal fluids are increased, and the supra-renal capsules are enlarged and engorged with blood; (e) the virulence of the organism or its toxin is completely neutralised by a simultaneous dose of diphtheria antitoxin. For purposes of rapid diagnosis, $(a)$ and $(b)$ are relied upon.

\section{Scarlet Fever}

That the essential cause of scarlet fever is a micro-organism there can be little doubt. But up to the present time no organism has been definitely isolated which fulfils the postulates of Koch in respect to specificity of bacteria. Various organisms have, however, been described as associated with the disease. Edington, Fränkel, Freudenberg, Klein, Kurth, Gordon, Baginsky, Class, and others have described organisms which they believed to be etiologically related to the disease. At present, however, it can only be said that these bacteria have been found associated with scarlet fever, but are not yet proved to be its cause. The organism which appears at present to be

* Trans. Jenner Inst. Prevent. Med. (First Series), 1897, p. 7 et seq.

† Guy's Hospital Report, 1898, vol. liii., p. 55.

Jour. of Med. Research, 1902, vol. ii., p. 128.

\$ Rep. of Local Govt. Board, 1901-02, pp. 418-39 (Gordon); Jour. of Hyg., 1904, pp. 299-316. 
the most likely cause of the disease is the Streptococcus scarlatince of Gordon. Probably the organisms isolated by Baginsky and Class are different forms of the same streptococcus.

As regards dissemination, it has long been known that scarlet fever, like small-pox, is most commonly spread by direct infection through the medium of infected clothing and other articles, or materials handled by the patient. The means by which infection has thus been carried are manifold, and need not claim our attention here. As we have seen, in 1870 a wider field of conveyance of scarlet fever was revealed by the investigations of Dr M. W. Taylor of Penrith. While studying an outbreak of scarlet fever, he observed that the main incidence of the disease fell upon customers of a certain milk-shop where scarlet fever was existent. Since that date abundant evidence has been fortheoming to show that to the channels of infection previously recognised, that of conveyance by milk must be added. Scarlet fever is disseminated in many ways from person to person, and also by the vehicle of "fomites." The virus is not diffusible, but is evidently tenacious of life. Infected garments that have been put aside for months have been known to originate an outbreak of the disease. Linen has been known on many occasions to infect laundresses. There is no evidence that the virus can be conveyed by water. As a rule, probably the infection of scarlet fever is not greatly spread by aërial connection, but by articles (toys, books, bed-clothes, letters, etc.), and such infected articles if set aside in stagnant air, at a moderate temperature, and in the absence of daylight, may retain the infection, like garments, for months.

Infectivity begins at the earliest stage of the attack, but is probably greatest when the fever is at its highest. In most cases the patient is free from infection at the end of six weeks. There is now strong evidence that at least the later desquamation is not infective. Probably the infection lingers longest in the nasal, tonsillar, buccal, and pharyngeal mucus, and especially in any chronic discharge from those mucous membranes. Discharges from the ear may retain infection for months.*

It is most probable that milk obtains its infection of scarlet fever from being brought into contact with persons suffering, as a rule, from the early and acute stages of the disease.

Streptococcus Scarlatinæ (Klein and Gordon). Streptococcus Conglomeratus (Kurth).- The organism was isolated from the blood, nasal and tonsillar discharge of persons suffering from scarlet fever in its earlier and later stages. Not from urine or skin. It has been isolated from blood of persons dying from scarlet fever. Assumed to be identical with streptococcus isolated from diseased udders of cows and from their milk. Found by Klein in ulcerations of teats and udders of

* See also Report to Metropolitan Asylums Board on Return Cases of Scarlet Fever, by W. J. Simpson, M.D., 1901, p. 24 ; also Brit. Med. Jour., 1902, vol. ii., p. 445 (M. H. Gordon). 
certain cows. Morphology.-A streptococcus; polymorphic; showing tendency to oval and rod-shaped elements, especially in impression preparations. Presence of wedge-shaped, spindle-like, rod-shaped elements in agar and gelatine, and the characteristic of coherent conglomeration differentiate this streptococcus from others of the same genus. Irregularity in size and shape of elements; every transition between coccus and bacillus. Coccus shape prevails in bouillon, the bacillary being more common on agar. The streptococcus is stained by simple stains and Gram's method. Cultural characters: Bouillon.-At $37^{\circ} \mathrm{C}$. after 24 hours, the medium remaining clear, a single, thick, white-grey mass, or several smaller masses, appear at the foot of the tube; coherent on shaking the tube, floats through the medium as a flattened bun-like body. Kurth pointed out that when this mass was examined under the microscope, a conglomerate appearance was present. The mass is cohesive. Gelatine plates and tubes.-Slow growth, forming small grey colonies, circular or oblong, with firm edge, and consisting of closely-set coherent mass of cocci. Older colonies become nodular. Non-liquefying. In gelatine at $37^{\circ} \mathrm{C}$. the same appearances occur as in bouillon, but often more marked. Chain formation from these cultures is more marked than in ordinary streptococcus. In gelatine at $37^{\circ} \mathrm{C}$. this organism grows similar to S. longus. Agar plates and tubes. - Three types of colonies occur after 24 hours : (a) grey, granular, irregularly-outlined tuberculated colonies ; (b) colonies of similar kind, but having confluent appearance without tubercles; (c) younger and smaller colonies which have a fine frilling of chains around a more compact coherent centre. The most useful feature for differential purposes is the granular, glossy, coherent centre, combined with tuberculation. Grows more slowly than $S$. pyogenes, and on the whole its colonies on agar are smaller, more opaque, and more irregular than those of the other streptococci present. Milk.-Rapid coagulation; produces acid. Sometines fails to clot milk. A firm, solid clot forms Litmus milk, as a rule, within the first 2 days at $37^{\circ} \mathrm{C}$. After 24 hours the acid-production is very strong, and commonly, when there is a clot as well, the lower half of the tube is yellow-white-the top layer being pink. This decolorisation of lower half of litmus milk is due to a reducing action of the streptococcus. Chain formation occurs more than in bouillon. The four chief diagnostic features are : (1) the sediment growth in broth cultures; (2) the rapid coagulation of milk; (3) the acid reaction in litmus milk; (4) the character of the agar colonies. Pathogenesis.-Pathogenic for mice and rabbits. After passing through the mouse, the streptococcus takes on a bacillary form (Gordon), and other modifications, including the diminution of conglomeration, occur. Its virulence differentiates this streptococcus from streptococci present in non-scarlatinal throats, except $S$. pyogenes, which is more virulent to white mice than $S$. conglomeratus. Klein holds that this $S$. conglomeratus is causally related to scarlet fever in man, and is wholly distinct from S. pyogenes. Gordon has isolated the latter from the secretion on the surface of the tonsil in a case of clinically mild, uncomplicated scarlatina. It has also been found like the $S$. conglomeratus in the nasal and aural discharge of scarlet fever patients. Gordon believes that both streptococci may play a part in the causation of scarlet fever, but that $S$. conglomeratus is the more important of the two, and that it occupies a position in the bacteriological kingdom between $S$. pyogenes and $B$. diphtheriae.*

\section{Typhoid Fever}

Typhoid fever is an acute infectious disease characterised clinically by continuous fever, with diarrhoea and other symptoms, and anatomically by a more or less extensive ulceration of 'the Peyer's patches in the intestine (ileum), with swelling of the mesenteric glands and enlargement of the spleen. The lesion of importance is the ulceration of the bowel, partly on account of its origin, partly on account of

For full record of Gordon's researches, see Reports of Medical Officer to Loc. Gov. Bd., 1898-99, pp. 480-93 ; 1899-1900, pp. 385-457 ; and 1900-01, pp. 353-404. 
its result. The pathogenetic action of the bacilli is obscure, but there can be no doubt that the ulcers in the intestine are directly or indirectly the result of the specific bacillus. When the bacilli reach the intestine they multiply, and, penetrating the mucous and submucous coats, set up the changes, which lead, first to hyperæmia, then to infiltration, and finally to ulceration of Peyer's patches. Some of the bacilli pass into the blood, collecting in the spleen and other glands. Whether in the bowels or in the organs of the body, the bacilli produce their toxins, and as a result of their action, inflammation and fever follow. The inflammation in the intestine leads, in conjunction with the irritation produced by the ulcers, to increased peristalsis, and therefore diarrhoa. Hence the excreta of a typhoid patient have two characteristics. They are usually abundant and frequent: and they are charged with large numbers of the bacilli of typhoid fever. It is, however, necessary to guard against the idea that typhoid fever is a local disease of the intestine, or even chiefly so. In ordinary cases, it is true, the intestinal lesions form the starting-point of the disease, but the bacilli rapidly become generalised, and are found in the most varied parts of the body, and not uncommonly in the blood itself. Such a state of things leads to a condition not remote from septicæmia, and this may occur with little or no local lesion in the intestinal tract. The reason why the bacilli of typhoid are not found in greater number in the blood, is probably in part due to the fact that in ordinary cases the blood is not a favourable medium for their growth, and in part to the fact that they are rapidly eliminated or excreted. "Any conception of the disease," writes Dr Horton-Smith, "which regards it merely as affecting the alimentary canal, can no longer be maintained. On the contrary, so far from considering it an intestinal disease, pure and simple, we should rather look upon it as a modified form of septicæmia. It is septicæmia in that always, and in all cases, the bacilli pass into the blood, and then into the various organs, and in that the symptoms, excepting so far as they are intestinal, are referable to the poisons there produced. It is a modified form, however, in that in nearly all cases there is a definite local and primary disease, whence the secondary dissemination of the micro-organism takes place."*

Whilst it has been held that typhoid infection can pass out of the infected person by means of the sweat, the expectoration, the fæces and the urine, it is only the latter two which need be considered as a rule. Typhoid stools should always be considered infective, both in the early and late stages, and the bacilli have even been found in the stools fifteen days after the temperature has become normal. Further, it is possible that the typhoid bacillus may be distributed Smith.

* Brit. Med. Jour., 1900, i., pp. 827-34 (Gulstonian Lectures, 1900), P. Horton- 
by persons not suffering from the disease. It is believed that the virus of typhoid fever is chiefly distributed by the contents of the alimentary canal, and this view is so universally held that it is unnecessary to elaborate it.

The urine is the other chief excretion by which the bacilli of typhoid fever are voided from the body. Horton-Smith has demonstrated that the urine of typhoid patients contains the bacilli of the disease in the proportion of one in every four cases. He has also shown, that, as a rule, it is towards the end of the disease, or during convalescence, that this condition occurs. Further, whilst it is always difficult to find the bacilli in the stools, in the urine it is generally easy, for when they are present they are nearly always in pure culture, and not uncommonly they are present in such extraordinary numbers that one cubic centimetre may contain many thousands of micro-organisms (Horton-Smith). Cammidge found 37 per cent. of all the typhoid urines examined contained the bacillus. In one case the organism was found eight months after convalescence. In London, Horton-Smith found typhoid bacilli present in the urine in 25 per cent. of all cases examined. Working in Boston, Richardson obtained a positive result in 22.5 per cent. of the cases examined. Both the last-named investigators found the bacilli present, in certain cases, in such large numbers that the urine was rendered turbid by their presence. Nor are such cases rare. Out of the cases in which the specific bacillus was present in the urine, in as many as twelve it was present to the degree of turbidity, and in only two was the urine described as "clear" (Horton-Smith). Referring to the stage of the disease in which the bacilli appear in the urine, they have been found as early as the thirteenth day from the commencement, and as late as the fourteenth day of convalescence (Horton-Smith). Speaking generally, the condition is rare before the third week of the disease. The duration of this specific bacilluria also varies. The shortest duration recorded by Horton-Smith was eight days, but in four other cases it had not disappeared until after the lapse of twenty-one days, twentyfive days, thirty days, and seventy days. The phenomenon of typhoid bacilli in the urine probably occurs because one or more bacilli find their way into the bladder, and there commence rapid growth in the urine within the bladder, which medium is by no means unfavourable to the multiplication of the bacillus (Horton-Smith).

From these facts there are two broad deductions which concern the bacteriologist and epidemiologist:-First, that enteric fever occurs as a result of infection by the typhoid bacillus; and secondly, that the typhoid bacillus leaves the body of the infected person through two chief channels, namely, the urinary and alimentary systems. It has been shown further, that the typhoid bacillus is 
capable of a saprophytic existence in soil, dust, water, milk, and other natural media. It can survive in ordinary earth for two months, on sterilised linen for sixty days, on woollen cloth for eighty days, in sterilised water one hundred and ninety-six days, in particular soils four hundred days (Martin, Firth and Horrocks, etc.). Therefore it follows that the organism may remain in the body for long periods, may pass from the body in urine or in fæces, and find its way into natural media, and from such media, sooner or later, back to man. The line of infection may be direct or indirect; but that it occurs there can be no doubt.

The Bacillus of Typhoid Fever (Eberth-Gaffky).-The evidence that Eberth's bacillus is the cause of typhoid fever consists in the main of three parts:-(1) The bacillus is found with almost invariable regularity in the spleen of persons dying of typhoid fever, when an adequate bacteriological examination is made. (2) Eberth's bacillus elaborates specific toxins. These toxins are for the most part intracellular, contained within the bacillus itself, and are chiefly set free when the latter is destroyed; and they are comparatively feeble compared with those of other pathogenic organisms; and to account for the clinical conditions of the disease the number of bacilli present in the infected body would have to be exceptionally large. This fact, coupled with the varying virulence of the bacillus, is all the more remarkable when it is remembered that not a few of the epidemics of the disease have arisen from a dose of poison, so excessively minute in itself, and so enormously diluted, as to appear out of all proportion to the number of persons attacked. It is possible that a few organisms introduced into the human body are able, under certain conditions, to multiply rapidly, and so bring about the same results as large dosage. Again and again it has been shown that considerable epidemics have arisen from a pollution of water so slight as to escape detection by any methods of chemical or bacteriological analysis at present known. (3) The blood serum of individuals suffering from typhoid fever has a specific agglutinative action upon the Eberth bacillus, similar to that observed in the blood serum of animals, rendered immune to this germ (compare also Pfeiffer's reaction). And whilst there is no evidence to suppose that animals suffer from typhoid fever as the disease occurs in man, there is evidence to show that under certain conditions, a disease, not unlike enteric fever, can be produced by inoculation of the $B$. typhosus into guinea-pigs, mice, rabbits, etc. (Fränkel and Simmonds). Klein has also recently demonstrated by inoculation, that the bacillus is able to multiply and develop in the lymph-glands of the calf. For all practical purposes, therefore, the B. typhosus of Eberth is now generally accepted as the causal agent in typhoid fever.

The channels of infection in typhoid fever are almost entirely 
concerned with the alimentary tract. Water, milk, shell-fish, fried fish, ice-cream, watercress, etc., have all been proved to be the vehicle of infection in spreading the disease. Personal contact may and does operate in spreading infection, and by this means food also may become contaminated. Flies are held to have acted as carrying agents in the Spanish-American War of 1898 and the Boer War of 1900-1902. Corfield records some dozen outbreaks of typhoid fever due to general insanitary conditions, 60 outbreaks to infected water, and a large number to minor channels.* The writer has collected 160 records of milk-borne outbreaks of the disease. $†$

In 1880-81 Eberth announced the discovery of the typhoid bacillus in cases of clinical enteric fever. In 1884 it was first cultivated outside the body by Gaffky. Since then other organisms have been held responsible for the causation of enteric (or typhoid) fever. In 1885 the $B$. coli communis was recognised, and it has been a matter of some debate among bacteriologists as to how far these two organisms are the same species, and interchangeable. There is evidence on both sides of the question, but bacteriologists generally regard the Eberth-Gaffky bacillus as the specific cause of typhoid fever, though complete proof is still wanting.

Under the microscope the bacilli appear as rods, $2-4 \mu$ long, $\cdot 5 \mu$ broad, having round ends. Sometimes threads are observable, being $10 \mu$ in length. In the field of the microscope the bacilli differ in length from each other, but are approximately of the same thickness. Round and oval cells constantly occur even in pure culture, and many of these shorter forms of typhoid appear to be identical in morphology with some of the many forms of $B$. coli. There are no spores. Motility is marked; indeed, in young culture the typhoid bacillus is the most active pathogenic germ we know. The small forms move about with extreme rapidity; the longer forms move in a vermicular manner. Its powers of motility are due to some five to twenty flagella of varying length, some of them being much longer than the bacillus itself. The flagella are both terminal and lateral, and are elastic and wavy.

The organism may be isolated from the ulcerated Peyer's patches in the intestine, from the spleen, the mesenteric glands, and the urine. Owing to the mixture of bacteria found elsewhere, it is generally most readily isolated from the spleen. The whole spleen is removed, and a portion of its capsule seared with a hot iron to destroy superficial organisms. With a sterilised knife a small cut is made into the substance of the organ, and by means of a sterilised platinum wire a little of the pulp is removed and traced over the

* The Milroy Lectures on Typhoid Fever, 1902.

$\dagger$ Bacteriology of Milk, 1903 (Swithinbank and Newman). 
○ 
surface of agar. The agar reveals a growth in about twenty-four hours at $37^{\circ} \mathrm{C}$., which is the favourable temperature. A greyish, moist, irregular growth appears, but it is invariably attached to the track of the inoculating needle. On agar plates the superficial colonies appear as circular spots, dull white by reflected light and bluish-grey by transmitted light. The deep colonies are opaque and finely granular. On gelatine the growth is much the same, but its irregular edge is, if anything, more apparent. There is no liquefaction and no gas formation. On plates of gelatine the colonies become large and spreading, with wavy margins. The whole colony appears raised and almost limpet-shaped, with delicate lines passing over its surface. When magnified, there is an appearance of transparent iridescence. The growth on potato is termed "invisible," and is of the nature of a potato-coloured pellicle, which appears to be moist, and may at a later stage become light brown in colour, particularly if the potato is fresh. Milk is a favourable medium, and is rendered slightly acid. No coagulation takes place. Broth is rendered turbid, and there is the formation of a sediment. The organism is stained with carbol-thionin blue, carbolic fuchsin, etc. It is decolorised by Gram's methods.

The problem of isolating the typhoid bacillus is greatly complicated by the fact that $B$. coli communis is a normal inhabitant of the alimentary canal, is widely distributed in nature, and is in many respects similar to the typhoid bacillus. (For full account of $B$. coli and its similarities to the typhoid bacillus, see pp. 46-51.)

We have pointed out elsewhere the relation between soil and typhoid. In water, even though we know it is a vehicle of the disease, the Bacillus typhosus has been only very rarely detected. The difficulties in separating the bacillus from water (like that at Maidstone, for example), which appears definitely to have been the vehicle of the disease, are manifold. To begin with, the enormous dilution must be borne in mind, a comparatively small amount of contamination being introduced into large quantities of water. Secondly, the group of the $B$. coli species considerably complicates the search.

Further, we must bear in mind a point that is frequently neglected, namely, that the bacteriological examination of a water which is suspected of having conveyed the disease is from a variety of circumstances conducted too late to detect the causal bacteria. The incubation period of typhoid we may take at fourteen days. Let us suppose a town water supply is polluted with some typhoid excreta on the 1st of January. Until the 14th of January there may be no knowledge whatever of the state of affairs. Two or three days are required for notification of cases. Several more days elapse generally before bacteriological evidence is demanded. Hence arises 
the anomalous position of the bacteriologist who sets to work to examine a water suspected of typhoid pollution three weeks previously. There can be no doubt that these three difficulties are very real ones. The solution to the problem will be found in the dictum that "a water in which sewage organisms have been detected in large numbers should be regarded with suspicion " as the vehicle of typhoid, even though no typhoid bacilli are discoverable. The chief of these sewage bacteria are believed to be Proteus vulgaris, Bacillus coli, Proteus Zenkeri, and Bacillus enteritidis sporogenes.

It may occur to the reader that, as the typhoid bacillus is, as far as we know, comparatively common, drinking water may frequently act as a vehicle to carry the disease to man. But, to appreciate the position, it is desirable to bear in mind the following facts. The typhoid bacillus is found, with other bacteria, in the excrement of patients suffering from the disease; it is short-lived; in waters there exist organisms which can exert an influence in diminishing its vitality; it is, so to speak, enormously diluted in waters; exposure to direct sunlight destroys it; and it has a tendency to be carried down stream, or in still waters settle to the bottom by subsidence. Even when all the conditions are fulfilled, it must not be forgotten that a certain definite dose of the bacillus is required to be taken, and that by a "susceptible" person.

\section{Epidemic Diarrhœa}

By "epidemic diarrhœe" (zymotic or epidemic enteritis) is meant a specific disease, which may be defined as an acute infective disease, affecting chiefly children under two years of age, occurring during the summer months in epidemic form, and characterised by the occurrence of diarrhœa, vomiting, and wasting, accompanied in severe cases by toxæmia and collapse. The disease is a large contributor to infant mortality, and in many urban distriets it is the most serious of all infant diseases, if measured by fatality.

The exact cause of epidemic diarrhœe is not at present known. In 1887 Ballard formulated certain propositions which have obtained general acceptance. They are as follow :-

"That the essential cause of diarrhœa resides ordinarily in the superficial layers of the earth, where it is intimately associated in the life-processes of some micro-organism not yet detected or isolated.

"That the vital manifestations of such organism are dependent, among other things, perhaps principally upon conditions of season and on the presence of dead organic matter, which is its pabulum.

"That on occasion such micro-organism is capable of getting abroad from its primary habitat, the earth, and having become air- 
borne, obtained an opportunity for fastening on non-living organic material, and of using such organic material both as nidus and as pabulum in undergoing various phases of its life-history.

"That in food, inside as well as outside the human body, such micro-organism finds, especially at certain seasons, nidus and pabulum convenient for its development, multiplication, or evolution.

"That from food, as also from the contained organic matter of particular soils, such micro-organism can manufacture by the chemical changes wrought therein through certain of its lifeprocesses a substance which is a virulent chemical poison; and that this chemical poison is, in the human body, the material cause of epidemic diarrhce." *

Bacteriology of Diarrhœa.-The three causal agents which Ballard mentions as playing a large part in the production of this disease are the soil, season, and food-and the causa causans is "some micro-organism not yet detected or isolated." It must be said that we have not got much further than this during the last fifteen years.

In 1885 Escherich published his classical researches on B. coli communis. He pointed out that the meconium of the newly-born infant is free from bacteria, but by the second day they are present in large numbers, and in the ordinary excreta of healthy infants he found chiefly two organisms, $B$. lactis cerogenes and $B$. coli communis. Of these the former was the more abundant in the upper part of the small intestine, and the latter in the lower part and in the colon, so that in the excreta $B$. coli was abundant, and $B$. lactis comparatively scarce. Booker, working in 1886 and onwards, found that the constant bacteria of the healthy excreta of the infant (B. coli and B. lactis arogenes) do not disappear in the excreta of diarrhœa. $B$. coli, however, does not predominate in the same degree, and $B$. lactis is present generally in greater numbers than in the healthy excreta. Booker examined the excreta of 31 children, and isolated 33 different species of bacteria. Many varieties of bacteria are sometimes found in individual cases of diarrhœa. The greatest number were found in cases of cholera infantum, and a larger number in catarrhal enteritis than in dysenteric enteritis. The actual number of individual bacteria was, he found, as great in the healthy excreta as in the diarrhœal excreta. Proteus vulgaris was found very generally, and in the most serious cases. No chromogenic bacteria were isolated, and cultures from a large number of green stools failed to develop green colonies. From these facts Booker concluded "that not one specific 1887.

* Supplement to the Report of the Medical Officer of the Local Government Board, 
organism, but many different varieties of bacteria, are concerned in the etiology of the summer diarrhœas of children.

From 1889-1895 Booker continued his studies, isolating bacteria from the rectum in 92 infants affected with epidemic diarrhœa, and also from the organs of 33 infants who died from this disease. $\mathrm{He}$ found the conditions for the development of bacteria in the intestine of infants affected with summer diarrhœa different from those in the healthy intestine of milk-fed infants, in that they favoured more varied bacterial vegetation, a rich growth of the inconstant species of intestinal bacteria, and a more uniform distribution through the intestine of the two constant varieties of healthy excreta bacteria ( $B$. coli communis and $B$. lactis ocrogenes). The first step in the pathological process, Booker believes to be a direct injury to the epithelium from abnormal or excessive fermentation and from toxic products of bacteria; and secondly, a general intoxication may be brought about indirectly through the production of soluble poisons. He holds that, bacteriologically and anatomically, three principal forms of summer diarrhœa of infants may be provisionally distinguished:-(i.) dyspeptic or non-inflammatory diarrhœa; (ii.) streptococcal gastro-enteritis; and (iii.) bacillary gastro-enteritis.* As a result of his extended researches, Booker came to a general conclusion which he expressed as follows:- "No single micro-organism is found to be the specific excitor of the summer diarrhoa of infants, but the affection is generally to be attributed to the activity of a number of varieties of bacteria, some of which belong to well-known species, and are of ordinary occurrence and wide distribution, the most important being the streptococcus (enteritidis) and Proteus vulgaris." The streptococcus termed S. enteritidis varies in morphology, and seems to be associated with two classes of cases, one of which simulates cholera, the other typical enteric fever. "Micrococci are present in all cases, sometimes in enormous numbers." $\dagger$ It may be added that Cumston, Holst, Escherich, and Hirsch have also laid emphasis upon the causal relationship of certain streptococci and diarrhœa.

Klein was one of the first workers to isolate an anaërobic organism from cases of epidemic diarrhœea. This organism, which he named $B$. enteritidis sporogenes, was found in three successive outbreaks of diarrhœa occurring among patients in St Bartholomew's Hospital. In the first two outbreaks the milk was evidently the channel of infection, in the third it was some rice pudding. The

* Johns Hopkins Hospital Reports, 1896, vol. vi., p. 253. See also a paper "On the Growth of Bacteria in the Intestine," by Lorrain Smith and Tennant-Brit. Med. Jour., 1902, vol. ii., p. 1941. Also Jeffries, Trans. American Pediatrics Society, vol. i., 1889 ; Baginsky, Archiv. f. Kinderheilkunde, xii., Nos. 1 and 2; Berliner klin. Woch., 1889 ; and Flexner and Holt's Rockefeller Inst. Rep., 1904.

$\dagger$ Johns Hopkins Hospital Reports, 1896, vol. vi., p. 251. 
P 


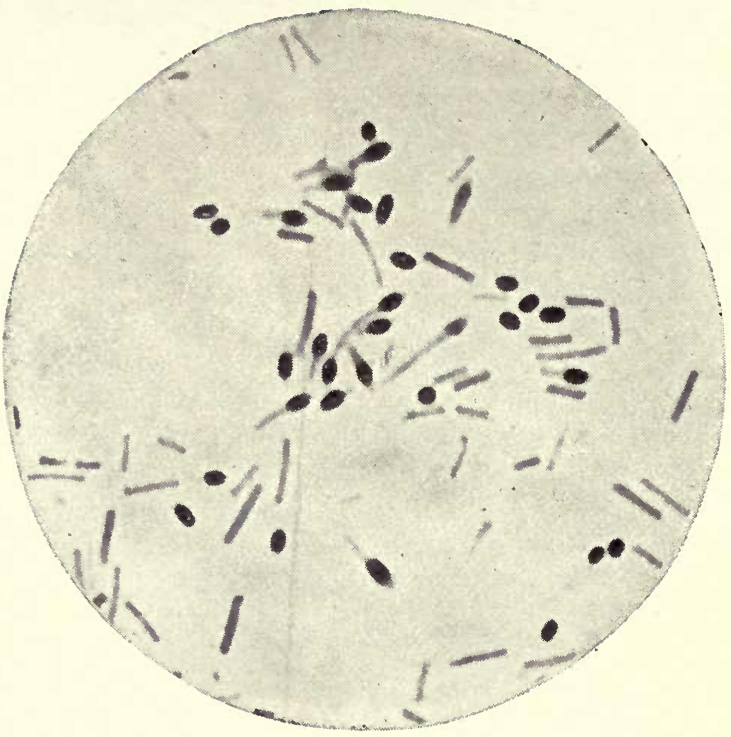

Bacillus enteritidis sporogenes. (Klein.)

Film preparation from serum culture, showing spores. $\times 2000$.

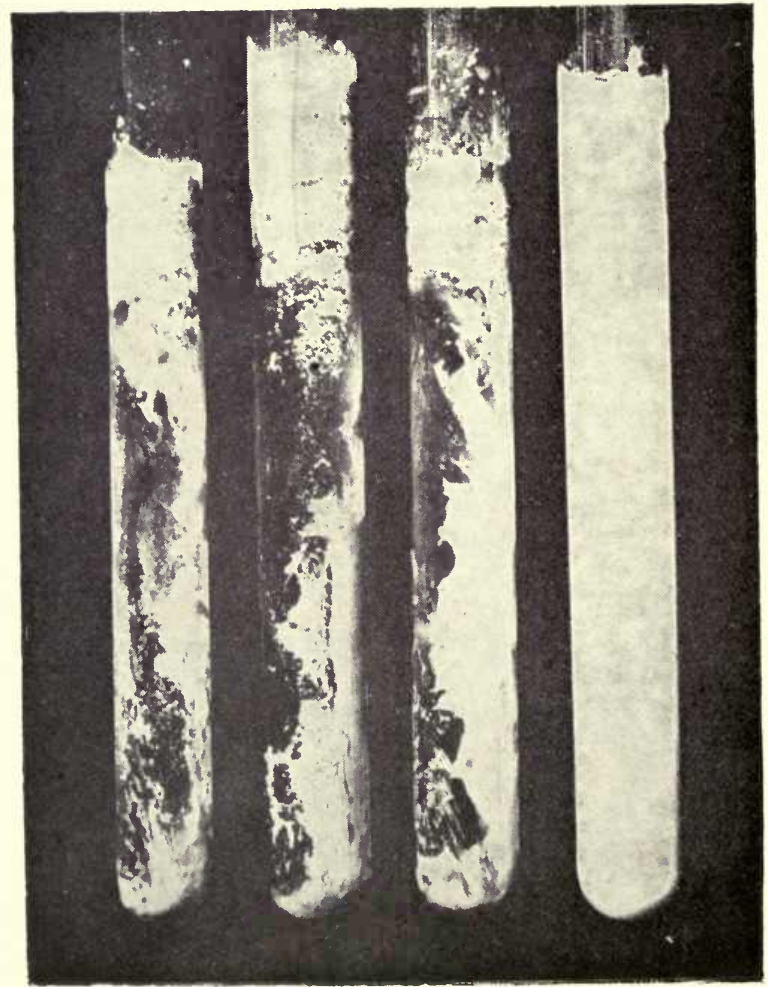

Anä̈robic Milk Culture, showing the "Enteritidis Change.' (K'lein.) From left to right, tubes contain $\frac{1}{10}, x_{00}^{\frac{1}{0}}, \frac{1}{100 \sigma}$, robod c.c. of Nottingham crude sewage. 
bacillus was carefully studied, and the main facts respecting it may be stated briefly here:-

B. enteritidis sporogenes (Klein) is an anaërobic bacillus : $1 \cdot 6-4 \cdot 8 \mu$ long, and $0.8 \mu$ broad ; stains by Gram's method and ordinary stains. Motile ; spore formation present; large, oval spores often situated near one end of bacillus ; grows well on gelatine and agar. In the former gas is produced and the gelatine liquefies. On agar there is also gas fermentation, and the colonies in the depth are round, white by reflected light, brown and granular in transmitted light. On the surface of agar plates flat, circular, moist, grey colonies appear in twenty-four to fortyeight hours ; thicker in centre than at margin, and showing granularity. Bacillus grows well in milk, producing the "enteritidis change." After thirty-six hours of anaërobic incubation at $37^{\circ} \mathrm{C}$. in milk, the cream is torn or disassociated by development of gas, so that the surface of the medium is covered with stringy, pinkishwhite masses of coagulated casein enclosing a number of gas-bubbles. The main portion of the tube of milk contains a colourless, thin, watery whey, with a few casein lumps here and there adhering to the sides of the tube. The whey has a smell of butyric acid, and is acid in reaction. It contains many bacilli. Pathogenesis-If 1 c.c. of milk whey containing the bacillus be injected into a guinea-pig (200 to 300 grammes), a swelling appears in six hours, extending over abdomen and thigh, and death occurs in eighteen to twenty-four hours. Post mortem : subcutaneous gangrene, with much sanguineous exudation, in which bacilli and spores will be found. Klein considers this organism to be the cause of epidemic diarrhœea.

$B$. enteritidis sporogenes is a widely-distributed organism, and occurs in normal and typhoid excreta, in sewage, manure, soil, dust, and milk.* The etiological relationship between this bacillus and epidemic diarrhœa has been called in question, and it is, of course, not proved-that the organism is the cause of the disease. On the other hand, it has been very frequently found in the mucous flakes of the dejecta in patients suffering from the disease, and in the outbreak produced by the consumption of cooked rice pudding it is difficult to understand how any organism except an anaërobe of highly resistant qualities could have produced the condition. It will be apparent, moreover, that $B$. enteritidis sporogenes fulfils in a somewhat exceptional degree the requirements suggested by Ballard.

That epidemic diarrhoa is caused by the $B$. coli either alone or in conjunction with other organisms, has been held by a number of authorities. Cumston, who investigated 13 cases of the disease, concluded that B. coli associated with Streptococcus pyogenes was the chief pathogenic agent concerned, and he claims that the virulence of $B$. col $i$ is exalted by the association. $†$ Lesage also formed the opinion that the disease was due to $B$. coli, and investigated the agglutinative properties of the serum of children suffering from epidemic diarrhoea on $B$. coli isolated from the intestine. He obtained a positive result in 40 out of 50 cases, and the serum of each of these 40 cases, also agglutinated samples of $B$. coli from 39 other children seized with

* Reports of Medical Officer of Local Government Board, 1897-98, pp. 210-51 ; 1902 , p. 406.

$\dagger$ International Medical Magazine, February 1897. 
the same disease.* Some of the most recent work on the relationship existing between $B$. coli and epidemic diarrhoea has been done by Delépine, who examined milk in the outbreak of epidemic diarrhœa which occurred in Manchester in 1894 (see p. 224), and has also examined a large number of town and country milks. His conclusion is that:-

"Epidemic diarrhœe of the common type occurring in this country is apparently, in the great majority of instances, the result of infection of food by bacilli belonging to the colon group of bacilli, and which are present at times in fæcal matter. It appears that this infection of food does not generally lead to serious consequences, unless the infection is massive from the first, or the food is kept for a sufficient length of time, and under conditions of temperature favouring the multiplication of these bacilli.

"Milk, which is the most common cause of epidemic diarrhœa in infants, is usually infected at the farm, or (through vessels) in transit. Of the bacilli of the colon group which are capable of rendering the milk infectious, those which do not produce a large amount of acid, and do not coagulate milk, are the most virulent, and are probably the essential cause of epidemic diarrhœea." $\dagger$

It is evident that our knowledge of the bacteriology of diarrhœa is not sufficiently established to permit of any very definite conclusion on the matter. It may be that the whole group of choleraic, enteric, and diarrhœal diseases are caused by a group of microorganisms having many similarities and relationships to each other; or it may be that different forms of diarrhœa have their own specific causal organism; or, lastly, it may be a question of association of organisms or of toxins which brings about the disease. + In any event, there is abundant evidence that epidemic diarrhoea is a bacterial disease in the same sense as typhoid fever.

Conditions favourable to Epidemic Diarrhœa.-Theprovisional results of Ballard's inquiry into the causation of epidemic diarrhœa may be stated as follows:-

"The summer rise of diarrhœal mortality does not commence until the mean temperature recorded by the 4 -foot earth thermometer has attained somewhere about $56^{\circ} \mathrm{F}$., no matter what may have been the temperature previously attained by the atmosphere or recorded by the 1 -foot earth thermometer. The maximum diarrhœal mortality of the year is usually observed in the week in which the temperature recorded by the 4 -foot earth thermometer attains its mean weekly maximum. The decline of the diarrhœal mortality is in this con-

* La Semaine Med., October 1897.

+ Jour. of Hygiene, 1903, vol. iii., No. 1, p. 90.

¥ See also Report of Medical Officer to Local Government Board, 1902, p. 395 (Martin), 404 et seq. (Klein). 
nection not less instructive, perhaps more so, than its rise. It coincides with the decline of the temperature recorded by the 4 -foot earth thermometer, which temperature declines very much more slowly than the atmospheric temperature, or than that recorded by the 1-foot thermometer; so that the epidemic mortality may continue (although declining) long after the last-mentioned temperatures have fallen greatly, and may extend some way into the fourth quarter of the year. The atmospheric temperature and the temperature of the more superficial layers of the earth, is little if at all apparent until the temperature recorded by the 4 -foot earth thermometer has risen as stated above; then their influence is apparent, but it is a subsidiary one."

In addition to these conditions of soil, Ballard and other workers have concluded that insanitation in the widest sense of the term favours epidemic diarrhœea. Density of population or houses upon an area, unclean soil, dusty surfaces, bad light, absence of ventilation, maternal neglect, etc., all have a share in creating an environment favourable to the disease. As we have seen, Delépine, like Ballard, attributes the disease in large measure to milk. Ballard believed that milk gained its infection by unsuitable storage and by the mode in which it was used. He found that "infants fed solely from the breast are remarkably exempt from fatal diarrhœa; that infants fed in whatever way with artificial food to the exclusion of breast milk are those which suffer most heavily from fatal diarrhœa; that children fed partially at the breast and partially with other kinds of food, suffer to a considerable extent from fatal diarrhœa, but very much less than those brought up altogether by hand; and that, as respects the use of 'the bottle,' it is decidedly more dangerous than artificial feeding without the use of the bottle." This view has been confirmed by Newsholme, Niven, Richards, the writer, and others.

Dr Newsholme of Brighton has published an interesting paper on the causation of epidemic diarrhœa. Some of his chief conclusions, which are now widely accepted, may be added:-

"(1) Epidemic diarrhoa is chiefly a disease of urban life.

Epidemic diarrhoea as a fatal disease, is a disease of the artisan and still more of the lower labouring classes to a preponderant extent. This is probably largely a question of social status per se; that is, it is due to neglect of infants, uncleanly storage of food, industrial occupation of mothers, etc. (3) Towns which have adopted the water-carriage system of sewerage have, as a rule, much less diarrhœa than those retaining other methods of removal of excrement. Towns with the most perfect scavenging arrangements, including the inethods of removal of house refuse, have the least epidemic diarrhœa. It has recently been suggested that epidemic diarrhoea is due to surface pollution derived from street dust, particularly dried horse- 
manure (Waldo). (5) The influence of the soil is a decided one. Where the dwelling-houses of a place have as their foundation solid rock, with little or no superincumbent loose material, the diarrhœal mortality is, notwithstanding many other unfavourable conditions and surroundings, low. On the other hand, a loose soil is a soil on which diarrhœal mortality is apt to be high (Ballard). The pollution of soil is probably the important element in the causation of diarrhœa in towns on pervious soils. (6) Given two towns equally placed so far as social and sanitary conditions are concerned, their relative diarrhoal mortality is proportional to the height of the temperature and the deficiency of the rainfall in each town, particularly of the third quarter of the year."

Dr Newsholme concludes that " the fundamental condition favouring epidemic diarrhoea is an unclean soil, the particulate poison from which infests the air and is swallowed, most commonly with food, especially milk." In other words, epidemic diarrhœa is a so-called " filth-disease," preventable by improved sanitation in the broadest meaning of the term.*

From the facts and suggestions quoted above, and they are but representative of many other similar views receiving the general support of epidemiologists, it will be evident that at the present time the cause of epidemic diarrhœa is to be found in four conditions, which may be expressed shortly as two propositions, thus: (1) Epidemic diarrhœa is a bacterial disease; (2) its occurrence depends, wholly or partly, upon surrounding temperature, deficiency of rainfall, and pollution of food, chiefly milk. The exact relationship which these conditions have to each other is not known. Some authorities hold that a certain temperature affects food, conducing towards creating in it injurious properties. Others believe that it is a question of pollution of mill by dust, which carries to the milk the causal micro-organisms, and that deficient rainfall favours this contamination, and increased temperature favours the growth and multiplication of the bacteria thus conveyed to the milk. As Dr Newsholme says, "Whatever be its mode of operation, a frequent fall of rain during the summer weeks, even though its total amount be not great, is one of the most effectual means of keeping down the diarrhœal death-rate"; $\dagger$ and whilst he considers temperature conditions of great importance, "rainfall is more important than temperature in relation to epidemic diarrhœa." Rain washes the air, if the expression may be allowed, and carries to the surface aërial dust. It, of course, also washes the surface of the soil and removes surface pollution, and with it micro-organisms capable of infecting infants, usually by food. Thus the relationship between these

* Public Health, 1899-1900, vol. xii., pp. 139-213.

$\dagger$ Annual Report on Health of Brighton, 1902, p. 48 
meteorological conditions and milk, though an open question, may be an essential one to the origin of the disease. Milk is probably a common vehicle of infection (Ballard, Delépine, Newsholme), and a number of outbreaks are now on record which appear to have been due to the consumption of contaminated milk. In 1892 Gaffky recorded an outbreak at Giessen,* in 1894 Niven reported on 160 cases of diarrhœa at Manchester, $\uparrow$ in 1895 and 1898 three outbreaks occurred at St Bartholomew's Hospital.+

The facts set forth above furnish sufficient indication of the appropriate methods of prevention.

\section{Suppuration and Abscess Formation}

The term suppuration is used to designate that general breaking down of cells which follows acute inflammation. An "abscess" is a collection, greater or smaller, of the products of suppuration, pus. Pus consists chiefly of two kinds of cells. First, leucocytes, which have immigrated to the part affected; and secondly, broken down and necrosed elements. Such an advanced inflammatory condition may occur in any locality of the body, and it will assume different characters according to its site. There are connected with suppuration, as causal agents, a variety of bacteria. Pus is not matter containing a pure culture of any specific species, but, on the contrary, is generally filled with a large number of different species, each playing a greater or lesser part in the process. The most important are as follows :-

1. The Staphylococcus group.-This species consists of micrococci arranged in groups, which have been likened to bunches of grapes (Plate 30, p. 398). They are the common organisms found in pus, and were, with other auxiliary bacteria, first distinguished as such by Professor Ogston of Aberdeen. There are several forms of the same species, differing from each other in certain respects. Thus we have the $S$. pyogenes aurreus (golden-yellow), albus (white), citreus (lemon), and others. They occur eommonly in nature, in air, soil, water, as well as on the surface of the skin, and in all suppurative conditions. The aureus is the only one credited with pathogenic virulence. It occurs in the blood in blood-poisoning (septicæmia, pyæmia), and is present in all ulcerative conditions, including ulcerative disease of the valves of the heart. The Staphylococcus cereus albus and S. cereus flavus are slightly modified forms of the S. pyogenes aureus, and are differentiated from it by the fact of their being non-liquefying. They produce a wax-like growth on gelatine.

* Deut. Med. Woch., vol. xviii. p. 14.

† Annual Report Medical Officer of Health, Manchester, 1894.

+Report of Medical Officer of Local Government Board, 1895-96, pp. 197-204; ibili., 1897-98, p. 235; and ibid., 1898-99, p. 336. 
Staphylococcus pyogenes aurcus, the type of the species, is grown in the laboratory on all ordinary media at room temperature, though more rapidly at $37^{\circ} \mathrm{C}$. Liquefaction sets in at a comparatively early stage, and subsequently we have in gelatine test-tube cultures a floceulent deposit of a bright yellow amorphous mass, and in gelatine plates small depressions of liquefaction with a yellow deposit. The organism renders all media acid, and coagulates milk. Its thermal death-point in gelatine is $58^{\circ} \mathrm{C}$. for ten minutes, but when dry considerably higher. Outside the body it may retain vitality for
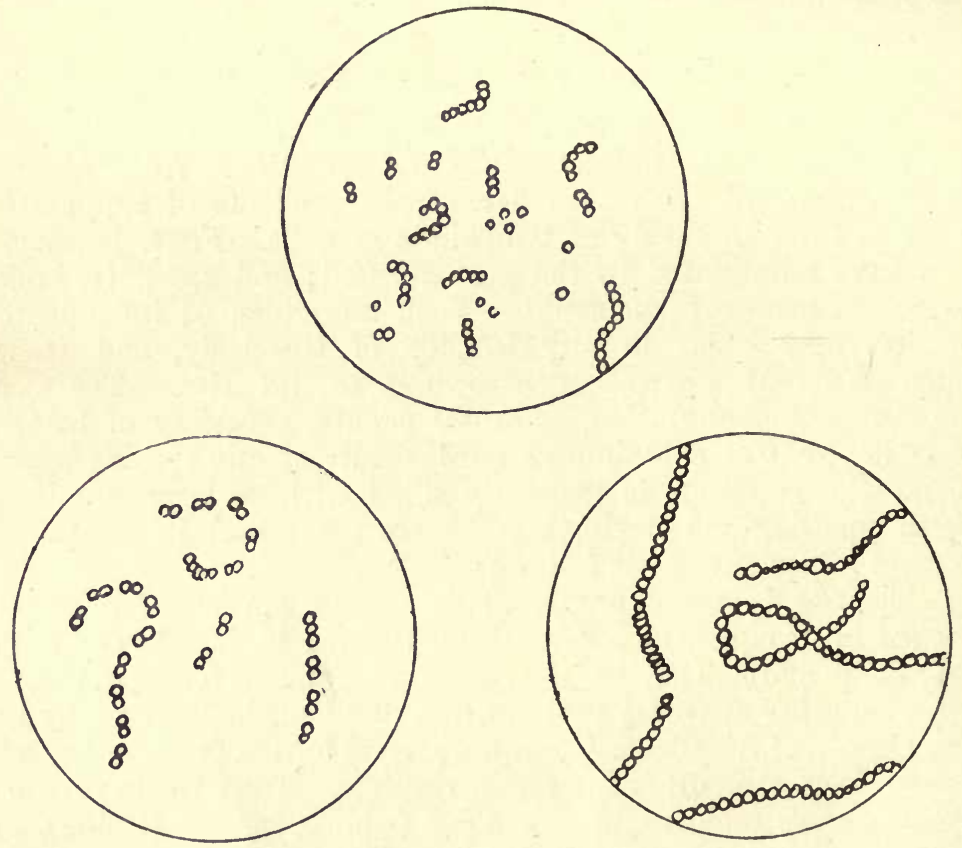

FiG. 25.-Diagram of Types of Streptococci.

months. It stains by Gram's method. It is a non-motile and a facultative anaërobe; but the presence of oxygen is necessary for the production of much pigment. Its virulence readily declines.

2. Streptococcus pyogenes. - In this species of micrococcus the elements are arranged in chains. Most of the streptococci in pus, from different sources, are probably of one species, having approximately the same morphological and biological characters. Their different effects are due to different degrees of toxic virulence; they are generally more virulent when associated with other bacteria, for example, the Proteus family.

The chains vary in length, consisting of more elcments when 
cultured in fluid media (hence $S$. longus and $S$. brevis). They multiply by direct division of the individual elements, and in old cultures it has been observed that the cocci vary in form and size (involution forms). This latter fact gave support to the theory that streptococcus reproduced itself by arthrospores, or "mother-cells."

In culture upon the ordinary media, Streptococeus pyogenes is comparatively slow-growing, producing minute white colonies on or about the sixth day. It does not liquefy gelatine, and remains strictly localised to the track of the inoculating needle. Like the staphylococcus, it readily loses virulence. The thermal death-point is, however, lower, being $54^{\circ} \mathrm{C}$. for ten minutes. Marmorek has devised a method by which the virulence may be greatly increased, and he holds that it depends upon the degree of virulence possessed by any particular streptococcus as to what effects it will produce. By the adoption of Marmorek's methods, attempts have been made to prepare an antitoxin.

Streptococeus pyogenes has been isolated from the membrane in cases of diphtheria, and from small-pox, scarlet fever, vaccinia, and other diseases. In such cases it is probably not the causal agent, but merely associated with the complications of these diseases. Suppuration and erysipelas are the only pathological conditions in which the causal agency of Streptococcus has been sufficiently established.

3. The Bacillus pyocyaneus occurs in green pus, and is the cause of the coloration. Gessard was the first to prove its significance, and he described two varieties. It is a minute, actively motile, nonsporulating bacillus, which occasionally complicates suppuration and produces blue-green pus. It stains with the ordinary aniline stains, but is decolorised by Gram's method. Oxygen is necessary for pigmentation, which is due to two substances: pyocyanin, a greenishblue product extracted with chloroform, and pyoxanthose, a brown substance derived from the oxidation of the former pigment. Both these colours are produced in cultivation outside the body. On gelatine the colour produced is green, passing on to olive. There is liquefaction. On potato we generally obtain a brown growth (compare B. coli, B. mallei, and others). The organism grows rapidly on all the ordinary media, which it has a tendency to colour throughout. It. will he remembered that when speaking of the antagonism of organisms, we referred to the inimical action of B. pyocyaneus upon the bacillus of anthrax.

4. Micrococcustetragonus. - This species occurs in phthisical cavities, and in certain suppurations in the region of the face. The micrococcus usually occurs in the form of small tetrads. A capsule is generally present. 
It is a non-liquefying organism, pathogenic for white mice (producing septicæmia). It grows on ordinary laboratory media, producing a viscid tenacious culture.

5. B. coli communis and many putrefactive germs commonly occur in suppurative conditions, but they are not restricted to such disorders (see p. 46).

6. Micrococcus gonorrhoce (Neisser, 1879).-This organism is more frequently spoken of as a diplococcus. It occurs at the acute stage of the disease (and in the purulent secretion of gonorrhœal conjunctivitis), but is not readily differentiated from other similar diplococci except by laboratory methods. Each element of the diplococcus presents a straight or concave surface to its fellow. A very marked concavity indicates commencing fission. The position which these diplococci take up in pus is intracellular, and they are arranged more or less definitely around the nucleus. In chronic gonorrhœa

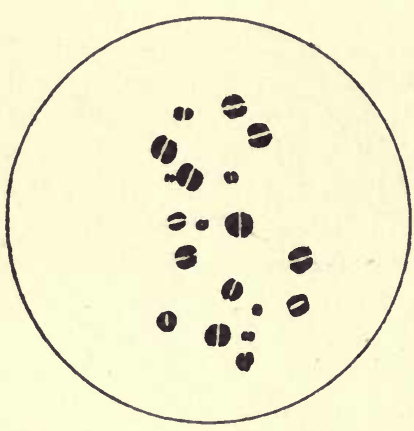

FIG. 27.-Diagram of Gonococcus. the diplococci are diminished in number. Difficulty has often been found in cultivating the organism in artificial media outside the body. Wertheim and others have suggested special formulæ for the preparation of suitable media, but it is a comparatively simple matter to secure cultures on agar plates smeared with human blood from a pricked finger. The plate is incubated at $37^{\circ} \mathrm{C}$. At the end of twenty-four hours small raised grey colonies appear, which at about the end of four days show adult growth. The margin is uneven, and the centre more opaque than the rest of the colony. This diplococcus is readily killed, and sub-cultures must be frequently made to retain vitality and virulence. Light, desiccation, and a temperature of $55^{\circ} \mathrm{C}$. all act germicidally. The organism stains readily with Löffler's blue, but is decolorised by Gram's method. It is more or less strictly parasitic to man, and has been definitely proved to be the cause of gonorrhœa. A toxin has been separated. The shape, size, character of growth, intracellular position, and staining properties of the gonococcus assist in differentiating it from various similar diplococci.* An organism not greatly different from the gonococcus is the diplococcus intracellularis meningitidis isolated by Weichselbaum from cases of cerebro-spinal meningitis. It occurs in the interior of leucocytes.

Such are the chief organisms associated with suppuration. In the condition known as septicomia, these organisms multiply in the

* See Trans. Jenner Inst. (First Series), A. G. R. Foulerton, F.R.C.S., pp. 40-81. 
blood, and give rise to general poisoning without abscess formation; in pyomia, however, multiple abscesses occur in various parts of the body, including internal organs. From the results of experiment it is now believed that suppuration in any form or degree is invariably the result of bacterial infection. But it is not known in what way bacteria exactly cause the condition; it may be due to extracellular toxins, or intracellular poisons, or to the bodies themselves setting up primary irritation, or to all three conditions. Positive chemiotaxis is probably the explanation of the immigration of the leucocytes.

\section{Anthrax}

This disease was one of the first in which the causal agency of bacteria was proved. In 1849 Pollender found an innumerable number of small rods in the blood of animals suffering from anthrax. In 1863 Davaine described these, and attributed the disease to them. But it was not till 1876 that Koch finally settled the matter by isolating the bacilli in pure culture and describing their biological characters.

It is owing in part to its interesting bacterial history, which opened up so much new ground in this comparatively new science, that anthrax has assumed such an important place in pathology. But for other reasons, too, it claims attention. It appears to have been known in the time of Moses, and was perhaps the disease described by Homer in the First Book of the Mliad. Rome was visited by it in 740 B.C.

Anthrax is an acute disease, affecting sheep, cattle, horses, goats, deer, and man. Cats, white rats, and Algerian sheep are immune. Swine become infected by feeding on the offal of diseased cattle (Crookshank).

Clinical Characters.-In most instances the first intimation of an outbreak of anthrax is the discovery of a dead animal in the pasture or byre. The animal may have been left a few hours earlier in apparent good health; at least, there may have been nothing to attract attention, or give warning of the near approach of death. Occasionally there are, however, premonitory symptoms of an attack of anthrax which can be recognised by an expert. The affected animal is dull, and disinclined to move. If the case occurs in a herd at pasture the fact is sometimes indicated by the separation of the sick animal from the rest. The affected animal will occasionally cease to feed, and stand with its head bent towards the ground, and sometimes a little blood is discharged from the nostrils and also with the fæces. Close attention will enable the observer to detect an occasional shiver and trembling of the limbs, which passes rapidly over the body, and then ceases. The shivering fits may then become more frequent, and perhaps, while these signs are being noted, the animal will suddenly roll over on its side, and, after a few violent struggles, expire. On close inspection, especially in the case of swine, it will often be found that there is a good deal of swelling under the throat extending down the neck; and the swollen part will at first be hot and tender to the touch, but as the disease progresses it becomes insensitive and cold. 
The post-mortem signs are mainly three: The spleen is greatly enlarged and congested, is dark red in colour, friable to the touch, and contains enormous numbers of bacilli; the skin may show exudations forming dark gelatinous tumours; and the blood remains fluid for some time after death, is black and tar-like, contains bubbles of air, and shows other degenerative changes in the red corpuscles, whilst the small blood-vessels contain such vast quantities of bacilli that they may be ruptured by them. Particularly is this true in the peripheral arteries. Many of the organs of the body show marked congestion.

The bacilli of anthrax are square-ended rods $1 \mu$ broad and 4-5 $\mu$ long. In the tissues of the body they follow the lines of the capillaries, and are irregularly situated. In places they are so densely packed as to form obstructions to the onward flow of blood. In cultures they occur in chains end to end, having, as a rule, equal

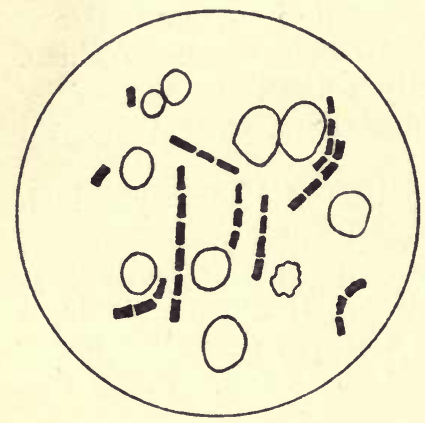

Fig. 28.-Diagram of Bacillus of Anthrax and Blood Corpuscles. interbacillary spaces. But long filaments and threads also occur. The exact shape of the bacillus depends, however, upon staining and spore formation. Both these factors may very materially modify the normal shape. The spores of anthrax are oval endospores, produced only in the presence of free oxygen, and at any temperature between 18 and $41^{\circ} \mathrm{C}$. On account of requiring free oxygen, they are formed only outside the body. The homogeneous protoplasm of the bacillus becomes granular; the granules coalesce, constituting spores. Each spore possesses a thick capsule, which enables it to resist many physical conditions which kill the bacillus. When the spore is ripe, or has exhausted the parent bacillus, it may either take on a resting stage, or under favourable circumstances commence germination, very much after the manner of a seed. The spores may infect a farm for many months; indeed, cases are on record which appear to prove that the disease on a farm in the autumn may, by means of the spores, be carried on by the hay of the following summer into a second winter. Thus, by means of the spores, the infection of anthrax may cling to the land for very long periods, even for years. Spores of anthrax can withstand 5 per cent. carbolic acid or 1-1000 corrosive sublimate for more than an hour; even boiling does not kill them at once, whilst the bacilli without their spores are killed at $54^{\circ} \mathrm{C}$. in ten minutes. When the spores are dry they are much more resistant than when moist. The persistence of the anthrax bacillus is due to its spores.

The bacillus is aërobic, non-motile, and liquefying. Broth 
cultures become turbid in thirty-six hours, with nebulous masses. of threads matted together. The pellicle which forms on the surfaces affords an ideal place for spore formation. Cultures in the depth of gelatine show a most characteristic growth. From the line of inoculation delicate threads and fibrillæ extend outwards horizontally into the medium. Liquefaction commences at the top, and eventually extends throughout the tube. On gelatine plates small colonies appear in thirty-six hours, and on the second or third day they appear, under a low power of the microscope, not unlike matted hair. The colonies after a time sink in the gelatine, owing to liquefaction. On potato, agar, and blood serum the anthrax bacillus grows well (Plates 17 and 22).

Channels of Infection. 1. The Alimentary Canal.-This is the usual mode of infection in animals grazing on infected pasture land. A soil suitable for the propagation of anthrax is one containing abundance of air and proteid material. Feeding on bacilli alone might possibly not produce the disease, owing to the germicidal effect of the gastric juice. But spores can readily pass uninjured through the stomach, and produce anthrax in the blood. Infected water, as well as fodder, may convey the disease. Water becomes infected by bodies of animals dead of anthrax, or, as was the case once at least in the south-west of England, by a stream passing through the washing-yard of an infected tannery. Manure on fields, litter in stalls, and infected earth, may all contribute to the transmission of the disease. Darwin pointed out the services which are performed in superficial soils by earthworms bringing up casts; Pasteur was of opinion that in this way earthworms were responsible for continually bringing up the spores of the anthrax bacillus from buried corpses to the surface, where they would reinfect cattle. Koch disputed this, but more recently Bollinger has demonstrated the correctness of Pasteur's views by isolating anthrax contagium from 5 per cent. of the worms sent him from an anthrax pasture. Bollinger also maintains that flies and other insects may convey the disease from discharges or carcases round which they congregate.

Alimentary infection in man is a rare form, and it reveals itself in a primary diseased state known as mycosis intestinalis, an inflamed condition of the intestine and mesenteric lymph-glands.

2. Through the Skin.-Cutaneous anthrax, when it occurs in the human subject, goes by the name of malignant pustule, and is caused by infective anthrax matter gaining entrance through abrasions or ulcers in the skin. This local form is obviously mostly contracted by those whose occupation leads them to handle hides or other anthrax material (butchers and cleaners of hides), and it naturally affects the skin of the hands, forearms, face, or back (as it occurs amongst hideporters). Two or three days after inoculation a red pimple appears, 
which rapidly passes through a vesicular stage until it is a pustule. Concomitantly, we have glandular enlargement (the pustule acting as a centre of subcutaneous œdema), general malaise, and a high temperature. Thus from a local sore a general infection may result. Unless this does occur, the issue is not likely to be fatal, and the bacilli will not gain entrance into the blood. The spleen is usually not affected, and the organs generally contain few or no bacilli. When a fatal issue occurs, it is due to the absorption of toxins. Early excision of the pustule is usually followed by recovery.*

3. Respiratory Tract.-In man, this is perhaps the commonest form of all, and is well known under the term "wool-sorters' disease," or pulmonary anthrax. This mode of infection occurs when dried spores are inhaled in processes of skin-cleaning. It frequently commences as a local lesion, affecting the mucous membrane of the trachea or bronchi, but it rapidly spreads, affecting the neighbouring glands, which become greatly enlarged, and extending to the pleura and lung itself. The lung shows collapse and œedema leading to pulmonary embarrassment. There is also fever. Such cases, as a rule, rapidly end fatally. Even in wool-sorters' disease the bacilli do not become widely distributed.

Preventive Methods.-As a rule, anthrax carcases are better not opened and exposed to free oxygen. An extended postmortem examination is not necessary. A small prick, for example, in the auricular vein will extract enough blood to examine for the anthrax bacilli, which are driven by the force of the blood current to the small surface capillaries. This occurs, of course, only when the disease has become quite general, for in the early stage the healthy blood limits the bacilli to the internal organs. In such cases examination of the blood of the spleen is necessary. The chief source of danger is the infection by anthrax blood or discharges (containing sporulating bacilli) of the field, farm-yard, byres, etc., and it is therefore necessary for thorough disinfection to be carried out if infection has occurred. Burning the entire carcase in a crematorium would be the ideal treatment. As such is not generally feasible, the next best thing is to bury the carcase deeply with lime below and above it, and rail in the area to prevent other animals grazing off it.

In the German Special Rules relating to the establishment and management of horse-hair spinning-mills, factories for hair and bristle dressing, and brush factories of all kinds, $\uparrow$ it is laid down that disinfection may be done in one of the three following ways: (1) by

* Accidental infection with anthrax has been held to be an accident to employés under the Workmen's Compensation Act, 1897 (Courts of Appeal), Justice of the Peace, May 7, 1904, vol. Ixviii., p. 193.

† Order dated October 22, 1902, under Industrial Code (Gewerbeordnung), $120^{\mathrm{e}}$. 

letting a current of steam act on the material for not less than half an hour, at a temperature of $218^{\circ} \mathrm{F}$.; (2) by boiling for not less than one hour in a solution containing 2 per cent. potassium permanganate, bleaching it afterwards with a solution containing 3 to 4 per cent. of sulphurous acid; or (3) by boiling in water for not less than two hours. A number of other regulations are included in the Order.* Various experiments have been carried out in this country with a view to determining the most effectual methods of disinfection. $\dagger$ Boiling does not appear to be always effective, and is, moreover, frequently impracticable owing to the damage it causes. In steam disinfection of hôrse hair and similar materials, a temperature of $230^{\circ} \mathrm{F}$. for 30 minutes is as effective as higher temperatures, but the hair must be loose and not closely packed in bundles. Probably one of the most practical methods for disinfection of hair is to soak it for twenty-four hours in a solution of one part of corrosive sublimate in a thousand parts of warm water (Klein). But apart from actual disinfection of the material, considerable protection is afforded by (a) the avoidance of horse hair from Russia, Siberia, and China, and wool from Persia (from which sources most infection is derived), unless it is guaranteed as thoroughly disinfected; (b) by compelling employés to wash with soap and hot water before leaving work or taking food, the more general the washing, as a rule, the greater the security obtained; $(c)$ the use of fans creating a down-draught to remove dust when sorting; and $(d)$ the exclusion of workpeople suffering from cuts or scratches of the skin from processes in which they are likely to come into contact with dust from horse hair.

Anthrax covers a wide geographical area all over the world, and no country seems altogether exempt. In Germany as many as 3700 animals have been lost in a single year. In 1903 there were 761 outbreaks of anthrax in Great Britain, in which 1127 animals were attacked. This is the largest return recorded since the passing of the Anthrax Order in 1886.

\section{Pneumonia}

Some of the difficulty which has surrounded the bacteriology of inflammation of the lungs is due to the confusion arising from supposing that attacks of the disease differed only in degree. Pneumonia, however, has various forms, arising now from one cause, now from another. The lobar or croupous pneumonia is associated with two organisms: Fränkel's diplococcus and Friedländer's pneumo-bacillus. Acute catarrhal pneumonia generally arises as a secondary complication to other disease, such as diphtheria, influenza, bronchial affections, ete. Septic pneumonias are also not

* Annual Report of Chief Inspector of Factories and Workshops, 1902, p. 214.

† Ibid., 1900, 1902, and 1903. 
specific, but secondary or mechanical. Other bacteria in addition to the two named have from time to time been held responsible for pneumonia, a streptococcus receiving, at one time, some support. But whilst opinion is divided as to the rôle of various extraneous and concomitant bacteria in lung disease, importance is attached to Fränkel's and Friedländer's organisms.

The diplococcus of Fränkel is a small oval diplococcus found in the "rusty" sputum of croupous pneumonia. It is non-motile, non-liq̉uefying, aërobic, and facultatively anaërobic. When examined from cultures the diplococci are frequently seen in chains, not unlike a streptococcus, and there is some reason to suppose that this form gave rise to the belief that it was another species; when examined from the tissues, sputum, or pus, it possesses a capsule, like an unstained halo (stained by MacConkey's method), but in culture this is lost except in gelatine at $37^{\circ} \mathrm{C}$. (Gordon). Involution forms occur. The diplococcus is difficult to cultivate, but grows on glycerine agar and blood serum at body temperature. . On ordinary gelatine at room temperature it does not grow, or if so, very slightly. The ideal fluid is a slightly alkaline liquid medium, and in twenty-four hours a powdery growth will occur in such broth. On potato there is apparently no growth. The pneumococcus always requires a temperature of about blood-heat for its maximum development. It rapidly loses its virulence on solid media, and is said to be non-virulent after three or four sub-culturings. A temperature of 54-58 ${ }^{\circ}$ C. for a few minutes kills the bacteria, but not the toxin. This, however, is removed by filtration, and is therefore probably intracellular. It is attenuated by heating to $70^{\circ} \mathrm{C}$. This diplococcus stains by Gram's method (see Plate 22, p. 318).

Fränkel's diplococcus occurs, then, in the acute stage of true croupous pneumonia, in company with streptococci and staphylococci. It is by far the most frequently present organism in croupous pneumonia. It also occurs in the blood in certain suppurative conditions, in pleurisy and inflammation of the pericardium, and sometimes in diphtheria, and therefore it is not peculiar to pneumonia.

Fränkel's organism is said to be frequently present in the saliva of healthy persons. Inflammation depresses the resistant vitality of the tissues, and thus affords to the diplococcus present in the saliva an excellent nidus for its growth.*

Friedländer's Pneumo-bacillus is a capsulated oval coccus, assuming the form of a small bacillus. It is inconstant in pneumonia, unequally distributed, and scarce; it is aërobic, and facultatively anaërobic;

* For further particulars respecting the pneumococcus, see Practitioner, March 1900, pp. 280-304 (J. W. Eyre); and Brit. Med. Jour., 1902, vol. ii., pp. 1585, 1646, 1704, 1765 (Croonian Lectures on Natural History and Pathology of Pneumonia, by J. W. Washbourn). 
it occasionally occurs in long forms and filaments; it is non-motile, non-liquefying, and has no spores; it does not stain by Gram's method, which stain is therefore used for differential diagnosis ; it will grow fairly well in ordinary gelatine at $20^{\circ} \mathrm{C}$; ; and it is a denitrifying organism, and also an actively fermentative one, even fermenting glycerine. It is not unlike $B$. coli communis, and to distinguish it from that organism it should be remembered that the $B$. coli is motile, never has a capsule, produces indol, and does not ferment glycerine.

It is now generally held that Fränkel's diplococcus is the chief factor in the causation of croupous pneumonia, and probably plays an important part in other forms of the disease. In the septic pneumonias the different suppurative organisms are found, and sometimes in ordinary pneumonias these organisms may be the causal agents.

\section{Influenza}

In 1892, during the pandemic of influenza, Pfeiffer discovered a bacillus in the bronchial mucus of patients suffering from the disease. It is one of the smallest bacilli known, and frequently occurs in chains not unlike a streptococcus. Canon obtained the same organism from the blood. In the bronchial expectoration it can retain its virulence for as long as a fortnight, but it is quickly destroyed by drying. The bacillus is aërobic, nonmotile, and up to the present spores have not been found. It is non-motile, and does not stain by Gram's method. It has no capsule. It grows somewhat feebly in artificial media, and readily dies out. Blood serum, glycerine agar, blood agar, and gelatine have all been used at blood-heat. It does not grow at room temperature. On blood agar colonies appear in twenty-four hours in the form of minute circular dots, almost transparent. The bacilli die out quickly in cultures. Pfeiffer's bacillus appears most abundantly at the height of the disease, and disappears with convalescence. It is said not to appear in any other disease. It is chiefly found in the respiratory passages in cases of influenza, and is usually isolated from nasal secretion and the masses of greenish-yellow bronchial sputum. The bacilli may persist after recovery of the patient.

\section{Actinomycosis}

This disease affects both animals and man. As Professor Crookshank has pointed out, it has long been known in this country, but its various manifestations have been mistaken for other diseases or have received popular names.*

* Bacteriology and Infective Diseases (1896), pp. 413-447. Professor Crookshank's Reports to the Agricultural Department of the Privy Council constitute a most complete account of this disease. See also Trans. Jenner Institute (Second Series), 1899 , p. 17. 
Here mention can only be made of the most outstanding facts concerning the disease. It is caused by the "ray fungus," or Streptothrix actinomyces, one of the higher bacteria which, growing on certain cereals, may gain entrance to the tissues of man and beast by lacerations of the mucous membrane of the mouth, by wounds, or by decayed teeth. Barley has been the cereal in question in some cases. The result of the introduction of the parasite is an "infective granuloma." This is, generally speaking, of the nature of an inflammatory tumour composed of round cells, epithelioid cells, giant cells, and fibrous tissue, forming nodules of varying sizes. In some cases they develop to large tumours, in others they soon break down. Actinomycosis resembles tuberculosis in some of its tissue characters.

In the discharge or pus from human cases of the disease small sulphur-yellow bodies may be detected, and these are tufts of "clubs" which are the broken-down rays of the parasite; for in the tissues which are affected the parasite arranges itself in a radiate manner, growing and extending at its outer margin and degenerating behind. In cattle the centre of the old ray becomes caseated, or even calcified. In the human disease abundant "threads" are formed as a tangled mass in the middle of the colony. As clubs characterise the bovine actinomycosis, so threads are the feature of the human form of the disease. But in both there is a third element, namely, small round cells, called by some spores, by others simply cocci. They are probably formed from the filaments, but authorities are not yet agreed as to the precise significance and rôle of these round cells. The life-history of the micro-organism may be summed up thus: "The spores sprout into excessively fine, straight or sinuous, and sometimes distinctly spirilliform, threads, which branch irregularly and sometimes dichotomously. The extremities of the branches develop the club-shaped bodies. The clubs are closely packed together, so that a more or less globular body is formed, with a central core composed of a dense mass of threads" (Crookshank). (Plate 13, p. 140.)

In man the disease manifests itself in various parts according to the point of entrance. It has occurred in the mouth, vertebræ, osophagus, intestine, liver, kidneys, lungs, etc. When occurring in the mouth, it attacks the lower jaw most frequently. In one recorded case the disease was localised to the bronchi, and did not even extend into the lungs. It was probably contracted by inhalation of the parasite. The disease may spread to distant parts by means of the blood stream (metastatic abscesses), and frequently the abscesses are apt to burrow in various directions. The chronic inflammatory change usually ends in suppuration.

In the $o x$ the discase remains much more localised, is more formative, and frequently occurs in the lower jaw, palate, or tongue. 
In the latter site it is known as "wooden tongue," owing to the hardness resulting. The skin and subcutaneous tissues are also a favourite seat of the disease, producing the so-called wens or clyers so commonly seen in the fen-eountry (Crookshank). Actinomycosis in eattle is especially prevalent in river valleys, marshes, and on land reelaimed from the sea. The disease oceurs at all seasons, but perhaps more commonly in autumn and winter. It is more frequently met with in young animals. The disease is probably not hereditary nor readily communieated from animal to animal.

The Streptothrix Actinomyces may be cultivated, like other parasites, outside the body. Gelatine, blood serum, agar, glyeerine agar, and potato have been used for this purpose. After a few days on glycerine agar at the temperature of the blood, small, white, shining colonies appear, which increase and coalesce. In about ten days' time the culture often turns a bright yellow, though it may remain white or even take on a brown or olive tint. The entire mass of growth is raised, dry, corrugated, and erinkled, and composed almost exclusively of threads. In its early stage small baeillary forms occur, and in its later stage coccal forms. True clubs never occur in pure cultures, although the threads may occasionally show bulbous endings.

\section{Glanders}

Glanders in the horse and ass, and sometimes by communication in man also, is caused by a short, non-motile, aërobic bacillus, named, after the old Roman nomenclature (malleus), Bacillus mallei. It was diseovered in 1882 by Löffler and Schütz. It is found in the nasal discharge of glandered animals. In appearanee, the bacillus is not unlike B. tuberculosis, except that it is shorter and thicker. The beading of the bacillus of glanders, like that in tubercle, does not denote spores. B. mallei can be cultivated on the usual media, especially on glycerine agar and potato. On the last-named medium at blood-heat it forms a very characteristic honey-like growth, which later becomes reddish-brown. High temperature is usually necessary.

In the horse glanders may affect the nasal mueous membrane, forming nodules which degenerate and emit an offensive discharge. From the nose, or nasal septum, as a centre, the disease may spread to surrounding parts. It may also occur as nodules in and under the skin, and involving the superficial lymph vessels and glands, when it is known as "farcy." Persons attending a glandered animal may contract the disease, often by direct inoculation. Horned cattle are immune.

In man glanders occurs in two forms, an acute and a ehronic. The site is, of course, usually on the hand or arm. The aente form has the appearanee of a "poisoned wound," locally, and there are 
also the general symptoms of pyæmia, and an eruption on the surface of the body. Such cases usually terminate fatally. The chronic form results in local ulceration and involvement of the lymphatics. It may at any time become acute.

The glanders bacillus is not quickly destroyed by drying, but it possesses comparatively feeble resistance to heat $\left(55^{\circ} \mathrm{C}\right.$. for ten minutes), and antiseptics ( 5 per cent. carbolic in three minutes). It differs widely from the tubercle bacillus in staining properties. Gram's method and that of Ziehl-Neelsen are inapplicable. Carbolthionin blue is the best stain to use. (Plate 13, p. 140.)

Mallein is a substance analogous to tuberculin, and is made by growing a pure culture of $B$. mallei in glycerine-veal broth in flat flasks, with free access of calcined air. After a month's growth the culture is sterilised, filtered, concentrated, and mixed with an equal volume of a 5 per cent. solution of carbolic acid. The dose is 1 c.c., and it is used, like tuberculin, for diagnostic purposes. If the suspected animal reacts to the injection, it is suffering from glanders. Reaction is judged by three signs, $(a)$ a rise of temperature $2-3^{\circ} \mathrm{C}$., (b) a large "soup-plate" swelling at the site of inoculation, and (c) an enlargement of the lymphatic glands.

In 1903 there were in Great Britain as many as 1463 outbreaks of glanders in which 2490 horses were attacked. This is the highest number of outbreaks since 1892, when they numbered 1657. The prevalence of the disease is localised often to certain counties and districts. In 1903, 855 of the 1463 outbreaks occurred in the county of London. 


\section{CHAPTER X}

\section{TUBERCULOSIS AS A TYPE OF BACTERIAL DISEASE}

Pathology and Bacteriology of Tuberculosis-The Bacillus of Koch-Animal Tuberculosis, Bovine, Avian, etc.-Bovine and Human Tubercle Bacilli compared -Intercommunicability-Diagnosis of Bovine Tubercle-The Prevention of Tuberculosis-Pseudo-Tuberculosis-Acid-fast Bacteria Allied to the Tubercle Bacillus : in Man, in Animals, in Butter and Milk, in Grass-Differential Diagnosis-Streptothrix Group.

Tuberculosis is from several points of view the type of bacterial disease which most concerns the public health. Its bacteriology is perhaps more worked out than that of any other disease. Its prevalence in all parts of the world, and among animals as well as man, makes it a disease of vital importance and interest to man. Moreover, the growth of our knowledge respecting it has led to the introduction of methods of prevention, and the world is beginning to understand that a scientific control of this disease is becoming possible. For these reasons it is desirable to treat somewhat fully of the chief facts respecting it.

\section{Pathology and Bacteriology*}

As far back as 1794, Baillie drew attention to the grey miliary nodules occurring in tuberculous tissue, which gave rise to the term "tubercles." This observation was confirmed by Bayle in 1810. In 1834 Laennec described all caseous deposits as "tubercles," insisting upon four varieties:-

* A detailed study of tuberculosis from its pathological and bacteriological aspect will be found in $L a$ Tuberculose et son Bacille, part i., Straus, Professor à la Faculté de Médecine de Paris; and in Tuberculosis, by Professor Cornet, edited by W. B. James and A. Stengel, 1904. 
1. Miliary, which were about the size of millet seeds, and generally occurring in groups.

2. Crude, miliary tubercles in yellow masses.

3. Granular, similar to the last, but scattered.

4. Encysted, a hard mass of crude tubercle with a fibrous or semi-cartilaginous capsule.

The "tubercle" possesses a special structure, although it is not always apparent, and certain cell-forms occur in it and give it a more or less characteristic appearance.

The typical lesion is a nodule of granulation tissue, as small as the size of a millet seed. The centre consists of one or more multinucleated cells known as giant cells, immediately surrounded by a zone of slightly elongated cells with a somewhat faintly-staining nucleus, termed epithelioid cclls, owing to their origin. These cells in their turn are surrounded by another zone of small round cells which have but little cell protoplasm, yet contain a deeply-staining nucleus, and are known as lymphoid cells. They are apparently identical with lymphocytes. The whole nodule is inflammatory tissue produced as a result of the action of a specific irritant, namely, the tubercle bacillus.

It was not till 1865 that the specific nature of tuberculosis was asserted by Villemin. Burdon Sanderson (1868-9) in England confirmed his work, and it was extended by Cohnheim, who a few years later laid down the principle that all is tubercular which by transference to susceptible animals is capable of inducing tuberculosis, and nothing is tubercular unless it possesses this property.

Klebs (1877) and Max Schiller (1880) described masses of living cells or micrococci in many tuberculous nodules in the diseased synovial membrane of joints and in lupus skin. In 1881 Toussaint declared that he had cultivated from the blood of tubercular animals and from tubercles an organism which was evidently a micrococcus, and in the same year Aufrecht stated that the centre of a tubercle contained small micrococci, diplococci, and some rods. But it was not till the following year, 1882, that Koch discovered and demonstrated beyond question the specific Bacillus tuberculosis.

It is now held to be absolutely proved that the introduction of this bacillus, or its spores, is the one and only essential agent in the production of tuberculosis. Its recognised manifestations in the body of man are as follows:-Tuberculosis in the lungs = acute or chronic phthisis; in the skin = lupus; ${ }^{*}$ in the mesenteric glands $=$ tabes mesenterica; in the brain =hydrocephalus; in lymphatic glands $=$ scrofula ${ }^{*}$

The disease may occur generally throughout the body, or it may

* There are, obviously, differences of virulence between these conditions and pulmonary tubercle. 
occur locally in the lungs, liver, glands, intestine, larynx, bones, kidneys, spleen, and other parts. thus:-

We may summarise the history of the pathology of tubercle

1794. Baillie drew attention to grey miliary nodules occurring in tuberculosis, and called them "tubercles."

1834. Laennec described four varieties: miliary; crude; granular ; encysted.

1843. Klencke produced tuberculosis by intravenous injection of tubercular giant cells.

1865. Villemin demonstrated infectivity of tubercular matter by inoculation of discharges; Cohnheim, Armanni, Burdon Sanderson, Wilson Fox, and others showed that nothing but tubercular matter could produce tubereulosis.

1877. Living cells were found in tubercles, "micrococci" (Klebs, Toussaint, Schiller).

1882. Koch isolated and described the specific bacillus, and obtained pure cultivations (1884).

\section{The Bacillus of Koch}

Biology. - The B. tuberculosis of artificial culture is usually an unbranched, slender, immotile rod, 1.5 to $4 \mu$ long and $\cdot 4 \mu$ broad, often slightly bent. In sputum and tissues the bacillus may appear branched and in thread forms. The protoplasm of the bacillus consists of fat and wax (26 per cent.), protamin (24 per cent.), nucleoproteid (23 per cent.), nucleic acid (8 per cent.), and the remainder of mineral and proteinoid (chitin) substances. The protoplasm is frequently vacuolated and irregularly segmented, and this becomes particularly obvious after staining. As to staining, the bacillus is acid-proof, and stains well with Ziehl-Neelsen or Gram. Klein and Marmorek have shown that very young tubercle bacilli are not resistant to acid and alcohol. Growth does not occur in the absence of oxygen, is most favoured by a temperature varying from $29^{\circ} \mathrm{C}$. to $42^{\circ} \mathrm{C}$., and is at all times slow on artificial media. In sputum and in tissues it will be found that many of the bacilli are straight with rounded ends; others are slightly curved. They are usually solitary, but may occur in pairs, lying side by side or in small masses. They are chiefly found in fresh tubercles, more sparingly in older ones. Some lie within the giant cells; others lie outside. When stained, they appear to be composed of irregular cubical or spherical granules within a faintly-stained sheath. In recent lesions the protoplasm appears more homogeneous, and only takes on the segmented or beaded character in old lesions, pus, or sputum. As a rule, the capsule stains. There are no flagella. So far as is known, 
the bacillus tuberculosis discovered by Koch is the only immediate cause of all forms of human tuberculosis. In the majority of cases the micro-organism is met with in the form of slender rods, but under certain conditions at present imperfectly understood, the micro-organism may show filaments, true dichotomous branching and club formation, and, in the tissues, especially in experimental tuberculosis, it may assume a radiate arrangement-characters which from a taxonomic point of view bring it into close relation with a large group of microorganisms variously designated Streptothriceæ, Oospora, Nocardiaceæ, and more recently Actinomycetes (Lachner-Sandoval). According to all experience, the tubercle bacillus is an aërobic facultative parasite, which grows extremely slowly outside the body, and the fact that for its growth it requires a relatively high temperature is against the supposition that the tubercle bacillus multiplies extra-corporeally, at least in temperate climates.

Morphological differences are found under different circumstances, and within limits variation occurs according to the environment. The filaments, threads, and true branching forms of old cultures have been met with, though only occasionally, in sputum. Clubbed actinomycotic forms have also been described. On these facts some bacteriologists are disposed to look upon the tubercle bacillus as belonging to the higher bacteria (Plate 18).

Cultivation on Various Media.-Koch inoculated solid blood serum with tubercular matter from an infected lymphatic gland of a guineapig, and noticed the first signs of growth in ten or twelve days in the form of whitish, scaly patches. These enlarged and coalesced with neighbouring patches, forming white, roughened, irregular masses. The blood serum is not liquefied. Nocard and Roux showed that by adding 5 to 8 per cent. of glycerine to the media commonly used in the laboratory, such as nutrient agar or broth, better growth is obtained. In glycerine broth abundant growth appears at the end of seven or eight days, and eventually cultures taken from glycerine broth will be found to grow well in ordinary bouillon. A pellicle generally forms. On glycerine agar, minute crumb-like colonies of whitishyellow colour appear in six to twelve days. Later, the whole growth turns browner in colour, and is sometimes dry, sometimes moist, in appearance depending on age of culture, and consistence of medium. Ultimately, the discrete colonies coalesce and form a lichenous growth. By continuous sub-culture on glycerine agar the virulence of the bacillus is diminished. But in fifteen days after inoculation of the medium the culture equals in extent a culture of several weeks' age on blood serum. In alkaline broth to which a piece of boiled white of egg was added, Klein obtained copious growth, and found that continued sub-culturing upon this medium also lessens the virulence. On potato the tubercle bacillus grows well in crumb-like masses. 


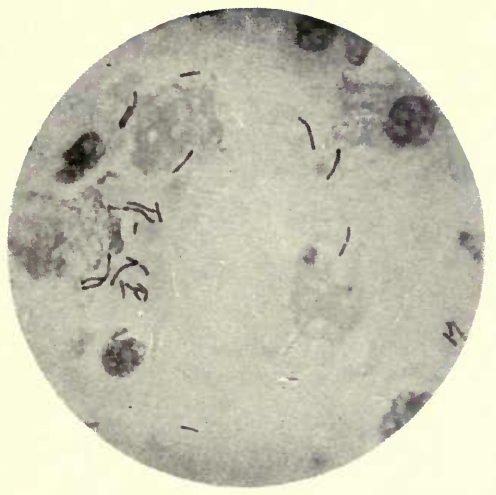

Bucillus tuberculosis.

In sputum from a case of human phthisis. Stained by Ziehl-Neelsen method. $\times 1000$.

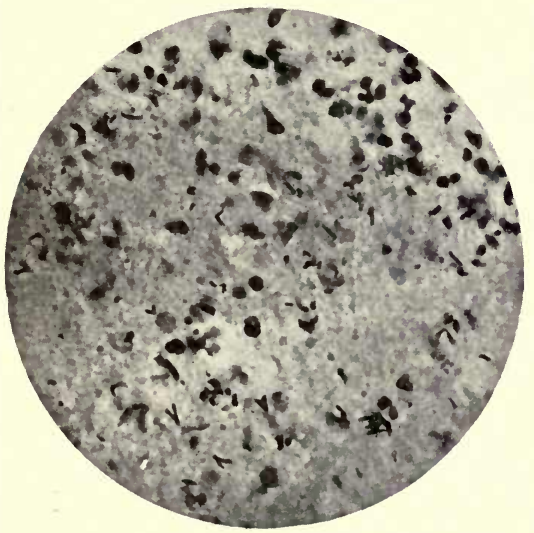

Bacillus tuberculosis.

From edge of caseous patch in human lung. $\times 750$.

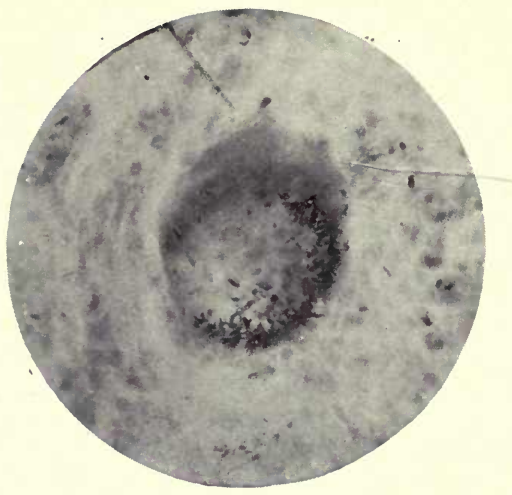

Bacillus tuberculosis.

Giant cell. Bacilli in situ within the cell. Stained by Ziehl-Neelsen method.

$\times 1000$.

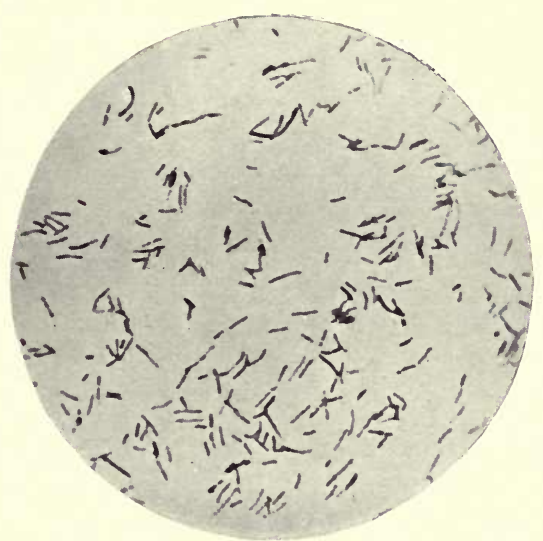

Bacillus tuberculosis.

Film preparation from glycerine-glncose-agar culture, 4 weeks at $37^{\circ} \mathrm{C}$. Stained with carbol fuchsin. $\times 1000$. 


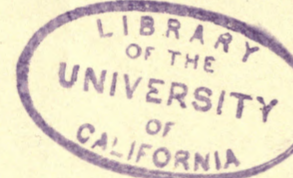


Spore formation.-In very old cultivations spore-like bodies can be observed both in stained and unstained preparations, but neither the irregular granules within the capsule nor the unstained spaces between the granules are spores (Babes and Crookshank). That the bacilli probably possess spores is believed on account of their behaviour under certain circumstances. For example, tubercular sputum when thoroughly dried retains its virulent character. Even cultures of tubercle artificially dried retain their virulence. Now, no sporeless bacillus is known at present which can withstand thorough desiccation. Again, non-spore-bearing bacilli are killed with a less exposure to heat than that which is required to destroy tubercular sputum. Koch, Lingard, Klein, and others long ago pointed out the resistance of the bacilli of tubercle to solutions of perchloride of mercury and to heating in suspension in salt solution, whilst sporeless bacilli succumbed to the same treatment. So that it is commonly believed that $B$. tuberculosis produces spores, even though such have not been demonstrably proved.

Koch and other bacteriologists have declared the bacillus to be a "true parasite." Koch based this view upon the belief which he entertained that the bacillus can only grow between $30^{\circ} \mathrm{C}$. and $41^{\circ} \mathrm{C}$., and therefore in temperate zones is limited to the animal body, and can only originate in an animal organism. "They are," he said, "true parasites, which cannot live without their hosts. They pass through the whole cycle of their existence in the body." But at length Koch and others overcame the difficulties and grew the bacillus as a saprophyte. Schottelius * has observed that tubercle bacilli taken from the lung of phthisical persons buried for years still retains its virulence and capability of producing tuberculosis upon inoculation. He further showed that tubercular lung kept in soil (enclosed in a box) revealed a marked rise in temperature. Klein quotes these experiments as indications that "tubercle bacilli are not true parasites, but belong to the ectogenic microbes which can live and thrive independently of a living host."

It has now been abundantly proved that the tubercle bacillus is capable of accommodating itself to circumstances much less favourable than had been supposed; as regards temperature and environment. For it is now known that it is possible to grow the bacillus upon glycerine agar at $28^{\circ} \mathrm{C}$. $\left(82^{\circ} \mathrm{F}\right.$.), obtaining an ample culture which develops somewhat more slowly than on blood serum, and to a less extent than at $37^{\circ} \mathrm{C}$. Sheridan, Delépine, Czaplewski, Ransome, Beevor, and others have also been successful in obtaining growths at room temperature both in summer and winter. Moeller succeeded in growing the bacillus at $20^{\circ} \mathrm{C}$., after passing it through a blindworm.

Centralblatt. f. Bact, und Parasit, vol, vii., p. 9. 
The Relation of the Bacillus to the Disease.-Having considered the structure of "tubercles" and the chief biological facts of the tubercle bacillus, we may now ask: How does the bacillus set up the changes in normal tissues which result in tubercular nodules? In arriving at a solution of this problem, we are materially aided if we bear in mind the fact that when such an organism is present in the tissues it has a double effect. First, there is an ordinary inflammatory irritation; and, secondly, there is a specific change set up by the toxins of the bacillus. Many authorities believe that the process is, generally speaking, as follows:-Directly the invading bacilli find themselves in a favourable nidus they commence multiplication. In the course of a few days this acts as an irritant upon the surrounding connective-tissue cells, which proliferate, and become changed into the large cells known as epithelioid cells. At the periphery of this collection of epithelioid cells, we have a congested area filled with lymphocytes drawn thither by the process of inflammation and constituting the zone of lymphoid cells. The production of the bacillary poisons changes the epithelioid cells in the centre of the nodule, some of which become fused together, whilst others expand and undergo division of nucleus. By this means we obtain a series of large multinucleated cells, giant cells. Thus is formed the typical "tubercle." But if the disease is very active, this soon caseates and breaks down in the centre. In a limb we get a discharge; in a lung we get an expectoration. Both discharge and expectoration arise from a breaking down of the new cell formation. Previously to breaking down we have in a fully developed nodule commencing at the periphery where the normal tissue is, healthy tissue, then the inflammatory zone of lymphoid cells, then epithelioid cells, and in the centre giant cells, containing nuclei and bacilli. The sputum or the discharge will, during the acute stage of the disease, at all events, contain countless numbers of the bacilli, which may thus be readily detected, and their presence used as evidence of the disease. It is obvious that if the centre of the nodule degenerates and comes away as a purulent discharge, a cavity will be left behind. By degrees this small cavity becomes enlarged, as is frequently the case in the lung, which particularly lends itself to such a condition. Hence, though at the outset the affected part of a tubercular lung becomes solid, ultimately the affected part becomes a cavity, unless repair sets in, and by growth of fibrous tissue the commencing cavity is obliterated.

The exact period of giant cell formation depends on the rapidity of the formative inflammatory processes. Thus different conditions occur. Giant cells are a constant feature of interstitial tubercles in connective tissue, but in uncomplicated caseous tubercular pneumonia there may not be found a single giant cell in 
a whole lung. Some authorities look upon giant cell formation as a sign of chronicity of the process. Further, in some of the lower animals, the giant cells become packed with tubercle bacilli, while in man it frequently occurs that few or none at all are found. When the giant cells do contain bacilli they are usually arranged in one of four ways: $(a)$ polar, $(b)$ zonal, $(c)$ mixed, or $(d)$ at the periphery of the giant cell. The breaking down of the nodule is partly due to the bacterial poisons, and partly to the nodule being non-vascular, owing to the fact that new capillaries cannot grow into the dense nodule, and the old ones are occluded by the growth of the nodule.

At first the disease is local, owing to the unfavourable action of the blood, to phagocytic action, or to the fewness of the number of bacilli absorbed. From the local foci of disease the tuberculous process spreads chiefly by three channels:-

(a) By the lymphatics, affecting particularly the glands. Thus we get tuberculosis set up in the bronchial, tracheal, mediastinal, and mesenteric glands, and so frequently present as to be a characteristic of the disease. This is the common method of dissemination in the body, and by this channel the virus of tuberculosis is carried along with the stream of lymph and infects progressively the lymph vessels and glands. It may also be propagated along the lymphatics in an opposite direction to the lymph stream.

(b) By the blood-vessels, by means of which bacilli may be carried to distant organs. But this channel is comparatively rare. Blood is not a favourable medium for the tubercle bacillus.

(c) By continuity of tissues, that is by infective giant cell systems encroaching upon neighbouring tissues, or discharge from lungs or bronchial glands obtaining, for example, entrance to the gullet and thus setting up intestinal disease.

It has been abundantly proved that the respiratory and digestive systems are those principally affected by the tubercle bacillus. Wherever the bacilli are arrested, they excite formation of granulations or miliary tubercular nodules, which increase and eventually coalesce. The lymphatic glands which collect the lymph from the affected region are earliest affected, always the nearest first, and for a time the disease may appear to be appreciably stopped on its invading march. Each lymphatic gland acts as a temporary barrier to progress until the disease has broken its structure down. It remains "local," in spite of increase in number and importance of the foci of disease, as long as the bacilli have not gained access to the body generally.

Channels of Infection.-The common methods of invasion by 
which the tubercle bacillus gains access to the human body are three, namely, through the skin, and through the alimentary and respiratory systems. A great variety of cases of skin infection are on record, although the uninjured epidermis affords a fairly reliable protection, so that simple contact with tuberculosis sputum does not suffice to produce infection if the skin be uninjured. The exact means and occasion of entry are innumerable. Wounds play a great part in rendering possible the invasion by tubercle bacilli. Infection by the alimentary tract takes place in a variety of ways. The bacilli may be carried in with air in mouth-inspiration, by dirty objects placed in the mouth (in children), by kissing tuberculous persons, or by the ingestion of infected food. Thus, we may have tuberculosis of the mouth and tonsils, of the stomach, and of the intestine and other abdominal organs, including the mesenteric glands. Elsewhere we remark upon the comparative rarity of primary abdominal tuberculosis in man, though the disease is more common in animals.

The chief channel of infection is, of course, the respiratory tract, and the two means by which tubercle bacilli thus reach the body are $(a)$ inhalation of the dust of dried tuberculous sputum, and (b) the inhalation of moist particles from the cough-spray of a phthisical patient. Wherever tuberculous sputum is allowed to dry the risks are great that the dust so produced may be inhaled in a virulent form, and lodging at one or more points may set up varying degrees of tuberculosis. This broad fact is based upon experimental and clinical evidence. Tuberculosis has been produced experimentally in animals in this way, and there is clinically the overwhelming frequency of tuberculosis of the lungs among men exposed to just such a manner of infection. But Koch, Flügge, and others have shown that not only is sputum a source of infection when dried and pulverised, but also when disseminated by coughing, shouting, etc., in the form of minute moist particles of spray. Koch exposed rabbits, guinea-pigs, rats, and mice to an infected spray for half an hour on three successive days, and produced tuberculosis in every animal. Heymann found that such spray particles from human beings inoculated into guinea-pigs produced tuberculosis. Most of the droplets are large and settle rapidly, but some may remain suspended in the air for more than an hour, retaining, of course, their virulent properties. Heymann found the duration of life of the bacilli in these droplets was eighteen days in the dark, and three days when exposed to light. Under ordinary circumstances and an absence of draughts, the zone of danger from a coughing consumptive extends to a distance of about three feet. It must be remembered that the tubercle bacilli in the moist particles of cough-spray. are probably of higher virulence than those in dried sputum dust, and therefore it seems reasonable to suppose that the cough-spray is the most 
dangerous channel of infection in tuberculosis.* At the same time experience shows that the degree of infectivity of phthisis is not a very high one. It is a truly infective disease, but not an extremely infectious disease. It may be rightly described as sub-infectious. $†$

Toxins of the Tubercle Bacillus.-Many investigators have isolated products from pure cultures of the tubercle bacillus. These have comprised chiefly albumoses, alkaloids, various extractives, and inorganic salts. Koch isolated "tuberculin" from cultures of tubercle bacillus upon glycerine broth by means of evaporation and precipitation with alcohol. Buchner obtained by trituration and compression of fresh tubercle bacilli a substance termed "tuberculo-plasmine." But of the real nature of the toxins of the tubercle bacillus little is known.

\section{Bovine Tuberculosis}

Cattle come first amongst animals liable to tubercle. Horses may be infected, but it is comparatively rare, and among small ruminants the disease is rarer still. Dogs, cats, and kittens may be easily infected. Amongst birds, fowls, pigeons, turkeys and pheasants the disease assumes almost an epidemic character. Especially do animals in confinement die of tubercle, as is illustrated in zoological gardens.

Bovine Tuberculosis.-Respecting the lesions of bovine tuberculosis, it will be sufficient to say that nothing is more variable than the localisation or form of its attacks. The lungs and lymphatic glands come first in order of frequency, next the serous membranes, then the liver and intestine, and lastly the spleen, joints, and udder (Nocard). The anatomical changes in bovine tubercle are mostly found in the lungs and their membranes, the pleuræ. It also affects the abdomen and its chief organs, the peritoneum, and the lymphatic glands. In both of these localities a characteristic condition is set up by small grey nodules appearing on the pleura and peritoneum, the nodules, increasing in size, giving an appearance of "grapes." Hence the condition is called grape disease, or Perlsucht. The organs, as we have said, are equally affected, and when we add the lymphatic glands we have a fairly complete summary of the form of the disease as it occurs in cattle. In about half of all cases the lungs and serous membranes become simultaneously affected, in about one-third the lungs alone; and in about one-fifth the serous membranes alone (Friedberger and Fröhner). As has been pointed out by Martin,

* For a discussion on the channels of infection in tuberculosis, see Carnet, Tuberculosis, 1904, pp. 96-282; Flügge, Zeitschrift für Hyg. u. Jufek., Band xxxviii., 1901.

† Koch, Etiology of Tuberculosis; in Brit. Med. Jour., 1903, i., p. 593 (Hillier), will be found a useful summary of modern views on the question. 
Woodhead, and others in their evidence before the Royal Commission, the organs, glands, and membranes are the common sites for tubercle, not the muscles (or meat).

The following table records the findings of Geddes, who in 19011902 was sent by the American Government to examine by means of tuberculin some of the chief breeds of British dairy cattle.*

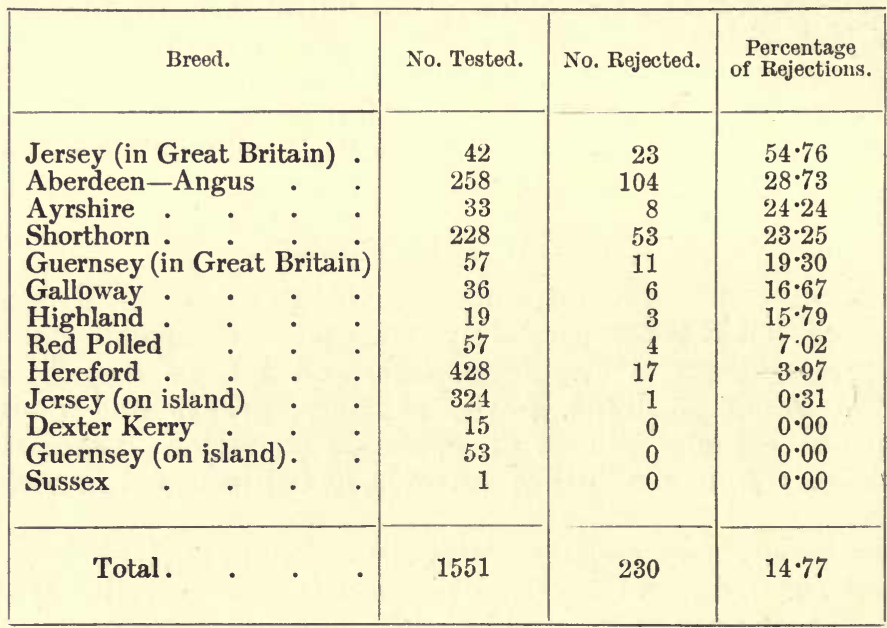

Eliminating the tests on Guernsey and Jersey, the proportions of reactions among the tests made in Great Britain and Ireland were as follow:-in 1901, 13.67 per cent.; in 1902, 20.97; and for both years, 17.92. Hopkins examined 571 Shorthorns and found the percentage of positive reaction was 23.0 as compared with Geddes's result of $23 \cdot 25 . \dagger$

Tuberculosis of the udder is comparatively rare. Out of 100 tuberculous cows not more than 3 or 4 have tuberculosis of the udder (Bang). The disease occurs as a diffuse, slightly hard, enlargement, generally unaccompanied by fever or tenderness of the organ. Usually only one quarter is attacked, and that generally a posterior quarter. The gland lobules become hypertrophied, and the larger milk-ducts contain yellowish caseous masses full of bacilli. As the condition advances, there is a considerable increase of the interlobular connective tissue (interstitial mastitis) of the nature of a sclerosis, and firm tubercles of various sizes begin to appear. Consequent upon these changes the udder becomes nodular, and hard

* Nineteenth Annual Report of the Bureau of Animal Industry, 1902, p. 551.

$\uparrow$ Report of Minister of Agriculture, Dominion of Canada, 1902, p. 134. 
and tough. Miliary tubercles appear in the walls of acini, and enormous deposits of bacilli may be found in the udder. Simultaneously with these changes, the mammary lymphatic glands (pudic glands) lying above the posterior region of the udder became enlarged, indurated, and caseous. The disease may advance slowly or with great rapidity. But finally the condition is such that the glandular tissue of the udder is, as it were, smothered by the hypertrophy and fibrous transformation of the interstitial connective tissue. The large excretory ducts become blocked by granulations or fibrous growth outside them, or by caseous masses inside. This stage inevitably leads to milk suppression (see also p. 203).

It should not be forgotten that tuberculosis of the udder is associated with tuberculosis of the internal organs. It is almost invariably secondary. It may exist with mild or advanced disease of the internal organs. Its diagnosis is all the more difficult owing to the fact that there may be no symptoms. Generally, opinion must be guided by the local condition of the udder, coupled with the condition of the milk. It may occur as a slow, painless growth only evident when advanced, or it may increase with extraordinary rapidity. This latter fact makes it desirable that every animal suffering from tuberculosis of however mild a character should be strictly eliminated from dairy stock. The three points usually emphasised for diagnosis of tuberculous udder disease are- $(a)$ abnormal milk from one quarter, generally a posterior quarter; $(b)$ some hardness, toughness, or irregularity of the udder; and $(c)$ enlargement of supra-mammary glands.* The best diagnostic of general tuberculosis is the tuberculin reaction.

Changes in Mille from a Tuberculous Udder.-One of the first signs of abnormality is the diminution in the yield. Previously to this it is said there is an actual increase in the quantity of milk. As soon as the disease begins to have effect, there is a definite decline in the yield. For example, a cow which in health gave, say, fifteen litres of milk, falls to one half or one quarter of that amount. The milk also changes in consistence, becoming thin, watery, and serous. At the same time the colour may turn to yellow, and the flocculi and flakes which occur in milk from a healthy udder are present in larger size. As the yield diminishes, the consistence of the fluid becomes more and more irregular, the flocculi predominating. If such milk be allowed to stand in a vessel, a deposit of solid matter, composed of these fragments, settles down, leaving a superficial layer of thin fluid at the top. Finally, the consistence becomes seropurulent and then purulent. Hence, previously to suppression we get a thick yellow purulent fluid, having an alkaline reaction, coagulated casein, and diminution of lactose. As a rule, tubercle * See also Reporl of Royal Commission on Tuberculosis, 1896, part iii., pp. 41, 42. 
bacilli are readily found, and whether that be so or not the milk is highly infective.*

The Entrance of the Bacillus into Milk.-There are two main sources of the tubercle bacilli found in milk, namely, a bovine source and a human source. The two common channels respectively are a tuberculous udder and a phthisical lung. From the former, milk may derive a direct and abundant supply of tubercle bacilli; from the latter, milk may become indirectly contaminated by the particulate matter of dried sputum.

Tuberculosis may be introduced into healthy cows in a variety of ways. The most common method is by means of a tuberculous animal, from the excretions and discharges of which infection may be conveyed to soil, water, air, fodder, and general surroundings. In this way not only other animals cohabiting with a tuberculous animal become infected, but premises, stables, and utensils may also become infected. The milk of a tuberculous animal may also be consumed by other animals on the farm, and so a vicious circle of infection is completed. Ravenel has shown that by the cough of a tuberculous cow tubercle bacilli may be distributed. Of thirty-four examinations carried out on five tuberculous cows, tubercle bacilli were detected on twenty occasions. One of the cows constantly coughed up a tenacious mucus containing large numbers of tubercle bacilli. $†$ The saliva as well as the bronchial mucus of tuberculous cows has been found to contain abundant bacilli, and by licking her udder it is possible for a tuberculous cow to convey tubercle bacilli to its exterior surface.

The excreta also are infective when lesions are located in the alimentary canal. In tuberculosis affecting the alimentary canal of the cow (1 per cent. of the cases), it is thus possible to get contamination of the milk, indirectly, from the excreta. The mucous membrane of the intestine, especially the colon, sometimes shows tubercular ulcers, which are less frequently observed in the abomasum. Tubercles may also develop under the mucous membrane, and serosa of the stomach and intestines. In these ways arises a condition of intestinal tuberculosis, which in its acute or ulcerating stage will cause the excreta to be loaded with tubercle bacilli. Any onc familiar with a cowshed will at once recognise how readily milk might become infected under such circumstances, which, though undoubtedly exceptional, must not be overlooked.+ In these ways stalls may become infected and transmit the disease to fresh herds stabled in such premises. Nor are herds unstabled always free from

* See also Report of Royal Commission on Tuberculosis, 1896, part iii., p. 142.

+ Commonwealth of Pennsylvania, Bulletin 75 (Pearson and Ravenel), 1901, p. 82. Heron). 
tuberculosis, as has been recently stated. A number of observers have shown that whilst it is true that ill-ventilated, dark, damp cowsheds predispose to infection, milch cows living entirely in the open do not, on that account, escape the disease.* It depends upon infection in the herd, that is, upon contagion. But it is probable that, through more than any other channel, the udder is the most common one for the conveyance of infection. When the udder is affected, the milk invariably contains large numbers of bacilli, and it will be understood when one cow in a herd is so diseased, the entire volume of mixed milk from the herd may be contaminated. The presence of the bacilli in the milk is not always proportionate to the extent of the disease in the animal, especially when diagnosed clinically. The reason of this is the difficulty of clinical diagnosis between chronic interstitial mastitis and tuberculous udder. There can, however, be little doubt that the chief source of tubercle bacilli in milk is the tuberculous udder.

Finally, milkers affected with phthisis may readily infect the milk, either by the repulsive habit of spitting on their hands prior to milking, or by dried expectoration in cowshed, dairy, or milk-shop. After distribution, milk is exposed in a variety of ways to dust, and it cannot be doubted that such dust does at times contain particulate matter derived from dried tubercular expectoration, and that therefore in this way also it is possible for milk to become infected.

The Bovine and Human Tubercle Bacillus Compared.-The morphology of the bacilli in cultures of bovine origin is more uniform and constant than in cultures from man. The bovine bacilli are thick, straight, and short, seldom more than $2 \mu$ in length, and averaging less (Theobald Smith). In the early generations many individuals are seen which are oval, their length not more than double their breadth. They are less granular than those from a human source. They stain evenly and deeply with carbol-fuchsin, beading being almost always absent from young cultures, and often from old ones. In culture they have fairly constant and persistent peculiarities of growth and morphology (Ravenel).

The human bacilli are, on the other hand, much longer, thinner, and tend to increase in length in sub-cultures. They are generally more or less curved, sometimes showing S-shaped forms. They stain less intensely with carbol-fuchsin, but beading is generally seen, even in early growths, and is often very well marked.

The above characteristics are most evident and persistent in cultures grown on blood serum. On glycerine agar, glycerine bouillon, and glycerine potato, bovine and human tubercle bacilli approach each other in cultural features and morphology much more closely, and by continued cultivation the differences tend to become

* Report on Bovine Tuberculosis, Government of New Zealand, 1900 (Gilruth). 
obliterated. Bovine cultures are more difficult to isolate than human, are apt to grow as discrete colonies in the first culture, and for several generations grow in a thin layer which somewhat resembles ground glass. The optimum temperature and the thermal deathpoint are practically the same in both forms.

The human bacillus, as a rule, grows somewhat more easily and abundantly from the first, and will grow well on glycerine agar in sub-cultures made directly from the original growth on blood serum. All attempts to obtain a like result with the bovine organisms have failed. In artificial culture the human bacillus rapidly loses virulence. The bovine bacillus grows as a film on blood serum, whereas the human bacillus produces warty growths.

The morphological distinctions tend to disappear also in the tissues of susceptible animals. We may inoculate a typical bovine culture, and in a short time obtain from the various organs long and much beaded bacilli simulating the human variety (Hueppe).

The most striking dissimilarity is, however, seen in the action of the bacilli from the two sources on animals. By whatever method of inoculation, the bovine bacillus, as a rule, possesses a much greater pathogenic power than the human bacillus for all animals on which it has been tried (Villemin, Ravenel, and others), the on] exceptions being possibly those animals, like guinea-pigs, which are so extremely susceptible to both types that it is difficult to draw very much distinction between them. Dorset and other workers hold that in bovine and human tuberculosis we have to do with organisms differing usually in virulence, but between which there is no other essential distinction.*

\section{Intercommunicability of Human and Bovine Tuberculosis}

Since the discovery by Koch in 1882 of the tuberele bacillus, it has generally been held that tuberculosis in man and animals is one and the same disease. + Villemin (1865) was the first to maintain this identity on the results of inoculation of bovine and human tubercular matter into small animals. Chauveau (1868) carried out similar experiments upon eattle. + Both workers were successful in transmitting the disease, which produced similar effects in the inoculated animals. Many other workers have obtained like results, which were more or less uniformly in support of the view that the identity of bovine and human tuberculosis was

* Trans. British Congress on Tuberculosis, 1901, vol. iii., pp. 553-81. See also experiment of Kossel and others, to which reference is made on p. 344, and U.S. Dep. of Agriculture, 1904, Bull. 52 (Dorset).

$\dagger$ Kruse, Pansini, Fischel, Johne, etc. See also Twelfth and Thirteenth Annual Reports of the Bureau of Animal Industry, Washington, 1895-96 ('Theobald Smith).

† Congrès pour l'étude de la Tuberculose, Paris, 1888 . 
a thing to be accepted as a proved and fundamental proposition. Not only have various workers separately arrived at that conclusion, but the conclusions of the Royal Commission on Tuberculosis, 1895, included the following words:- "We find the present to be a convenient occasion for stating explicitly that we regard the disease as being the same disease in man and the food animals, no matter though there are differences in the one and the other in their manifestations of the disease; and that we consider the bacilli of tubercle to form an integral part of the disease in each, and (whatever be its origin) to be transmissible from man to animals, and from animals to animals. Of such transmission there exists a quantity of evidence, altogether conclusive, derived from experiment." *

Whilst there was up to 1901 almost entire unanimity of opinion amongst various workers in respect to this identity, it should not be supposed that there was unanimity in respect to the degree of pathogenicity. It was, in fact, conceded on all hands that tuberculosis was a more virulent disease in animals than in man, and that the bacillus in the two species differed in various respects as to morphological, biological, and pathological properties (Theobald Smith, Dinwiddie, Frothingham). In 1901, however, Dr Koch expressed the opinion that, "human tuberculosis differs from bovine, and cannot be transmitted to cattle," + and that bovine tuberculosis was scarcely, if at all, transmissible to man. On the same occasion counter-evidence was produced by MacFadyean, † Ravenel, $§$ Crookshank, $\|$ and many others.

As a result of experiment, Koch felt " justified in maintaining that human tuberculosis differs from bovine, and cannot be transmitted to cattle." He further concluded that bovine tuberculosis was scarcely, if at all, transmissible to man. It will be at once obvious that these two conclusions, that human tuberculosis is not transmissible to cattle, and that bovine tuberculosis is not transmissible to man, are of profound and far-reaching importance. Now if it were found on further investigation that these conclusions were correct, the prevention of human tuberculosis would be greatly simplified, and the precautionary measures hitherto adopted for protecting human food from infection with animal tuberculosis need not be enforced with the same stringency as at present, or, at least, would require considerable modification. Tा

* Report of Royal Commission on Tuberculosis, 1895, part i., p. 10, par. 23.

† Trans. Brit. Cong. on Tuberculosis, 1901, vol. i., p. 29.

¥Ibid., vol. i., p. 79.

$\S 1 b i d$. , vol. i., p. 91, and vol. iii., p. 553.

\|l Ibid., vol. i., p. 92.

If It would not necessarily be justifiable to say that in this event such precautionary measures might be "altogether.withdrawn," as has been suggested, for it will be understood that tuberculous meat and milk from animals might still be unwholesome and unfit for the food of man, even though there was evidence to show that the exact specific disease was incommunicable. Presumption would always be against the consumption of meat or milk plus disease products, whether tubercle bacilli or not, for such food is not of the quality and nature reasonably expected by the purchaser. Various non-specific diseases of animals cause meat to be unfit for the food of man. 
The evidence furnished by Dr Koch for the conclusion that human tuberculosis is not communicable to animals is briefly this:-Nineteen young cattle which had stood the tuberculin test (and were therefore presumably free from tuberculosis) were treated as follows :- Six were fed with tubercular human sputum almost daily for seven or eight months. Four repeatedly inhaled great quantities of bacilli which were distributed in water and scattered with it in the form of spray. The remainder (9) were infected in various ways with pure cultures of tubercle bacilli taken from human tuberculosis, or tubercular sputum direct from consumptive patients. In some cases the bacilli or sputum were injected under the skin, in others into the peritoneal cavity, and in others into the jugular vein. None of these 19 cattle showed any symptoms of disease. After six to eight months they were killed, and in their internal organs not a trace of tuberculosis was found. The result was utterly different, however, when the same experiment was made on cattle free from tuberculosis with tubercle bacilli from bovine sources. In this case virulent tuberculosis rapidly supervened. Further, an almost equally striking distinction between human and bovine tuberculosis was brought to light by a feeding experiment with swine. Six young swine were fed daily for three months with the tubercular sputum of consumptive patients. Six other swine received bacilli of bovine tuberculosis with their food daily for the same period. The animals that were fed with sputum remained healthy and grew lustily, whereas those that were fed with the bacilli of bovine tuberculosis soon became sickly, were stunted in their growth, and half of them died. After three months and a half the surviving swine were all killed and examined. Among the animals that had been fed with sputum no trace of tuberculosis was found, except herc and there little nodules in the lymphatic glands of the neck, and in one case a few gray nodules in the lungs. The animals, on the other hand, which had eaten bacilli of bovine tuberculosis had, without exception (just as in the cattle experiment), severe tubercular diseases, especially tubercular infiltration of the greatly enlarged lymphatic glands of the neck and of the mesenteric glands, and also extensive tuberculosis of the lungs and the spleen. The difference between human and bovine tuberculosis appeared not less strikingly in a similar experiment with asses, sheep, and goats, into whose vascular systems the two kinds of tubercle bacilli were injected. Dr Koch also stated that other experiments in former times, and recently in America, have led to the same result.

In support of his second contention, namely, that bovine tuberculosis is not transmissible to man, Dr Koch points out that the direct experiment upon human beings is, of course, out of the question, and hence it is necessary to rely upon indirect evidence. Dr Koch, therefore, reasons as follows : Tuberculosis, caused by meat or milk, can be assumed with certainty only when the intestine suffers first, i.e., when a so-called "primary tuberculosis" of the intestine is found. If bovine tubercle bacilli are capable of causing disease in man there are abundant opportunities for the transference of the bacilli from one species to the other, and cases of primary intestinal tuberculosis from consumption of tuberculous milk ought therefore to be of common occurrence. "But such cases," he maintains, "are extremely rare." In support of this view Dr Koch stated that he had only seen 2 cases; that only 10 cases had been met with in the Charité Hospital in Berlin ; and that out of 3104 post-mortems of tubercular children, Biedert observed only 16 cases. Reference was also made to other similar evidence.

Finally, Dr Koch maintained that " though the important question whether man is susceptible to bovine tuberculosis at all is not yet absolutely decided, and will not admit of absolute decision to-day or to-morrow, one is, nevertheless, already at liberty to say that if such a susceptibility really exists the infection of human beings is but a very rare occurrence."

Such, then, was the position of the question at the end of 1901. It may be convenient here to add the chief reasons for supposing that bovine and human tuberculosis are one and the same disease, and intercommunicable :-

1. That the tubercle bacillus of bovine tuberculosis possesses characteristics of shape, size, staining, and cultivation on artificial media similar to, and in the opinion of many authorities almost identical with, the tubercle bacillus of human origin. 
2. That in specially prepared and suitable media artificial cultures of the tubercle bacillus from bovine and human sources have produced indistinguishable effects when they have been employed to infect a variety of animals, which would seem to indicate that the conditions produced are only variations of one and the same disease.

3. That tuberculin* produces a specific reaction in tuberculous cattle, whether human or bovine tubercle bacilli have been employed in its preparation.-(MacFadyean.)

[It will be seen that these three reasons have relation to the theory of the identity of bovine and human tuberculosis.]

4. That because the tubercle bacillus derived from bovine sources is, either by inoculation or ingestion as food, admittedly very virulent and dangerous for such diverse species of animals as the rabbit, horse, dog, pig, sheep, and cow, it is highly probable that it is also dangerous to man. $\dagger$ For it is well known that the majority of disease-producing bacteria are harmful to only one or two species of animals, but those disease-producing bacteria that are common to all the domesticated animals are also able to produce disease in man.

5. That the statistics and percentages set forth by Dr Koch with regard to primary intestinal tuberculosis cannot be accepted as representing universal experience. For example, in two separate reports from two children's hospitals in London and Edinburgh dealing with 547 cases of death from tuberculosis in children, it appears that $29 \cdot 1$ per cent. and $28 \cdot 1$ per cent. of the cases respectively primary infection appeared to have taken place through the intestine. But quite apart from statistics, the whole question of such primary intestinal tuberculosis (which Dr Koch held as the only acceptable evidence of tuberculous infection through milk and meat) is fraught with many difficulties and fallacies, and is at present sub judice. It has been shown by Professor Sidney Martin and others that primary intestinal tuberculosis may not be, by any means, an invariable criterion of tubercular infection by means of food (vide infra).

6. That there are on record a number of cases in which there appeared to be substantial evidence to show that persons had contracted tuberculosis, directly or indirectly, by means of milk or mest. It is obvious that such cases, unless occurring with extraordinary frequency, are only of relative value. Moreover, there are other channels of infection to eliminate, and this it is often impossible to do.

7. That the results obtained from the inoculation of human tubercle into animals by Dr Koch cannot be accepted as in complete accord with universal experience. In England alone somewhat similar experiments have been performed, having positive results. Several years ago Professor Crookshank carried out such an experiment. He obtained sputum containing numerous tubercle bacilli from an advanced case of

* Tuberculin is a product of the artificial cultivation of the tubercle bacillus (human or bovine) now used as a diagnostic injection test into cattle. If such cattle are suffering from tuberculosis they "react" (giving high temperature, swelling at the point of inoculation, etc.); if not so suffering, they do not react.

$\dagger$ See the researches of Villemin (1865), Klebs, Chauveau (1867), Gerlach, Günther and Harms (1870-1873), Bollinger, and others. Further, Fríedberger and Fröhner state in their Veterinary Pathology that Wesener compiled reports up to 1884 of 369 feeding experiments, the positive and negative results of which were about equal in number. From this compilation it appears that $(a) 71$ animals, among which guineapigs and swine proved most susceptible, were experimented upon with human tubercular matter; $(b) 180$ experiments were made with tubercular matter from cattle; (c) the flesh of tuberculous cattle was given on 32 occasions as food, with the result that pigs were found to be more susceptible than other animals, and that dogs were unaffected; and $(d)$ the milk of tuberculous cows was given as food in 86 cases. From these experiments it was found that in the scale of comparative racial susceptibility the herbivora (cattle, sheep, goats) proved highest, then swine, and after these guinea-pigs and rabbits. Carnivorous animals were little affected. Bovine tubercular matter was found to possess the greatest power of infection, then came the sputum of tuberculous men, then the milk of tuberculous animals, and lastly, tuberculous flesh. 
human consumption. This was injected into the peritoneal cavity of a healthy calf. The animal became ill and died forty-two days after inoculation from pyomia (bloodpoisoning). On post-mortem examination it was found that there were abundant signs of generalised tuberculosis. ${ }^{*}$ This calf was not tested with tuberculin pre. viously to the experiment. Professor Sidney Martin carried out a number of experiments for the Royal Commission on Tuberculosis, + amongst which three out of four calves fed on human tuberculous sputum contracted the disease.

In 1902 Koch again emphasised the comparative rarity of primary intestinal tuberculosis in the human being, and the local, as distinguished from the general, infective nature of accidental bovine inoculation of man (tuberculosis verrucosa cutis). In isolated cases the nearest lymph glands might become affected, but the disease remained nevertheless a local one. Dr Koch further expressed the view that if bovine tuberculosis was transmissible to man by means of the milk of cows with tuberculous udders, it would be reasonable to suppose that "groups of illnesses" would occur, in a manner analogous to other infective diseases, though the circumstances would differ owing to the different length of the incubation periods. By way of illustrating the non-infectivity of bovine tubercle bacilli conveyed by milk, Koch points out $(\alpha)$ that bovine tubercle bacilli must be taken into the human system very frequently, as 1 to 2 per cent. of all milch cows suffer from tuberculous udders; $(b)$ that in addition to being drunk in considerable quantity and for long periods, such milk is also widely distributed; $(c)$ that domestic sterilisation of milk does not occur to any appreciable extent; $(d)$ that the same may be said of the large dairies; and finally $(e)$ that if milk under such circumstances is dangerous, the butter derived from it will also be dangerous. For these reasons he maintained that any resulting disease must be widespread. Yet Koch has found "instead of the countless cases," which we ought to expect, "two groups of illnesses and 28 isolated cases of illness." On examination he finds most of these recorded cases not free from objection. To carry conviction as to milk-borne tuberculosis, Koch maintains, that the following conditions must be fulfilled:-(i.) Certain proof of tubercle in the person affected; (ii.) exclusion of other sources of infection; (iii.) the condition of all the consumers of the suspected milk; (iv.) the exact source of the suspected milk, particularly in respect to the disease of the udder of the cow yielding the milk. Finally, he concludes that all that can be said at present is that the injurious effects of milk infected with bovine tuberculosis and its products are not proven.

On the other hand, many other workers have been investigating

* Bacteriology and Infective Diseases-Edgar M. Crookshank, 1896, pp. 389-391.

+ Report of the Royal Commission appointed to inquire into the Effect of Food derived from Tuberculous Animals on Human Health, 1895, part iii., Appendix, pp. 18 and 19. 
the matter in Europe and America, and Delépine, ${ }^{*}$ Hamilton, $†$ Orth, and Behring + are amongst those who have obtained positive results. Hamilton was able again to establish the truth of Martin's statement, that not infrequently tuberculosis occurred in animals fed on tubercular sputum without affecting the mesenteric and other intestinal glands upon which Koch relied as indication of positive results.

The fundamental feature of Behring's theory based upon his experiments, the results of which are entirely opposed to those of Koch, is that tuberculosis in animals and in human beings represents different varieties of the same disease, and that it is transferable, especially by the agency of tuberculous milk. $\mathrm{He}$ distinguishes in this respect between adults and infants, and maintains that while the former, except under special conditions of the digestive organs, may safely partake of unsterilised milk, infants are particularly liable to infection from this source. Experiments made on newborn foals, calves, guinea-pigs, and other animals show that the mucous membrane of the intestines at that stage of their development is like "a filter with very large pores," and that the bacilli of infection pass through it into the blood precisely as if the animals had been inoculated with the poison. In subsequent stages of their development these animals are provided by nature with a mucous membrane which tends to exclude the danger of infection. Behring is convinced that the same holds true of infants and adults, and that a large portion of mankind is infected in infancy with the germs of tuberculosis derived from cows' milk. In support of his assertion he adduces statistics both of anatomical and of pathological investigation.

This latter evidence tending to show the transmission of tuberculosis to man by means of milk and meat, is of the same character as that upon which the Royal Commission relied when it reported:- “We cannot refuse to apply, and we do not hesitate to apply, to the case of the human subject, the evidence (of transmission of the disease) thus obtained from a variety of animals that differ widely in their habits of feeding-herbivora, carnivora, omnivora. As regards man, we must believe that any person who takes triberculous matter into the body as food incurs some risk of acquiring tuberculous disease." § And again, "We have obtained ample evidence that food derived from tuberculous animals can produce tuberculosis in healthy animals. In the absence of

* Brit. Med. Jour., 1901, ii., p. 1224.

$\dagger$ Trans. of Highland and Agricult. Soc. of Scotland, 1903, and Public Health, 1903 , p. 689.

$\ddagger$ Deut. Med. Woch., 1903.

$\S$ Report of Royal Commission, 1895, part i., p. 10, par. 22. 
direct experiments on human subjects we infer that man also can acquire tuberculosis by feeding upon materials derived from tuberculous food-animals." *

Viewing all the facts, there can be little doubt but that this conclusion is the right one from the point of view of the public health. Various circumstances have in all probability contributed to render unsuccessful or irregular in result the numerous feeding experiments which have been made. The tissues of animals differ greatly in susceptibility to tuberculosis; the infective material is exposed to the digestive juices which are, in measure, germicidal, and yet not equally so; the virulence of the infective material itself varies enormously, as does the virulence between different generations or races of tubercle bacilli. Hence it comes about that one animal may eat with its food a certain amount of tuberculous material, and yet not develop tuberculosis, whilst another animal of the same species might quickly develop the disease, which would in all probability show itself at the animal's weakest point, and not always necessarily in the intestine. Further, there is another point which should not be overlooked, namely, the subsequent treatment of the inoculated animal. Whilst it is essential to prove that the animal to be inoculated is free from tuberculosis, it should be remembered that in taking very healthy animals for experiment, and in subsequently treating them in what may be termed an "ideal" fashion, some of the very conditions essential to the production of the disease in ordinary life are removed. As in men, so in cattle and other animals, it may be presumed that abundance of good food and fresh air, and, in general, an ideal environment, tend to counteract the effect of the inoculated or communicated virus. Thus such experiments as those stated above may not always fairly represent the modes of transmission of the disease as they occur in ordinary life. It is not the "very healthy" animal of a herd, well housed and fed, which contracts tuberculosis.

As a result of the wide differences of opinion revealed by the pronouncement of Koch's views, special Commissions of Inquiry were instituted in Germany, Great Britain, and other countries, in addition to the individual research work to which reference has been made. As this book has been passing through the press, reports of these inquiries have been made by the German Imperial Health Office and the Royal Commission on Tubereulosis, appointed in 1901 by the British Government. The conclusions are briefly as follows:-

Kossel, Weber, and Heuss, who carried out a comparative research upon tubercle bacilli of different origins, made a number of experiments on calves by injecting some forty different strains of human bacilli and fifteen strains from bovine, fowl, and swine sources. They con-

* Report of Royal Commission, 1895, part i., p. 20, par. 77. 
clude as a result of these experiments that in a preponderating number of cases of human tuberculosis, tubercle bacilli were found distinguishable from the bovine bacilli of Perlsucht in cows morphologically, culturally, and in pathogenic properties, but that exceptionally in man tubercle bacilli occur which cannot be distinguished. They hold, nevertheless, that the possibility of infection in man, under certain circumstances, by milk from tuberculous udders is proved. They found that generalised tuberculosis was produced in animals by injecting strains of tubercle bacilli obtained from tuberculous diseases in children.*

The Royal Commission appointed in this country has also issued an interim report (1904), signed by Sir M. Foster and Professors Sims Woodhead, Sidney Martin, MacFadyean, and Boyce. The Commission was appointed to inquire and report with respect to tuberculosis:(1) Whether the disease in animals and man is one and the same; (2) whether animals and man can be reciprocally infected with it; and (3) under what conditions, if at all, the transmission of the disease from animals to man takes place, and what are the circumstances favourable or unfavourable to such transmission.

The first line of inquiry upon which they entered may be stated in their own words, as follows:-

What are the effects produced by introducing into the body of the bovine animal (calf, heifer, cow), either through the alimentary canal as food, or directly into the tissues by subcutaneous or other injection, tuberculous material of human origin, i. e., material containing living tubercle bacilli obtained from various cases of tuberculous disease in human beings, and how far do these effects resemble or differ from the effects produced by introducing into the bovine animal, under conditions as similar as possible, tuberculous material of bovine origin, i.e., material containing living tubercle bacilli obtained from cases of tuberculous disease in the cow, calf, or ox?

We have up to the present made use, in the above inquiry, of more than twenty different "strains" of tuberculous material of human origin, that is to say, of material taken from more than twenty cases of tuberculous disease in human beings, including sputum from phthisical patients, and the diseased parts of the lungs in pulmonary tuberculosis, mesenteric glands in primary abdominal tuberculosis, tuberculous bronchial and cervical glands, and tuberculous joints. We have compared the effects produced by these with the effects produced by several different strains of tuberculous material of bovine origin.

In the case of seven of the above strains of human origin, the introduction of the human tuberculous material into cattle gave rise at once to acute tuberculosis, with the development of widespread disease in various organs of the body, such as the lungs, spleen, liver, lymphatic glands, etc. In some instances the disease was of remarkable severity.

In the case of the remaining strains, the bovine animal into which the tuberculous material was first introduced was affected to a less extent. The tuberculous disease was either limited to the spot where the material was introduced (this occurred, however, in two instances only, and these at the very beginning of our inquiry), or spread to a variable extent from the seat of inoculation along the lymphatic glands, with, at most, the appearance of a very small amount of tubercle in such organs as the lungs and spleen. Yet tuberculous material taken from the bovine animal thus affected, and introduced successively into other bovine anumals, or into guinea-pigs from which

* T'uberkulose-Arbeiten aus dem Kaiserlichen Gesundheitsamte, Heft i., 1904, p. 34. 
bovine animals were subsequently inoculated, has, up to the present, in the case of five of these remaining strains, ultimately given rise in the bovine animal to general tuberculosis of an intense character; and we are still carrying out observations in this direction.

We have very carefully compared the disease thus set up in the bovine animal by material of human origin with that set up in the bovine animal by material of bovine origin, and so far we have found the one, both in its broad general features and in its finer histological details, to be identical with the other. We have so far failed to discover any character by which we could distinguish the one from the other; and our records contain accounts of the post-mortem examinations of bovine animals infected with tuberculous material of human origin, which might be used as typical descriptions of ordinary bovine tuberculosis.

The results which we have thus obtained are so striking, that we have felt it our duty to make them known, without further delay, in the present interim report.

The Commission defer to a further report all narration of the details of their experiments, as well as all discussions, including those dealing with the influence of dose and of individual as well as racial susceptibility, with questions of the specific virulence of the different strains of bacilli, with the relative activity of cultures of bacilli and of emulsions of tuberculous organs and tissues, and with other points.

Meanwhile they have thought it their duty to make this short interim report, for the reason that the result at which they have arrived, namely, that tubercle of human origin can give rise in the bovine animal to tuberculosis identical with ordinary bovine tubcreulosis, seems to them to show quite clearly that it would be most unwise to frame or modify legislative measures in accordance with the view that human and bovine tubercle bacilli are specifically different from each other, and that the disease caused by the one is a wholly different thing from the disease caused by the other.

In this final conclusion as to administrative measures both German and British Commissions agree. They also agree as to the intercommunicability of bovine and human tuberculosis.**

Diagnosis of Bovine Tuberculosis. - There are three methods of diagnosis-clinical, bacteriological, and by means of tuberculin.

(a) Clinical.-When tuberculosis affects the lungs and respiratory organs generally, it is accompanied by a frequent cough, but no fever. There is disturbance of the respiration, the breathing being quickened by slight exertion or excitement, and the cough stimulated by changes of temperature. The departure from the normal in the relative length of the inspiratory and expiratory movement (the expiration being markedly prolonged) can be readily seen as a rule, and not uncommonly in these cases a rough harsh sound may be heard in the throat during respiration. By auscultation it is possible sometimes to detect dull portions of the lung surrounded by areas of increased resonance. The vesicular murmur is loúder and harsher (Salmon). 
than normal over the upper half of the chest, and is particularly marked during expiration. Usually the superficial glands in the throat, those between the jaws, and under the ear or of the udder are swollen and hard. The animal may continue for months in an apparently healthy condition. When the disease is abdominal and the glands and organs in the belly are chiefly affected, the symptoms of defective nutrition are early evident, namely, emaciation, lessened milk secretion, indigestion, breathlessness, and more or less rapid failure in general health. In these cases the udder should be especially examined.

It should be noted that clinical diagnosis of tuberculosis, especially of udder tuberculosis, does not enable us to judge whether tubercle bacilli are secreted along with the milk of the cow in question. The bacteriological test and tuberculin are necessary. The former is sometimes tedious, and the latter remains at present our only quick and sure method.

(b) Bacteriological Examination.-This method of diagnosis can be applied at once in suspected udder disease by examination of the milk; or, as recommended by Nocard, a trocher may be used by which a small fragment of tissue from the indurated portion of the udder may be obtained for examination. Mucus or discharges from throat, wounds, and ulcers may also be examined and assist in diagnosis. The only sure method of bacteriological examination is by inoculation of animals. Microscopical and cultural tests are unreliable.

(c) Tuberculin.- - In recent years the method of testing herds for tuberculosis by means of tuberculin has come into vogue, and it will be necessary to refer briefly to this subject. The discovery by Koch, in 1890 , of the production of fever, indicated by a rise in temperature, in tuberculous animals into which he injected a sterilised glycerine extract of pure cultures of tubercle bacilli, while it produced no effect whatever when the animals were free from that disease, furnished us with a simple but fairly reliable diagnostic agent.

Tuberculin is a soluble product of cultures of tubercle bacilli, of which a glycerine extract is made, which is sterilised by heat and filtered through porcelain, so that it contains no living germs, and therefore cannot produce tuberculosis in animals injected with it. It has, therefore, no effect on healthy animals; in some cases the disease is aggravated by it when it exists, but it cannot be produced by it. The lymph must not be exposed to sunlight; it must not be frozen, and must be kept well corked to exclude air.

Koch's "old tuberculin" is made from glycerine-veal broth cultures of $B$. tuberculosis by means of evaporation and precipitation with.alcohol. The liquid cultures are thus concentrated to one-tenth 
of their original bulk, and then passed through a Chamberland filter. The brown and viscid filtrate is the tuberculin. Buchner and Römer pointed out that the proteins of other bacteria have a similar effect upon tuberculosis, that is, cause a reaction with rise of temperature. In 1897, Koch was able to improve his tuberculin, and under the name "Tuberculin T. R." recommended a new preparation. In point of fact, the new preparation takes three forms, distinguished by the letters T. A. (alkaline tuberculin), T. O. (upper tuberculin, Germ. ober), and T. R. (residual tuberculin). T. A. is extracted from a young and virulent culture of $B$. tuberculosis by means of a one-tenth normal solution of caustic soda, and the solution is filtered. The reaction on inoculation is intense, and may be accompanied with abscesses. Accordingly, its clinical use is open to objection. T. O. and T. R. are prepared by vigorously pounding in a mortar dried cultures of the tubercle bacilli and then adding distilled water. The emulsion is thoroughly centrifugalised. The clear, opalescent fluid collecting at the upper part of the tube contains no tubercle bacilli, and constitutes in the first centrifugalisation T. O. The débris or residuum of tubercle bacilli remaining at the bottom of the tube is used for the production of T. R. This residue is dried, triturated with distilled water, and centrifugalised repeatedly until hardly any residue remains. Twenty per cent. of glycerine is then added to both preparations for purposes of preservation. T. R. alone is used clinically.

The method of use is as follows: The animals are kept a day or two in their byres, and the temperature is taken to standardise the normal, which is generally about $102 \cdot 2^{\circ} \mathrm{F}$. The tuberculin is then injected (30-40 centigrammes), and if the animal be tuberculous, there is a rise in temperature of $1 \frac{1}{2}^{\circ}$ to $3^{\circ}$. The fever usually begins between the twelfth and fifteenth hour after injection, and lasts several hours. The more nearly the temperature approaches $104^{\circ} \mathrm{F}$, the more reason is there to suspect tuberculosis (Bang). The duration and intensity of the reaction, however, has not a direct relation to the number or gravity of the lesions, but the same dose in healthy cattle causes no appreciable febrile reaction. The tuberculous calf reacts just as well as the adult, but the dose used is generally smaller.

Tuberculin injection has no bad effects on the secretion of milk, either in quantity or in quality. The consensus of opinion of those most experienced is that it does not lessen the secretion of milk in dairy cattle, consequently they may be tested even when in full milk without disturbing its secretion, unless it be during the few hours of its absorption. It does not cause abortion in cows, or sterility in bulls.

It is the quickest and most reliable method of diagnosis of 
bovine tuberculosis which we possess. Schütz maintains that failures in diagnosis by tuberculin injection only amount to 2.9 per cent. Obviously, it may fail in animals which are highly tuberculous, owing to the fact that their tissue already contains so much tuberculin that they are unable to respond any longer to the tuberculin test. If there is any lesion whatever in the udder, and there is a reaction to tuberculin, the milk from that cow should not be used.*

\section{Tuberculosis of Other Animals}

Tuberculosis of the Pig is less common than that of cattle, but not so rare as that of the calf (Nocard). In nine out of ten cases the pig is infected by ingestion, particularly when fed on the refuse from dairies and cheese factories. The disease follows the same course as in cattle, but generalisation is more common and more rapid. Lesions of the abdominal organs occur in almost every case. The glands, particularly those of the throat, are markedly affected. The finding of the tubercle bacillus is difficult, and the only safe test is inoculation. The massive lesions are often thinly scattered, rich in giant cells, and containing few bacilli. The disease usually assumes the acute or "galloping" form, and not infrequently emaciation is absent and the dead pork meat possesses a healthy and fat appearance. The internal organs and glands are the chief sites of disease. The whole carcase should be condemned. In a pork carcase seized by the writer in Finsbury in 1904, the retro-pharyngeal, submaxillary, cervical, and mediastinal glands were enormously enlarged and caseous, the deep glands of the body and the udder and its glands were also affected, and the joints of the right fore foot were ankylosed owing to tuberculous infiltration. The internal organs in this case were a mass of tubercle.

Sheep are very rarely affected with tuberculosis, though there is evidence which goes to prove that very long confinement in limited space with tuberculous cattle might result in transmitting tuberculosis to sheep. One of the few cases on record in this country was met with by the writer, and has been described by Foulerton. $\dagger$ This was a half-bred, emaciated ewe having both lungs extensively consolidated, and containing numerous tubercles which were also present on the pleuræ. The liver, spleen, both kidneys, and lymphatic glands were affected. An emulsion, made from one of the affected glands, inoculated into the guinea-pig, caused death from generalised tuberculosis. The sheep, though rarely attacked, is not naturally immune.

\footnotetext{
* For a discussion as to the practical use of the tuberculin test, see Trans. Brit. Cong. on Tuberculosis, 1901, vol. ii., pp. 235-78 (Delépine).

+ Transactions of the Pathological Society of London, 1902, vol. liii., pt. iii., p. 428.
} 
Tuberculosis in the Horse is relatively very rare. It attacks the organs of the abdominal cavity, especially the glands; it affects the lung secondarily as a rule. The cases are generally isolated ones, even though the animal belongs to a stud. Nocard holds that the bacillus obtained from the pulmonary variety is like the human type, whilst the abdominal variety is more like the avian bacillus.

Dog.-Nocard says: "If the dog can become tuberculous from contact with man, the converse is equally true. Infection is at any rate possible when a house-dog scatters on to the floor, carpet, or bed, during its fit of coughing, virulent material, which is rendered extremely dangerous by drying, especially for children, its habitual playmates. The most elementary prudence would recommend the banishment from a room of every dog which coughs frequently, even though it only seems to be suffering from some common affection of the bronchi or lung." *

Birds. - Tuberculosis is a common disease among birds of the poultry-yard: poultry, pigeons, turkeys, pea-fowl, guinea-fowl, etc. They are infected almost exclusively through the digestive tract, generally by devouring infected secretions or organs of previous tubercular fowls, and though very susceptible in this way, birds can consume large quantities of phthisical sputum without becoming tubercular. Whatever the position or form of avian tuberculosis, the bacilli are present in enormous numbers, and are often much shorter but sometimes longer than those met with in tuberculous mammalia, and grow outside the body at a higher temperature $\left(43^{\circ} \mathrm{C}\right.$.). They are said also to be more resistant and of quicker growth. The species is probably identical with Koch's bacillus, though there are differences (Maffucci). In the nodule, which is larger than in human tuberculosis, there are few or no giant cells, and it does not so readily break down. Guinea-pigs and other animals are not so readily infected with avian tubercle as mammalian. The writer has prepared a number of histological specimens illustrating the comparative pathology of tuberculosis, particularly in birds which have died of the disease in the Zoological Gardens, London, including guan, quail, ostrich, rhea, currasow, swan, cuckoo, vulture, goose, eagle, fowl, pheasant, parrot, etc. In most cases the disease affects the organs of the alimentary canal, especially the liver. The lungs are rarely affected except secondarily. The disease frequently develops rapidly like an acute infective disease, and the bacilli may often be found in the tissues arranged in large colonies, as in leprosy in the human tissues.

The bacillus of avian tubercle differs from the organism of tuberculosis in mammals in the appearance of the cultures and the temperature conditions. Fischel, who worked under Huppe's direction, says both micro-organisms are of one and the same kind

* Animal Tuberculosis, p. 129. 


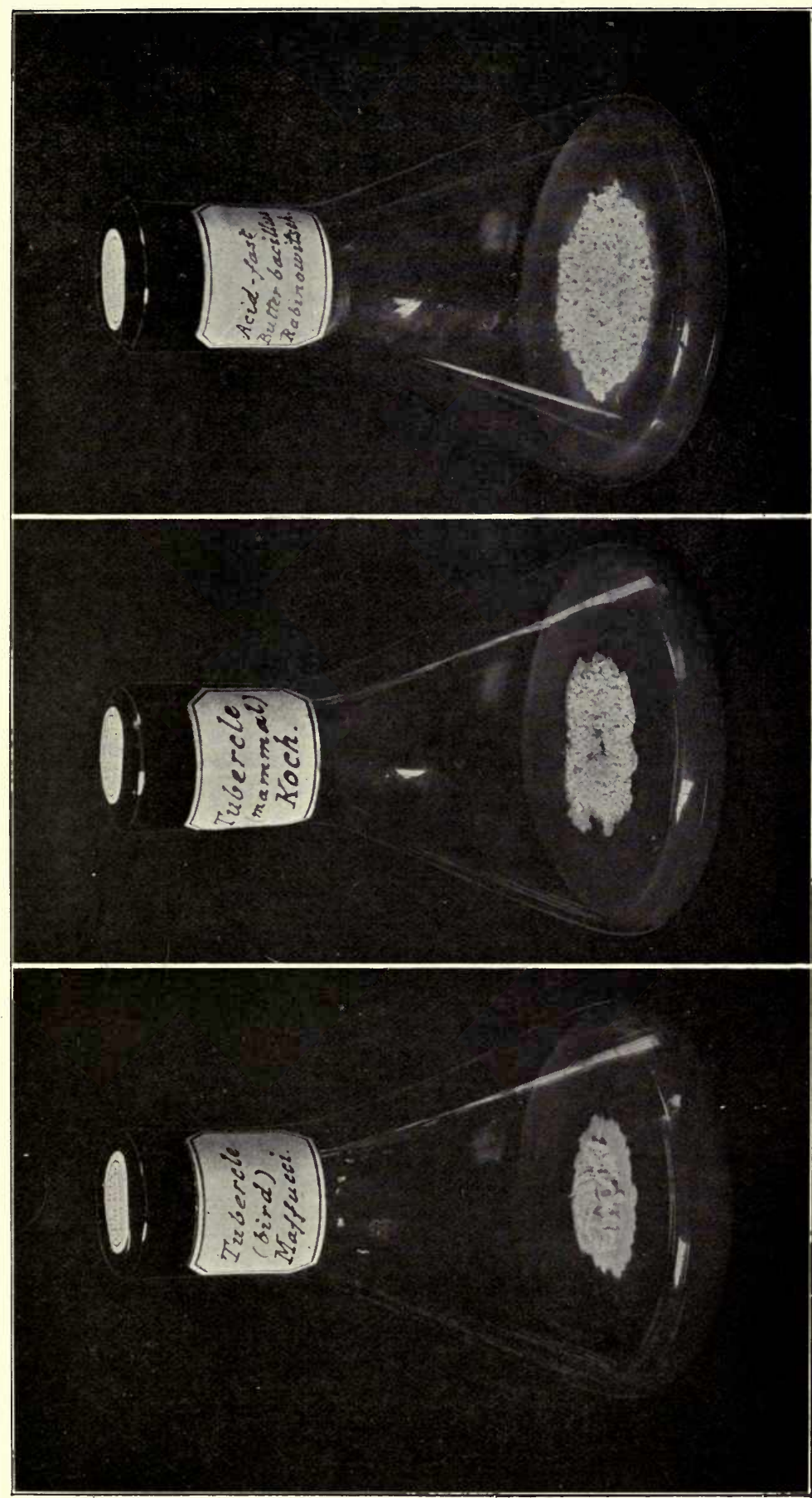

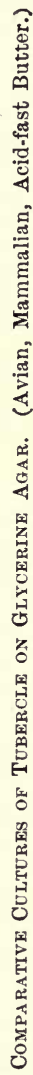


CALIFORNIA 
as regards nutritive media. In consequence of the different physiological nutritive media of the colder mammalian bodies on the one hand, and the warmer avian bodies on the other, a distinction has arisen between the two kinds. By artificial cultivation Fischel succeeded in bringing about approximation in outer qualities between the two bacilli; he succeeded in getting the organism of tuberculosis in mammals accustomed to a higher temperature, and in given nutritive media he obtained a resemblance in the appearance of the cultures, but as regards pathogenesis he could not transfer one to the other. Fischel states that he was able, with the organism of avian tuberculosis, to bring about a general tuberculosis in a guineapig, but the cultures started out of the organs of this animal were not identical with those of avian tuberculosis.

Tuberculosis in cold-blooded animals is in the same way to be regarded as a modification of the tuberculosis in mammals. Bataillon and Terre succeeded in cultivating tuberculosis in mammals and birds by means of passing it through the body of a frog at room temperature. Lubarsh was able to modify tuberculosis in mammals by passage through the body of a frog, so that cultures taken from the spleen of a frog grew at a temperature of as much as $28-30^{\circ}$. Dubard also cultivated cultures taken from fish inoculated with mammalian tuberculosis which also thrived at room temperature. Moeller was able to produce cultures from the spleen of a slow-worm inoculated with sputum containing tubercle bacilli, which flourished at $20^{\circ}$; but which ceased to grow at a temperature of $30^{\circ}$ and over. The cultures resemble in appearance those of avian tuberculosis, grow at room temperature, and are moist and thick.

It should be added that certain abnormal and tubercle-like conditions have been met with in the carp, and from such conditions a bacillus morphologically and tinctorially similar to the tubercle bacillus has been isolated.

Most bacteriologists maintain that the $B$. tuberculosis of Koch is the common denominator in all tubercular disease, whatever and wherever its manifestations, in all animals. The bacillus, they hold, may, however, experience profound modifications owing to successive passages through the bodies of divers species of animals. But if the modifications which it undergoes as a result of transmission through birds, for example, are profound enough to make the bacillus of avian tubercle a peculiar variety, though not a distinct and separate species, of Koch's bacillus, they are not enough, it is generally believed, to make these bacilli two distinct species. We may, therefore, take it for granted that tuberculosis is one and the same disease, generically, with various manifestations, common to man and animals, intercommunicable, and having but one vera causa, the B. tuberculosis of Koch. 


\section{The Prevention of Tuberculosis}

At the present time much attention is being directed to the administrative and personal control of tuberculosis. How greatly this is needed in so preventable a disease is evident from a perusal of the following table from the Registrar-General's reports:-

\section{ENGLAND AND WALES}

\section{ANNUAL DEATH-RATES FROM ALL TUBERCULAR DISEASES}

The following is a table of death-rates to a million living (England and Wales), 1880-1901 (Reg.-Gen. Annual Reports):-

\begin{tabular}{|c|c|c|c|c|c|c|c|c|c|c|c|c|c|c|}
\hline & & & & 1880 & 1881 & 1882 & 1883 & 1884 & 1885 & 1886 & 1887 & 1888 & 1889 & 1890 \\
\hline Tabes Mesente & ica & . & - & 370 & 284 & 313 & 289 & 310 & 251 & 300 & 253 & 240 & 269 & 265 \\
\hline Tubercular Me & ingit & & • & 330 & 276 & 264 & 262 & 264 & 253 & 257 & 236 & 239 & 234 & 240 \\
\hline Phthisis . & • & . & • & 1869 & 1825 & 1850 & 1880 & 1827 & 1770 & 1739 & 1615 & 1568 & 1573 & 1682 \\
\hline Other Forms & . & . & . & 129 & 145 & 153 & 160 & 170 & 157 & 177 & 179 & 174 & 183 & 189 \\
\hline \multirow[t]{2}{*}{ Total } & . & - & - & 2698 & 2530 & 2580 & 2591 & 2571 & 2431 & 2473 & 2283 & 2221 & 2259 & 2376 \\
\hline & & & & 1891 & 1892 & 1893 & 1894 & 1895 & 1896 & 1897 & 1898 & 1899 & 1900 & 1901 \\
\hline \multirow{2}{*}{\multicolumn{3}{|c|}{$\begin{array}{l}\text { Tabes Mesenterica } \\
\text { Tubercular Meningitis }\end{array}$}} & - & 251 & 242 & 265 & 192 & 243 & 196 & 201 & 202 & 198 & 185 & 188 \\
\hline & & & - & 247 & 227 & 226 & 211 & 222 & 210 & 213 & 213 & 203 & 198 & 183 \\
\hline Phthisis . & . & . & . & 1599 & 1468 & 1468 & 1385 & 1398 & 1307 & 1341 & 1317 & 1336 & 1333 & 1264 \\
\hline Other Forms & . & . & . & 203 & 199 & 186 & 185 & 200 & 179 & 175 & 184 & 174 & 186 & \begin{tabular}{|l|}
372 \\
\end{tabular} \\
\hline Total & • & . & . & 2300 & 2136 & 2145 & 1973 & 2063 & 1892 & 1930 & 1916 & 1911 & 1902 & 1807 \\
\hline
\end{tabular}

Tabes mesenterica is a term applied to tuberculosis of the alimentary canal and mesenteric lymph glands.

Tubercular meningitis is the name of the same disease as it affects the membranes of the brain (acute hydrocephalus).

Phthisis is the term applied to "consumption," or tubercle in the lungs.

These figures show a marked decline in the three worst forms of the disease. But this decline is less marked in tabes mesenterica 
than in phthisis or tubercular meningitis, i.e. less in the kind of tubercle loeated in the abdomen chiefly. Fortunately, the State is beginning to realise its duty in regard to preventive measures. The abolition of private slaughter-houses, the protection of meat and milk supplies, the condemnation of tuberculous milch cows, and suchlike measures, fall obviously. within the jurisdiction of the State rather than the individual, and claim the earnest and urgent attention of the public health departments of Government.*

Methods of Prevention.-A consideration of the various facts set forth in this chapter will suggest the best means of the prevention of consumption. These means depend upon broad principles of sanitation and personal health rather than on bacteriological niceties or theoretical considerations. What is required has been stated briefly by Sir Hermann Weber as:-(a) Purity and free circulation of air; (b) sufficiency of good and pure food; $(c)$ well-constructed and ventilated sunny rooms in houses situated on dry and pure soil; and $(d)$ the maintenance of the resisting power of the body and its different organs. $†$ These general desiderata are to be secured by practical preventive methods, of which the following are some of the more important:-

1. Personal hygiene and the maintenance of a high degree of resistance in the human tissues is a matter which must rest with the individual rather than the State, which can only exert its influence, generally speaking, on the environment of the individual. Below will be found a number of particulars as to the disease, stated in simple form, and many of which bear a direct relation to the management of personal conditions. The healthy life with sufficient food, exercise, etc., is what is necessary to maintain healthy tissues. Mention may, however, be made of the abuse of alcohol and the neglect of simple ilinesses as two most powerful factors in the creation of conditions in the body favourable to tubercle infection.

2. Only second in importance is general sanitation and the creation of a healthy environment for the individual and the community, and in this, and subsequent methods, much of the preventive work in tuberculosis is centred. Such conditions as dust in the air, overcrowding in houses, too great a density of houses on the area, illventilated and unclean rooms and workshops, etc., exert an indirect influence of great force in the propagation of tuberculosis, and therefore the reduction and abatement of these conditions serves as a means of prevention. The right enforcement of the Housing of the Working Classes Acts, the Public Health Acts, the Building Acts,

* See the Harben Lectures, November, 1898, by Sir Richard Thorne Thorne, Medical Officer to the Local Government Board; also the Reports of the Royal Commissions on Tuberculosis, 1896, 1898, and 1903.

+ Tuberculosis, 1899, vol. i., p. 9. 
and the Factory and Workshop Act are therefore matters of great importance in the prevention of this disease.

3. Food has also been shown to be infected in a greater or less degree with the virus of tuberculosis, and though the disease is not spread so greatly through this channel as in other ways, it is nevertheless necessary to protect the public from tuberculous food, especially meat and milk. The Public Health Acts (1875 and 1891) give powers of seizure of diseased food, and the Dairy, Milkshops, and Cowsheds Order of 1885 , and its amendments, operate in the direction of the control of the milk supply. These latter Orders should be unified, and much more vigorously enforced than has been the case in the past.

4. Lastly, there are certain measures of great importance which concern the avoidance of infection from diseased persons. The consumptive is the chief agent in the spread of consumption. Therefore, anything which lessens the degree of his contagiousness is a means of prevention. The first requirement is evidently knowledge of the existence of cases of phthisis, and this may be obtained in various ways, e.g., through hospitals or private practice, through poor-law institutions, or by voluntary or compulsory notification. Voluntary notification was first adopted in this country by the Local Authorities in Brighton, Manchester, and Finsbury, and is now in vogue in many districts. The results are not wholly satisfactory, but are better than no information at all. Compulsory notification has recently been instituted for an experimental period in Sheffield.* The cases of phthisis being known, the next steps are supervision, disinfection of sputum, house, and clothes, and, if practicable, isolation and treatment of suitable cases. Sanatoria act partly as therapeutic agencies, partly as prophylactic agencies. Much is now being done in civilised countries in these directions, and many sanitary authorities carry out disinfection regularly, and make various efforts to prevent consumptives infecting their neighbours or fellow-workmen.

Still, after all, the prevention of phthisis is in no small degree a matter of personal hygiene and precautions to be exercised by the people themselves.

Hence we hail with satisfaction the recent endeavours to educate public opinion. In order to simplify this matter, we have placed in a footnote a series of statements embodying some of the chief facts which every individual in our crowded communities should know. $†$

* Sheffield Corporation Act, 1903, sect. 45.

$\dagger$ 1. Tuberculosis is a disease mainly affecting the lungs (consumption, decline, phthisis) of young adults and the bowels of infants (tabes mesenterica). It may affect any part of the body, and its manifestations are very various. It also affects animals, particularly cattle, by whom it may be transmitted to man.

2. Its direct cause is a microscopic vegetable cell, known as the $B$. tuber- 


\section{Pseudo-tuberculosis}

In 1899 the Pathological Society of London urged that this term should be discarded. It is used here not as concerned with diseases

culosis, discovered by Koch in 1882. This fungus requires to be magnified some hundreds of times before it can even be seen. When it gains entrance to the weakened body it sets up the disease, which is an infectious, or sub-infectious, disease, though different in degree to the infectiousness of, say, measles.

3. Trade influence and occupation, in some cases, undoubtedly predisposes the individual to tubercle. Cramped attitudes, exposure to dampness or cold, ill ventilation, and exposure to inhalation of dust of various kinds, all act in this way. In support of the evil effect of each of these three conditions much evidence could be produced.

4. Overcrovding has a definite influence in propagating tubercular diseases. The agricultural counties without large towns, like Worcestershire, Herefordshire, Buckinghamshire, and Rutland, are the counties having the lowest mortality from tuberculosis; whilst the crowded populations in Northumberland, South Wales, Lancashire, London, and the West Riding suffer most. Speaking more particularly, the overcrowded areas of London, such as Southwark, Shoreditch, Finsbury, Holborn, and Central London generally, show very high tubercular death-rates.

5. Tuberculosis is not increasing.-During the last thirty years it has shown, with few exceptions, a steady decline in all parts of England. "Consumption" is most fatal in comparatively young people (fifteen to forty-five years), whilst "tabes" and other forms of tubercle are fatal chiefly to young children. These forms have not declined so much as the lung form. The mortality in consumption of males has since 1866 been in excess of that of females. The age of maximum fatality from consumption is later than in the past, which is probably due to improved hygiene and treatment.

6. This decline has been due not to any special repressive measures-for few have been carried out-but to a general and extensive social improvement in the life of the people, to an increase of knowledge respecting tuberculosis and hygiene, to an enormous advance in sanitation, and to more efficient land drainage.

7. Not all persons are equally liable to consumption, some being much more susceptible than others. We have mentioned the predisposing influence of certain trades. There is also heredity, which acts, as we have said, in transmitting a tubercular tendency, rarely, if ever, the actual virus of the disease ; there is, thirdly, the debilitating effect of previous illness or chronic alcoholism ; there is, fourthly, the habitual breathing of stagnant, polluted air; and, fifthly, there are the conditions of the environment, such as dampness and darkness of the dwelling. Such influences as these weaken the resisting power of the tissues, and thus afford a suitable nidus for the bacillus conveyed in milk, or by the inspiration of infected dust or mucus.

8. Consumption may be arrested if taken in time. In cases where the lungs are half gone, and consist of large cavities, it is obvious that curability is out of the question. But if the disease can be properly treated in its earliest stages, there is considerable likelihood of recovery, or at least of arrest of the disease.

9. The breath is not dangerous, as far as we know, but there is danger from discharges of any kind from any infected part, whether lungs or bowels; for such discharges, when dry, may readily pollute the air, and either the bacilli or spores be inhaled into the lungs. The breath itself in coughing, shouting, etc., may emit a fine spray of mucus or saliva, in which the bacilli may be suspended, and thus convey infection.

10. The chief channels of personal infection in the spread of the disease amongst a community are two: (a) dried tubercular sputum or "cough spray" (or other tubercular discharges); (b) infected milk or meat. As for milk and meat, boiling the former and thoroughly cooking the latter will remove all danger. In any case, there is evidence to show that infection from milk or meat is nothing like so common as infection from sputum or mucous particles from a consumptive patient. 
simulating tuberculosis (protozoal infections, parasitical disease, etc.), but to designate a pathological condition set up by a special group

11. The expectoration is infective. - This is one of the commonest modes of infection, and to it is held to be due the large amount of respiratory tuberculosis (consumption, phthisis). The expectoration from the lungs must contain, from the nature of the case, a very large number of bacilli. As a matter of fact, a single consumptive individual can cough up in a day millions of tubercle bacilli. When expectoration becomes dry, the least current of air will disseminate the infective dust, which can by that means be readily reinspired. Infective saliva on pavcments and floors, as well as on handkerchiefs, or even in books, may thus become a source of danger to others. The discharges from the bowels of infants suffering from the disease also contain the infective material.

12. Milk, though a much more likely channel for conveyance of tubercle than meat, is only or chiefly virulent when the udder is the seat of tuberculous lesions. The consumption of such milk is only dangerous when it contains a great number of bacilli and is ingested in considerable quantity. Practically, the danger from using raw milk only exists for those persons who use it as their sole or principal food, e.g., young children. All danger is avoided by boiling or pasteurising the milk.

At the same time there is an increasing amount of evidence forthcoming at the present time which goes to prove that milk is not infrequently tainted with tubercle (see pp. 204-206). The tuberculin test should be applied to all milch cows, and the infected ones isolated from the herd. They need not necessarily be slaughtered. Milk supplies should be more strictly inspected.

13. 'There are several methods by which meat infection can be prevented. In the first place, herds should be kept healthy, and tubercular animals isolated. Cowsheds and byres should be under sanitary supervision, especially as regards overcrowding, dampness, lack of light, and uncleanliness. Public slaughter-houses under a Sanitary Authority would undoubtedly be advantageous. Meat inspection should also be more strictly attended to ; efficient cooking, and avoidance of "roll " meat which has not been thoroughly cooked in the middle, are also wise measures to adopt.

14. Consumptive patients may diminish their disease.-Dr Arthur Ransome* has laid down five axioms of hygiene for phthisical patients which, if followed, would materially improve the condition of such persons. At Davos, St Moritz, Nordrach, Nordrach-on-Mendip, and many other places where they have been practised, the beneficial change has been in many cases extraordinary.

(1) Abundance of light, nutritious, easily digested food, which must comprise a large allowance of fat; small meals, but frequent.

(2) An almost entirely open-air life, with as much sunshine as can be obtained.

(3) Suitable clothing, mostly wool.

(4) Cleanliness, and bracing, cold-water treatment.

(5) Mild but regular exercise.

15. Consumptive patients may also assist in preventing the spread of the disease. - In the first place, they should follow the hygienic directions just mentioned, because the fulfilment of such conditions will materially lessen the contagiousness of the disease. Next, the expectoration must never be allowed to get dry. A spitting-cup containing a little disinfectant solution (one teaspoonful of strong carbolic acid to two tablespoonfuls of water) should always be used, or the expectoration received into paper handkerchiefs which can be burnt. Spoons, forks, cups, and all such articles should be thoroughly cleaned before being used by other persons. The patient should not sleep in company with another, and should occupy, if possible, a separate bedroom. Isolation hospitals for consumptives, as for patients suffering from the ordinary infectious diseases, are now being established.

16. House influence has some effect, both directly and indirectly, upon tubercular diseases. Damp soils, darkness, and small cubic space in the dwelling-house exert a very prejudicial effect upon tubercular patients. Sir Richard Thorne Throne + has

* Arthur Ransome, M.D., F.R.S., Treatment of Phthisis.

+ Practitioner, vol. xlvi. 
of bacilli of which the chief is the B.pseudo-tuberculosis of Pfeiffer.* Other workers have described very similar organisms.

described the favourable house for such persons as one built upon a soil which is dry naturally or freed by artificial means from the injurious influences of dampness and of the fluctuations of the ground water. The house itself should be so constructed as to be protected against dampness of site, foundations, and walls. Upon at least two opposite sides of the dwelling-house there should be enough open space to secure ample movement of air about it, and free exposure to sunlight. Lastly, it should be possible to have free movement of air by day and night through all habitable rooms of the house. It is clear many inhabited houses could not stand these tests; but effort should be made to approach as near to such a standard as possible.

17. Tubercle-infected Houses. - Many authorities have demonstrated the fact that dust in houses may contain the tubercle bacillus, and that thus, presumably, persons may become infected. In 1904, Klein found living tubercle bacilli in the sweepings of the floors of public-houses, and some fifteen years ago Cornet published the result of his investigations into the infectivity of the dust found in the dwellings of consumptives in Berlin, and some work of a similar character has been done in England by Coates.*

These investigations consisted of bacteriological examinations of dust collected in houses of three types:-

(a) Dirty houses in which a consumptive patient is living who takes no precautions to dispose of his expectoration, but spits freely upon the floor and into his pocket handkerchief. In $66^{\circ} 6$ per cent. of these houses virulent tubercle bacilli were found showing the large amount of dangerous infective material present in an infected house.

(b) Clean houses in which a patient is living who is not sufficiently careful as to the disposal of his sputa. In 50 per cent. of these instances the bacillus was found. It is evident that ordinary household cleanliness alone is insufficient to prevent the accumulation of infective material in rooms occupied by a consumptive.

(c) Very dirty houses in which there had been no case of consumption for some years. In this class of house no tubercle bacilli were present, showing that virulent dust found in classes 1 and 2 must have been due to the presence of the consumptive patient.

Taking the first two classes, the average of houses infected was 61 per cent. Cornet's similar work resulted in finding 71 per cent. tubercle infected.

It was ascertained that the dust nearer the floor than the ceiling possessed the greatest virulency. It was also shown that the infective dust was most virulent in cases where the access of sunlight and free circulation of air was prevented, while, conversely, the beneficial effect of light and air was demonstrated even in the dirtiest houses. Instances were given of the dangers attaching to infected rooms, and the risk to healthy occupants and their successors. According to Koch, "it is the overcrowded dwellings of the poor that we have to regard as the real breeding-places of tuberculosis."

18. Sunlight and fresh air are the greatest enemies to infection.

19. Disinfection is necessary after death from phthisis, and should be as complete as after any other infective disease. Compulsory notification of fatal cases and compulsory disinfection have been officially ordered by the Prussian Government. In this country, also, absolute disinfection should always be insisted upon after phthisis. Walls, floors, carpets, curtains, etc., should be strictly disinfected. Spraying with 1-100 solution of chloride of lime, or other similar disinfectant, is the best method (see p. 444).

* The pathology and etiology of pseudo-tuberculosis is fully treated of by Klein in the Supplement to the Twenty-Ninth Annual Report of the Local Government Board, 18.99-1900, pp. 355-384. See also Annales de l'Institut Pasteur, 1894, No. 4, and Jour. of Path. and Bact., 1898, pp. 160-181 (Muir).

Trans. Brit. Cong. on Tuberculosis, 1901, vol. ii., pp. 88-101. 
The B. pseudo-tuberculosis (Pfeiffer) resembles B. coli, and occurs as short, small bacilli, cylindrical, and with round ends. Its manner of grouping is singly, or in couples or chains; sometimes filamentous forms and long chains occur in bouillon culture. It stains by alkaline Loffler's methyl-blue, and also by Gram's method (Klein). It is non-motile. In bouillon, in twenty-four hours a well-marked granular cloudiness appears, and small flocculi float through the liquid. Imperfect pellicle after several days' growth. No general turbidity. On gelatine the growth resembles $B$. coli, but the colonies are more circumscribed and granular, and later, they become tuberculated. Growth is slow, and the colonies become more opaque, whiter, and less spread out than $B$. coli. No gas is formed in gelatine shake cultures. There is no liquefaction of gelatine. On agar minute grey-white flat colonies appear. Stroke and stab cultures are similar to $B$. coli, but not so luxuriant. There is limited growth on potato, which forms a thin layer with crenated thicker margin of a whitish-yellow colour. It grows well in milk, but leaves it unaltered. Pathogenesis-Guinea-pigs inoculated subcutaneously with a small quantity of culture die in a few weeks. Their organs are found to be studded with yellow-white nodules containing the bacillus in pure culture. These nodules develop more rapidly than true tuberculosis, but do not contain any giant cells. If fed with food contaminated with this organisin, similar nodules develop in the walls of the intestine and mesenteric glands. Klein believes that: "The presence of the B. pseudo-tuberculosis in milk may probably play a part in causing pseudo-tuberculous disease in the human subject."

Klein found this bacillus present in 2 out of 5 samples of London milk,* and in 8 out of 100 samples of country milk delivered in London.† Delépine found that out of 450 samples of milk, lesions produced by pseudo-tubercle bacilli were met with four times. It seems not unlikely that this group of bacilli includes several varieties bearing a close general resemblance to each other, but possessing slightly different properties. They gain access to milk in all probability by some accidental contamination. The milk itself remains unaltered in appearance, though it becomes alkaline. As regards differential diagnosis, it may be said that the pseudo-tubercle bacillus is not acid-fast, nor is it similar to B. tuberculosis in morphological or cultural characters. The pathological changes set up by it, and which form its chief claim to be considered as in any way related to tuberculosis, differ from that disease in showing an absence of giant cells in the nodules, absence of the true tubercle bacilli, copious presence of the pseudo-tubercle bacilli, and a more rapid development of disease.

\section{Acid-Fast Bacilli allied to the Tubercle Bacillus}

We may here suitably consider the group of organisms morphologically and tinctorially similar to the bacillus tuberculosis. This group is known as that of the acid-fast bacilli, on account of the fact that in staining by the Ziehl-Neelsen method (see p. 459) these organisms possess, like the tubercle bacillus, the power of

* Report of Local Government Board, 1899-1900, p. 360, and 1900-1901, p. 332.

$\dagger$ Jour. of Hygiene, 1901, vol. i., p. 83. 
resisting decolorisation by the acid following the red stain.* In England such bacilli are termed acid-fast, in Germany saurefeste, and in France acidophile. The group is one of great importance, partly on account of the ease with which its members may be mistaken for the "true" tubercle bacillus, and partly on account of the relationship which appears to exist between them and the tubercle bacillus. Some bacteriologists hold that possibly these acid-fast bacilli represent a saprophytic stage in the life-history of the true tubercle bacillus.

In his description of the tubercle bacillus, Koch foretold the probability of other acid-fast organisms being discovered, and some fourteen years after, in 1896, Koch and Petri actually demonstrated the occurrence of such bacilli in the butter and milk of Berlin. In the years immediately following, Rabinowitsch, Korn, Coggi, Tobler, and others, found further organisms of this nature in such articles of food. In 1898 Moeller showed that these acid-fast bacilli occurred naturally in animals and plants. Dust, grass, hay, manure, and similar substances yielded them, and now it is known that a considerable family of these bacteria exists. It should, however, be understood that the group is provisional only. Further knowledge may reveal facts which would considerably modify present views.

Classification of Acid - fast Bacilli.-These bacilli may be divided provisionally and for convenience into four chief subdivisions :-

(a) The acid-fast bacilli of other diseases or conditions affecting man (e.g., B. leproe, B. smegmatis, B. of syphilis of Lustgarten, etc.). Other non-tubercular acid-fast bacilli have been found in lung gangrene (Fränkel), in the nasal cavities (Karlinski), in excreta, and in certain chronic eye diseases, etc.

(b) The acid-fast bacilli occurring in other animals (e.g., $B$. tuberculosis avium of Maffucci; the B. tuberculosis piscium of Dubard, Bataillon, Terre; B. tuberculosis ranicola of Lubarsch; B. tuberculosis anguicola of Moeller, etc.).

(c) The acid-fast bacteria of butter and milk (e.g., B. laticola planus, perrugosum, Friburgense, etc.), of Petri, Moeller, Rabinowitsch, Binot, Markl, Coggi, Tobler (Nos. i.-v.), Grassberger, and Korn (Nos. i. and ii.).

(d) The acid-fast bacilli of grass (e.g., B. phlei or Timothy bacillus, and Grass bacillus, No. ii., of Moeller), the "manure bacillus" of Moeller, the urine bacillus of Marpmann.

All these various organisms are morphologically and in staining

* Acid-fastness is due, in all probability, not to fat in the bacillus, but to a substance of the nature of wax, which can be extracted by acid-alcohol, ether, or other wax solvents. For a discussion of this subject, see Trans. British Congress of Tuberculosis, 1901, vol. iii., pp. 498-502 (Bulloch). 
properties allied to the $B$. tuberculosis. The chief characters of most of them are referred to under Tuberculosis, Leprosy, etc., but it will be necessary to describe briefly the character of others.

(a) Acid-fast Bacilli of Human Origin.-The leprosy bacillus will be described subsequently (see p. 398).

The smegma bacillus was first discovered by Tavel and Alvarez, in 1885 , in the normal preputial smegma, and also in the secretion of the outer skin, particularly where a collection of epithelium may occur, as in the fold of the groin, between the toes, etc. The discovery of the bacillus was incidentally made in investigating an observation of Lustgarten, in 1884, on the syphilis bacillus. Morphologically and tinctorially, the smegma bacillus closely resembles the syphilis bacillus of Lustgarten. This fact discounted the importance attached to Lustgarten's discovery, and subsequent investigations show that Lustgarten's bacillus has not been found in sufficient numbers, or with sufficient constancy, in the syphilitic tissue.

The smegma bacillus, according to Tavel and Alvarez, is morphologically exceedingly like the tubercle bacillus, and can be stained by the same methods. Inoculation experiments on animals were without result, nor were the authors able to obtain a pure culture. Laser and Czaplewski have cultivated (the former from the secretion of syphilitic affections, the latter from gonorrhœal pus) micro-organisms resistant to acids, similar to diphtheria bacilli, which have been declared by both authors to be identical with the smegma bacillus. Fränkel only calls those micro-organisms smegma bacillus which first attracted attention by their great resemblance to tubercle bacilli. This resemblance is wanting in the cultures of Czaplewski and Laser, and in his own cultures, which he described later. In form and other characters they are much more like a pseudodiphtheritic bacillus. Moeller agrees with Fränkel. Moeller was not able to get a pathogenic effect in guinea-pigs either with the diphtheroid bacillus cultivated in pure culture from smegma, or with the genuine smegma bacillus containing cutaneous secretion in abundance. In this way the smegma bacillus differs from other acid-fast bacilli. He found human serum the best culture medium for smegma bacillus. The morphology differs according to media, especially in milk cultures. Moeller found the bacillus to be absolutely acid-fast and alcohol-fast, and this property is not much diminished by age or media. The organism is strongly aërobic, and grows slowly. On glycerine agar at $37^{\circ} \mathrm{C}$. dull grey-white scales of growth occur, and on potato dull white-grey colonies. Growth is rapid in milk, and the milk is not coagulated.

With respect to differential diagnosis, especially in reference to urogenital tuberculosis, the smegma bacillus is undoubtedly of great importance. 
PLATE 25.

Acid-fast bacillus from butter of Berlin

(Petri-Rabinowitsch).

Flask culture on glycerine-agar-3 months at $22^{\circ} \mathrm{C}$.

Single colony-actual size.

Acid-fast bacillus from milk of Belzig

(A. Moeller).

Flask culture on glycerine-agar- 3 months at $22^{\circ} \mathrm{C}$.

Single colony-actual size. 
properties allied to the $B$, fulderchlosis. The efiet sharacters of most of them are referred to moder Tuberenlonis, Leprosy, etc., but it will be necessary to desoribe brietty the chatseber of others.

(a) Acid-fast Baellil of Human Origin. - The leprosy bacitlus will be deseribed sulfirgd to toffud mont enlliond fasit-biah

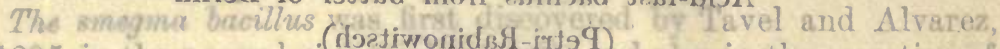

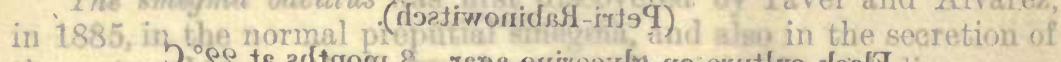

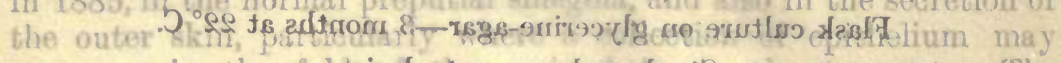
cocur, as in the folosia frutos-cynolos sfgrriz the toes, eto. The discevery of the bacillus was incidenitaly mado in investigating an observation of Lustgarten, in 1884 . wh the syphilis bacillus, Morphologically and tinctorially, the smegrma bincillus closely resembles the syphilis hacillus of Inowgsthr, This faot discounted

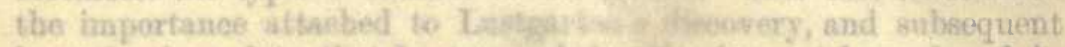
investigations ahow that faraterass

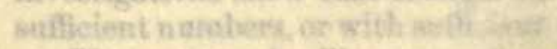

Thes mencerus taerilitak

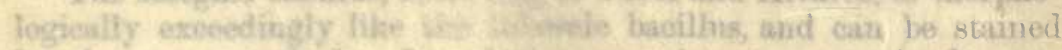
by the sume methoris. Tum tixase experiments on animals were withont restilt, nor were the suldesta able to obtain a pure eulture. Laser and Czaplewski have oulkscat the former from the secretion of syphilitio affections, the latter frise ponorrhoial pes) zatcro-organisms resistant to acids, similar te ciphtheris becilli, which have been declared by both authors to fo identical with the smegma bacillus. Fränkel only calls those asioro-organisms smegma bacillus which first attescted attention by thehr great resemblence to tuberele bacilli. This resemblance is wanting in the eultures of Craplewski and Laser, and in his owr cultures, which he doscribed later. In

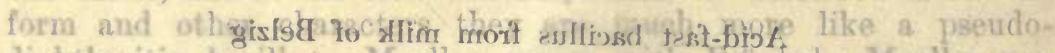

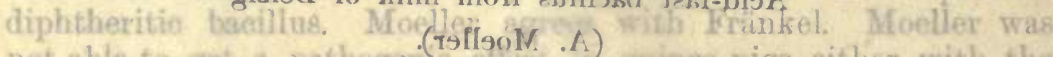

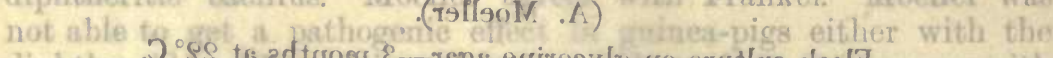

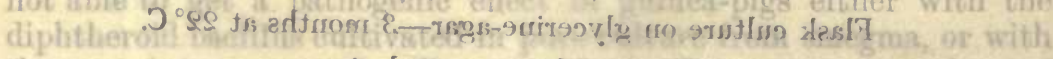

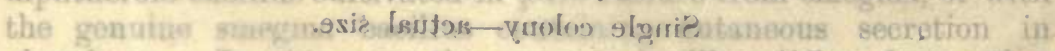

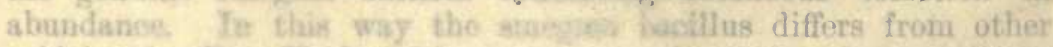
acid-fast baeilhi. Ho found human serasu the best culture mediuile for stmegrua bucillus. The morphotheg distors according to media, especially in mitk oultures. Moellez found the bacillus to be absolutely acid-fast and alcohol-fast, asal this property is not much diminished by age or media. The organien is strongly aẻrobio, and grows slowly. On glyeerine agar at 37.0 . dull grey-white seales of growth oovur, and on potato dull white-grey colonies. Growth is rapid in milk, and the snilk is not coagalated.

With regreot to differential diagnosis, especially in reference-t.

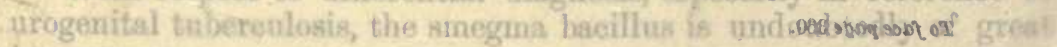
importance. 
Nenfeld and others have obtained acid-fast organisms from smegma, and Neufeld concludes that some of these may be described as similar to $B$. diphtherioe and others to the tubercle bacillus.

(b) Acid-fast Tubercle Bacilli in Animals. - The members of this group are provisionally assumed to be related to the tubercle bacillus. Reference is made to these organisms under Tuberculosis (see p. 349).

(c) Acid-fast Bacilli in Butter and Milk.--Several years after Koch's discovery of the tubercle bacillus, species of bacteria were found possessing acid-fast properties, but it was not until 1896 that Koch and Petri isolated such organisms from the milk and butter of Berlin, and in the following year Lydia Rabinowitsch carried out her research on the same subject. In 1899, Korn discovered two bacilli in the butter of Freiburg, Binot a bacillus in the butter of Paris, and Coggi a bacillus in the butter of Milan, all four of which were acid-fast. In the same year Grassberger published a statement upon acid-fast organisms occurring in butter and margarine. In 1900, Beck and Santori met with similar organisms in milk; and in 1901, Maria Tobler, Markl, and Moeller and Jong isolated acid-fast bacilli from both butter and milk. All these organisms were bacilli, but they showed much variation in form and polymorphism, some appearing to be like $B$. diphtherioe, and others like actinomyces. The staining properties were, in all cases, those of the true bacillus of Koch, except that the power of resistance to decoloration by acid was rather less.* Swithinbank has cultured many of these organisms upon different media, and has found them to show various modifications in form, chromogenicity, vitality, polymorphism, etc. By a series of cultures, he showed the various characters of ten of these acid-fast species compared with the human and bovine tubercle bacillus, all the cultures having been grown on the same media, for approximately the same length of time, under precisely the same conditions. The cultures were in each case composed of one colony only, and admirably revealed the differences between the species. These acid-fast bacilli live and develop on all ordinary media at room temperature and blood-heat, preferably under aërobic conditions. They do not form indol or liquefy gelatine, nor do they possess much pathogenic action. $†$

The Butter Bacillus of Petri-Rabinowitsch.-Morphologically, this organism is like the ordinary tubercle bacillus, though somewhat shorter and thicker. It stains in the same manner, but grows readily at room temperature and rapidly at blood-heat. It is non-motile.

* Report of Medical Officer of Local Government Board, 1900-1, pp. 331-3.

† See Bacteriology of Milk (Swithinbank and Newman); in The Journal of State Medicine, 1903-4, will be found a useful summary of present knowledge of acid-fast bacteria, by A. C. Coles; Potet's work should also be consulted. 
The cultures appear as moist, thick, creamy, wrinkled layers of growth on the surface of the medium. The bacillus renders broth turbid and acid. Indol is also produced, and a disagreeable odour. The growth is slow on gelatine, occurring as small granular colonies. It is nonliquefying. On glycerine agar, growth is abundant, rapid, and characteristic. It occurs as a creamy film-of a light golden colourmoist, thick, and much wrinkled (see Plate). It possesses a glistening appearance, and an unpleasant odour These characters disappear after much sub-culturing. Milk is not coagulated. A dull dry growth generally occurs on potato. The organism possesses less virulence when inoculated in pure culture. But when inoculated with, or without, butter, it has clearly defined effects. Giant cclls, nests of epithelioid cells, and typical tuberculous caseation are, according to Rabinowitsch, never to be found in the foci of disease set up by this bacillus. None of the animals injected with this bacillus reacted to tuberculin. The intra-peritoneal injection of pure cultures often produces a formation of nodules in the abdominal organs which frequently heal. If, however, the animals are killed in three or four weeks, the following characteristics are found, namely, slightly distended abdomen, more or less severe peritonitis, nodules on mesentery and beneath the intestinal serosa, mesenteric glands enlarged, and liver, spleen, and kidneys showing small nodules with yellowish exudation. When the butter itself containing the organisms is used, a fatal result often follows the injection after three to fifteen days. Similar changes to the above have occurred, but of a more intense degree. Rabinowitsch found rabbits insusceptible in contrast to guinea-pigs. It is not known whether this bacillus is in any degree pathogenic for man. But probably such is not the case. It appears to be widely distributed in nature, as 60 per cent. of butter samples in Berlin were found to contain it. The only satisfactory way to differentiate this bacillus from the tubercle bacillus is by inoculation of animals.

Moeller isolated a somewhat similar organism from milk, which is generally known as Mocller's Milch Bacillus. It was found in pasteurised milk at Belzig, and is almost identical in morphology to the tubercle bacillus. It is acid-fast, non-motile, and grows at room temperature as well as blood-heat. Broth becomes but little turbid, and there is no deposit. Surface membrane of fatty aspect and amber colour, adherent to tube walls, is sometimes formed. On gelatine plates and tubes a white wrinkled culture of creamy nature occurs, and on glycerine agar, after about three weeks, the growth is white, uniform, and of a creamy nature, though at times slightly wrinkled, and dry. In old cultures it is dry, or glazed, and of a yellowish colour, which later turns to a reddish tint, the culture itself becoming of a wrinkled appearance. Frequently the raised 
PLATE 26.

Acid-fast bacillus from butter of Friburg

(Bacillus Friburgensis-Korn 1.)

Flask culture on glycerine-agar- 3 months at $22^{\circ} \mathrm{C}$.

Single colony-actual size.

Acid-fast bacillus from butter of Friburg (Korn II.)

Flask culture on glycerine-agar-3 months at $22^{\circ} \mathrm{C}$. Single colony-actual size. 
The esitures sppear as moist, thick, creamy, wrinkled layers of growth va the earlisee of the medimn. The bacilins renders broth turbid and acich. Indel is also produced, and a disagreeable odour. The growth is abov on gelatine, oecurring as small granulàr colonies. It is nonliquofying. On glyoerine agar, growth is abundant, rapid, and char-

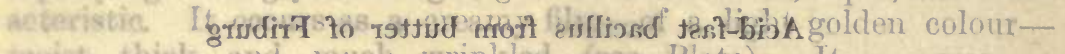

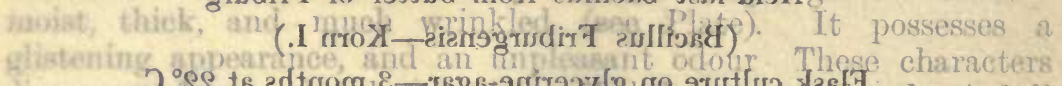

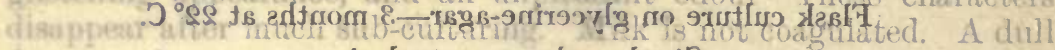

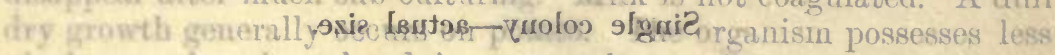
viealenee when inoenlated in pures eulture. But when inoculated with, or without, butter, it has clowely defined éffects. Giant cells, neste of epithelioid cells, and tyisical tuberoulous caseation are, assording to Rabinowitsch, never bi he found in the foei of disease ses. sp by this bacillus. None of the animals injeoted with this lwastivs nuted to tuberculin. The intra-peritoneal injection of pure sculbeys nften prodnees a formation of nodules in the abdominal

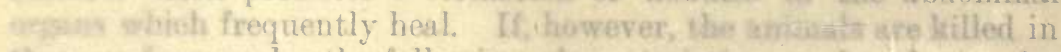
Aliron of four weeks, the following characteristios are found, namely, slichtily distended abdomen, more of less severe peritonitis, nodules on rowontary and beneath the intastinal serosa, mesenteric glands enlarged, and liver, spleen, and kidneys showing small nodules with yellowish exudation. When the butter itself containing the organisms is used, a fatal result often follows the injection after three to fifteen days Similar changes to the atove have occurred, but of a more intessed degree. Rabinowitsch found rabbits insusceptible in contrast to guinas-jiga. It is not known whether this bacillus is in any dingree patiogenic for man. But probubly strch is not the case. It uppears to be widely distributed in nature, as 60 per cent. of butter

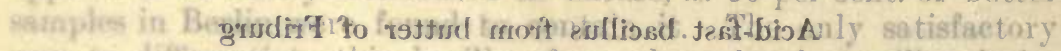
way thi difforeariate this b (.H imo $\pi)^{m}$ the tubercle bacillus is by

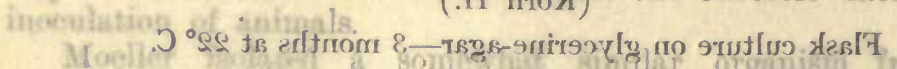

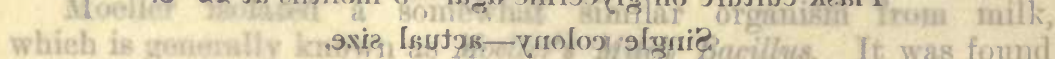
in pasterurined matk at Belzig, and is almost identical in morphology to the tuberele basillus. It is acid-last, non-motile, and grows at room tempernture as well as blood-heat, Broth becomes but little turbid, and there is no deposit. Surface membrane of fatty aspect and amber colour, adherent to tube walls, is sometimes formed. On achistine plates and tubes a white wrinkled culture of creamy nature werters, and on glycerine agar, after about throe weeks, the growth is whabe moifom, and of a oreamy nature, though at times slightly whichel, and dry. In old eultures it is dry, or glazed, and of a yochounde colour, which later turns to a redilish tint, the eulture

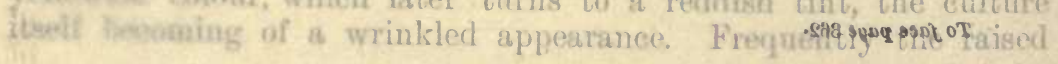




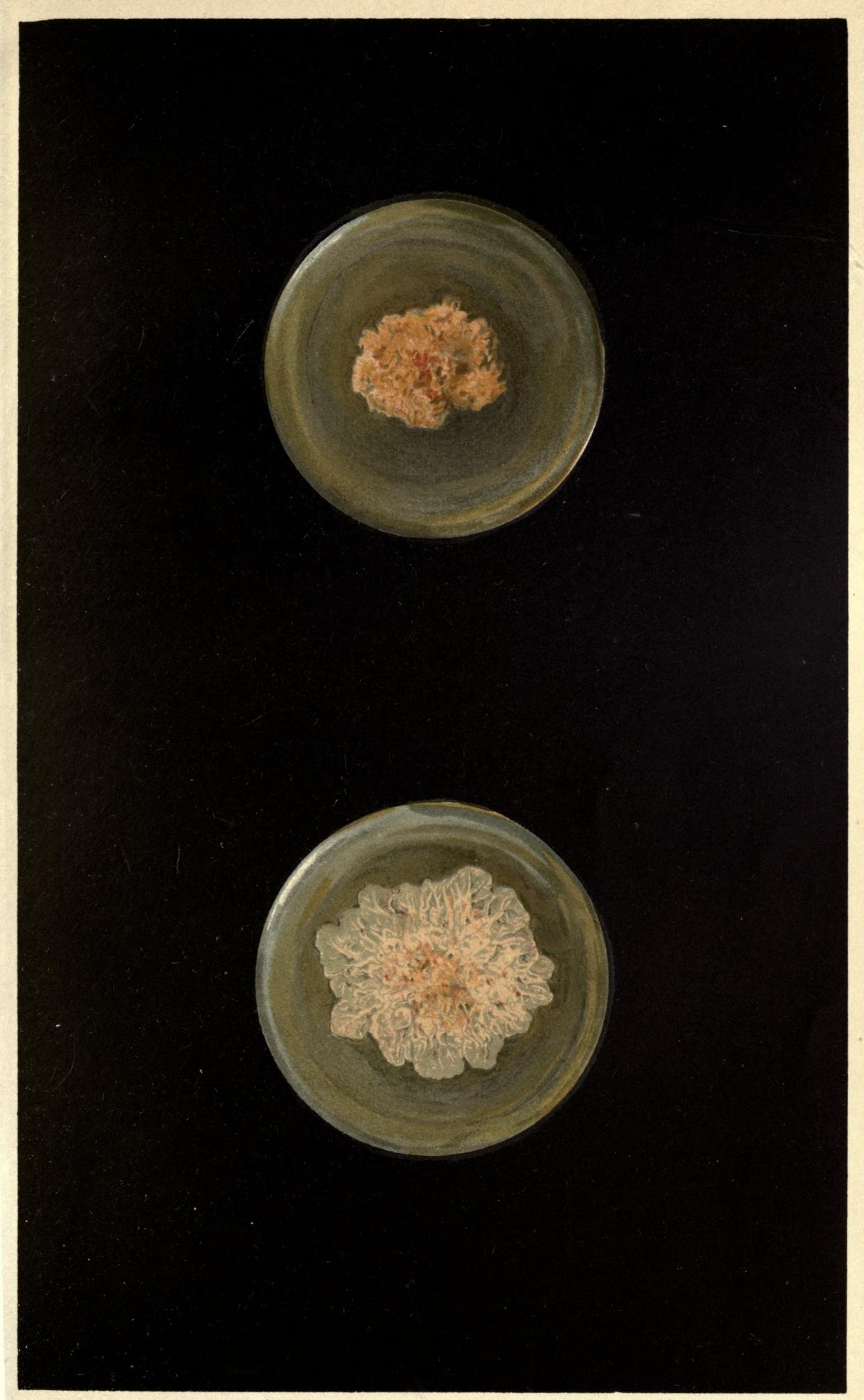


LISRART UNIVERSITY

CALIFORNIA 
"granular" centre is surrounded by a light blue zone, but slightly raised above the medium (see Plate); on glycerine potato, there is at first a white creamy growth very little raised above the surface of the medium, but as it grows older the culture becomes wrinkled and of a deep yellowish tint, almost red. The bacillus grows quickly and luxuriantly in milk, forming an ochre-yellow ring round the surface edge of the medium. The organism was found to produce nodules in the organs of inoculated animals.

Korn isolated two acid-fast bacilli from Friburg butter. B. friburgensis, No. 1, varies in morphology under different circumstances. In preparations made from the organs of an inoculated animal, the bacilli resemble in shape and size the B.tuberculosis of Koch. In bouillon, the shape very much resembles the $B$. coli, but is a little longer and slightly curved. On agar the bacilli are slightly thinner. In old cultures upon agar and serum, they assume the aspect of "Coccothrix." Upon potato they appear under form of cocci, diplococci, and specially of short, stout bacilli slightly curved. Upon cooked beetroot at the end of three or four days the organisms resemble staphylococci. They are shorter when grown at ordinary temperature than when grown at $37^{\circ} \mathrm{C}$. In culture media the older growths generally exhibit some orange or red coloration, though on almost all media the growth is at first white and non-wrinkled. Upon glycerine-agar a thick, white, brilliant growth occurs with deposit in the water of condensation, which is soon covered with a veil adherent to the walls of the tube. Later on the growth becomes slightly folded. At the age of about three weeks the culture is creamy, presenting a few, light folds, and of yellow-orange colour. In older cultures the growth is very abundant, raised considerably above the surface, and irregularly folded and convoluted, the colour varying in depth from light to dark orange or even red brick (see Plate). Milk is not coagulated, but at the end of three weeks possesses a yellowish-browu colour. Subcutaneous or intraperitoneal injection of pure cultures produce only an abscess at site. If injected with butter itself in white mice, granulations are produced in thoracic and abdominal viscera, showing no giant cells, but commencing caseation.

B. friburgensis, No. 2, consists of a small rod two or three times longer than broad, often irregular and sometimes clubbed. It is much less sensitive to decolorisation by acid than No. 1; grows feebly on ordinary laboratory media, with the exception of glycerine agar, in which growth appears in twenty-four hours, and eventually becomes abundant. The culture is creamy, glistening, and a yellowish colour (see Plate). Milk turns of a dirty red colour in about three weeks time.

Markl, Tobler, Coggi, Binot, and Grassberger have also isolated 
acid-fast bacilli from butter. Brief reference may be made to the organisms discovered by the two last-named workers.

Binot's Butter Bacillus is in morphological and tinctorial characters similar to the tubercle bacillus. It differs in cultural features. Binot describes it as producing a thick, viscous, creamy layer in broth, from which "stalactites" grow down into the liquid. It grows on gelatine (which it does not liquefy), producing thin creamy irregular surface colonies. On glycerine-agar plates and tubes white colonies appear, becoming straw-coloured, and finally orange. They may attain diameter of two-frane piece or larger; and have a bright glistening surface, and are very adherent to medium. On the surface they soon become wrinkled and irregular, and have scalloped edges. Chromogenic characters are miore marked if the growth is exposed to air and light (see Plate). On potato, a scanty growth occurs, which is at first moist, and of a clear yellow colour. On glycerine potato an abundant homogeneous growth occurs, of an opaque yellow colour, turning to orange. Irregular nodosities appear on the colony. The bacillus produces tuberculous-like changes in animals in which it is inoculated.

The Butter Bacillus of Grassberger is very similar to the other members of this group, except that it produces, especially on gelatine, but also on glycerine agar, a dry, much-wrinkled growth not unlike some forms of mould, and of a deep rose colour. There is no liquefaction. The wrinkles in the large colonies appear as characteristic markings (see Plate). Milk is coloured throughout by the organism, but not coagulated. There is also a surface growth and deposit, both rose coloured. On potato the growth is similar to tubercle bacillus, but of a deep rose tint.

(d) Acid-fast Bacilli in Grass, Hay, and Manure.-This second group of acid-fast bacilli associated with milk (marked $(d)$ in the classification above) is often designated as that of the grass bacilli. They were first cultured on Timothy grass (Phleum pratense), which is much valued for feeding cattle.* Since then, however, this grass bacillus has been found in various places, and it or its allies have been isolated from cattle fodder, hay, hay-dust, manure, milk and its derivatives. Morphologically, this bacillus (B. phlei) is similar to the tubercle bacillus, slender and slightly curved. It contains highly-stained granules and oval, clear spaces; often grows in threads; and is branched, and sometimes has clubbed swellings at one end. It is acid-fast in staining, and grows best at incubation temperatures on the ordinary culture media. The colonies become visible in thirty-

* Cat's tail or Timothy grass (Phleum pratense). Although well known to the British grower this grass is more extensively cultivated in the United States, where it was introduced from Britain, nearly a century ago, by Mr Timothy Hanson, after whom it is named Timothy grass. 
PLATE 27.

Acid-fast bacillus from butter of Paris (Binot).

Flask culture on glycerine-agar-3 months at $22^{\circ} \mathrm{C}$. Single colony - actual size.

Acid-fast bacillus from butter of Vienna

(Grassberger).

Flask culture on glycerine-agar-3 months at $22^{\circ} \mathrm{C}$. Single colony - actual size. 


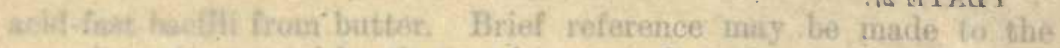
onganiscan diakowozod by the two la'st-nataed workers.

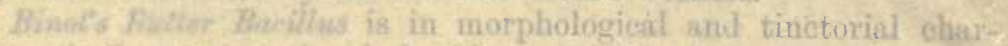

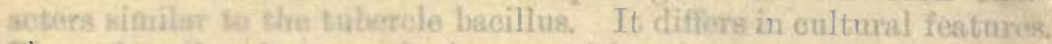
Binot degoritus it an prowlucing a thiok, viscons, oreamy layer in broth, frobs whichs" staluntites" grow down inte the liguid. It grows

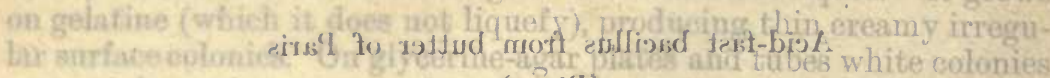
appear, beomaing straw-colon:e(forrid) finally orange. They mey

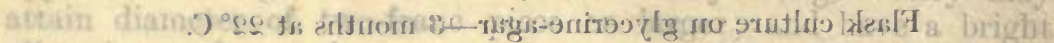

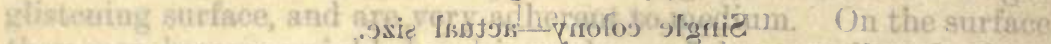
they sow become wrinkled and irregular, and have sealloped edges. Chensachanic characters are more marked if the growth is exposed to aif and light (see Plate). On potato, a scanty growth ovcurs, which is at first moist, and of a clear yellow colour. On glycerine patato wh whindant homogenewns growth ocours, of an opaque yellow colout: furning to orange. Irregaism, nodosities appear on the colony. Thes: busillus produeses tubcrutons-like changes in wnimais in which it is inoculated.

The Buttor Baoilhes of Qrassberger is very similar to the other members of this gmip; exeept that it produces, especially on gelatine, but also on glycerine agar, a dry, much-wrinkled growth not unlike some forms of mould, and of a deep rose colour. There is no liquefaction. The wrinkles in the lurge colonies appear as characteristio markings (ree Plate). Milk is colouret tbronghout by the organism, but not coagnlated. There is also a snxface growth and deposit, both rose colonsid, On potato the growth is similar to tubercle bacillus, but of a deran roge tint:

(d) Acid-fast Bacilli in Geass, Hay, and Manure,-This seconi group of actil-fast becilli assoejintoy with milk (marked (d) in the

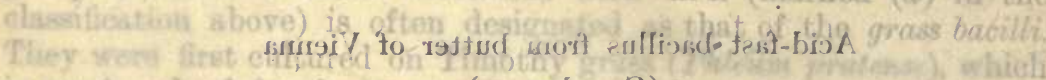
is wheh walued for feeding en (rogtoderesti))

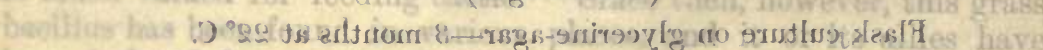

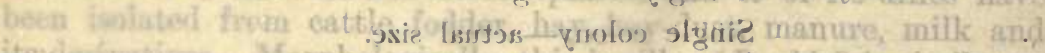

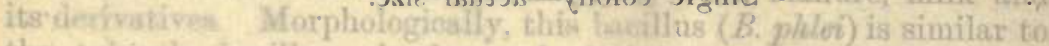

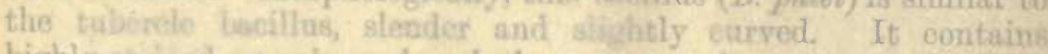
highly-stuinod granules sad oval, clear mpuces; often grows in threads. and is bratuched, and sametimeg hos chabbed swetlings at one end. It is achitast in shatning, and grows ben at incubation temperatures on' the octinary ene me media. The colunies become visible in thirty.

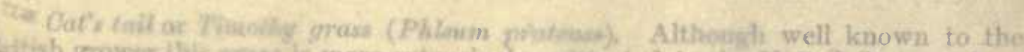
Ratish grower this mas is more extensively seitikated in thin United States, where

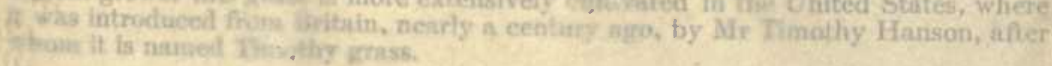



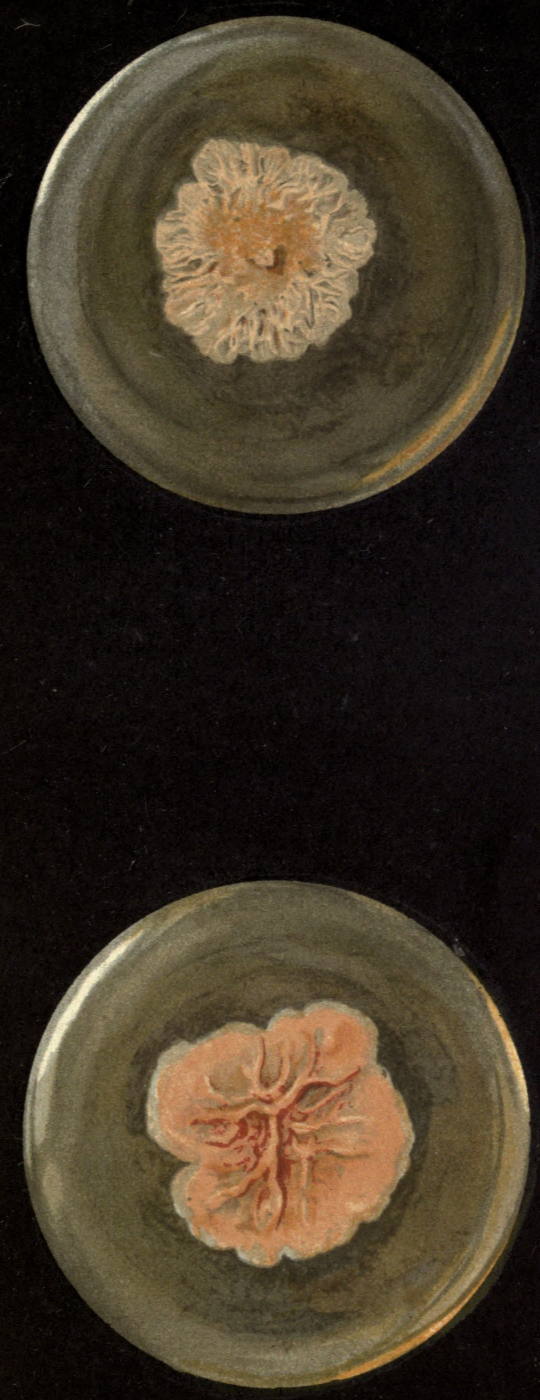

six hours, are scale-like and greyish-white or yellow in colour. It grows best on glycerine agar. It grows slowly in milk, but does not coagulate it. Under certain conditions its growth in artificial media is very similar to the tubercle bacillus, which, however, does not thrive at room temperature. As regards pathogenic properties, the grass bacillus is almost identical with the Petri-Rabinowitsch butter bacillus in its effects on guinea-pigs. It has somewhat different effect on rabbits, producing a condition difficult to distinguish from true tuberculosis (Lubarsch). Giant cells, epithelioid cells, and caseation are all said to occur. In all animals injected with the grass bacillus a negative reaction to tuberculin is obtained. Moeller has isolated a grass bacillus, No. 2 (from the dust of a hay-loft), which he considers essentially different from the Timothy bacillus. The colonies are moist and sticky, become confluent, and are yellow in colour. It loses its acid-fast properties in old cultures. Its pathogenic properties are most marked when cultured in milk. It frequently shows marked polymorphism. In culture it is like the butter bacilli. Freymuth has shown that the changes this organism sets up in coldblooded animals are indistinguishable from true tuberculosis. An acid-fast bacillus similar to grass bacillus, No. 2, has recently been isolated by Moeller from milk. A variety of the grass bacillus has also been found by Moeller in the excreta of animals, and is therefore termed the manure bacillus (mist bacillus). This acid-fast bacillus has been isolated from the excreta of cattle and other animals, and bears a morphological and tinctorial resemblance to the Timothy bacillus, whilst in cultures it is like grass bacillus, No. 2. On agar these organisms grow in a similar manner, but bouillon does not become turbid with the growth of the mist bacillus. It has certain pathogenic properties. When injected into guinea-pigs, nodules resulted; but they contained few epithelioid cells. The true radial arrangement of the bacilli occurs, however. It is possible that most of the acid-fast bacilli found in milk and butter have their origin in the soil or vegetation.

Differential Diagnosis.-This brief record of the acid-fast bacilli is enough to show that there exist a large number of bacilli, which on occasion may be present in milk or milk products, having characters which ally them closely to the tubercle bacillus. Moeller holds that the primitive form is the grass bacillus, and that the butter bacillus, manure bacillus, etc., are varieties thereof. The main points of distinction between this group and the true tubercle bacillus are five.

First, the tubercle bacillus shows a fairly uniform manner of growth.

Secondly, it requires incubation temperature.

Thirdly, it is unique with respect to its excessively slow growth. 
Fourthly, it is as regards growth and propagation a parasite.

Fifthly, on inoculation it produces pathological cellular changes distinct from the nodular new growths following inoculation of acid-fast bacilli. In particular this is true, as far as is known at present, in regard to the human organism.

In a sentence, the acid-fast bacilli differ from the tubercle bacillus in three main particulars, viz.: morphology of culture, conditions of development (chromogenicity, rapidity of growth, range of temperature within which they flourish), and their feebler pathogenic properties. From these facts it follows that however great the degree of similarity between these various acid-fast bacilli, and however much it is possible by artificial cultivation to modify the morphology of the various forms, there is sufficient difference to enable a differential diagnosis to be made if all the biological characters are ascertained, and most of all the pathogenic properties. Hence the importance of the inoculation test being applied to acid-fast and tubercle-like organisms detected in milk or butter.

As a simple method of differential diagnosis, Moeller suggests that the smegma, sputum, or other secretion should be mixed with nutritive bouillon, and kept at about $30^{\circ} \mathrm{C}$. If in two or three days there is a visible increase in the bacteria resistant to acids, it is certain that it is not the genuine tubercle bacillus, which requires $37^{\circ} \mathrm{C}$. Sometimes in sputum, mixed with certain nutritive media, the tubercle bacillus increases at incubation temperature. This proliferation, due in all probability to the importation of globulin-like substances from the body, is, however, exceedingly small, and ceases altogether after, at the latest, forty-eight hours; whilst in the pseudo-tubercle bacilli a persistent further proliferation takes place at $30^{\circ}$.

The pathological differences from Koch's bacillus are that inoculation with acid-fast bacilli gives rise to no "giant cells," no epithelioid cell clusters, and no tuberculous caseation. Nodular lesions occur suggestive of tubercle, but according to Potet,* and Abbot and Gildersleive $\dagger:(a)$ they constitute a localised lesion only, having no tendency to dissemination, metastasis, or progressive destruction of tissue by caseation; (b) they tend to terminate in suppuration like ordinary abscesses; (c) when occurring as result of intravenous inoculation they appear in the kidney, rarely in the lung and other organs; and $(d)$ the form of granuloma set up is similar to actinomyces.

This group of organisms is one of considerable importance to the milk bacteriologist, and in all investigations dealing with the tubercle bacillus, or with milk and its produets, it is essential that the bacilli met with should be clearly differentiated from the tubercle bacillus.

* Etude sur les Bactéries dites Acidophiles (Potet, Paris, 1902), pp. 188-194.

† The University of Pennsylvania Medical Bulletin, June, 1902. 


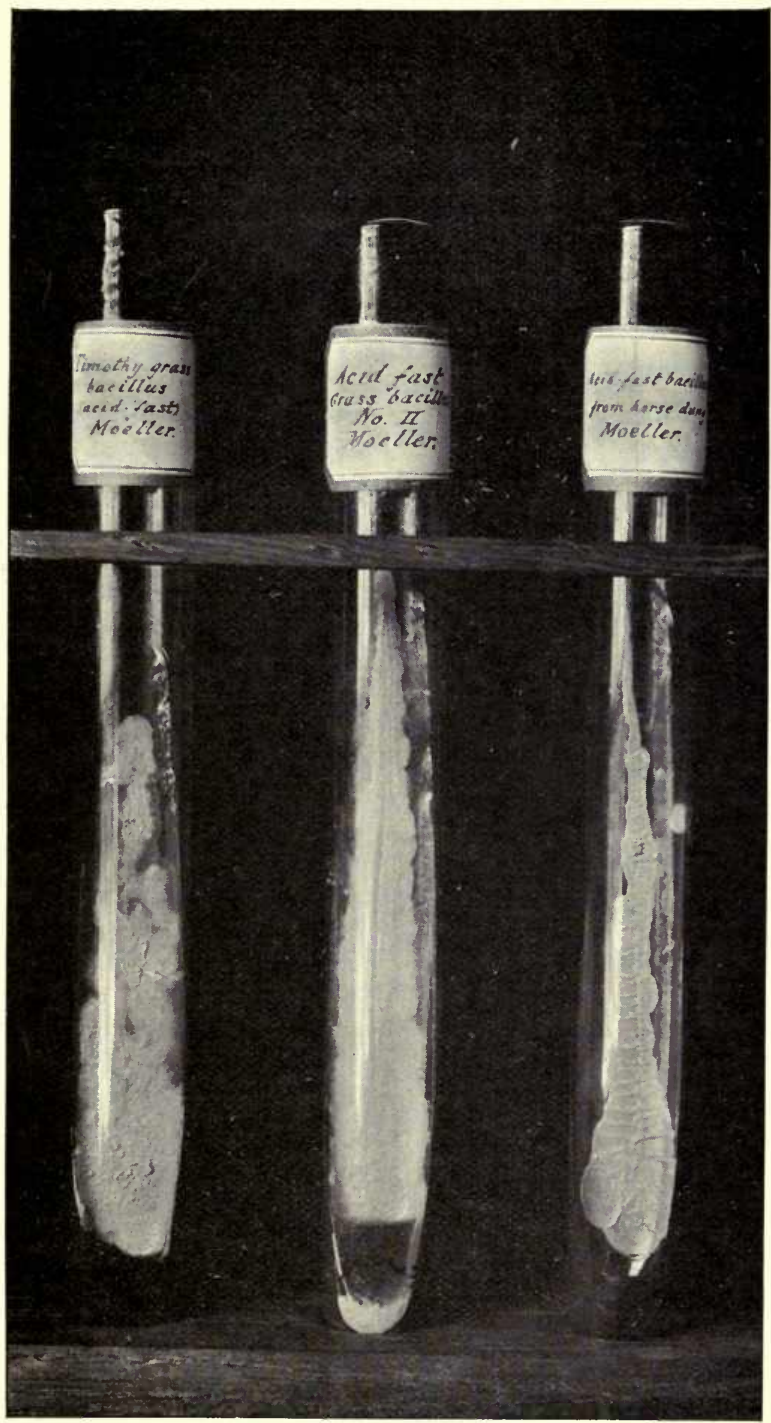

Comparative Cultures of Acid-Fast Bacteria on Glycerine Agar. (Timothy Grass Bacillus, Møller's Grass Bacillus, the Mist Bacillus). (Grown and photographed by Swithinbank). 


\section{LIBRART
OFTHE UNIVERSITY}

CALIFORNIA 
Insufficient care has been taken in this respect up to the present. Any such organism found should be compared in cultural and pathogenic properties with the human tubercle bacillus, the bovine bacillus of pseudo-tuberculosis, and the various acid-fast organisms, and not simply accepted on tinctorial properties as a tubercle bacillus.

Acid-fast Streptothrix Group.-Recently, considerable attention has been given to the group of "higher bacteria" known as the streptothricece. Seen in its mature form, a streptothrix appears to consist of a tangled mass of mycelial threads, some short bacillary forms, and spores. The life cycle is completed by the growth or sprouting of spores by which a mycelium is developed. This branches off in various degrees and directions, and probably sprouts itself, and so produces, with the development arising from spores, a fresh mycelial growth. The mycelium may undergo fragmentation, and thus bacillary forms occur. Streptothrix is usually readily stained by Gram's method, but several species are acid-fast. Foulerton and Price Jones found this to be characteristic of $S$. Nocardii, S. caproe, S. hominis (Sabrazes), and S. Eppingeri, in older culture, and then only the mycelial threads and not the spores. Twenty-one other species were not acid-fast.* The germs of streptothrix usually grow better in culture media at $37^{\circ} \mathrm{C}$. than at $22^{\circ} \mathrm{C}$, and better aërobically than anaërobically. Pigment production occurs in some forms, and certain species liquefy gelatine. One of the best media to use is maltose-peptone agar, but potato, peptome broth, gelatine, or milk are also used. A number of workers have now isolated species of streptothrix from natural media, some of which are declared to be acid-fast. Many of the group are pathogenic to man or animals. $S$. actinomycis (actinomycosis), $S$. bovis communis, $S$. madurce ("Madura-foot"), and the disease known as "farcins des bœufs" are examples. The organisms found in lachrymal concretions, alveolar abscesses, and similar conditions, especially in relation to the jaws, are probably frequently streptothrical in origin. These may gain access to the tissues through carious teeth (by air or food). Infection may also occur through the tonsils, or cutaneously (as in Madura-foot).

A description of Streptothrix antinomyces will be found on a subsequent page. Here two forms of Streptothrix isolated by Foulerton from cases of disease may be described.

\section{Streptothrix luteola (Foulerton).}

Isolated from a case of sloughing keratitis in a girl of 12 years.

Staining Characteristics. - Stains well by Gram's method in young cultures;

* Truns. of the Path. Soc. of London, 1902, vol. liii., pt. i., p. 65. 
cultures up to three months old show no acid-fast portions when stained by the ZiehlNeelsen method.

Cultural Characteristics.-Grows freely in presence of oxygen, very scanty growth under anaërobic conditions; on all media, except potato, growth is distinctly more active at $37^{\circ} \mathrm{C}$. than at $22^{\circ} \mathrm{C}$; old cultures, especially those in peptone beef broth, have a faintly feculent odour. On gelatine at $22^{\circ} \mathrm{C}$.-Streak culture : obvious growth is seen on third day, at first of an opaque white appearance, later may show a very faint yellowish tinge. Growth sinks slowly into the medium, which gradually liquefies. Stab culture : growth occurs along track of needle in the form of superimposed colonies, which have a somewhat flocculent appearance. On peptone-maltose agar.-After 24 hours' incubation at $37^{\circ} \mathrm{C}$. there is some indication of growth ; at $22^{\circ} \mathrm{C}$. no obvious growth occurs within this period. After 72 hours' incubation at $37^{\circ} \mathrm{C}$. there is a fair amount of growth, and rather less after incubation at $22^{\circ} \mathrm{C}$. The growth, at first of a faint drab or whitish tint, after longer incubation, becomes usually of a faint yellowish colour. Cultures may yield a free formation of aërial hyphæ, giving a snowy appearance to the surface of the growth, or the surface may assume a reticulated appearance, without any efflorescence. (For microscopic appearances, see Plate 29.) On inspissated horse-serum.-Growth is comparatively scanty on this medium, appearing after 72 hours' incubation at $37^{\circ} \mathrm{C}$. At the end of twenty-eight days' incubation at $37^{\circ} \mathrm{C}$. there is a dry, wrinkled, drab-coloured growth, which has sunk slightly into the medium; no liquefaction. On potato.-Growth on this medium is equal at temperature $37^{\circ} \mathrm{C}$. and $22^{\circ} \mathrm{C}$.; at the end of 48 hours' growth appears as a brownish or faintly yellowish stain on the medium; later, growth usually assumes a café au lait colour; there is no pigmentation or erosion of the medium. Surface efflorescence is seen only in cultures incubated at $37^{\circ} \mathrm{C}$., and not in cultures at $22^{\circ} \mathrm{C}$. In peptone beef broth.-After 48 hours' incubation at $37^{\circ} \mathrm{C}$. the appearance of some filmy growth is seen at the bottom of the tube; in older cultures growth appears as flocculent, more or less discoid colonies. No pigmentation. (For microscopic appearances, see Plate 29.) In alkaline litmus milk.-Medium at end of 72 hours' incubation at $37^{\circ} \mathrm{C}$. is of a faint pink colour; no coagulation. The pink colour changes to a dirty white, and the milk clears gradually from the surface downwards, becoming at last of a brown colour. Growth on peptone agar is about equal to that on peptone-maltose agar; growth on glycerine agar ( 1 per cent. glycerine) is less free than it is on either of the two preceding media. Diastatic action.-No action of the sort is manifested within fourteen days' incubation at $37^{\circ} \mathrm{C}$. Resistance to heat.-Sporulating cultures resist exposure to moist heat at $70^{\circ} \mathrm{C}$. for 20 minutes, but are destroyed by an exposure to the same temperature for 30 minutes. Pathogenicity for lover animals.-Not found pathogenic for rabbits (intra-venous and intra-peritoneal inoculation), for guinea-pigs (intra-peritoneal inoculation), or for tame mice (intra-peritoneal and subcutaneous inoculation).

\section{Streptothrix hominis (Foulerton).}

Isolated from a case of pulmonary infection in a woman (especially from subcutaneous abscesses).

Staining Characteristics.-Takes Gram's stain. A three-months' old culture from glycerine-peptone agar showed no acid-fast portions when stained by the ZiehlNeelsen method.

Cultural Characteristics.-Growth was obtained in peptone-beef broth and on solid media under ordinary aërobic conditions; no growth occurred on tubes of peptone agar and glycerine-peptone agar incubated under anaërobic conditions. Growth is more active at $37^{\circ} \mathrm{C}$. than at $22^{\circ} \mathrm{C}$. On peptone agar.-Growth very slow and scanty; after several weeks' incubation small whitish, heaped-up, drylooking colonies, resembling somewhat the growth of B. tuberculosis, are seen. On glycerine agar and maltose-peptone agar.--The amount of growth is very much the same as in the last case, and of much the same appearance. On inspissated horseserum and inspissated ox-serum.-Growth is very scanty; the colonies sink slightly into the medium. On potato. - No growth was obtained. In peptone-beef broth. Small globular colonies appear in the depth of the broth after about six days' incu- 


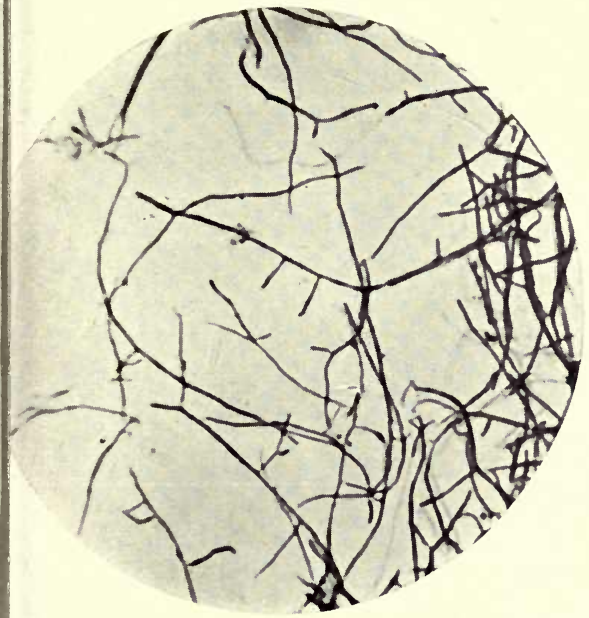

tothrix luteola (Foulerton). Film preparation from eptone-beef-broth culture, 14 days at $37^{\circ} \mathrm{C} . \times 1000$.

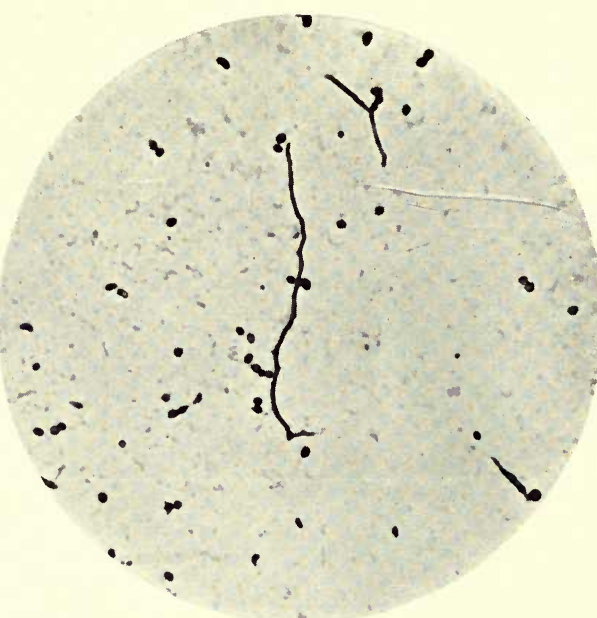

Streptothrix luteola (Foulerton). Film preparation from maltose agar culture, 6 weeks at $37^{\circ} \mathrm{C} . \quad \times 1000$.

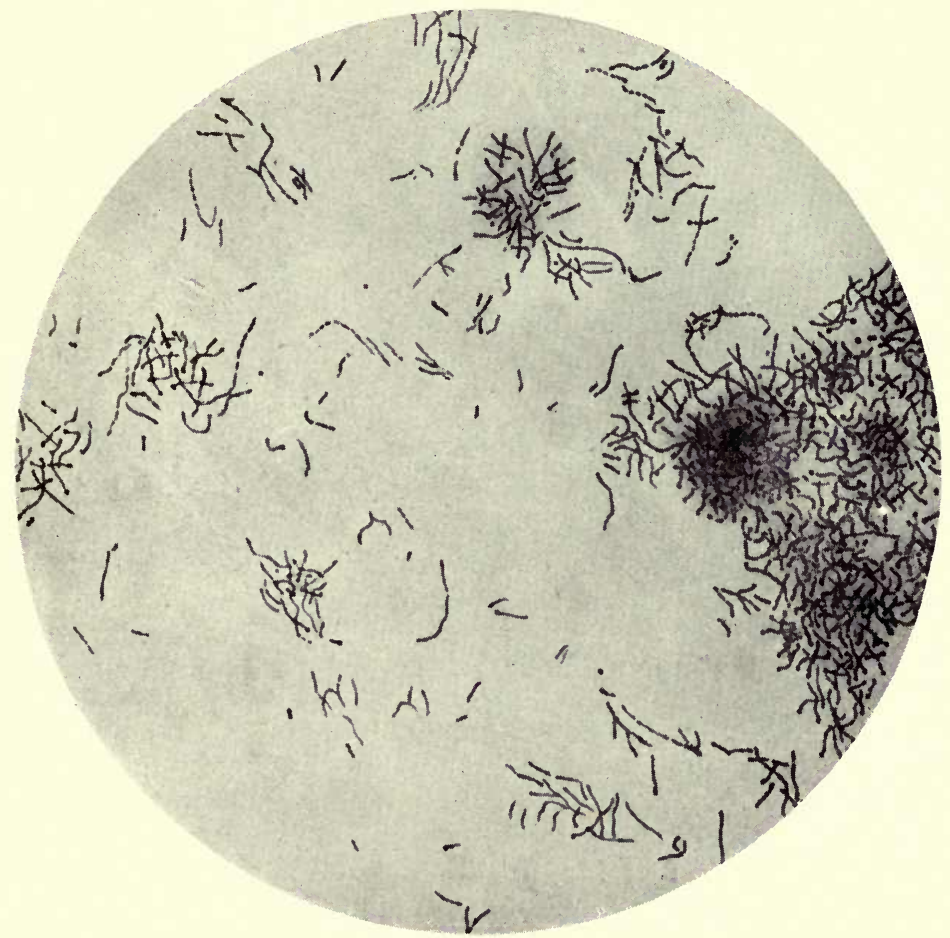

Streptothrix hominis (Foulerton). From pus of small abscess in chest-wall. Stained by Gram's methor.

[To face page 368 . 


\section{LIBRA \\ UNIVERSITY}

Q. कि

QF 
bation at $37^{\circ} \mathrm{C}$. These do not increase much in size, and after four weeks' incubation are not much larger than a pin's head; there is a tendency for the colonies to cohere in flocculent masses. The growth is whitish in colour. Pathogenicity for lower animals. - Two rabbits received intra-peritoneal injections of broth cultures, but there were no obvious effects. *

Other acid-fast members of the pathogenic streptothrix group have been isolated by Birt and Leishman from a case of pleural effusion; by Eppinger from an abscess of the brain; by MacCallum from peritoneal pus; by Nocard in "farcin du bœuf." These organisms can, as a rule, be differentiated from the tubercle bacillus by morphological, cultural, and tinctorial properties.

\section{General Note on Differentiation of Acid-fast Organisms}

The acid-fast bacillus, pseudo-tubercle bacillus, and acid-fast streptothrix may all be found to resemble the tubercle bacillus in greater or less degree. It has been suggested that they are all of the streptothrix genus. Whilst they are all acid-fast they are not equally resistant, and this fact assists in their differentiation. Broadly, none of these forms can resist decolorisation with 25 per cent. sulphuric acid for more than sixteen hours, whereas the tubercle bacillus can withstand decolorisation for seventy-two hours (Coles). Hence, if film preparations be made in the usual way, stained for seven minutes with hot carbol fuchsin, and then decolorised with 25 per cent. sulphuric acid for sixteen hours, the only bacilli remaining red are tubercle bacilli. If this method fail, cultivation and inoculation tests must be applied. The former by culturing in broth at $30^{\circ} \mathrm{C}$. (shows growth in three days in non-tubercle bacilli), the latter by inoculation of guinea-pigs. And here, as elsewhere, it is necessary to observe all the characters before forming a diagnosis.

* An excellent statement on the general characteristics and pathogenic action of the genus Streptothrix will be found in the Trans. of the Path. Soc. of London, 1902, vol. 53, part i., pp. 56-127 (Foulerton and Price Jones). 


\title{
CHAPTER XI
}

\author{
THE ETIOLOGY OF TROPICAL DISEASES
}

Malaria : Forms of Malarial Fever, the Mosquito Theory, Prevention of Malaria-

Cholera: Methods of Diagnosis-Plague: Symptoms, Rats and Plague, Bacteriology, Administrative Considerations - Leprosy - Yellow Fever Malta Fever-Sleeping Sickness-Beri-beri.

IT is now generally accepted that the future prosperity of the AngloSaxon race depends upon the measure to which it is able to control the Tropies. For it is obvious that that great middle band of the globe which we term the Tropics is increasingly one of the most valuable and important areas for colonisation in the world. Yet, though the rewards are great, the risks and penalties are also great. The coloniser from temperate regions knows this to his cost. Malaria and plague and cholera, to speak of no other tropical diseases, have made irretrievable claims upon him. Recently it has come to be recognised that much of this great loss is preventable, and ought therefore to be prevented. The establishment of Schools of Tropical Medicine in London, Liverpool, and other places, and the practical means adopted for preventing cholera epidemics and stamping out plague and malaria, are examples of the new sense of responsibility which is stimulating nations and governments to do their utmost to bring under control those scourges of pestilence, which have made the Tropics so often the grave of the white man.

The channels of infection in tropical diseases are various. Unhealthy surroundings, diet, the soil, bad water, and parasite hosts (as, for example, rats in plague and mosquitoes in malaria), seem to be the chief. But there is much yet to be done in the investigation of the causes of certain tropical diseases.

We may now enter upon the consideration of five typical diseases mostly limited to the Tropics: (1) Malaria; (2) Cholera; (3) Plague; (4) Leprosy; (5) Yellow Fever. It is apparent that many bacterial 
diseases are "cosmopolitan." Tuberculosis, for example, may occur in all parts of the world; so may pneumonia or typhoid. But the five diseases named above are in a greater or lesser degree endemic in tropical regions.*

\section{Malaria}

The term malaria (lit., bad air) is often applied rather to a group of fevers than to one specific affection. Such fevers have certain points in common. One common feature is that, with few exceptions, the disease originates in the blood. A second feature is the elaboration of a black or brown pigment from the hæmoglobin present in the blood corpuscles. And a third common character is that these diseases are produced not by bacteria but by homatozoa, that is to say, protozoa which can live and perform their functions in the blood. The term "malaria" should, however, be reserved for the specific disease caused by the malarial parasite.

For many years the group of diseases represented by malaria were designated miasmatic, owing to the belief that they were caused by some damp and unhealthy condition of the soil, from which emanated a miasm or soil ferment. Thus was explained their prevalence on and around marshy tracts of land, and their prevention by land drainage. Whilst these two latter features remain true, a new interpretation has been placed upon them.

In 1880 Laveran first discovered and described parasites in the blood cells of malarial patients, and on further investigation it was soon found that these assumed many different forms. These differences depend upon the kind of fever and the stage of fever.

The reasons for believing that Laveran's bodies-though they have not yet been cultivated outside the human body-are the specific cause of malaria are briefly these:-(1) The parasites found in the blood of malarial patients of all countries are the same. Such parasites are not found in healthy persons. (3) Their development fully accounts for the production of the melanœmia and malarial pigmentations of viscera owing to the melanin-forming property of the parasite. (4) The phases in the development of such parasites corresponds with the clinical course of malaria (Golgi). (5) Quinine, which cures malaria, kills the parasite. (6) Malaria. can be conveyed by the introduction of this parasite into the blood

* The term endemic indicates that a disease affects people within a certain geographical limit, and which seems therefore to arise from local or particular causes. Epidemic indicates that a disease attacks a large number of people at the same time and approximately in the same place. Whereas a pandemic is the same with an indefinitely wide distribution. 
of man, and the parasite reappears in the blood of the individual so inoculated. It is interesting in this connection to observe the negative results of the recent attempts of Koch to inoculate the higher apes with malaria in Batavia, as reported by the German Colonial Office.* Laveran's bodies have been variously classified as knowledge of them has grown. It is now agreed that these parasites belong to the Sporozoa, to the order of Homocytozoa, and to the genus of Homamoba.

Now if we examine a sample of human blood from, say, the benign tertian form of malaria, we shall find not different parasites as in three forms named below, but different stages in the evolution of one parasite. These different stages are normal and regular, and not accidental or chance forms, and for the sake of convenience we may summarise them seriatim thus :-

1. Early Form of Parasite.-Looking through the microscope, we shall readily observe large numbers of blood corpuscles, and in some of these, and possibly many of them, there will be apparent certain irregularities. In the first place, the protoplasm of the affected corpuscles is paler than that of the healthy cells. Next, within the protoplasm will be seen the parasite (amcbula), containing possibly a few specks of black pigment, and of more or less irregular outline, sometimes nearly filling the whole blood corpusele. This body is motile, and moves about like an amoba inside the corpuscle, in the tertian fever with great rapidity. As it increases in size, the corpuscle becomes paler. The largest of these spherical forms are outside the cells (extra-corpuscular spores, enhæmospores), and move about free in the blood plasm. But the smaller ones are generally inside the blood cells (intra-corpuscular amœbula). They live at the expense of the hæmoglobin in the corpuscle, and ultimately change it into pigment (melanin).

2. Concentration of the Pigment.-After the parasite has gained its mature form as regards size, an increase and concentration of the pigment occurs. The body of the parasite now fills the corpuscle, and the pigment which before existed in specks, or diffusely, becomes gathered together towards the centre of the parasite.

3. The third change in the evolution of the amobula is segmentation. By this process the parasite splits up into segments; the tertian fever forming much smaller and more numerous segments than the quartan. This segmentation gives rise to what is known as the "rosette body."

4. In reality these segments are sporocytes, new amœboid bodies, which, by the rupture of the eaten-out corpuscle, become diffused freely in the blood. Many of these "spores" are supposed to pass to the spleen, some are absorbed by phagocytes or scavenging cells,

* Deutsche Medicinische Wochenschrift, February 1900. 
but, in a few hours, many others reappear in the blood and inaugurate another stage.

5. The Infection of the Corpuscles.-The spores now attach themselves to healthy blood corpuscles, slowly pass into their interior, and set up a precisely similar series of changes; the actively amœboid stage, the increase of size and pigmentation, the concentration of the pigment, the mature form, and the segmentation resulting in the rosette body, and eventual escape of sporocytes. In this way the multiplication of the parasite is carried on in the human host. This is known as the Cycle of Golgi. Each paroxysm of malaria is related to the evolution cycle of a generation of these parasites-probably many millions in number-the commencement of each paroxysm coinciding with the maturation of a generation of parasites. The severity of a paroxysm in a given type of fever is also in direct relation to the number of parasites in the blood. It does not necessarily follow that the gravity of the case is in proportion to the intensity of the paroxysms.

Malaria is characterised by marked intermittency, which is usually divided clinically into three leading forms :-

(a) Quartan, depending upon a parasite which takes seventy-two hours to pass through its cycle of development, and produces fever every third day. The corpuscles invaded do not become so much decolorised, hypertrophied, or altered in shape as in other forms. The parasite shows distinctly less amoboid movement, and is not so delicate in structure or definition as in the Tertian varieties,
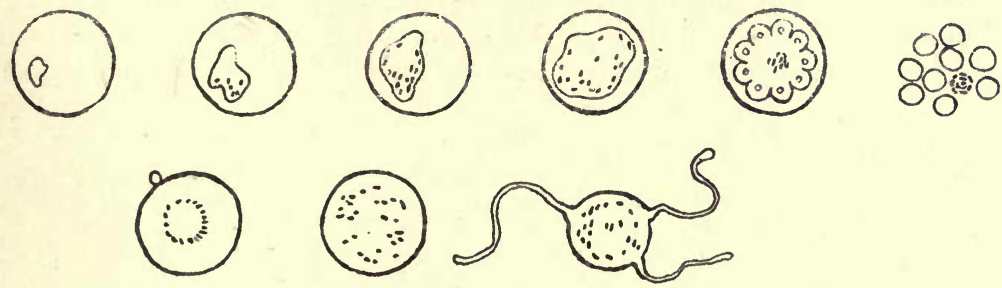

Fig. 29.-Quartan Malaria Parasite.

though it carries a large amount of dark brown, pigmented material, which is coarse in grain. The developed sporocyte has what is described as a "daisy-head" appearance. The six to fourteen spores are rounded in form, and possess a well-defined nucleus. Quartan fever is relatively much more common in temperate and subtropical latitudes than in the tropics.

(b) Benign or Mild Tertian.-In this fever the parasite takes forty-eight hours to complete its cycle. The amobula is actively motile inside the corpuscle, giving rise to great and rapidly-changing 
irregularities in the condition of the corpuscle, which becomes swollen, pale in colour, and may show deeply-stained "spots." The pigment granules are finer than in the quartan parasite. The final
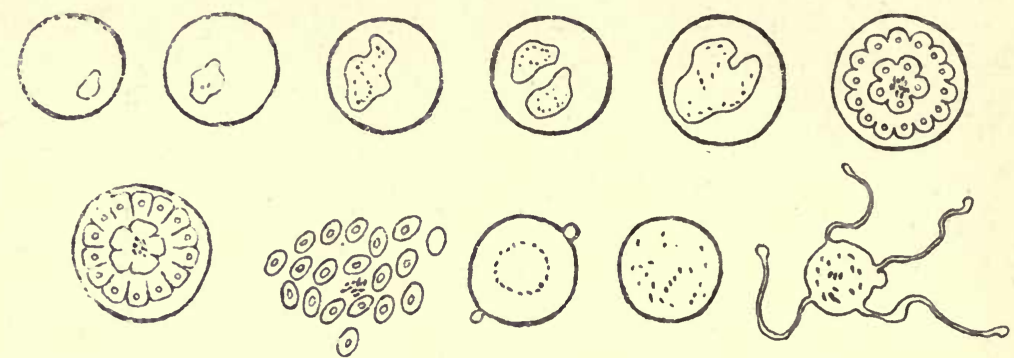

Fig. 30.-Tertian Malaria Parasite.

decolorisation of the corpuscle is very marked. The "rosette body" or sporocyte in this species is composed of some fifteen to twenty-five spores, small, smooth, and oval; the gametes are spherical. The benign tertian parasite is probably the commonest form found in malaria, and is widely distributed over the globe.

(c) The Malignant Infections (æstivo-autumnal, malignant quotidian, malignant tertian). The amsebuloe in these conditions are much smaller than in the benign types, but may occur in prodigious numbers, and their movements are very active. The organism causes considerable modifications in the shape and size of the corpuscle, which has a tendency to shrivel. It is not filled by the parasite in the same degree as in the other forms. Sporulation occurs in the spleen
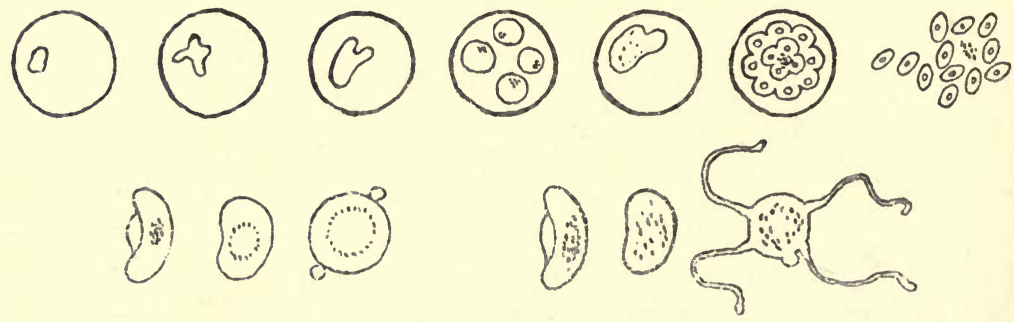

Fıa. 31.-Maliguant Malaria Parasite.

and other internal organs, and not in the blood, and, therefore, the sporocytes in this form are not found in the blood in the usual way. The most distinctive feature of all is that the malignant parasites (gametes) form "crescents," and attack a larger proportion of red corpuscles than in the other forms. The classifications, minor 
subdivisions, and clinical nomenclature have passed through a variety of changes. The three old divisions have been retained here for convenience. Sir Patrick Manson has suggested the following classification :-
A. Benign $\cdot\left\{\begin{array}{l}\text { Quartan } \\ \text { Tertian }\end{array}\right\}=\begin{gathered}\text { In which the parasites do } \\ \text { not form crescents. }\end{gathered}$
B. Malignant $\left\{\begin{array}{l}\text { Quotidian-with pigmentation } \\ \text { Quotidian-without pigmentation } \\ \text { Sub-tertian }\end{array}\right\}=\begin{aligned} & \text { In which the para- } \\ & \text { sites do form } \\ & \text { crescents. }\end{aligned}$

Now in some forms of malarial fever, namely, the malignant infections, the spherical bodies or mature form immediately prior to segmentation into rosette bodies, do not actually show segmentation, but assume the form of crescents lying inside the blood corpuscle, the hæmoglobin of which has been absorbed. Between the poles of the crescent may be seen the membrane of the blood corpuscle, the crescent being folded somewhat on itself. These crescents represent the form of the parasite which requires to enter the body of the mosquito in order to attain development. The crescents do not, as a rule, appear in the blood until about one week from the onset of the fever, and are the first stage of the extra-corporeal phase of the parasite. They are termed the gametocytes, and are of two kinds, male and female. It will be necessary to follow the development of each kind separately.

The microgametocyte, or male gamete, is the parasite in crescent form, with the delicate membrane of the containing blood corpuscle at first investing it, as described above. These crescents are hyaline in appearance, and the motionless pigment is loosely arranged. The crescent eventually absorbs or exhausts the blood corpuscle, and becomes a free body in the blood serum. Next, it changes by becoming kidney-shaped, then round at the poles, thicker, more ellipsoidal, and eventually spherical. The pigment granules now become mobile, and eventually assume most active movement and become diffused throughout the whole sphere, and almost immediately thereafter the sphere itself becomes agitated, and from its circumference shoot out long flagella (microgametes). This flagellated parasite is a weird-looking, "octopus-like" body, with long lashing tentacle-like flagella, and containing in its centre a mass of moving pigment granules. The flagella or microgametes are of the nature of spermatozoa, and fulfil a similar function, and are in length some three or four times the diameter of the microgametocyte. They are unpigmented, and may bear at their extremities bulbous swellings. They break off and become free in the blood, continuing their active movements.

The flagellated body may be produced from free spheres of the 
quartan and benign tertian parasite (which do not produce crescents) as well as from crescents. But it is never seen in fresh blood immediately after being drawn from the body. It only appears after the blood has left the body for twenty minutes or half an hour. Such a striking transformation of a free sphere or a crescent is evidently a stage of great importance, and two different explanatory theories have been advanced to account for it. Some have held it to be a degenerative change in the parasite-that the coldness of the outside air has killed it, that its contortions and wriggling flagella are but its death struggles, and that the active movement of its pigment particles are but Brownian movements of dead granules. Other authorities, and particularly Sir P. Manson, have declared the flagellated body to be a vital evolutionary change-a normal step in the life of the parasite, the first stage in its life-history outside the human body, the extra-corporeal homologue of the intra-corporeal sporulating body. It is now agreed that these microgametes or flagella are the essential sporulating bodies of an extra-corporeal phase, and that their function is the impregnation of the female gametocyte.

The macrogametocyte, or female gamete, is the second kind of gamete, and starts its course in much the same way as the male cell. It also is a crescent inside the blood cell, which it eventually breaks down, and thus becomes free in the blood serum. Instead of being hyaline it is granular, and the pigment is situated more centrally. It eventually becomes ellipsoidal, and then spherical. The protoplasm of the female crescents stains more deeply than that of the male crescents; the pigment is more closely grouped together, generally in ring form, in the centre of which will be seen one or occasionally two large masses of chromatin. At its maturity as a macrogametocyte, two small polar bodies or excrescences or papilla are seen on its circumference, and it is at this site that impregnation by the free flagellum (male cell or microgamete) is effected. The result is the zygote, or travelling vermicule. In 1897, MacCallum observed this impregnation actually taking place in a case of human malaria, and others have observed it in one of the malaria-like organisms of birds, the halteridium. After its entry into the female cell the flagellum became quiescent, and the pigment became collected at the posterior end of the cell, which then assumed the shape of a spear head, and became the actively-motile zygote.

The Mosquito Phase. - Just as the segmentation body eventually splits up into spores for the further propagation of the parasite in the blood of the malarial patient, so the flagellated body provides for the propagation of the parasite in some living host outside the human body; for, as is well known, parasites pass from one host to 
another. There is now complete evidence to show that the host is the Mosquito. It is therefore necessary to consider briefly the outstanding features of the Mosquito Phase.

The Mosquito is widely distributed, especially in tropical

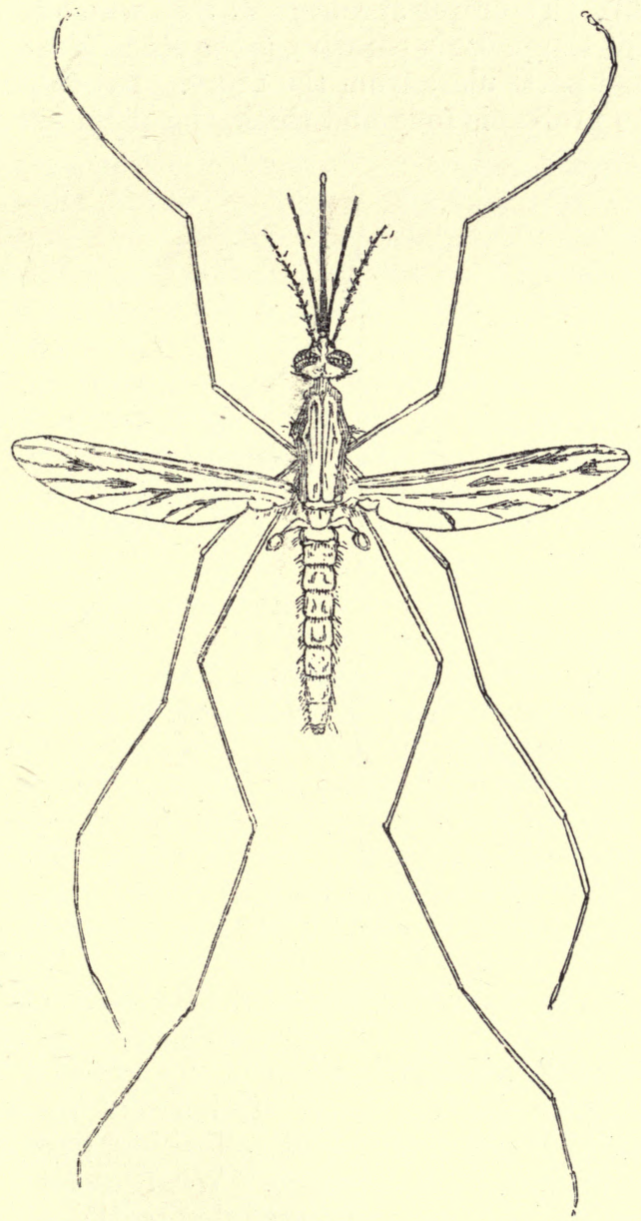

F1G. 32.-Anopheles maculipennis $\$$ (Meigen).

countries, and is generally classified under the genus Culicido, of which probably a large number of varieties exist. The Culex is the commonest species. Its larvæ live almost everywhere in warm countries, inhabiting any pot, tub, well, cistern, broken bottle, or, 
indeed, anything in which a little water can lodge. They are almost domestie animals. The palpi are short, the wings unspotted, the proboscis thin, the thorax large, and the larvæ have breathing tubes. They prefer to lie in artificial eolleetions of water. When at rest, for example on a wall, the body of Culex is found parallel to the wall (see fig. 33). The rarer species, and that which has been proved to be the host of the malaria parasite, is the Anopheles. This differs in various essential particulars from the Culex. In Anopheles the body is slim, but the proboscis long and thick, the palpi are long, and the
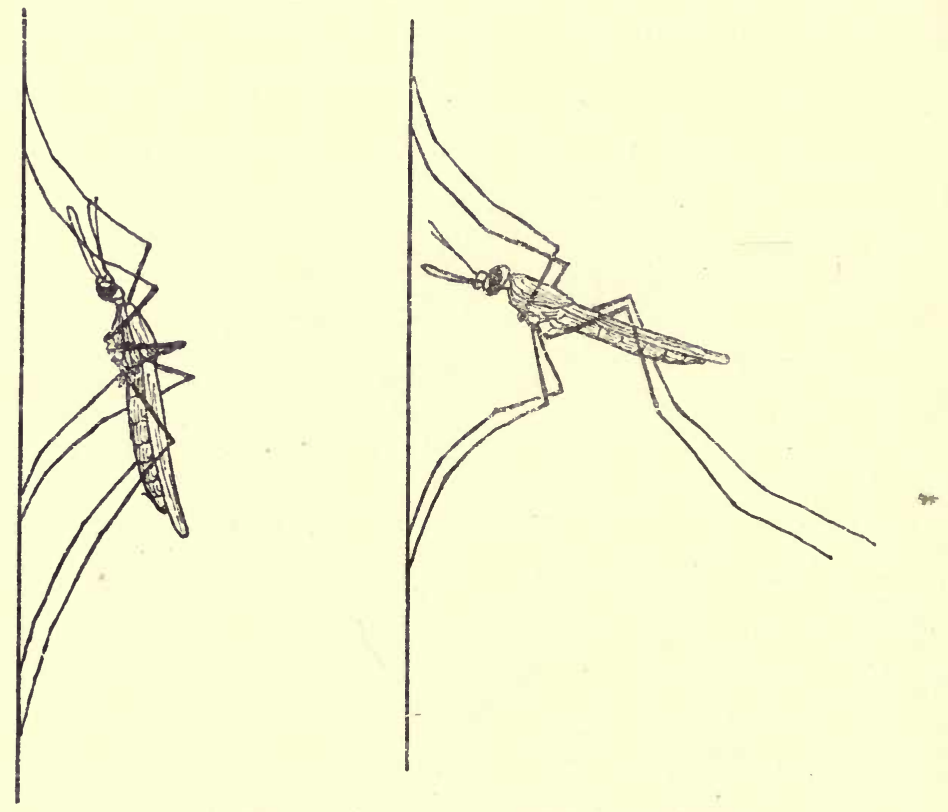

FIG. 33.-Diagram of Culex and Anopheles on Wall (Ross).

wing is "dappled" with dark spots on its anterior margin. The larvæ have no breathing tubes, and lie horizontally (not vertically as in Culex) in the water of puddles, looking like bits of brown stick or thorns floating on the surface. When at rest on a wall, the axis of its body is almost at right angles to the wall, so that the head of the Anopheles is direeted towards the wall, whilst the body projects out into the room. Culex has been briefly designated a "pot-breeding" mosquito, whilst one of the features of Anopheles is that it is "puddle-breeding." Its favourite haunts are slow, small streamlets containing algæ; small, shallow, natural puddles with confervoid growths in the water; or stagnant and fairly permanent 
collections of rain-water. The larvæ cannot live when the puddles in which they breed dry up. Nor does Anopheles appear to favour swamps containing deep water. The puddles they select are in immediate proximity to houses, where the adult mosquitoes may frequently pass to find human beings or cattle, from whom they may derive their nourishment. As is well known, it is the female mosquito which is the blood-sucker. After she has filled herself with blood, she retires to some dark, sheltered spot near a stagnant puddle, and after a few days deposits her eggs (from 200-400 in number) in a mass on the surface of the water. Then she dies and falls into the water beside her eggs. The eggs give rise (sometimes in sixteen hours time) to the tiny swimming larvæ, which feed greedily and grow rapidly, shed their skins, and become nymphoe or pupce. Eventually, the shell of the nympha cracks along its dorsal surface, and the young mosquito emerges. Standing on the raft of its empty pelt, it dries its wings and flies away.* Very soon it also lays eggs. The entire cycle from egg to egg is about fifty days. The three conditions necessary for the multiplication of malarious mosquitoes are $(a)$ high atmospheric temperature, $75^{\circ}$ to $104^{\circ} \mathrm{F}$.; (b) collections of water, fresh or brackish; and $(c)$ the presence in the breeding pools of low forms of animal and vegetable life.

Having now gathered the outstanding facts concerning the mosquito, we may return to the part which it plays in the propagation of malaria. The method which nature elects for the liberation of any given parasite is generally one that is reasonably regular and frequent in its operations. Hence, it occurred to Laveran and Manson that as the malarial organism is a passive blood parasite, its escape from the human body might be effected on the same principle that the escape of the passive muscle parasites is effected. As the latter obtain their opportunity by being swallowed by some flesh eater, Dr Manson reasoned that the blood parasite might obtain similar liberty by being swallowed by some blood-eater, some suctorial insect such as the sand-fly or mosquito. He was still further led to the mosquito theory owing to the parallel conditions which he had already found to exist in the case of the analogous mosquito phase of Filaria sanguinis. $\dagger$ The mosquito phase then is the extra-corporeal stage of the life-history of the malaria parasite which takes place within the body of the mosquito, and we may now briefly follow the course of events in the further development of the flagellated body inside the mosquito.

Whilst the mosquito theory was largely the suggestion of Sir

* For further particulars as to mosquito Anopheles, see Brit. Med. Jour. 1901, i., p. 195 ; Jour. of Hygiene, 1901, i., pp. 4-77, 269, and 451, also vols. 1902 and 1903.

$\dagger$ Goulstoniun Lectures, 1896. Sir Patrick Manson. Brit. Med. Jour., 1896, i., 641 et seq., and 1900, i., 328. 
P. Manson, the practical investigation and final elucidation of it were in great measure due to the patient observanee and skill of Ronald Ross, at that time a Surgeon-Major in the Indian Medical

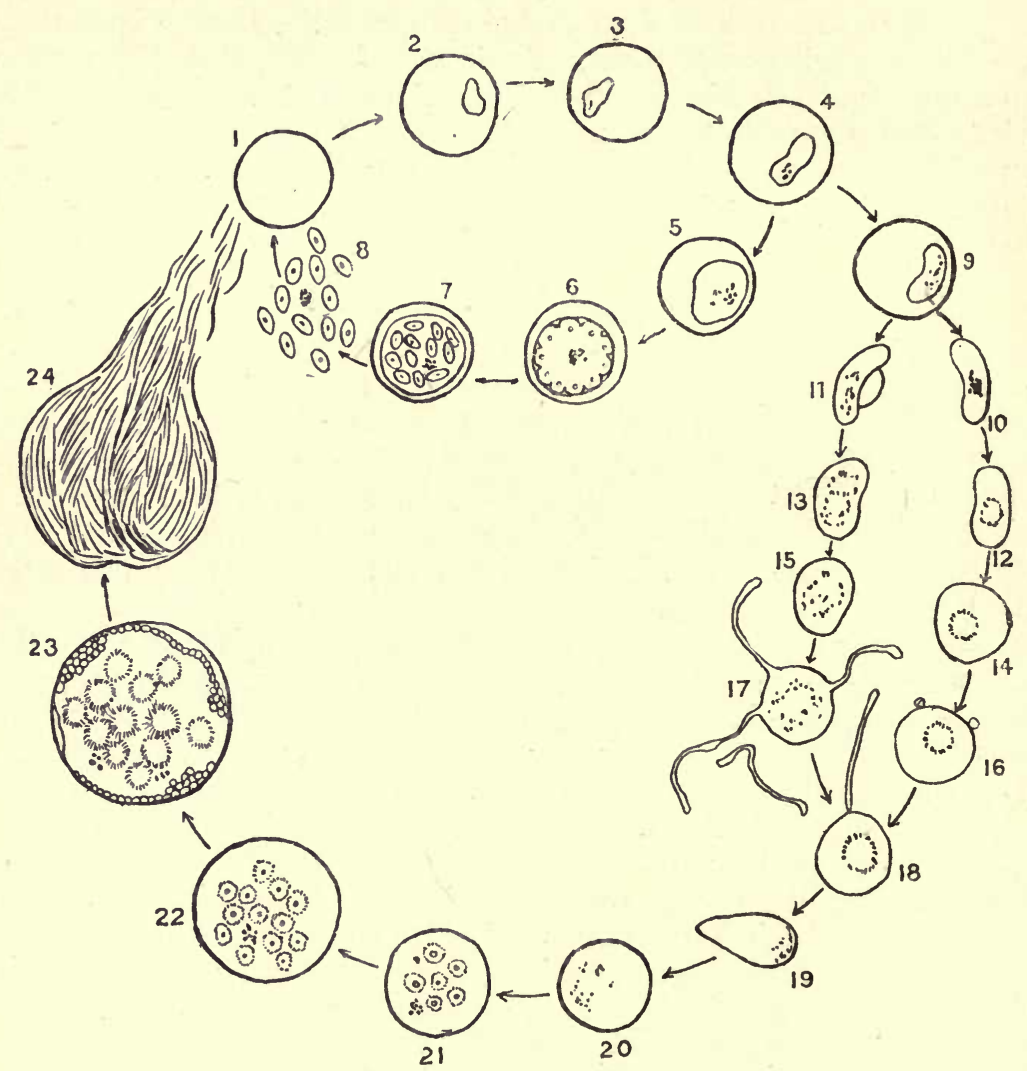

Fig. 34.-Scheme showing Human and Mosquito Cycles of the Malaria Parasite (Manson).

1.8, the human or endogenous or asexual cycle; 9.24 , the mosquito or exogenous or sexual cycle. 1 , Normal red blood corpuscle; $2-5$, blood corpuscles containing an amœbula ; 6.8 , sporocytes; 9 , young gametocyte; $11,13,15,17$, microgametocytes or male gametes; $10,12,14,16$, macrogametocytes or female gametes; 18, female gametocyte being impregnated by microgametes; 19 , travelling vermicule; 20 , young zygote; 21,22 , zygotomeres; 23 , blastophore ; 24 , mature zygote.

Service. His chief contribution to the solution of the problem was the discovery of the malarial parasite in the tissues of the mosquito. He found that in the body of mosquitoes fed upon malarial human blood 70 per cent. of the crescent forms of the parasite, as we have seen one of the latent forms of the flagellated body, were transformed 
into the flagellated body. This transformation occurred in a proportion of instances very much greater than occurs in malarial blood spread in the ordinary way on a slide and exposed to the air. $\mathrm{He}$ found, also, that the flagella broke away from the flagellated body, yet he was unable to trace what became of the free flagella. But in a "dapple-winged" mosquito (anopheles) fed in the same way with malarial blood containing crescents (summer-autumn fever), he discovered, embedded in the tissues of the stomach wall, certain peculiar oval cells containing the same pigment as the malarial parasite in the human blood. This was, in fact, the extra-corporeal form of the human parasite after impregnation, namely, the zygote. Next he discovered pigmented cells in the body tissues of mosquitoes fed upon sparrows' blood, affected with a similar parasitical condition to malaria (proteosoma); and from one step to another he demonstrated the evolution of the mosquito phase of these parasites. Ross made it evident that the cycle of extra-corporeal development of the parasite, as we have already seen, is carried on inside the body of the mosquito. After malarial blood is shed or swallowed by the mosquito, the changes already described take place. Practically, all the crescents become spheres within a few minutes of being taken into the stomach of the mosquito, then the male gametes become flagellated, and the female gametes become impregnated. According to Ross, the condition which brings about the transformation of crescents into flagellated bodies is not low temperature, nor exposure to air, nor contact with the wall of the mosquito's stomach, but abstraction of the water from the serum of the blood.

However that may be, the changes resulting from impregnation result in the mosquito's stomach in the production of the zygote or fertilised cell. This body is a travelling vermicule, and on or about the second day it penetrates the stomach wall and becomes encysted between the muscle fibres. The number of zygotes produced in the mosquito after its feed on malarial blood varies widely, sometimes being few, sometimes many. In the enlarging encysted cell there now come to be developed a number of cells known as zygotomeres, which as development proceeds become blastophores filled with filiform spore cells (sporozoits or germinal rods or zygotoblasts). Ultimately, the zygote becomes thus transformed into a cyst (sporocyst) packed full of zygotoblasts. When fully developed, at about the eighth or ninth day after the mosquito ingested the malarial blood, the sporocyst measures about 60 micromillimetres in diameter. About the twelfth day it bursts, discharging the zygotoblasts, which are, of course, "spores" or reproductive elements, into the body cavity and fluid of the mosquito, and spreading from thence they become accumulated in the large veneno-salivary gland, and are thus in a position to be injected along with its secretion into the human 
subject next bitten. These zygotoblasts are the actual source then of infection of man, and on arriving in the human blood the parasite (as spores) attacks the blood cells, and thus commences the intracorporeal or human phase described above. The mosquito phase occupies a time varying between six to sixteen days or longer, depending on temperature and other factors.

Such, in outline, is the mosquito theory of malaria. No one supposes that the last word has been said. But sufficient is now known to make it certain that the mosquito phase is a fact of essential importance in the conveyance of the disease to man. In the first place, the malarial parasite has been found repeatedly in the body of the mosquito, and in the second place the crucial experiment of inoculation has been performed, and has yielded a positive result. Infected mosquitoes were brought from the Roman Campagna, and Dr Thorburn Mason and Mr George Warren consented to be bitten, and thus contracted malaria. There yet remain gaps to be filled up in our knowledge of the disease, but there can be little doubt that future work will further establish and elaborate the prinoiples of the mosquito theory, and the lines of prevention will of necessity follow the new facts now proved.*

As concerns preventive medicine, the new facts may be summarised in three propositions:-(1) Malaria is caused by a number of microscopical parasites which live and propagate themselves in the blood. (2) These parasites are carried from infected persons to healthy ones by the agency of the genus of mosquitoes termed Anopheles. (3) These mosquitoes breed chiefly in shallow and stagnant terrestrial waters.

Examination of Malarial Blood (see Appendix, p. 485).

\section{The Prevention of Malaria}

The new knowledge respecting malaria indicates the only adequate preventive methods. The malarial parasite gains access to the human subject by means of mosquito bites, and, as far as is known, in no other way. Hence the methods of prevention must be directed mainly against the mosquito :-

1. The prevention of mosquito-breeding.

2. The destruction of mosquitoes.

3. Avoidance of being bitten by mosquitoes.

4. The use of quinine.

1. The Prevention of Mosquito-breeding.--In order to prevent the breeding of mosquitoes, it is necessary to eradicate all possible breeding-places. As we have seen, such places are tanks, cisterns, vessels of stagnant water, ditches, small pools, buckets, cocoa-nut

* For full account of malaria, see Tropical Diseases (Manson), 1903, pp. 1-173. 
shells, tius, cans, pots, etc., wherever stagnant water readily collects, especially near houses. The larvæ of Culex float when at rest on the surface of the water, suspended by their tails and with their heads hanging downward; when disturbed, they wriggle to the bottom. The larvæ of Anopheles float flat on the surface like small sticks, and when disturbed they wriggle on the surface with a backward skating movement. The former are usually present in artificial collections of water, such as pots, broken bottles, cans, etc., whilst Anopheles prefer natural collections of water, chiefly rain-water puddles which do not dry up quickly, or which contain green water-weed. Such being the breeding-places, prevention is simple. Collections of stagnant water must not be permitted. Search must be made for them, vessels must be emptied and puddles brushed out with a broom, and small pools drained and filled in. Water must not be allowed to collect anywhere near the house. Land drainage is an obvious preventive of the first importance.

Cisterns and similar necessary collections of water may be protected by paraffin or petroleum, for these by lying on the surface of the water prevent the pupæ of the mosquito from reaching the surface at the time of transformation. When water is required, it may be drawn off from the bottom of the cistern. The surface of paraffin may be renewed once a fortnight or oftener if necessary. Paraffin (kerosene oil) also acts as a culicicide, destroying the larvæ by choking their air tubes. The essential condition in any scheme for the sanitary improvement of a malarious region is that the eggs, larvæ, and nymphæ of the mosquito should be exterminated in that region. Covering small collections of water with healthy soil, accompanied by thorough drainage, inasmuch as they remove at the same time both the water and the atmospheric air, the two indispensable elements of mosquito life, are the best preventive methods.*

2. The Destruction of Mosquitoes.-Smoke from a wood fire or damp tobacco leaves, or sulphur, or other gaseous disinfectants may be used for this purpose. Kerosene may be used as recommended above for killing the larvæ. Individual mosquitoes should be killed whenever possible.

3. Avoidance of being Bitten by Mosquitoes.-For the protection of the person from attack by mosquitoes, there are a variety of contrivances, from mosquito nets to mosquito-proof houses. Mosquito nettings on the bed should invariably be used in malarious countries. There are several points as to the effectual use of such nets. In the first place, the net should be square, should be hung inside a framework, tucked carefully under the mattress all round and not allowed to hang down, and stretched tight so as to allow air to pass in easily.

* See also Brit. Med. Jour. 1900, vol. i., pp. 300-306 (Celli); and 1901, vol. i., pp. 193-203 and p. 240 . 
In the second place, the ronf should be made of netting similar to the other parts of the net, and not of cloth. Thirdly, during the day, when the net is not being used, it should be hung up in such a way as to prevent mosquitoes entering it. Fourthly, the mesh of the net should be sufficiently fine to effect its purpose, and should contain no rents, holes, or other apertures. Where punkahs are available, they should swing above the mosquito net.

Again, habits of life, especially temperateness and moderation, not sleeping in the open air, living as far as possible in healthy houses, not frequenting native quarters after sunset, and not associating with native children, are methods by which to avoid being bitten. It is now well known that in malarious towns and districts the great majority of native children harbour the malarious parasites in their blood, and therefore segregation is a necessary preventive method. Europeans' houses should be built at a distance from the native quarters.

The experiments of Sambon and Low, of Celli, of Grassi, of Fermi, and Tonsini, and of the Red Cross Society of Italy, have demonstrated beyond all question that it is practicable to construct habitable houses which shall be mosquito-proof.*

Lastly, probably some protection is obtained by means of perfumes, washes, pomades, soaps, etc., though these should not be relied upon. Certainly flannel clothing is a great advantage.

4. Quinine.-When it is too late for preventive measures the time has come for isolation, disinfection of rooms containing infected mosquitoes, and treatment. A person with malaria is always a risk to other persons, and should be isolated as far as practicable. Infected rooms should be disinfected with gaseous disinfectants, such as sulphur or formic aldehyde. Quinine is the specific remedy in treatment. It acts not only as an antipyretic but as a specific drug, destroying the parasite in the blood. For an ordinary intermittent fever the dose of quinine may be 10 grains, given when the sweating stage commences, followed by 5 grains every six or eight hours for a week, and with a view of preventing relapse 5 grains three times every fifth, sixth, or seventh day for two or three months. Five to ten grains of quinine twice a week or oftener, tends to prevent or abate incipient malarial infection.

\section{Cholera}

This word is used to denote a group of diseases rather than one specific well-restricted disease. In recent years it has become customary to speak of Asiatic cholera and British cholera, as if, indeed, they were two quite different diseases. But, as a matter

* Practitioner, March 1901, p. 262. 
of fact, we know too little as yet concerning either form to dogmatise on the matter. Until 1884 practically nothing was known about the etiology of cholera. In that year, however, Koch greatly added to our knowledge by isolating a spirillum from the intestine, and in the dejecta of persons suffering from the disease.

Cholera has its home in the delta of the Ganges. From this endemic area it spreads in epidemics to various parts of the world, often following lines of communication. Cholera is generally conveyed by means of water. It is a disease which is characterised by acute intestinal irritation, manifesting itself by profuse diarrhœa and general systemic disturbance accompanied by collapse, cramps, cardiac depression, subnormal temperature, and suppression of urine. The incubation period varies from only a few hours to several days. In the intestine, and setting up its pathological condition, are the specific bacteria, in the general circulation their toxic products bringing about the systemic changes.

The spirillum of Asiatic cholera (Koch, 1884) generally appears in the body and in artificial culture, broken into bacillary elements known as "commas." These are curved rods with round ends, showing an almost equal diameter throughout, and sometimes united in pairs or even in chains (spirillum). The latter rarely occurs in the intestine, but may be seen in fluid cultures. The common site for Koch's comma is in the intestinal wall, crowd-

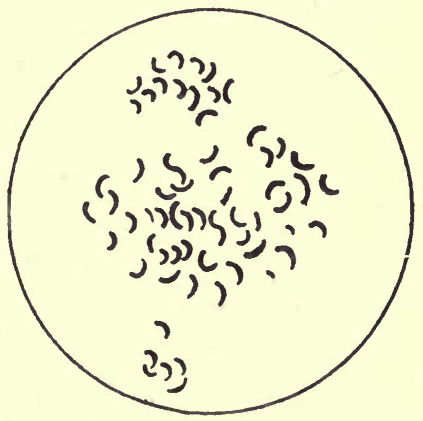

FIG. 35.-Diagram of the Comma Bacillus of Cholera. ing the tubules of the intestinal glands situated between the epithelium and the basement membrane, abundant in the detached flakes of mucous membrane, and free in the contents of the intestine. The bacilli are present in enormous numbers, and lie usually with their long axes in the same direction, giving the "fish in stream" appearance (Koch). The bacilli do not occur in the blood, nor are they distributed in the organs of the body. They occur mostly in the lower intestine.

The bacillus is actively motile, and possesses at least one terminal flagellum. The organism is aërobic, and liquefies gelatine. It stains readily with the ordinary aniline dyes, but does not stain by Gram's method. It does not produce spores, though certain refractile bodies inside the protoplasm of the bacillus in old cultures have been regarded as such. The virulence of the bacillus is readily attenuated, and both the virulence and morphology appear to show in different localities and under different conditions of artificial 
cultivation a large variety of involution forms. Unless the organism is constantly being sub-cultured, it readily dies. Acid, even the $\cdot 2$ per cent. present in the gastric juice, readily kills it. Prolonged drying, or heating to $55^{\circ} \mathrm{C}$. for sixty minutes, or treatment with weak chemicals has the same effect. The bacilli, however, have comparatively high powers of resistance to cold. Unless examined by the microscope in a fresh and young stage, it is difficult to differentiate Koch's comma from many other curved bacilli.

Its characters in culture are not always distinctive. Microscopically, the young colonies in gelatine appear as cream-coloured, irregularly round, and granular. Liquefaction sets in on the second day, producing a somewhat marked "pitting" of the medium, which soon becomes reduced to fluid. In the depth of gelatine, the growth is very characteristic. An abundant, white, thick growth exactly follows the track of the needle, here and there often showing a break in continuity. Liquefaction sets in on the second day, and produces a distinctive "bubble" at the surface. The process proceeds steadily, at first a funnel-shaped liquefaction resulting, and then in the course of a week or two all the gelatine may be reduced to fluid. On agar Koch's comma bacillus produces within twenty-four hours a thick greyish irregular growth. On potato, especially if slightly alkaline and incubated at $37^{\circ}$ C., an abundant brownish layer is formed. Broth and peptone water are favourable media, and at $37^{\circ} \mathrm{C}$. a general turbidity occurs with the formation on the surface of a pellicle, containing spirilla in active motility. In milk it rapidly multiplies, curdling the medium, with production of acid. Unlike $B$. coli, it does not form gas, but, like $B$. coli, it produces large quantities of indol, and a reduction of nitrates to nitrites. Hence the indol test may be applied by simply adding to a peptone culture several drops of strong sulphuric acid, when in the course of several hours, if not at once, there will be produced a pink colour, "the cholera red reaction," due to the formation of nitroso-indol. Although the bacillus readily loses virulence, and its resistance is little, it retains its vitality for considerable periods in moist cultures, upon moist linen, or in moist soil. In cholera stools kept at ordinary room temperature the cholera bacillus will soon be outgrown by the putrefactive bacteria. The same is true of sewage water.

The lower animals do not suffer from any disease exactly similar to Asiatic cholera, and hence it is impossible to fulfil the postulate of Koch dealing with animal inoculation. In this respect it is like the typhoid bacillus. It is, however, provisionally accepted that Koch's bacillus is the cause of the disease. The four or five other bacteria which have from time to time been put forward as the cause of-cholera have comparatively little evidence in their support. It 
is less from these and more from several spirilla occurring in natural waters that difficulties of diagnosis arise.

The reasons for believing Koch's bacillus to be the cause of cholera are four: (a) its constant presence in cases of the disease; $(b)$ the results of accidental infection with this bacillus; $(c)$ the agglutinative and protective properties of the serum of cholera patients; and $(d)$ the result of Haffkine's preventive inoculation.

There appears to be evidence to show that comma bacilli may be introduced into the alimentary canal without producing the disease, unless there be some injury or disease of the wall of the intestine (Peiffer). Desquamation of the intestinal epithelium seems an essential factor in the production of the disease in man. It need hardly be added that the bacillus acts, like other pathogenic bacteria, by the production of toxins (Peiffer), which appear to be intracellular. At present very little is known of their chemical nature. Brieger separated cadaverin and putrescin and other bodies from cholera cultures, and other workers have separated a toxalbumin.

\section{Methods of Diagnosis of Cholera:-}

1. The nature of the evacuations and the appearance of the mucous membrane of the intestine afford striking evidence in favour of a positive diagnosis. Nevertheless, it is upon a minute examination of the flakes and pieces of detached epithelium that reliance must be placed. In these flakes will be found abundance of bacilli having the size, shape, and distribution, of the specific comma of cholera. The size and shape have been already referred to. The distribution of comma bacilli ("fish in stream") in the flakes of watery stools is, when present, somewhat characteristic of Asiatic cholera, and may greatly aid in a correct diagnosis. But unfortunately, it is not always present, and then search for other characters must be made.

2. The appearance of cultivation on gelatine, to which reference has been made, is of diagnostic value, and the growth on agar and in peptone solution.

3. The "cholera red reaction." It is necessary that the culture and the sulphuric acid be pure for successful reaction.

4. The intra-peritoneal injection in guinea-pigs is followed by abdominal distention, subnormal temperature, and other characteristic symptoms.

5. Isolation from water is, according to Dr Klein, best accomplished as follows: A large volume of water (100-500 c.c.) is placed in a sterile flask, and to it is added so much of a sterile stock fluid containing 10 per cent. peptone, 5 per cent. sodium chloride, as will make the total water in the flask contain 1 per cent. peptone and .5 per cent. salt. Then the flask is incubated at $37^{\circ} \mathrm{C}$. If cholera vibrios are present in the water, however few, it will be found 
after twenty-four hours' incubation that the top layer contains actively motile vibrios, which can now be isolated readily by gelatineplate culture.

6. To demonstrate in a rapid manner the presence of cholera bacilli in evacuations, even when present in small numbers, a small quantity must be taken up by means of a platinum wire and placed in solution containing 1 per cent. of pure peptone and 5 per cent. sodium chloride (Dunham's solution). This is incubated as in the case of the water, and in twelve hours is filled with a turbid growth, which when examined by means of the hanging drop shows characteristic bacilli.

7. Pfeiffer's Test.-Take a loopful of six hours' agar culture of suspected cholera bacilli, and add it to 1 c.c. of ordinary broth containing 001 c.c. of anti-cholera serum (see p. 423). The mixture is injected intra-peritoneally into a guinea-pig of 250 grammes. In 20-30 minutes a drop of peritoneal fluid is withdrawn and examined microscopically for comma bacilli, when, if the reaction is positive, it will be found that the spirilla have broken down into granules.

\section{Plague}

This disease, like anthrax and leprosy, has a long historical record behind it. As the Black Death, it decimated the population of England in the fourteenth century, and visited the country again in epidemic form in the middle of the seventeenth century, when it was called the Great Plague. It is highly probable that these two scourges and the recent epidemic in the East are all forms of one and the same disease. As a matter of fact, it is difficult to be sure what was the exact pathology of a number of the grievous ailments which troubled our country in the Middle Ages, but from all accounts bubonic plague and true leprosy were amongst them. The former came and went spasmodically, as is its habit; the latter dragged through the length of several centuries.

There are four chief varieties of plague: first, the bubonic form, the most common and typical; the lymph glands are chiefly affected in the groin, the axilla, or the neck; secondly, the septicomic form in which the bacillus reaches the blood; thirdly, the pneumonic, in which the lungs are mainly affected; and fourthly, pestis minor, in which the affection of the glands stops short of the septicæmic stage, and even the local symptoms are slight. There are certain symptoms common to all forms of plague, when at all severe.

Symptoms of Plague.-An ordinary attack of plague usually begins three to five days after exposure to infection. Such attack may develop gradually, but, generally, there is sudden onset with much fever, as indicated by a high temperature, rapid pulse, headache, hot 
skin, and thirst. The eyes are injected as if inflamed; the expression, at first haggard, anxious and frightened, becomes subsequently vacant, listless, and dull; the utterance is thick, and the gait unsteady as in one under the influence of drink. Mental aberration develops quickly. There is frequently a marked tendency to faint. The tongue is at first covered with a moist white fur except at the edges, which are red, but later on it becomes dry and of a mahogany colour. Vomiting and nausea are present from the onset. Sleeplessness is a characteristic symptom.

The most distinctive sign of plague is the presence of swellings, or "buboes" as they are called, in the groin, armpit, or neck. These "buboes," which led to the disease being called "bubonic plague," and which have no relation to venereal complaints, appear as a rule on the second or third day of the disease. They occur as large, smooth, tense swellings. They are usually painful and tender on pressure, and in size they vary from that of an almond to that of an orange. Later on they may "gather" and burst like an ordinary abscess. There may appear about the body purple spots, and even "carbuncles."

But buboes are not an essential feature of plague. Cases occur in which these manifestations of the disease are greatly delayed or even absent, as, for instance, in "pneumonic," "gastric," and "septicæmic" plague; forms of the malady which may be mistaken respectively for inflammation of the lungs, typhoid fever, or acute blood poisoning. Plague in these forms is always grave; not only because of the fatality of the cases but for the reason that they, especially the "pneumonic," are highly infectious to other persons. It is important, therefore, that in localities where plague is present or is threatened, cases of anomalous illness of the above sorts be without loss of time brought under medical supervision.

Besides the forms of plague already referred to, there is yet another, namely, the so-called "ambulant form." In plague of this description the affected person is hardly ill at all, presenting no definite symptoms perhaps beyond indolent, though painful, swellings in groin or armpit. Such plague cases may nevertheless be instrumental in spreading the disease, and any persons therefore who, having been possibly exposed to plague, exhibit these symptoms, should be isolated and watched medically until the nature of their malady has been definitely ascertained.

The sudden onset, the marked prostration, the mental aberration, the splitting headache, vomiting and nausea, backache, the rise in temperature, the furred tongue, when taken in conjunction with tenderness and pain in some one of the groups of glands, are sufficient to arouse suspicion as to the case being one of plague.

The distribution of plague at the present time is fortunately a 
somewhat limited one, namely, a definite area in Asia known as the "Plague Belt." From Mesopotamia, as a sort of focus, the disease spreads northwards to the Caspian Sea, westwards to the Red Sea, southwards as far as Central India, and eastwards as far as the China Sea. This constitutes the "belt," but the disease may take an epidemic form, and is readily, though very slowly, conveyed by infection or contagion. It appears to be infectious by means of infective dust, and contagious by prolonged and intimate contact with the plague-stricken.

Rats and Plague.--Rats have been shown to be the agents for conveying the disease from port to port, and even infecting man. It is probable that rats are not the only agency acting in this way.* Nevertheless, it is true that rats contract the disease more readily than any other animals, and that when suffering from it they may spread the infection. How it is thus spread is not known. Cantlie and Yersin have pointed out that previously to an epidemic of plague rats die in enormous numbers, and Manson has declared that rats supply "the best and most probably the initial opportunity" for the bacillus of plague. "Were I asked," he continues, "how I would protect a state from plague, I would certainly answer, exterminate the rats as a first and most important measure." But, to be effective, this measure must be employed in anticipation of the advent of the disease. "When the rats are tumbling about drunk with plague it is too late." We may quote Sir P. Manson's simile of the position of the rat in epidemic plague. "I would compare a plague-threatened, but as yet not invaded, city," he says, "to a grate in which the fire is laid all ready for lighting. There is the refractory though combustible coal on top, there is the greasy paper and dry, resinous, inflammable wood underneath, and there is the lighted match ready to be employed. Drop the match on the top of the coal; it flickers for a second and goes out-the coals do not catch fire. But apply it to the paper and sticks underneath, and in a moment there is a blaze: the sticks are consumed, the coals catch, and in a little while the fire burns merrily. The coals will now burn by themselves, or, if they threaten to go out, another stick or two will quickly revive the fire. In my simile the coals stand for the human inhabitants, the sticks for the rodent inhabitants, and the lighted match for the plague germ that has dodged the quarantine intended to protect that threatened city. No sticks, no fire; no rats, no plague epidemic." †

Dr Doriga, the Principal Medical Officer of Health for Venice, has set forth a brief résumé of the chief facts relating to the

\footnotetext{
* See Indian Plague Commission Report, 1902, and Report on Plagne at Sydney, 1903 (Ashburton Thompson).

† Brit. Med. Joner., 1899, vol. ii., p. 924.
} 
agency of rats and mice in the spread of plague, which is as follows :-**

"1. Kitasato and Yersin, and many others after them, have found the specific bacillus of plague in the dead bodies of rats and mice collected in houses in which cases of the disease subsequently broke out among the occupants, or in the streets of infected towns. They have also placed beyond question the great susceptibility of these rodents to the bacillus.

" 2 . In all the towns of India manifest examples of contagion from mice to men have been observed. At Bombay, in certain establishments where the dead bodies of rats were found, it has been noticed that the persons who collected them alone contracted plague, although many other work-people were engaged at the same place.

" 3 . The first cases of the disease have sometimes appeared in warehouses where wheat, cotton seed, or other substances likely to attract rats were stored. At Kurachee, where the warehouses are situated in streets without dwelling-houses, the first sufferers were the caretakers.

"4. Well-constructed and well-maintained houses, i.e. where rats cannot find harbour, nearly always remain free from plague. This same immunity was demonstrated by Rennie at Canton, in 1894, among the occupants of boats anchored in the river. On the other hand, is to be observed the permanence of infection in the houses of poor natives, notwithstanding the removal of the residents and furniture and the most rigorous disinfection, because of reinfection by means of mice.

"5. The epidemics at Bombay, Kurachee, and Karad were chiefly localised in quarters where the disease had broken out amongst rats. The spread of infection in other parts of these same towns was regularly preceded by the immigration and death of rats, and its diffusion always corresponded to the route of travel taken by these rodents in their migrations.

" 6 . In healthy countries adjoining infected, the disease broke out amongst the inhabitants without the importation of a single (human) case, but was preceded by the immigration of rats from an infected place.

"7. In many countries and towns the development of the epidemic among the inhabitants followed a month after the importatation of the first cases, or after the death of fugitives arriving from infected localities. During the interval the plague had been propagated by mice.

"8. Lastly, the mode of infection and propagation of plague on certain ships proved that the rats on board had been the vehicles of

\footnotetext{
* "The Prevention of Plague through Suppression of Rats," Revue d'Hygiène, August 1899.
} 
contagion." * It should be added that Doriga's views are not universally held, and were not fully accepted by the Indian Plague Commission.

Quite recently, attempts have been made in Paris, with the consent of the Prefect of the Seine, to exterminate rats wholesale, in order to protect the city from an epidemic of plague. $†$

The Bacteriology of Plague is one of the latest additions to the science. During the Hong Kong epidemic in 1894, Kitasato, of Tokio, demonstrated the cause of plague to be a bacillus. This was immediately confirmed by Yersin, and further proved by the isolation in artificial media of a pure culture of a bacillus able, by means of inoculation, to produce the specific disease of bubonic plague.

The bacillus was first detected in the blood of patients suffering from the disease. It takes the form of a small, round-ended, oval cell $(0.7 \mu$ broad by $1.5 \mu$ long), with marked polar staining, and hence having an appearance not unlike a diplococcus. In the middle there is a clear interspace, and the whole is surrounded with a thick capsule, stained only with difficulty. The organisms are often linked together in pairs or even chains (especially in fluid cultures), and exhibit polymorphic forms. In culture the bacillus may be coccal or bacillary in form. Involution forms occur in old cultures, and also, more rapidly, when 2-5 per cent. of sodium chloride is added to the medium. On such salt-agar the involution forms are very marked. The bacillus is non-motile (Plate 30, p. 398).

The plague bacillus grows readily on the ordinary media at bloodheat, producing smooth, shining, circular cream-coloured colonies, with a wavy outline, which eventually coalesce to form a greyish film. The colonies slip about on the agar when touched with the platinum wire. If melted butter (or ghee) or oil be added to bouillon, this bacillus grows in "stalactite" form, that is, the growth starts on the under surface of the fat globules, and extends downwards in the form of pendulous string-like masses which readily break off if the tube is slightly shaken. The following negative characters help to distinguish the bacillus: There is no growth on potato; milk is not coagulated; gelatine is not liquefied; Gram's method does not stain the bacillus; and there are no spores. The bacillus is readily killed by heat or by desiccation over sulphuric acid at $30^{\circ} \mathrm{C}$. Both in cultures and outside the body the bacillus loses virulence. To this may be attributed possibly the variety of forms of the plague bacillus which differ in virulence. But it has great powers of resistance against cold.+

On gaining entrance to the human body the bacillus affects in

* For a general discussion of the subject of plague in the lower animals, see Brit. Med. Jour., 1900, i. pp. 1141 and 1216.

+ Brit. Med. Jour., 1900, vol. i., p. 722.

¥ For further particulars as to cultured characters of B. pestis, see Brit. Med.Jour., 1902 , ii., 956. 
particular two organs, the spleen and the lymph glands (bubonic plague). The latter become inflamed in groups, commencing generally with the inguinal (60 per cent.) followed by the axillary (17 per cent.). The buboes consist usually of masses of inflamed and enlarged lymph glands, attended with hæmorrhage and often with necrotic softening. The spleen suffers from inflammatory swelling, which may affect other organs also. In both places the bacilli occur in enormous numbers. In the pulmonary form the lung is affected with broncho-pneumonia. This form of plague is said to be always fatal. Kitasato considers that the bacillus may enter the body by the three channels adopted by anthrax, namely, $(a)$ the skin, $(b)$ alimentary canal, and (c) respiratory tract. But the vast majority of cases arise from infection through the skin. Infection through the alimentary canal is still doubtful. Soil, clothes, and contaminated articles generally are the agencies of infection. As stated already, rats play an important part in the propagation of the disease. The Indian Commission hold that suctorial insects are practically of no importance as transmitters of infection.

Haffkine has prepared a vaccine to be used as a prophylactic (see p. 424), and the Indian Plague Commissioners have recently reported on its effects. Inoculation with this vaccine appears sensibly to diminish the incidence of the plague attacks on the inoculated population, although the degree of protection is not perfect. The disease is four times more numerous among the uninoculated than among the inoculated. The fatality of the attacks is also diminished in the inoculated. Protection does not begin till a few days after the inoculation, but it lasts many weeks and even months. It may here be added that the means of stamping out plague are the ordinary methods of notification, isolation, and disinfection. The latter should include destruction of the patient's clothes, and the scraping of the walls, and, in India, burning of the earthen floor of his dwelling. The soil and dwellings are among the chief sources of infection, and therefore require most attention.

As to the infectivity of plague, it is now generally held that the bubonic form is, as a rule, dangerous from the excretions, and only in the last stages of the disease; that the primary pneumonic form is highly infective; that houses in which plague patients or plague rats have died, and in which clothing has been soiled by excretions, are infective; and that there is much more danger from living in an infected house than from coming into contact with a plague patient. Plague is a disease which is specially favoured by insanitation within the walls of houses as contrasted with insanitation outside houses, relating, for example, to drainage, removal of refuse, etc. Rats, merchandise, clothing, etc., may each and all play a part in the conveyance of plague from one village to another, or one country to 
another. But the chief agency for spreading the disease to uninfected places consists of travellers. The lines of human communication are followed by the infection in a marked degree, especially lines of steamship and railway.

Plague is essentially a "filth disease," and it is frequently preceded by famine. Temperature and overcrowding exert an influence upon it. The areas affected by the disease in the Middle Ages in the seventeenth century, and in 1894-96, are alike in being characterised by filth and overcrowding. There is little fear, speaking generally, of the plague ever flourishing under Western civilisation, where the conditions are such that even when it appears there is little to encourage or favour its development.*

Administrative Considerations. - Plague will niot readily fasten on any section of a population which is properly housed, cleanly, and generally, in a sanitary sense, well-to-do ; rather it will especially affect, if it obtains foothold in a district, insanitary areas such as are peopled by the poorest class, and where overcrowding of persons in houses and lirt and squalor of dwellings and of inhabitants tend to prevail.

In these circumstances, and from an administrative point of view, the following facts respecting plague should be borne in mind :-

(1) Plague has an incubation period of three to five (in exceptional cases of perhaps eight to ten) days.

(2) Plague is wont, especially in its earlier manifestations, to assume a mild form, or even to present anomalous symptoms, tending to confound it with other and more innocent diseases.

(3) Plague in all its forms must needs be regarded as personally infective.

(4) Plague affects rats as well as the human subject; it may, indeed, be found, causing mortality among these lower animals antecedent to its definite invasion of the population. There can be no doubt that the rat and man are, as regards plague, reciprocally infective.

Although Local Authorities should be on their guard against plague, when cases occur at the ports or elsewhere in these islands, it is not intended to suggest that there exists, under these circumstances, cause for alarm. There can be no doubt that, in this country, hygienic conditions and methods of dealing with infectious diseases are far in advance of those of former centuries wherein plague was repeatedly epidemic in our populations; they are in advance, too, of those in localities abroad, where plague has shown itself formidable in recent years. During the past fifty years there

* The most complete account of plague hitherto published is The Report of the Indian Plague Commission, 1902, vols. i.-viii. (see in particular vol. v.). 
has occurred in England and Wales a large diminution in the mortality from most diseases of the infectious class, and in the same period typhus fever has declined almost to extinction. This latter disease is that which, as regards the conditions under which it becomes prevalent, most closely resembles plague. Wherefore it may be confidently anticipated that the measures of sanitary improvement, of isolation and of disinfection, which have been found effectual against indigenous disease such as typhus, will, if promptly and thoroughly brought to bear, be equally effectual against plague.

First among measures requisite for control of plague is prompt notification to the local authority of all cases of the disease occurring in their district. As a rule, the first cases of an outbreak will require bacteriological diagnosis in addition to or auxiliary to clinical diagnosis.

Secondly, in the event of plague being detected in any district, the measures to be taken to prevent its spread are, generally speaking, those which are available against the more ordinary epidemic diseases. These measures include prompt removal of the sick persons to hospital and their isolation therein; the destruction or thorough disinfection of all infected articles, with the effectual disinfection also of the invaded dwelling-place; the keeping under observation during ten days after detection of each plague case all persons who have been in contact with the patient; house to house visitation for the discovery of unreported or suspicious cases; the abatement as speedily as possible of all insanitary conditions in the locality which may tend to the sprear of the disease; and, in the case of death, the prompt disposal of the body, with all due precantions against its becoming a source of infection.

Thirdly, an essential measure of precaution, in view of the observed relation between plague in rats and plague in the human subject, will be the prompt destruction of all rats in districts threatened or invaded by plague, care being taken that their carcases are collected and burnt without being unduly handled.*

When treated in a well-appointed hospital, with plentiful fresh air and proper attention to cleanliness and disinfection, plague, except in its pneumonic and septicæmic forms, shows but small infective power; and that therefore doctors and nurses in attendance on the sick run but little risk of contracting the disease. Nevertheless, these and other persons brought into close relation with plague may be afforded protection against infection by submitting themselves to protective inoculation ten days before contact with plague cases.

* Danysz has suggested the killing of rats by infecting them with an organism fatal to them, Brit. Med. Jour., 1904, vol. i., p. 947. 


\section{Directions for Obtaining and Forwarding for Bacterioscopic Examina- tion Material from Suspected Plague Cases}

\section{A.-From the Living Person.}

1. Clean with soap and water, and then with alcohol, the last phalanx of either the second or third finger. When dry, or after mopping with a clean cloth, put a piece of tape round the proximal end of the last phalanx, so as to cause venous congestion. Prick the palmar surface of this phalanx with a sterile needle, and immediately take up the exuding blood in two sterile capillary tubes such as are used for collecting vaccine lymph. These tubes when charged should be sealed at both ends.

2. When there is a discharging bubo, collect fluid therefrom in capillary tubes, as in the case of blood. When this discharge is not of a sufficiently fluid character for collection in this way, place some of it in a small glass-stoppered phial, previously well washed out with alcohol, care being taken that no alcohol remains in the phial.

3. If expectoration be obtainable, collect some in a phial in the manner prescribed in section 2.

[In blood, discharge, or expectoration, cover glass preparations should be made and stained by simple stains, and by Gram's method. The plague bacillus does not stain by Gram's method. Cocci, streptococci, and diplococcus pneumonice do stain by Gram's method. Cultivations and inoculations must also be made.]

\section{B.-From the Dead Body.}

1. Cut out any inflamed lymph gland, together with some of its surrounding tissue, and place the whole in a wide-mouthed glass-stoppered bottle previously well washed out with alcohol, care being taken that no alcohol remains in the bottle. 'The bottle should have the stopper well secured and sealed.

2. Obtain also a piece of the spleen, dealing with it in the same manner.

All suspected plague material should be carefully packed, so as to avoid risk of breakage.

\section{Leprosy}

This ancient disease is said to have existed in Egypt 3500 B.c., and was comparatively common in India, China, and even in parts of Europe 500 B.c. We know it has existed in many parts of the world in the past, in which regions it is now extinct. Some of the earliest notices we have of it in this country come from Ireland, and date back to the fifth and sixth centuries. Even at that period of time also various classical descriptions of the disease had been written and various decrees made by Church councils to protect lepers and prevent the spread of the disease, which was often looked upon as a divine visitation. In the tenth century leprosy was prevalent in England; it reached its zenith in the thirteenth century, or possibly a little earlier, and declined from that date to its extinction in the sixteenth. But even two hundred years later leprosy was endemic in the Shetlands, and it is recorded that in 1742 there was held a public thanksgiving in Shetland on account of the disappearance of leprosy. The last leper living in the Shetlands was admitted to the Edinburgh Infirmary in 1798. 
At one time or another there were as many as 200 institutions in the British Isles for the more or less exclusive use of lepers. Many of these establishments were of an ecclesiastical or municipal character, and owing to the fact that diagnosis was not accurately or carefully made, it is certain that these institutions frequently housed persons suffering from diseases other than leprosy. Bury St Edmunds, Bristol, Canterbury, London, Lynn, Norwich, Thetford, and York were centres for lepers. Burton Lazars and Sherburn, in Durham, were two of the more famous leper institutions.

Elsewhere, the writer has furnished the evidence obtainable in support of the view that true leprosy (elephantiasis grocorum) was prevalent in England in the Middle Ages.* It existed there anterior to the crusades, which per se had little or no effect in spreading the disease in England. It was generally supposed from the eleventh century that leprosy was a contagious and hereditary disease, and that it depended upon these two characters for its extension in England. But probably such was not the case, for it is fairly certain that strict segregation was never carried out. The disease as an endemic disease reached its zenith in the thirteenth century or earlier, and declined till final extinction in the eighteenth. In England itself it disappeared approximately in the sixteenth century. Probably the famine of 1315 , and the Black Death of 1349 , materially assisted in the extermination of lepers. The disease being diffused neither by contagion nor heredity has under favourable hygienic circumstances a tendency to die out. Hence the decline and final extinction of leprosy in Great Britain was due to this general tendency under favouring circumstances, viz., to an extensive social improvement in the life of the people, to a complete change in the poor and insufficient diet, and to general sanitary advancement.

At the present time the distribution of the disease is mostly Asiatic. Norway contains about 1200 lepers, Spain a smaller number. Scattered through Europe are perhaps another 2000 to 3000 , in India 100,000 , and a number in Japan. The Cape possesses a famous leper hospital on Robben Island, with a number of patients. The disease is also endemic in the Sandwich Islands.

Descriptions of the pathological varieties of leprosy have been very diverse. The classification now generally adopted includes three forms: the tuberculated, the ancesthetic, or (maculo-anæsthetic), and the mixed. Lepra tuberculosa is that form of the disease affecting chiefly the skin, and resulting in a nodular tuberculated growth or a diffuse infiltration. It causes great disfigurement. The ancesthetic form causes a destruction of the nerve fibres, and so p. 108.

* The Decline and Extinction of Endemic Leprosy in the British Islands, 1895, 
produces anæsthesia, paralysis, and what are called "trophic" changes. Not infrequently patches occur on the skin, which appear like parchment, owing to this trophic change. Bullæ may arise. When the tissue change is radical or far advanced, considerable distortion may result. The mixed variety of leprosy, as its name implies, is a mixture of the two other forms.

The Bacteriology of Leprosy.-The B. leproe was discovered by Hansen in 1874. He found it in the lepra cells in the skin, lymph glands, liver, spleen, and thickened parts of the nerves. It is common in the discharge from the wounds of lepers. It is conveyed in the body by the lymph stream, and has rarely been isolated from the blood (Köbner).

The bacillus is present in enormous numbers in the skin and tissues, and has a form very similar indeed to B. tuberculosis. It is a straight rod, and showing with some staining methods marked beading, but with others no beading at all. It measures $4 \mu$ long and $1 \mu$ broad. Young leprosy bacilli are said to be motile, but old ones are not. Neisser has maintained that the bacillus possesses a capsule and spores. The latter have not been seen, but Neisser holds that this is the form in which the bacillus gains entrance to the body. There is a characteristic which fortunately aids us in the diagnosis of this disease in the tissues, and that is the arrangement of the bacilli, which are rarely scattered or isolated, as in tubercle, but gathered together in clumps and colonies. The bacilli oceur for the most part inside the round cells, but they are also found free in the lymphatics, inside connective tissue cells, and in the walls of blood-vessels. A few may often be found in the hair follicles or glands of the skin, or even in the epithelium. The bacilli also occur in the lymphatic glands and in the internal organs. The brain and spinal cord are almost always exempt. But recent research has made it evident that the distribution in the tissues may be more widespread than was formerly supposed. Bordoni-Uffreduzzi, Carrasquilla (1899) and Campana, claim to have isolated the bacillus and grown it on artificial media, the two former aërobically on peptone-glycerine blood serum, at $37^{\circ} \mathrm{C}$., the latter anaërobically. Other workers have been unable to obtain successful results. Cultivated bacteria from the organs of lepers, described rather later by Babes, and still more recently by Czaplewski, differ from the genuine bacillus of leprosy in their incomplete resistance to acids. Both authors maintain that the bacteria cultivated by them resemble the bacilli of diphtheria. In any case it is very doubtful whether these bacteria cultivated from leprosy are the genuine Bacillus lepro. Hence it is not possible to study the bacteriology of leprosy at all completely; inoculation experiments also have not proved successful. Nevertheless there is little doubt that leprosy is a bacterial disease 


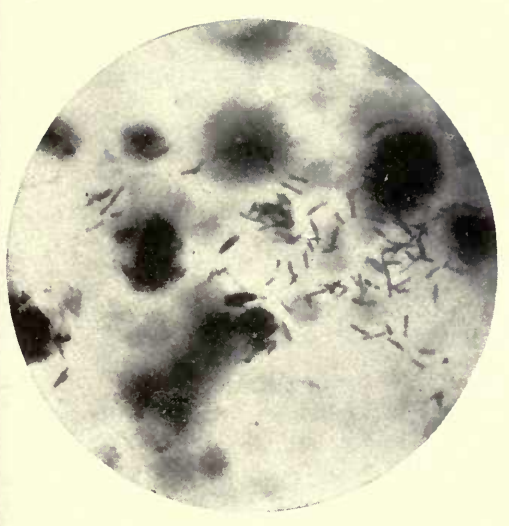

Bacillus lepro.

Discharge from sore of leper. Stained by Ziehl-Neelsen method.

$\times 1000$.

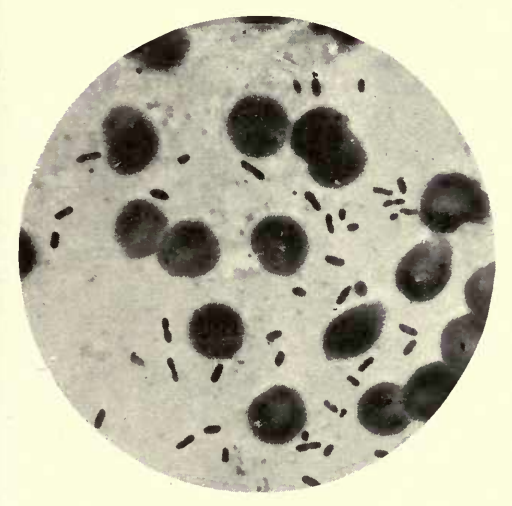

Bacillus of Plague (B. pestis bubonica).

From liver of plague-stricken rat. $\times 1000$.

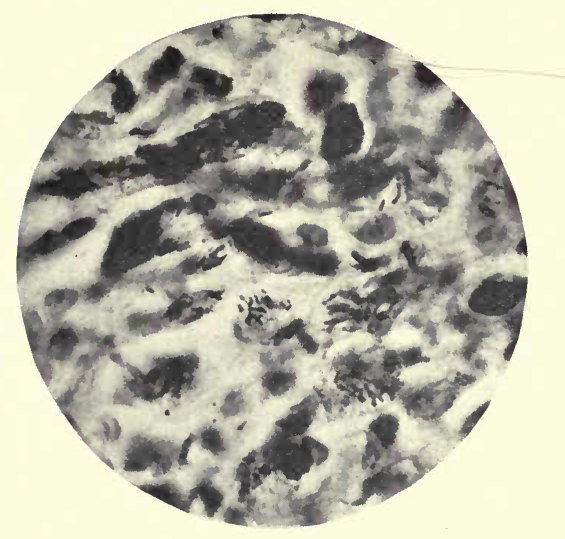

Bacillus lepree.

Lepra cells containing bacilli. From lobule of ear of leper. Stained by Ziehl-Neelsen method.

$\times 1000$.

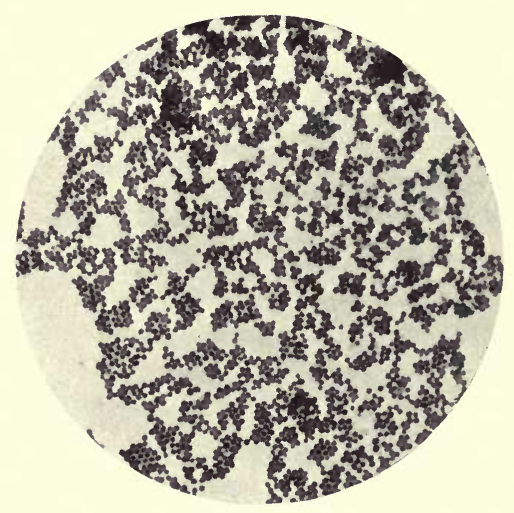

Staphylococcus pyogenes aureus.

$\times 1000$. 


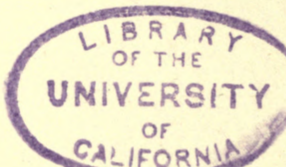


produced by the bacillus of Hansen. Bordoni-Uffreduzzi maintains that the parasitic existence of the $B$. leproc may alternate with a saprophytic stage. This may be of importance in the spread of the disease. There is evidence in support of the non-communicability of the disease by heredity or contagion. Segregation does not appear always to result in a decline of the disease, as we should expect if it were purely contagious. Ehlers, of Copenhagen, has, however, as recently as 1897 reaffirmed his belief in the contagiousness of leprosy; Virchow, on the other hand, declared that it was not highly contagious. There is evidence to show that persons far advanced in the disease may live in a healthy community, and yet not infect their immediate neighbours. Indeed, the transmission of the disease is still an unsolved problem. Mr Hutchinson maintains that diet, particularly uncooked or putrid fish, is a likely channel. Deficiency of salt, telluric and climatic conditions, racial tendencies, social status, poverty, insanitation, drinking-water, even vaccination, have all secured support from various seekers after the true channel by which the bacillus gains entrance to the human body. The real mode of transmission is, however, still unknown. The decline and final extinction of leprosy in the British Islands was, as we have stated, probably due in part to the natural tendency of the disease to die out, and in part to a general and extensive social improvement in the life of the people, to a complete change in the poor and insufficient diet, and to general sanitation.

At the Leprosy Congress held in Berlin in 1897, Hansen again emphasised his belief that segregation was the cause of the decline of leprosy wherever it had occurred. But there appears to be evidence to show that leprosy has declined where there has been no segregation whatever, and therefore, however favourable to decline such isolation may be, it would seem not to be an actually necessary condition. At the same Congress Besnier declared in favour of the infective virus being widely propagated by means of the nasal secretion. Sticker states that the nasal secretion contains myriads of lepra bacilli, especially in the acute stages of the disease, and Besnier and Sticker have pointed out how frequently and severely the septum nasi and skin over the nose is affected in leprosy. Several leprologists in India have recorded similar observations. These facts appear to support Besnier's contention, that the disease is spread by nasal secretion.

We may add here the conclusions arrived at by the English Leprosy Commission * in India:-

" 1 . Leprosy is a lisease sui generis; it is not a form of syphilis

* Dated 1890-91. The Commissioners were the late Beaven Rake, M.D., G. A. Buckmaster, M.D., the late Prof. Kanthack, of Cambridge, the late Surgeon-Major Arthur Barclay, and Surgeon-Major S. J. Thomson. 
or tuberculosis, but has striking etiological analogies with the latter.

"2. Leprosy is not diffused by hereditary transmission, and, for this reason and the established amount of sterility among lepers, the disease has a natural tendency to die out.

" 3 . Though in a scientific classification of diseases, leprosy must be regarded as contagious, and also inoculable, yet the extent to which it is propagated by these means is exceedingly small.

"4. Leprosy is not directly originated by the use of any particular article of food, nor by any climatic or telluric conditions, nor by insanitary surroundings, neither does it peculiarly affect any race or caste.

" 5 . Leprosy is indirectly influenced by insanitary surroundings, such as poverty, bad food, or deficient drainage or ventilation, for these by causing a predisposition increase the susceptibility of the individual to the disease.

"6. Leprosy, in the great majority of cases, originates de novo, that is, from a sequence or concurrence of causes and conditions dealt with in the Report, and which are related to each other in ways at present imperfectly known."

The practical suggestions of the Commission for preventive treatment included voluntary isolation, prohibition of the sale of articles of food by lepers, leper farms, orphanages, and "improved sanitation and good dietetic conditions" generally. Serum-therapy has been attempted on behalf of the French Academy of Medicine, but without success. Many forms of treatment ameliorate the miserable condition of the leper, but up to the present no curative agent has been found.

\section{Yellow Fever}

This disease is admitted to be one of the most terrible of tropical diseases. Fortunately, its area of endemicity is comparatively limited. When, however, it breaks out, especially on board ship, its high percentage of fatality is well known.

A number of investigators, from the beginning of last century down to the present time, have been at work on the cause of yellow fever. Sanarelli, the Director of the Institute of Hygiene, in the University of Montevideo, in South America, is one of the more recent workers, and he has isolated a bacillus which he believes is the causal agent of the disease. The bodies of those who die of yellow fever are, however, either so free from organisms, or so entirely invaded by organisms, that the $B$. icteroides is difficult to discover. Moreover, led by the clinical signs of the disease-c "black vomit" and other gastro-intestinal phenomena - investigators have, à prior $i$, supposed that the digestive canal was the seat of the disease, and 
therefore the probable locality of the causal bacillus; whereas, as Sanarelli pointed out, the $B$. icteroides must be sought for in the blood and tissues, and not in the alimentary canal. But even thus the difficulties are not wholly removed. For it happens that this organism may only be found in comparatively small numbers, and certainly at the beginning of the disease multiplies very little in the human body. Its influence upon the body, too, appears to be such that the tissues of a yellow fever patient become the hunting ground of vast numbers of secondary infective bacteria.

This bacillus ( $B$. icteroides) may be obtained from the small capillaries-in, say, the liver-by incubation at favourable temperature $\left(37^{\circ}\right.$ C. $)$. It is a short bacillus with round ends, like $B$. coli. It is motile, and possesses 4-8 flagella. It develops sufficiently well for all practical purposes on the ordinary media. On agar at blood-heat it grows well-a grey, iridescent, smooth layer, with regular margins; and on the same medium, at the temperature of the room, it produces in twenty-four hours characteristic colonies not unlike drops of milk. It grows on gelatine without liquefaction. The organism is a facultative anaërobe, decolorised by Gram's method; ferments sugar, but does not coagulate milk until after some weeks. It appears strongly to resist drying. Direct sunlight kills it in seven hours; but it is said to be able to live for some time in sea water. The organism can be isolated from the living patient as well as the dead body.*

Sanarelli has maintained that atmospheric transmission is the common channel of infection in yellow fever. As everyone knows, it is a disease which, when once installed on board ship, seems to cling to it tenaciously, more particularly in the hold, magazines, merchandise, and in all close and restricted quarters. Humidity, heat, and want of light and ventilation have been, until recently, the supposed conditions necessary to the conveyance or harbouring of yellow fever. Sanarelli has further suggested that moulds must be considered "the natural protectors of the specific agent of yellow fever." $\uparrow$ By a series of interesting experiments, he demonstrated the stimulating effect which moulds have upon gelatine-plate cultures of this bacillus in the laboratory. Outside the laboratory, in houses and on ships, the conditions favouring the growth of moulds appeared also to be the conditions favouring yellow fever. For instance, humidity, heat, and scanty aëration are highly favourable to mould growth, and thus, according to Sanarelli, to yellow fever. To these factors, also, is supposed to be due the unhealthiness of Rio Janeiro. During the yellow fever epidemic in Montevideo in 1872, the

* Brit. Med. Jour., 1897, vol. ii., p. 7 (Prof. G. Sanarelli), 1900, vol. i., p. 334, and The Medical News (New York), 9th December 1899.

+ Brit. Med. Jour., 1897, vol. ii., p. 11. 
inhabitants of the houses facing north were attacked much more than others, and it was found that both these houses and the streets in which they stood were distinguished by an exceptional degree of humidity.

In 1901 the United States Army Commission reported, after extensive investigations into the etiology of yellow fever, that whilst $B$. icteroides was not always present in cases of yellow fever, the blood of the patient appeared to contain the virus, whatever it was, and retained it after being passed through a Berkefeld filter. The Commission further reported that the disease was not communicable by direct contact with those suffering from the disease, but was probably communicated by mosquitoes in a similar way to malaria. The species of mosquito found capable of carrying the infection in this way is the Stegomyia fasciata. Though the matter was not proved, nor the nature of the virus determined, preventive measures were adopted in Havana on the mosquito hypothesis, with the remarkable result that the disease was stamped out. Guitéras of Havana has carried out further experiments which confirm many of the Commission's findings, and, in particular, the transmission of the disease by mosquitoes.*

In 1902 a United States Army Expedition was appointed to reinvestigate the subject, and it reported in 1903. The chief conclusions reached were as follows : (1) Bacteriological examination of the blood of persons with uncomplicated yellow fever during life, as well as of organs and blood immediately after death, is negative. (2) The mosquito known as Stegomyia fasciata, when allowed to suck the blood of a yellow fever patient after the lapse of forty-one hours after the onset of the disease, and subsequently fed on sugar and water for twenty-two days can, if permitted to bite a non-immune person, produce a severe attack of the disease. (3) Stegomyia fasciata, contaminated by sucking the blood of a yellow fever patient, and then killed, cut into sections and appropriately stained, presents with regularity a protozoan parasite, Myxo-coccidium stegomyioe, which can be traced through a cycle of developments from the gamete to the sporozoite. (4) Stegomyia fasciata, fed on the blood of a person with malarial fever, on normal blood, or artificially, does not harbour the myxo-coccidium.

The etiology of yellow fever, therefore, remains at the present time sub judice, but the probabilities are that the disease is mosquitoborne and due not to bacteria but to sporozoal parasites.

There are other tropical diseases to which brief reference must be made.

Malta Fever (Mediterranean fever) is common along the coast of the Mediterranean and on its islands. It also occurs elsewhere. In 1886 Bruce cultivated from 
the spleen of patients dead of the disease an organism now known as the Micrococcus melitensis. Clinically, Malta fever is a disease of long duration and variable symptoms, including remitting fever. Perspiration, pains, swelling of joints, enlargement of the spleen, etc., are among the common signs. Micrococcus melitensis is a small, round, or slightly oval coccus, singly, in pairs, or chains. Does not stain by Gram's method. Can be cultivated on agar at $37^{\circ} \mathrm{C}$. from the spleen; colonies appear about third day as small, round, slightly raised growths, old cultures assume a buff tint. Addition of nutrose hastens growth of culture. On gelatine growth is very slow; there is no liquefaction. In broth there is a turbid growth, without pellicle formation.

Cultures kept at $22^{\circ} \mathrm{C}$. retain their vitality for fourteen months, but the organism dies in about five days in sterile, fresh, and sea water and urine, but remains active for longer in sterile milk, and for sixty-nine days in dust. The organism is present in the peripheral blood in all cases during the early stages, and in severe pyrexial relapses. It has recently been isolated from the urine of patients. The disease appears to be inoculable in animals.

Sleeping Sickness. - Investigations point to the conclusion that sleeping sickness is caused by the entrance into the blood, and thence into the cerebro-spinal fluid, of a species of trypanosoma (probably the Trypanosoma gambiene, discovered by Forde and described by Dutton), which is transmitted from the sick to the healthy by a species of tsetse fly (Glossina palpalis), and by it alone; that, in short, sleeping sickness is a human tsetse-fly disease. From a series of carefully controlled and minutely observed experiments, carried out by Bruce, Nabarro, and Grieg, it was discovered that monkeys inoculated with cerebro-spinal fluid from sleeping sickness patients, or with blood from natives not as yet showing symptoms of sleeping sickness, but containing a similar parasite, sickened and died with all the symptoms of sleeping sickness.

From the analogy of the closely related disease in cattle, the nagana or tsetse fly disease of South Africa, it was suspected that in sleeping sickness a like method of infection took place. It has been demonstrated by experiment that not only were these flies, fed on sleeping sickness cases, capable of conveying the disease to healthy monkeys, but that the freshly caught flies from an infected area, without any artificial feeding, were also capable of conveying the disease.

It was further discovered by a carefully-organised investigation that this fly, like its congener the tsetse fly of South Africa, is confined to well-defined areas, and that these areas correspond absolutely with the distribution of sleeping sickness; whereas, in regions where no Glossina palpalis is found, although other biting flies abound, there is no sleeping sickness. Moreover, an examination of a large number of individuals in the sleeping sickness areas and the non-sleeping sickness areas respectively, revealed the fact that, while a large percentage (28) of the inhabitants of the sleeping sickness areas have in their blood the trypanosoma already referred to, in not a single case taken from inhabitants of non-sleeping sickness areas was this parasite found." The only other human trypanosome at present known is that occurring in trypanosomiasis.

Dysentery is another tropical disease in which the etiology has not been finally worked out. Endemic or tropical dysentery is possibly due to Amoba coli. Epidemic dysentery is more probably due to Bacillus dysenterioe, and sporadic and parasitic dysentery is due to various parasites, such as Balantidium coli and the Bilharzia. B. dysenterice is a short rod, often occurring in pairs; non-motile; does not stain by Gram's method; does not curdle milk nor liquefy gelatine. $\nmid$ The researches

* See also Brit. Med. Jour., 1903, vol. i., p. 1431; and vol. ii., pp. 1343 and 1427 ; and Lancet, 1904 (July), p. 290, for a résumé.

$\dagger$ For a full discussion of the subject, see Brit. Med. Jour., 1901, vol. ii., p. 786, "A Comparative Study of the Bacilli of Dysentery"; and 1903, vol. i., p. 1315, "Amœbic Dysentery in India"; and Report of Royal Commission on Dysentery, 1903. For description of B. dysenteria, see Report of Medical Officer to Local Govt. Bd., 1901-02, p. 396 ; Brit. Med. Jour., 1904, vol. i., p. 1002 ; Edin. Med. Jour. (June), 1904, p. 489 (Eyre). 
of Shiga, Kruse, Flexner, and others point to acute dysentery being caused by the specific bacillus $B$. dysenteria, or some member of that group of organisms. Mott holds that "asylum dysentery" is identical with tropical dysentery, and both conditions are in all probability of bacillary origin.*

Beri-beri. - The first medical writer to describe beri-beri, and by that name, was Dr J. D. Malcolmson, F.R.S., of the Madras Medical Service, in a paper published in 1835. Sir Joseph Fayrer, F.R.S., wrote on it, identifying one form of it with the barbiers of the earlier European travellers. $\dagger$ The disease is endemic in Western India, in the Indian Archipelago, and throughout the coasts of Further India and Upper India, or China and Japan. It is practically confined to the labouring classes where they are vegetarians. Dr Wallace Taylor traces it to a microscopic spore infecting rice ; and other observers consider it a " place disease." The salient fact is that it almost exclusively attacks those who are engaged in hard labour on insufficient nourishment, and it may be defined as the scurvy of the tropics. It is marked by extreme weakness and dropsical distension of the abdomen, limbs, and face, both symptoms developing so rapidly as to alarm alike the sufferer and those attending to him. Hence its name beri, meaning "debility," and the reduplication of it, beri-beri signifying " extreme," " alarming," " fatal," debility.

The disease is in all probability a germ disease but possibly not communicable from man to man. It may be that the germ resides in soil or rice, or houses, and surroundings of beri-beri localities, and produces a toxin which on being absorbed produces a disease having many similarities with alcoholic neuritis (Manson). This may be the explanation of the view that beri-beri is a " place disease." Pekelharing and Winkler hold that they have isolated a bacterium which is the cause of the disease, but their views iave not been generally accepted.

* See also Bacteriological and Clinical Studies of the Diarrhoeal Diseases of Infancy, by Flexner and Emmett Holt (Rockefeller Inst. Rep.), 1904.

+ Practitioner (January), 1877; see also Report on Prison Alministration in Burma, 1878. 


\section{CHAPTER XII}

\section{THE QUESTION OF IMMUNITY AND ANTITOXINS}

Bacterial Products-Toxins-Question of Immunity-Kinds of Immunity-Theories of Immunity-Applications of Immunity-Vaccination for Small-pox : Effect of Vaccination-Pasteur's Treatment for Rabies-Inoculations for Cholera, Typhoid, and Plague-Antitoxin Treatment of Diphtheria and its Effects.

THE term natural immunity is used to denote natural resistance to some particular specific disease. It may be due to species of animal, or age, or individual idiosyncrasies. We not infrequently meet with examples of this freedom from disease. Certain species of animals do not, as a rule, take certain diseases. For example, cholera and typhoid, which affect man, do not affect the lower animals. Swine plague, which affects swine, does not affect man. The white rat is immune to anthrax, which readily attacks cattle. Such examples might easily be multiplied. Children, again, are susceptible to certain diseases and insusceptible to certain others to which older people are susceptible. The young of the lower animals also are susceptible to diseases which do not attack adult animals. We know, too, that some individuals have a marked protection against certain diseases. Some persons coming in the way of infection at once fall victims to the disease, whilst others appear to be proof against it.

It is only in recent times that any intelligent explanations have been offered to account for these phenomena. The most recent, and that which appears to have most to substantiate it, is known as immunity due to antitoxins. To understand the nature of antitoxins it is necessary to consider briefly the products of bacterial activity. They are chiefly seven:- 
1. Pigment.-We have already seen that many organisms exhibit their energy in the formation of various pigments. These are, as a rule, "innocent" bacteria. Oxygen is required for the production of pigment by some of these species, absence of light by others, and they all vary according to the medium upon which they are growing. Red milk, blue milk, and green pus are illustrations of materials owing their colour to pigment produced by bacteria. Chromoporous bacteria are those in which the pigment is diffused out into the surrounding medium; chromophorous bacteria are those in which the pigment is stored in the cell protoplasm of the organism.

2. Gas.-A large number of the common bacteria, like B. coli, produce gas in their growth; hydrogen $(\mathrm{H})$, carbonic acid $\left(\mathrm{CO}_{2}\right)$, methane $\left(\mathrm{CH}_{4}\right)$, sulphuretted hydrogen $\left(\mathrm{H}_{2} \mathrm{~S}\right)$, and even nitrogen (N) being formed by different bacteria.

3. Acids.-Lactic, acetic, butyric, etc., are common types of acids resulting from the growth of bacteria.

4. Liquefying Ferment.-As we have seen, bacteria may also be classified with regard to their behaviour in gelatine medium, as to whether or not they produce a peptonising ferment which liquefies the gelatine.

5. Phosphorescence.-Some species of bacteria, for example, certain species in sea-water, possess the power of producing light (photogenic bacteria).

6. Many organisms are capable of producing indol (a substance formed by bacterial action from proteids by alimentary decomposition), or other metabolic substances as end-products.

7. Organic Chemical Products.-When a pathogenic bacillus grows in the body, it produces as a result of its metabolism certain poisonous substances termed toxins. These may occur in the blood as a direct result of the life of the bacillus, or they may occur as the result of a ferment produced by the bacillus. Toxins are of various kinds, and by their effect upon the blood and body tissues they cause the symptoms of the various diseases. We know, for instance, that a characteristic symptom common to many diseases is fever, which is produced by the action of the albumoses (bodies allied to the albumins) upon the heat-regulating centres in the brain. Whenever we have a bacillus growing in the body which has the power of producing a toxin albumose, we obtain fever as a result of that product acting upon the brain. Albumoses, as a matter of fact, cause a number of symptoms and poisonous effects, but the mention of one as an illustration will suffice. Toxins act, broadly speaking, in two ways. They have a local effect 
and a specific effect, as the two following illustrations will make evident:-

(1) They have a local action, as, for example, in the formation of an abscess. The presence of the causal bacteria in the tissue brings about very marked changes. There is a multiplication of connective tissue corpuscles, an emigration of leucocytic cells, a congestion of blood corpuscles. These elements contribute towards creating a swelling and redness, and pain results owing to the subsequent pressure upon the nerve endings. We have, in short, a state of inflammation. It is then that the toxin commences its local action. The oldest cells in the mass of congestion will break down, and necrosis or death will rapidly set in. The connective tissue cells, leucocytes, blood corpuscles, etc., will thus lose their form and function, and become pus. The local breaking down of these gatherings of cells into fluid matter is believed to be the work, not of the bacteria themselves, but of their toxins.

(2) Toxins may be absorbed and distributed generally throughout the body. When this occurs they produce degenerative changes in muscles, in organs, and in the blood itself. Of such a change diphtheria is an example. The bacillus occurs in a false membrane in the throat, and occasionally other parts. It first causes the inflammatory condition giving rise to the membrane, and then it breaks it down. In the body of the membrane the bacillus appears to secrete a ferment which by its action and interaction with the body cells and proteids, chiefly those of the spleen, produces albumoses and an organic acid (Martin). These latter bodies are the toxins. They are absorbed, and pass throughout the body. As a result, we get the frequent pulse and high temperature of fever: the toxins irritate the mucous membrane of the intestine, and cause various fermentative changes in the contents of the intestines, therefore we get the symptoms of diarrhœa: they penetrate the liver, spleen, and kidney, setting up fatty degeneration and its results in these organs : they finally affect many of the motor and sensory nerves, breaking up their axis cylinders into globules, and producing the characteristic paralysis. Loss of weight naturally follows many of these degenerative or wasting changes. Thus, then, we have some of the chief changes set up by the toxins, and these changes constitute the leading symptoms in the disease as it is known clinically. In addition to the presence of the specific bacillus in the membrane, we also have a number of other organisms, like the B. coli, Streptococcus pyogenes, and various staphylococci, diplococci, etc. Each of these produces, or endeavours in the midst of keen competition and strife to produce, its own specific effect. Thus we obtain the complications of diphtheria, such as various suppurative and septic conditions. The 
whole of this compound process may be tabulated roughly as follows:-**

Bacillus coli.

Staphylococci.

Diplococci.

Streptococci.

Toxins.

Suppurative glands, septic poisoning, etc.
BACILLUS OF DIPHTHERIA = primary infective agent. Inflammatory changes and fibrinous exudation.

Ferment in membrane = secondary infective agent.

Passes through body, and ) $\left.\begin{array}{l}\text { by digestion of proteids } \\ \text { produces }\end{array}\right\} \begin{aligned} & \text { ALBUMOSES ; } \\ & \text { AN ORGANIC ACID. }\end{aligned}$

1. Fever.

2. Diarrhœa.

3. Loss of body weight.

4. Fatty degeneration.

5. Degeneration of peripheral nerves and resulting paralysis.

Such is the specific effect of toxins in diphtheria. The same principles apply with equal force in tetanus, typhoid, etc., the differences being in degree of virulence, specificity, mode of onset, and portions of the body affected.

Sidney Martin suggested a provisional classification of bacterial toxins as follows:- +

1. Poisons secreted by the bacterium itself $=($ ferment? $)$ toxin?)

2. Products of digestive action of bacterium = albutracellular

3. Final non-proteid products = animal alkaloid;

4. Poisons present in the body of the bacillus bacterial poisons. Such occur, for example, in the tubercle bacillus and the cholera vibrio.

The toxins of bacteria are of a kind which cannot be fully expressed chemically, but only pathologically. They are probably of a ferment nature in diphtheria and tetanus. The arguments in support of that view are-(1) that they act in infinitesimal doses; (2) that they may act slowly and produce death after many days by profoundly affecting the general nutrition; and (3) that they are sensitive to the action of heat in a way that no chemical poisons are known to be. If they are considered as ferments, they must be

* It should be distinctly understood that this table is merely schematic and provisional. The details of toxin production and its effect are of course still open to revision and amendment.

$\dagger$ Sidney Martin, M.D., F.R.S., F.R.C.P., Croonian Lectures delivered before the Royal College of P'hysicians, June 1898. 
substances which have a peculiar affinity for certain tissues of the body on which they produce their special toxic effect. Hitherto, all attempts at the separation of such bacterial ferments have been without success, and for other reasons also the whole question of such ferments must be left open at present. Sidney Martin and others have demonstrated that many of the extra-cellular toxins are albumoses or bodies of a similar nature. They are non-crystallisable, soluble in water, precipitated along with the proteids by concentrated alcohol, relatively unstable, having their toxicity diminished or destroyed by heat, light, or certain chemical agents. As for the products of digestion, they are formed either by the bacillus ingesting the proteid and discharging it as albumose, or the digestion occurs by means of a ferment secreted by the bacillus in the body of the individual or animal suffering from the disease.

It is now held by some that the virus of anthrax produces albumoses and an alkaloidal substance (Martin), the former producing fever, the latter œdema, congestion, and local irritation. Hankin arrived at the view that the bacillus first produces a ferment and then elaborates albumoses. In tetanus the bacillus produces a secretion of non-proteid toxin which causes the convulsions. The albumoses present in this disease are probably due to the secretory toxin. Ehrlich has isolated a spasm-producing toxin (tetanospasmin), and a crude poison capable of destroying red blood cells (tetanolysin). The nature of the tetanus toxin is not determined, but it is known that it is a most powerful poison, probably less than $\frac{1}{2} \frac{1}{50}$ th of a grain being poisonous to man. In diphtheria, too, we have a secretory poison in the membrane and in the tissues, and an albumose which is possibly the result of the secretion. But the true chemical nature of the diphtheria toxin is also still unknown. In typhoid fever intra-cellular bacillary poisons exist, and a toxalbumin has been obtained which has pathogenic effects of an indefinite character. The toxins of the typhoid bacillus appear to have little digestive effect.

Summary of Toxic Effects.-The action of bacteria as disease producers depends (1) upon the effects of the presence of the bacteria themselves, and (2) upon their power of forming, directly or indirectly, certain chemical organic products known as toxins. The effects of the bacteria, though very diverse, may be classified generally as of a necrotic or a separative character, leading to increased functional activity at first (such as phagocytosis), and subsequently to increased formative activity (such as cell growth and subdivision). In most diseases the lesion has a special site (as in typhoid fever), and the body generally is only affected indirectly. This localisation may be due to specific action, or to point of entrance of the bacillus (as in malignant pustule). Secondarily to tissue 
changes, the body metabolism is affected owing to the distribution of toxins, and it is to this cause that the chief symptoms of disease are due.

\section{The Question of Immunity}

However the details of the modus operandi of the formation of toxins are finally settled, we know that there comes a time when the disease symptoms vanish, the disease declines, and the patient recovers. In past times this was explained by saying that the disease had exhausted itself, having gone "through" the body. In a sense that idea is probably true; but recently a number of investigators have applied themselves to this problem, and with some promising results. And it is now known that, as a result of the action of the toxins in the body tissues, powers of resistance are stimulated or conferred in or upon the body cells affected. What has been found to be true of lower animals by experimentation is now known to be true of the human body. It has, therefore, become possible to inoculate resistant blood serum into toxic blood with the result of opposing the toxins, and bringing about a condition of resistance, and ultimately, recovery. Or, in other words, one of the means of defence against the invasion of such organisms which is possessed by the animal body is the capacity to manufacture, and set free in the blood stream, substances which combine with the toxins and so render them inert. By habituating a large animal, such as the horse, to the action of toxin in increasing quantities, cells or fluids of its body can be thereby so stimulated to produce and throw into the blood stream antitoxins in excessive quantity, that the serum of the animals may contain sufficient excess for its useful employment as a remedy for the disease in man or animals. From such results it is but a step to protective inoculation.

Various protective inoculations against anthrax, for instance, were practised as early as 1881, and the protected animals remained healthy. In 1887 Wooldridge succeeded in protecting rabbits from anthrax by a new method, by which he showed that the growth of the anthrax bacillus in special culture fluids gave rise to a substance which, when inoculated, conferred immunity. In 1889 and 1890 Hankin and Ogata worked at the subject, and announced the discovery in the blood of animals which had died of anthrax of substances which appeared to have an antagonistic and neutralising effect upon the toxins of anthrax and upon the anthrax bacilli themselves. These substances, they afterwards found, were products of the anthrax bacillus. Behring and Kitasato arrived at much the same results in tetanus and diphtheria. In 1890 they showed that the blood serum of an animal which had been immunised against 
tetanus was capable, when injected into other animals, of protecting them not only against poisoning with tetanus toxin but also against infection with living tetanus bacilli. They also proved that, under certain conditions, a curative action could be demonstrated in animals which already presented symptoms of tetanus infection. Similar, though less striking, results were described in the case of diphtheria. The next step was to isolate these substances, and separating them from the blood, investigate still further their constitution. A number of workers were soon occupied at this task, and Buchner, Hankin, the Klemperers, Roux, Sidney Martin, and others have added to our knowledge respecting these toxinopposing bodies now known as antitoxins. Some believed these bodies were a kind of ultratoxin-substances of which an early form was a toxin; others held that, as the toxins were products of the bacteria invading the tissues, the antitoxins were of the nature of ferments produced by the resisting tissues. A third view is that possibly antitoxins may be the result of an increased formation of molecules normally present in the tissues. Finally, antitoxins came to be looked upon as protective substances produced in the body cells as a result of toxic action, and held in solution in the blood, and there and elsewhere exerting their influence in opposition to the toxins. These antitoxic bodies gradually increase in the blood and tissues, and their action falls into two groups: (a) antitoxic, which counteract the effects of the poison itself; and (b) antimicrobic, which counteract the effects of the bacillus itself. "In one and the same animal the blood may contain a substance or substances which are both antitoxic and antimicrobic, such, for example, as occurs in the process of the formation of the diphtheria and tetanus antitoxic serums" (Sidney Martin). Antitoxin must, therefore, be looked upon as a normal constituent of the living cells which is produced in increased quantity. Of the chemical nature of toxins and antitoxins, very little is known. Martin and Cherry have come to the conclusion that toxins are probably of the nature of albumoses, and antitoxins probably have a molecule of greater size, and may be allied to the globulins. Antitoxin has been shown to appear in the various secretions of the body as well as in the blood, though in a less concentrated state.

The relation of the antitoxin to the toxin, and its mode of antagonism, is probably one analogous to chemical union. The two bodies unite to form an inert compound possessing no toxic or pathogenic effect. It is found that a definite period of time elapses before the effect of the toxin is neutralised, and that it takes place more rapidly in strong solutions than in weak, and in warm temperature than in cold, which all goes to confirm the view that 


\section{THE QUESTION OF IMMUNITY AND ANTITOXINS}

such union is the mode of antagonism.* The progress of disease is, therefore, a struggle between the toxins and the antitoxins: when the toxins are in the ascendency we have an increase of the disease; when the antitoxins are in the ascendency we have a diminution of disease. If the toxins triumph, the result is death; if the antitoxins and resistance of the tissues triumph, the result is recovery.

Different Kinds of Immunity.-We have gathered, then, that whenever bacteria, introduced into the blood and tissues, fail to multiply or produce infection (as in saprophytic bacteria, or in immunity of a particular animal from a specific microbe), this inability to perform their rôle is brought about by some property in the living blood serum which opposes their life and action; and further we have seen that this protective property is exhaustible according to the number of bacteria, and differs with various species of bacteria, and in different animals. Buchner designated these protective bodies, held in solution in the blood, alexines, and regarded them as belonging to the albuminous bodies of the lymph and plasma. Alexines are naturally produced antitoxins; ordinary antitoxins are acquired alexines. Hence we have the well-known terms "natural" and "acquired" immunity. Of the former we have already spoken. The latter, acquired immunity, is a protection not belonging to the tissues of individuals naturally and as part of their constitution, but it is acquired during their lives as a further protection of the tissues. This may happen in one, or both, of two ways. Either it may be an involuntary acquired immunity, or a voluntary acquired immunity, a natural attack of disease, or an artificial attack due to inoculation. Small-pox, typhoid fever, even scarlet fever, are diseases which rarely attack the same individual twice. That is because each of these diseases leaves behind it, so to speak, its antitoxic influence. Hence the individual has involuntarily acquired immunity against these diseases. An example of voluntary acquired immunity is also at hand in the old method of preventive inoculation for small-pox, or variolation. This was clearly an inoculation setting up an artificial and mild attack of small-pox, by which the antitoxins of that disease were produced, and protected the individual against further infection of small-pox; that is to say, it was a voluntary acquired immunity. This form of artificial production of protection is artificial immunity. It may be

* Ehrlich has shown that the antitoxic power of these anti-bodies varies widely, and is not uniform. Moreover, antitoxins are specific in their action. He suggests that the ultimate toxin molecule contains two unsatisfied affinities, one of which can - combine with antitoxin (haptophorous), and the others having a toxic action (toxophorous). These groups under certain conditions can lose none of their combining power, the toxophorous being more readily weakened than the haptophorous. The weakened toxins are termed toxoids or toxones. 
convenient to marshal together these various terms in a table as follows :-

Immunity in $\operatorname{man}\left\{\begin{array}{c}=\text { a condition of protection or insusceptibility to certain } \\ \text { diseases. }\end{array}\right.$

1. Natural immunity $=$ constitutional protection produced by alexines.

Acquired naturally (involuntary) produced by antitoxins formed by an attack of the disease. Acquired artificially (voluntary) $=$

(a) Active immunity, produced by direct inoculation of the weakened bacteria or weakened toxins of the disease, e.g. vaccination, or Pasteur's treatment of rabies, or Haffkine's

2. Acquired immunity $=\left\{\begin{array}{c}\text { Pasteur's treatment of rabies, or Haff kine's } \\ \text { inoculation for cholera. } \\ \text { (b) Passive immunity, produced by inoculation, }\end{array}\right.$ not of the disease or of its toxins, but of the antitoxins produced in the body of an animal suffering from the specific disease. These antitoxins combine in some way with the toxins, and so avert their harmful effects. An example of passive immunity occurs in diphtheria antitoxin.

\section{Theories of Immunity}

We may now consider shortly how these new facts were received, and what theories of explanation were put forward to explain continued insusceptibility to disease. It had, of course, been known for a long time that one attack of small-pox, for example, in some degree protected the individual from a subsequent attack of the same disease. To that experience it was now necessary to add a large mass of experimental evidence with regard to toxins and antitoxins. The chief theories of immunity which have been propounded are as follows :-

1. The Exhaustion Theory.-The supporters of this view argued .that bacteria of disease circulating in the body exhausted the body of the supply of some pabulum or condition necessary for the growth and development of their own species (Pasteur).

2. The Retention Theory.-This theory, on the contrary, was based upon the view that there were certain products of micro-organisms of disease retained in the body after an attack which acted antagonistically to the further growth in the body of that same species, as occurs in a test-tube culture.

3. The Acquired Tolerance Theory.-Some have advanced the theory that, after a certain time, the human tissues acquired such a degree of tolerance to the specific bacteria or their specific products, that no result followed their action in the body. The tissues became acclimatised to the disease.

4. The Phagocyte Theory.-This theory, which gained so many adherents when first promulgated by Metchnikoff, attributes to 


\section{THE QUESTION OF IMMUNITY AND ANTITOXINS}

certain cells in the tissues the powers of "scavenging," overtaking germs of disease, and absorbing them into their own protoplasm. This, indeed, may be actually witnessed, and had been observed before the time of Metchnikoff. But he it was who applied the observation to the destruction of pathogenic organisms. He came to the conclusion that the successful resistance which an animal offered to bacteria depended upon the activity of these scavenging cells, or phagocytes. These cells are derived from various cellular elements normally present in the body: leucocytes, endothelial cells, connective tissue corpuscles, and any and all cells in the body which possess the power of ingesting bacteria. If they were present in large numbers and active, it was argued, the animal was insusceptible to certain diseases; if they were few and inactive, the animal was susceptible. It appears that the bacteria or other foreign bodies in the blood which are attacked by the phagocyte become assimilated until they are a part of the phagocyte itself. Metchnikoff explained how the phagocyte is able to encounter bacteria when both are circulating through the blood. It is guided, he holds, in this attack on the organisms by the power of chemiotaxis. The bacteria elaborate a chemical substance which attracts the phagocyte, and this is termed "positive chemiotaxis." But it may occur that the chemical substance produced by the bacteria may have an opposite, or repellent, effect upon the leucocytes, in which case we have "negative chemiotaxis." Metchnikoff distinguishes two chief varieties of phagocytes which become active in disease: $(a)$ the microphages, which are the polynuclear leucocytes of the blood, and $(b)$ the macrophages, which include the larger hyaline leucocytes, connective tissue cells, etc. It is now known that blood serum, from which all leucocytes (phagocytes) have been removed, possesses immunising effects as before, it is therefore clear that such effect is a property of the serum per se, and not wholly or only due to the scavenging power of certain cells in it. Metchnikoff explains this fact by stating that the phagocytes possess digestive ferments (cytases) which may be set free in the blood serum, giving it its bactericidal properties. Metchnikoff admits that antitoxin and toxin form a neutral compound, but holds also that acquired resistance of body cells is of importance in toxin immunity.

5. Ehrlich's Side Chain Theory.-Ehrlich looks upon a molecule of protoplasm as composed of a central atom cell with a large number of side chains of atom groups. The central cell is the mother cell, the side chains are receptors, that is, cells having combining affinity with food stuffs by which nutriment is brought to the mother cell. These receptors are of two kinds, those having power of combining with molecules of simple constitution, and those having power of breaking up compound bodies by ferment action for the purposes of assimilation. Now if toxins be introduced into the 
system they are fixed to the receptors by their haptophorous elements, and their toxophorous elements are therefore free, and if in sufficient numbers or amount produce the toxic changes. If the dose of toxin molecules is small, the mother cell is able to throw off the receptor plus the toxin $(\mathrm{R}+\mathrm{T})$, which thereby becomes free in the blood. The central atom group, however, is able to produce new receptors, which in their turn come to be free in the blood. As a result of repeated loss, the regeneration of receptors becomes an overregeneration, and the excess of unfixed receptors become free in the blood, constituting antitoxin molecules. When forming part of the mother cell the receptors anchor the toxin which is thus able to set up toxic effects in the body cells and tissues, but when the receptors are free in the blood $(\mathrm{R}+\mathrm{T})$, we have an inert compound, and therefore no toxic effect. This ingenious theory of Ehrlich explains the facts of antitoxic effect better than any other, and though not established, and still requiring much more elucidation, is the theory which mostly holds the field at the present time.

\section{The Application of the Principles of Immunity}

We propose now to consider in some detail four illustrations of the application of the facts concerning immunity to the prevention or treatment of disease, viz., vaccination, Pasteur's treatment of rabies, antityphoid and antiplague inoculation, and antitoxin inoculation for diphtheria. The vaccination in small-pox is an inoculation of the virus of an attenuated form of the disease; the rabies inoculation is a transmission of the vital products of the attenuated disease; the typhoid and plague inoculations are of pure cultures of living virus from outside the body; and the diphtheria inoculation is the introduction of antitoxins (passive immunity).*

\section{Vaccination for Small-pox}

In 1717, Lady Mary Wortley Montagu described the inoculation of small-pox as she had seen it practised in Constantinople. So greatly was she impressed with the efficacy of this process, that she had her own son inoculated there, and in 1721, Mr Maitland, a surgeon, inoculated her daughter in London. This was the first time inoculation was openly practised in England. + For one hundred and twenty years small-pox inoculation (or variolation, as it is more

* See also Serums, Vaccines, and Toxines, W. C. Bosanquet, 1904.

† The friend of Addison and Pope, who married Mr Edward Wortley Montagu in 1712, and on his appointment to the ambassadorship of the Porte in 1716 went with him to Constantinople. They remained abroad for two years, during which time Lady Wortley Montagu wrote her well-known Letters to her sister the Countess of Mar, Pope, and others.

$\ddagger$ Crookshank, History and Pathology of Vaccination. 
correctly termed) was practised in this country, until by Act of Parliament in 1840 it was prohibited. There were different methods of performing variolation, but the most approved was similar to the modern system of arm-to-arm vaccination, the arm being inoculated, by a lancet in one or more places, with small-pox lymph instead of, as now, with vaccine lymph. As a rule, only local results or a mild attack of small-pox followed, which prevented an attack of natural small-pox. But its disadvantage is apparent: it was in fact inoculating small-pox, and it was a means of breeding small-pox, for the inoculated cases were liable to create fresh centres of infection.

In 1796, Edward Jenner, who was a country practitioner in Gloucestershire, observed that those persons affected with cow-pox, contracted in the discharge of their duty as milkers, did not contract small-pox, even when placed in risk of infection. Hence he inferred that inoculation of this mild and non-infectious disease would be protective against small-pox, and would be preferable to the process of variolation then so widely adopted in England. Jenner therefore suggested the substitution of cow-pox lymph (vaccine) in place of small-pox lymph, as used in ordinary variolation.

It should not be forgotten that variolation was thus the first work done in this country in producing artificial immunity, and was followed by vaccination, which was only partly understood. Even to-day there is probably much to learn respecting it. Vaccination may be defined as active immunisation by means of a weakened form of the specific virus causing the disease. The nature of the specific virus of both small-pox and cow-pox awaits discovery. Burdon Sanderson, Crookshank, Klein, Copeman, and others have demonstrated bacteria in cow-pox or vaccine lymph, and in 1898 Copeman announced that he had isolated a specific bacillus and grown it upon artificial media.* Numerous statements have been made to the effect that a specific bacillus has been found in small-pox also. But neither in small-pox nor cow-pox is the nature of the contagium really known.†

These facts, however, did not remove the suspicion which had hitherto rested upon vaccine lymph as a vehicle for bacteria of other diseases which by its inoculation might thus be contracted. A few remarks are therefore called for at this juncture upon the work of Copeman and Blaxall, in respect to what is known as glycerinated calf lymph. Evidence has been forthcoming to substantiate in some measure the distrust which many persons have from time to time

\footnotetext{
* An exhaustive account of vaccine may be found in the Milroy lectures, delivered in 1898 at the Royal College of Physicians by S. Monckton Copeman, M.D., Brit. Med. Jour., 1898, vol. i., pp. 1185, 1245, 1312 ; see also paper on the "Bacteriology of Vaccinia and Variola," Brit. Med. Jour., 1902, vol. ii., pp. 52-67. 1898 .

† Crookshank, Bacteriology and Infective Diseases; Virchow, The Huxley Lecture,
} 
felt in the vaccine commonly used in vaccination, hence the new form as above designated. This retains the toxic qualities required for immunity, but is so produced that it possesses in addition three very important advantages: namely, it is entirely free from extraneous organisms, it is available for a large number of vaccinations, and it retains full activity for eight months. It is prepared as follows : -A calf, aged three to six months, is kept in quarantine for a week. If then found upon examination to be quite healthy, it is removed to the vaccinating station, and the lower part of its abdomen antiseptically cleaned. The animal is now vaccinated upon this sterilised area with glycerinated calf lymph. After five days the part is again thoroughly washed, and the contents of the vesicles, which have of course appeared in the interval, are removed with a sterilised sharp spoon, and transferred to a sterilised bottle. This is now removed to the laboratory, and the exact weight of the material ascertained. A calf thus vaccinated will yield from 18 to 24 grams of vaccine material. This is now thoroughly triturated and mixed with six times its weight of a sterilised solution of 50 per cent. chemically pure glycerine in distilled water. The resulting emulsion is aseptically stored in sealed tubes in a cool place. At intervals during four weeks it is carefully examined bacteriologically until by agar plates it is demonstrably free from extraneous organisms, when it is ready for distribution.

The Effect of Vaccination.-The Royal Commission on Vaccination, 1896, concluded (p. 90) that the protection vaccination affords against small-pox may be stated as follows :-

"(1) That it diminishes the liability to be attacked by the disease. (2) That it modifies the character of the disease and renders it less fatal and of a less severe type. (3) That the protection it affords against attacks of the disease is greatest during the years immediately succeeding the operation of vaccination. It is impossible to fix with precision the length of this period of highest protection. Though not in all cases the same, if a period is to be fixed, it might, we think, fairly be said to cover in general a period of nine or ten ycars. (4) That after the lapse of the period of highest protective potency, the efficacy of vaccination to protect against attack rapidly diminishes, but that it is still considerable in the next quinquennium, and possibly never altogether ceases. (5) That its power to modify the character of the disease is also greatest in the period in which its power to protect from attack is greatest, but that its power thus to modify the disease does not diminish as rapidly as its protective influence against attacks, and its efficacy during the later periods of life to modify the disease it still very considerable. (6) That revaccination restores the protection which lapse of time has diminished, but the evidence shows that this protection again diminishes, and that to ensure the highest degree of protection which vaccination can give the operation should be at intervals repeated. (7) That the beneficial effects of vaccination are most experienced by those in whose case it has been most thorough. We think it may be fairly concluded that where the vaccine matter is inserted in three or four places it is more effectual than when introduced into one or two places only, and that if the vaccination marks are of an area of half a square inch they indicate a better state of protection than if their area be at all considerably below this."

These findings are well illustrated in the returns of the London Epidemic of Small-pox which occurred in 1901-2. These returns are the most recent evidence as to the protection afforded by vaccination. They are as follows :- 


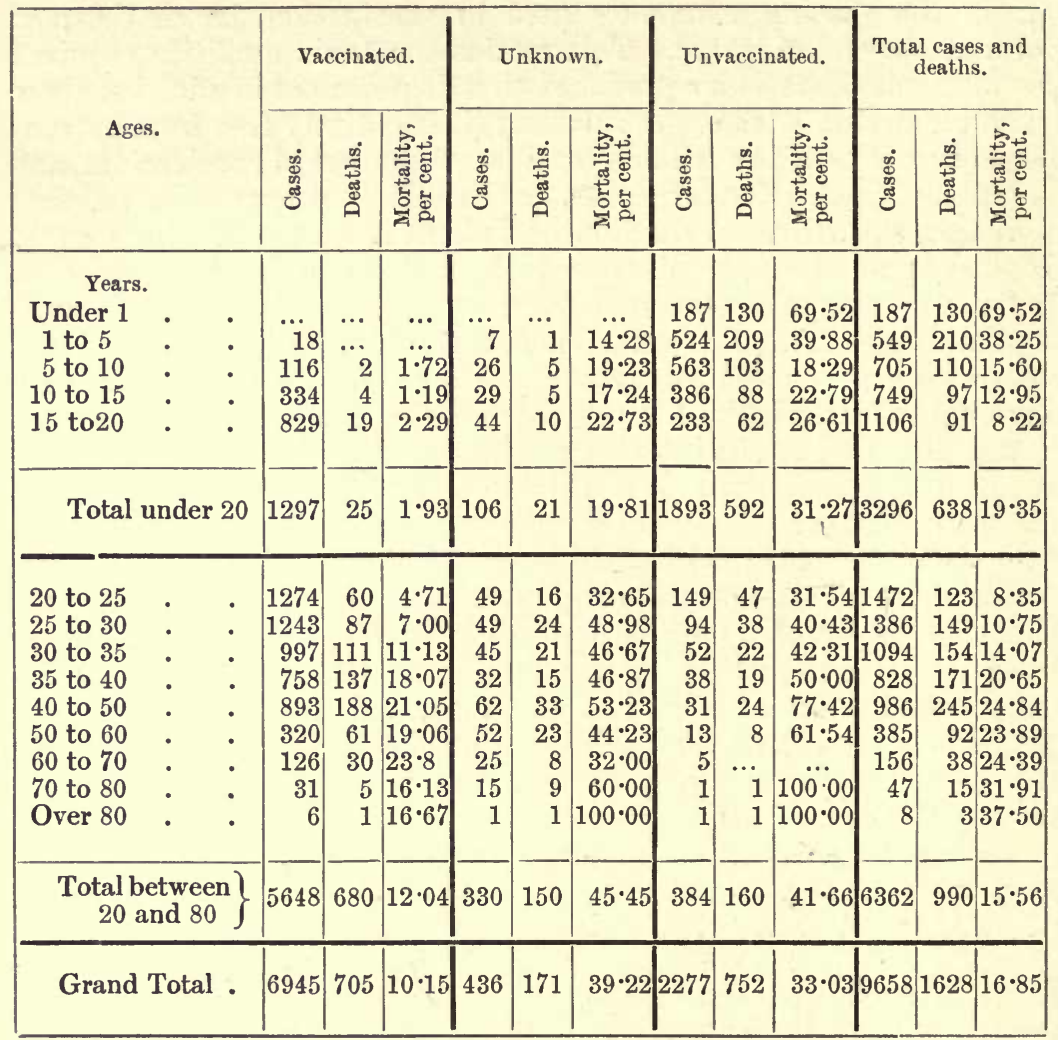

From these figures it will be seen :-

(a) That under five years of age 18 cases of small-pox occurred in children who had been vaccinated, whilst 711 cases occurred in children who had not been vaccinated. (b) That under ten years of age the cases of small-pox in vaccinated persons had a mortality percentage of $1 \cdot 72$, and the unvaccinated cases had a mortality percentage of over 42 per cent. (c) That under twenty years of age the cases of small-pox occurring in vaccinated persons had a mortality percentage of 1.93 , and the unvaccinated cases had a mortality percentage of $31 \cdot 27$. $(d)$ That over twenty years of age the cases of small-pox occurring in vaccinated persons numbered 5648 , and there were 680 deaths, giving a mortality percentage of $12 \cdot 04$; whereas the cases occurring in unvaccinated persons were 384,160 of whom died, giving a mortality percentageof $41 \cdot 66$. The larger number of cases of small-pox in vaccinated persons is, of course, due to the fact that by far the larger proportion of the population at that age-period have, at some time or other in their lives, been vaccinated. Three broad facts stand out with clearness:-(1) That small-pox among the vaccinated is nowadays mainly a disease of adults, because children are protected by primary vaccination and adults are not protected by revaccination. (Ninety-two per cent. of the vaccinated cases were over fifteen years of age.) (2) That among the unvaccinated, small-pox is still, in great measure, a disease of the young as it was in prevaccination days. (Seventy-three per cent. of the unvaccinated cases were under fifteen years of age.) (3) That the mortality rate among the vaccinated is at all 
ages much less than among the unvaccinated, and that this difference is very striking and complete in children because of their recent vaccination.

Those who advocate vaccination and revaccination as protective in a greater or lesser degree against small-pox do so upon three main grounds. In the first place, they claim that, other things being equal, persons who have been vaccinated (especially within ten years) are less liable to attack from small-pox. This is abundantly established by the figures quoted above. In the second place, they claim that persons who have been vaccinated, and yet, on account of their greater number in the population, and, therefore, their consequent greater probability of infection, are attacked by small-pox, do not die so readily from the disease as those who have not been vaccinated. This claim also is more than proved in the returns quoted above. In the third place, they claim that the protection afforded by vaccination depends upon the efficiency of the vaccination. This may be measured, as is frequently done, by the number of marks, but it is more satisfactorily measured by area of vaccination mark (i.e. area of cicatrix). The return of the Metropolitan Asylums Board respecting this point is given below, and a study of it will amply prove the claim made.

\begin{tabular}{|c|c|c|c|}
\hline & Admissions. & Deaths. & $\begin{array}{l}\text { Mortality, } \\
\text { per cent. }\end{array}$ \\
\hline $\begin{array}{l}\text { Vaccinaten CAsEs- } \\
\text { Area of Cicatrix: } \\
\text { Half and upwards of half } \\
\text { square inch } \\
\text { Area of Cicatrix: } \\
\text { One-third, but less than half } \\
\text { square inch } \\
\text { Area of Cicatrix: } \\
\text { Less than one-third square } \\
\text { inch } \\
\text { Area of Cicatrix: } \\
\text { Not recorded }\end{array}$ & $\begin{array}{r}835 \\
860 \\
87\end{array}$ & $\begin{array}{r}131 \\
162 \\
33\end{array}$ & $\begin{array}{r}7 \cdot 34 \\
15 \cdot 69 \\
16 \cdot 87 \\
37 \cdot 93\end{array}$ \\
\hline Totals of Vaccinated Class & 6945 & 705 & $10 \cdot 15$ \\
\hline $\begin{array}{l}\text { Unknown and Doubtful Class . } \\
\text { Unvaccinated Class . }\end{array}$ & $\begin{array}{r}436 \\
2277\end{array}$ & $\begin{array}{l}171 \\
752\end{array}$ & $\begin{array}{l}39 \cdot 22 \\
33 \cdot 06\end{array}$ \\
\hline Grand Totals & 9658 & 1628 & $16 \cdot 87$ \\
\hline
\end{tabular}

\section{Pasteur's Treatment of Rabies}

Rabies is a disease affecting dogs (in Western Europe)* and wolves (in Russia), and can be transmitted to other animals (chiefly mammals and especially the Carnivora) and man. Infection may be conveyed from the rabid animal by biting (which is the most frequent mode), by licking raw surfaces, by suckling, and possibly by the ingestion by animals of the flesh of other animals which have died from the disease.

* In the decennium 1891-1903, 1555 dogs in Great Britain were reported as suffer. ing from rabies, of which only 29 cases occurred during the last five years. The marked decline in recent years is attributed to the effects of the muzzling order and stricter inspection of ownerless vagrant dogs. See also Year-Book of Department of Agriculture, U.S.A., 1900. 
Although rabies was mentioned by Aristotle, and has been studied by a large number of workers since, the contributions of Pasteur have been greater than all the other additions to our knowledge of the disease put together. Professor Rose Bradford has pointed out that Pasteur's discoveries concerning rabies may be said to be four in number: $(a)$ that the virus was not only in the saliva, but also in the central and peripheral nervous system, yet absent from the blood; (b) that the disease was most readily inoculated in the nervous system; $(c)$ that by suitable means the virus could be attenuated; and $(d)$ that by means of an attenuated virus preventive and even curative methods might be adopted.

The disease takes two chief forms: (1) furious rabies, and (2) paralytic or dumb rabies. The former is more common in dogs. The animal becomes restless, has a high-toned bark, and snaps at various objects; sometimes it exhibits depraved appetite. Briefly, the animal passes from a melancholy to a maniacal and then a paralytic state, ending in coma and death. In man, the incubation period is fortunately a very long one, averaging about forty days. Nervous irritability is the first sign; spasms occur in the respiratory and masticatory muscles, and the termination is similar to rabies in the dog. The symptom of fear of water is a herald of coming fatality.

Although a number of the workers at the Pasteur Institute and elsewhere have addressed themselves to the detection of a specific microbe, none has as yet been found, although, in the opinion of Pasteur, such an agent may be suspected as the cause.

Pathologically, rabies and tetanus are closely allied diseases, and the recent remarkable additions to our knowledge of the latter disease only make the similarity more evident. There are in rabies three chief sets of post-mortem signs. First, and by far the most important, are the changes in the nervous system. Here we find patches of congestion in the brain, and breaking down of the axis cylinders of the nerves. The stomach, in the second place, exhibits hæmorrhagic changes, not unlike acute arsenical poisoning. Thirdly, the salivary glands show a degenerative change in a breaking down of their secretory cells. Roux has pointed out that in life the saliva of a mad dog becomes virulent three days before the appearance of the symptoms of disease. The poison appears to be present mainly in the nervous system and the saliva; it is not present in the blood.

The method of treatment by inoculation was introduced by Pasteur. Before his time cauterisation of the wound was the only method adopted. But if more than half an hour has elapsed since the bite, cauterisation is of little or no avail. The basis of Pasteur's treatment was the difference in virulence obtainable in spinal cords 
infected with rabies. Pasteur found that drying the cord led to a lessening of its virulence, just as certain other conditions increased its virulence. Next he established the fact that subcutaneous injection of a weak virus, followed up with doses of ever-increasingly virulent cords, immunised dogs against infection or inoculation of fully virulent material. From this he reasoned that if he could establish a standard of weakened virulence he would have at hand the necessary "vaccine" for the treatment of the disease.

Subsequent research and skilled technique resulted in a method of securing this standard, which he found to be a spinal cord dried for fourteen days. The exact details of preparation of this vaccine are as follows: The spinal cords of two rabbits dead of rabies are removed from the spinal canal in their entirety by means of snipping the transverse processes of the vertebræ. Each cord is divided into three more or less equal pieces, and each piece, being snared by a thread of sterilised silk, is carefully suspended in a sterilised glass jar. At the bottom of the jar is a layer, about half an inch deep, of sterilised calcium chloride. The jars are then removed to a dark chamber, where they are placed at a temperature of $20-22^{\circ}$ C. in wooden cases. Here they are left to dry. Above each case is a tube of broth, to which has been added a small piece of the corresponding cord, in order to test for any micro-organism that may by chance be included. In case of the slightest turbidity in the broth, the cord is rejected. Fourteen series of cords are thus suspended on four-

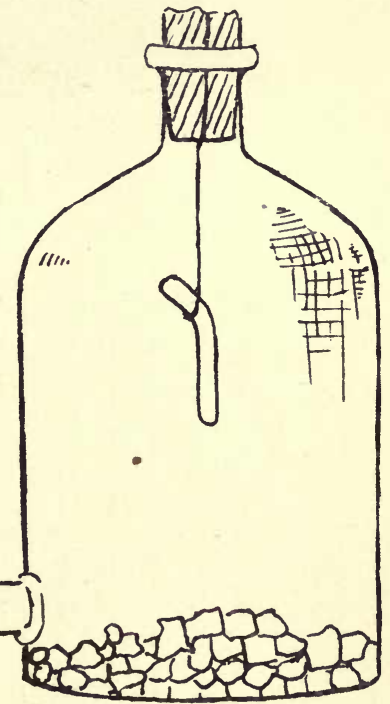

Fig. 36.-Suspended Spinal Cord. In drying jar containing Calcium Chloride. teen consecutive days. The first, second, and third are found to be of practically equal virulence, but from the third to the fourteenth the virulence proportionally decreases, and on the fifteenth day the cord would be practically innocuous and non-virulent. When treatment is to be commenced, obviously the weakest-that is, the fourteenth day-cord is used to make the "vaccine," and so on in steadily increasing doses (as regards virulence) up to, and including, a thirdday cord. The fourteenth-day cord is therefore taken, and a small piece cut off and extracted in 10 c.c. of sterile broth, which are placed in a conical glass and covered with two layers of thick filterpaper, the glass with its covering having been previously sterilised 
by dry heat. When the patient bitten by the rabid animal is prepared, 3 c.c. of this broth emulsion of spinal cord are inoculated by means of a hypodermic needle (under aseptic precautions) into the flanks or abdominal wall. On the following day the patient returns for an inoculation of a cord of the thirteenth day, and so on until a rabid cord emulsion of the first three days has been inoculated. As a matter of practice, the dosage depends upon the three recognised classes of bites, viz. (1) bites through clothing (least severe); (2) bites on the bare skin of the hand; (3) bites upon the face or head, most severe owing to the vascularity of these parts. An example of each, which the writer was permitted to take in the Pasteur Institute, may be here added to illustrate the usual practice.

Inoculation Treatment for Persons affected with Rabies.

\begin{tabular}{|c|c|c|c|c|c|c|c|c|c|c|c|c|c|c|}
\hline \multicolumn{5}{|c|}{$\begin{array}{l}\text { 1. For those Bitten through } \\
\text { Clothes. }\end{array}$} & \multicolumn{5}{|c|}{$\begin{array}{l}\text { 2. For those Bitten on } \\
\text { Uncovered Skin of Hands, etc. }\end{array}$} & \multicolumn{5}{|c|}{$\begin{array}{l}\text { 3. For those Bitten on Face } \\
\text { or Head. }\end{array}$} \\
\hline \multicolumn{3}{|c|}{$\begin{array}{c}\text { Days of } \\
\text { Treatment. }\end{array}$} & 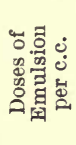 & 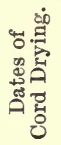 & \multicolumn{3}{|c|}{$\begin{array}{l}\text { Days of } \\
\text { Treatment. }\end{array}$} & 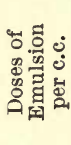 & 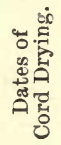 & \multicolumn{3}{|c|}{$\begin{array}{l}\text { Days of } \\
\text { Treatment. }\end{array}$} & 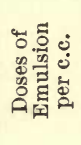 & 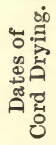 \\
\hline \multicolumn{3}{|c|}{1 at 11 a.m. } & 3 & 14 & \multicolumn{3}{|c|}{1 at 11 a.m. } & 3 & 14 & \multirow{2}{*}{\multicolumn{3}{|c|}{1 at 11 a.m. }} & 3 & 14 \\
\hline 1 & , & ,, & 3 & 13 & & , & ,, & 3 & 13 & & & & 3 & 13 \\
\hline 2 & ", & ," & 3 & 12 & & ," & & 3 & 12 & \multicolumn{3}{|c|}{1 at 3 p.m. } & 3 & 12 \\
\hline 2 & , & , & 3 & 11 & & , & , & 3 & 11 & \multicolumn{3}{|c|}{$1,, \quad}$, & 3 & 11 \\
\hline 3 & ," & ," & 3 & 10 & & ," & , & 3 & 10 & \multicolumn{3}{|c|}{2 at 11 a.m. } & 3 & 10 \\
\hline 3 & , & , & 3 & 9 & & , & , & 3 & 9 & \multicolumn{3}{|c|}{$2 \quad,, \quad}$, & 3 & 9 \\
\hline 4 & ," & ," & 3 & 8 & & ," & ," & 3 & 8 & \multicolumn{3}{|c|}{2 at 3 p.m. } & 3 & 8 \\
\hline 4 & , & , & 3 & 7 & & , & , & 3 & 7 & \multirow{2}{*}{\multicolumn{3}{|c|}{2 ,", }} & 3 & 7 \\
\hline 5 & , & , & 3 & 6 & 5 &, & , & 3 & 6 & & & & 3 & 6 \\
\hline 5 & ," & ", & 3 & 6 & 5 & , & , & 3 & 6 & \multicolumn{3}{|c|}{3 at $11 \mathrm{a} . \mathrm{m}$. } & 3 & 6 \\
\hline 6 & , & ," & 3 & 5 & 6 & ," & , & 3 & 5 & & , & & 3 & 5 \\
\hline 7 & , & , & 3 & 5 & 7 & , , & , & 3 & 5 & & , & & 3 & 5 \\
\hline 8 & , & , , & 3 & 4 & 8 & , & , & 3 & 4 & 6 & ,, & & 3 & 4 \\
\hline 9 & , & , & 2 & 3 & 9 & , & , & 2 & 3 & 7 & , & & 2 & 3 \\
\hline 10 & , & , , & 3 & 5 & 10 & , & , & 3 & 5 & 8 & ," & & 3 & 4 \\
\hline 11 & ," & ., & 3 & 5 & 11 & ," & ,, & 3 & 5 & 9 & ," & & 3 & 3 \\
\hline 12 & ," & ,, & 3 & 4 & 12 & ," & ," & 3 & 4 & 10 & ," & & 3 & 5 \\
\hline 13 & ," & ," & 3 & 4 & 13 & ," & , & 3 & 4 & 11 & ," & & 3 & 5 \\
\hline 14 & , , & , & 3 & 3 & 14 &, & & 3 & 3 & 12 & & & 3 & 4 \\
\hline 15 & ," & , & 3 & 3 & 15 & , & , & 3 & 3 & 13 & & & 3 & 4 \\
\hline & $\ldots$ & & $\ldots$ & $\ldots$ & 16 & ," & , & 3 & 5 & 14 & ," & & 3 & 3 \\
\hline & $\ldots$ & & $\ldots$ & $\ldots$ & 17 & , & , & 3 & 4 & 15 & , & & 3 & 3 \\
\hline 2 & $\cdots$ & & $\ldots$ & $\ldots$ & 18 & ," & ," & 3 & 3 & 16 & ,", & & 3 & 5 \\
\hline & $\cdots$ & & $\ldots$ & $\ldots$ & & $\ldots$ & & $\ldots$ & $\ldots$ & 17 & & & 3 & 4 \\
\hline x & $\ldots$ & & $\ldots$ & $\ldots$ & & $\ldots$ & & $\ldots$ & $\ldots$ & 18 & , & & 3 & 3 \\
\hline & $\cdots$ & & $\ldots$ & $\ldots$ & & $\ldots$ & & $\ldots$ & $\ldots$ & 19 & ", & & 3 & 5 \\
\hline & $\ldots$ & & $\ldots$ & $\ldots$ & & $\ldots$ & & $\ldots$ & $\ldots$ & 20 & , & & 3 & 4 \\
\hline & $\cdots$ & & $\cdots$ & $\ldots$ & & $\ldots$ & & $\ldots$ & $\ldots$ & 21 & , & & 3 & 3 \\
\hline
\end{tabular}


Effect of Treatment.-It may be well to add the returns of inoculation made at the Pasteur Institute, Rue Dutot, Paris, as above described, and the mortality rate resulting. The record is as follows :-

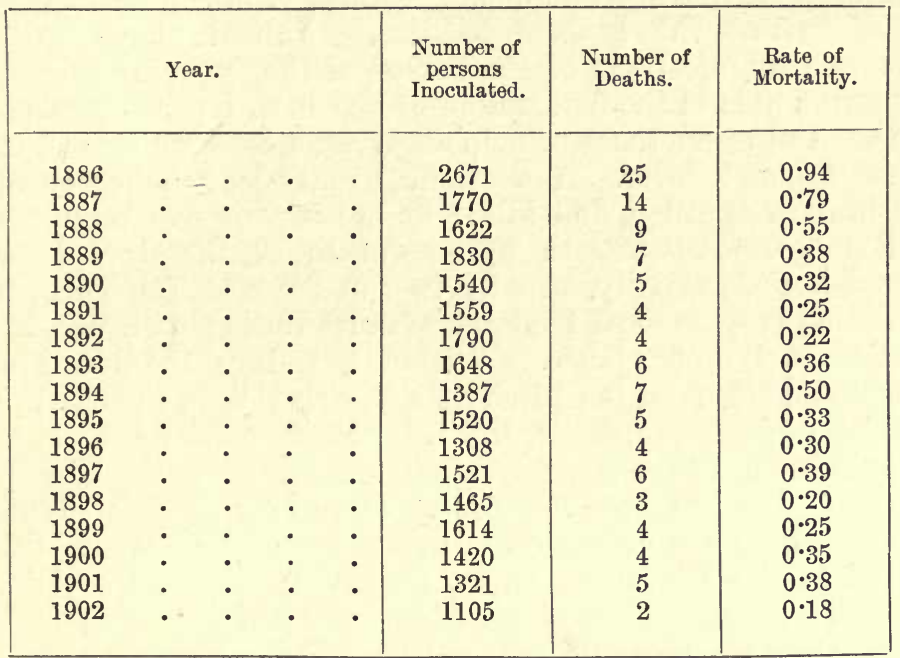

Of the 1105 persons under treatment in 1902, 9 were English, 2 Spaniards, 2 Russians, and one each Greek, Dutch, and Swiss-making 16 foreigners, 1089 French. The diminution in the number of French patients, as compared with several preceding years, is explained by the opening of anti-rabic institutes at Lille, Marseilles, Montpellier, Lyons, and Bordeaux, at one or other of which persons residing in the neighbourhood of those towns have been sent instead of going to Paris.

Pasteur's treatment of rabies by inoculation of emulsions of dried spinal cord is, therefore, a "vaccination" of attenuated virus, resulting in antitoxin formation, to the further protection of the individual against rabies.

\section{Inoculations for Cholera, Typhoid, and Plague}

Anti-Cholera Inoculation.-Inoculating cholera virus against cholera has been made illegal, like variolation was in 1840 . But Haffkine has prepared two vaccines. The weak one is made from pure cultures of Koch's spirillum of Asiatic cholera, attenuated by growth to several generations on agar or broth at $39^{\circ} \mathrm{C}$., or by passing a current of sterile air over the surface of the cultures. The strong one, virus exalté, is from similar culture the virulence of which has been increased by passage through guinea-pigs. One cubic centimetre of the first vaccine is injected hypodermically into the flank, and the second vaccine three or four days afterwards. The immunisation is prophylactic, not remedial, and its action takes effect five or six days after the second vaccine has been injected. 
Anti-Typhoid Inoculation.-It is now known that the serum of persons who have recovered from typhoid fever, and the serum of animals artificially immunised against virulent typhoid bacilli, protect against the typhoid bacillus. Animals have now been immunised by injections of the toxins of the typhoid bacillus; and their serum aids in the destruction of the bacilli which produce the toxins. Acting on these principles, Wright has prepared a vaccine against typhoid fever. A virulent twenty-four hours culture is emulsified in bouillon, and killed by heating for five minutes at $60^{\circ}$ C. For use, one-twentieth to one-fourth of the dead culture is injected hypodermically, usually in the flank.* The effect of the inoculation is some local tenderness and swelling with enlargement of adjacent lymph glands. Within ten days the blood of the inoculated person begins to show a positive Widal reaction, owing to its immunising properties, and it is also bactericidal in vitro.

Haffkine's Preventive Inoculation for Plague.-In plague the same plan has been followed. Luxurious crops of Kitasato's plague bacillus are grown on ordinary broth with the addition to the surface of a film of oil or fat ("ghee"). Under the globules of fat flakes of plague culture grow like stalactites, hanging down into the clear broth. The culture is kept at $25^{\circ} \mathrm{C}$. These are, every few days, shaken to the bottom, and a second crop grows on the undersurface of the fat. In the course of six weeks a number of such crops are obtained and shaken down into the fluid, until the latter assumes an opaque milky appearance. The purity of this culture is controlled by transferring with a sterile pipette a small quantity to a dry agar tube, and noting the appearance of the growth by reflected light through the thickness of the agar. The culture is now, unlike the cholera vaccine, exposed to a temperature of $65^{\circ} \mathrm{C}$. for one hour in a water-bath, and a small quantity of carbolic acid is added ( 5 per cent.), by which processes the bacilli are killed. The dose is 5 to 10 c.c. This preparation has the advantage of being easily prepared, obtainable in large quantities, and requires no animals in its preparation. When inoculated, it produces local pain and swelling at the site of inoculation, and general reactive symptoms such as fever. From a careful analysis of the results of this inoculation, it is shown that the efficacy of the prophylactic depends upon the virulence of the bacillus culture from which the vaccine is prepared, and upon its dose and ability to produce a well-marked febrile reaction. It appears to be more effective in the prevention of deaths than of attacks. $\dagger$

* For methods employed in preparation of the vaccine, see Brit. Med. Jour., 1900 , vol. i., p. 122 (Wright).

†Proc. Roy. Soc., 1900; Report of Medical Officer to Local Government Board, 1902, pp. 357-94. 
The Indian Plague Commission concluded that (1) inoculation sensibly diminishes the incidence of plague attacks on the inoculated population, but the protection afforded is not absolute; $(2)$ inoculation diminishes the death-rate among the inoculated population; (3) inoculation does not appear to establish protection until after some days; and (4) protection is conferred for a considerable number of weeks and possibly for months. Finally, the Commission recommend that under the safeguards and conditions of accurate standardisation and complete sterilisation of the vaccine, and the thorough sterilisation of the syringe in every case, inoculation should be encouraged wherever possible, and in particular among disinfecting stuffs, and the attendants of plague hospitals.

\section{Antitoxin Inoculation for Diphtheria}

We may now consider an illustration of passive immunity. This, it will be remembered, may be defined as-a protection (against a bacterial disease) produced by inoculation, not of the disease itself, as in small-pox inoculation, nor yet of its weakened toxins, as in rabies, but of the antitoxins produced in the body of an animal suffering from that particular disease. Examples of this treatment are increasing every year. The chief examples are to be found in Diphtheria, Tetanus, Streptococcus, and Pneumococcus.

To be of value, antitoxins must be used as early as possible, before tissue change has occurred and before the toxins have, so to speak, got the upper hand. When the toxins are in the ascendency the patient suffers more and more acutely, and may succumb before there has been time for the formation in his own body of the neutral compound of toxin and antitoxin. If he can be tided over the "crisis," theoretically all will be well, because then his own antitoxin will eventually gain the upper hand. But in the meantime, before that condition of affairs, the only way is to inject antitoxins prepared in some animal's tissues whose disease began at an earlier date, and thus add antitoxins to the blood of the patient, early in the disease, and the earlier the better, for, however soon this is done, it is obvious that the toxins begin their work earlier still. It should not be necessary to add that general treatment must also be continued, and indeed local germicidal treatment, e.g. of the throat in diphtheria and the poisoned wound in tetanus. Further, in a mixed infection, as in glandular abscesses with diphtheria, it must be borne in mind that the antitoxin is specific, and may therefore probably fail to reduce the complication which must be treated separately.

In the production of antitoxins, an animal is required from whose body a considerable quantity of blood can be drawn without injurious effect. Moreover, it must be an animal that can stand 
an attack of such diseases as diphtheria and tetanus. Such an animal is the horse. Now, by injecting into the horse $(a)$ living organisms of the specific disease, but in non-fatal doses, or (b) dead cultures, or $(c)$ filtered cultures containing no bacteria and only toxins, we are able to produce in the blood of the horse first the toxins and then by natural processes the antitoxins of the disease in question. The non-poisonous doses of living organisms can be attenuated, by various means. Dead cultures have not been much used to produce immunity except by Pfeiffer. In actual practice the third method is much the most general, viz., filtering a fluid culture free from bacteria, and then inoculating this in everincreasing doses. The preparation of diphtheria antitoxin may be taken as an example, but what follows would be equally applicable to other diseases, such as tetanus:-

1. To obtain the Toxin.-First grow a pure culture of the KlebsLöffler bacillus of diphtheria in large flasks containing "Löffler's medium," or a solution made by mixing three

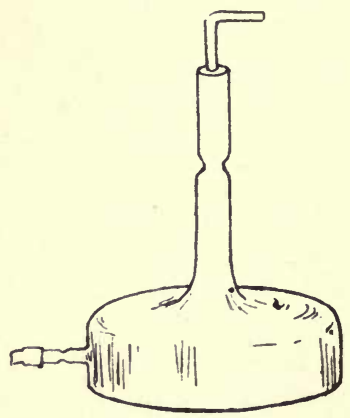

Fic. 37.-Flask used for Prepara. tion of the Toxin of Diphtheria. parts of blood serum with one of beef broth, and adding 1 per cent. of common salt $(\mathrm{NaCl})$ and 1 per cent. of peptone. An alkaline medium is necessary, and a free supply of oxygen and the presence of a large proportion of peptone in the medium favours a high degree of toxicity. The bouillon must be glucose-free. The flask, thoroughly sterilised before use, is now plugged with sterile cotton-wool and incubated at $37^{\circ} \mathrm{C}$. for three weeks. Sterile air may be passed over the culture periodically, thereby aiding the growth. After the lapse of about a month a scum of diphtheria growth will have appeared over the surface of the fluid. This is now filtered through a Chamberland filter into sterilised flasks, and some favourable antiseptic added to ensure that nothing foreign to the toxin shall flourish. The flasks are kept in a cool place in the dark. Here, then, we have the product, the toxin, ready for injection into the horse.

The power of the toxins is estimated by subcutaneous injection of varying amounts into a number of guinea-pigs, and the minimum lethal dose (M.L.D.) is obtained. The standard M.L.D. is the smallest amount which will kill a 250-gram guinea-pig in four days. According to Behring, a normal M.L.D. (expressed as D.T.N. ${ }^{1}$ ) is 01 c.c.; a toxin of which 02 is M.L.D. will therefore be expressed as D.T.N. ${ }^{5}$

2. Immunisation of the Horse.-The general principle is that 
the animal is treated with increasing doses of the particular poison. The toxins, which have been previously tested on small animals, such as rabbits and guinea-pigs, are injected subcutaneously, intramuscularly, or intravenously. At first either very minute doses of weak toxins, or toxins which had been modified by chemical agents, or in other ways, are employed. In the case of tetanus, in the early stages the toxin is usually modified by being treated with iodine. The injection of the toxin may be followed by swelling at the site of inoculation, loss of appetite, general malaise, and rise of temperature. When these have passed off the animal receives a second, rather larger injection, and in this way the quantity of toxin is increased until within a few months the horse is capable of tolerating many thousand multiples of what would be a lethal dose if given as a first injection. When the serum has reached the strength suitable for clinical use, blood is withdrawn from time to time by venesection.

It is evident that only healthy horses are of service in providing healthy antitoxin, even as healthy children are necessary in arm-to-arm vaccination. To provide against any serious taint, the horse is tested for glanders (with mallein) and for tuberculosis (with tuberculin). The dose of the injection of toxin is at the commencement about $\frac{1}{10}$ c.c., or a little more. The site of the inoculation is the apex of the shoulder, which has been antiseptically cleaned. After the first injection there is generally a definite febrile reaction and a slight local swelling. From $\frac{1}{10}$ or $\frac{1}{2}$ c.c. the dose is steadily increased, until at the end of two or three months* perhaps as much as 300 c.c. (or even half a litre) may be injected without causing the reaction which the initial injection of $\frac{1}{10}$ c.c. caused at the outset. This shows an acquired tolerance of the tissues of the horse to the toxic material. After injecting 500 c.c. into the horse without bad effect, the animal has a rest of four or five days.

3. To obtain the Antitoxin.-During this period of rest the interaction between the living body cells of the horse and the toxins results in the production in the blood of an antitoxin. By means of a small sterilised cannula, five, or eight, or even ten litres of blood are drawn from the jugular vein of the horse into sterilised flasks or jars. As used in Paris, the top of the jar is closed by two paper coverings before it is sterilised. Then it is again covered with a further loose one. Before use the loose one is removed and replaced by a metal (zinc) lid, which has been separately sterilised. This metal lid contains an aperture large enough for the tube which

* To shorten this period Dr Cartwright Wood has adopted a plan by which time may be saved, and 200 c.c. injected, say, within the first two or three weeks. This is accomplished by using a "serum toxin" (containing albumoses, but not ferments) previously to the broth toxin. 
conveys the blood from the cannula to pass through. The tube, therefore, passes through the metal lid and two paper covers, which it was made to pierce. When enough blood has passed into the vessel the tube is withdrawn, and the metal lid slightly turned. Thus the contained blood is protected from the air.* The jar containing the blood (which contains the antitoxins) is next placed in a dark, cool cellar, where it stands for separation of the clot. During this time the blood naturally coagulates, the corpuscles falling as a dense clot to the bottom, and the faintly yellow serum rising to the top. The serum, or liquor sanguinis, averages about 50 per cent. of the total blood taken. Sometimes antiseptic (' 3 per cent. carbolic acid) is added with a view to preservation. It is generally filtered (through a Berkefeld) before bottling for therapeutic use, and examined bacteriologically as a test of purity, for sterility and for absence of toxicity, and for antitoxic value.

The latter step is the estimation of the antitoxic power of the serum, or what is termed the "standardising" of the serum. This is accomplished by testing the effect of various quantities upon a certain amount of toxin. Ehrlich has adopted as the immunity unit the amount of antitoxic serum which will neutralise a hundred times the minimum lethal dose of toxin, the serum and toxin being mixed together, diluted up to 4 c.c., and injected subcutaneously. A normal antitoxic serum is one of which 1 c.c. contains an immunity unit.

Process of Standardisation of Antitoxins.-This matter will be best illustrated by an illustration, as follows:-

Stage 1. - Varying amounts of a toxin are added to a definite amount of antitoxin, i.e. to 1 Ehrlich unit, and injected into a guinea-pig of 250 grammes. That mixture, which kills the guinea-pig in four days, is held to contain 1 M.L.D., over and above the amount of toxin required to neutralise 1 antitoxin unit. The total amount of toxin used to bring about the death of the guinea-pig in four days = the standard toxin for that particular standardisation.

Stage 2.- Varying quantities of the antitoxin to be tested are added to the standard toxin and injected into guinea-pigs. That mixture, which kills the guineapig in four days as before, contains 1 M.L.D. of toxin over and above that neutralised by the added antitoxin. The amount of toxin used in Stages 1 and 2 is the same, therefore the amount of antitoxin in Stages 1 and 2 must be equal. In Stage 1, 1 Ehrlich unit was used, therefore the amount of antitoxin in Stage 2 which, with the standard toxin killed the guinea-pig in four days, also contains 1 Ehrlich unit.

Example. Stage 1.

25 c.c. Toxin +1 Ehrlich Antitoxin Unit

"26 c.c.

27 c.c.

28 c.c.

,

,

9

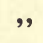

,9

,
- Guinea-pig alive fifth day.

$$
\text { ", died fourth day. }
$$$$
\text { , died first day. }
$$

$\therefore \quad 27$ c.c. Toxin $=$ Standard toxin, containing 1 M.L.D. of toxin after neutralisation with 1 Antitoxin unit.

* At the conclusion of the operation the cannula is removed from the jugular vein and the wound is closed by the valvular character of the slit in the skin and vein, and the elasticity of the wall of the vein. No stitching or dressing is required. 
Example.

Stage 2.

$\frac{1}{100}$ c.c. Antitoxic Serum to be tested $+\cdot 27$ c.c. Toxin . Guinea-pig alive fifth day. $\frac{1}{200}$ c.c.

$\frac{1}{300}$ c.c.

$\frac{1}{400}$ c.c.

, $\quad$,

, ,

, :

", died fourth day.

", died first day.

$\therefore$ The mixture of $\frac{1}{300}$ c.c. of Antitoxic Serum +27 c.c. Toxin, killing guineapig in four days, contains 1 M.L.D.

$\therefore \frac{1}{300}$ c.c. Antitoxic Serum $=1$ Ehrlich unit.

$\therefore 1$ c.c. $=300$ Ehrlich units.

4. The Use of Antitoxin.-The antitoxin is now ready for injection into the patient who has contracted diphtheria, and in whose blood toxins are in the ascendency, and under which the individual may succumb. They are injected in varying doses, as we have already pointed out. As large a dose should be given as practicable. A common first dose varies from 2000 to 5000 units. For prophylactic purposes a smaller dose is administered (500, and for children under two years of age, 300 units). Early administration is of great importance. The flank between the crest of the ilium and the last rib and the lower part of the abdomen are generally selected as the sites of injection, but any region with loose subcutaneous connective tissue is suitable. The injections should be subcutaneous. In performing the injection strict asepsis must be observed. The syringe must be well washed and boiled before use. The skin must be well cleansed with soap and water, and afterwards treated with an antiseptic such as a 1 in 1000 corrosive sublimate solution, or 1 in 20 carbolic acid solution. The antitoxin of diphtheria has been used on various recent occasions as a prophylactic in outbreaks of the disease, and it is now considered as one of the practicable means for controlling an epidemic. Antitoxin inoculation played a greater or less part in the checking of diphtheria outbreaks at Cambridge,* Colchester, $†$ Kempston, + and other places. In the Cambridge outbreak antitoxin was supplied free for prophylactic use in the case of those who had come into contact with actual cases of diphtheria, or where those who, not being ill, were known by bacteriological examination of the throat to be harbouring the diphtheria bacillus. Thus free bacteriological examination of the throat of suspected or known "contacts" was first carried out. In the cases yielding positive results antitoxin was injected. At Cambridge 500 units of antitoxin were given in such contact persons; at Kempston 1000 units was the dose. The general result is that mortality has been lessened, and that in fatal cases there has been a considerable lengthening of the period of life. Moreover, the whole clinical course of the disease is greatly modified, and its severity reduced.

* Jour. of Hygiene, 1901, vol. i., pp. 228 and 487.

† Ibid., 1902, vol. ii., p. 170.

¥ Report on an Outbreak of Diphtheria at Kempston, p. 21. 


\section{Effect of Diphtheria Antitoxin Inoculation}

The following summary of the Antitoxin Treatment of all forms of Diphtheria at the Hospitals of the Metropolitan Asylums Board, 1895-1903, compared with the results obtained before the adoption of that treatment,* affords striking evidence of the efficacy of diphtheria antitoxin :-

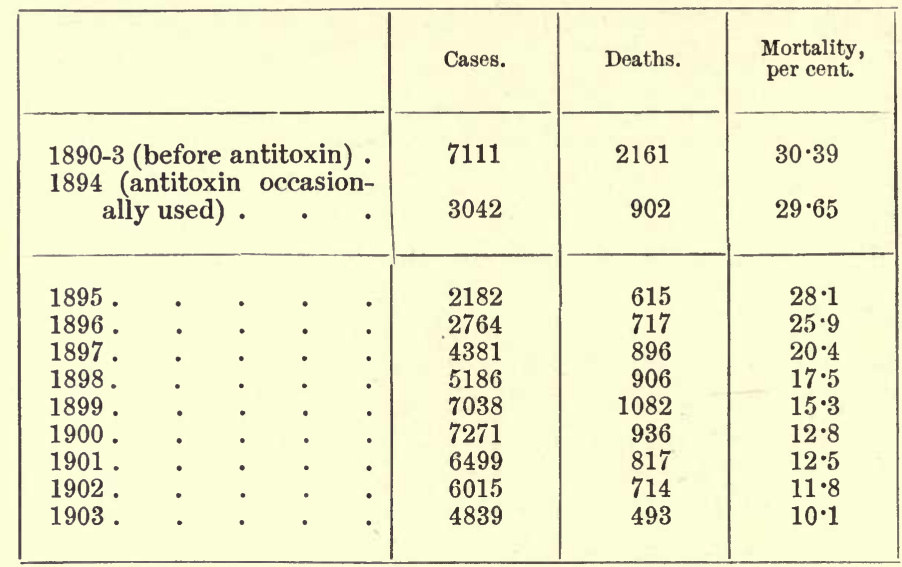

The value is particularly noticeable among children. Amongst cases in the first year of life the rate has fallen from 61.8 to $37 \cdot 8$; in the second year from 63.1 to 35.4 ; in the third year from $55 \cdot 1$ to 26.4 ; in the fourth year from 48.3 to 22.9 ; and in the fifth year from $39 \cdot 6$ to $20 \cdot 7$.

At the Brook Hospital, Shooters Hill, Woolwich (Metropolitan Asylums Board), Dr MacCombie has kept records since 1897 showing the results of the antitoxin treatment on all the cases at the hospital, with special reference to the day of the disease on which treatment began, in order to illustrate the effect of early administration :-

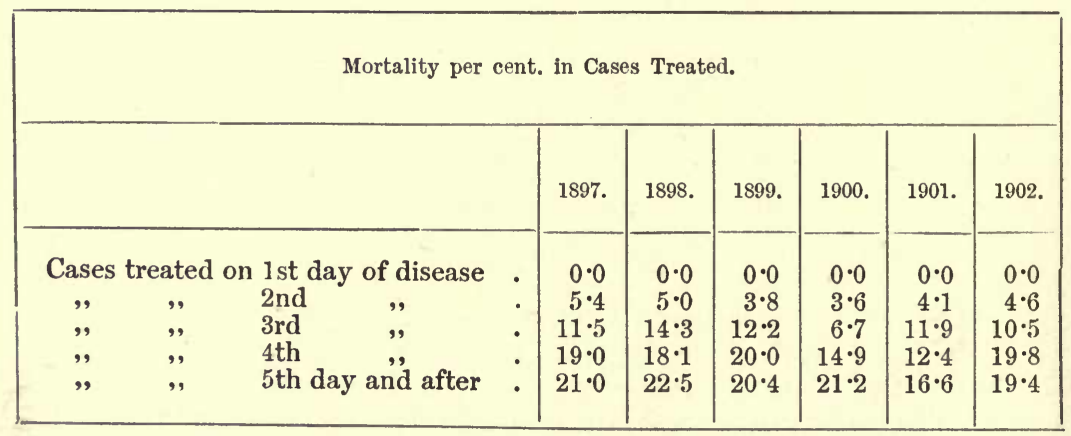

* Annual Report of Metropolitan Asylums Board, 1902, p. 172.

+ For the most complete account of diphtheria antitoxin and its effects, see Report on the Bacteriological Diagnosis and Antitoxic Serum Treatment of Cases admitted to the Hospitals of the Metropolitan Asylums Board, 1895-6, by Professor Sims Woodhead, M.D. 
" During the past six years," Dr MacCombie reports, " the total number of cases treated with antitoxin has been 4202 . Not a single death has taken place among the cases that came under treatment on the first day of disease, and among those coming under treatment on the second day of disease, the mortality has not exceeded $5 \cdot 4$, and has been as low as $3 \bullet 6$. While among those that came under treatment later the average mortality is very much higher. Were it possible to secure the admission to hospital of all cases on the first or second day of illness, the lives of a large number of patients would thereby be saved." Dieudonné has collected similar returns to the foregoing table from four or five different sources. The importance of early administration is therefore widely established.

* Annual Report of Metropolitan Asylums Board, 1902, p. 208 ; see also Report, 1903, p. 218. 


\title{
CHAPTER XIII
}

\author{
DISINFECTION
}

General Principles-Means of Disinfection: by Heat; by Chemicals-Practical Disinfection: Rooms, Walls, Bedding, Clothing, Excreta, Books, Linen, Stables, etc.-Disinfection of Hands-Disinfection after Special Diseases : Phthisis, Small-pox, Scarlet Fever, Diphtheria, Typhoid, Plague.

THE object of modern bacteriology is not merely to accumulate tested facts of knowledge, nor only to learn the truth respecting the morphology and life-history of bacteria. These are most important things from a scientific point of view. But they are also a means to an end; that end is the prevention of preventable diseases and the treatment of any departure from health due to micro-organisms. In a science not a quarter of a century old, much has already been accomplished in this direction. The knowledge acquired of, and the secrets learned from, these microscopic vegetable cells which possess such potentiality for good or evil have been, in some degree, successfully turned against them. When we know what favours their vitality and virulence, we know something of the physical conditions which are inimical to their life; when we know how to grow them, we also know how to kill them.

We have previously made a brief examination of the methods which are adopted for opposing bacteria and their products in the tissues and body fluids. We must now turn to consider shortly the modes which may be adopted in preventive medicine for opposing bacteria outside the body.

It will be clear at once that we may have varying degrees of opposition to bacteria. Some substances kill bacteria, and are thus 432 
germicides; other substances prevent their development and resulting septic action, and are termed antiseptics. The word disinfectant is used more or less indiscriminately to cover both these terms. A deodorant is, of course, a substance removing the odour of evilsmelling putrefactive processes. These are the four common designations of substances able to act injuriously on bacteria and their products outside, or upon the surface of, the body. But a moment's reflection will bring to mind two facts not to be forgotten. In the first place, an antiseptic applied in very strong dose, or for an extended period, may act as a germicide; and, vice versa, a germicide in too weak solution to act as such may perform only the function of an antiseptic. Moreover, the action of these disinfecting substances not only varies according to their own strength and mode of application, but it varies also according to the specific resistance of the protoplasm of the bacteria in question. Examples of the latter are abundant; for instance, between the bacillus of typhoid fever and the spores of anthrax there is an enormous difference in power of resistance. In the second place, there are the physical conditions injurious to the development of bacteria. At a low temperature bacteria do not multiply at the same rapidity as at blood-heat. Within the limits of a moist perimeter the air is, to all intents and purposes, germ-free. Direct sunlight has a definitely germicidal effect in the course of time upon some of the most virulent bacteria we know. In a certain sense these three examples of physical conditions-low temperature, moist perimeter, direct sunlight-may become first antiseptics and then germicides. Yet for a limited period they have no injurious effect upon bacteria. These would seem to be very simple points, and calling for little comment, yet the pages of medical and sanitary journals reveal not a few keen controversies upon the injurious action of certain substances upon certain bacteria, owing to the discrepancies of necessity arising between results of different skilled observers who have been carrying out different experiments with different solutions of the same substance upon different protoplasms of the same species of bacteria. We feel no doubt that in these pioneering researches much labour has been to some extent misspent, owing to the neglect of a common denominator. Only a more accurate knowledge of bacteria or a recognised standard for disinfecting experiments can ever supply such common denominator. Species of bacteria for comparative-observation-experiments into the action of chemical or physical agents must be not only the same species, but cultured under the same conditions, and treated by the agent in the same manner, otherwise the results cannot be compared upon a common basis, or with any hope of arriving at comparable conclusions. 
In 1884 was issued from the English Local Government Board one of the first adequate statements respecting the principles of disinfection, as applied to the facts known respecting bacteria.* In that report Dr Franklin Parsons arrived at the following important conclusions: $(\alpha)$ that all infected articles which could be treated by boiling water could not be so well disinfected in any other way as by simply boiling for a few minutes; (b) that for articles which could not be so treated, high pressure steam, with complete penetration, was most satisfactory; and $(c)$ where articles would be injured by either boiling or steam, dry heat at $240^{\circ} \mathrm{F}$., if sufficiently prolonged, would be effectual. He found that with the exception of anthrax spores all the infected materials he experimented upon were destroyed after an hour's exposure to dry heat at $220^{\circ} \mathrm{F}$., or five minutes exposure to steam at $212^{\circ} \mathrm{F}$. Anthrax spores required four hours' dry heat at $220^{\circ} \mathrm{F}$. Dry heat penetrates very slowly into bulky and badly conducting articles, such as bedding. Parsons also pointed out that at or above $250^{\circ} \mathrm{F}$. "scorching" occurred, and above $212^{\circ} \mathrm{F}$. many kinds of stains were fixed in fabrics, so that they could not be removed by washing. He advocated that the standard of true disinfection should be the destruction of the most stable infective matter known.

Previously to this period, experiments had shown the efficacy of washing articles in boiling water, and Koch had shown the value of corrosive sublimate. He had also shown the inefficacy of dry heat, and of a number of chemical substances which it had been supposed were disinfectants.

In 1887 the Committee on Disinfectants of the American Public Health Association reported a number of findings, as the result of experiment, which crystallised known facts. For infectious material containing spores or sporulating bacilli they recommended burning, steam under pressure $105^{\circ} \mathrm{C}$. for ten minutes, boiling in water for thirty minutes, chloride of lime 4 per cent., and mercuric chloride 1-500. If such material did not contain spores, or sporulating bacilli, a 2 per cent. solution of chloride of lime sufficed, also mercuric chloride 1-2000, carbolic acid 5 per cent., chlorinated soda 10 per cent., and sulphur if 3 to 4 lbs. per 1000 cubic feet, and exposure not less than twelve hours. For excreta the Committee advised chloride of lime 4 per cent., for soiled underclothing, bed linen, etc., burning, boiling, or immersion for four hours in mercuric chloride (1-2000), or carbolic acid (2 per cent.). For washing furniture or hands the same-solution of carbolic acid; for disinfecting the bodies of the dead carbolic acid ( 5 per cent.), chloride of lime $(4$ per cent.), or mercuric chloride (1-500); and for washing surfaces in 
sick rooms and hospitals, 2 per cent. carbolic, or 1-1000 solution of mercuric chloride.*

More recently a number of experiments have been carried out in Europe and America as to the efficacy of certain chemical substances, and reference will be made subsequently to some of the results. Much of the evidence has been of a conflicting nature which is due, as we have said, to varying conditions, strengths of disinfectants, and resistance of organisms.

Two or three years ago several workers at Leipzig $\dagger$ drew up simple directions, the adoption of which would considerably assist in securing a common standard for disinfectant research. They were as follows:-

1. In all comparative observations it is imperative that molecularly equivalent quantities of the reagents should be employed.

2. The bacteria serving as test objects should have equal powers of resistance.

3. The number of bacteria used in comparative observation should be approximately equal.

4. The disinfecting solution should always be used at the same temperature in comparative experiments.

5. The bacteria should be brought into contact with the disinfectant with as little as possible of the nutrient material carried over. (This obviously will depend upon the object of the research.)

6. After having been exposed to the disinfectant for a fixed time, they should be freed from it as far as possible.

7. They should then be returned in equal numbers to the respective culture medium most favourable to the development of each, and kept at the same, preferably the optimum, temperature for their growth.

8. The number of surviving bacteria capable of giving rise to colonies in solid media should be estimated after the lapse of equal periods of time. +

\section{Means of Disinfection}

We may now mention shortly some of the commoner methods and substances adopted to secure efficient disinfection. They are all divisible, according to Buchanan's standard, into two groups:-

1. Heat in various forms;

2. Chemical bodies in various forms.

In practical disinfection it is necessary to inhibit or kill microorganisms without injury to, or destruction of, the substance harbour-

* Sternberg's Bacteriology, p. 201 et seq.

+ Zeitschr. f. Hy. und Inf. Krank., xxv.

¥ See also "Standardisation of Disinfectants," by Rideal and Walker, Jour. of Sanit. Inst., 1903. 
ing the germs for the time being. If this latter is of no moment, as in rags or carcases, cremation or burning is the simplest and most thorough treatment. But with mattresses and beddings, bedclothes and garments, as well as with the human body, it is obvious that as a rule something short of burning is required.

\section{Disinfection by Heat}

From the earliest days of bacteriology heat has held a prominent place as a means of disinfection. But it is only in comparatively recent times that it has been fully established that moist heat is the only really efficient form of heat disinfection. Boiling at atmospheric pressure $\left(100^{\circ} \mathrm{C}\right.$. or $212^{\circ} \mathrm{F}$.) is the oldest form of moist heat disinfection, and because of the simplicity of its application it has gained a large degree of popularity. But it must not be forgotten that mere boiling $\left(100^{\circ} \mathrm{C}\right.$.) may not effectually remove the spores of all bacilli, and obviously boiling is not applicable to furniture, mattresses, and similar objects. For such objects hot-air ovens were used in former days. But it was found that such dry heat disinfection $\left(150^{\circ} \mathrm{C}\right.$. for an hour) injured articles of clothing, etc., and yet left many organisms and spores untouched, as the degree of temperature was rarely, if ever, uniform throughout the substance being treated. The failures following in the track of these methods were an indication of the need for some form of moist heat, viz., steam.

When water is heated certain molecular changes take place, and at a certain temperature $\left(100^{\circ}\right.$ C., $212^{\circ} \mathrm{F}$.) the water becomes steam, or vapour, and on very little cooling, or on coming into contact with cooler bodies, will condense and give off its latent heat. But if the vapour is heated, it will become practically a gas, and will not condense until it has lost the whole of the heat, i.e. the heat of making water into vapour plus the heat of making vapour into gas. A gas proper is, then, the vapour of a liquid of which the boiling point is substantially below the actual temperature of the gas. But we know that the temperature at which it boils depends on the pressure to which it is subjected (Regnault's law). Hence in reality "steam at any temperature whatever may be a vapour proper, provided the pressure is such as prevents the liquid from boiling below that temperature." In such a condition of vapour it is termed saturated steam, or steam at or near its condensation point. Steam at any pressure is "saturated," when it is at the boiling-point of water for that pressure. But if it is at that same pressure further heated, it becomes practically a gas, and is called superheated steam, or steam heated above its natural condensation point. The former can condense without cooling; the latter cannot so condense at the 
same pressure. Saturated steam condenses immediately it meets the object to be disinfected, and gives out its latent heat; superheated steam acts by conduction, and not uniformly throughout the object. Its advantage is, that it dries moistened objects. It differs physically from saturated steam, because it does not condense (and give out its latent heat), until its temperature falls. Therefore, as a disinfecting power, superheated steam is much less than saturated steam, it has less heat in it, so to speak, and it has less penetrative power. One further term must be defined, namely, current steam. This is steam escaping from a disinfector as fast as it is admitted, and may be at atmospheric or higher pressures. The disinfecting temperature which is now commonly used as a standard is an exposure to saturated steam of $115^{\circ}$ C. for thirty minutes.

A number of different kinds of apparatus have been invented to facilitate disinfection at this standard on a large scale. All the larger Sanitary Authorities are now supplied with some form of steam disinfector, though many are not furnished with high-pressure disinfectors. Professor Delépine has pointed out that a current of steam at low pressure may disinfect completely.* Whilst such simple current-steam machines have thus been demonstrated as efficient bactericides, for practical purposes it is important to have disinfectors, $(a)$ capable of giving temperatures considerably above $100^{\circ} \mathrm{C}$., (b) of simple construction, $(c)$ having a constant steam power of uniform temperature and rapid penetration, and $(d)$ containing, when in action, a minimum of superheated steam. In addition to these characters of a first-rate steam disinfector, two other important points in actual management should be borne in mind, namely, the air must be completely ejected from the disinfection chamber before the results due to steam are obtained, and some sort of automatic indicator giving a record of each disinfection is indispensable.

The five chief types of steam disinfectors in common use are, the Washington Lyon, the Goddard, Massey, and Warner, the Equifex (Defries), the Thresh, and the Reck. $†$

Washington Lyon's apparatus consists of an elongated boiler having double walls, with a door at each end. The body of the apparatus is in a "jacket" for the purpose of preventing loss of heat and for "drying" disinfected articles after the process. The whole is large enough to admit of bedding and mattresses, and generally is so arranged that one end opens into one room, and the other end opens into another room. This convenient position admits of inserting infected articles from one room and receiving them disinfected into

* Jour. of State Med., December 1897, p. 561.

+ Full particulars of these various disinfectors may be obtained by communicating with the makers. Elaborate catalogues are now issued with illustrations and details of working. 
the other room. Possible reinfection is thereby removed. Steam is admitted into the jacket at a pressure of between 20 and 25 lbs., and its penetrating power may be increased by intermitting the pressure during the disinfection. At the end of the operation a partial vacuum is created, by which means much of the moisture on the articles may be removed. In some cases a current of warm air is admitted before disinfection in order to diminish the extent of condensation.

The Equifex (Defries) contains no steam jacket, but coils of pipes are placed at the top and bottom of the apparatus, with the object of imparting to the steam as much heat as is lost by radiation through the walls of the disinfecting chamber, and at the same time of preventing undue condensation, and to be available for drying. The air is first removed by a preliminary current of steam, after which steam at a pressure of $10 \mathrm{lbs}$. is intermittently introduced (for about five minutes), and allowed to escape. The object of this proceeding is to remove air from the pores of the articles to be disinfected by the sudden expansion of the film of water previously condensed on their surface.

The apparatus introduced by Thresh was constructed with a view of overcoming the objection to some of the other machines, that bulky articles retained a large percentage of moisture, thus necessitating the use of some additional drying apparatus. A central chamber receives the articles to be disinfected, and is surrounded by a boiler containing a solution of calcium chloride (carbonate of potash is now used) at a temperature of $225^{\circ} \mathrm{F}$. This is heated by a small furnace, and the steam given off is conducted into the central chamber. Owing to the dissolved potash the temperature of the steam given off when the solution boils is several degrees higher than ordinary steam. The steam is not confined under any pressure except that of the atmosphere. When the steam has passed for a sufficient length of time, it is readily diverted into the open air. Hot air is now introduced, and at the expiration of an hour the articles may be taken out disinfected and as dry as they were when inserted. The apparatus is comparatively inexpensive, and not of a complicated nature. The current steam is saturated, and at a temperature a few degrees above the boiling-point. The apparatus is now made in various forms, portable or otherwise.

There are many other forms of steam disinfector, including the apparatus by Goddard, Massey, and Warner, the disinfector of Delépine, and that of Reck and others, and each has its enthusiastic supporters. 


\section{Disinfection by Chemical Substances}

The effects of chemical. substances as solutions, or in spray or gaseous form, upon bacteria have been observed from the earliest days of bacteriology. To some decomposing matter or solution a disinfectant was added and sub-cultures made. If bacteria continued to develop, the disinfection had not been efficient; if, on the other hand, the sub-culture remained sterile, it was assumed that disinfection had been complete. From such rough-and-ready methods large deductions were drawn, and it is hardly too much to say that no branch of bacteriology contains such a mass of unassimilated and unassimilable statements as that relating to research into disinfectants. Most of the tabulated and recorded results. are conspicuous in having no standard as regards bacterial growth. Yet without such a standard results are not comparable.

Silk threads, impregnated with anthrax spores, were placed in bottles containing carbolic acid of various strengths, and at stated periods threads were removed and placed in nutrient media, and development or otherwise observed. But, as Professor Crookshank pointed out, this method is fallacious, the thread being still wet with the solution when transferred to the medium, and thus the culture was modified or even inhibited altogether.* It is unnecessary for us here to discuss every mode adopted by investigators in similar researches. We may, however, point out that the most approved methods at the present time are based more or less upon two simple modes of exposure. In one a known volume of recent broth culture of an organism grown under specified conditions is used, and to this is added a measured quantity of the antiseptic. At stated periods loopfuls of the broth and antiseptic mixture are sub-cultured in fresh-sterilised broth, and resulting development or otherwise closely observed. The other method is practised in dealing with volatile bodies. In such cases a standard culture is made of the organism in broth at a standard temperature. Into this are dipped small strips of sterilised linen. When thoroughly impregnated, these are removed from the broth and subsequently dried over sulphuric acid in a vacuum at $38^{\circ} \mathrm{C}$. These may now be exposed for a longer or shorter period to the fumes of the antiseptic in question, and broth cultures made at the end of the exposure. It is obvious that a very large number of modifications are possible of these two simple devices for testing the bactericidal power of chemical substances. It should be remembered that here, perhaps, more than anywhere else in bacteriological research, careful "control" experiments are absolutely necessary.

Recently, Ainslie Walker suggested various conditions of experi-

* Bacteriology and Infective Diseases, p. 35. 
mentation with a view to obtaining comparable results. First, he recommended the use of a well-known disinfectant giving regular and consistent results, such as pure phenol. Secondly, the source and age of the culture used is of importance. If the culture be in broth, Walker suggests the following procedure: to 5 c.c. of a twenty-four hours' blood-heat culture of the organism add 5 c.c. of the dilute disinfectant. Shake and take sub-cultures at definite intervals in suitable media. Incubate for at least two days at $37^{\circ} \mathrm{C}$. If an agar culture be preferred, take up part of the growth on the point of a platinum needle, and distribute it evenly in sterilised water. The resulting emulsion may be used in place of the broth culture. Thirdly, Walker emphasises the importance of working with the same organism for comparable results.*

A substance, to be a satisfactory disinfectant, should, according to Andrewes, possess five characters: $(a)$ it should be germicidal within a reasonable time-limit; $(b)$ it should not possess chemical properties which unfit it for ordinary use; $(c)$ it should be soluble in water, or capable of giving rise to soluble products in contact with the material to be disinfected; $(d)$ it should not produce injurious effects on the human tissues; and (e) it should not be too costly in proportion to its germicidal value. $\dagger$

Mineral acids (nitric, hydrochloric, sulphuric), especially concentrated, are all germicides, but owing to their corrosive action their application is limited.

A number of bodies, such as chloroform and iodoform, have been much advocated as antiseptics. The cost of the former and odour of the latter have, however, greatly militated against their general adoption.

Chloride of lime is a powerful disinfectant. Professor Sheridan Delépine and Dr Arthur Ransome have demonstrated its germicidal effect as a solution (1 per cent.) applied directly to the walls of rooms inhabited by tuberculous patients. $\neq$ Coates confirmed these results in houses in Manchester infected by consumptives. Chlorinated lime ought to be used which will yield not less than 33 per cent. of available chlorine. It may also be used in solid form for decomposing matter, excreta, etc.

Mercuric chloride (corrosive sublimate) has been an accepted germicide for some time. But the experiments of Behring, Crookshank, and others, have proved that the weaker solutions $(1-4000)$ cannot be relied upon. This is, in part, due to the fact that

* Practitioner, 1902, lxix., p. 523.

† Many useful hints and suggestions as to testing disinfectants, and on the whole process of disinfection will be found in Lessons in Disinfection and Sterilisation, by F. W. Andrewes, M.D., F.R.C.P., 1903, p. 81 et seq. ; see also Brit. Med. Jour., 1904, ii., p. 13.

¥ Brit. Med. Jour., 1895, vol. i., p. 353. 
it forms in albuminous liquids an albuminate of mercury which is inactive. Dilute solutions have the further disadvantage of being unstable. Various authorities recommend a solution of 1-500 as a germicide, and much weaker solutions are of course antiseptic. An ounce each of corrosive sublimate and hydrochloric acid in 3 gallons of water makes an efficient disinfectant.

Potassium permanganate is, of course, the chief substance in Condy's fluid, as zinc chloride is in Burnett's disinfecting fluid. A 5 per cent. of the former and a $2 \frac{1}{2}$ per cent. of the latter are germicidal. Solutions are used for street-cleansing.

Boracic acid is used as an antiseptic with which to wash sore eyes, or preserve tinned foods or milk. It is not a strong germicide (it inhibits rather than kills), but an unirritating and effective wash. Many cases of its addition to milk have found their way into the law courts owing to cumulative poisoning, and as a rule its use as a food perservative should be deprecated.

Carbolic acid has come much into prominence as an antiseptic since its adoption by Lister in antiseptic surgery. It is cheap, volatile, and effective. One part in 40 is antiseptic, and 1 in 20 germicidal. As a wash for the hands the former is used, and a weaker solution for the body generally. Carbolic soap and similar toilette combinations are now very common. At one time it appeared as if corrosive sublimate would take the place of carbolic acid as an antiseptic solution, but a large number of experiments have confirmed opinion in favour of carbolic. Crookshank found that carbolic acid, 1 in 40, acting for only one minute, was sufficient to destroy Streptococcus pyogenes, S. erysipelatis, and Staphylococcus pyogenes aureus, and in the strength of 1 in 20 carbolic acid completely sterilised tubercular sputum when shaken up with it for one minute. Klein, Houston, and Gordon, and other workers have found a 5 per cent. solution of carbolic to be a reliable disinfectant for almost all bacteria.

Cresol, a member of the phenol series, is a good disinfectant and the active element in lysol, Jeye's fluid, creolin, izal, and other similar substances, which have been recently introduced and have proved efficacious as disinfectants.

Sulphurous acid is one of the commonest disinfectants employed for fumigation - the old orthodox method of disinfecting a room in which a case of infective disease had been nursed. It is evolved, of course, by burning sulphur. For each thousand cubic feet from 1 to 5 lbs. of sulphur is used, and the walls may be washed with carbolic acid. Dr Kenwood carried out some experiments in 1896 which appeared to support a belief in the disinfecting power of sulphur fumes.* But he has since advocated formaldehyde as preferable. $\mathrm{He}$ found that the B. diphtherioe was not killed by sulphur though

Brit. Med. Jour., 1896 (August), p. 439. 
markedly inhibited, when the sulphurous gas $\left(\mathrm{SO}_{2}\right)$ did not much exceed 25 per cent. But the bacillus was killed where the sulphur fumes exceeded 5 per cent. Both these results had reference to the $\mathrm{SO}_{2}$ in the air in the centre of the room at a height of 4 feet, and after the lapse of four hours. There can be little doubt that thoroughly fuming a sealed-up room with sulphur in a moist atmosphere, and leaving it thus for twenty-fours, is generally if not always, efficient disinfection. Moreover, its simplicity of adoption is greatly in its favour. Anyone can readily apply it by purchasing a few pounds weight of ordinary roll sulphur and burning this in a saucer in the middle of a room which has had all its crevices and cracks in windows and walls blocked up with pasted paper.* But it is almost useless as a gaseous disinfectant unless used in a particular way. The following seem to be the only lines upon which anything like adequate disinfection can be secured by means of sulphur:-

1. The room to be disinfected must be effectually sealed up.

2. Not less than $3 \mathrm{lbs}$. of sulphur should be used for every 1000 cubic feet.

3. Twenty-four hours should elapse between the time of lighting the sulphur and the unsealing of the room.

4. The air in the room should be damp during the process, and this may be achieved by steam, or spraying the walls with water, or suspending wet blankets. By this means sulphurous acid is formed, which is the essential part of the process.

5. At the end of the twenty-four hours the doors and windows should be kept wide open for at least one, and if possible for two, days.

6. Furniture and fixtures should, as far as possible, be wiped down with a damp cloth soaked in carbolic or some other disinfectant solution. Dry dusting or sweeping should be strongly deprecated. The walls may be stripped in cases where they are very dirty or where there has been a recurrence of a disease. Sulphur fumigation is not sufficient in disinfection after consumption.

The conclusions of Dr Novy respecting the efficacy of sulphur fumes as a disinfectant may be added. He urges that "sulphur fumes possessed little or no action on most bacteria when in a dried state. If, however, the specimens are actually wet, they will be destroyed except in the state of the resistant forms, such as spore stage and tubercle bacilli. For tubercle bacilli or sporecontaining material, wet or dry, it is of no value. It can be used for the disinfection of rooms which have been infected with ordinary disease organisms. From 3 to $6 \mathrm{lbs}$. of sulphur must be burned in each 1000 cubic feet of space. The walls, floors, and articles should be sprayed with water. The room should be made

* See also Public Health, 1900, p. 438 et seq. 
perfectly tight, and should be kept closed at least twenty hours." * Calmette states that sulphur vapour under pressure may be relied upon for the disinfection of ships, ete.

Recently, formalin has come much into favour as a room disinfectant. Formalin is a 40 per cent. solution in water of formaldehyde, a gas discovered by Hofmann in 1869. This gas is a product of imperfect oxidation of methyl alcohol, and may be obtained by passing vapour of methyl alcohol, mixed with air, over a glowing platinum wire or other heated metals, such as copper and silver. Its formula is $\mathrm{CH}_{2} \mathrm{O}$, and it is a colourless gas with a pungent odour, and having penetrating and irritating properties particularly affecting the nasal mucous membrane and the eyes of those working with it. It is readily soluble in water, and in the air oxidises into formic acid $\left(\mathrm{CH}_{2} \mathrm{O}_{2}\right)$. This latter substance occurs in the stings of bees, wasps, nettles, and various poisonous animal secretions. Formalin is a strong bactericide even in dilute solutions, and, of course, volatile. Its use should be restricted to disinfection of articles injured by heat (furs, etc). A solution of 1-10,000 is said to be able to destroy the bacilli of typhoid, cholera, and anthrax. A teaspoonful to 10 gallons of milk is said to retard souring. When formalin is evaporated down, a white residue is left known as paraform. In lozenge form this latter body is used by combustion of methylated spirit to produce the gas. Hence we have three common forms of the same thing-formalin, formic aldehyde, paraform-each of which yields formic acid, and thus disinfects. The vapour cannot in practice be generated from the formalin as readily as from the paraform.

By a variety of ingenious arrangements, formic aldehyde has been used by a large number of observers during the last two or three years. We may refer to four modes of application: 1. The sprayer produces a mixture of air and solution for spraying walls, ceilings, floors, and sometimes garments. There are a number of different forms of spraying apparatus such as the Equifex, the Mackenzie, the Robertson, etc. 2. The autoclave (Trillat's apparatus). In this apparatus a mixture of a 30-40 per cent. watery solution of formaldehyde and calcium chloride (4-5 per cent.) is heated under a pressure of three or four atmospheres, and the almost pure, dry gas is conducted through a tube passing through the keyhole of the door into the sealed-up room. 3. The paraform lamp (the Alformant). The principle of this lamp is that the hot, moist products from the combustion of methylated spirit act upon the paraform tablets, converting them into gas. 4. Lingner's apparatus consists of a ring boiler in which steam is generated and driven into a reservoir filled

* Tenth Report of State of Maine Board of Health, 1898, p. 365. This report contains a digest on the whole subject of disinfection. 
with formalin or glyco-formal (30 per cent. formalin with 10 per cent. glycerine), which is thus vaporised and ejected in the form of a fine spray through four nozzles. A room is thereby speedily filled with a dense formalin vapour. After four hours exposure, Houston and the writer found that B. pyocyaneus, Staphylococcus pyogenes aureus, and various saprophytic organisms were killed.* Klein and the writer found that $B$. anthracis and the tubercle bacillus were killed by the same means. Rideal claims that the resistant spores of anthrax may be killed when 7.5 c.c. of formalin per cubic metre (85 grammes of formaldehyde per 1000 cubic feet) are vaporised with not less than four times its volume of water, and that exposure need not exceed six hours. $†$ Klein, Houston, and Gordon found that B. typhosus, B. diphtherioe, and certain suppurative organisms were killed by means of the alformant lamp method. + It is agreed that the gas is harmless to colours, metals, leather, and polished wood. The vapour acts best in a warm atmosphere. As for its action on bacteria, it may be said that it compares favourably with any other disinfectant.

Many observers have not recommended formaldehyde on account of its professed lack of penetrating power. Professor Delépine, however, states that it possesses "penetration powers probably greater than those of most other active gaseous disinfectants. $B$. coli, B. tuberculosis, B. pyocyaneus, and Staphylococcus pyogenes aureus were killed in dry or moist state, even when protected by three layers of filter-paper." § In Professor Delépine's opinion, the vapours of phenol, izal, dry chlorine, and sulphurous acid have, under the same conditions, given inferior results. Since 1898 a number of experimenters have confirmed these opinions. It is extremely important that that disinfectant should be used which is the most suitable one for the particular purpose at issue. A germicidal substance which under certain conditions, and in relation to one species of organism may be practically useless, may under other conditions be most efficacious.

\section{Practical Disinfection $\|$}

To disinfect a room, seal up cracks and crevices, spray the walls with water, and burn, say, 3-6 lbs. of roll sulphur for every

* Practitioner, 1902, vol. Ixix., p. 328.

+ Jour. of Sanitary Institute, 1903, vol. xxiii., part iv.

$¥$ Report of Medical Officer of London County Council, 1902.

$\S$ Jour. of State Med., 1898 (November), p. 541.

\| For hints in the detail management of disinfection, the reader is recommended to study $A$ Practical Guide to Disinfection, by Rosenau and Allan, 1903; Lessons in Disinfection, by F. W. Andrewes, 1903; the Practitioner, 1902, p. 300 (Houston); Rideal's Disinfection and Disinfectants, 1904; and Public Health, 1901, pp. 558-570. 
1000 cubic feet of space.* Let the room remain sealed up for twenty-four hours, then be freely opened. Formaldehyde gaseous disinfection may be used as described above. But it would appear that neither sulphur or formaldehyde are always reliable in disinfecting after tuberculosis. The most important point is to cleanse surfaces, and probably the most efficient disinfection of a room is by Lingner's apparatus (glyco-formal, 1 litre to every 1000 cubic feet), coupled with spraying or washing surfaces with germicidal solution.

To disinfect walls, floors, etc., wash or spray with chloride of lime solution (1-100), izal (1-100), formalin (2-100), or carbolic acid (1-40). The last-named solution may be used to wipe down furniture. These disinfectants may be used after sulphur fuming. Formic aldehyde may also be used by autoclave or Lingner's apparatus.

To disinfect bcdding, etc., the steam sterilisation secured in an efficient apparatus is the best $\left(115^{\circ} \mathrm{C}\right.$. for thirty minutes). Rags and infected elothing, unless valuable, should be burnt.

To disinfect garments and wearing apparel.-If possible, steam in an efficient steriliser; if that be not available, such articles should be washed in a disinfectant solution ( 5 per cent. carbolic), or fumed with formic aldehyde (Lingner's glyco-formal apparatus).

To disinfect excreta or putrefying solutions, enough disinfectant should be added to produce in the solution or matter being disinfected the percentage of disinfectant necessary to act as such. Adding a small quantity of antiseptic to a large volume of fluid or solid is as useless as pouring a small quantity of antiseptic down a sewer with the idea that such treatment will disinfect the sewage. The mixture of the disinfectant with the matter to be disinfected must contain the standard percentage for disinfection. Chloride of lime is a common substance for use in this way $\left(\frac{1}{4} \mathrm{lb}\right.$. to a gallon of water) or in a 4 per cent. solution. Potassium permanganate (1-100), and carbolic (5 per cent.), and many manufactured bodies containing them, are also widely used. Corrosive sublimate (1-500), izal (1-100), copper sulphate (1-20), lysol, cresol, or creolin (1-40), have all been found efficacious (Houston). Drs Hill and Abram recommend that the excreta and disinfectant be thoroughly mixed, and stand for at least half an hour. $\dagger$ For various reasons they particularly advise chinosol as the most convenient disinfectant for this specific purpose. But subsequent experience has perhaps hardly supported this recommendation.

Antiseptics for wounds.-Carbolic acid (1-40) or corrosive sublimate $(1-1000)$ are commonly used in surgical practice. Boracic

* The measurement of cubic space is, of course, made by multiplying together in feet the length, breadth, and height of a room.

† Brit. Med. Jour., 1898 (April), p. 1013. 
acid is one of the most unirritating antiseptics which is known. It may be used in saturated watery solution (1-30) or dusted on copiously as fine powder. It is especially applicable to open wounds, and as an eye-wash.

Boots, books, leather-covered articles, etc., should be disinfected by dry heat or formalin (preferably Lingner's apparatus).

Infected linen should be steamed or boiled, but if that is not available, immersion for one hour in corrosive sublimate (1-500) or for twenty-four hours in the same solution 1-1000. Less powerful germicides have, however, been found successful, e.g. izal (1-100), carbolic acid (1-100).

Cups, saucers, plates, spoons, knives, forks, etc., should all be disinfected in boiling water.

Rags in bales can only be disinfected by steam.

Pens, byres, stables, trucks, vans, markets, etc., are best treated with some form of sprayer (e.g. Equifex hot-spray disinfector) or distributor (e.g. the chloros distributor). Ships also may be treated by this apparatus or by means of the Newcastle disinfecting hulk (Goddard, Massey, and Warner).

Disinfection of the Hands.-To a surgeon, the disinfection of the hands is a matter of vital importance. There are many opportunities for conveying bacteria on the hands, which naturally come in the way of dust and dirt, and so carry organisms in the cracks of the skin surface, in the sebaceous glands, under the nails, and even in the substance of the epithelium. This was demonstrated by Lockwood in 1896, and again by Freeman in 1899. Subsequent experiments confirmed the fact of the difficulty of completely freeing the skin of the hands from micro-organisms. In 1902, Dr Schaeffer of Berlin, whilst recognising that absolute asepticism of the hands is not possible, showed by experiments that it is possible to render the hands so free from organisms during a surgical operation that the danger of wound contamination is exceedingly small. Collins has pointed out (1904) that much depends upon vigorous scrubbing, clean nail-brushes, and hot water. Soap, water, and carbolic acid (1-20), permanganate of potash, corrosive sublimate (1-1000), lysol, and many other similar antiseptic solutions have beell used with more or less satisfactory results. Schaeffer, however, decides in favour of the hot-water-alcohol method (96 per cent. spirit), the chief advantage of which is that it removes organisms from the skin rather than killing them on the skin. Mikulicz advocates spirit-soap as cheaper than alcohol, but apparently the difference in expense in this country is not great, and the spiritsoap leaves the hands in a slippery condition. It may be pointed out that washing in spirit rather than antiseptics preserves the smooth surface of the skin and results in no roughness. 


\section{Disinfection in or after Special Diseases}

Disinfection after Phthisis.-The following statement was drawn up in 1901, by Drs Newsholme, Niven, and the writer, for the National Association for the Prevention of Consumption and other forms of Tuberculosis. It may serve as a basis for practical disinfection of rooms, etc., after phthisis :-

"The necessity for disinfection in consumption is based on wellestablished facts. The essential cause of consumption and of all other forms of tuberculosis is a living microbe, the B. tuberculosis, though the condition of the bodily health of the individual greatly influences the resistance to the disease and the prospect of recovery from it.

"The disease is always contracted by taking into the system the microbes causing it, which are derived solely from persons or animals suffering from the same disease. These microbes may be taken in infected milk or less commonly in infected flesh.

"The most frequent source of infection, however, is the discharges and particularly the phlegm (spit or expectoration) of a consumptive person. These discharges whilst moist are not likely to be scattered, but if allowed to dry they become broken up into dust, and are then extremely dangerous. There is little or no risk of contracting consumption directly from the breath of a consumptive person, but the phlegm infects everything upon which it fallshandkerchiefs, books, papers, linen, floors, carpets, furniture, etc., and is then readily inhaled by healthy persons. This is the chief means by which consumption is spread from person to person.

"On these facts rest the important question of disinfection. In preventing a consumptive person from spreading the disease, two sets of preventive measures are required:-1st, the removal or destruction of the infective matter already disseminated by the patient's discharges, especially by his phlegm; and, 2nd, the prevention of future dissemination. For the latter purpose the main object is not to permit any discharge to become dry before being destroyed. Before the consumptive person has learned the personal precautions which must be taken, and up to the time when he has been trained to carry them out carefully, he has probably distributed a considerable amount of infective matter. This is especially liable to accumulate in a dangerous form at home, where the space is small, and light and ventilation are defective. Infective particles will be found in greatest abundance on and uear the floors, on ledges, and in room-hangings. But the personal clothing and bedclothes will also have become infected. Hence it is necessary to disinfect the floor, walls, and ceiling of the rooms occupied by the patient, as well as the furniture, carpet, bedclothes, etc. 
"When this has been done, if the personal precautions advised are carried out by the consumptive, further disinfection should not be needed.

"It is, however, difficult to make sure that personal precautions are fully carried out, and rooms should therefore be subsequently cleaned at least once in six months, the floors being scrubbed with soft soap, the furniture washed, the walls cleaned down with dough, and the ceiling whitewashed.

"Confined workshops in which a consumptive has worked for some time should be cleansed, and a notice in reference to spitting should be suspended in all workshops. The latter precaution should also be observed in all public-houses and common lodging-houses, both of which require special attention to cleansing.

"Disinfection of rooms which have been occupied by consumptive patients may be secured in various ways, but the following are the practical rules which must underlie any methods adopted:-

"1. Gaseous disinfection of rooms, or 'fumigation' as it is termed, by whatever method it is practised, is inefficient in such cases.

" 2. In order to remove and destroy the dried infective discharges, the disinfectant must be applied directly to the infected surfaces of the room.

"3. The disinfectant may be applied by washing, brushing, or spraying.

"4. Amongst other chemical solutions used for this purpose, a solution of chloride of lime ( 1 to 2 per cent.) has proved satisfactory and efficient.

" 5 . In view of the well-established fact that it is the dust from dried discharges which is chiefly infective, emphasis must be laid upon the importance of thorough and wet cleansing of infected rooms.

"6. Bedding, carpets, curtains, wearing apparel, and all similar articles belonging to or used by the patient, which cannot be thoroughly washed, should be disinfected in an efficient steam disinfector.

"7. After all necessary measures of disinfection have been carried out, the essential principle governing the subsequent control of a case of consumption is that all discharges, of whatever kind (especicially expectoration from the lungs), should under no circumstances be allowed to become dry."

In Manchester and other places, where disinfection after phthisis is regularly practised, a solution of chlorinated lime of the strength of $1 \frac{1}{2}$ ounces to the gallon is used. The wall-paper is thoroughly saturated with this solution, applied with a soft brush or spray, 
and is then, where necessary, stripped from the walls. The bare walls, the ceiling, and floor are washed over several times with the solution, and any articles of furniture which will admit of such treatment are similarly washed over. Articles of clothing, bedding, etc., are taken away to be disinfected in the steam disinfector.

In houses in Manchester which are in a clean condition, and where it is certain that there has been no direct soiling of the walls or floors with sputum, and where the infectious dust, if present, has come from soiled pocket-handkerchiefs or articles of clothing, the chlorinated lime method of disinfection is not considered necessary, and the method of disinfection recommended by Esmarch is practised:- The wall-paper is rubbed well with crumb of bread, or with dough kneaded to a proper consistency. Floors, painted walls, and woodwork are washed with soap and water, and ceilings are limewashed. In addition, bedding, articles of clothing, etc., are either disinfected by steam or washed with boiling water.

This method of disinfection, when properly carried out, was found to remove practically all dust from a room, so that little or no dust can be obtained by subsequently rubbing the wall-paper with a sterilised sponge. The method, however, requires a certain amount of care to make sure that all dust is removed from the walls, especially from the angles and corners, and to properly rub down a fair-sized room takes a considerable time. It is useless in cases where the paper is directly soiled with sputum. Owing to the mucus which it contains, the dried sputum sticks tenaciously to the paper, in spite of repeated rubbing with dough.

This method of rubbing the walls with dough is an excellent way of periodically cleaning a room, so as to keep it free from dust.

After Small-Pox.-It is necessary that disinfection be very thoroughly done. As a rule, the walls of the room used by the patient must be "stripped and cleansed." Fumigation with formic aldehyde and vigorous spraying of walls are usual. All bedding and wearing apparel must be steamed, and if very unclean, burnt.

After Scarlet Fever.-The room used by the patient should be disinfected in the ordinary way. Infection may be conveyed by clothing, carpets, table-cloths, bell-ropes, etc., and such things must receive attention. Infection is also probably conveyed by the peeling skin, and even more so by the throat secretions. All discharges from the mouth and nose, and also those from the ear when affected, should be received on rags or thin paper handkerchiefs and burned. The seat of infection may also be directly attacked by the use of disinfectant gargles, of which chlorine water is one of the best. During desquamation the skin may be oiled, and occasionally washed in warm carbolic solution (1-40). 
After Diphtheria.-The bacillus of diphtheria is non-sporulating, and has comparatively little resistance against disinfectants. Ordinary means of disinfection are therefore sufficient. Local disinfectants should be used for the throat until bacteriological examination is negative to the Klebs-Löffler bacillus, which may persist in the throat for long periods. The throat may be painted with a solution of perchloride of mercury (1-500); 15 to 20 minims of such a solution would be a suitable amount to use for a single application. Gargles or sprays may be employed, consisting of chlorine water, or permanganate of potash (1-300). The throat and nose discharges should be received on rags which can be burned.

After Typhoid Fever and Cholera.-Bedding and articles which have come into contact with the patient require attention in typhoid fever and cholera. The disinfection of the excreta (fæces and urine) is the most important item. These discharges should not be passed into the house drains until disinfected. They should stand for some hours thoroughly mixed with the disinfectant before being considered disinfected. Chloride of lime (1-500 of the total mixture), izal (1-200 of the total mixture) and carbolic acid (1-40 of the total mixture) are all used in this way. If there is no house-drainage or water-carriage system, the excreta should be treated as above, and deeply buried remote from any well or water-course. The nurse's hands must be kept thoroughly cleansed (thorough washing with hot water, soap, and perchloride solution, 1-1000), especially before meals.

After Plague.-The detailed arrangements for the removal of cases and disinfection of infected tenements after plague should be under the personal supervision of the medical staff, and may be detailed as follows:

(a) Removal of patient to hospital.

(b) Removal of "contacts" to reception house, and kept under medical observation for fourteen days.

(c) Fumigation of infected house by liquefied sulphur dioxide or formic aldehyde from twelve to twenty-four hours, the disinfectant being used in proportion to the cubic space dealt with.

(d) After the fumigation the house is entered; all articles of clothing, etc., to be removed are first of all thoroughly wetted with 2 per cent. solution of formalin (1 gallon 40-per-cent. solution formaldehyde to 50 gallons water), or 2 per cent. chloride of lime, then wrapped up in sheets soaked in the same fluid and removed to the sanitary wash-house. There all articles which cannot be boiled or steamed, or treated with formaldehyde, are burned.

(e) The walls, ceiling, flooring, woodwork, etc., and furniture of the infected house are also sprayed with the formalin solution (1 gallon to 50 gallons water) or chloride of lime. 
(f) All rooms in the infected dwelling are cleansed; the lobbies, stairs, and landings being dealt with by formaldehyde or chloride of lime solution.

(g) Courts of such dwellings are watered with chloride of lime solution.

(h) Ash-pits have contents watered with same, and then removed and burned. 



\section{APPENDIX}

\section{NOTES ON TECHNIQUE}

Synopsis of Technique:-General Methods of Examination; Staining Methods; Flagella; Spores, etc.-Bacteriological Diagnosis-Examination of WaterExamination of Milk - Bacteriological Diagnosis in Special Diseases Examination of Malaria Blood-Examination of Oysters-Examination of Sewage-Miscellaneous.

\section{General Elementary Methods of Examination}

W гтн the exception of pathological tissue and similar insoluble substances, the common practice in bacteriology is to reduce as far as possible the article to be examined to a fluid, that is to say, it is chiefly fluids which can be systematically examined by the methods of bacteriology. Water, milk, sewage, urine, blood, etc., are at once in a condition to make examination available, but cheese, butter, foods, soil, pus, dust, etc., require to be reduced to fluid, or washed in fluid media, preparatory to examination. Thus soil particles may be washed and macerated in sterilised broth, and the broth examined for contained organisms. It will, on this account, be most convenient in the first place to consider the application of bacteriological methods to the examination of fluids.

The principle underlying the ordinary technique is the solidification of fluid gelatine at or below room temperature. If a drop of contaminated water, for example, be added to a tube of 10 c.c. of liquid gelatine, thoroughly mixed, and then the contents of the tube poured out into a Petri plate (or other shallow glass dish) and allowed to solidify, we shall have scattered through

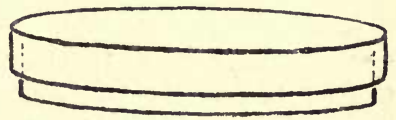

FIG. 38.-Petri Dish. the solid film of gelatine the contained bacteria, in a favourable medium for their growth and multiplication. Such a plate will be protected from the air and incubated at a regulated temperature. This is the principle of Koch's Plate Method. In the course of two or three days the film of gelatine on the plate becomes covered with colonies of germs, consisting of countless individual bacteria gathered round the 453 
parent organism which found its way thither from the drop of contaminated water. The next step is to examine these quantitatively and qualitatively.

1. Naked-Eye Observation of the Colonies.-By this means, at the very outset certain facts may be obtained, viz., the size, elevation, configuration, margin, colour, grouping, number, and kinds of colonies, all of which facts are of importance, and assist in final determination as to the quantity and character of the organisms present in the original drop of

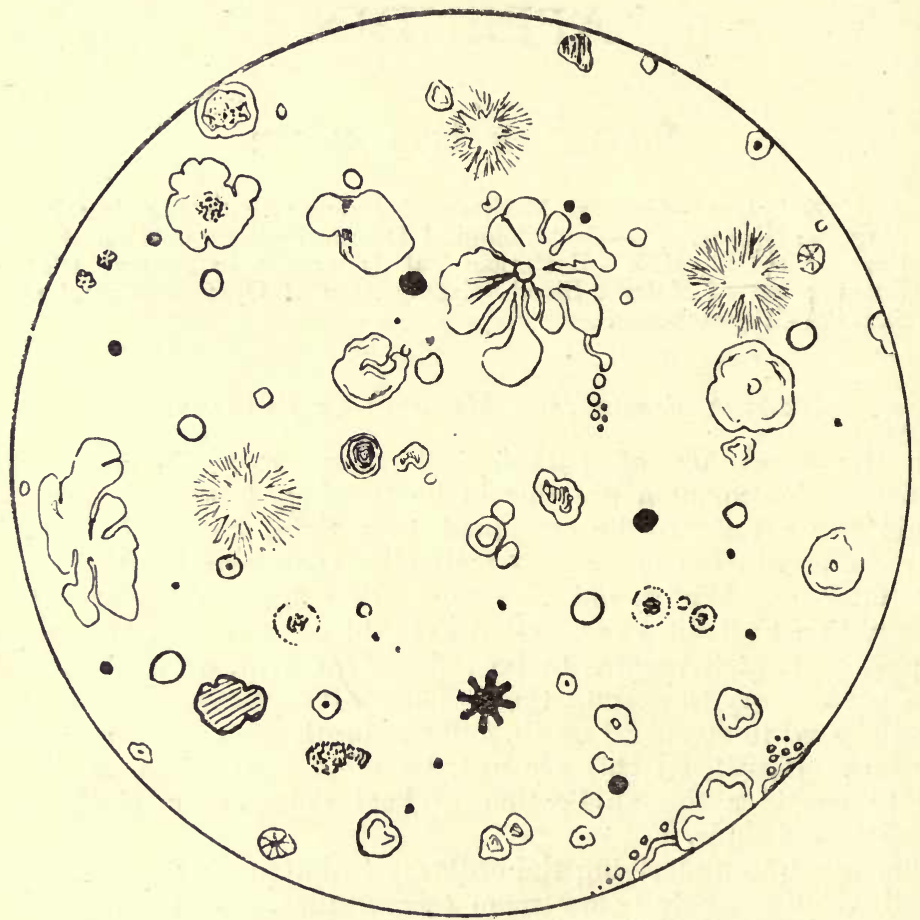

Fra. 39.-A Diagram of Colonies of Bacteria on a Gelatine Plate.

water. Moreover, in the case of gelatine medium (owing to the fact that it is liquefiable by ferments), one is able to observe whether or not there is present what is termed liquefaction of the gelatine. Some organisms produce in their development a peptonising ferment which breaks down gelatine into a fluid condition. Many have not this power, and hence the characteristic is used as a diagnostic feature.

2. The Microscopic Examination of Colonies (under low magnification, $\times 60-100$ ) confirms or corrects that which has been observed by the naked eye. Micro-organisms, when growing in colonies, produce cultivation features which are peculiar to themselves (especially is this so when 
growing in test-tube cultures), and in the early stages of such growths a low power of the microscope or a magnifying glass facilitates observation.

3. The Making of Cover-glass Preparations : (a) unstained-" the hanging drop"; (b) stained-single stains, e.g., gentian-violet, methyleneblue, fuchsin, carbol-fuchsin, etc.; or double stains by Gram's method, by Ziehl-Neelsen's method, etc. 'This third part of the investigation is obviously to prepare specimens for examination under the microscope.* "The hanging drop" is a simple plan for securing the organisms for microscopic examination in a more or less natural condition. A hollow ground slide (i.e. a slide with a shallow depression in it) is taken, and a small ring of vaseline placed round the edge of the depression. Upon the under-side of a clean cover-glass is placed a drop of dis-

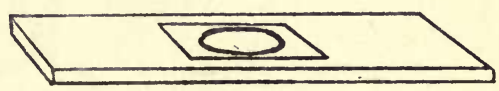

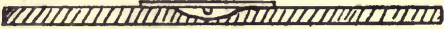

Fig. 40.-The Hanging Drop.

tilled water, and this is inoculated with the smallest possible particle taken from one of the colonies of the gelatine plate on the end of a sterilised platinum wire. The cover-glass is then placed upon the ring of vaseline, and the drop hangs into the space of the depression. Thus is obtained a view of the organisms in a freely moving condition, if they happen to be motile bacteria. In ordinary practice the hollow slide may be dispensed with, and an ordinary slide used.

With regard to staining, it will be undesirable here to dwell at length upon the large number of methods which have been adopted. The

A good microscope is essential. It should have objectives of 1 inch, 1 , and $\frac{1}{12}$ (oil immersion). A white light, and proper adjustment of the substage condenser and draw-tube are also necessary. A lens of ${ }_{12}^{1}$ th inch focal depth is the usual power required for the study of bacteria, although in some cases a lens of a focal length of $\frac{1}{18}$ th inch, or even stronger, is desirable. Streptothrix actinomyces, which belongs to the higher bacteria, is better seen with a power of $\frac{1}{6}$ th inch than with one of $\frac{1}{12}$ th inch. The principle of the immersion lens is the filling up of the space between the lens and the cover-glass with a material whose refractive index is the same as that of the lens, so that there will be no loss of illumination by the rays of light passing through media of different powers of refraction, while proceeding from the object to the lens. The power of a microscope varies not only according to that of the lens, but also according to the power of the eye-piece. Thus the magnifying power of a 1-inch objective in Swift's microscope varies, according to the strength of the eye-piece and to the fact that the draw-tube is closed or extended, from 25 to 140 diameters; a $\frac{1}{6}$ th inch objective, from 175 to 690 diameters; and a $\frac{1}{12}$ th inch objective, from 385 to 1627 diameters. As a high-power lens gives a picture which has comparatively very little "depth" of focus, it is necessary to place the object under examination in as nearly the same plane as possible. Hence the material to be investigated should be reduced to an extremely thin film.

The object should also be in the optical axis of the instrument, and secured in position by means of the spring clips. In using the oil immersion lens, the body tube of the microscope must be screwed down until the lens is in contact with the oil and nearly touching the coverslip. The substage condenser must be screwed up flush with the stage. The best light must be obtained by adjustment of the mirror, and fine focus must be used. A skilful use of the microscope depends, of course, upon an understanding of its parts and upon practice. 
"single stain" may be shortly mentioned. It is as follows. A clean cover-glass or slide is taken (cleaned with nitric acid and alcohol, or bichromate of potash and alcohol), and a drop of distilled water placed upon it. This is inoculated with a particle of a colony on the end of a platinum needle, and a scum is produced. The film is now "fixed" by slowly drying it over a flame. When it is thus dried, a drop of the

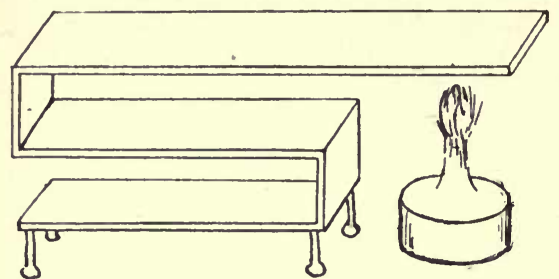

FIG. 41.-Drying Stage for Fixing Films. selected stain (e.g. gentianviolet) is placed over the film and allowed to remain for a few seconds. It is then washed off with clean water, and the specimen dried, and mounted in Canada balsam. The organisms will now appear under the microscope as violet in colour, and will thus be more clearly seen than when unstained.

"Double staining" is adopted when it is necessary to stain the organisms one colour and the tissue in which they are situated a contrast colour. The chief methods will be mentioned subsequently.

4. Sub-culture of Colonies. - The plate method was introduced by Koch in order to facilitate isolation of species. In a flask it is impossible to isolate individual species, but when the growth is spread over a comparatively large area, such as a plate, it is possible to obtain separate detached colonies, and this being done, the colonies may be replanted, by means of a platinum wire, in fresh media; that is to say, a sub-culture may be made, each organism cultivated on its favourable medium and its manner of life closely watched. For example, a water may contain six species of bacteria. On the plate these six species would reveal themselves by their own peculiar growth. Each would then be isolated and placed in a separate tube, on a favourable medium, and at a suitable temperature. Thus each would be a pure culture; i.e., one, and only one, species would be present in each of the six tubes. By this simple means an organism can be isolated and cultivated in the same sort of way as in floriculture. From day to day the habits of each of these six species may be observed, and probably at an early stage of their separate existences it would be possible to determine to what species they belonged. If not, further microscopic examination could be made, and, if necessary, secondary or tertiary sub-cultures.

5. Inoculation of Animals. - It may be necessary to observe the action of supposed pathogenic organisms upon animals. There is no means of testing the pathogenic power of an organism, except by learning by experiment, whether or not it produces disease. As a matter of fact, an immense amount of bacteriological investigation can be carried on without inoculating animals; but, strictly speaking, as regards many of the pathogenic bacteria, this test is the only reliable one. Nor would any responsible bacteriologist be justified in certifying a water as healthy for consumption by a large community if he were in 


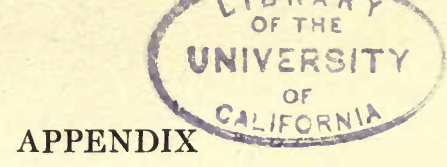

doubt as to the disease-producing action of any of the contained organisms.

By working through some such scheme as the above, it is possible to detect what quantity and species of organisms, saprophytic or parasitic, a water or similar fluid contains. For, observe what information has been gained by following out these five steps in procedure. We have learned the form (whether bacillus, micrococcus, or spirillum), size, consistence, motility, method of grouping, and staining reactions of each micro-organism; the characters of its culture, colour, composition, presence or absence of liquefaction or gas formation, its rate of growth, odour, or reaction; and, lastly, its effect upon living tissues. Here, then,
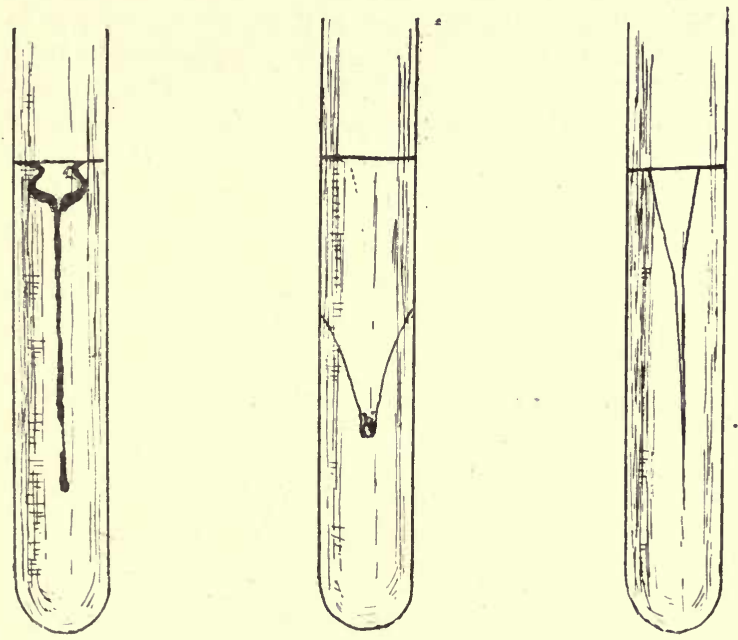

Fia. 42.-Types of Liquefaction of Gelatine.

are ample data for arriving at a satisfactory conclusion respecting the qualitative estimation of the drop of water under examination.

As to the quantitative examination, that is fulfilled by counting the number of colonies which appear, say by the third or fourth day, upon the gelatine plates. Each colony has arisen, it is assumed, from one individual, so that if the colonies be counted, though we do not thereby know how many organisms there are upon the plate, it is known approximately how many organisms there were when the plate was first poured out, which are the figures we require, and which can at once be multiplied up and returned as so many organisms per drop, or if the quantity of water were measured, per c.c.

When counting colonies in a Petri's dish, it is sufficient to divide the circle into eight equal divisions, and counting the colonies in the average divisions, multiply up and reduce to the common denominator of 1 c.c. (or a Pakes' or Jeffer's Counting Disc may be used). For example, 
if the colonies of the plate appear to be distributed uniformly, we count those in one of the divisions. They reach, we will suppose, the figure of $60 ; 60 \times 8=480$ micro-organisms in the amount taken from the suspected water and added to the melted gelatine from which the plate was made. Let us suppose this amount was $\cdot 25$ c.c. Then the number of microorganisms in the suspected water is $60 \times 8=480 \times 4=1920$ m.o. per c.c.

Double Staining Methods.-These are various, and are used when it is desired to stain the bacteria one colour, and the matrix or ground substance in which they are situated another colour. Two of the common methods are those of Ziehl-Neelsen and Gram. They are as follows :-

Gram's Method.-The primary stain in this method is a solution of aniline gentian-violet (saturated alcoholic solution of gentian-violet 30 c.c., aniline water 100 c.c.), or Nicolle's carbol-gentian-violet, which stains both ground substance and bacteria in purple. The preparation is next immersed in the following solution for thirty or forty seconds :-

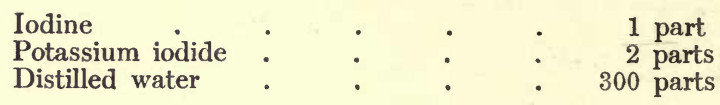

In this short space of time the iodine solution acts as a mordant by chemical combination, fixing the purple colour in the bacteria, but not in the ground substance. Hence, if the preparation be now (when it has assumed a brovn colour) washed in alcohol (methylated spirit), the ground substance slowly loses its colour and becomes clear. But the bacteria retain their colour, and thus stand out in a well-defined manner. Cover-glass preparations decolorise more quickly than sections of hardened tissue, and they should only be left in the methylated spirit until no more colour comes away. The preparation may now be washed in water, dried, and mounted for microscopic examination, or it may be double-stained, that is, immersed in a contrast stain which will lightly colour the ground substance. Eosin or Bismarck brown are commonly used for this purpose. The former is applied for a minute or two, the latter for five minutes, after which the specimen is passed through methylated spirit (and preferably xylol also) and mounted. The result is that the bacteria appear in a dark purple colour on a background of faint pink or brown. Carbol thionine blue, picro-carmine, and other stains, are occasionally used in place of the aniline gentian-violet, and there are other slight modifications of the method. The application of the method of Gram to coverslips and ordinary slide specimens for the microscope may be shortly stated thus :-

1. Allow two or three drops of the gentian-violet stain to fall upon slide and remain in contact with the film for five seconds. 2. Wash off the stain with the iudine solution applied from a drop bottle for five or six seconds. The film should then be black or dark brown. 3. Wash off the iodine solution with a mixture of 1 part acetone and 2 parts alcohol absolute, but allow to remain in contact for two or three seconds only. 4. Wash off with absolute alcohol, applied until no more stain comes 
away. 5. Wash in water, blot off superfluous water, and set aside to dry. If thought desirable, the preparation may be counter-stained by the application of a very weak solution of Ziehl-Neelsen.

The method of Gram enables us to classify bacteria into two great groups. Certain organisms when coloured with a basic stain in aniline or carbolic acid solution, and afterwards treated with a special mordant of which iodine is the base, resist decolorisation by means of absolute alcohol or other like reagent. Others, on the contrary, when treated in the same fashion, readily give up their stain and decolorise when treated with such reagents. The Bacillus anthracis may be taken as a type of the former, the Bacillus typhosus of the latter.

Nicolle's Modification of Gram's Method, used in the staining of diphtheria bacillus, consists in substituting carbolic acid for aniline water, and in the use of a stronger iodine solution, and of acetone in the decolorising fluid. Take 10 c.c. saturated alcoholic solution of gentianviolet, and add 100 c.c. of a 1 per cent. solution of carbolic acid. The iodine solution consists of iodine 1 gramme, potassium iodide 2 grammes, and distilled water, 200 c.c. Place the film in the stain for five minutes, then pass directly into iodine solution for five seconds, and decolorise by passing rapidly through a mixture of one volume of acetone with four volumes of absolute alcohol. This removes all unfixed stains at once. The specimen is then dehydrated in xylol, allowed to dry, and mounted in balsam.

Ziehl-Neelsen Method.-Here the primary stain is a solution of carbol-fuchsin :-

Basic fuchsin .

Absolute alcohol

Carbolic acid

Distilled water

It is best to heat the dye in a sand bath, in order to distribute the heat evenly. The various stages in the staining process are as follows:-(a) The cover-glass with the dried film upon it is immersed in the hot stain for one to three minutes. (b) Remove the cover-glass from the carbol-fuchsin, and, after washing in water, place it in a capsule containing a 33 per cent. solution of nitric acid to decolorise it. Here its redness is changed into a slate-grey colour. (c) Wash in water, and alternately in the acid and water, until it is of a faint pink colour. (d) Now place the cover-glass for a minute or two in a saturated aqueous solution of methylene-blue, which will counter-stain the decolorised ground substance blue. (e) Wash in water. $(f)$ Dehydrate by rinsing in methylated spirit, dry, and mount. A pure culture of bacteria will not necessarily require the counter-stain (methylene-blue). Sections of tissue may require twenty to thirty minutes in a primary stain (carbol-fuchsin).

This stain may be used for the bacillus of tubercle, with the modification necessary to separate the bacillus of tubercle from other organisms (leprosy, etc.) with similar staining properties. This modification is to wash in absolute alcohol, after the carbol-fuchsin stain has been 
used, until all the colour has entirely disappeared. Then decolorise in 25 per cent. acid solution for a few seconds, wash in water and alcohol and acid alternately, and counter-stain as usual. Honsell recommends acid alcohol (absolute alcohol 97 per cent., $\mathrm{HCl} 3$ per cent.) for ten minutes before counter-staining. With a little practice, the staining of the bacillus of tubercle when present in pus or sputum becomes a simple and accurate method of diagnosis. A small particle of sputun or pus is placed between two clean cover-glasses, and thus pressed between the finger and thumb into a thin film. This is readily dried and stained as above. But washing in alcohol and acid is not a reliable method of differentiation between the tubercle bacillus and other acid-fast organisms. Animal inoculation is the only reliable test.

Examination of Moulds.-The examination of hyphomycetes or mould fungi is, for differentiation purposes, best carried out on the Petri dish itself, where the construction of the microscope will admit of this being placed on the stage.

By the following method there is but little difficulty in recognising the various species, and an excellent demonstration is given of the hyphæ with interstitial cells, and germinating conidia of the Oidium lactis, the conidiophore and sclerotium of the Pencillium glaucum, or the ramified mycelium, sporangia, and germinating zygospores of the various species of Mucor, without disturbance of the growth. By means of a finely drawn pipette allow to fall upon the centre of the mould colony a small drop of aqueous solution ( 1 per cent.) of eosin. It is necessary to exercise a little care in this, or the liquid will at once run off the colony on to the surrounding medium. Place carefully upon the centre of the drop a thin cover-glass, and press in order to obtain close contact. Remove the Petri dish to the stage of the microscope, and examine the margins of the growth with a sixth objective.

If the construction of the microscope will not allow examination on the Petri dish, or if a permanent specimen is desired, the following method can be recommended:-Detach by means of a pair of finepointed forceps a portion of the young growth, holding it by the base, and place it carefully on a slide. Place near it one drop of ammoniated alcohol, and bring this in contact with the specimen by means of a finely pointed needle. The absorption of the alcohol will allow the subsequent penetration of the tissues by the liquids employed. Drop on to the preparation a small quantity of Fleming's solution, and allow it to remain for four or five minutes. Wash carefully with water, cover with a coverglass, and examine.

To make a permanent preparation, replace the water with glycerine by placing a drop of the latter at one side of the cover-glass, and absorb the water from the other by means of filter-paper. Dry carefully with filter-paper damped with alcohol, and ring with paraffin.

Fleming's Solution

Chromic acid, 1 per cent. Osmic acid, 2 ,". Glacial acetic acid 


\section{Flagella Staining}

Successful staining of flagella is a matter of practice, and of careful and exact technique. Whatever the method of staining adopted, the preparation of the film is the same, and too much care cannot be exercised at this stage. The slides should in no case have been previously used, and they should be most carefully cleaned in the manner described on p. 487. When taken out of the alcohol, the slide should be carefully dried and wiped with a clean piece of old cambric, without handling with the bare fingers. It should then be passed several times through the flame, and set aside to cool.

Preparation of the Film.-1. The cultures for examination should be upon agar, and should not be less than six, or more than twelve hours old, if incubation has taken place at $37^{\circ} \mathrm{C}$. If incubated at $20^{\circ}$, slightly older cultures may be employed (twelve to twenty hours).

2. Transfer from the young culture a small loopful by means of the platinum needle, to a test-tube containing from 30 to 40 c.c. of sterile water at room temperature. Or the emulsion may be made in a capsule with a few c.c. of distilled water. Hold the loop in the water for a few moments without shaking, until the water shows a slight turbidity. Do not shake or handle the tube roughly. Incubate the emulsion for five hours at $37^{\circ} \mathrm{C}$. or for twelve to twenty-four hours at $20^{\circ} \mathrm{C}$.

3 . With a finely looped pipette, take up a small quantity of the surface water from the inoculated tube, and distribute it in small droplets, upon the slide.

4. Place aside to dry, carefully covered from chance of dust. When dry, the staining can be proceeded with, according to the method adopted. Do not fix the films in the flame; the flagella are apt to be injured thereby, and it will be found that the subsequent manipulations will cause the organisms to adhere sufficiently to the slide.

\section{Staining the Film}

The three ordinary methods practised in this country are :-

(1) Pitfield's Method (Muir's modification).

The following solutions are required :-

A. The Mordant.

Tannic acid, 10 per cent. aqueous solution - $\quad 10$ c.c.

Corrosive sublimate, saturated aqueous solution . $\quad 5$ c.c.

Alum, saturated aqueous solution . - . . 5 c.c.

Carbol-fuchsin (Ziehl) . $\quad$ - . . 5 c.c.

The above must be thoroughly mixed and the precipitate which forms must be allowed to deposit. The clear supernatant fluid is then drawn off with a pipette and placed in a clean dropping bottle. The mordant will remain good for one or two weeks, but not longer. It should be centrifugalised before use.

B. The Stain.

Alum, saturated aqueous solution

Gentian-violet, saturated alcoholic solution

25 c.c.

5 c.c.

Filter twice. The stain must be freshly prepared.

The film is prepared as described above. The mordant is then dropped on to 
the slide and heated gently over the flame until the steam begins to rise. Allow to steam for from one to two minutes; wash well in running water and dry carefully. When thoroughly dry, apply a sufficient quantity of the stain, and heat as before, allowing to steam for two minutes. Wash in distilled water, dry, and examine.

(2) Van Ermengem's Method.

Three solutions are required in this method:-

A. Fixing Solution.

Osmic acid, 2 per cent. aqueous solution .

Tannin, 20 per cent. solution

To each 100 c.c. of this mixture add 4 to 5 drops of glacial acetic acid. The colour of this solution should be violet, and the solution should be filtered before use.

B. Sensitising Solution.

Nitrate of silver $\quad$. $\quad . \quad$. $\quad .0 .5$ aqueous solution

This solution should be kept in the dark, and filtered before use.

C. Reducing Solution.

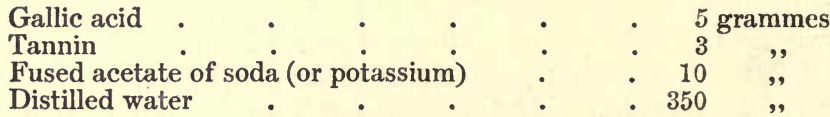

(a) Cover the film with solution "A," and allow to act for five minutes at $37^{\circ} \mathrm{C}$., or one hour at room temperature. Or heat gently until steam rises, and allow the staining fluid to act for five minutes.

(b) Wash well with distilled water, then in absolute alcohol, and then again in distilled water.

(c) Treat with solution "B," and allow it to act for thirty seconds, keeping the fluid in movement on the slide.

$(d)$ Allow the fluid to run off the slide, and without washing treat with " $\mathrm{C}$ " for thirty seconds in the same manner.

(e) Allow fluid to run off, and again treat with " B" until the preparation begins to turn black.

$(f)$ Wash in distilled water, mount in water, and examine under the microscope. The method is not wholly satisfactory.

A simple method is as follows :-

(3) Night-Blue Method (M'Crorie).

Place 2 or 3 drops of the emulsion on an absolutely clean slide, and dry at room temperature. It is not necessary or desirable to fix by heat. The stain is made by mixing 10 c.c. of night-blue, saturated alcoholic solution, 10 c.c. of a saturated aqueous solution of potash alum, and 10 c.c. of a 10 per cent. aqueous solution of tannin. The stain must be filtered before use. The slides, as prepared above, are stained with this for two minutes in the hot incubator, and then washed gently in running water. It may be found best to change the blue stain several times during the two minutes. A counter-stain may be used if desired, and one of the best is aniline gentian-violet. This should be applied for about a minute, after which a cover-glass may be fixed over the film with Canada balsam. In such a preparation the bacilli will be stained violet and the flagella blue. Better results may sometimes be obtained by staining deeply with the blue (ten minutes), and then decolorising to the necessary extent in dilute methylated spirit.

\section{The Staining of Spores}

The following are the methods commonly adopted :-

(1) Möller's Method.

(a) Prepare the film as usual, fix and dry, observing the precautions taken in preparing milk specimens. 
(b) Treat with alcohol for two minutes, and then with chloroform for two minutes ; wash in water.

(c) Treat with chromic acid, 5 per cent. aqueous solution, for from one to two minutes ; wash and dry.

$(d)$ Pour on freshly filtered carbol-fuchsin and warm gently till it steams ; allow it to act for ten minutes and wash off with water.

(e) Decolorise with sulphuric acid (5 per cent.) and water alternately, to remove the carbol-fuchsin from the bacilli but not the spores.

(f) Dry and counter-stain with Löffler's blue until the film is of a faint bluish tint. Wash off stain, dry and examine. The spores will be stained red and the bacilli blue.

(2) Ziehl-Neelsen Methód.

(a) Stain the film as for tubercle bacilli.

(b) Decolorise with 1 per cent. aqueous solution of sulphuric acid, or alcohol 2 parts, acetic acid 1 per cent., 1 part.

(c) Counter-stain with Löffler's blue.

(d) Wash, dry, and examine.

(3) Abbott's Method.

Prepare films in usual way, and stain with Loffler's alkaline methylene-blue, heating gently till steam rises (5 minutes). Then wash in water and decolorise with nitric acid, 2 per cent. alcoholic ( 80 per cent.) solution, washing again in water. Counter-stain with eosin, 1 per cent. aqueous solution. Wash, dry, and mount. The spores are blue and the bacilli red.

Bacteriological Diagnosis.-The following points must be ascertained in order to identify any particular micro-organism :-

(1) Its morphology : shape, size, etc. (bacillus, coccus, spirillum, etc.); the presence or absence of involution forms ; motility, by the unstained cover-glass preparation ("hanging drop"); note presence of flagella; presence of spores, their appearance and position. Staining reaction; whether or not the organism stains by Gram's method.

(2) Cultural Characters. - The character of the growth upon various media (gelatine, agar, milk, potato, blood serum, broth, and special media); the presence or absence of liquefaction in the gelatine culture ; its power of producing pigment, acid, gas, indol, ferments, phenol, etc.

(3) Biology : whether it is aërobic or anaerrobic ; its powers of resistance to external agencies; agglutination reaction, etc.; pathogenesis, its effect upon animal tissues and the course of the disease produced; its toxins, etc.

\section{BACTERIOLOGICAL EXAMINATION OF WATER}

Collection of Samples.-Water from streams or wells should be collected in glass bottles or flasks closed with glass stoppers previously sterilised (at $150^{\circ} \mathrm{C}$. for three hours), or washed out with pure sulphuric acid. When the latter method is adopted, the bottle should be well rinsed with the water which is to be examined before the sample is taken. In taking the sample, the bottle should be held below the surface before the stopper is removed, in order to obtain a sample of the main body of water and not the surface water only. If it is an ordinary water supply through pipes or from a cistern, the tap should be turned on and the water allowed to run for a few minutes before taking the sample: and 
the same principle applies to a well not in regular use. Such a well should be pumped for some time before taking the sample. For obtaining samples from a considerable depth Miquel's apparatus may be used, or, if that is not available, a weighted bottle.

After collection, the bottle should be at once stoppered, labelled, and packed in ice and sawdust for transport to the laboratory, or placed in one of the various ice cases now in use (Delépine's or Pakes'). Below $5^{\circ} \mathrm{C}$, organisms do not multiply in water, and therefore it is important to keep samples previous to examination at a low temperature. In all cases where it is possible, the water should be examined at once after collection.

Physical Examination.-The temperature and reaction of the water should first be tested, and an examination made of any deposit or suspended matter. Bubbles of gas, if present, should be noted. The colour, character, and amount of particulate matter in suspension or sediment should be observed and noted; turbidity, odour, flavour and taste, peatiness, etc., should all be noted. A record of the quantity of the sample, its source, and the date and time of its collection is also important. A microscopical examination of the matter obtained by filtration followed by centrifugalisation may also yield important facts.

Bacteriological Examination.-This divides itself naturally into two divisions-(a) a quantitative examination, and (b) a qualitative examination.*

\section{(a) Quantitative Examination}

The sample should be gently mixed, and plate cultivations made. Take five tubes of 10-15 c.c. of gelatine and five Petri dishes, and melt the medium of the former in a water bath. The gelatine should be well liquefied, but not overheated. The Petri dishes should be of even surface, equal size, and properly sterilised. Take a 1 c.c. sterilised

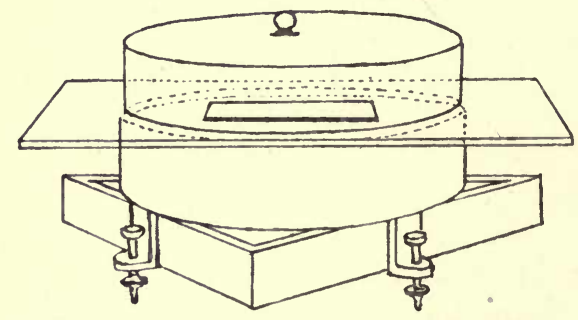

FIG. 43.-Levelling Apparatus for Koch's Plate.

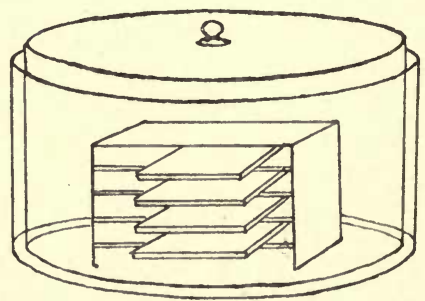

FIG. 44.-Moist Chamber for Koch's Plate.

pipette accurately calibrated, and pass it into the bottle, removing the necessary quantities of water. As a rule, 0.5 c.c., 0.2 c.c., 0.2 c.c., 0.1 c.c., and 0.1 c.c. are suitable quantities for each of the five plates. Add these quantities to the five tubes of liquefied gelatine, and gently

* An admirable illustration of how to examine a water is furnished in the Report of Medical Officer to Local Government Board, 1901-2, pp. 494-547 (Houston). 
mix and pour into the Petri dishes. Allow the gelatine to set; and incubate at $22^{\circ} \mathrm{C}$. for as long as possible before complete liquefaction occurs. Count the colonies which appear after forty-eight hours incubation (agar), take the average at the period of maximum growth (gelatine 4-5 days), multiply up according to the fraction of a c.c. which has been used, and return as so many organisms per cubic centimetre, stating medium, period and temperature of incubation, etc. It is advisable that each quantity of water from which the fractional part is added to the gelatine should be taken up separately, and not that 1 c.c. of water should be taken up and the fractional amounts, say of $0 \cdot 5,0 \cdot 2$, and $0 \cdot 1$ c.c., be added to the gelatine. If Koch's plates are used they should be allowed

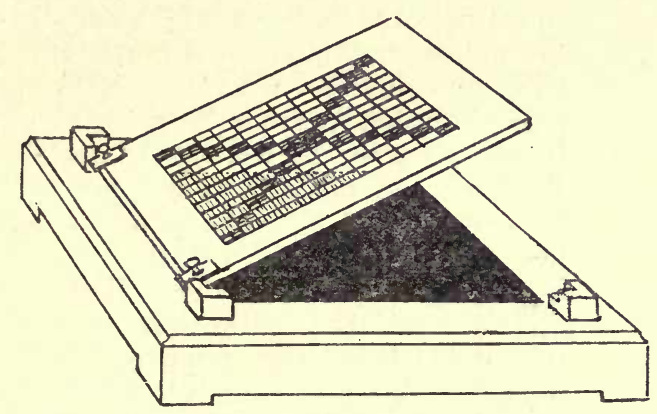

Fia. 45.-Wolfhïgel's Counter.

to set on the levelling apparatus, then placed in the moist chamber for incubation at $22^{\circ} \mathrm{C}$., and the colonies counted by means of Wolfhügel's counter (see Figs. 43, 44, and 45).

\section{(b) Qualitative Examination}

At the time of making the gelatine plates for quantitative examination, several agar, and litmus-lactose agar, plates may be made for qualitative purposes. The plates must be poured immediately after inoculation of liquefied agar with small quantities of the water, as below $40^{\circ} \mathrm{C}$. the agar will resolidify. When poured, the agar plates should be placed on cold "stone or metal, and then incubated at blood-heat. On the second or third day colonies will have appeared, and these should be studied and sub-cultured (as pure cultures) on suitable media.

Valuable facts as to the quality of the water may also be obtained from an examination of the five gelatine plates, particularly in respect of the liquefying organisms, which should be counted as carefully as any other colonies, and noted separately as well as in the total number of colonies present. But in addition to the facts obtained from gelatine and agar plates, other methods must be adopted in order to obtain information respecting the quality of the water.

Take a sterilised Berkefeld porcelain filter, and pump or aspirate through it 1000-2000 c.c. of the water under examination, and with a 
sterilised brush, transfer the particulate matter which has collected on the candle into 10 c.c. of sterile water or broth. This is now a concen-

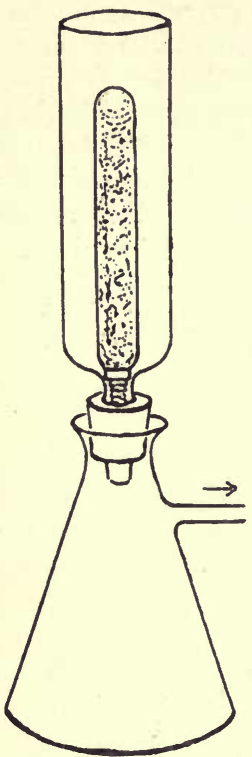

FIa. 46.-Filter in position for filter-brushing method. tration or emulsion of the organismal content of the litre of water, and may be used for examination for special organisms.

(a) B. enteritidis sporogenes.-Place 0.5 or 1 c.c. of the concentrated water in each of three tubes of 10-15 c.c. of fresh sterilised milk. It is important to use fresh milk, recently boiled, and cooled down before inoculation. After inoculation with the water to be examined, put the three tubes into the water bath for fifteen minutes at $80^{\circ} \mathrm{C}$., and after allowing them to cool, place them in a Buchner's tube or cylinder containing freshly-prepared pyrogallic solution (pyrogallic acid, 120 grains, strong liquor potassæ, 10 c.c.). Accurately seal up the Buchner, and place it, containing the tubes, in the incubator at $37^{\circ} \mathrm{C}$. The next day, or in forty-eight hours, examine for $B$. enteritidis sporogenes. If that organism is present, the following characteristic appearances-the enteritidis change-will be apparent (Klein). The cream of the milk will be torn and altogether dissociated by the development of gas, so that the surface of the medium becomes covered with stringy white masses of coagulated casein, enclosing a number of gas bubbles. The main portion of the tube formerly occupied by the milk will contain a colourless thin watery whey, with a few lumps of casein adhering here and there to the sides of the tube (see Plate 21, p. 307). If the tube be opened, there will be found to be an odour of butyric acid and an acid reaction. If some of the contents of the tube are stained, as slide preparations, the bacilli will be seen.

(b) B. coli communis (p. 46).-Take from $0 \cdot 1$ to 0.5 of the concentrated or sample water, and add to tubes of phenolated gelatine ( .05 per cent. phenol), or litmus-lactose agar, and make plates.

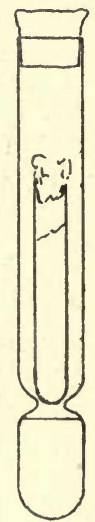

Fic. 47.-Buchner Tube. Colonies developing in these plates (red in latter medium) should be suspected of being $B$. coli communis, and tested accordingly ;

$O r$, inoculate from the concentrated or sample water, three tubes of Parietti's broth,* and incubate at $37^{\circ} \mathrm{C}$., and those tubes which show

* Parietti's Formula consists of-phenol, 5 grams; hydrochloric acid, 4 grams ; distilled water, 100 c.c. To 10 c.c. of broth, $0 \cdot 1-0 \cdot 3$ c.c. of this solution is added. The tube is then incubated in order to test its sterility. If it be sterile, a few drops of the suspected water are added, and the tube reincubated at $37^{\circ} \mathrm{C}$. for twenty-four hours. If the water contains the $B$. typhosus or $B$. coli, the tube will show a turbid growth. 
PLATE 31.

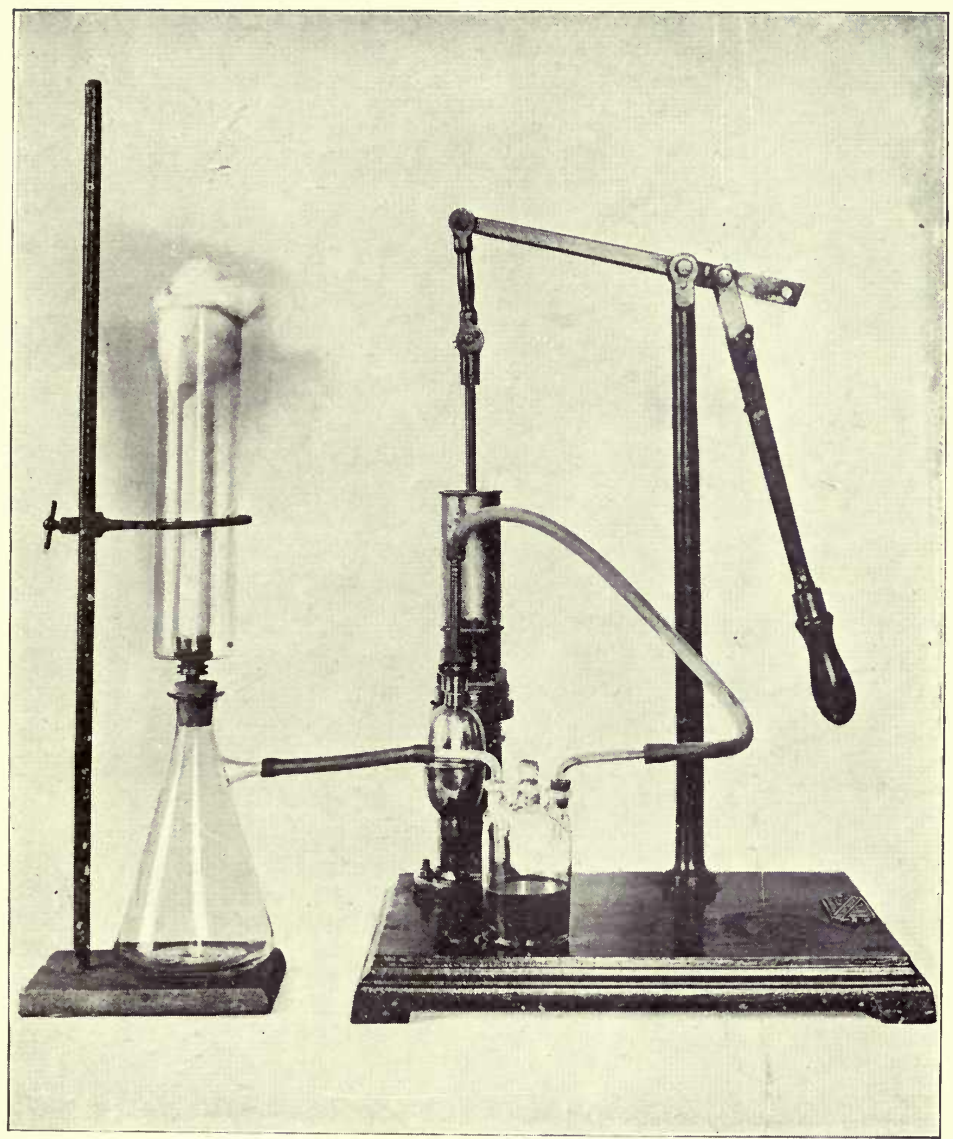

Apparatus for Filtering Water to facilitate its Bacteriological Examination. (The filter-brushing method). 


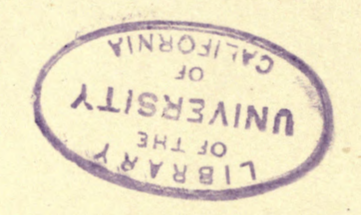


growth in one to three days should be plated out on ordinary or phenolated gelatine and colonies of $B$. coli examined for. Some authorities recommend incubating Parietti's tubes at $42^{\circ} \mathrm{C}$., a temperature favourable to $B$. coli but unfavourable to ordinary water organisms ;

Or, tubes of glucose-formate bouillon (meat infusion, 1 per cent. peptone, 0.5 per cent. salt, 2 per cent. glucose, and 0.4 sodium formate) may be inoculated with 0.1 to 0.5 c.c. of the water, and incubated in Buchner's tube at $42^{\circ} \mathrm{C}$., and the tubes which show turbidity in 24 hours may be plated out on gelatine or glucose-litmus agar and $B$. coli, if present, thus isolated (Pakes' method);

$\mathrm{Or}$, inoculate tubes of $\mathbf{M}^{\prime}$ Conkey's medium, bile-salt-glucose peptone, with 1.5 , or 10 c.c. of the water. "When this test yields negative results, the absence of $B$. coli and of glucose fermenting coli-like microbes may be accepted without reserve" (Houston) (see p. 484);

Or, place 1-20 c.c. of the water, or $\cdot 01-5$ of the suspension, into tubes of bile-salt solution, and incubate at $37^{\circ} \mathrm{C}$. aërobically for twenty-four hours, or at $42^{\circ} \mathrm{C}$. anaërobically (in Buchner's tubes) for twenty-four hours; and if, after this period, there is $(a)$ presence of growth, $(b)$ formation of acid, or $(c)$ formation of gas, plate out on gelatine agar or bile-saltlactose-peptone agar, and sub-culture coli-like colonies on suitable media ;

$\mathrm{Or}$, incubate at $38^{\circ}$ C. 1 c.c. of the water in a Smith's fermentation tube with glucose broth. If after twelve hours' growth gas collects, it may be $B$. coli, and the species must be further tested. If there is no gas there is probably no $B$. coli.

In the examination for $B$. coli, the important media for sub-culturing are as follows: gelatine shake cultures * (for gas production and liquefaction), glucose gelatine or glucose agar (for gas production), milk or litmus milk (for acidity and coagulation), peptone water (for indol), $\dagger$ and potato. Elsner medium, $\ddagger$ neutral-red agar, lactose and maltose media may also be used.

* "Shake Cultures."-To 10 c.c. of melted gelatine, a small quantity of the suspected organism is added. The test-tube is then shaken and incubated at $22^{\circ} \mathrm{C}$. In this medium the $B$. coli have opportunity for gas production.

tThe Indol Reaction. - Indol and skatol are amongst the final products of digestion in the lower intestine. They are formed by the growth, or fermentation set up by the growth, of certain organisms. Indol may be recognised on account of the fact that with nitrous acid it produces a dull red colour. The method of testing is as follows. The suspected organism is grown in pure culture in broth or peptone water (or Dunham's solution), and incubated for forty-eight hours at $37^{\circ} \mathbf{C}$. Two c.c. of a 01 per cent. solution of potassium nitrite is added to the test-tube of broth culture. Now 1 c.c. of concentrated sulphuric acid (unless quite pure, hydrochloric should be used) is run down the side of the tube. A pale pink to dull red colour appears almost at once, or in a few minutes, and may be accentuated by placing the culture in the blood-heat incubator for half an hour. The presence of much dextrose (derived from the meat of the broth) inhibits the reaction. $B$. typhosus does not produce indol, and therefore does not react to the test; $B$. coli and the bacillus of Asiatic cholera do produce indol, and react accordingly.

$\ddagger$ Elsner's Medium. - This special potassium-iodide-potato gelatine medium is used for the examination of typhoid excreta. It is made as follows : 500 grams of potato gratings are added to 1000 c.c. of water; stand in ice-chest for twelve hours, and filter through muslin; add 150 grams of gelatine; sterilise and add enough deci- 
Differential Diagnosis of B. coli.-The general characters of B. coli will be found in the text of the present volume, but it may be stated for diagnostic purposes that most reliance should be placed upon the following characters. (But all characters must be taken into consideration in forming an opinion.) $B$. coli produces a characteristic growth on gelatine plate, smooth, milk-white colonies; produces gas in lactose, saccharose and glucose media; is motile, non-liquefying (up to the 14th day) and does not stain by Gram; produces acid curdling of milk within four days at $37^{\circ} \mathrm{C}$. The production of a yellowish-green fluorescence in neutral-red agar shake culture and the production of indol in peptone water or broth (but without pellicle) are further tests relied upon by some. The Lawrence method (State Board of Massachusetts) of testing for B. coli includes the following seven tests : (a) characteristic appearance on agar streak, $(b)$ growth on litmus-lactose agar, $(c)$ gas production in dextrose broth, $(d)$ coagulation of milk, $(e)$ production of nitrites in nitrate broth, $(f)$ production of indol in Dunham's solution, and $(g)$ non-liquefaction of gelatine.*

$B$. lactis aërogenes is similar to $B$. coli, but coagulates milk much more slowly and is non-motile.

B. Gaertner and its allies ferment glucose but not lactose in litmus milk; cultures are generally acid at first, and subsequently alkaline, and there is no coagulation.

$B$. typhosus produces no gas in any media, does not coagulate milk, stains by Gram, and serum diagnosis is also practicable.

Proteus group are similar to $B$. coli, except that they liquefy gelatine and are slow in curdling milk.

(c) B. typhosus may be examined for by adopting exactly the same methods as for $B$. coli. Its detection in, and isolation from, water supplies is so difficult as to be well-nigh impossible. The condition of a water is, however, ascertainable short of an absolute test for B. typhosus, valuable though that would be.

(d) For the detection of the cholera spirillum, add 10 c.c. of peptone solution (10 per cent. peptone, 20 per cent. gelatine, and 5 per cent. salt) to 90 c.c. of the water to be tested. Incubate at $37^{\circ} \mathrm{C}$. After twelve to twenty-four hours incubation, examine loopfuls from the surface pellicle for spirilla; or plate out loopfuls of the pellicle on gelatine and agar; or test for cholera red reaction and Pfeiffer's reaction and agglutination test; or culture emulsion from Berkefeld filter in peptone water, and then plate out on gelatine and agar from tubes showing pellicle.

(e) For the detection of Streptococci, plate out the emulsion obtained from the Berkefeld filter on agar, and incubate at $37^{\circ} \mathrm{C}$. After forty-

normal caustic soda until only faintly acid; add white of egg; sterilise and filter. Before use add a gram of potassium iodide to every 100 c.c. Filter, and sterilise a $100^{\circ} \mathrm{C}$. for twenty minutes on three successive days. Upon this acid medium common water |bacteria will not grow, but B. typhosus and B. coli flourish-the former like " small clear droplets," the latter as dark brown globular masses. and 280 ,

* Report of State Board of Health, Massachusetts, 1901, p. 400; ibid., 1902, pp. 262 
eight hours examine the plate with a lens, and pick out the minute colonies, streptococci, and sub-culture in broth, and incubate at $37^{\circ} \mathrm{C}$. Stain by Gram's method, and, if necessary, further sub-culture.

$(f)$ Sewage organisms and the organisms indicative of surface pollution should also be examined for. If they be present in the water, it may be taken as proved that such water has been recently polluted, and should be condemned. Crude sewage generally contains in 1 c.c.: (a) 1 to 10 million bacteria ; (b) 100,000 B. coli (or closely allied forms); (c) 100 spores of $B$. enterilidis sporogenes; and (d) 1000 streptococci (Houston). Further, so minute a quantity as $\frac{1}{1000}$ of a c.c. of crude sewage is usually sufficient to produce "gas" in a gelatine "shake" culture in twenty-four hours at $20^{\circ} \mathrm{C}$, and the inoculation of animals with crude sewage always leads to a local reaction and not uncommonly results in death. These three organisms, B. coli, B. enteritidis sporogenes, and streptococci have been termed the "microbes of indication." These bacteria are wholly, or relatively, absent from pure water, and their presence, at all events in considerable numbers, must be taken as indicating recent animal pollution.* $B$. coli is a most accurate measure of intestinal pollution, and far greater information as regards the sewage pollution of water can be gathered by its estimation, than by simply counting the total number of organisms present in water. It is an intestinal parasite, and tends to perish in other media. $\dagger$ When it is present in a small stream, contamination from houses can be traced. $\ddagger$

Thresh has suggested the following scheme of examination of a water as one furnishing the minimum amount of information which will enable anyone to say positively that a water is fæcally contaminated :-

(1) The detection of the presence of organisms of intestinal type ; (2) the isolation and identification of $B$. coli; and (3) the detection of the presence of spores of $B$. enteritidis sporogenes. The process he recommends is as follows:- $(a)$. Make bile-salt broth cultures with 1, 5, 10, and 20 c.c. of the water to be examined. After twenty-four hours the tube containing the smallest quantity of water showing acid and gas formation is selected for further examination. If after forty-eight hours there is no such reaction, no further examination is made. (b) Two or three loopfuls of the culture are added to 10 c.c. of sterilised water, and a loopful of the solution is spread over a plate of bile-salt-lactosepeptone agar containing neutral red and made faintly alkaline to litmus. This plate is incubated for twenty-fours at $37^{\circ} \mathrm{C}$., and the colonies produced carefully examined. As the $B$. coli communis ferments lactose with the production of acid, any colonies of this organism will be of a red colour and be surrounded by a haze, formed by the precipitation of the bile acids. As this haze may not be apparent at the end of twentyfour hours, types of all the red colonies are taken for the further examination. (c) Each colony so selected is used to inoculate a tube of lactose-peptone-bile-salt-litmus solution, and after twenty-four hours' incubation, if acid and gas is produced, the growth is examined micro-

* Second Report of Royal Commission on Sewage Disposal, 1902, pp. 26 and 27.

+ Ibid., p. 99. $\ddagger$ lbid., p. 109 . 
scopically to ascertain if the bacillus is motile or not (or spore-bearing), and it is then treated by Gram's method to ascertain whether it retains the stain. The results being recorded. (d) The turbid fluid is used to inoculate-Litmus milk, for acid and clotting; glucose-neutral-red agar, for gas and fluoresence; gelatin (stab or streak), for absence of liquefaction ; and peptone solution, for indol. If the B. coli is present, all the reactions indicated, with the possible exception of the production of fluorescence, will be produced by one or more of the colonies selected. The water is tested for the presence of $B$. enteritidis sporogenes in the ordinary way. It should be added that such an examination as the above fails to obtain information upon the general constitution or the bacteriological flora of a water which is obtained by the additional means of the gelatine plate method, and whilst of value for rapid use, should not be substituted for the systematic study of a water.

REPORT OF THE COMMITTEE APPOINTED BY THE ROYAL INSTITUTE OF PUBLIC HEAL'TH TO CONSIDER THE STANDARDISATION OF METHODS FOR THE BACTERIOSCOPIC EXAMINATION OF WATER, 1904.

All the members of the committee are in agreement that the minimal number of procedures should be :

(a) Enumeration of the bacteria present on a medium incubated at room temperature $\left(18^{\circ}-22^{\circ} \mathrm{C}\right.$. $)$.

(b) Search for B. coli, and identification and enumeration of this organism if present.

The committee regard these procedures as an irreducible minimum in the bacterioscopic analysis of water. The majority of the committee recommend in addition :

(c) Enumeration of the bacteria present on a medium incubated at blood-heat $\left(36^{\circ}-38^{\circ}\right.$ C. $)$.

(d) Search for and enumeration of streptococci.

The committee do not think it necessary as a routine measure to search for the B. enteritidis sporogenes, but are agreed that in special or exceptional instances it may be advisable to look for this organism.

The Collection of the Sample. - No special precautions beyond those generally recognised are suggested for taking the sample. The samples should be collected in sterile stoppered glass bottles having a minimal capacity of 60 c.c. In special instances it may be desirable to have much larger quantities.

Unless examined within three hours of collection the sample must be ice-packed.

('The committee recognise that under all circumstances the sooner the water is examined after collection the more reliable are the results obtained.)

Media to be employed for Enumeration. - The choice of medium lies between distilled-water gelatin, nutrient gelatin, distilled-water agar, gelatin agar, and nutrient agar. The reaction of the medium is of importance.

For enumeration at room temperature, any of these media may be employed; but for enumeration at blood-heat, an agar or gelatin agar must be used.

The Americans seem to be using an agar medium only, and although on the ground of simplicity it might be desirable to use a single medium for enumeration under all circumstances-e.g. a distilled-water agar-it is felt by the committee that gelatin media frequently give indications of value that are lacking with agarviz., liquefaction of the medium by many organisms and the more characteristic appearance of the colonies in it; gelatin is therefore recommended.

Since with a polluted water (detection of pollution being the ultimate aim in 
water examination) nutrient gelatin gives a relatively larger number of colonies than distilled-water gelatin, nutrient gelatin should be used when one gelatin only is employed. At the same time, it is recognised that cultures in distilled-water gelatin compared with cultures in nutrient gelatin often give useful indications. Thus with an unpolluted water the number of colonies is usually relatively larger in distilled-water gelatin than in nutrient gelatin ; with a polluted water the converse is the case. Therefore the use of both gelatins (distilled-water and nutrient) is desirable, sets of plates being made with each medium.

Similarly, it was felt by many members of the committee that a comparison of the ratio of the number of organisms developing at room temperature to those developing at blood-heat gives useful indications. With a pure water this ratio is generally considerably higher than 10 to 1 , with a polluted water this ratio is approached, and frequently becomes 10 to 2,10 to 3 , or even less. The actual number of organisms growing at blood-heat is also of considerable value apart from any question of ratio. Therefore it is suggested that plates of nutrient agar should also be employed and incubated at blood-heat.

In certain instances it is true that this ratio may be unreliable. Thus with surface waters, especially in tropical countries (as pointed out by Major Horrocks), varieties of the B. fluorescens liquefaciens and non liquefaciens and B. liquefaciens may be abundant and grow well at blood-heat.

\section{Preparation and Reaction of Media for Enumeration}

(a) Distilled-Water Gelatin.-Ten per cent. gelatin in distilled water, and brought to a reaction of +10 (Eyre's scale).

(b) Nutrient Gelatin.-Ten per cent. nutrient gelatin, preferably made with meat (beef) infusion and Witte's peptone, and brought to a reaction of +10 (Eyre's scale).

In hot weather it may be necessary to increase the percentage of gelatin.

Some members of the committee advocate the use of meat extracts in place of meat infusion, on the score of convenience and uniformity of composition, Brand's Essence being recommended as the best. It is the general opinion, however, that Liebig's Extract is less suitable for this purpose.

(c) For enumeration at blood-heat it is recommended that nutrient agar should be employed, being prepared with the same constituents as nutrient gelatin, but substituting $1 \frac{1}{2}$ per cent. of powdered agar for the gelatin. Reaction $+10$.

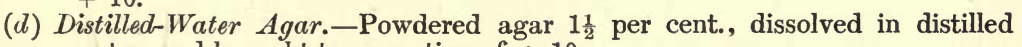
water, and brought to a reaction of +10 .

Owing to the changes which occur in the reaction of the medium on keeping, the media employed should preferably be not more than three weeks old.

Amounts to be Plated, Size of Dishes, etc.-Gelatin.-For an ordinary water amounts of $0.2,0.3$, and 0.5 c.c. may be plated in Petri dishes of not less than 10 centimetres diameter, preferably done in duplicate.

Agar.-Two plates may be made with 0.1 and 1.0 c.c., and are preferably duplicated.

In dealing with an unknown water, and in all cases of doubt, additional sets of plates should be prepared with a dilution of the water (made with sterilised tapwater) of ten or hundred fold, according to circumstances.

The amount of the medium in a plate should be 10 c.c.

The sample must be thoroughly shaken and mixed in all cases before plating:

Temperature of Incubation. - (a) Room temperature $=18-22^{\circ}$ C. ; (b) blood-heat $=$ $36-38^{\circ} \mathrm{C}$.

Counting.-Counting to be done with the naked eye, preferably in daylight, any doubtful colony being determined with the aid of a lens or low-power objective.

Time of Counting.-Gelatin plates should be counted at the end of seventy-two hours; but in all cases the plates should be inspected daily, in order that the count may be made earlier should liquefaction render this necessary. hours. 


\section{Search for B. Coli}

\section{Method.-The committee recommend either-}

(a) The glucose-formate broth method of Pakes.

(b) The bile-salt broth method of M'Conkey.

Incubation anaërobically at $42^{\circ} \mathrm{C}$. increases the chances of success with either medium, and is strongly recommended.

It has also been suggested that the neutral-red (Grübler's) glucose broth medium may be employed.

The committee do not regard with favour the Parietti method, or the use of carbolic acid media.

Quantity of Water to be Examined.-As a routine 50 c.c. should be the minimal quantity examined for the presence of the $B$. coli, quantities from a minimum of $0 \cdot 1$ c.c. to a maximum of 25 c.c. being added to the tubes of culture media.

The committee are of opinion that it is preferable to add the water directly to the tubes of culture medium, even with the larger amounts, rather than first to concentrate by filtration through a porcelain filter (the filter-brushing method). The culture media recommended may be diluted with at least an equal volume of the water without interfering with their cultural properties, and large tubes or small flasks may be used for the larger amounts.

In the case of the bile-salt-lactose-peptone water, the medium may for the larger amounts be prepared of double strength.

Isolation of $\boldsymbol{B}$. coli, if Present.-If indications of the presence of the $\boldsymbol{B}$. coli be obtained in the preliminary cultivations, the organism must be isolated and identified.

This may be done by making surface cultures on plates of either $(a)$ litmus-lactose agar, reaction +10 ; $(b)$ bile-salt agar ; $(c)$ nutrose agar of Conradi and Drigalski ; or $(d)$ ordinary nutrient gelatin.

The best medium of all is, probably, the nutrose agar of Conradi and Drigalski. Agar media have the advantage of saving time.

Identification of, and Tests for, the B. coli.-Having obtained coli-like colonies on the plates made from the preliminary cultivations of the water, sub-cultures must be made in order to identify the organism. The following, at least, should be made :

(a) Surface agar at $37^{\circ} \mathrm{C}$. The abundant growth so obtained enables many subcultures and preparations to be made if required.

(b) Stab and surface cultures in gelatine. This may be done in the same tube.

(c) Litmus milk incubated at $37^{\circ} \mathrm{C}$.

(d) Glucose litmus medium.

(e) Lactose litmus medium.

( $f$ ) Peptone water for indol reaction.

Characters of the $B$. coli. - The $B$. coli is a small motile, non-sporing bacillus, growing at $37^{\circ} \mathrm{C}$. as well as at room temperature. The motility is well observed in a young culture in a fluid glucose medium. It is decolorised by Gram's method of staining. It never liquefies gelatin, and the gelatin cultures should be kept for at least ten days in order to exclude a liquefying bacillus. It forms smooth, thin surface growths and colonies on gelatin, not corrugated, growing well to the bottom of the stab (facultative anaërobe).

It produces permanent acidity in milk, which is curdled within seven days at $37^{\circ} \mathrm{C}$. It ferments glucose and lactose, with the production both of acid and of gas.

The typical $B$. coli must conform to the above description and tests.

It generally also forms indol (best obtained in peptone-water cultures), gives a thick yellowish-brown growth on potato (greatly dependent on the character of the potato), sometimes (about 50 per cent.) ferments saccharose, changes neutral-red (Grübler's), and reduces nitrates, and half the gas produced by it from glucose is absorbable by $\mathrm{KOH}$; and these tests, if time and opportunity permit, may be performed in addition to the foregoing.

The committee recognise that atypical $B$. coli are met with, but in the present state of our knowledge hesitate to make any suggestion with regard to their significance. 


\section{Streptococci}

The committee consider that it is a distinct advantage to search for streptococci. They may be looked for by making hanging-drop preparations of the fluid media employed for the preliminary cultivation of the $B$. coli (glucose-formate broth, etc.). The presence or absence of streptococci in these tubes gives also a quantitative value to the examination, just as in the case of $\mathrm{B}$. coli, and the result obtained should be stated. The streptococci should be isolated (best carried out on nutrose agar plates), and their characters determined.

\section{B. Enteritidis Sporogenes}

As already stated, the committee do not consider that it is essential as a routine procedure to search for the $B$. enteritidis sporogenes, though in certain instances it may be of advantage to do so. A negative result in such cases is probaby of more value than a positive one.

This report is the outcome of prolonged deliberations, and every point has been carefully considered and discussed by the members of the committee.

In conclusion, the committee suggest that if the above recommendations were to be adopted by all engaged in the bacteriological examination of water it would conduce to uniformity of results, and would render comparable the data obtained by different observers. An addendum might be added to a report on an analysis conducted on these lines, to the effect that the analysis had been carried out in conformity with the procedures recommended by the committee of The Royal Institute of Public Health, 1904.

The committee beg to acknowledge their great indebtedness to Professor R. Tanner Hewlett, M.D., D.P.H., upon whom the great burden of the work of the committee has devolved.

R. Tanner Hewrete, M.D., M.R.C.P.,

RUPERT BOYCE, M.B., F.R.S., Chairman. Hon. Secretary.

\section{BACTERIOLOGICAL EXAMINATION OF MILK*}

Physical examination (temperature, reaction, colour, cream, deposit, specific gravity, etc.) of the milk should be made if necessary. The microscopical examination of the milk before and after centrifugalisation or sedimentation will likewise often yield useful results.

1. Plate Cultivation.-Dilute as required, and make plate cultivations in Petri dishes or flat-bottomed flasks. Six or more gelatine plates should be made and incubated at room temperature. Plates should also be made with nutrient agar for incubation at $37^{\circ} \mathrm{C}$. Other media may also be used. The plates should be counted on the second, third, and fourth days, and the necessary sub-cultures made. Agar plates incubated wholly at $18^{\circ}$ or $22^{\circ} \mathrm{C}$., will in the long run show more colonies than whell incubated at $37^{\circ} \mathrm{C}$. and then at $22^{\circ} \mathrm{C}$. or at $37^{\circ} \mathrm{C}$. throughout.

2. Anaërobic Cultivation.-At the same time that the primary aërobic plate cultivations are made, similar plates should be made on lactose gelatine and lactose agar for anaërobic culture (see p. 117).

3. Primary T'ube Cultivation.-Take ten tubes of 10 c.c. of the milk

* For further particulars concerning the bacteriological technique in milk examination, see Bacteriology of Milk, by Swithinbank and Newman, 1903, pp. $30-115$. 
under examination, and place three of them in the incubator at room temperature and three of them at $37^{\circ} \mathrm{C}$. Place four of them in a water bath heated to $80^{\circ} \mathrm{C}$. for fifteen minutes, and then enclose each of the four tubes in a Buchner's tube. These primary cultures may be tested in forty-eight hours for $B$. coli, the presence of indol, and $B$. enteritidis sporogenes.

4. Secondary or Sub-cultures.-From the primary cultivations, make sub-cultures on selected media for the isolation of organisms making their appearance on the plates, or what is often preferable, make a set of plates for qualitative examination only.

5. Examination for Special Micro-organisms.-The milk must be centrifugalised or the particulate matter allowed to gravitate by sedimentation. It is, as a rule, useless to attempt examination microscopically or otherwise without first using the centrifuge or sedimentation flask. The deposit is then to be stained for the particular organism for which search is being made (see p. 476).

For centrifugalisation, take two or three samples of the milk under examination to the amount of about 40 c.c. each, and place it in the sterilised tubes of the centrifuge. In these tubes the milk may be centrifugalised for ten or fifteen minutes at 3000 revolutions a minute. At the end of such a period the milk in each tube has separated into three layers-at the top there is a dense layer of cream, at the bottom there is the sediment or "slime" containing all the particulate matter, between these two is the separated milk. Aspirate off the cream by means of a sterile glass tube connected with an aspirator or vacuum pump, and examine separately; aspirate all the separated milk except 2 c.c. The remaining sediment is so compact and dense that the tube may now be inclined and the sediment fully exposed without displacement. By means of a sterilised platinum loop a small portion may be taken up and spread on the surface of half a dozen slides, and stained. The remainder of the sediment is well mixed with the 2 c.c. of milk and used for inoculation of guinea-pigs.

For sedimentation, take two conical sedimentation glasses and fill them with the milk under examination, allowing them to stand in the refrigerator for twelve to fourteen hours. It is customary to add a few small carbolic crystals to each flask. On the completion of sedimentation the milk has separated into three main strata: the cream at the top, the sediment at the apex of the flask, and the separated milk in the middle. The cream and milk may then be carefully decanted, and the sediment will be available for examination.

\section{Staining Methods in Milk Examination}

The only difficulty which presents itself in the preparation of milk for the microscope is the simultaneous staining of the casein and fat as well as the organisms which may seriously confuse the issue. Hence the removal of the two former substances is recommended, as follows :-

(a) Staining after Clearing with 5 per cent. Acetic Acid.-The slides are 
thoroughly cleaned in the ordinary way, and immediately before use are again washed with equal parts of alcohol and ether. Several loopfuls of the milk to be examined are now placed on the slide and allowed to dry at the temperature of the room, being protected from the air by means of a small glass cover. When the film is dry it is fixed, preferably with alcohol and ether, as described below. It is then washed alternately with a 5 per cent. solution of acetic acid and distilled water until there is but little apparent film left upon the slide, which is then dried between layers of fine filter-paper. The specimen may now be stained by means of any of the ordinary aniline dyes, washed in distilled water, again dried, and examined under the microscope. Ether, chloroform, various strengths of alcohol, and other clearing agents may be used if preferred.

(b) Saponification.-If it be desired to retain the background of casein and fat, it will be found best to saponify the milk in the following manner:-Prepare the film of milk as before, but before drying it add an equal number of loopfuls of a sodium carbonate or sodium hydrate solution ( 5 per cent. to 50 per cent. dilution). The loopfuls of milk and soda solution should be placed in immediate proximity to each other on the slide, and thoroughly mixed by means of the platinum loop. By this means an even distribution of the bacteria is obtained. The film is then dried by gentle heating, stained, washed, and cleared with xylol. The result will be that the organisms will be stained more deeply in colour than the background of saponified matter.

(c) Clearing with Acetic Acid after Saponification.-The best preparations are obtained by a combination of the above methods. For this purpose the films are prepared exactly as in the ordinary saponification method above described, but as soon as the films have become saponified, instead of at once proceeding to stain with the desired dye, the film is thoroughly cleared by several alternate washings with the 5 per cent. solution of acetic acid and distilled water. The subsequent procedure is as in $(a)$.

Methods of Fixation.-The object of fixing is to coagulate the albuminous material, and cause perfect adhesion of the prepared film to the slide. The following alternative methods are recommended:-

(a) Heat.-Holding the glass slide by one extremity between the thumb and index finger of the right hand, pass it, film side upwards, gently through the flame three times, allowing the under surface to rest on the back of the left hand between each passage.

(b) Alcohol-ether.-Place one or two drops of a mixture of equal parts of absolute alcohol and ether upon the dried film, and allow it to evaporate.

(c) Formal-alcohol.-Formalin 1 part, absolute alcohol 9 parts. Leave in contact for from three to four minutes, wash well in water, blot off excess of moisture, and stain.

(d) Perchloride of Mercury.-Saturated aqueous solution. Leave in contact with the film for four or five minutes. Wash off with a stream of water, and apply Gram's iodine solution in order to dissolve out any 
formed crystals of the salt. Wash again in water, blot off excess of moisture, and apply stain. This fixing agent should be used on all occasions when dealing with morbid material or cultures of a specially virulent nature.

\section{Methods of Examination for Special Micro-organisms in Milk}

Bacillus pseudo-tuberculosis of Pfeiffer (found in London milk by Klein).-By the centrifuge or by sedimentation in an ice-chest for twenty-four hours, obtain the particulate matter of the milk to be examined. Inoculate 2 c.c. into a guinea-pig subcutaneously or intraperitoneally. In the course of three to four weeks caseo-purulent nodules will occur in the inguinal glands (if subcutaneously inoculated), or in the omentum and pancreas and other organs (if intra-peritoneally). Cultures may be obtained best from glands, spleen, pancreas, or liver. Examine the nodules by staining and culture. They will have the following characters if the disease be pseudo-tuberculosis : (a) Absence of giant cells ; $(b)$ absence of the true tubercle bacillus; $(c)$ presence of large numbers of $B$. pseudo-tuberculosis; and $(d)$ signs of a rapid and not a slow development.

Method of Staining.-Make films in the ordinary way and stain with Löffler's methylene-blue, heating the stain till it steams (Klein). Wash in distilled water. Nodules may be hardened in Müller's fluid and spirit, and sections cut and stained by placing in Löffler's blue for twenty-four hours and counter-staining in a mixture of eosin and methylene-blue. Löffler's blue may also be used for staining the bacillus in milk-films made from sediment. Gram's method is also applicable, but the bacillus is not acid-fast, and will not hold the ZiehlNeelsen stain.

Bacillus diphtheriæ.-By centrifuge or sedimentation obtain the particulate matter of the milk under examination and inoculate it into a guinea-pig. Sub-culturing from the tissues of the guinea-pig, or, having obtained sediment as above, inoculate six tubes or plates of Löffler's medium (ox serum 3 parts, veal broth 1 part-the broth to contain glucose 1 per cent., peptone 1 per cent., and sodium chloride 0.5 per cent.). Upon this medium the Klebs-Löffler bacillus grows rapidly in twelve to twenty hours, producing scattered nucleated, round, white colonies which later become yellow.

Method of Staining.-Gram's method as modified by Nicolle (see p. 459) will be found the most satisfactory, but the methylene-blue solution of Löffler is often used. This consists of 30 c.c. of a saturated alcoholic solution of methylene-blue added to 100 c.c. of a 01 per cent. solution of caustic potash. By this stain the striped appearance of the bacilli of older cultures on blood serum is obtained more readily than by other methods.

Neisser's Method for Differentiation of the Diphtheria Bacillus.-This method consists in applying two stains as follows. Stain I. is made of 1 gramme of methylene-blue dissolved in 20 c.c. of a 95 per cent. alcohol, 
and 950 c.c. of distilled water. To this is added 50 c.c. of glacial acetic acid. Stain II. consists of 2 grammes of vesuvin dissolved in 1000 c.c. of boiling distilled water. Both stains are filtered before use. Prepare films in usual way, and stain with No. I. for thirty seconds. Wash in water and then stain with No. II. for thirty seconds. Wash, dry, and mount. The bacilli are stained brown by vesuvin and the metachromatin granules blue-black. Some bacteriologists place great reliance upon the diagnostic value of Neisser's stain for the diphtheria bacillus from blood serum cultures and from swabs. In the latter case the stain is sometimes used as a "rapid method of diagnosis." It is not, however, absolutely reliable.

Streptococcus in Milk.-By centrifuge or sedimentation obtain the particulate matter of the milk. Take a sterilised platinum loop, dip in the sediment, and remove a drop of it. Distribute this in a testtube containing 1 to 2 c.c. of sterile salt solution. Inoculate agar plates with a drop of this dilution, and incubate at $37^{\circ} \mathrm{C}$. When the colonies appear, sub-culture those resembling streptococcus colonies in bouillon, and on blood serum. Sub-culture from the bouillon in milk, gelatine, and agar, carefully noting the characters of the growth, etc. Or guinea-pigs may be inoculated in the subcutaneous tissue of the groin or intra-peritoneally. An acute purulent inflammation will be set up in the exudation of which streptococcus will occur in large numbers.

Method of Staining.-Gram's method is the most satisfactory. Next to Gram's stain the most useful is Löfler's blue. It may be noted that most of the putrefactive organisms do not hold Gram's stain.

Bacillus coli communis. - (a) Dilute the milk to be examined 500 or 1000 times. Take a sterilised brush, dip it in the dilution, and brush over the surface of six agar plates without recharging the brush. Incubate at $42^{\circ} \mathrm{C}$., and sub-culture the coliform colonies (bouillon, milk, litmus milk, gelatine "shake" cultures, bile-salt-glucose-peptone, etc.).

(b) Take six tubes of phenol bouillon ( 0.05 per cent. of carbolic acid), and inoculate them with crude or diluted milk. Those which show abundant turbidity after twenty-four to forty-eight hours at $37^{\circ} \mathrm{C}$. may be plated out on phenol gelatine, incubated at $20^{\circ} \mathrm{C}$., and the coli colonies sub-cultured; or diluted milk may be at once plated out on phenol gelatine, and colonies sub-cultured on such media as will show the characteristics of the organisms.

The main characters of the $B$. coli group of organisms may be briefly restated here, though particulars will be found elsewhere in the present volume:-(1) They are non-sporing and non-liquefying; (2) they rarely stain by Gram's method; (3) they are motile; (4) they produce acid and gas in glucose and lactose media; (5) they produce acid in milk, and usually coagulate it; (6) they grow well at a temperature of $42^{\circ} \mathrm{C}$. Referring to the isolation of $B$. coli, Houston writes: "No test based on observation of a change or changes produced in the nutrient medium, and supposed to be characteristic of $B$. coli, can compare with isolation from plate cultivations of the microbes suspected to be $B$. coli, and the 
subsequent attentive study of the biological characters of pure cultures of these bacteria grown in various media."*

Bacillus enteritidis sporogenes of Klein.--Take six tubes containing 15 c.c. of fresh milk and sterilise them by boiling for half an hour. Rapidly cool them by placing them in a beaker

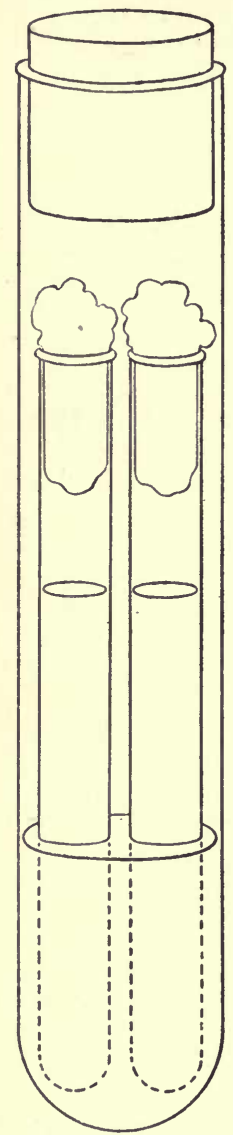

Fia. 48. - Another form of Buchner Tube. of cold water, add to each tube 1 c.c. of a 1 in 500 dilution of the milk to be examined, or if it be preferred 0.1 c.c. of the crude milk. Heat the inoculated tubes at $80^{\circ} \mathrm{C}$. for fifteen minutes. Then remove and cool, and place in Buchner tubes or cylinder containing freshly prepared mixture of pyrogallic acid and potassium hydrate solution. Seal the Buchner tubes or cylinder with great care, making it absolute. Place the Buchner apparatus, including the milk tubes, in the incubator at $37^{\circ} \mathrm{C}$. After forty-eight hours take out the tubes, and examine them for the $B$. enteritidis sporogenes. If necessary, inoculate guinea-pigs subcutaneously with 1 c.c. of the whey, which in a few hours causes swelling at the point of inoculation, and extensive gangrene of the subcutaneous and muscular tissues with sanguineous exudation; the animal dies in twenty-four or thirty hours. The B. butyricus of Botkin may produce similar changes in milk tubes, but it has no pathogenic action. Milk may be examined directly by placing 20 c.c. in tubes and treating as above. For the "enteritidis change" in the milk, see p. 307.

Bacillus tuberculosis.-Obtain the sediment of the milk under examination and inoculate 2 c.c. of it into the subcutaneous tissue of the guinea-pig. In about four weeks' time, local if not general tuberculosis will have been set up. Take some of the discharge and stain it after the Ziehl-Neelsen method. The sediment of tuberculous milk may be stained forthwith, without inoculation, by the same method, and in some cases the tubercle bacillus may be thus detected, but, generally speaking, the only sure test is inoculation of animals. The pathological process is slower than in pseudo-tuberculosis, and on examination the diseased tissues show giant cells and numerous tubercle bacilli arranged within the giant cell (see Plate 23, p. 328).

Method of Rabinowitsch for Tubercle Bacillus in Butter.-The butter is placed in sterile conical glass in the incubator at $37^{\circ} \mathrm{C}$., where on melting it will arrange itself in two layers. Three c.c. of the supernatant fatty liquid are injected into the peritoneal cavity of a guineapig. A similar quantity of the deposit is treated in a like manner, 
and finally, two or three other animals are inoculated intra-peritoneally with the semi-liquid substance obtained on mixing together the two layers into which the butter was formed. At the end of expiration of seventy days the animals which have not already succumbed are sacrificed, and a careful post-mortem examination is made. Microscopic preparations and cultures are made from any organs affected. The latter, taken together with the general aspect of the lesions, will, in the majority of cases, be sufficient to enable a diagnosis to be made between the true bacilli of tuberculosis, and other acid-fast organisms resembling it. Bacilli which resists in a moderate or somewhat feeble manner decolorisation by acids, which develop rapidly at a temperature of $37^{\circ} \mathrm{C}$., and grow feebly at ordinary room temperature, which exhibit chromogenic properties in culture, and give rise in the guinea-pig to lesions which are not characteristically those of tuberculosis, must be regarded as organisms of the acid-fast group, non-pathogenic for man, though possibly related in some degree to the true bacillus of tuberculosis (see also p. 358 ).

Another method is that indicated by Roth. Five grammes of butter are vigorously shaken up in sterile water, and the whole is then centrifugalised. A fat-free deposit is thus obtained, and given quantities of this are injected into animals in the ordinary manner,

\section{Special Methods}

Examination of Colostrum.-Colostrum is the term applied to the first milk yielded by the cow after parturition. It differs considerably from ordinary milk, and generally appears as a thick, turbid, yellowish, viscid fluid. When examined under the microscope, it is found to contain, in addition to the ordinary milk corpuscles, peculiar conglomerations of very minute fat granules which are hence known as colostrum corpuscles. The chief chemical differences between colostrum (or beastings) and milk are mainly three. First, colostrum is deficient in casein. Secondly, it is proportionately rich in albumen. Thirdly, it contains nearly three times more salts than milk. Probably it is this excess of salts that usually causes it to exert a purgative effect upon the newborn calf, and thus to remove the meconium which has accumulated in the foetal intestine.

The difficulties of bacteriological examination of such a subject as colostrum are considerable. At the outset, a fair sample is only obtainable by adopting the following precautions: (a) The teats and udder to be cleansed ; $(b)$ milking to be carried out as soon after calving as possible, when the calf has sucked; $(c)$ the first part of the "milking" to be discarded, and the last part only to be examined. When the colostrum reaches the laboratory, it must be diluted in precisely the same manner as thick cream. After abundant dilution treat the solution in the ordinary way, by staining preparations for the microscope, plating out on various media, and sub-culturing.

Bacteriological Examination of Butter.-Take a quarter of a 
pound of butter and place it in a sterilised flask with 150 c.c. of sterile salt solution. Place the flask in the water bath at about $35^{\circ} \mathrm{C}$., and shake gently until the butter has melted. The contents of the flask now appear as a milk-like emulsion. A small quantity of this mixture may be used for plate cultivation on gelatine and agar, as in milk. The remainder should be placed in a sedimentation flask in the refrigerator for twenty-four hours. By this means the particulate matter of the butter, including the contained organisms, are deposited. After removing the superficial solidified fat by means of a sterile spatula, the turbid fluid may be decanted, and the sediment collected for microscopical examination or the injection of guinea-pigs.

Examination of Cheese.-With a knife previously sterilised by pissing through the flame, cut off from the piece of cheese under examination a thin slice parallel to the surface. Remove this, and with a second sterile knife cut perpendicularly downward from the bared surface. Pass down into the latter cut a coarse sterile platinum needle, of which a small portion near the extremity has been slightly roughened with a file.

Inoculate with this needle a sufficient number of tubes of bouillon from which plate cultivations can subsequently be made for isolation purposes, and placed under both aërobic and anaërobic conditions.

Examination of Milk for Pus Cells.-Place 10 c.c. of the milk to be examined in each tube of the centrifuge (Plate 5, p. 74) and centrifugalise for two minutes. Pour off the supernatant fluid, and with a sterilised needle or pipette take up a small quantity of the sediment remaining in the tube. Spread the sediment evenly over the surface of an ordinary glass slide, and dry over the flame of a Bunsen burner or on the drying stage. Wash the fixed film with ether (or alternately with absolute alcohol and ether) until all the superfluous fat is removed, and stain. The preparation may be stained $(a)$ by one of the ordinary solutions such as Löffler's blue, etc.; or (b) by Gram's method. Examine under the microscope with a $\frac{1}{12}$ th oil immersion lens.

Inoculation of Guinea-pigs in Milk Examination.-It will be sufficient to remark that the simplest forms of inoculation are all that are usually required in milk investigation, namely, the intra-peritoneal and subcutaneous. In some cases it may be sufficient to inoculate a few c.c. of the original milk; but, as a rule, it is advisable to centrifugalise, or use the sedimentation flask containing about 250 c.c. From the deposit or sediment two guinea-pigs may be inoculated, the one subcutaneously in the groin, the other intra-peritoneally. Particularly is this necessary in making a reliable and exhaustive search for the $B$. tuberculosis. Microscopic examination alone for this organism is not reliable (see p. 478). The details of the process as carried out in practice are as follows :-

After centrifugalisation the deposit is mixed with the 2 c.c. of milk remaining in the tube after aspiration of that which is superfluous. Two guinea-pigs (of say 250 grammes weight each) are taken and inoculated with the deposit from about 40 c.c. of milk. The fluid is inoculated subcutaneously on the inner side of the leg under strict aseptic precau- 
tions (the skin having been washed with 1-1000 corrosive sublimate, and shaved). In less than a fortnight's time, if the inoculated milk contained a considerable number of tubercle bacilli, typical infection of the popliteal and inguinal glands can be detected. If the milk contained very few bacilli the infection is much slower (fifth week). After the animal has been killed the presence of the tubercle bacilli can be detected in the inguinal glands and the spleen. Some workers make it a rule to inoculate two guinea-pigs from the sediment of the milk, one receiving half of the sediment subcutaneously in the groin, the other receiving the other half intra-peritoneally.

\section{BACTERIOLOGICAL DIAGNOSIS IN SPECIAL DISEASES}

1. Diphtheria.-Obtain a piece of the membrane or a "swab" from the throat. Take a piece of stout iron wire and twist a piece of cotton wool round one end of it, and insert in a test-tube, and sterilise. By means of such a swab obtain a rubbing of the suspected throat. Then scraping off from the swab sufficient material for $(a)$ a microscopic examination, $(b)$ smear the swab over the surface of agar and blood serum media, and finally $(c)$ place in a tube of sterilised broth. Thus we have material for a film preparation, for cultivation, and for animal inoculation. Make the film in the usual way, and stain with Nicolle's modification of Gram (see'p. 458) or Neisser's stain (see p. 476). Examine under the microscope. The value of examining such a preparation microscopically depends upon the experience of the bacteriologist.

Of culture media, blood serum is perhaps the best, but, if no serum tubes can be had, an egg may be used. It should be boiled hard, the shell chipped away from one end with a knife sterilised by heating, and the inoculation made on the exposed white surface; the egg is then placed, inoculated end downwards, in a wine-glass of such a size that it rests on the rim and does not touch the bottom. A few drops of water may with advantage be put at the bottom of the glass to keep the egg moist. The preparation is kept in a warm place for twenty-four to forty-eight hours, and then examined. The examination, of course, consists in staining and preparing specimens for the microscope, and observing the form, arrangement, and characters of the organism or organisms present. The same is done for cultures on agar or blood serum. On the latter the colonies show characteristic growth. A small piece of the membrane may be detached, washed in water, and stained for the bacilli.

To differentiate the true or Klebs-Löfler bacillus from the pseudo or Hofmann bacillus, note especially that Hofmann's bacillus is plumper, shorter, and thicker in the middle than the true diphtheria bacillus. It also stains more regularly, grows better on alkaline potato, and produces an alkaline reaction in neutral litmus agar or bouillon incubated for two days at $37^{\circ} \mathrm{C}$. It is non-pathogenic for guinea-pigs, whereas the KlebsLöffler bacillus is pathogenic.

2. Tetanus. - The detection of the bacillus of tetanus in the dis- 
charge of a tetanic wound is not always easy. Make preparations, and stain with carbol-fuchsin. Drumstick-shaped, spore-bearing bacilli are to be looked for. If a small piece of tissue is available, sections should be prepared and double-stained. Cultivations should also be made from the discharge in blood serum or glucose agar incubated at $37^{\circ} \mathrm{C}$. for forty-eight hours. Then keep the culture at $80^{\circ} \mathrm{C}$. for twenty to thirty minutes, to kill all non-sporing bacilli. Sub-culture in glucose gelatine in hydrogen at $22^{\circ} \mathrm{C}$., and examine in five days. Animal inoculation (mice and guinea-pigs) is generally necessary.

To isolate the tetanus bacillus from soil, proceed as follows:-Make an emulsion of the soil in sterilised water. Expose it to $80^{\circ} \mathrm{C}$. for twenty minutes. Add 1 c.c. of the emulsion to each of three tubes of glucose-formate broth, and incubate anaërobically in Buchner's tubes at $37^{\circ} \mathrm{C}$. After twenty-four hour's' incubation, inoculate guinea-pigs subcutaneously, using $0 \cdot 1$ c.c., and observe results. Also make glucose-agar plates from the same emulsion (after heating to $80^{\circ} \mathrm{C}$.), and incubate anaërobically in Bulloch's apparatus.

3. Tuberculosis. - The tubercle bacillus is an acid-fast organism, stained by Ziehl-Neelsen method. But several allied organisms possess the same tinctorial properties, and therefore inoculation into a guinea-pig is frequently necessary for diagnosis. Sputum, however, is generally accepted as proved to be tubercular if bacilli having the morphology and staining properties of the tubercle bacillus are present.

4. Typhoid.-Widal's Application of Grïber's Reaction. This diagnostic test depends upon the effect which the blood serum of a person suffering from typhoid fever has upon the $B$. typhosus. The effect is twofold. In the first place, the actively motile $B$. typhosus becomes immotile; and secondly, there is an agglutination, or grouping together in colonies, of the B. typhosus. Neither of these features occur if healthy human blood serum is brought into contact with a culture of the typhoid bacillus.

The method of using the test is as follows:-

(a) Collection of Serum.-Wash the lobe of the patient's ear with antiseptic ( 2 per cent. lysol), and by rubbing render the ear hyperæmic. Wash with methylated spirit and dry. Puncture the vein of the lobe with a sterilised needle or lancet, and collect the issuing blood in a pipette. Hold one end in contact with the bleeding point, and lower the other end. By gravity the blood will enter the pipette; if not, gentle suction may be applied. When full to the shoulder, remove the pipette, and placing the clean end to the lips, draw the blood gently but completely into the body of the pipette. Now seal the ends in a flame, and let the pipette lie horizontally till the blood is coagulated.

(b) Dilution of Serum.-Place the pipette in the vertical position, preferably in an ice-chamber, and in a few hours the clear serum, free from corpuscles, will collect at the lower end, ready for dilution. If necessary, centrifugalise to obtain corpuscle-free serum. There are several methods of dilution used in practice, but broadly they are divisible into two, a rough-and-ready dilution and an exact measured dilution. 
The rough dilution is to take of the corpuscle-free serum to be examined one drop. Dilute it with nine parts of neutral bouillon. Mix on a slide or cover-glass a drop of this one-tenth dilutiou of serum one or more drops of typhoid broth cultivation of eighteen to twenty-four hours' growth. The serum and culture are thoroughly mixed together in the trough of a hollow-ground slide, and a single drop is taken, placed upon an ordinary clean slide, and a cover-glass superimposed; or the mixture may be made on the cover-glass and superimposed on the slide.

The measurement method is to dilute the serum by exact quantities, giving say, a 10 per cent., a 1 per cent., and a 0.1 per cent. dilution; or three mixtures containing respectively 50 per cent., 5 per cent., and 0.5 per cent. of serum. The 50 per cent. dilution is made by adding equal loopfuls of serum and of a typhoid broth culture on a slide or cover-slip. The 5 per cent. is made by diluting $10 \mathrm{c.m}$. (measured by graduated hæmatocytometer) of the serum, with $90 \mathrm{c.m}$. of the broth culture in a small sterilised test-tube. After thoroughly mixing, one loopful of this dilution (now 10 per cent.) is mixed with one of cultivation. The 0.5 per cent. is made by first diluting $10 \mathrm{c.m}$. of the 10 per cent. serum with 90 c.m. of sterile broth in a small test-tube, and then mixing equal loopfuls of this diluted serum and of the broth culture.

(c) The 'Typhoid Culture used should be one sub-cultured from a virulent culture, and should be a broth or agar culture of about eighteen to twentyfour hours; and, if preferred, may be filtered before use to remove any normally agglutinated masses of bacilli before commencing the test.

(d) The Reaction.-The reaction is positive if the bacilli have become grouped together tightly into clumps (agglutination), leaving the field between the clumps free from bacilli. Immotility will also be present. The reaction time is half-an-hour (see Plate 20). In his first experiments, Widal used a test-tube in the following manner:-The blood to be tested is diluted by one part of it being added to fifteen parts of broth in a test-tube. The mixture is inoculated with a drop of a typical B. typhosus culture. The tube is then incubated at $37^{\circ} \mathrm{C}$. for twenty-four hours, after which it is examined. If the reaction be positive, the broth appears comparatively clear, but at the bottom of the test-tube a more or less abundant sediment will be found. This is due to the clumps of bacilli having fallen owing to gravity. If, on the other hand, the reaction is negative, the broth will appear more or less uniformly turbid. This method is not as satisfactory as the one described.

Some bacteriologists use two dilutions, 1 in 20 and 1 in 40 , with a time limit of one hour for each case. The reactions obtained are interpreted as follows:-Where both dilutions show clumping and loss of motility at the end of the hour a diagnosis of "enteric fever" is made; but if the reaction is present only in the 1 in 20 dilution, a guarded opinion is given and the case stated to be "probably enteric fever" ; if both preparations are unchanged, the case is reported as "probably not enteric fever."

In the measured dilutions it may be said that if in half-an-hour there 
is a positive result with the 50 per cent., 5 per cent., and 0.5 per cent., the case is undoubtedly one of typhoid fever, and if in half-an-hour there is no reaction in all three, the result is definitely negative. Intervening degrees of reaction must each be judged on its own merits, and a subsequent examination made.

From the compilation of a large number of cases, the New York Health Board concludes that Widal's reaction is present in typhoid fever:-

From the fourth to seventh day in 70 per cent. of the cases.

From the eighth to fourteenth day in 80 per cent. of the cases.

During the third and fourth weeks in 90 per cent of the cases.

It is absent throughout in 5 to 10 per cent. of the cases.

Widal's reaction persists in the blood for months, or even years, but after three or four months is usually feeble.

\section{Differentiation of B. Typhosus}

On p. 48 will be found some of the chief distinguishing tests for the typhoid bacillus, which produces no gas in any media, does not coagulate milk, and stains by Gram's method. McConkey's test for B. coli may also be used. The medium which he makes use of is bile-saltlactose agar, which is prepared as follows: To 1000 c.c. of tap-water in a flask are added 2 per cent. of peptone, 0.5 per cent. of sodium taurocholate, and 1.5 per cent. of agar. The flask is autoclaved at $105^{\circ}$ to $110^{\circ} \mathrm{C}$. for one and a half hours. The mixture is then cooled, mixed with white of egg, and filtered; then 1 per cent. of lactose is added. The medium is distributed into test-tubes, 10 c.c. in each, which are sterilised by steaming for 15-20 minutes on each of three successive days. Plates are made and incubated at $42^{\circ} \mathrm{C}$. for forty-eight hours. There is a marked difference between the colonies of the organisms of the typhoid group and those of the colon group. Of the typhoid group the surface colonies are small, round, raised, and semi-transparent, the deep one lens-shaped, white, and opaque, the medium remaining clear. Of the colon group the surface colonies are roundish or irregular, with flattened tops, opaque, white, with a yellow or orange spot in the centre ; a few have a haze round them. The deep colonies all have a haze round them, and are lens-shaped and orange-white. The haze is due to precipitation of the sodium taurocholate by acid produced by fermentation of the lactose. McConkey and Hill * have further modified this method by the use of a bile-salt broth, composed as follows : Sodium taurocholate, 0.5 per cent.; glucose, 0.5 per cent.; peptone, 2 per cent. ; water, 100 c.c. The constituents are dissolved by heat, and the mixture is filtered. After filtration, sufficient neutral litmus is added to give a distinct colour, and the medium is then distributed into Durham's fermentation tubes. These are ordinary test-tubes containing a piece of light-glass tubing, about an inch in length, closed at the upper end.

* Thompson Yates Laboratories Report, 1901, vol. iv., part i., p. 151. 
This acts as a miniature gas-holder if fermentation of the medium occurs. The tubes are finally steamed for twenty minutes for each of three successive days. For the examination of water 1 c.c. is added to each tube, and several are inoculated and incubated at $42^{\circ} \mathrm{C}$. for fortyeight hours. If the colon bacillus be present, the medium becomes uniformly red, and is permeated with small gas bubbles, while the little tule is filled with gas. Subsequently, plates may be made from the tubes with the bile-salt agar medium.

\section{Examination of Malarial Blood}

1. Fresh Blood.-Thoroughly clean a cover-glass and wash a finger of the patient. Then prick the finger and squeeze out a drop of blood. This first drop of blood should be rejected. But when a second smaller drop appears, just touch its surface with the clean cover-glass. Now place the cover-glass on a clean slide, but do not exert any pressure upon it. Under the weight of the cover-glass the blood will now spread out into a very thin film. On examination under the microscope or by the naked eye it will be seen that the blood corpuscles have, roughly speaking, assumed the following zones:-

(a) A zone of scattered corpuscles immediately surrounding a central portion empty of corpuscles and devoid of colour. This "scattered" zone is composed of isolated, compressed, and much expanded corpuscles.

(b) Outside this first zone is one composed of corpuscles just touching each other by the margin. This has, therefore, been called the single layer zone.

(c) The third zone lies still further outside, and is composed of heaped-up corpuscles, overlapping each 'other and often in rouleaux. Beyond them is the area of free hæmoglobin, and valuable as enabling the observer to see if there are pigment parasites present in the blood.

The ordinary pigmented amoeboid forms of the parasite will generally be found in the single layer zone, whilst the flagellated bodies, if present, will be seen chiefly in zone $(c)$.

Pigmented leucocytes may appear anywhere in the field of the microscope.

The intra-corpuscular parasites may generally be detected because of their amoboid movements, pigmentation, feeble definition, and effect upon the corpuscle containing them.

2. Stained Preparation.-Whilst it is always best to examine malarial blood in a fresh state if possible, it is generally desirable to make more permanent preparations. This may be done as follows:-Make upon a clean slide a very thin film of the malarial blood (by drawing a needle or edge of cigarette paper over film). Allow it to dry in the air. Then wash the slide containing the dried film with weak acetic acid (say two or three drops of glacial acetic to an ounce of water) to clear the hæmoglobin. This may also be accomplished by dropping on the slide a little alcohol, which may be dried up in several minutes' time with 
filter-paper. After either of these methods has been adopted, stain the film for thirty seconds with a concentrated aqueous solution of methyleneblue (or the following solution for the same period of time: Borax, 5 parts; methylene-blue, 2 parts; water, 100 parts). Wash in water, dry with filter-paper, and mount in xylol-balsam under a cover-glass.* Löffler's blue or carbol thionin may be used. For double staining, Jenner's, Romanowsky's, or Leishman's stains may be used.

To demonstrate flagella, proceed as follows:-Take a piece of thick blotting-paper, $3 \times 1 \frac{1}{2}$ inches, with a round hole in the middle the size of an ordinary cover-glass. Moisten the blotting-paper and place it on a clean slide. Take a drop of the blood on another slide which has been breathed upon, and invert it on the blotting-paper (moist cell). In thirty minutes separate and dry the blood-film on both slides by gentle warming over the lamp. Fix with absolute alcohol, which may be allowed to evaporate or be dried with filter-paper. Wash with acetic acid (15 per cent.) to dissolve out the hæmoglobin, wash in water, and dry as before. Stain the dried film with carbol-fuchsin (20 per cent.) for six to eight hours. Wash and mount as before.

\section{Bacteriological Examination of Oysters}

Particular attention should be paid to the $(a)$ washings of the shell, (b) the liquor in the pallial cavity, and (c) the contents of the alimentary canal of the oyster. The two latter are the chief parts for examination in the ordinary course, and to obtain knowledge of the contained bacteria the method to adopt is as follows:-

Method.-Thoroughly cleanse the oyster shells by scrubbing with soap and water, rinse under the tap, and again in sterile water. Also the hands of the bacteriologist should be thoroughly cleansed and rinsed in antiseptic (e.g. 1-1000 corrosive sublimate) and sterile water. Now lay the oysters on the table with the flat shell uppermost, and open with a sterile knife. Pour the pallial liquor into a sterilised flask or capsule, and cut up the body of the oyster, adding the pieces to the liquor or to another flask. Add to the flasks of liquor and of oyster pieces, or to the one flask containing both sufficient sterile water (100 c.c. or 1000 c.c. as desired). The emulsion is now to be cultured as follows, adding in each case suitable quantities of the emulsion, e.g. (10 c.c. or 5 c.c. or 1 c.c. or 5 c.c.) :-Three tubes of broth (for indol formation); three tubes of phenolated broth (for B. coli and its allies, and also for secondary plate cultivation); three tubes of $\mathbf{M}^{\prime}$ Conkey medium, bile-salt-glucose peptone (for B. coli and its allies, coloration and gas); three tubes of freshly sterilised milk, heated after inoculation to $80^{\circ} \mathrm{C}$. for 15 minutes, and cultured anaërobically (for B. enteritidis sporogenes); three tubes of litmus milk (for acid and clotting); three gelatine "shake" cultures (for gas production); three plates of phenolated gelatine and three of ordinary gelatine; and three plates of agar for incubation at $37^{\circ} \mathrm{C}$. For quantitative estimation of colonies on the

* See also Tropical Diseases (Manson), p. 46. 
plates, it will, of course, be necessary to multiply up according to degree of dilution of the pallial liquor with sterile water in making the emulsion in the first instance.*

Examination of Urine.-Urine is examined in the same way as water or sewage effluent. Plates (gelatine and agar) and sub-cultures are made in the usual way. The urine should also be centrifugalised and the sediment carefully examined by microscope and culture, and if necessary inoculated into guinea-pigs. The organisms chiefly to be looked for are B. typhosus (in cases of typhoid fever), B. tuberculosis, septic organisms, and $B$. coli.

Examination of Ice-cream.-Ice-cream usually contains vast numbers of bacteria. It is examined in the same way as milk, and requires high dilution before examination.

Examination of Meat, Fish, etc.-Mince a portion of the unsound meat or potted meat or fish by aid of sterile scissors and forceps, and make an emulsion in broth in a flask at $42^{\circ} \mathrm{C}$. (for thirty minutes). Shake. Pipette off 10 c.c. of extract for inoculation of animals. Make plates and further tube cultures of the emulsion. Incubate duplicates anaërobically in Bulloch's apparatus. Feed animals on portions of the samples.

\section{Methods of Examination of Sewage and Sewage Effluents}

The sample of sewage or effluent to be examined must be collected in the same manner as in water.

1. Physical Examination.-Take note of quantity, colour, character and amount of deposit and suspended matter, reaction, temperature, bubbles of gas, etc.

2. Dilution.-This must be carried out as in the examination of milk, 500-1000 times.

3. Quantitative Examination.-Make plates on Petri dishes, gelatine for incubation at $20^{\circ} \mathrm{C}$, and agar at $37^{\circ} \mathrm{C}$. Sewage is rich in intestinal germs, most of which grow luxuriantly at blood-heat.

4. Qualitative Examination.-The three chief organisms of sewage are: (a) B. coli (p. 46), (b) B. enteritidis sporogenes (pp. 156 and 307), and (c) sevcage streptococcus (p. 155). It is necessary, therefore, to examine particularly for these organisms. It may also be necessary to estimate quantitatively for $B$. coli and $B$. enteritidis sporogenes. $\dot{\dagger}$

5. Subsidiary Differential Tests.-Inoculation of animals test; production of gas in gelatine "shake" cultures in twenty-four hours at $20^{\circ} \mathrm{C}$. ; acid clotting of litmus milk in twenty-four hours at $37^{\circ} \mathrm{C}$.; greenishyellow fluorescence in neutral-red broth cultures in twenty-four hours at $37^{\circ} \mathrm{C}$. ; the production of indol within five days at $37^{\circ} \mathrm{C}$. ; and the bile-salt broth test (growth, gas, and acid).

* A large number of methods and modes of experiment in the investigation of Oysters will be found in the appendices of the Fourth Report of Royal Commission on Sewage Disposal, 1904, vol. iii., pp. 191-309 (Houston).

$\dagger$ For methods, see Royal Commission on Sewage, Second Report, 1902, p. 140 (Houston). 


\section{To Clean Glass Apparatus}

Test-tubes and flasks may be washed in a bucket with hot water and soap powder or soda, or boiled in the same. They should then be cleaned with test-tube brushes and inverted for draining. Before use they must be sterilised. Pipettes may be treated in the same way, and then rinsed through with rectified spirit, and sterilised in the hot-air oven. When test-tubes and pipettes are infected, they should be treated in a similar manner, and also placed in strong disinfectant or nitric acid (5 per cent.). Greasy slides should be placed in alcohol and acid ( 5 per cent. $\mathrm{HCl}$ or $\mathrm{H}_{2} \mathrm{SO}_{4}$ ) for several hours, and then rinsed in water. Greasy cover-slips may be treated in the same way, or boiled in chromic acid (10 per cent.) and washed in acid alcohol and water.

\section{Choice of Medium}

This must be left very largely to individual experience and the objects of the investigation. In a general way the constituents of the various media described indicate the purposes to be obtained. The general standard liquid media are bouillon and milk, the solid media are gelatine (for room temperature cultivation) and agar (for blood-heat). In tropical countries a combination of the two may be used. Further, just as gelatine is a solid bouillon, so gelatinised milk may be used when a solid milk medium is required. For anaërobes glucose and formate media are commonly used. There are, of course, various media used for different species of organisms. For the streptothrix group including $B$. tuberculosis, glycerine media and potato are used. To isolate the $B$. typhosus, carbolised media and Elsner are taken. Chromogenic bacteria nearly always grow well on potato. The use of litmus milk, beer wort, wort gelatine, milk agar, etc., is sufficiently designated in the names of the media.

Preservation of Media.-Media may be kept in good condition for. months if a few simple precautions are borne in mind. The tubes or flasks containing the medium must be effectually sealed, either with caps, corks, or paraffin. The store of media must then be kept in a closed metal box, and in a cool dark place.

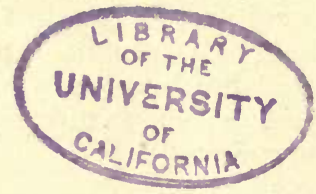




\section{IN D E X}

Anscess formation, 311

bacteria of, 312-313

Acetous fermentation, 102

Acid-fast bacteria, 358-369

classification of, 359

of human origin, 360

of butter and milk, 361

of grass and manure, 364

differential diagnosis, 365

streptothrix, 367

Actinomycosis, 321

Aërobic organisms, 23

Agar, 16

Air, bacteriology of, 76-91

dust and bacteria, 76

examination of, 73-75

moisture and bacteria, 79

of sewers, 82

currents and bacteria, 84

expired, 79-81

of workshops, 85

bacteria and gravity, 83

of bakehouses, 86

standard of bacteria in, 79, 91

of railway tubes, 88,90

pathogenic bacteria in, 91

of House of Commons, 88

passages, bacteria in, 80

Alcohol, formation of, 96

Alcoholic fermentation, 96

Alexines, 412

Alformant lamp for disinfection, 443

Algæ in water, 35

Ammoniacal fermentation, 110

Amylolytic ferments, 95

Anaërobic organisms, 23

methods of culture, 117-119

in hydrogen, 117

in glucose agar, 118

in Fränkel's tube, 118

in Buchner's tube, 118 489
Andrewes on air of central railway tube, 90

Aniline dyes, 455, 458

Antagonism of organisms, 30

Antibiosis, 29

Anthrax, 315-319

clinical characters of, 315

pathology of, 316

spores of, 316

bacillus of, 316

in sewage, 177

channels of infection, 317

Antiseptics, 433

definition of, 433

some of the chief, $439-444$

Antitoxins, 405

preparation of, 425

use of, 429

unit of, 428

effect of, 430

Appendix on technique, 453

Arthrospores, 12, 13

Artificial purification of water, $64-70$

Ascospores, 14, 98

Asiatic cholera, 384

Association of organisms, 29

Attenuation of virulence, 31

Autoclave, 25

Bacillus, definition of, 88

aceti, 102

acidi lactici, 105, 106, 196

anthracis, 316

aquatilis, 45

botulinus, 269

butyricus, 108-110

capillareus, 155

cloacce fluorescens, 155

coli communis, 46-51, 56-60, 154, 466, 477

tests for, $48-51,466$ 
Bacillus-

diphtheria, 288

enteritidis of Gaertner, 269

enteritidis sporogenes, $45,154,156$, 307

fuorescens liquefaciens, 45

fluorescens non-liquefaciens, 45

fluorescens stercoralis, 155

fusiformis, 155

friburgensis, Nos. 1 and 2, 363

of cholera, 385

of Binot, 364

of diarrhœea, 305

of dysentery, 403

of Grassberger, 364

of influenza, 321

laclis erythrogenes, 45, 200, 201

lactis pituitosi, 200

lactis viscosus, 200

liquefaciens, 45

mallei, 323

membranous patulus, 155

of leprosy, 398

phlei, 364

of glanders (mallei), 323

mesentericus, 45

of Mœller, 362

mycoides, 45

of malignant œdema, 144

No. 41,245

of Rabinowitsch, 361

of symptomatic anthrax, 142

of plague, 392

prodigiosus, 200

pseudo-tuberculosis, 358

pyo-cyaneus, 157

pyogenes cloacinus, 155

radicicola, 134

saponacei, 200

smegmatis, 360

subtilis, 45

subtilissimus, 155

synxanthus, 201

of tetanus, 141, 481

of tubercle, 327,337

typhosus, 48, 301

of yellow fever, 400

Bacteria, action of, 285

composition of, 9

Bacteria, in sewage, 151-158

and wheat supply, 131

and fixation of nitrogen, 131-139

in cheese-making, 241

in the dairy, 178-251

products of, 406

and disease, 280

the higher, 6,8

in soil, 116

Bacterial action, 285
Bacterial action-

diseases of plants, 32

treatment of sewage, 162-177

Bacterio-purpurin, 10

Bacteroids, 136

Bakehouses, bacteria in, 86

Ballard on soil and disease, 145

on epidemic diarrhœa, 304,308

Beer diseases, 110-113

Berkefeld filter, 71

Beri-beri, 404

Biogenesis, 2

Biology of bacteria, 1

Bitter fermentation, 112

Blood serum, 16

Blue milk, 201

Booker on bacteria of epidemic diarrhœa, 306

Boracic acid, 441

Boyce and others on bacteriological examination of water, 470-473

Bread, bacteria in, 276-279

sour, 277

mouldy, 278

sticky, 278

red, 279

Broth, 16

Brownian movement, 11

Bubonic plague, 388-396

Buchner's tube, 118, 466, 478

Butter bacilli, the, 361-364

Butter, bacteria in, 241

making, 242-246

examination of, 479

bacterial flavouring of, 242

Butyric fermentation, 107

Carbol-Fuchisin, 455,459

Carbol-gelatine, 16

Carbolic acid as a germicide, 441

Carbonic acid gas and bacteria, 85-91

Caries, dental, 80

Carson's dairy farm, 232

Cellulose, 10

Chamber, moist, 464

Channels of infection in disease, 284 abnormal, 251

Cheese, bacteria in, 241

making, 246

examination of, 480

poisonous, 251

Chemical products of bacteria, 406 substances as disinfectants, 439-444 
Chemical products of bacteria-

and bacteriological examination of water compared, 55

tests for nitrification, 128-132

Chemiotaxis, 11

Chloride of lime as a germicide, 440

Cholera, 384

bacillus of, 385

diagnosis of, 387

and filtration, 66

and milk, 223

Chromogenic bacteria, 406

Clams and bacterial infection, 266

Clark's process, 64

Classification, 5

Clowes on bacterial treatment of sewage, 164

Coccus, definition of, 6

Cockles and bacterial infection, 263-266

Collingridge on ice-cream poisoning, 274

Colon bacillus, see $B$. coli communis, 46

Comma bacillus, 385

Commensalism, 133

Commissions on food preservatives, 230 leprosy, 399

plague, 394, 425

sewage disposal, 49, 157, 161, 162, $172,177,261,262,469,485$

tuberculosis (1898) 270, (1895) 339, 343, (1901) 345

vaccination, 417

Composition of bacteria, 9

Conditions affecting bacteria in water, 60

Contact beds for sewage, 169

Contagion, 284

Contamination, organisms of, 56

Corrosive sublimate as disinfectant, 440

Counter (Wolfhügel), 465

Cover-glass preparations, 455

Cream, bacteria in, 240

Crenothrix polyspora, 36

Cresol as a germicide, 441

Cultivation beds, 169

Culture media, 16

Cultures, anaërobic, 117

hanging drop, 455

plate, 453

pure, 456

shake, 467

sub-culture of, 456

Decomposition bacteria, 123
Delépine on bacteria in milk, 192-194

Denitrifying bacteria, 123

Dental caries, 80

Deodorants, 433

Desiccation, 18

Diagnosis, 463

Diarrhœea of infants, 304

and milk, 223

conditions favourable to, 304, 308

and soil, 308

bacteria of, 305

Diphtheria, 287-296

antitoxin of, $425-431$

bacillus of, 288

bacillus in throat, 293

toxins of, 426

prevention of, 294

and milk supply, 211

and school influence, 292

pseudo-bacillus of, 295

diagnosis of, 290,481

Diplococcus, definition of, 7

of gonorrhœa, 314

in pneumonia, 320

Directions for estimating disinfectants, etc. , 434

Disease, production of, $280-287$

Diseases of beer, 110-113

of plants, 32

conveyed by water, 53

and soil, 145

Disinfectants, 439

Disinfection, 432-451

means of, 435

by heat, 436

by chemicals, $439-444$

of a room, 444

of walls, 445

of bedding, 445

of garments, 445

of excreta, 445

of wounds, 445

of hands, 446

of books, etc., 446

of stables, vans, etc., 446

after phthisis, 447

after small-pox, 449

after scarlet fever, 449

after diphtheria, 450

after typhoid, 450

after cholera, 450

after plague, 450

standards of, 434

Domestic purification of water, 70

Doriga on rats and plague infection, 390

Dunham's solution, 388 


\section{INDEX}

\section{Dysentery, 404}

EARTH temperatures and disease, 145, 308

Effluents, 158, 175

Elsner's medium, 467

Endospores, 12, 14

Enteric fever (see typhoid), 298-304

Enzymes, 94

Equifex disinfector, 438

Examination, bacteriological-technique of, $453-463$

air, 73

cholera, 387

diphtheria, 481

fish, 485

ice-cream, 485

leprosy, 398

malarial blood, 485

meat, 485

milk, $473-481$

oysters, 484

sewage, 485

soil, 117

tetanus, 481

typhoid, 482

tubercle, 482

urine, 485

water, $463-473$

yeasts, 97

Extracellular poisons, 408

External conditions, effect of, on bacteria, 15

Feruentation, 92-115

kinds of, 94

acetous, 102

alcoholic, 96-102, 198

ammoniacal, 110

butyric, 107, 197

lactic acid, 104, 196

Ferments, organised, 94

unorganised, 94

chromogenic, 200

curdling, 104, 197

bitter, 112-199

slimy, 199

soapy, 200

Films, 100

Filters, domestic, 71

sterilisation of, 72

Filtration of milk, 228-230

method of air-examination, 74

Filtration of water, $65-72$

Filter-beds, 65

Firth and Horrocks on pathogenic bacteria in soil, 148
Fission, 12

Fixing specimens, 475

Flagella, 11 staining, 461

Food, bacteria in, 178, 253

Formaldehyde and formalin, 443

Forms of bacteria, 6

Foulerton on pollution of water, 63 on bacteria in oysters, 260

on streptothrix group, 367

Fowler on bacterial treatment of sewage, 174

Fractional sterilisation, 24

Fränkel's pneumococcus, 320

Frankland on bacteria in water, 37 on filtration of water, 65

Freezing, effect of, on bacteria, 18

Friedländer's pneumo-bacillus, 320

$\mathrm{G}_{\text {AS, }}$, production of, 406

Gathering-ground, 34

Gelatine, 16

carbol, 466

liquefaction of, 457

Gemmation, 98

Gentian-violet, aniline, 455

Germicidal temperatures, 23-25

Germicides, 439-444

Gilbert on nitrification, 134

Ginger-beer plant, 137

Glanders, 323

Gonorrhœea, 314

Gram's method of staining, 458

Nicolle's modification of, 459

Gravity, influence on bacteria, 83

Gypsum block, 98

HжмосутоzoA, 372

Hæmamœba, 372

Haldane on ventilation of workshops, 85

Hanging-drop cultivations, 455

Hansen's method of dilution, 99

Heat as steriliser, 23-25

Heredity, 284

Hesse's method of air examination, 74

Hewlett and others on bacteriological examination of water, $470-473$

High yeasts, 101

Higher bacteria, 6, 8

Horrocks and Firth on soil and disease, 148 
Horrocks on classification of water bacteria, 44

Hot-air steriliser, 24, 25

Houston on streptococci in water, 51 on pathogenic bacteria in soil, 148 on sewage bacteria, $153,157,175,177$ on bacteria in oysters, 261

Hydrogen cultivation, 23

Hydrophobia, treatment of, 419

IcE, bacteria in, 274

Ice-cream, bacteria in, 272-274 manufacture of, 272 examination of, 485

Immunity, 405-431 acquired, 413 active, 412

Ehrlich's side chain theory, 414 artificial, 412

general principles, 405-412

natural, 413

passive, 413

theories of, 413

in small-pox, 415

in rabies, 419

in typhoid, 424

in plague, 424

in diphtheria, 425

in cholera, 423

Incubators, 17

Indol, formation of, 467 testing for, 467

Industries and bacteria, 113-115

Infection, channels of, 284

Influenza, 321

Inoculation, 456

Interpretations of bacteriology, 55

Intracellular poisons, 31

Inversive ferments, 95

Involution forms, 9

JonDAN's classification of water bacteria, 44

KePHIR, 136, 198

Kipp's apparatus for producing hydrogen, 23

Klebs-Löffler bacillus, 288

Klein on bacteria in oysters, 259 on bacteria in cockles, 263

on bacillus enteritidis sporogenes, 156 , 307

Koch's plate method, 453

steam steriliser, 24,25

tubercle bacillus, 327 et seq.

postulates, 281
Koch on filtration of water, 66

on inter-communicability of tuberculosis, 339 et seq.

comma bacillus, 385

bacillus of tubercle, 327,337

views on tuberculosis, $338-346$

Koumiss, 198

LACrIC acid fermentation, 104, 196

Leguminosæ, fixation of nitrogen by, 131

Leprosy, 396-400

history of, 396

forms of, 397

bacillus of, 398

Light, influence upon bacteria, 18-22

Lingner's apparatus for disinfection, 443

Liquefaction of gelatine, 457

Liquid hydrogen and bacteria, 18

Lloyd on Cheddar cheese-making, 249

Low yeasts, 101

Lymph, glycerinated calf, 416

Lyon's, Washington, disinfector, 437

Maceration industries, 113

Malaria, 371-384

kinds of, 373,375

cycle of Golgi, 380

parasites in, 372

microgametocytes of, 375

macrogametocytes of, 376

and mosquitoes, 376

anopheles of, 377

culex and, 378

examination of blood, 485

preventive measures, $382-384$

mosquito breeding, 382

destruction of mosquitoes, 383

bites of mosquitoes, 383

quinine, 384

Malignant œdema, 144

bacillus of, 144

Mallein, 324

Malta fever, 402

Manchester sewage treatment, 170-174

Manson on malaria, 375 et seq. on plague, 390

Martin on soil and disease, 147 on tuberculosis, 341-342

Mastitis, 184

M'Conkey's bile-salt method, 467,484

Meat and bacterial infection, 267-272 examination of, 485

poisoning bacteria, 269

tuberculous, 270

decomposed, 270

Media, culture, 16 
Merismopedia, 8

Metabiosis, 29

Metachromatic granules, 10

Metchnikoff on phagocytosis, 414

Methods of examination, 453

Micrococcus, definition of, 6 aquatilis, Freudenreichii, 199 gonorrhace, 314

tetragonus, 313

viscosus, 199

Milk, bacteriology of, 178-251 composition of, 195

incubation period for bacteria in, 179 sources of pollution, 181-184

number of bacteria in, 184-194

influence of time and temperature upon bacteria in, 185-194

fermentation bacteria in, 196-202

kinds of bacteria in, 194

disease-producing power of, 202

lactic acid fermentation of, 196

butyric fermentation of, 197

coagulation fermentation of, 197

alcoholic fermentation of, 198

anomalous fermen'ation of, 199

and tuberculosis, 203-207

and typhoid, 207-211

and cholera, 223

and epidemic diarrhoea, 223-226

and diphtheria, 211-214

and scarlet fever, 214-217

and sore-throat illnesses, $219-223$

and thrush, 218

character of milk-borne disease, 218 prevention of milk-borne disease, 226

method of protection, 227

control of milk supply, 227-240

filtration of, 230

refrigeration of, 228

straining of, 228

sterilisation of, 231

of Liverpool, 205

pasteurisation of, 231

results of, 235

summary of control, 236

products, bacteria in, 240-251

examination of, 473-481

specialised milks, 237

and economic bacteria, 240-249

and municipal depôts, 237

chromogenic fermentation of, 200

Miquel's method of air examination, 74

Modes of bacterial action, 25

Mohler on tuberculosis of the udder, 203

Moist chamber, 464

Moisture necessary for bacteria, 18
Morphology of bacteria, 6

Motility, 11

Mosquitoes and malaria, 376

Mycoderma aceti, 103

Mycoprotein, 9

Nasal passages, bacteria in, 80

Natural purification of water, 60-64

Needles, platinum, 17

New soil science, 138

Newsholme on conditions favourable to diphtheria, 291

on causation of epidemic diarrhœen, 309

on disinfection after phthisis, 447

Nitric organism, 128

Nitrification, 125-131

chemistry of, 125

stages in, 129

bacteria of, 129

Nitrifying organisms, cultivation of, $12 \%$, 128

Nitrogen, fixation of, 131-139

Nitrogen-fixing bacteria, 131

Nitrous organism, 126

Niven on disinfection after phthisis, $44 \tau$

Nodules on roots, bacteria in, 133

Ocean bacteria, 36

Oidium albicans, 218

Oxygen necessary for bacteria, 23

Oysters and typhoid fever, 253-263

poisoning, symptoms of, 256

infection, 257

and disease, prevention of, 262

examination of, 484

PAKES' formate broth method, 467

Paraform for disinfection, 443

Parasitism, 25

Parietti's method, 466

Pasteur on fermentation, 93

Pasteur's treatment of rabies, 419

Pasteur filter, 71

Pasteurisation of milk, 231-236

Pathogenic bacteria, in soil, 140 in water, 53

Perlsucht, 333

Petri dishes, 453

Phagocytosis, 413-414

Phosphorescence, 22

Pigment, formation of, 406

Place of bacteria in nature, 4 
Plague, 388-396

varieties of, 388

symptoms of, 388

and rats, 390

distribution of, 389

bacillus of, 392

administrative control of, 394

vaccination for, 424

diagnosis of, 396

Plant diseases, 32

Plasmolysis, 9

Plate cultures, 453

Platinum needles, 17

Pleomorphism, 9

Pneumonia, 319 bacteria of, 320

Pneumo-bacillus, 320

Pneumococcus, 320

Polymorphism, 9

Postulates, Koch's, 281

Potato medium, 16

Pouchet's aëroscope, 74

Power on milk-borne scarlet fever, 214

Products of bacteria, 406

Proteolytic ferments, 95

Proteus family, 154, 45

cloacinus, 154

vulgaris, 154

Zenkeri, 154

mirabilis, 154

Pseudo-diphtheria bacillus, 295

Pseudo-tuberculosis, 355-358

Purification of water, $60-72$ natural, 60-64

domestic, 70

artificial, $64-70$

Pus, 311

Pyocyanin, 313

Pyoxanthose, 313

Quantivative standard of water bacteria, 42

air bacteria, 77

milk bacteria, 191

soil bacteria, 116

Quarter-evil, 142

clinical characters, 143

RAliss, treatment of, 419

forms of, 420

pathology of, 420

results of treatment, 423

Red milk, 200

Reproduction of bacteria, modes of, 11

Retting, 113
Rivers, natural purification of, 38, 60-64

Robertson on soil and typhoid, 147

Ross on malaria, 380

Rotch's specialised milk, 239

Russel on butter-making, 244

on cheese-making, 247

Saccharomycetes, biology of, 97

methods of examination, 99

anomalous, 99

apiculatus, 102

aquifolii, 102

cerevisice, 101

conglomeratus, 102

ellipsoideus 1., 101, 102

ellipsoideus 1I., 102

exiguus, 102

Hansenii, 102

illicis, 102

Ludroigii, 99

mycoderma, 102

pastorianus 1., 102

pastorianus 11., 102

pastorianus, 111., 102

pyriformis, 102

Sand filtration of water, 65

Saprophytes, 25

Sarcina, 8

Savage on bacteria of made soil, 149

Scarlet fever, 296

milk and, 214

bacteria of, 296

streptococcus in, 297

Sedgwick's method of air analysis, 75

Seed and soil, 26

Sedimentation, 62, 64

Septic processes, 311

tank, 165-169, 174

Sewage, organisms in, 151-158

bacterial treatment of, 162-17 7

constitution of, 151

examination of, 154,485

organic matter in, 152

inorganic matter in, 152

number of bacteria in, 153

kinds of bacteria in, 154

spores in, 153

streptococcus of, 155

pathogenic bacteria in, 155

nitrification and denitrification in, $157,158,166$

aërobic and anaërobic bacteria in, $157,158,166$

disposal of, 159

chemical treatment of, 160

biological treatment of, 158, 160-177 
Sewage-

irrigation of, 161

intermittent filtration, 160

effluents and pathogenic organisms, 175

London treatment of, 164

Manchester treatment of, 170

Sutton treatment of, 169

Exeter treatment of, 165

septic tank method of treating, 165169

contact bacteria beds method, 169

Leicester treatment of, 173

effect of bacterial treatment of, 175

Sewer air, 82

and toxicity of bacteria, 83

Shake cultures, 467

Shell-fish and bacteria, 253-266

Sleeping sickness, 403

Small-pox, 416

Smith, Graham, on air of House of Commons, 88

Smith, Horton, on typhoid urine, 300

Soil, bacteriology of, 116-150

bacteria in, 116

composition of, 119-122

denitrification in, 123

examination of, 117-119

and typhoid fever, 146-148

and tetanus, 140

polluted, 149

kinds of bacteria in, 119

nitrification in, 125-131

nitrogen-fixing bacteria in, 131-139

and its relation to disease, 145

pathogenic bacteria in, 140

classification of bacteria from, 119, 120

symbiosis in, 132, 136

Sorensen's dairy farm at York, 228

Species of bacteria, 28

Specificity of bacteria, 28

Spirillum, definition of, 88

of cholera, 385

of Obermeier, 8

Spontaneous generation, 3

Sponges, 114

Spores, kinds of, 12

resistance of, 12-15

staining of, 462

of yeasts, $98-99$

Staining methods, 455-463

Standard of sterilisation, 24

Staphylococcus, 8, 311

cereus albus, 311
Staphylococcus-

pyogenes aureus, 312

pyogenes albus and citrens, 311

Steatolytic ferments, 95

Steam, as a disinfector, $436-438$

disinfectors, 437

steriliser, 24

saturated, 436

superheated, 436

current, 437

Sterilisation, 23-25

methods of, 23-25

Streptococcus, 7

in milk, 297

in water, 51

in sewage, 155

of scarlet fever, 297

pyogenes, 312

conglomeratus, 297

Hollandicus, 199

Streptothrix group, 367

actinomyces, 321

hominis, 368

eppinger, etc., 369

luteola, 367

Structure of bacteria, 6

Sub-cultures, 456

Sulphurous acid as a germicide, 441

Suppuration, 311

Swine fever, 272

Swithinbank and author on milk, $188-190$

Symbiosis, 29, 132, 136

Symptomatic anthrax, 142

TABLE of economic bacteria in soil, 120

Temperature, influence of, on bacteria, 17

T'etanus, 140-142

toxin of, 141

bacillus of, 141,481

Thermophilic bacteria, 17

Thresh's disinfector, 438

Thresh on bacteriological examination of water, 469

Thrush, 218

Tobacco-curing, 114

Toxins, 406-412

Tropical diseases, 370-404

Tuberculin, 347

Tuberculosis, 325-358

pathology of, 325

varieties of, 326

history of, 326

conveyed by the air, 79,331

and the milk supply, 335 
Tuberculosis-

of the udder, 334

giant cells in, 330

bacillus of, 327,337

bovine, 333,337

diagnosis of bovine, 346

cultivation of bacillus of, 328

spores of, 329

relation of bacillus to disease, 330

temperature for growth, 329

toxins, 333

of horse and dog, 350

of cold-blooded animals, 351

of animals, 349-351

of pig, 349

of birds, 350

of sheep, 349

prevention of, 352-357

disinfection in cases of, 447

decline of, 352

and overcrowding, 355

channels of infection in, 331

inter-communicability of, 338-346

and house influence, 356

pseudo-, 355-358

Typhoid fever, 298-304

bacillus of, 301

effect of light on bacillus, 20

pathology, 298

bacillus compared with $\boldsymbol{B}$. coli, 48

bacillus in sewage, 175

bacillus in drinking-water, 303

tests for bacillus of, $48,468,482,484$

and soil, 145

conveyed by the air, 78, 81

and milk supply, 207-211

Tyrotoxicon, 251

UDDEn tuberculosis, 203, 334

Unit of antitoxin, 428

Urea, 120

Vaccination, 415-419

effect of, 417

Vaccines, 415-425

plague, 424
Vaccines-

cholera, 423

small-pox, 415

Vaccinia, 415

Vacuolation, 9

Variolation, 415

Virulence, attenuation of, 31

WARRINGTON on nitrification, 123 et seq.

Washington Lyon disinfector, 437

Water, bacteria, classification of, 44 bacteria in, 33,44 collection of samples, 33

number of bacteria in, $34,36,42$, 56

examination of, $463-473$

organisms of contamination, 56

pathogenic organisms in, 53,56

multiplication of bacteria in, 34

natural purification of, 60-64

river, bacteria in, $36-42$

artificial purification of, $64-70$

B. coli in, 46-60

filtration of, 65

ordinary bacteria in, 45

domestic purification of, 70

London, bacteria in, 38-42

quantitative standard in, 42

quality of, 44-60

sewage bacteria in, 45

pollution of, $54-60$

sea, bacteria in, 36

Watercress, bacteria of, 279

Wheat supply and bacteria, 131

Widal reaction, 482

Wolfhügel's counter, 465

Wool-sorters' disease, 318

Yeasts, 14, 97-102

Yellow fever, 400-402

bacteria in, 400

and mosquitoes, 402

Yellow milk, 201

Ziehl-NeELSEN stain, 459

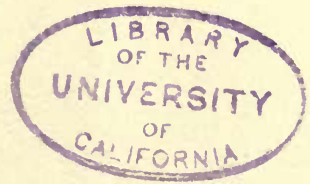


PRINTED BY

OLIVER AND BOYD

EDINBURGH 





